\title{
Monograph
}

urn:1sid:zoobank.org:pub:89FA8DDF-F4B9-417A-A5AF-B2BC9660E024

\section{The 'red-tailed' Lasioglossum (Dialictus) (Hymenoptera: Halictidae) of the western Nearctic}

\author{
Joel GARDNER ${ }^{1, *} \&$ Jason GIBBS ${ }^{2}$ \\ ${ }^{1,2}$ Department of Entomology, University of Manitoba, 12 Dafoe Rd., \\ Winnipeg, Manitoba, R3T 2N2, Canada. \\ ${ }^{*}$ Corresponding author: clickbeetle3364@gmail.com \\ 2Email: jason.gibbs@umanitoba.ca \\ ${ }^{1}$ urn:1sid:zoobank.org:author:AA15FE6E-921A-46D6-BC67-88E067F283D9 \\ ${ }^{2}$ urn:1sid:zoobank.org:author:BA42A49F-3EBC-4679-8F03-A58E798106B1 \\ 1 (1) https://orcid.org/0000-0003-1843-466X \\ 2 (1) https://orcid.org/0000-0002-4945-5423
}

\begin{abstract}
Sweat bees in the subgenus Lasioglossum (Dialictus) are one of the most diverse and abundant bee taxa, and a critically important component of bee biodiversity. Yet, the most basic taxonomic knowledge of these bees is lacking in many regions. As a step towards a better understanding of the L. (Dialictus) of the western Nearctic region, a revision of the 'red-tailed' L. (Dialictus) species was completed. Thirty-six species were revised, 20 of which are described as new, and two names are treated as junior subjective synonyms. Descriptions, figures, distribution maps, floral hosts, and keys to species for females and males are provided. The following 20 species are described as new: Lasioglossum (Dialictus) arenisaltans sp. nov., L. (D.) argammon sp. nov., L. (D.) austerum sp. nov., L. (D.) cactorum sp. nov., L. (D.) cembrilacus sp. nov., L. (D.) clastipedion sp. nov., L. (D.) clavicorne sp. nov., L. (D.) decorum sp. nov., L. (D.) festinum sp. nov., L. (D.) imbriumbrae sp. nov., L. (D.) julipile sp. nov., L.(D.) lilianae sp.nov., L. (D.) meteorum sp. nov., L. (D.) miltolepoides sp. nov., L. (D.) minckleyi sp. nov., L. (D.) perditum sp. nov., L. (D.) rufornatum sp. nov., L. (D.) spivakae sp. nov., L. (D.) tessellatosum sp. nov., and $L$. (D.) torrens sp. nov. Previously unknown males of $L$. (D.) clematisellum (Cockerell, 1904), L. (D.) droegei Gibbs, 2009, L. (D.) kunzei (Cockerell, 1898), and L. (D.) pallidellum (Ellis, 1914) are described and figured for the first time. Lasioglossum (Dialictus) clarissimum (Ellis, 1914) (=Halictus clarissimus Ellis, 1914) and L. (D.) perexiguum (Sandhouse, 1924) (= Halictus (Chloralictus) perexiguus Sandhouse, 1924) are new subjective junior synonyms of L. mesillense (Cockerell, 1898) (= Halictus nymphalis var. mesillensis Cockerell, 1898). A lectotype specimen is newly designated for L. mesillense, for which the location of the type material has not previously been known. The following five new records for Mexico are reported: L. clematisellum, L. droegei, L. eophilus (Ellis, 1914), L. kunzei, and L. pallidellum.
\end{abstract}

Keywords. Apoidea, DNA barcodes, new species, revision, taxonomy.

Gardner J. \& Gibbs J. 2020. The 'red-tailed' Lasioglossum (Dialictus) (Hymenoptera: Halictidae) of the western Nearctic. European Journal of Taxonomy 725: 1-242. https://doi.org/10.5852/ejt.2020.725.1167 


\section{Introduction}

Sweat bees (Hymenoptera: Halictidae) are one of the most abundant and species-rich components of native bee communities. Halictidae are commonly collected by any method appropriate for bees (Morse 1960; Sakagami et al. 1967; Allred 1969; Tommasi et al. 2004; Batáry et al. 2010; Smith et al. 2012), but especially with pan traps or 'bee bowls' (Toler et al. 2005; Campbell et al. 2007; Gonçalves \& Oliviera 2013; Buri et al. 2014; Geroff et al. 2014; Joshi et al. 2015; Griffin et al. 2017; Onuferko et al. 2017; Rhoades et al. 2017b; Lettow et al. 2018; Portman et al. 2020), in which they can exceed 90\% of individuals (Droege et al. 2010; Gonzalez et al. 2020).

Lasioglossum Curtis, 1833 is the largest genus of bees, and L. (Dialictus) Robertson, 1902 (sensu Gibbs 2018a) is its largest subgenus (Ascher \& Pickering 2020). There are 385 species of L. (Dialictus) known worldwide, 257 of these from North America (Ascher \& Pickering 2020). Most recent studies of bee biodiversity involve pan-trapping, so Halictidae often dominate bee collections, and L. (Dialictus) often dominate the Halictidae (Campbell et al. 2007; Patenaude 2007; Minckley 2008).

Although L. (Dialictus) are an unavoidable and critical part to Nearctic bee biodiversity, thorough revisions only exist for Canada (Gibbs 2010) and the eastern United States (Gibbs 2011). In the western United States and northern Mexico, the focal area of this work (hereafter generally referred to as 'western'), no comprehensive revision has been completed. The only substantial taxonomic works for western L. (Dialictus) are Gibbs (2010), which includes western Canada and is applicable to some of the northwestern United States, or Sandhouse (1924), which does not cover a well-defined area but includes a large number of western species. P.H. Timberlake wrote keys to the L. (Dialictus) of California, the Rocky Mountains, and perhaps other regions, including many new species descriptions. However, these were never published, and some of his original manuscripts may have been lost.

DNA barcodes are available for many western species (Gibbs 2010, 2018b), and could be used for identification even without keys. However, many of these species are undescribed and/or species whose limits have not been fully resolved. In addition, recent and rapid diversification in Lasioglossum (Gibbs et al. 2012b; Grab et al. 2019) confounds analysis of DNA barcodes in certain species complexes (Gibbs $2018 b$ ). For this reason, Gibbs (2018b) recommends using morphology and geography alongside DNA barcode-based identifications.

Due to these difficulties, numerous studies in the western Nearctic are necessarily published in which significant numbers of $L$. (Dialictus) specimens are not identified to species (Ayala et al. 1993; Frankie et al. 2005; Minckley 2008; Tepedino et al. 2008; Hung et al. 2017; Kuhlman \& Burrows 2017; Rhoades et al. 2017a; Messinger-Carril et al. 2018; Ballare et al. 2019; Galbraith et al. 2019). This weakens the conclusions of such studies and makes comparison with other studies impossible without access to voucher specimens.

Lasioglossum is one of the most interesting taxa for studying the evolution of social behaviour (Michener 1974; Schwarz et al. 2007) because many closely-related species span a broad spectrum of social complexity (Plateaux-Quénu 1959; Michener 1960; Sakagami \& Munakata 1972; Richards 2000) with multiple reversals to solitary behaviour (Danforth et al. 2003; Gibbs et al. 2012b). The subgenus Dialictus spans nearly the full behavioural range known in Lasioglossum as a whole, from solitary (Michener \& Lange 1958; Knerer 1969; Packer 1994), semisocial (Eickwort 1986), to eusocial (Michener \& Lange 1958; Michener \& Wille 1961; Batra 1964, 1987; Michener 1966; Stockhammer 1967; Wille \& Orozco 1970; Packer 1992), and socially parasitic (Wcislo 1997). Much can be learned from L. (Dialictus) alone, but little is known of the nesting or social biology of western species, largely due to a lack of basic taxonomic studies enabling separation of species. Work on eastern species did not begin in earnest until Mitchell (1960) revised the bees of the eastern United States. 
The fact that we know so little about such a large group of bees is concerning, given the importance of bees as pollinators for crops (Klein et al. 2007; Reilly et al. 2020) and wildflowers (Ollerton et al. 2011). It is especially concerning that we know so little about the sweat bees in this particular region, because the deserts of the southwestern United States and northern Mexico are a major world bee biodiversity hotspot (Michener 1979). The range of habitats in the western Nearctic, from deserts to forests over a wide range of elevations, could allow for many types of behaviour. Up to ten species are still only known from the type specimen. It is imperative that a revision of the western L. (Dialictus) is completed, so that knowledge of the western fauna can be brought up to the same level and the same standards as in the rest of the Nearctic.

\section{The 'red-tailed' Lasioglossum (Dialictus)}

This work is part of ongoing research aimed at a comprehensive revision of the western L. (Dialictus). We have chosen to begin with an informal grouping sometimes called the 'red-tailed' L. (Dialictus); that is, those species in which the metasoma attains a red or orange colour, as opposed to the usual black, brown, or metallic green. The function of red colouration in the metasoma is unknown, but it seems to be correlated with hot, dry habitats, especially sandy ones. Some plausible hypotheses are that it is related to thermoregulation or camouflage, but this has not been investigated.

Although no comprehensive species-level phylogeny of Lasioglossum or L. (Dialictus) has been made, it is clear from partial phylogenies (Gibbs et al. 2012a) and preliminary results from DNA barcodes (this paper) that red/orange colouration of the metasoma has evolved independently numerous times in L. (Dialictus) and other subgenera of Lasioglossum, including Austrevylaeus Michener, 1965 (Michener 1965); Callalictus Michener, 1965 (Michener 1965); Capalictus Pauly, Gibbs \& Kuhlmann, 2012 (Pauly et al. 2012); Chilalictus Michener, 1965 (Michener 1965); Hemihalictus Cockerell, 1897 (Pesenko 2007; Gibbs et al. 2013); Parasphecodes Smith, 1853 (Smith 1853; Michener 1965); and Sphecodogastra Ashmead, 1899 (Pesenko 2007; Gibbs et al. 2013). The colour of the metasoma can vary from orange to black even within a single species (Fig. 1; Murao \& Tadauchi 2011; Gibbs et al. 2013). Despite this, it is useful to treat the red-tailed $L$. (Dialictus) as a group of convenience. Specimens with red metasomas are easy to pick out among others with the naked eye, even by non-entomologists, and could be one of the first levels of morphospecies sorting performed. The keys presented herein would then be a valuable tool for identification of those morphospecies, and a logical first step towards breaking down the western L. (Dialictus) taxonomic impediment.

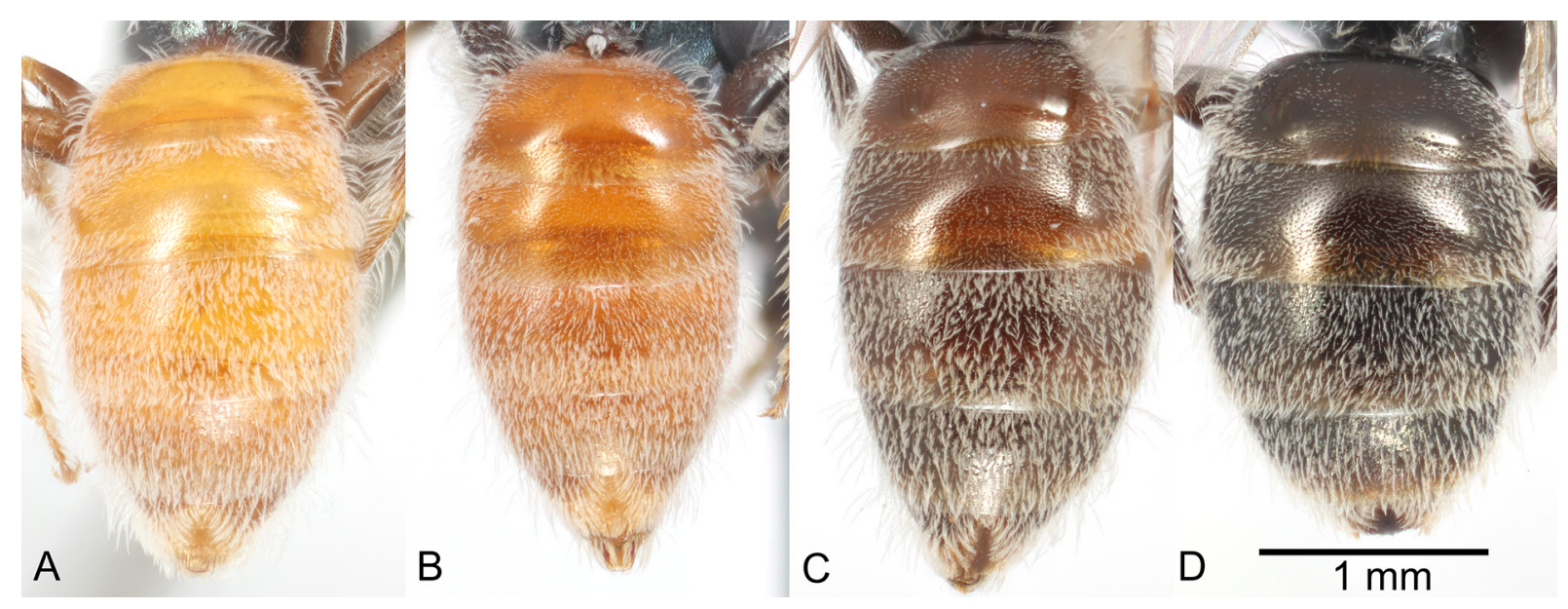

Fig. 1. Colour variation in the metasoma of Lasioglossum (D.) hudsoniellum (Cockerell, 1919). A. Yellow-orange. B. Red-orange. C. Reddish brown. D. Black. "Red-tailed" Lasioglossum (Dialictus) are considered to be any species with natural colour matching A or B. 


\section{Material and methods}

\section{Taxon concepts}

The taxon concept for L. (Dialictus) follows the classification in Gibbs (2018a). This concept of L. (Dialictus) differs from past authors who placed most weak-veined Lasioglossum in Evylaeus Robertson, 1902 (Ebmer 2002; Pesenko 2007) or Halictus Latreille, 1804 (most authors prior to Michener 1944), or considered parasitic species and/or species with three submarginal cells as the separate genera or subgenera Paralictus Robertson, 1901 and Chloralictus Robertson, 1902 (Robertson 1901, 1902; Michener 1944; Mitchell 1960; Sandhouse 1923, 1924). It also differs from some authors (Gibbs 2010; Michener 1990, 2007), which included a large number of non-metallic species in L. (Dialictus) that are now placed in L. (Hemihalictus).

Species delimitation was done using the evolutionary species concept (Wiley \& Mayden 2000) and an integrated taxonomic approach as outlined in DeSalle et al. (2005).

\section{Definition of red-tailed Lasioglossum (Dialictus)}

The red-tailed Lasioglossum (Dialictus), as defined herein, include all known species that naturally have some degree of bright, bold red, orange, or yellow colour on the metasoma (Fig. 1A-B). They do not include species that only attain an indistinct reddish brown (Fig. 1C). The current work does, however, treat both sexes (if known) of all these species, even if one sex is not known to ever be red-tailed.

In rare cases, museum specimens (especially old ones) of species that normally have a dark metasoma can appear to be red through fading or poor preservation (for example, the type of Halictus stultus Cresson, 1872 (= L. imitatum (Smith, 1853)), and species that normally have a red metasoma can appear to be dark as the inner surface of the integument is stained black by the rotted contents of the metasoma. Faded specimens will often be bleached throughout, making it evident that they do not belong to the red-tailed group.

If dark-coloured or ambiguous specimens are identified using the current work, it is recommended that they be compared to or associated with a series including some red-tailed specimens.

\section{Geographic scope}

The focal area of this work is the western Nearctic region, which is approximately defined as North America west of $97^{\circ}$ longitude, excluding the tropical coastal regions of Mexico. Effort was focused on the southwestern United States and northern Mexico, as the Canadian fauna was revised by Gibbs (2010), and the western Great Plains share many species with the east, which was revised by Gibbs (2011). Some specimens from Neotropical regions were examined for the purpose of determining distribution limits, and when Neotropical species were judged likely to occur close to the Nearctic, or be confused with Nearctic species, they were included.

\section{Excluded species}

At least two red-tailed L. (Dialictus) species within the geographic scope of the current work are included in the key to species, but are not treated further. Lasioglossum hunteri (Crawford, 1932) is part of the parvum-tegulare species complex and will be treated in more depth in a forthcoming revision of that group. Traditionally, any western $L$. (Dialictus) with a red metasoma and an enlarged, punctate tegula has been referred to L. hunteri. However, DNA barcodes in combination with morphology indicate that numerous species, described and undescribed, can have this combination of characters. At present, the true L. hunteri seems restricted to the southern Great Plains east of the Rocky Mountains and west of the Mississippi River. 
The male of Lasioglossum aquilae (Cockerell, 1898) has a bright orange metasternum, but is also not treated beyond the key to species. This species is a member of the "Augochlora-like L. (Dialictus)" treated by Engel et al. (2007). These are relatively large, coarsely sculptured, mostly Neotropical species (L. aquilae is the only one known to occur in the Nearctic) and are not considered by us to be true L. (Dialictus). Published phylogenies including L. figueresi Wcislo, 1990 (another member of this group) indicate that it renders L. (Dialictus) polyphyletic (Danforth et al. 2003; Gibbs et al. 2012a, 2012b, 2013), and additional unpublished data support this finding. Other red-tailed Augochlora-like L. (Dialictus) exist (L.aspasia Smith, 1879 and at least two undescribed species), but these seem to be strictly Neotropical. The subgeneric placement of the Augochlora-like L. (Dialictus) will be formally addressed in a forthcoming work.

Four red-tailed $L$. (Dialictus) species occur on the east coast of the United States, only reaching as far west as Mississippi (L. arantium Gibbs, 2011, L. nymphale (Smith, 1853), L. stuartense (Mitchell, 1960), and L. surianae (Mitchell, 1960)). The reader is referred to Gibbs (2011) for identification of these species.

\section{Specimen examination and description}

Specimens were examined under a dissecting microscope at 30-80 $\times$ magnification (depending on the size of the specimen) and illuminated with a standard desk lamp with a $13 \mathrm{~W}$ fluorescent bulb (60W incandescent equivalent) set to one side. These bulbs provide very diffuse light, which is ideal for seeing punctures and surface sculpture. LED and fiber optic lights are not recommended for examining L. (Dialictus) because the integument is usually highly reflective, and such focused light creates more glare that can obscure characters. If such light must be used, then glare can be reduced by covering the light source with diffusion paper (Gibbs 2020).

Morphological terms and measurements follow standard usage in Michener (2007), with Lasioglossumspecific additions by Gibbs (2010). Surface sculpture terms follow Harris (1979). Hair length is given in units of median ocellus diameters (OD).

Puncture density is given in terms of the range of interspaces (i) between punctures, in units of puncture diameters (pd). Punctures are said to be 'crowded' when there are few or no distinct interspaces; often this tight packing results in some punctures becoming polygonal rather than round, and this condition can blend into reticulate, rugulose, or areolate sculpture (Fig. 2A). This is contrasted with 'dense ( $\mathrm{i}<1 \mathrm{pd})$ ', in which all punctures are round and separated by at least narrow interspaces (Fig. 2B). The terms 'dense' and 'sparse' are an indication of whether most interspaces are toward the upper or lower end of

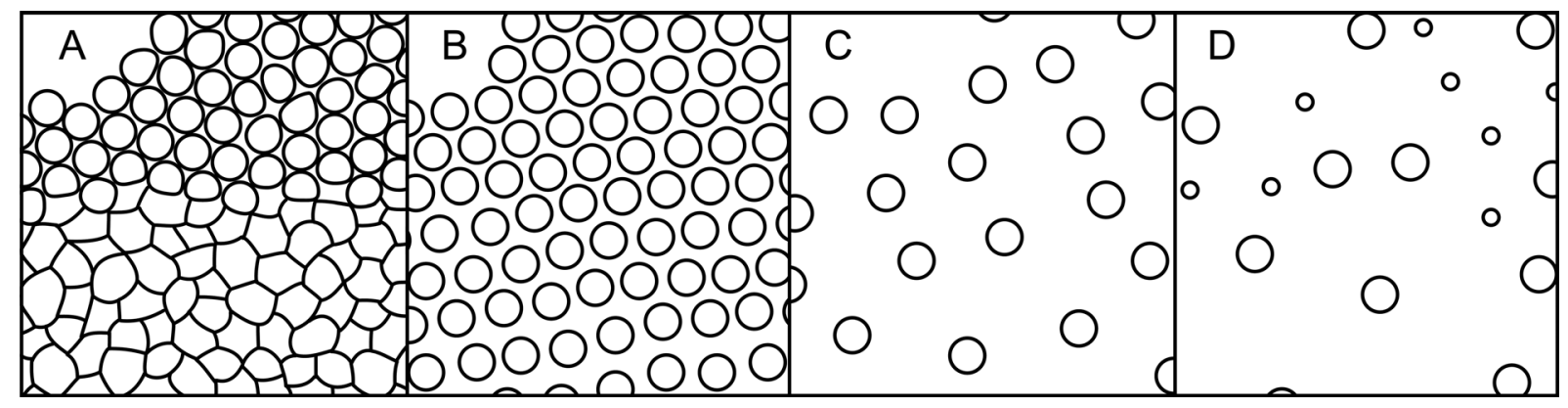

Fig. 2. Examples of punctation in Lasioglossum (Dialictus). A. Crowded ( $\mathrm{i}=0 \mathrm{pd}$ ) with few or no distinct interspaces in upper half to ruguloso-punctate in lower half. B. Dense $(\mathrm{i}<1 \mathrm{pd})$ but distinct. C. Moderately dense in upper half to moderately sparse in lower half $(i=1-2 \mathrm{pd})$. D. Sparse $(i=1-3 \mathrm{pd})$ and diversopunctate in upper half. 
the given range. 'Moderately dense ( $\mathrm{i}=1-2 \mathrm{pd}$ )' punctures are therefore separated mostly by about $1 \mathrm{pd}$, with a few interspaces up to $2 \mathrm{pd}$, and 'moderately sparse ( $\mathrm{i}=1-2 \mathrm{pd})$ ' punctures are mostly separated by about $2 \mathrm{pd}$ (Fig. 2C). There are almost always a few punctures separated by only 1 or $2 \mathrm{pd}$, so a large range such as 'sparse (i=1-6 pd)' should be interpreted as closer to $6 \mathrm{pd}$ on average. An additional term, diversopunctate, is occasionally used to refer to punctation where the punctures vary greatly in size (Fig. 2D). It is derived from the species L. diversopunctatum (Ellis, 1914), which exemplifies this character.

Male genitalia were extracted for examination by relaxing a specimen in a sealed large glass jar containing water and ethanol (to prevent mold growth) for 1-7 days (depending on the age of the specimen) until it was flexible enough to manipulate the metasoma without breaking. A handmade tool consisting of a wooden handle with a minuten pin glued to the end with the tip bent to a small hook was inserted into the tip of the metasoma, the hooked minuten was caught on the genital capsule, and then the genital capsule was pulled out until it was entirely visible. Male genitalia were not examined for all species studied, because some were represented by only one male or a very small number of very old males, and the risk of causing accidental damage to the specimens was deemed too high.

Descriptions also refer to the apicolateral denticles of the clypeus in the female. These are small, usually inconspicuous rounded projections at the apicolateral corners of the margin of the clypeus (Fig. 3B); in a few species they can be larger and more acutely pointed (Fig. 3A), which is occasionally useful for identification.

Species accounts are organized into the following sections: 1) Nomenclatural summary including synonymies; 2) Diagnosis for identification confirmation and comparison with similar species; 3) Etymology of the specific epithet (omitted if previously published); 4) List of material examined; 5) Complete description (omitted for species described in Gibbs 2009 and 2010); 6) Summary of known geographic range; 7) List of known floral host records; 8) Discussion of DNA barcodes; 9) Additional comments. For the material examined, label data is given in a standardized format. If GPS coordinates were not recorded on the label, they were estimated using Google Earth; this is indicated by enclosing the coordinates in square brackets ('[]'). Coordinates were usually estimated to two decimal digits, but up to three are given for localities that were possible to locate more precisely, or fewer for very vague localities such as entire counties. Verbatim label data is only given for newly designated type material. New rows on a label are indicated with a single forward slash ('/) and additional labels are indicated with a double forward slash ('//'). Plant classification was updated for floral host lists using the World Flora Online

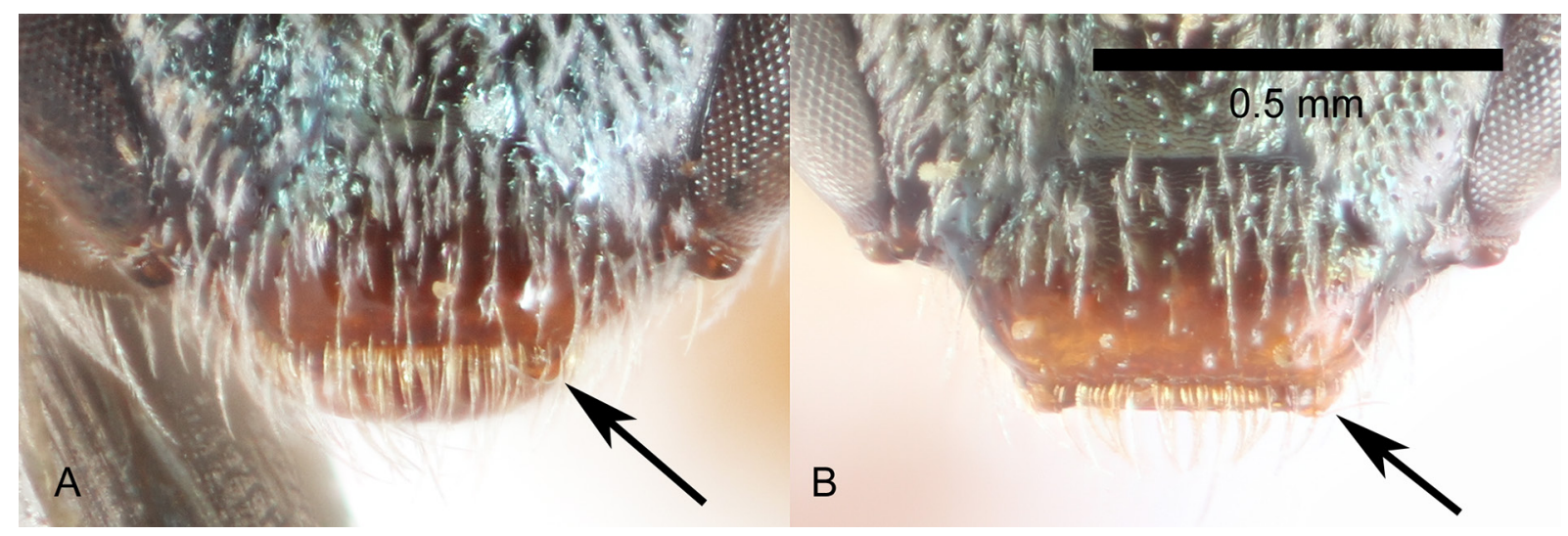

Fig. 3. Clypeus apicolateral denticles. A. Lasioglossum (D.) minckleyi sp. nov., acute points. B. L. (D.) arenisaltans sp. nov., low knobs. 
database (WFO 2020). Additional floral host records were obtained from Moure \& Hurd (1987) and Gibbs $(2009 b, 2010,2011)$ (indicated by the abbreviations MH87, G09, G10, and G11, respectively).

\section{Morphometrics}

Several morphometric measurements were made on ten specimens (if available) per sex, per species, for each species that was described. These are listed and defined in Table 1, and head measurements are shown in Fig. 4. Clypeus and supraclypeal area length/width ratios were not measured for males, as these areas are often obscured by very dense tomentum, and antennal measurements were not included for females, as they were not found to be informative. Measurements were made using either an ocular micrometer or by measuring pixels on images taken from a microscope-mounted camera, at the highest possible magnification for which the structure being measured fit in the viewing area, and with both ends of the structure in focus.

Morphometric data were analyzed with redundancy analysis (RDA) with the vegan package ver. 2.5.2 (Oksanen et al. 2018) in R ver. 4.0.0 (R Core Team 2020). A permutation test was performed to test for significant differences among species, and ordination plots were generated to visualize the results. The ordination plots were used to look for morphologically similar species pairs which might be difficult to identify qualitatively, but with diagnostic quantitative differences. The data were re-analyzed with linear discriminant analysis (LDA) and leave-one-out cross-validation in the R MASS package (Venables \& Ripley 2002) to assess the chances of one species being misclassified as another based solely on the morphometric data. Species forming separate clusters on the RDA plot and which were very seldom misclassified as each other in the LDA were candidates for possessing previously unnoticed diagnostic characters. For these species, the specimens and raw data were re-examined to assess practical utility of the characters, and if they were found to be useful, they were added to the diagnoses.

Finally, for species with $<50 \%$ classification accuracy in the female, some qualitative data were added to see if this could improve clustering and corroborate species hypotheses. Qualitative characters used are also shown in Table 1. This expanded data set was converted to a Gower distance matrix using the R cluster package (Maechler et al. 2019), which combines quantitative and qualitative measures into a single quantitative metric. Partitioning around medioids (PAM), a clustering method, was then

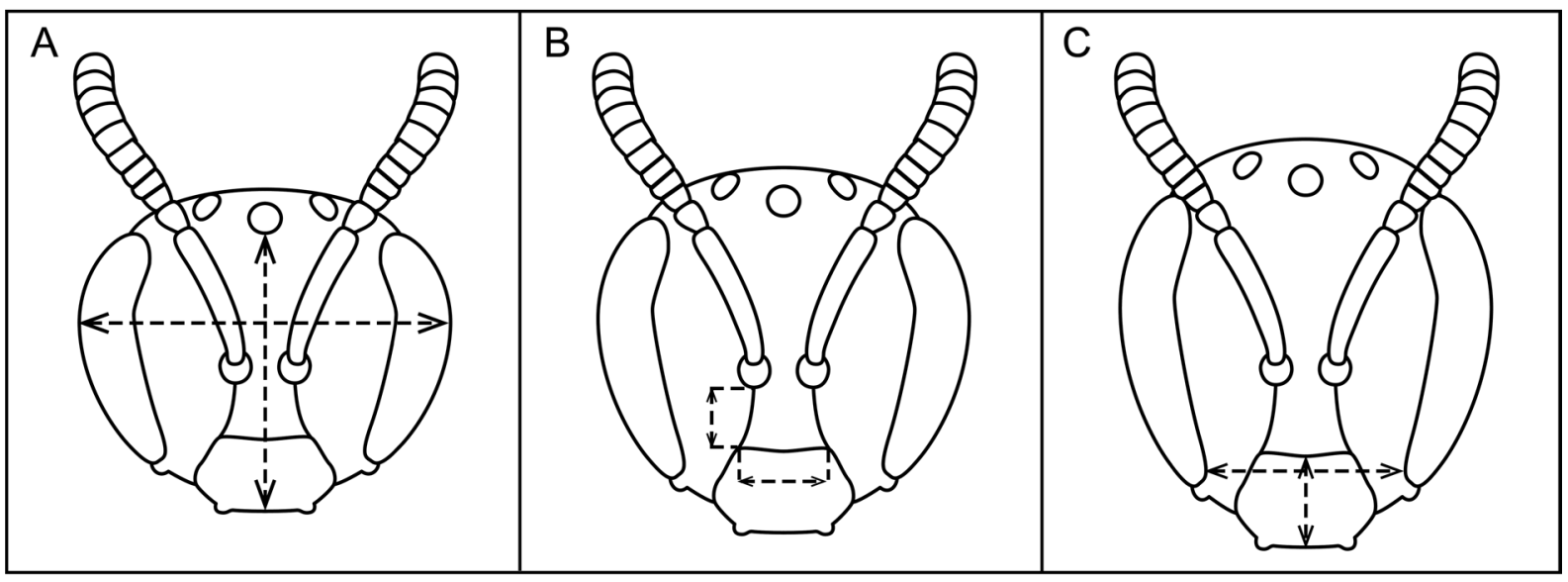

Fig. 4. Head morphometric measurements and examples of variation in face length/width ratios. A. Face short (length/width ratio $\sim 0.75$ ). Dotted lines and arrows show face length and width measurements. B. Face round (length/width ratio $\sim 0.85$ ). Dotted lines and arrows show supraclypeal area length and width measurements. C. Face long (length/width ratio $\sim 0.95$ ). Dotted lines and arrows show clypeus length and lower interocular distance measurements. 
Table 1. Morphometric measurements used in species descriptions and their definitions. Type ' $T$ ' characters are quantitative and were used in all analyses; type ' $L$ ' characters are qualitative and were only used in PAM clustering with Gower distance matrix.

\begin{tabular}{|c|c|c|}
\hline Measurement & Type & Definition \\
\hline Intertegular distance & $\mathrm{T}$ & Minimum distance between tegulae. \\
\hline Face length/width ratio & $\mathrm{T}$ & $\begin{array}{l}\text { Distance from lower edge of median ocellus to apical } \\
\text { margin of clypeus including flange, divided by maximum } \\
\text { width of head including eyes. }\end{array}$ \\
\hline Clypeal area length/width ratio & $\mathrm{T}$ & $\begin{array}{l}\text { Length of clypeus including apical flange (measured in } \\
\text { center), divided by lower interocular distance. }\end{array}$ \\
\hline $\begin{array}{l}\text { Supraclypeal area length/width } \\
\text { ratio }\end{array}$ & $\mathrm{T}$ & $\begin{array}{l}\text { Clypeoantennal distance divided by distance between } \\
\text { tentorial pits. }\end{array}$ \\
\hline F1:pedicel length ratio & $\mathrm{T}$ & $\begin{array}{l}\text { Maximum length of F1 divided by maximum length of } \\
\text { pedicel. }\end{array}$ \\
\hline F2:F1 length ratio & $\mathrm{T}$ & Maximum length of $\mathrm{F} 2$ divided by maximum length of F1. \\
\hline F2 length/width ratio & $\mathrm{T}$ & Maximum length divided by maximum width of F2. \\
\hline F9 length/width ratio. & $\mathrm{T}$ & Maximum length divided by maximum width of F9. \\
\hline Scutum length/width ratio & $\mathrm{T}$ & $\begin{array}{l}\text { Maximum length of scutum divided by intertegular } \\
\text { distance. }\end{array}$ \\
\hline Scutum:scutellum length ratio & $\mathrm{T}$ & $\begin{array}{l}\text { Maximum length of scutum divided by maximum length } \\
\text { of scutellum. }\end{array}$ \\
\hline Scutellum:metanotum length ratio & $\mathrm{T}$ & $\begin{array}{l}\text { Maximum length of scutellum divided by maximum length } \\
\text { of metanotum. }\end{array}$ \\
\hline $\begin{array}{l}\text { Metanotum:metapostnotum length } \\
\text { ratio }\end{array}$ & $\mathrm{T}$ & $\begin{array}{l}\text { Maximum length of metanotum divided by maximum } \\
\text { length of metapostnotum in dorsal view. (For species with } \\
\text { the metapostnotum gently rounded onto the posterior face } \\
\text { of the propodeum, the end point was estimated as the point } \\
\text { at which the propodeum drops out of focus.) }\end{array}$ \\
\hline Frons puncture density & $\mathrm{L}$ & Crowded $(i=0 \mathrm{pd})$, dense $(\mathrm{i}<1 \mathrm{pd})$, or sparse $(\mathrm{i} \geq 1 \mathrm{pd})$ \\
\hline Frons puncture size & $\mathrm{L}$ & $\begin{array}{l}\text { Normal (deep and distinct) or fine (not much bigger than } \\
\text { diameter of hair). }\end{array}$ \\
\hline Frons surface sculpture & $\mathrm{L}$ & Shiny or dull. \\
\hline Mesepisternum puncture density & $\mathrm{L}$ & $\begin{array}{l}\text { Crowded }(\mathrm{i}=0 \mathrm{pd}) \text {, dense }(\mathrm{i}<1 \mathrm{pd}) \text {, sparse }(\mathrm{i} \geq 1 \mathrm{pd}) \text {, } \\
\text { crowded dorsally and dense ventrally, or dense dorsally } \\
\text { and sparse ventrally. }\end{array}$ \\
\hline Mesepisternum puncture size & $\mathrm{L}$ & $\begin{array}{l}\text { Normal (deep and distinct), fine (not much bigger than } \\
\text { diameter of hair), obscure, or diversopunctate. }\end{array}$ \\
\hline Mesepisternum surface sculpture & $\mathrm{L}$ & Smooth and shiny, smooth and dull, or rugulose. \\
\hline Scutum puncture density & $\mathrm{L}$ & $\begin{array}{l}\text { Dense (majority of punctures } \mathrm{i}<1 \mathrm{pd} \text { ) or sparse (majority } \\
\text { of punctures } \mathrm{i} \geq 1 \mathrm{pd} \text { ). }\end{array}$ \\
\hline Scutum surface sculpture & $\mathrm{L}$ & Shiny, dull anteromedially, or completely dull. \\
\hline $\begin{array}{l}\text { Propodeum lateral surface } \\
\text { sculpture }\end{array}$ & $\mathrm{L}$ & Smooth and dull or rugulose. \\
\hline T1 anterior surface sculpture & $\mathrm{L}$ & Shiny, weakly coriarious, or strongly coriarious. \\
\hline Metasoma tomentum & $\mathrm{L}$ & Present/absent. \\
\hline Scutum hair plumosity & $\mathrm{L}$ & Mostly thin and simple or mostly densely plumose. \\
\hline Number of submarginal cells & $\mathrm{L}$ & 2,3 , or 2 in one wing and 3 in the other. \\
\hline
\end{tabular}


run on the Gower distance matrix with $\mathrm{k}=12$ (the number of species for which qualitative data were obtained), also in the cluster package (Maechler et al. 2019). Results from PAM were represented in two-dimensional space using T-distributed stochastic neighbour embedding (van der Maaten \& Hinton 2008; van der Maaten 2014) implemented in the R Rtsne package (Krijthe 2015). Accuracy of species predictions was assessed by comparing PAM clusters with a priori species identifications.

\section{DNA barcoding}

DNA was extracted from hind legs of preserved specimens (or a hind leg and mid-leg for small specimens) by placing the tissue in individual tubes or wells, and then crushing it with a sterile pestle. Tubes or the pestle (for 96-well plates) were dipped in liquid nitrogen to cool the tissue and make it brittle. For small numbers of specimens (less than 30), DNA was extracted using DNeasy Blood \& Tissue silica membrane spin column kits (Qiagen, Hilden, Germany) and a modified protocol developed for dried museum specimens by Crane (2013). For sets of 96 specimens, DNA was extracted using KingFisher Cell and Tissue DNA kits (Thermo Fisher Scientific Inc., Waltham, Massachusetts, United States), or MagMAX-96 DNA Multi-Sample kits (Thermo Fisher Scientific Inc., Waltham, Massachusetts, United States), on a KingFisher Flex magnetic particle processor (Thermo Fisher Scientific Inc., Waltham, Massachusetts, United States) and the manufacturer's default program. In all cases, the amount of elution buffer in the final step was reduced to increase the concentration of DNA.

PCR was run with 8.4-9.4 $\mu \mathrm{L} \mathrm{dH}_{2} 0,5 \mu \mathrm{L} 5 \mathrm{X}$ PCR buffer (Promega, Madison, Wisconsin, United States), $2.5 \mu \mathrm{MgCl}_{2}(25 \mathrm{mM})$ (Promega, Madison, Wisconsin, United States), $5 \mu \mathrm{dNTPs}$ (1 mM each) (Promega, Madison, Wisconsin, United States), $0.125 \mu \mathrm{L}$ GoTaq Flexi DNA polymerase (5 units/ $\mu \mathrm{L}$ ) (Promega, Madison, Wisconsin, United States), 1-2 $\mu \mathrm{L}$ DNA, and $1 \mu \mathrm{L}$ of each primer (forward and reverse) (Integrated DNA Technologies, Coralville, Iowa, United States) for a $25 \mu \mathrm{L}$ final reaction volume. For most specimens, primers used were the BarBeeF-MtD9 pair designed by Françoso \& Arias (2013) for amplification of the COI DNA barcode region in corbiculate Apidae. The reaction was run for 5:00 at $94^{\circ} \mathrm{C}$, followed by 35 cycles of $1: 00$ at $94^{\circ} \mathrm{C}, 1: 20$ at $41^{\circ} \mathrm{C}$, and $2: 00$ at $64^{\circ} \mathrm{C}$, and a final extension of 10:00 at $64^{\circ} \mathrm{C}$. This resulted in a maximum sequence length of $676 \mathrm{bp}$, excluding primer sequences (inclusive of the standard $658 \mathrm{bp}$ barcode region; Folmer et al. 1994). For a few specimens, the newer BEEf-BEEr primer pair designed by Creedy et al. (2020) was used, with reaction conditions described therein. This resulted in a maximum sequence length of $658 \mathrm{bp}$ (the standard barcode region). It was thought that these primers could have a greater success rate, as they were designed as a degenerate mix using data from a large range of bees.

PCR products were checked for success by gel electrophoresis. Samples showing visible bands in the expected size range were purified with $0.4 \mu \mathrm{L}$ of ExoSAP-IT (Thermo Fisher Scientific Inc., Waltham, Massachusetts, United States) per $1 \mu \mathrm{L}$ DNA and sent for Sanger sequencing. Sequencing was first performed by University Core DNA Services at the University of Calgary (Alberta, Canada), but switched to Eurofins MWG Operon (Toronto, Ontario, Canada) in June 2019 for cost savings. Sequencing was performed in both forward and reverse directions.

Successful sequences were trimmed and aligned in Geneious ver. 8.1 .7 (Kearse et al. 2012) and uploaded to the Barcode of Life Data System (BOLD; http://www.boldsystems.org) (Ratnasingham \& Hebert 2007), along with trace files and collection information. DNA barcodes generated by Joel Gardner using the methods described above were uploaded with sample IDs with the letters 'gard' followed by a unique four-digit number, a hyphen, and a state/province abbreviation. DNA barcodes for some redtailed L. (Dialictus) were available from previous studies (Gibbs 2009b, 2010, 2011) and unpublished data from Jason Gibbs. These data were generated following the protocols listed in Gibbs (2009b) and uploaded to BOLD using other sample ID formats. 
Distance metrics for DNA barcodes were calculated using the built-in functionality in BOLD. Diagnostic individual nucleotides were found using the nucDiag function in the spider package ver. 1.4-2 (Brown et al. 2012) in $\mathrm{R}$ ver. 4.0.0 (R Core Team 2020), after aligning the sequences first in BOLD and then making corrections by hand in Mesquite ver. 3.61 (Maddison \& Maddison 2019).

\section{Phylogeny}

A phylogeny of red-tailed L. (Dialictus) was generated to test the hypothesis that the red-tailed L. (Dialictus) are an unnatural grouping. COI barcode sequences of all available red-tailed species of L. (Dialictus) and representatives of other major L. (Dialictus) lineages were downloaded from BOLD and aligned. The alignment was trimmed to $654 \mathrm{bp}$ and gaps manually removed in Mesquite ver. 3.61 (Maddison \& Maddison 2019). Aligned sequences were analyzed with ModelFinder (Kalyaanamoorthy et al. 2017), which selected a GTR $+\mathrm{I}+\mathrm{G} 4$ ( $=\Gamma$ with 4 rate parameters) model of evolution. A Markov chain Monte Carlo (MCMC) tree search was then performed in MrBayes ver. 3.2.7 (Ronquist et al. 2012) for 30000000 generations, with L. (Austrevylaeus) sordidum (Smith, 1853) set as the outgroup. Other settings used were the program defaults (two simultaneous runs with one cold and three hot chains each, sampling every 5000 generations, and discarding the first $25 \%$ of trees as burn-in). The final tree was rooted at the outgroup node.

\section{Images}

Face, lateral and dorsal habitus, and metasoma images of all included species were generated with a Canon EOS 7D camera with MPE-65 lens (Canon, Ōta, Tokyo, Japan). Three wireless flash units were arranged at approximately 120 degree intervals around the specimen. A tube of diffusion paper was placed between the flash units and the specimen to provide a uniform, bright, diffuse light source, as for microscope examination. The camera was mounted on a StackShot system (Cognisys Inc., Traverse City, Michigan, United States) to obtain several images at varying focal depths, which were then processed for focus stacking in Helicon Focus software (Helicon Soft Ltd, Kharkiv, Ukraine). Male genitalia and some diagnostic characters were illustrated in Illustrator CS5 software (Adobe Inc., San Jose, California, United States), while examining the character using a microscope with ocular grid for scale reference.

\section{Mapping}

Distribution maps were generated from all georeferenced specimen records included in Material examined, with some additional records obtained from Gibbs (2009b, 2010, 2011), Heron \& Sheffield (2015) and Ascher (2020) to fill the gaps. These coordinates were imported into Maxent ver. 3.4.1 (Phillips et al. s.d.). Maxent predicts a species' distribution by maximizing entropy (more uniform spread) of a probability distribution of ecological niche occupancy, within constraints inferred from species presence records and environmental data (Phillips et al. 2006). Environmental data used included the 19 bioclimatic variables (based on temperature and precipitation), solar radiation, wind speed, and elevation available from WorldClim (Fick \& Hijmans 2017) and soil classification available from the FAO/UNESCO Digital Soil Map of the World (FAO/UNESCO 2007). These variables were thought to be most relevant to L. (Dialictus) foraging and nesting. All environmental data were at the 2.5 minute spatial scale, as this is close to the maximum estimated precision of most of the georeferenced specimen records. Solar radiation and wind speed data were converted from 12 monthly averages to yearly mean, minimum, maximum, range, and standard deviation $\times 100$ in QGIS software (QGIS.org 2020), which is consistent with the WorldClim bioclimatic variables. The soil map was downloaded in vector format and rasterized to the 2.5 minute spatial scale in QGIS (QGIS.org 2020).

Maps of each species' predicted probability of occurrence in North America, along with known collection records, were generated with soil type as categorical and all other variables as continuous, a regularization multiplier of 1 , and complementary log-log (cloglog; the probability that at least one 
individual is present in a quadrat/pixel; Phillips et al.2017) as output. Because there were a large number of environmental variables and (in many cases) relatively few presence records, which often resulted in an over-fit prediction, only linear and quadratic variables were used. This had the effect of 'spreading out' the predicted distribution and reducing patchiness. For species with very few presence records $(\leq 5)$ and a very large predicted distribution, the regularization multiplier was reduced to 0.5 to obtain a much more conservative prediction. For species with biased 'hot spots' of many nearby presence records and 'cold spots' of sparse presence records in areas of 0 probability, the presence records were thinned and the analysis re-run. Thinning was accomplished by rounding each coordinate to the nearest 0.1 degree and removing duplicate records, using the original coordinates associated with the remaining records for the final analysis.

Final maps were modified by adding lakes, national borders, and state/province borders downloaded from Natural Earth (https://www.naturalearthdata.com; accessed 31 Jul. 2020).

\section{Specimens examined}

Type material and other specimens were examined from the following institutions and individuals. Abbreviations used throughout the rest of the text are given first, with the full name and curator/lender (if applicable) given in parentheses afterward.

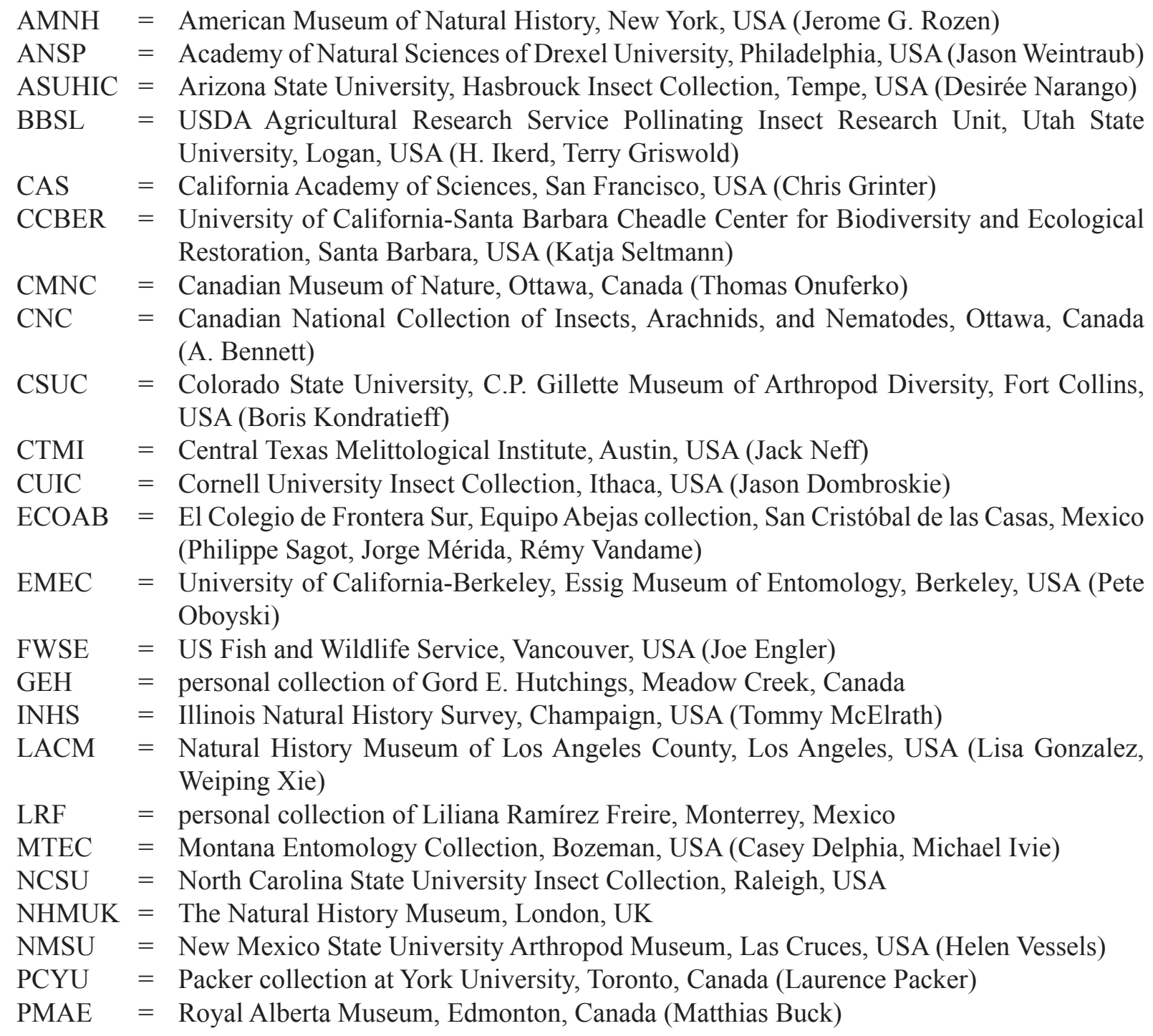




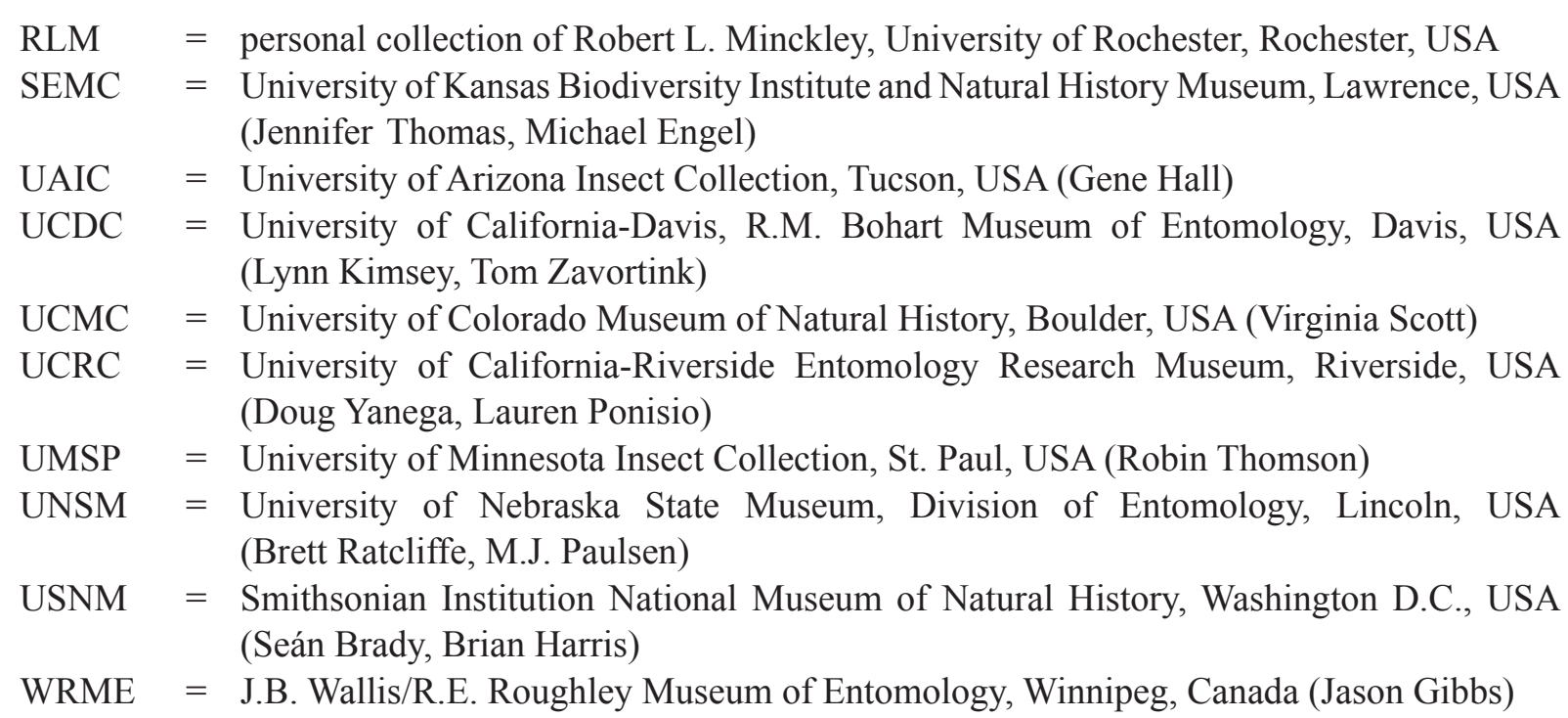

\section{Results}

In total, 39 species of red-tailed Lasioglossum (Dialictus) are recognized in North America following this work (40 if L. aquilae is considered a L. (Dialictus)). Twenty are described as new, and two names are treated as junior synonyms. Four of these species are restricted to the east coast of the United States, leaving 35 in the western Nearctic. However, the taxonomic limits of L. hunteri are pending further study. The remaining 34 species were revised in detail. All 34 of these species are known from the female, and 31 are also known from the male. Male genitalia were examined and illustrated for 17 species.

\section{Morphometrics}

A permutation test on the RDA indicated that there were significant morphometric differences among the species studied (females: $\mathrm{df}=23, F=10.56, p<0.001$; males: $\mathrm{df}=22, F=4.34, p<0.001$ ). In general, species form recognizably distinct clusters on the ordination plots, although there is overlap among some similar species (Fig. 5).

Leave-one-out cross-validation on the LDA indicated that the available morphometric data were generally poor for classifying closely similar species, although some morphologically distinct species were classified with $90-100 \%$ accuracy (Supplementary file 1; Supplementary file 2). Females of Lasioglossum arenisaltans sp. nov., L. cembrilacus sp. nov., L. festinum sp. nov., L. lilianae sp. nov., $L$. perditum sp. nov., and L. rufornatum $\mathrm{sp}$. nov. and males of $L$. cactorum sp. nov., L. festinum sp. nov., $L$. imbriumbrae sp. nov., and L. tessellatosum sp. nov. were accurately classified by the LDA. It should be noted that only two specimens were available for all of the aforementioned males, so the reliability of morphometric-based identifications might break down with additional specimens and data. The morphologically similar species pairs in female $L$. argammon sp. nov. $+L$. lilianae sp. nov. and $L$. mesillense $+L$. minckleyi sp. nov. were reliably distinguished by the LDA (if specimens were misclassified, they were not classified as the other species in the pair except in one L. minckleyi sp. nov. specimen) and previously unnoticed diagnostic characters were discovered for each.

In most cases, adding qualitative characters and analyzing the data as a Gower distance matrix with PAM resulted in more discrete clusters and equal or better accuracy of species identifications (Fig. 6, Supplementary file 3). Most species were correctly assigned $100 \%$ of the time, although species in the L. clematisellum species complex (described below) remained problematic, often resulting in over-inclusive clusters. Male L. perditum sp. nov. were split into two clusters, resulting in no cluster corresponding to $L$. clavicorne sp. nov. The only case where the addition of qualitative data resulted in worse accuracy was in male $L$. mesillense and $L$. minckleyi sp. nov. The best character for separating 

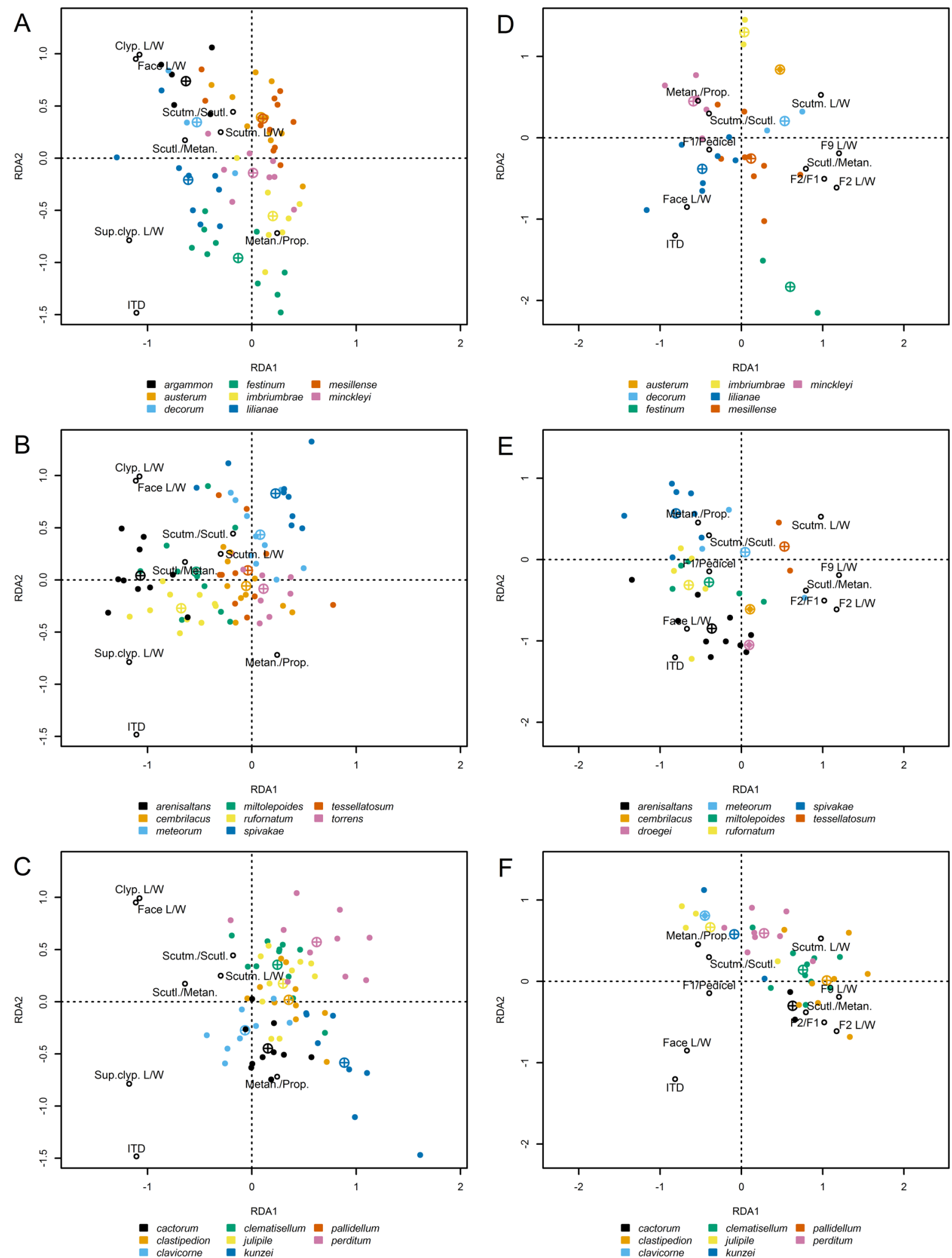

Fig. 5. Ordination plot of redundancy analysis of morphometrics. A-C. $q$ + $q$. D-F. $\widehat{\partial} \widehat{\partial}$. A-C and D-F depict the same plot and analysis; three groups of species are each shown separately for ease of interpretation. Characters are represented by open black circles. Crosshairs indicate centroids for species clusters. 

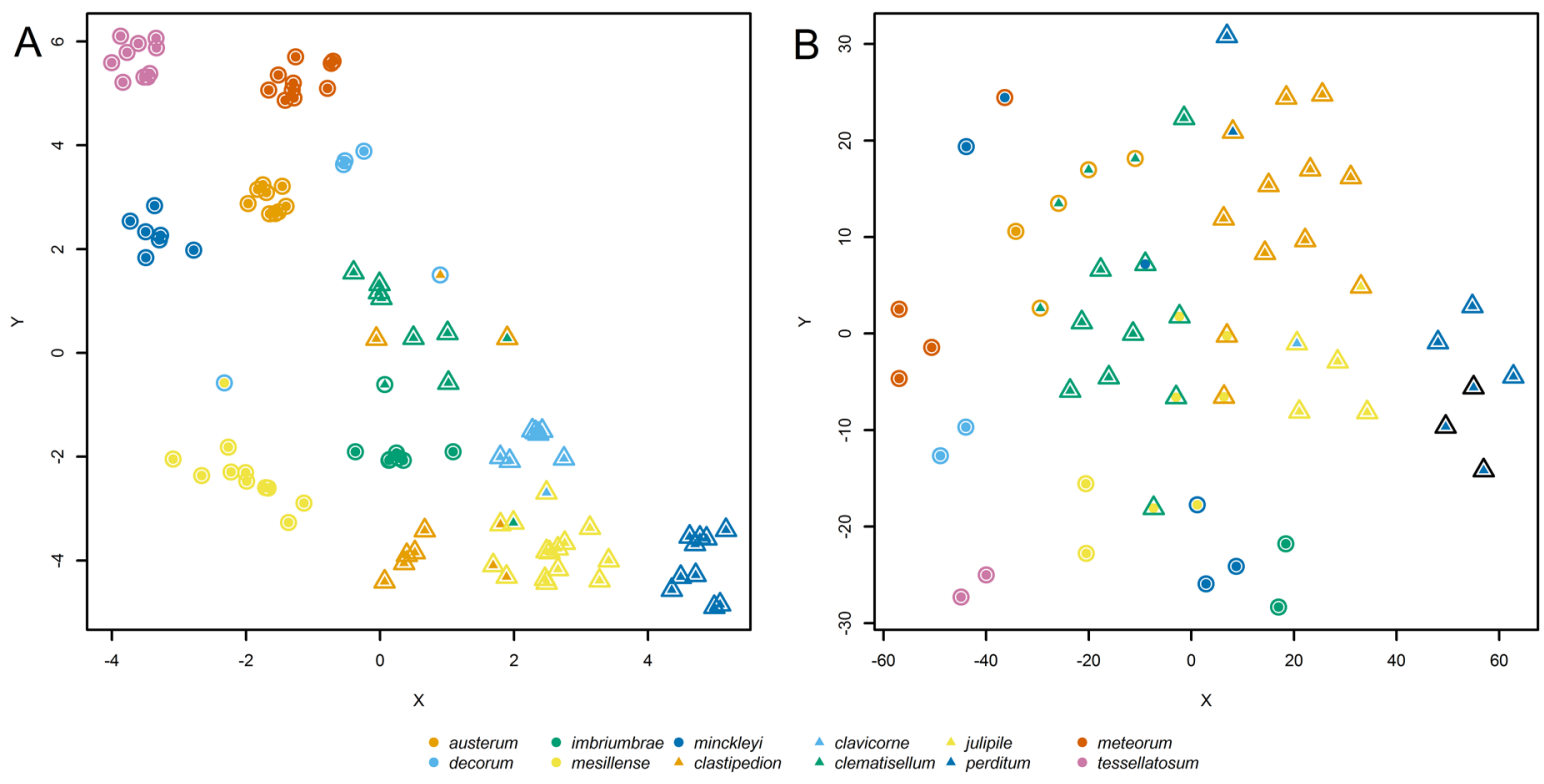

Fig. 6. Two-dimensional representation of clusters created by PAM. A. $q q$. B. $\widehat{\delta}$. Actual species identifications are indicated by the colour of filled centre points, and cluster assignments by PAM are indicated by borders around each point. Black borders in B indicate a second cluster corresponding to L. (D.) perditum sp. nov.

these species in the male is the length of the flagellum (a quantitative character), so it is reasonable to expect that adding qualitative characters of dubious utility could obscure species boundaries.

\section{DNA barcoding}

DNA barcodes were available for 31 species of red-tailed Lasioglossum (Dialictus) in all of North America, and 27 in the western Nearctic (Supplementary file 4). Twenty novel sequences were generated for this study and 172 sequences are available in total. Among the 27 western species, the mean intraspecific p-distance was $0.59 \%$ (range $0-4.42 \%$ ) and the mean interspecific $p$-distance was $8.45 \%$ (range 1.03-13.51\%) after excluding sequences less than $500 \mathrm{bp}$. Analysis on BOLD indicated the presence of a barcode gap (maximum intraspecific distance was less than distance to nearest interspecific neighbour in all cases). However, if eastern species are included, the barcode gap disappears in the case of L. hudsoniellum (Cockerell, 1919) and L. nymphale.

Barcodes for the 27 western species included 28 barcode index numbers (BINs; Ratnasingham \& Hebert 2013) assigned by BOLD. There were multiple BINs per species in several cases ( 3 for L. hudsoniellum, 2 for L. lilianae sp. nov., and 2 for L. tessellatosum sp. nov.). Lasioglossum griswoldi Gibbs, 2009 and L. tuolumnense Gibbs, 2009 shared the same BIN.

All 172 DNA barcodes for the red-tailed $L$. (Dialictus) species are available in the public BOLD dataset DS-GARDRED.

\section{Phylogeny}

Red-tailed species appeared in nearly every major clade of Lasioglossum (Dialictus) (Fig. 7), so that despite the limited data upon which this phylogeny is based, it is extremely unlikely that the red-tailed species of $L$. (Dialictus) are a monophyletic group. Several nodes had rather low posterior probability support values, and should be interpreted with great caution. However, many other nodes were supported by posterior probabilities up to $100 \%$. 


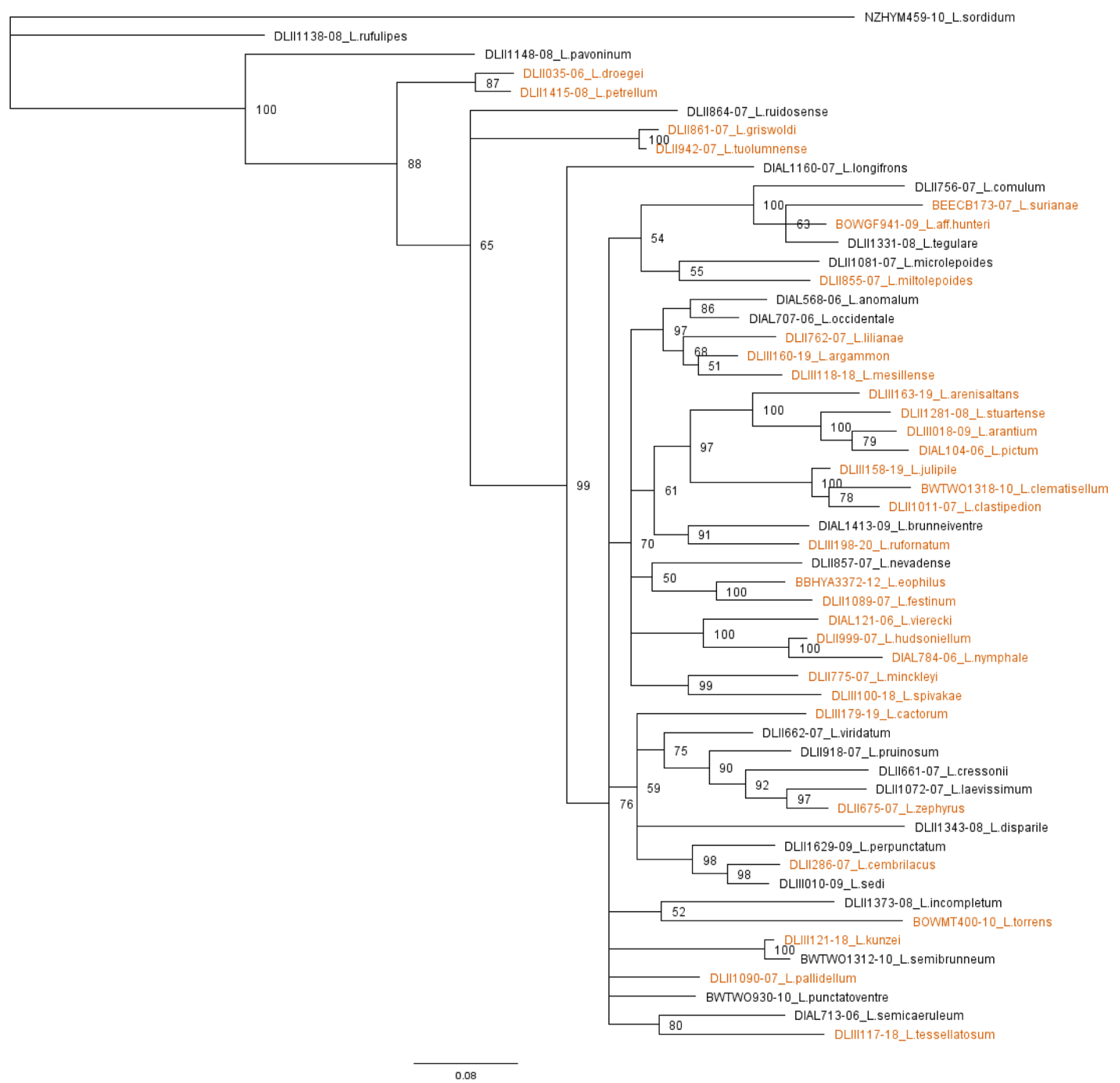

Fig. 7. 50\% majority-rule consensus tree of red-tailed Lasioglossum (Dialictus) and representatives of other major lineages of $L$. $(D$.) based on a Bayesian phylogenetic analysis of 654-bp CO1 barcode sequences downloaded from BOLD. MCMC tree search was run in MrBayes ver. 3.2.7 for 30000000 generations, using a GTR $+\mathrm{I}+\Gamma$ model of evolution, with L. (Austrevylaeus) sordidum (Smith, 1853) set as the outgroup. Node labels are posterior probabilities. Names in vermilion text are red-tailed species; BOLD process IDs are given before species names. In addition to the labeled red-tailed species, $L$. (D.) rufulipes (Cockerell, 1938) is hypothesized to be an additional lineage containing the red-tailed species $L$. (D.) testaceum (Robertson, 1897), for which CO1 barcodes were not available. As this phylogeny is based on very limited data, some relationships are likely misleading or incorrect (particularly those with low posterior probability); however, it serves to illustrate the point that the redtailed L. (Dialictus) are an unnatural grouping. 


\section{Taxonomy}

Class Insecta Linnaeus, 1758

Order Hymenoptera Linnaeus, 1758

Family Halictidae Thomson, 1869

Tribe Halictini Thomson, 1869

Genus Lasioglossum Curtis, 1833

Subgenus Dialictus Robertson, 1902

Paralictus Robertson, 1901: 229 (type species: Halictus cephalicus Robertson, 1892 (= Halictus cephalotes Dalla Torre, 1896: 57), by original designation).

Dialictus Robertson, 1902: 48 (type species: Halictus anomalus Robertson, 1892, by original designation and monotypy; given priority over Paralictus Robertson, 1901; International Commission of Zoological Nomenclature 1997).

Chloralictus Robertson, 1902: 248 (type species: Halictus cressonii Robertson, 1890, by original designation; given priority over Paralictus Robertson, 1901; International Commission of Zoological Nomenclature 1997).

Halictus (Gastrohalictus) Ducke, 1902: 102 (type species: Halictus osmioides Ducke, 1902, by monotypy).

Halictomorpha Schrottky, 1911: 81 (type species: Halictomorpha phaedra Schrottky, 1911, by original designation).

Rhynchalictus Moure, 1947: 5 (type species: Rhynchalictus rostratus Moure, 1947, by original designation).

Halictus (Smeathhalictus) Warncke, 1975: 88 (type species: Melitta smeathmanella Kirby, 1802, by original designation).

Lasioglossum (Afrodialictus) Pauly, 1984: 142 (type species: Halictus bellulus Vachal, 1909, by original designation).

Gnathalictus Moure, 2001: 493 (type species: Gnathalictus capitatus Moure, 2001, by original designation).

Evylaeus (Viridihalictus) Pesenko, 2007: 25 (type species: Halictus viridis Brullé, 1840, by original designation).

Evylaeus (Glauchalictus) Pesenko, 2007: 26 (type species: Halictus problematicus Blüthgen, 1823, by original designation).

Evylaeus (Virenshalictus) Pesenko, 2007: 26 (type species: Hylaeus virens Erichson, 1835, by original designation).

Evylaeus (Loethalictus) Pesenko, 2007: 26 (type species: Halictus loetus Brullé, 1840, by original designation).

Evylaeus (Aerathalictus) Pesenko, 2007: 27 (type species: Melitta aerata Kirby, 1802, by original designation).

Lasioglossum (Dialictus) arenisaltans sp. nov. urn:1sid:zoobank.org:act:2DAFB486-B93D-48D9-BE7C-AE0B5FB7C47D

Figs 3B, 8-10, 90A, 100A, 110B, 113B

\section{Diagnosis}

Females of Lasioglossum arenisaltans sp. nov. can be recognized by the frons and mesepisternum punctures crowded and indistinct, scutum very densely punctate $(\mathrm{i}<1 \mathrm{pd})$, face relatively long (length/ width ratio $\sim 0.85$ ), supraclypeal area dull, gena and postgena lineate, metapostnotum with strong anastomosing rugae and weak microsculpture, T2-4 with dark spiracular spots, and apical margin of 
the clypeus sometimes orange. They are most similar to L. pictum (Crawford, 1902), the southeastern US species L. nymphale, and worn specimens of L. vierecki (Crawford, 1902). Females of L. pictum have the face slightly shorter (length/width ratio $\sim 0.82$ ), scutum punctures sparser in part ( $\mathrm{i} \leq 1 \mathrm{pd}$ ), and apical margin of the clypeus never orange. Females of $L$. nymphale have T1 anterior surface lacking any appressed hairs or acarinarial fan. Females of $L$. vierecki have the metapostnotum with strong microsculpture and weak rugae, metasomal terga more densely punctate $(\mathrm{i} \leq 1 \mathrm{pd})$, and $\mathrm{T} 2$ lacking dark spiracular spots.

Males of L. arenisaltans sp. nov. can be recognized by the face being relatively long (length/width ratio $\sim 0.89$ ), frons punctures crowded and indistinct ( $\mathrm{i}=0 \mathrm{pd}$ ), hypoepimeron and mesepisternum densely punctate ( $\mathrm{i} \leq 1 \mathrm{pd}$ ), propodeum dorsolateral slope rugulose, flagellomeres long (F2 about 1.5 times as long as broad and 1.75 times as long as F1), rims of T1-3 with punctures usually not reaching the apical margin, scutum and metasoma mostly lacking tomentum, clypeus apical margin orange, and legs often entirely orange. They are most similar to L. lilianae sp. nov., L. festinum sp. nov., and L. pictum. Males of L. lilianae sp. nov. have the flagellomeres relatively short (F2 about 1.25 times as long as broad and 1.5 times as long as F1), scutum and metasoma with dense tomentum, and propodeum dorsolateral slope smooth. Males of $L$. festinum sp. nov. have the face slightly shorter (length/width ratio $\sim 0.86$ ) and rims of T1-3 punctate to the apical margin without change in sculpture across the premarginal line. Males of L. pictum lack extensive orange colouration of the clypeus apical margin, legs, and metasoma.

\section{Etymology}

The specific epithet arenisaltans is formed from the Latin noun 'arena' ('sand') and the present participle 'saltans' ('hopping'). It refers to this species' distribution in small, widely scattered sandy habitats, in which it seems to hop from place to place. An appropriate translation would be the sand-hopping sweat bee.

\section{Material examined}

\section{Holotype}

UNITED STATES - Texas - + ; Bailey Co., Muleshoe Wildlife Refuge, Grassland 1; $33.963^{\circ} \mathrm{N}$, 102.7282 W; 13 May 2013; S.J. Galdek leg.; BBSL.

[Verbatim label: USA:TX Bailey Co. Muleshoe Wildlife Refuge, Grassland1 N33.9630 W102.7282 13 May 2013 S.J. Galdek / Pantrap, fluor. Blue 1 (Row) / BBSL916563 // U.S.A. TX, Bailey County / MNWR-GR1 / N33.962972 W102.728167 / May 13, 2013 / S. Galdek USFWS // BOLD / gard0139-TX / DLIII163-19 // HOLOTYPE / Lasioglossum (Dialictus) arenisaltans Gardner and Gibbs]

\section{Paratypes}

MEXICO-Chihuahua • 1 क; 6 km S of Samalayuca; [31.28 N, 106.48 W]; 30 Aug. 1991; R. Ayala leg.; SEMC • 1 \%; same location as for preceding; 30 Aug. 1991; J.G. Rozen leg.; SEMC.

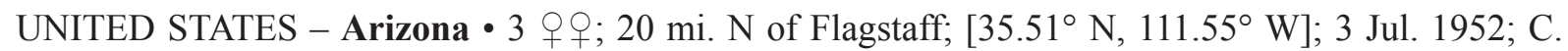
Liang, W. LaBerge, R.H. and L.D. Beamer leg.; SEMC • 1 क ; Forestdale; $\left[34.13^{\circ} \mathrm{N}, 110.1^{\circ} \mathrm{W}\right]$; 20 Jun. 1957; G. Butler and F. Werner leg.; UAIC. - Colorado • 1 + ; Weld Co., 11 mi. N of Roggen, jct. Rd 386 and Empire Intake Canal, swale with willow in rolling dunes; $40.2751^{\circ} \mathrm{N}, 104.2902^{\circ} \mathrm{W} ; 7-9 \mathrm{Jul} .1998$; M.E. Irwin and S. Fitzgerald leg.; CSUC • 3 q $\circ$; Roggen; $\left[40.17^{\circ} \mathrm{N}, 104.37^{\circ} \mathrm{W}\right] ; 26$ Aug. 1935;

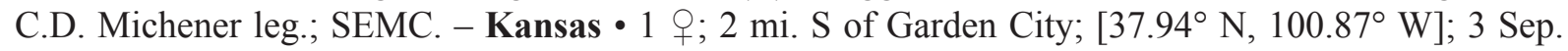
1951; C.D. Michener and W.E. LaBerge leg.; ex Helianthus petiolaris; SEMC 11 \%; 7 m. E of Garden City; [37.97 ${ }^{\circ}$ N $100.74^{\circ} \mathrm{W}$ ]; 12 Jun. 1952; Wille and Michener leg.; SEMC • 3 우 ; same location as for preceding; 12 Jun. 1952; Wille and Michener leg.; ex Monarda; SEMC • 1 क; 8 mi. E of Burdett; $\left[38.19^{\circ} \mathrm{N}, 99.38^{\circ} \mathrm{W}\right]$; 12 Jun. 1952; Wille and Michener leg.; ex Melilotus officinalis; SEMC • 8 우; 
9 mi. S of Garden City; [37.84 ${ }^{\circ}$ N, $100.87^{\circ}$ W]; 12 Jun. 1952; Wille and Michener leg.; ex Melilotus officinalis; SEMC • 1 क ; Williamsburg; [38.48 $\mathrm{N}, 95.47^{\circ} \mathrm{W}$ ]; 13 Jun. 1952; Wille and Michener leg.; ex Rhus glabra; SEMC. - Nebraska • 1 q; F. Crow Ranch, 25 mi. SW of Valentine; $\left[42.7^{\circ} \mathrm{N}, 100.9^{\circ} \mathrm{W}\right]$; 3 Sep. 1959; W.E. LaBerge leg.; ex Helianthus petiolaris; UNSM. - New Mexico • 2 $q$; DeBaca Co., 10 mi. S of Ft Sumner; [34.32 ${ }^{\circ}$ N, 104.25 W]; 19 May 1969; Brothers, Krueger and Michener leg.; ex

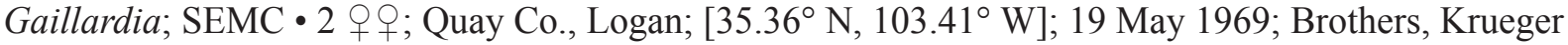
and Michener leg.; SEMC • 3 क 9 ; Chaves Co., Mescalero Sands; $\left[32.94^{\circ}\right.$ N, $103.95^{\circ}$ W]; 3 Jun. 1974; H.E. Evans leg.; CSUC - 1 क; Roosevelt Co., nr Boone Draw, 14 mi. SW of Portales; $\left[34.05^{\circ} \mathrm{N}\right.$, $103.52^{\circ} \mathrm{W}$ ]; 26 May 1972; Saul Frommer and N. Jorgrnsen leg.; UCRC • 1 ; Socorro Co., Sevilleta

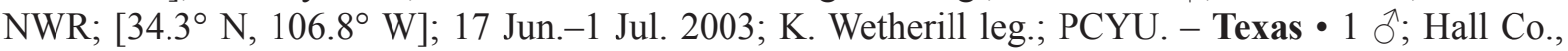

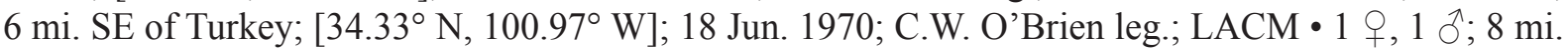

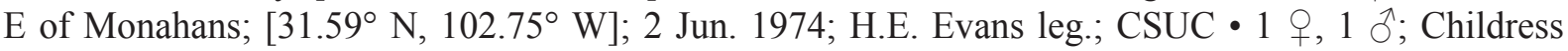

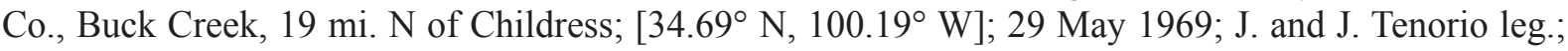

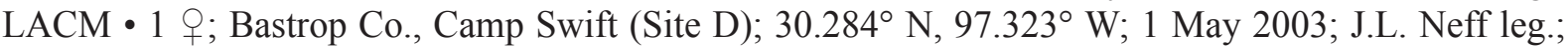
ex Hymenopappus artemisiifolius; CTMI - 2 qo ; Bastrop Co., Camp Swift Nat. Gd, $8.6 \mathrm{~km}$ N of

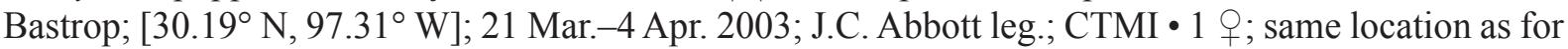
preceding; 17-31 Oct. 2003; J. C. Abbott leg.; CTMI 1 ○’; Bastrop Co., Camp Swift TNG; $30.28^{\circ}$ N,

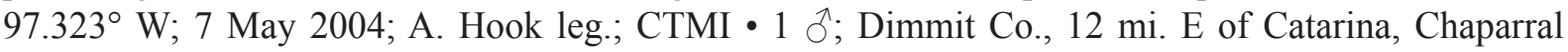
Wildlife Management Area; [28.3193 N, 99.4045 ${ }^{\circ}$ W]; 13 Jun. 1992; J.L. Neff and A. Hook leg.; ex

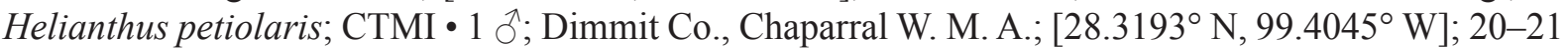
Apr. 1991; A.W. Hook leg.; CTMI • 1 o'; Dimmit Co., Chaparral W.M.A.; $28.3193^{\circ}$ N, $99.4045^{\circ}$ W; 13 Apr. 2001; A. R. Hook leg.; ex Acacia greggii; CTMI • 2 우; Gillespie Co., Fredericksburg; [30.28 $8^{\circ}$,

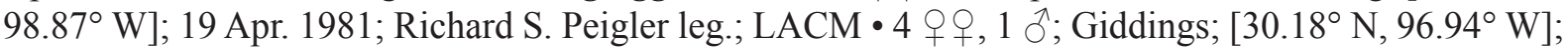
9 May 1954; R.H. Beamer leg.; ex Monarda; SEMC • 1 \%; same location as for preceding; 9 May 1954; R.H. Beamer leg.; ex Brazoria truncata; SEMC • 1 गे; same location as for preceding; 9 May 1954; R.H. Beamer leg.; ex Opuntia; SEMC • 1 q; Ward Co., Monahans; [31.59 N, $\left.102.89^{\circ} \mathrm{W}\right]$; 24 Jul. 1976; ex Mentzelia strictissima; LACM • 1 \&; Ward Co., Monahans Sandhills State Park; $31.64^{\circ}$ N, $102.819^{\circ}$ W; 17 Jun. 2006; J.L. Neff leg.; ex Hymenopappus flavescens; CTMI • 1 q; same location as for preceding; 22 Apr. 2006; J.L. Neff and A. Hook leg.; ex Phacelia integrifolia; CTMI • 1 क ; Ward Co., Monahans Sandhills S.P.; 31.64 N, 102.819 W; 832 m a.s.1.; 16 Sep. 2005; J.L. Neff and A. Hook leg.; ex Euphorbia missurica; CTMI 1 क; Ward Co., Monahans Sandhills S.P. campground area; $31.6367^{\circ} \mathrm{N}, 102.8169^{\circ} \mathrm{W}$; 13 Jun. 1998; J.A. LaFuente leg.; CTMI • 1 क; same location as for preceding; 13 Jun. 1998; E.B. Atarod, C.R. Nelson and Field Ent. class leg.; CTMI • 1 क; same location as for holotype; 14 May 2013; S.J. Galdek leg.; BBSL • 2 우; same location as for holotype; 30 Apr. 2013; S.J. Galdek leg.; BBSL • 1 q; same location as for holotype; 29 Apr. 2013; S.J. Galdek leg.; BBSL • 1 क; Bailey Co., Muleshoe Wildlife Refuge, Grassland $2 ; 33.9302^{\circ} \mathrm{N}, 102.4861^{\circ} \mathrm{W} ; 13$ May 2013; S.J. Galdek leg.; BBSL • 1 ô; Victoria; [28.81ํ N, $97^{\circ}$ W]; 16 May 1913; J.D. Mitchell leg.; ex Stillingia; LACM.

\section{Description}

\section{Female}

Colouration. Head and mesosoma blue to golden-green; clypeus apical colour black, reddish brown, or orange; labrum black, reddish brown, or orange; mandible orange with black basal spot or band and red tip; flagellum black to reddish brown dorsally, brown to orange ventrally; pronotal lobe reddish brown to orange; metasoma red to orange with dark spiracular spots on T3-4 and usually T2, T1-5 sometimes partially or entirely dark brown; legs reddish brown to orange; tegula orange; wing membrane hyaline or lightly infuscated, veins with subcosta dark brown, otherwise pale amber to brown.

PubesCence. Body hair colour golden to white. Tomentum dense on paraocular area, gena, pronotal collar and lobe, metepisternum, metanotum anterior margin, T1 laterally, T2 basolaterally, T3 throughout except medially, and T4 throughout; sparse on face below eye emargination, preëpisternum, hypoepimeron, 
and mesepisternum. Scutum hair thin to moderately plumose. Wing hairs dark or light, short and dense. Acarinarial fan complete, sparse. T2 fringes sparse, T3 fringes sparse.

SURFACE SCULPTURE. Clypeus punctures irregularly sparse $(\mathrm{i}<3 \mathrm{pd})$, sculpture shiny in apical half or more, tessellate basally; supraclypeal area punctures moderately dense to sparse $(i=1-4 \mathrm{pd})$, sculpture tessellate; paraocular area punctures crowded $(\mathrm{i}=0 \mathrm{pd})$, slightly sparser around antenna socket $(\mathrm{i} \leq 1 \mathrm{pd})$, sculpture shiny, becoming tessellate around antenna socket; frons punctures crowded ( $\mathrm{i}=0 \mathrm{pd})$, sculpture shiny; vertex punctures crowded laterally ( $i=0 \mathrm{pd}$ ), sparse between ocelli ( $\mathrm{i}=1-3 \mathrm{pd})$, sculpture shiny, sometimes weakly rugulose laterally; gena punctures moderately dense $(i=1-2 \mathrm{pd})$, sculpture shiny, becoming lineate posteriorly and ventrally; postgena sculpture lineate, sometimes becoming smooth and shiny anteriorly; tegula punctures absent; scutum punctures dense $(i<1 \mathrm{pd})$, sculpture tessellate, sometimes becoming shiny posterolaterally; scutellum punctures dense marginally and on median line ( $i<1 \mathrm{pd}$ ), sparse submedially ( $\mathrm{i}=1-3 \mathrm{pd}$ ), sculpture shiny to tessellate; metanotum sculpture shiny and finely, densely punctate ( $<<1 \mathrm{pd}$ ), becoming rugulose laterally; metapostnotum rugae strong, anastomosing, nearly reaching margin, sculpture imbricate; preëpisternum sculpture areolate-rugose; hypoepimeron punctures crowded $(\mathrm{i}=0 \mathrm{pd})$, sculpture shiny or imbricate; mesepisternum punctures crowded ( $\mathrm{i}=0 \mathrm{pd}$ ), sometimes becoming distinctly separated ventrally, sculpture shiny or imbricate; metepisternum sculpture lineate dorsally, ruguloso-punctate medially, imbricate ventrally; propodeum lateral face sculpture tessellate; propodeum posterior face sculpture tessellate; T1 anterior face sculpture shiny to coriarious; $\mathrm{T} 1$ dorsal surface punctures moderately dense, becoming sparse medially ( $\mathrm{i}=1-4 \mathrm{pd}$ ), absent or very sparse in large apicolateral oval patches, sculpture shiny to coriarious; T2 disc punctures moderately dense ( $\mathrm{i}=1-3 \mathrm{pd})$, disc sculpture shiny to coriarious, rim punctures fine, sparse $(\mathrm{i}=1-4 \mathrm{pd})$, sometimes obscure, rim sculpture shiny to coriarious.

Structure. Face length/width ratio 0.85 ( $\pm 0.02 \mathrm{SD}$ ). Clypeus projecting $\sim 67 \%$ below suborbital tangent; clypeal area length/width ratio $0.46( \pm 0.02 \mathrm{SD})$; apicolateral denticles very low rounded knobs; supraclypeal area length/width ratio $0.89( \pm 0.09 \mathrm{SD})$. Forewing with 3 submarginal cells; pronotal angle right-angled or nearly so; tegula shape normal. Intertegular distance $1.06( \pm 0.06 \mathrm{SD}) \mathrm{mm}$. Scutum length/width ratio 0.8 ( $\pm 0.02 \mathrm{SD}) ;$ scutum/scutellum length ratio 3.03 ( $\pm 0.14 \mathrm{SD}) ;$ scutellum/metanotum length ratio $1.82( \pm 0.15 \mathrm{SD})$; metanotum/metapostnotum length ratio $0.62( \pm 0.05 \mathrm{SD})$. Propodeum lateral carinae not reaching dorsal margin; oblique carina weak (sometimes virtually absent), straight. T2 depressed apical rim length about $50 \%$ of segment. $(n=10)$

\section{Male}

Colouration. Head and mesosoma blue-green to golden-green; clypeus apical colour orange; labrum orange; mandible orange with black basal spot and red tip; flagellum reddish brown dorsally, orange ventrally; pronotal lobe orange; metasoma orange to reddish brown with dark spiracular spots on T2-4; legs orange to reddish brown; tegula orange; wing membrane hyaline, veins with subcosta dark brown, otherwise orange to dark brown.

Pubescence. Body hair colour white. Tomentum dense on paraocular area, pronotal angle and lobe, and space between pronotal lobe and tegula; sparse on clypeus, supraclypeal area, gena, and episterna. Scutum hair thin to moderately plumose. Sterna hair short (0.75-1.25 OD), densely plumose, dense and erect. Wing hairs dark or light, short and dense.

SURFACE SCULPTURE. Clypeus punctures sparse ( $\mathrm{i}=1-4 \mathrm{pd}$ ), sculpture shiny; supraclypeal area punctures dense $(\mathrm{i} \leq 1 \mathrm{pd})$ to sparse $(\mathrm{i}=1-5 \mathrm{pd})$, sculpture shiny to tessellate; paraocular area punctures dense $(\mathrm{i} \leq 1 \mathrm{pd})$ to crowded $(\mathrm{i}=0 \mathrm{pd})$, sculpture shiny; frons punctures crowded $(\mathrm{i}=0 \mathrm{pd})$, sculpture shiny; vertex punctures dense $(i \leq 1 \mathrm{pd})$, sometimes sparse medially ( $i=1-4 \mathrm{pd})$, sculpture shiny, sometimes weakly rugulose medially; gena punctures moderately sparse ( $i=1-3 \mathrm{pd})$, sculpture shiny, becoming 


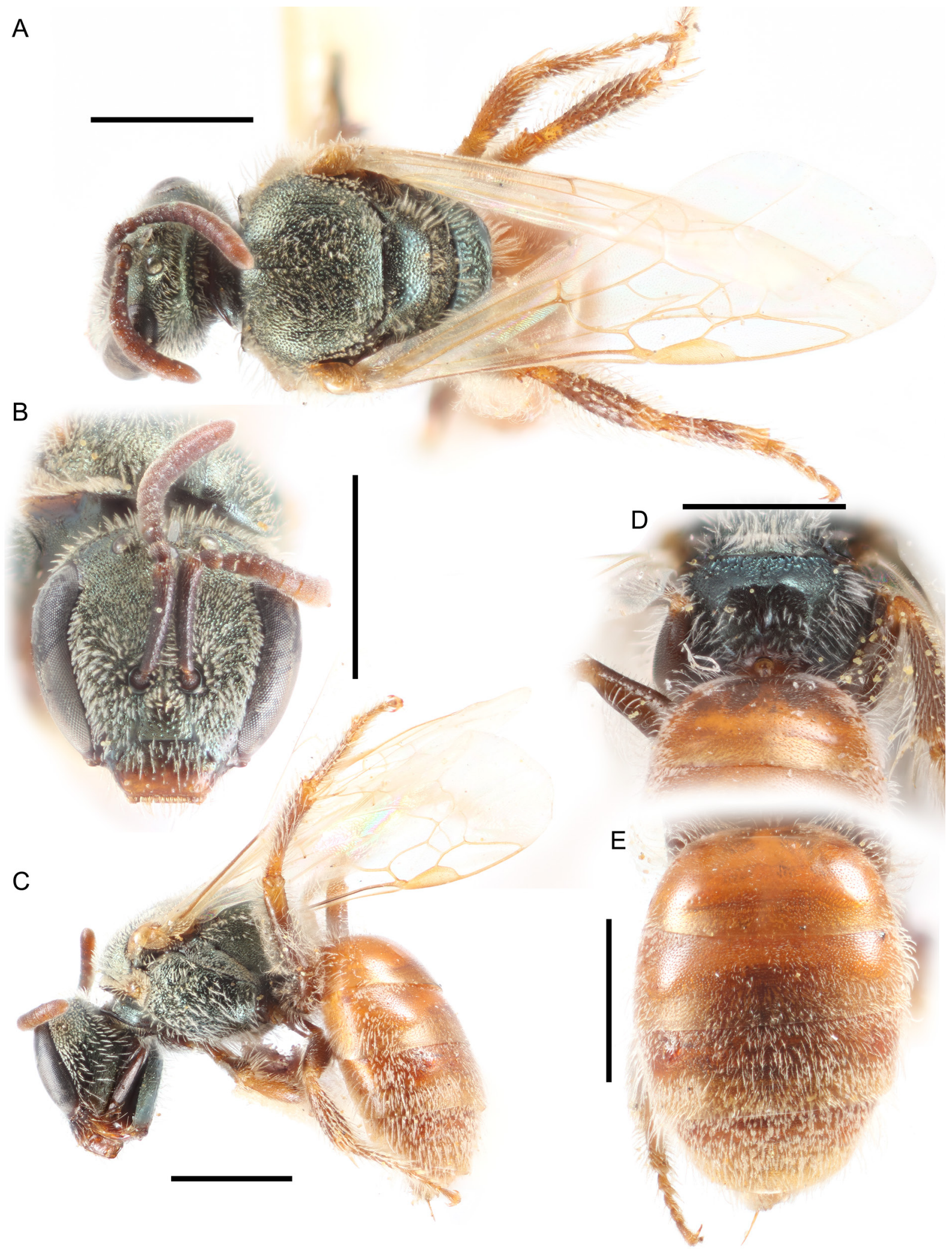

Fig. 8. Lasioglossum (D.) arenisaltans sp. nov., + . A. Dorsal habitus. B. Face. C. Lateral habitus. D. Propodeum. E. Metasoma. Scale bars: $1 \mathrm{~mm}$. 


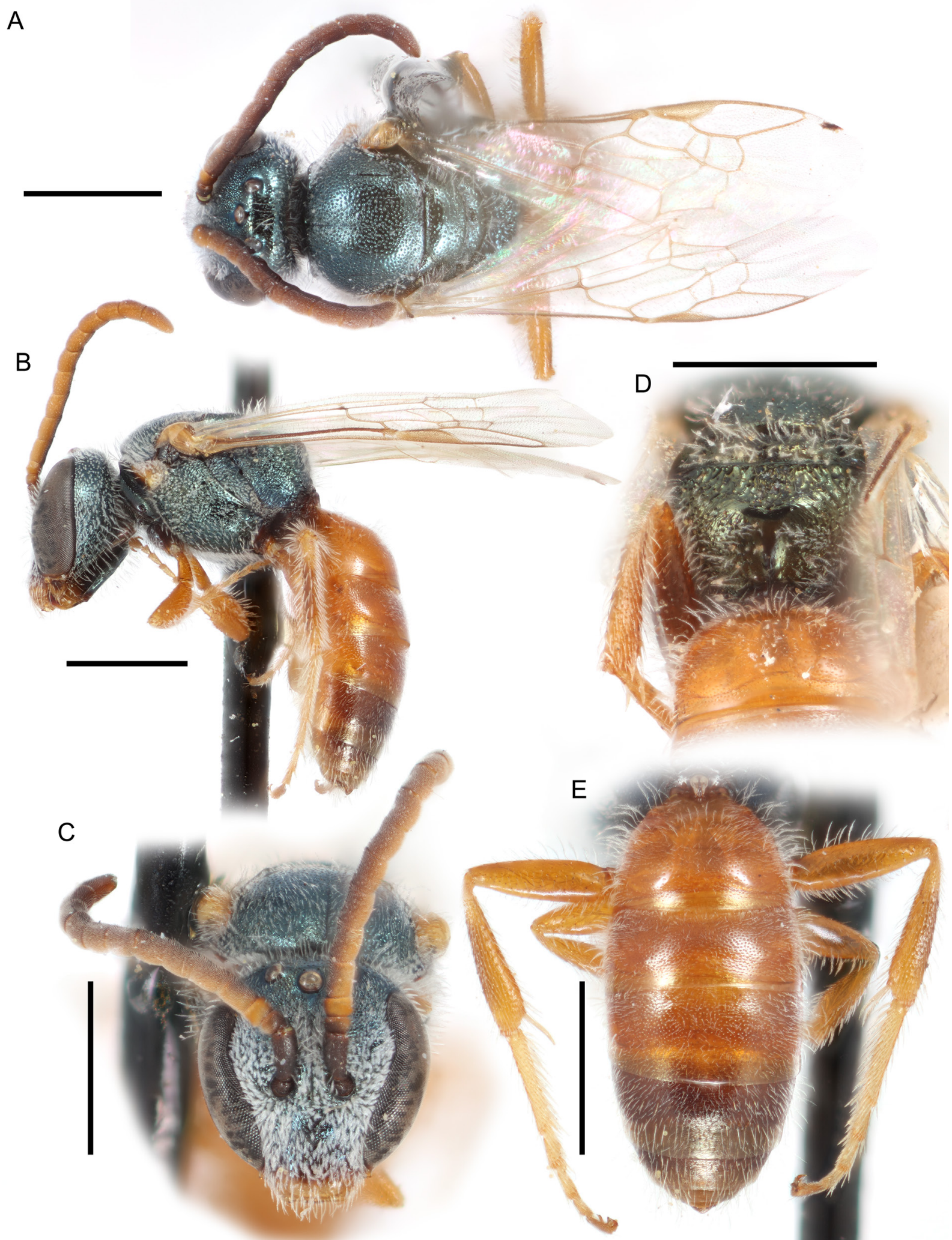

Fig. 9. Lasioglossum (D.) arenisaltans sp. nov., $\widehat{\partial}$. A. Dorsal habitus. B. Lateral habitus. C. Face. D. Propodeum. E. Metasoma. Scale bars: $1 \mathrm{~mm}$. 
ruguloso-lineate posteriorly and ventrally; postgena sculpture lineolate; tegula punctures absent; scutum punctures dense to moderately sparse ( $i \leq 2 \mathrm{pd})$, becoming slightly sparser submedially ( $i \leq 3 \mathrm{pd})$, sculpture shiny, becoming tessellate anteromedially; scutellum punctures dense marginally and on median line $(\mathrm{i}<1 \mathrm{pd})$, moderately sparse submedially $(\mathrm{i}=1-2 \mathrm{pd})$, sculpture shiny to tessellate; metanotum sculpture rugulose, sometimes becoming shiny and densely punctate medially $(\mathrm{i}<1 \mathrm{pd})$; metapostnotum rugae strong, subparallel, reaching margin, sculpture shiny to weakly imbricate; preëpisternum sculpture rugose; hypoepimeron punctures crowded $(\mathrm{i}=0 \mathrm{pd})$, sculpture shiny to weakly imbricate; mesepisternum punctures dense $(i \leq 1 \mathrm{pd})$, often becoming crowded dorsally ( $\mathrm{i}=0 \mathrm{pd})$, sculpture shiny to weakly imbricate; metepisternum sculpture ruguloso-lineate dorsally, areolate ventrally; propodeum lateral face punctures obscure, sculpture imbricate to rugose; propodeum posterior face sculpture shiny to weakly tessellate, becoming ruguloso-punctate laterally; T1 anterior face sculpture shiny; T1 dorsal surface punctures dense to moderately dense $(i \leq 2 \mathrm{pd})$, sparse in small apicolateral oval patches ( $\mathrm{i}=1-4 \mathrm{pd})$, and absent on apical rim medially, sculpture shiny; T2 disc punctures dense to moderately dense ( $\mathrm{i} \leq 2 \mathrm{pd}$ ), disc sculpture shiny, rim punctures moderately dense ( $\mathrm{i}=1-3 \mathrm{pd})$, absent apicomedially, rim sculpture shiny.

StRUCTURE. Face length/width ratio 0.89 ( $\pm 0.02 \mathrm{SD})$. F1:pedicel length ratio $1.19( \pm 0.14 \mathrm{SD}) ; \mathrm{F} 2: \mathrm{F} 1$ length ratio $1.77( \pm 0.25 \mathrm{SD}) ; \mathrm{F} 2$ length/width ratio $1.44( \pm 0.13 \mathrm{SD}) ; \mathrm{F} 9$ length/width ratio 1.19 $( \pm 0.11 \mathrm{SD})$. Forewing with 3 submarginal cells; pronotal angle obtuse; tegula shape normal. Intertegular distance $1.04( \pm 0.03 \mathrm{SD}) \mathrm{mm}$. Scutum length/width ratio 0.78 ( $\pm 0.02 \mathrm{SD})$; scutum/scutellum length ratio $2.93( \pm 0.24 \mathrm{SD})$; scutellum/metanotum length ratio $1.56( \pm 0.19 \mathrm{SD})$; metanotum/metapostnotum length ratio 0.66 ( $\pm 0.06 \mathrm{SD})$. Propodeum lateral carinae not reaching dorsal margin; oblique carina absent. $(n=10)$

Genitalia. As in Fig. 90A. Gonocoxite short, truncate. Gonostylus acutely pointed apically, with very few, very short hairs. Retrorse lobe broad, ovoid, covered in sparse short hairs.

\section{Range}

Sand dunes of the western Great Plains east to the Mississippi River, north to the Nebraska sand hills, south to the Rio Grande River, and west to northern Arizona (Fig. 10).

\section{Floral records}

ANACARDIACEAE R.Br. Rhus L. R. glabra L. • ASTERACEAE Giseke: Gaillardia • Helianthus: $H$. petiolaris Nutt. $\bullet$ Hymenopappus: $H$. artemisiifolius DC. $\bullet H$. flavescens A.Gray $\bullet$ BORAGINACEAE Juss. Phacelia Juss. P. integrifolia Torr. $\bullet$ CACTACEAE Juss. Opuntia Mill. • EUPHORBIACEAE Juss. Euphorbia L. E. missurica Raf. - Stillingia Garden - FABACEAE Juss. Acacia: A. greggii A. Gray • Melilotus: M. officinalis (L.) Pall. - LAMIACEAE Martinov: Brazoria Engelm. \& A.Gray: B. truncata (Benth.) Engelm. \& A.Gray • Monarda L. • LOASACEAE Juss. Mentzelia L. M. strictissima (Wooton \& Standl.) J. Darl.

\section{DNA barcodes}

Two sequences available (BOLD process IDs: DLIII163-19, DLIII164-19; BIN: BOLD:AEA1246). Lasioglossum arenisaltans sp. nov. differs from all other western red-tailed L. (Dialictus) by one fixed substitution: 150(C) (Supplementary file 4).

\section{Remarks}

Lasioglossum arenisaltans sp. nov. is apparently a sand dune specialist. All collection localities are in or near sand, some of them quite small and isolated. It has been collected as far west as Coconino County, Arizona, but is apparently rare west of the Rocky Mountains. 


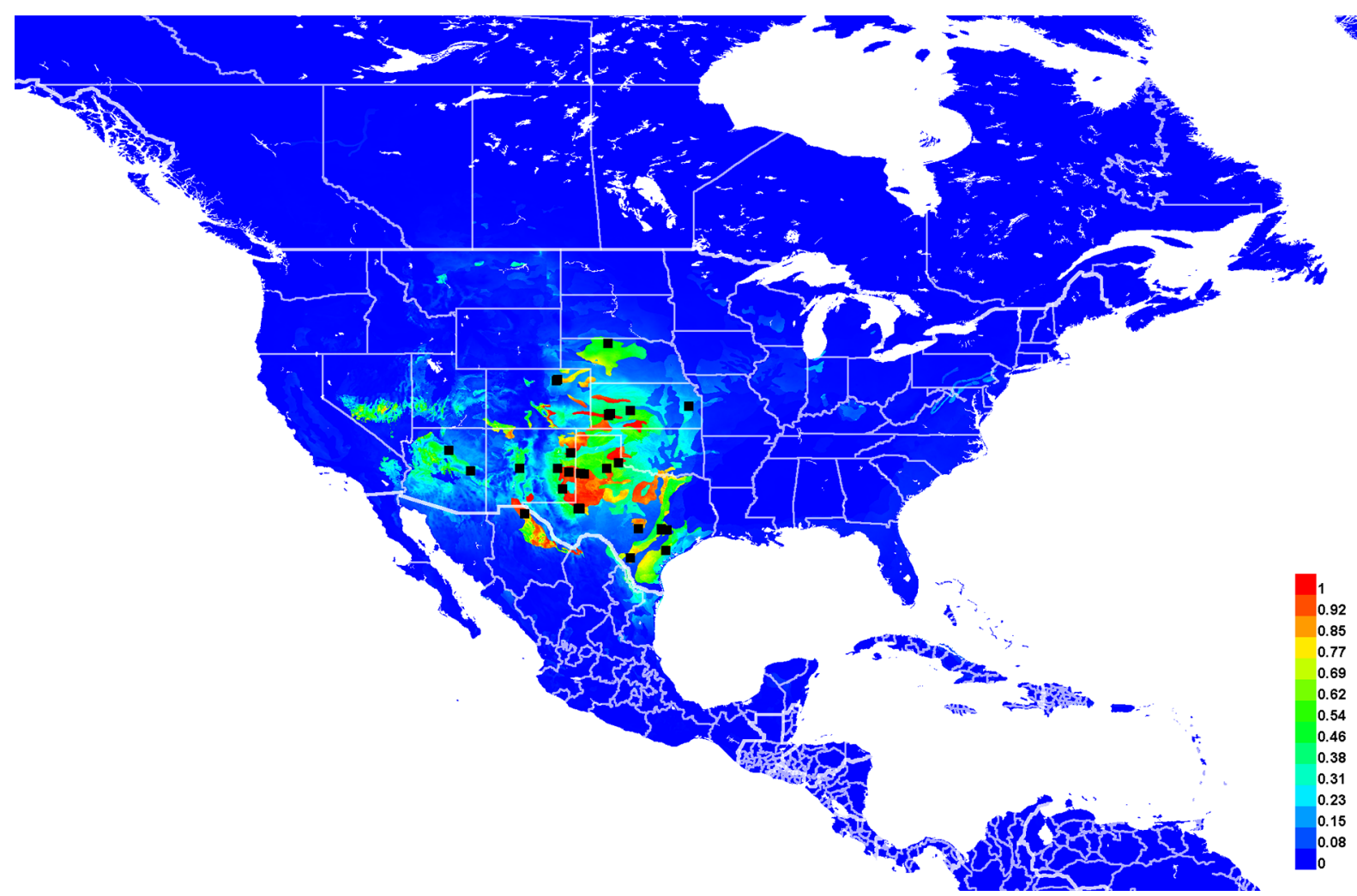

Fig. 10. Georeferenced collection records of Lasioglossum (D.) arenisaltans sp. nov. (black squares) and predicted distribution by maximum entropy ecological niche modeling in Maxent (colour shading). Warmer colours indicate higher cloglog probability of occurrence.

Lasioglossum (Dialictus) argammon sp. nov. urn:1sid:zoobank.org:act:AE9F3361-270B-4D1A-BA1C-83074AC69F50

Figs 11-12, 95B

\section{Diagnosis}

Females of Lasioglossum argammon sp. nov. can be recognized by the face relatively long (length/width ratio $\sim 0.91$ ), episterna and metasomal terga mostly covered in dense tomentum, T1 anterior surface and scutum anterior portion with dull microsculpture, frons shiny with dense but distinctly separated punctures, mesosoma mostly densely punctate $(\mathrm{i} \leq 1 \mathrm{pd})$ with sparser punctures $(\mathrm{i}=1-3 \mathrm{pd})$ limited to submedial areas of the scutum and scutellum, metasomal terga finely and moderately sparsely punctate ( $i=1-3$ pd), pronotal angle obtuse, T2-4 with dark spiracular spots, ocelli normal (separated by more than two thirds of one OD), and relatively small size (ITD usually $<1 \mathrm{~mm}$ ). They are most similar to L. lilianae sp. nov. and are somewhat similar to L. hudsoniellum. Females of L. lilianae sp. nov. have the scutum and T1 anterior surface almost entirely shiny, scutum more sparsely punctate ( $\mathrm{i}=1-4 \mathrm{pd})$, ocelli slightly enlarged (separated by less than two thirds of one OD), and are larger (ITD usually $>1 \mathrm{~mm}$ ). Females of $L$. hudsoniellum have T1 anterior surface shiny, metasomal terga usually with moderately large and dense punctures ( $\mathrm{i}=1-2 \mathrm{pd}), \mathrm{T} 2$ lacking dark spiracular spots, and pronotal angle nearly 90 degrees.

The male of Lasioglossum argammon sp. nov. is unknown, but it is probably similar to that of L. lilianae sp. nov. 


\section{Etymology}

The specific epithet argammon is formed from the Greek adjective 'argos' ('bright', 'white') and the genitive plural noun 'ammon' ('of sands'). It refers to this species' restricted distribution in and around the White Sands National Monument. An appropriate translation would be the White Sands sweat bee.

\section{Material examined}

\section{Holotype}

UNITED STATES - New Mexico • ${ }^{\circ}$; Otero Co., White Sands National Monument; [32.7 N, $106.3^{\circ} \mathrm{W}$ ]; 16 Jul. 1991; B. Alexander leg.; ex Lepidium; SEMC.

[Verbatim label: NEW MEXICO:Otero Co. / White Sands Nat'1 Mon. / 16 July 1991, B. Alexander / ex: Lepidium // HOLOTYPE / Lasioglossum (Dialictus) argammon Gardner and Gibbs]

\section{Paratypes}

UNITED STATES - New Mexico • 2 우; 25 mi. W of Tularosa; [33.07 N, 106.45 ${ }^{\circ}$ W]; 1 Jul. 1940;

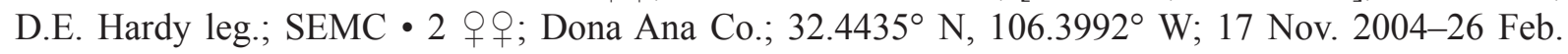
2005; S.L. Buchmann and A.J. Donovan leg.; RLM. - Texas • 1 क; Hudspeth Co., Salt Flats, N of Hwy 62/180; $31.7563^{\circ} \mathrm{N}, 104.9909^{\circ} \mathrm{W} ; 1105$ m a.s.1.; 26 Jun. 2010; J. D. Herndon leg.; BBSL.

\section{Description}

\section{Female}

Colouration. Head and mesosoma blue-green to olive green; clypeus apical colour reddish brown to orange; labrum reddish brown to orange; mandible orange with black basal spot and red tip; flagellum reddish brown dorsally, orange ventrally; pronotal lobe reddish brown to orange; metasoma reddish brown to orange with dark spiracular spots on T2-4; legs reddish brown with femur-tibia joints, base and apex of tibiae, and tarsi mostly orange; tegula pale amber to orange; wing membrane hyaline, veins with subcosta dark brown, otherwise pale amber to brown.

Pubescence. Body hair colour white. Tomentum dense on paraocular area, frons, gena, pronotal collar and lobe, space between pronotal lobe and tegula, margins of scutum, metanotum, episterna, T1 laterally, T2 basolaterally, and T3-4 throughout; sparse on clypeus and supraclypeal area. Scutum hair moderately plumose. Wing hairs light, long or short, and dense. Acarinarial fan complete, sparse. T2 fringes sparse, T3 fringes sparse.

SuRfACE SCULPTURE. Clypeus punctures dense to sparse ( $\mathrm{i}=1-4 \mathrm{pd}$ ), large and irregularly spaced apically ( $i<2$ pd), sculpture shiny; supraclypeal area punctures dense $(i \leq 1 \mathrm{pd})$, sculpture shiny; paraocular area punctures dense $(i<1 \mathrm{pd})$, sometimes crowded around antenna socket $(i=0 \mathrm{pd})$, sculpture shiny, sometimes weakly imbricate around antenna socket; frons punctures dense $(i<1 \mathrm{pd})$, sculpture shiny; vertex punctures dense ( $i \leq 1 \mathrm{pd})$, sometimes fine and sparse medially ( $i=1-3 \mathrm{pd})$, sculpture shiny; gena punctures fine, moderately dense $(i=1-2 \mathrm{pd})$, sculpture shiny; postgena sculpture shiny, becoming tessellate posteriorly; tegula punctures absent; scutum punctures dense ( $\mathrm{i} \leq 1 \mathrm{pd})$, becoming moderately dense to sparse submedially and anteromedially ( $\mathrm{i}=1-3 \mathrm{pd}$ ), sculpture shiny to weakly tessellate, becoming more strongly tessellate anteromedially; scutellum punctures dense marginally and on median line $(i<1 \mathrm{pd})$, sometimes finer and sparser anteriorly $(\mathrm{i}=1-2 \mathrm{pd})$, sparse submedially $(\mathrm{i}=1-3 \mathrm{pd})$, diversopunctate, sculpture shiny, becoming weakly tessellate marginally; metanotum sculpture shiny and finely, densely punctate ( $\mathrm{i}<1 \mathrm{pd}$ ), becoming weakly rugulose laterally; metapostnotum rugae strong, anastomosing, not reaching margin, sculpture tessellate; preëpisternum sculpture areolaterugulose; hypoepimeron punctures crowded $(\mathrm{i}=0 \mathrm{pd})$, sculpture shiny; mesepisternum punctures crowded ( $\mathrm{i}=0 \mathrm{pd})$, sculpture shiny; metepisternum sculpture ruguloso-lineate dorsally, imbricate ventrally; propodeum lateral face sculpture tessellate; propodeum posterior face sculpture tessellate; 


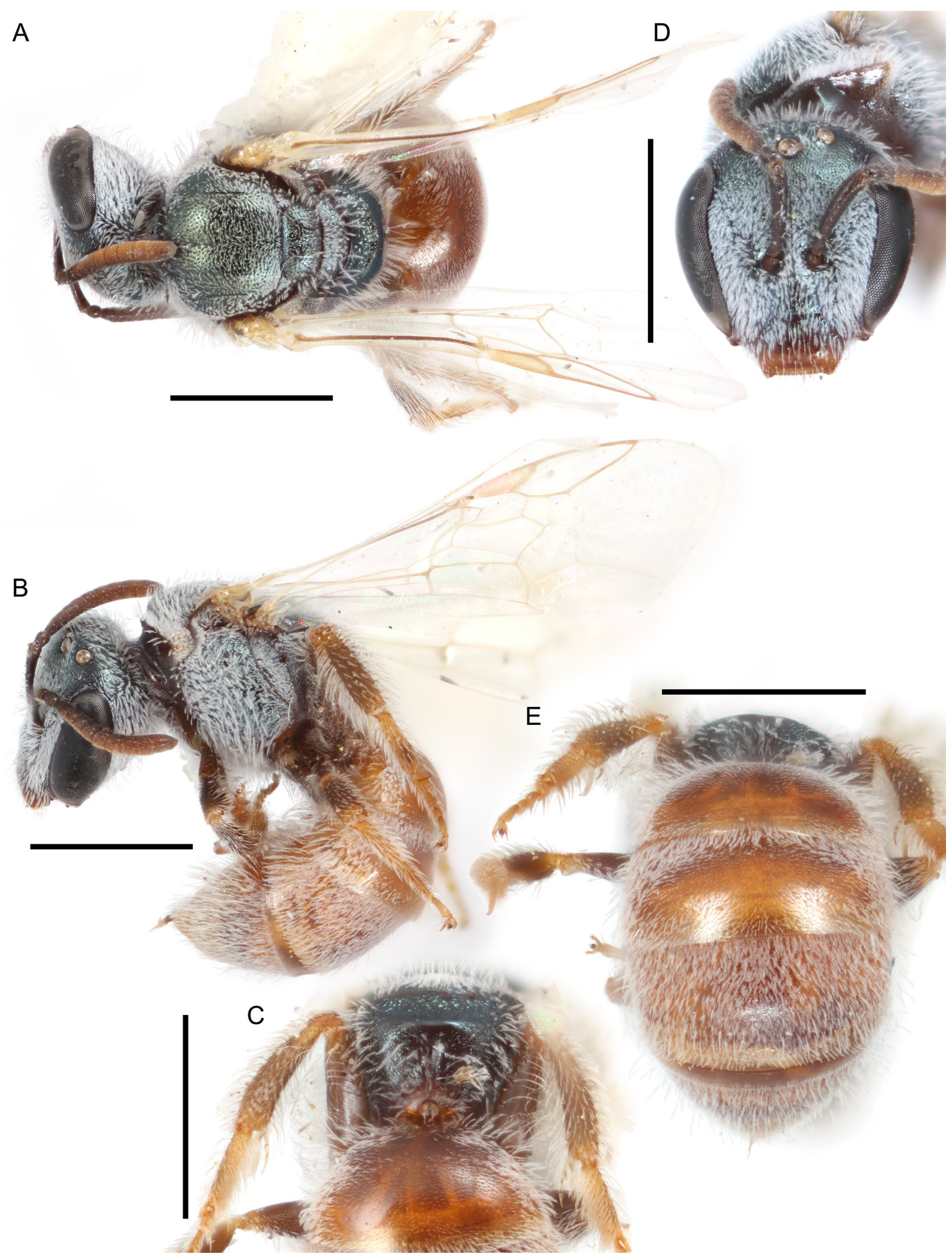

Fig. 11. Lasioglossum (D.) argammon sp. nov., holotype, +. A. Dorsal habitus. B. Lateral habitus. C. Propodeum. D. Face. E. Metasoma. Scale bars: $1 \mathrm{~mm}$. 
T1 anterior face sculpture coriarious; T1 dorsal surface punctures moderately sparse ( $\mathrm{i}=1-3 \mathrm{pd}$ ), absent or very sparse in large apicolateral oval patches, sculpture shiny; T2 disc punctures moderately sparse $(i=1-3 \mathrm{pd})$, disc sculpture shiny, rim punctures minute, sparse $(i=2-6 \mathrm{pd})$, rim sculpture shiny.

STRUCTURE. Face length/width ratio 0.91 ( $\pm 0.01 \mathrm{SD})$. Clypeus projecting $\sim 67 \%$ below suborbital tangent; clypeal area length/width ratio $0.42( \pm 0.05 \mathrm{SD})$; apicolateral denticles rounded knobs; supraclypeal area length/width ratio 0.81 ( $\pm 0.07 \mathrm{SD})$. Forewing with 3 submarginal cells; pronotal angle obtuse; tegula shape normal. Intertegular distance $0.86( \pm 0.05 \mathrm{SD}) \mathrm{mm}$. Scutum length/width ratio $0.83( \pm 0.05 \mathrm{SD})$; scutum/scutellum length ratio 3.14 ( $\pm 0.29 \mathrm{SD})$; scutellum/metanotum length ratio 1.65 ( $\pm 0.1 \mathrm{SD})$; metanotum/metapostnotum length ratio $0.58( \pm 0.06 \mathrm{SD})$. Propodeum lateral carinae not reaching dorsal margin; oblique carina absent. T2 depressed apical rim length less than $50 \%$ of segment. $(n=6)$

\section{Male}

Unknown.

\section{Range}

Gypsum dunes of the White Sands Desert of New Mexico, southeast to Hudspeth County, Texas (Fig. 12).

\section{Floral records}

BRASSICACEAE Burnett: Lepidium L.

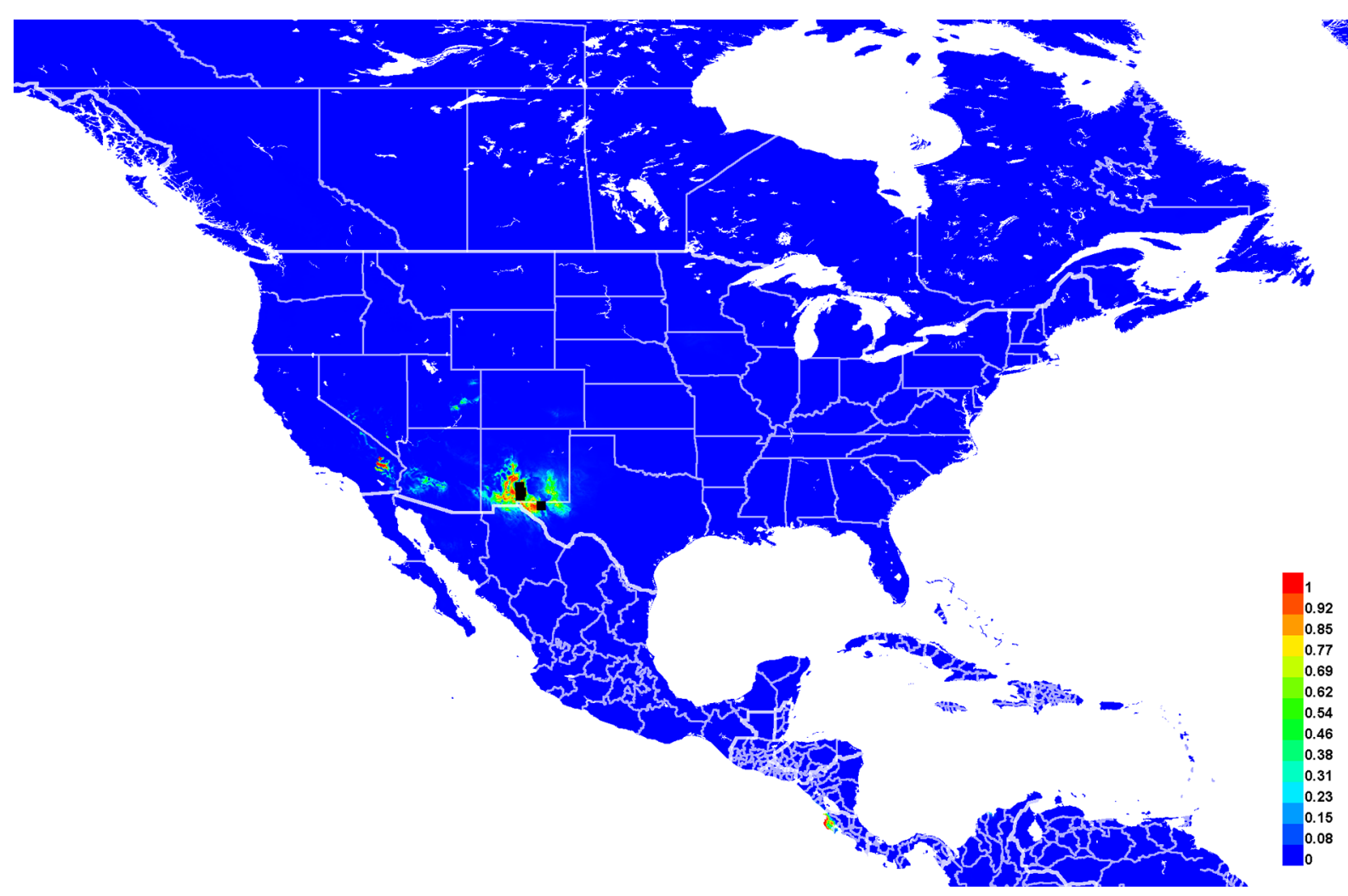

Fig. 12. Georeferenced collection records of Lasioglossum (D.) argammon sp. nov. (black squares) and predicted distribution by maximum entropy ecological niche modeling in Maxent (colour shading). Warmer colours indicate higher cloglog probability of occurrence. Due to the very small number of records, this distribution should be interpreted with caution. 


\title{
DNA barcodes
}

One sequence available (BOLD process ID: DLIII160-19; BIN: BOLD:AEA0446). This sequence has greater than expected divergence from L. lilianae sp. nov. (4.7\% p-distance), which appears to be closely related based on overall morphology. Phylogenetic analysis of DNA barcodes (Fig. 7) suggests that L. argammon sp. nov. may be more closely related to L. mesillense. Lasioglossum argammon sp. nov. has no unique fixed substitutions among all western red-tailed L. (Dialictus).

\section{Remarks}

Rare. Only six specimens are known, all from gypsum dunes in southern New Mexico and western Texas.

\author{
Lasioglossum (Dialictus) austerum sp. nov. \\ urn:1sid:zoobank.org:act:ADA77DE6-7290-46F5-A476-A932354952D5
}

Figs 13-15

\section{Diagnosis}

Females of Lasioglossum austerum sp. nov. can be recognized by the metasomal terga lacking tomentum, head and mesosoma mostly covered with dull microsculpture, T1 anterior surface coriarious, frons punctures fine and distinctly separated, mesepisternum moderately densely punctate ( $\leq 1 \mathrm{pd}$ ), and T2-4 with dark spiracular spots. They are most similar to L. mesillense and L. minckleyi sp. nov. Both of these species have the face and mesosoma mostly shiny and mesepisternum more densely punctate ( $i<1 \mathrm{pd}$ at least in dorsal half). In addition, females of $L$. mesillense often have the metasomal terga with basolateral tomentum, and females of L. minckleyi sp. nov. have the frons with slightly larger, crowded, indistinct punctures $(\mathrm{i}=0 \mathrm{~d})$.

Males of L. austerum sp. nov. can be recognized by the face short (length/width ratio $\sim 0.8$ ), mesepisternum shiny with punctures about 1 puncture diameter apart, flagellomeres relatively short (F2 about 1.75 times as long as F1 and 1.4 times as long as broad), and tomentum sparse on the face and largely absent elsewhere. They are most similar to L. mesillense, L. minckleyi sp. nov., and L. decorum sp. nov. Males of $L$. mesillense have the flagellomeres longer (F2 about 2 times as long as F1 and 1.6 times as long as broad), face below ocelli covered in dense tomentum, and metasomal terga often with basolateral patches of tomentum. Males of L. minckleyi sp. nov. have the mesepisternum more densely punctate $(\mathrm{i}<1 \mathrm{pd})$ and face below ocelli covered in dense tomentum. Males of $L$. decorum sp. nov. have the rims of T1-3 impunctate and face slightly longer (length/width ratio 0.86 ) with the clypeus more distinctly projecting below the eyes.

\section{Etymology}

The specific epithet austerum is a Latin adjective meaning 'austere', 'plain', or 'harsh'. It refers to this species' habitat in the sparsely populated, hot deserts of the Baja California peninsula, as well as its lack of tomentum. An appropriate translation would be the austere sweat bee.

\section{Material examined}

Holotype

MEXICO - Baja California • $q$; 10 mi. S of Punta Prieta; $\left[28.78^{\circ} \mathrm{N}, 114.16^{\circ} \mathrm{W}\right] ; 21$ Jun. 1938; Michelbacher and Ross leg.; CAS.

[Verbatim label: 10 mi. S. Punta Prieta, / L. Cal. VI-21-38 // Michelbacher \& Ross Collectors // HOLOTYPE / Lasioglossum (Dialictus) austerum Gardner and Gibbs] 
Paratypes

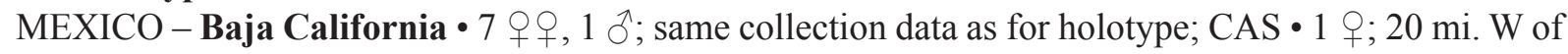
San Augustine; [24.1 ${ }^{\circ} \mathrm{N}, 110.6^{\circ} \mathrm{W}$ ]; 24 Sep. 1941; Ross and Bohart leg.; ex Eriogonum; CAS. - Baja California Sur $\bullet 1$; 15 mi. N of San Ignacio; $\left[27.49^{\circ}\right.$ N, $112.9^{\circ} \mathrm{W}$ ]; 29 Sep. 1941; Ross and Bohart leg.;

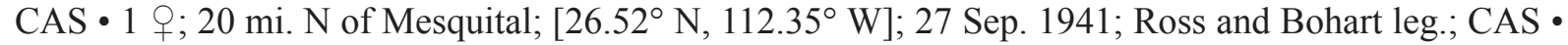
1 क; 20 mi. W of San Augustine; [24.1 ${ }^{\circ} \mathrm{N}, 110.6^{\circ} \mathrm{W}$ ]; 24 Sep. 1941; Ross and Bohart leg.; CAS • 1 क; $8 \mathrm{~km} \mathrm{~W}$ of Sta. Rita; [24.58 N, $111.54^{\circ} \mathrm{W}$ ]; 11 Jun. 1975; H. Evans, W. Rubink and D. Gwynne leg.; CSUC • 2 우 ; Concepcion Bay; $\left[26.55^{\circ} \mathrm{N}, 111.7^{\circ} \mathrm{W}\right]$; 25 Oct. 1941; Ross and Bohart leg.; CAS • 1 ㅇ; San Sebastian Bahia, San Nicholas; [26.6097 N, 111.57 W]; 19 May 1975; P. DeBach leg.; UCRC•

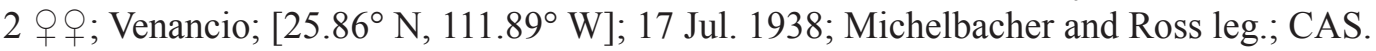

\section{Description}

\section{Female}

Colouration. Head and mesosoma blue-green to golden-green; clypeus apical colour black, reddish brown, or orange; labrum reddish brown to orange; mandible orange with black basal spot or band and red tip; flagellum reddish brown dorsally, brown to orange ventrally; pronotal lobe reddish brown to orange; metasoma orange with dark spiracular spots on T2-4; legs reddish brown with femur-tibia joints, base and apex of tibiae, and tarsi orange; tegula orange; wing membrane hyaline or lightly infuscated, veins with subcosta dark brown, otherwise brown to dark brown.

Pubescence. Body hair colour white. Tomentum dense on gena, pronotal collar and lobe, and space between pronotal lobe and tegula; sparse on paraocular area and episterna. Scutum hair thin. Wing hairs dark or light, short and dense. Acarinarial fan complete, sparse. T2 fringes sparse, T3 fringes sparse.

SURFACE SCULPTURE. Clypeus punctures large and irregularly sparse $(\mathrm{i}<3 \mathrm{pd})$, becoming fine and moderately dense in basal third ( $i=1-2 \mathrm{pd}$ ), sculpture shiny, becoming imbricate basally; supraclypeal area punctures sparse $(i=1-4 \mathrm{pd})$, sometimes absent ventromedially, sculpture tessellate, sometimes shiny medially; paraocular area punctures dense $(\mathrm{i}<1 \mathrm{pd})$, irregularly sparser around antenna socket $(\mathrm{i}<2 \mathrm{pd})$, sculpture tessellate around antenna socket, otherwise shiny; frons punctures dense $(\mathrm{i}<1 \mathrm{pd})$, sculpture tessellate or imbricate; vertex punctures dense laterally ( $\mathrm{i}<1 \mathrm{pd}$ ), sparse and sometimes obscure medially ( $\mathrm{i}=1-3 \mathrm{pd}$ ), sculpture imbricate, sometimes shiny medially; gena punctures sparse (i=1-3 pd), sculpture shiny, becoming tessellate and weakly lineate posteriorly; postgena sculpture tessellate and weakly lineate, becoming shiny anteriorly; tegula punctures absent; scutum punctures dense to moderately sparse $(i=1-3 \mathrm{pd})$, becoming slightly sparser submedially and anteromedially $(\mathrm{i}=1-4 \mathrm{pd})$, sculpture tessellate; scutellum punctures dense marginally and on median line $(\mathrm{i}<1 \mathrm{pd})$, sparse submedially $(i=1-4 \mathrm{pd})$, diversopunctate, sculpture tessellate, becoming shiny submedially; metanotum sculpture shiny or tessellate with fine, dense punctures $(i<1 \mathrm{pd})$, becoming sparse and obscure laterally; metapostnotum rugae strong or weak, anastomosing, usually not reaching margin, sculpture finely rugulose to reticulate; preëpisternum sculpture tessellate and weakly areolate-rugulose; hypoepimeron punctures dense $(\mathrm{i}<1 \mathrm{pd})$, obscure, sculpture weakly imbricate; mesepisternum punctures dense ( $\mathrm{i} \leq 1 \mathrm{pd})$, sculpture weakly imbricate; metepisternum sculpture lineate dorsally, weakly areolaterugulose medially, imbricate ventrally; propodeum lateral face sculpture tessellate; propodeum posterior face sculpture tessellate; T1 anterior face sculpture coriarious; T1 dorsal surface punctures fine, sparse $(i=1-4 \mathrm{pd})$, absent or very sparse in large apicolateral oval patches, sculpture shiny to coriarious; T2 disc punctures fine, sparse $(i=1-4 \mathrm{pd})$, disc sculpture shiny to coriarious, rim punctures fine, sparse $(\mathrm{i}=1-4 \mathrm{pd})$, obscure, rim sculpture coriarious.

StRUCTURE. Face length/width ratio $0.83( \pm 0.02 \mathrm{SD})$. Clypeus projecting $\sim 50 \%$ below suborbital tangent; clypeal area length/width ratio $0.45( \pm 0.04 \mathrm{SD})$; apicolateral denticles rounded acute points; supraclypeal area length/width ratio $0.65( \pm 0.04 \mathrm{SD})$. Forewing with 3 submarginal cells; pronotal angle obtuse; tegula shape normal. Intertegular distance $0.9( \pm 0.04 \mathrm{SD}) \mathrm{mm}$. Scutum length/width ratio 


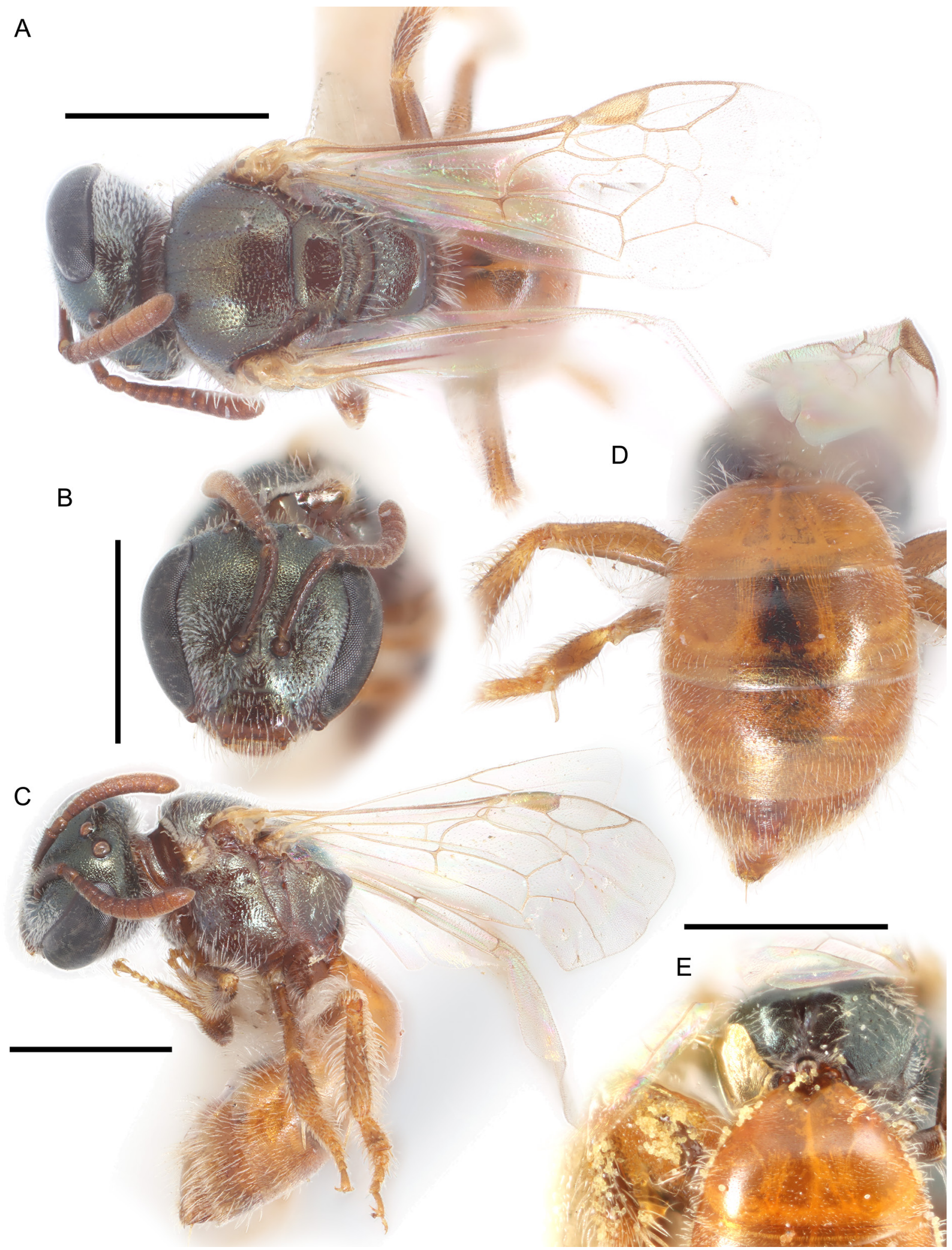

Fig. 13. Lasioglossum (D.) austerum sp. nov., ․ A. Dorsal habitus. B. Face. C. Lateral habitus. D. Metasoma. E. Propodeum. Scale bars: $1 \mathrm{~mm}$. 


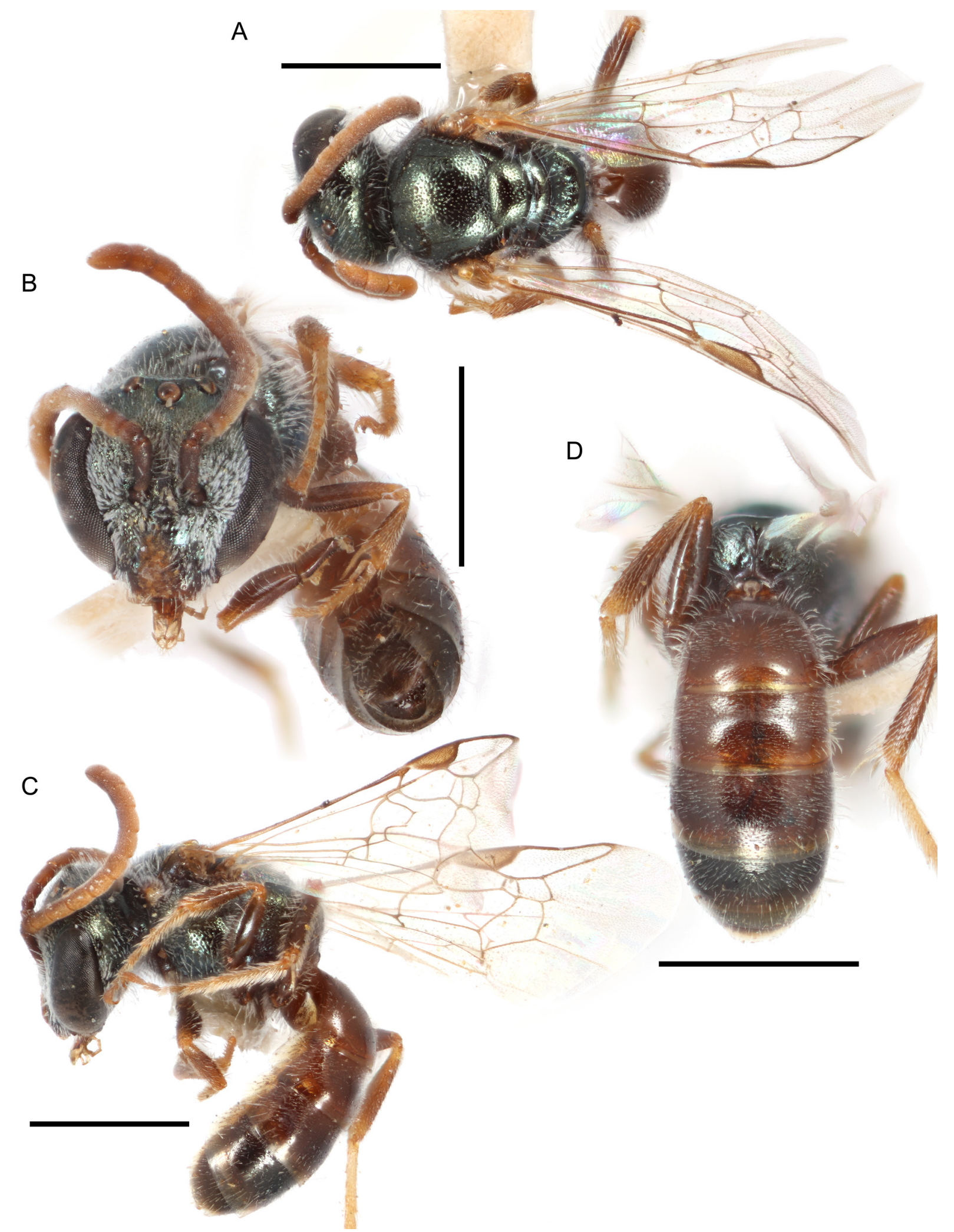

Fig. 14. Lasioglossum (D.) austerum sp. nov., ô. A. Dorsal habitus. B. Face. C. Lateral habitus. D. Propodeum and metasoma. Scale bars: $1 \mathrm{~mm}$. 
0.77 ( $\pm 0.03 \mathrm{SD}) ;$ scutum/scutellum length ratio 2.91 ( $\pm 0.18 \mathrm{SD}) ;$ scutellum/metanotum length ratio 1.54 ( $\pm 0.13 \mathrm{SD}$ ); metanotum/metapostnotum length ratio 0.66 ( $\pm 0.06 \mathrm{SD}$ ). Propodeum lateral carinae not reaching dorsal margin; oblique carina absent. T2 depressed apical rim length less than $50 \%$ of segment. $(\mathrm{n}=10)$

\section{Male}

Colouration. Head and mesosoma olive green; clypeus apical colour reddish brown; labrum reddish brown; mandible orange with black basal band and red tip; flagellum reddish brown dorsally, light brown ventrally; pronotal lobe reddish brown to orange; metasoma black to reddish brown with rims of terga and sterna and downcurved lateral areas of terga broadly translucent orange; legs reddish brown with femur-tibia joints, base and apex of tibiae, and tarsi orange; tegula orange; wing membrane hyaline, veins dark brown.

PubesCEnCE. Body hair colour white. Tomentum dense on paraocular area; sparse on face below eye emargination, gena, pronotal angle and lobe, and space between pronotal lobe and tegula. Scutum hair thin. Sterna hair short (1-1.5 OD), moderately plumose, sparse and erect. Wing hairs dark, short and sparse.

SuRfaCe SCULPTURE. Clypeus punctures dense ( $i \leq 1 \mathrm{pd})$, sculpture shiny; supraclypeal area punctures sparse ( $\mathrm{i}=1-3 \mathrm{pd})$, sculpture shiny; paraocular area punctures dense $(\mathrm{i} \leq 1 \mathrm{pd})$, sculpture weakly imbricate around antenna socket, otherwise shiny; frons punctures crowded ( $\mathrm{i}=0 \mathrm{pd})$, sculpture imbricate and finely areolate; vertex punctures dense and obscure laterally $(\mathrm{i}<1 \mathrm{pd})$, sparse and distinct medially $(i=1-3 \mathrm{pd})$, sculpture imbricate laterally, shiny medially; gena punctures sparse $(i=1-3 \mathrm{pd})$, sculpture shiny; postgena sculpture shiny and weakly lineate; tegula punctures absent; scutum punctures sparse ( $\mathrm{i}=1-3 \mathrm{pd})$, sculpture shiny, becoming weakly tessellate anteromedially; scutellum punctures moderately sparse ( $\mathrm{i}=1-2 \mathrm{pd}$ ), becoming dense marginally ( $\mathrm{i} \leq 1 \mathrm{pd})$, sculpture shiny; metanotum sculpture shiny and finely, sparsely punctate ( $\mathrm{i}=1-4 \mathrm{pd})$; metapostnotum rugae strong, subparallel, not reaching margin, sculpture weakly tessellate; preëpisternum sculpture areolate-rugulose; hypoepimeron punctures dense $(\mathrm{i}<1 \mathrm{pd})$, sculpture shiny; mesepisternum punctures dense $(\mathrm{i} \leq 1 \mathrm{pd})$, sculpture shiny; metepisternum sculpture rugoso-lineate dorsally, smooth and shiny ventrally; propodeum lateral face punctures obscure, sculpture rugulose; propodeum posterior face sculpture shiny; T1 anterior face sculpture weakly coriarious; T1 dorsal surface punctures sparse ( $\mathrm{i}=1-3 \mathrm{pd}$ ), sculpture shiny; T2 disc punctures sparse $(i=1-3 \mathrm{pd})$, disc sculpture shiny, rim punctures sparse medially $(i=1-4 \mathrm{pd})$, absent laterally, rim sculpture shiny.

STRUCTURE. Face length/width ratio 0.8. F1:pedicel length ratio 0.86; F2:F1 length ratio 1.75; F2 length/ width ratio 1.4; F9 length/width ratio 1.26. Forewing with 3 submarginal cells; pronotal angle obtuse; tegula shape normal. Intertegular distance $0.73 \mathrm{~mm}$. Scutum length/width ratio 0.92 ; scutum/scutellum length ratio 2.65; scutellum/metanotum length ratio 1.55 ; metanotum/metapostnotum length ratio 0.71 . Propodeum lateral carinae not reaching dorsal margin; oblique carina absent. $(\mathrm{n}=1)$

Genitalia. Not examined.

\section{Range}

Deserts of the Baja California peninsula from Punta Prieta to La Paz (Fig. 15).

\section{Floral records}

POLYGONACEAE Juss. Eriogonum Michx.

\section{DNA barcodes}

Not available. 


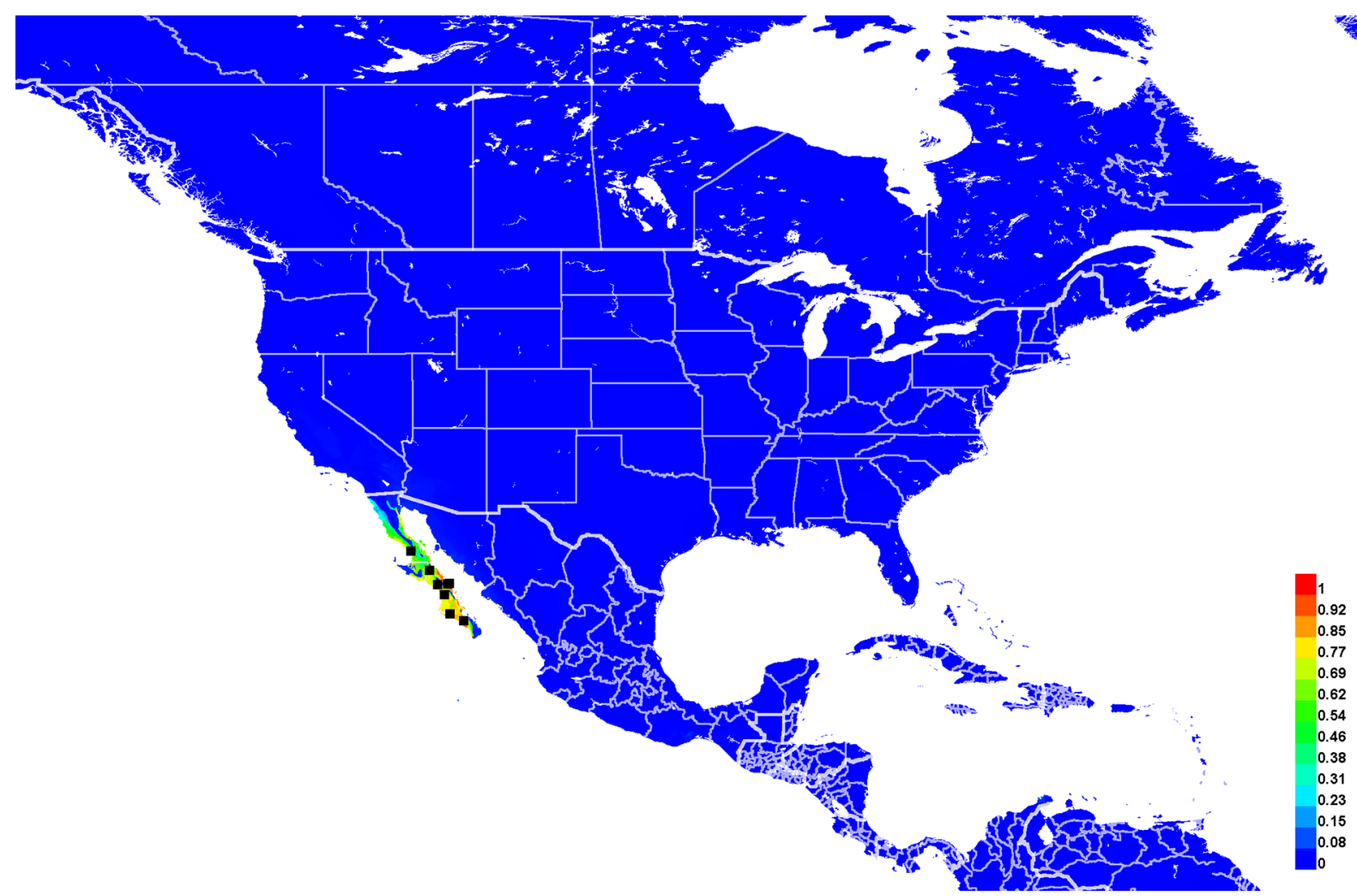

Fig. 15. Georeferenced collection records of Lasioglossum (D.) austerum sp. nov. (black squares) and predicted distribution by maximum entropy ecological niche modeling in Maxent (colour shading). Warmer colours indicate higher cloglog probability of occurrence.

Lasioglossum (Dialictus) cactorum sp. nov. urn:lsid:zoobank.org:act:A034807A-0DCB-48D2-9C47-EA6271D76562

Figs $16-18,93 \mathrm{C}, 114 \mathrm{~A}$

\section{Diagnosis}

Females of Lasioglossum cactorum sp. nov. can be recognized by the unusual rims of T1-3, which are extremely narrow, abruptly depressed, and with long and dense lateral hair fringes, head broad (face length/width ratio $\sim 0.83$ ) and often quite large, mesepisternum rugose or with very dense punctures at least dorsally ( $\mathrm{i}<1 \mathrm{pd}), \mathrm{T} 1$ without impunctate apicolateral oval patches, $\mathrm{T} 2-4$ with dark spiracular spots, and supraclypeal area sometimes black or golden-green medially. They are most similar to L. festinum sp. nov. and L. mesillense. Both of these species have the rims of T1-3 wide and flat and T1 with impunctate apicolateral oval patches. In addition, females of $L$. festinum sp. nov. have T2 lacking dark spiracular spots, and females of L. mesillense have the supraclypeal area always metallic blue-green.

Males of $L$. cactorum sp. nov. can be recognized by T1-4 strongly convex in lateral view, with very narrow, steeply depressed rims and no change in punctation or sculpture across the premarginal line, mesepisternum densely punctate $(\mathrm{i}<1 \mathrm{pd})$, propodeum dorsolateral slope smooth and distinctly punctate, face below eye emargination covered in dense tomentum, and metasoma entirely black. They are most similar to L. arenisaltans sp. nov., L. clematisellum, and L. minckleyi sp. nov. All of these species have the metasomal terga mostly flat in lateral view with sparser, less distinct punctures across the premarginal line. In addition, males of L. arenisaltans sp. nov. have the propodeum dorsolateral 
slope rugulose and metasoma often red-orange. Males of L. clematisellum are smaller (about $4 \mathrm{~mm}$ ), with the mesepisternum more sparsely punctate dorsally $(\mathrm{i}=1-2 \mathrm{pd}$ ), and propodeum dorsolateral slope weakly rugulose. Males of $L$. minckleyi sp. nov. have the face slightly shorter (length/width ratio $\sim 0.8$ ) and metasoma often partially red-orange.

\section{Etymology}

The specific epithet 'cactorum' is the Latin genitive plural of the noun 'cactus'. It refers to this species' frequent association with flowering plants in the family Cactaceae. An appropriate translation would be the cactus sweat bee.

\section{Material examined}

\section{Holotype}

UNITED STATES - Nevada • $q$; Clark Co., 2.2 mi. SSW of Mormon Well; $36.6147^{\circ} \mathrm{N}, 115.1111^{\circ} \mathrm{W}$; 2008 m a.s.l.; 14 Jun. 2004; E. Ahlstrom and D. Skandilis leg.; PCYU.

[Verbatim label: USA NV Clark Co. 2.2 mi. SSW Mormon Well 6588ft 11S E668922 N4053788 14Jun 2004 PnTrp, fl ylw, E. Ahlstrom, D. Skandilis / BBSL537603 // 2374F06 // HOLOTYPE / Lasioglossum (Dialictus) cactorum Gardner and Gibbs]

\section{Paratypes}

UNITED STATES - Arizona • 1 q; Maricopa Co., Mountain View Park; $33.5787^{\circ} \mathrm{N}, 112.0799^{\circ} \mathrm{W} ; 18$ May 2017; S.J. Hall et al. leg.; ASUHIC • 2 क o ; Grand Canyon Mile 1792; [ $\left.36^{\circ} \mathrm{N}, 112^{\circ} \mathrm{W}\right]$; $11 \mathrm{Jun}$. 1953; G.D. Butler leg.; UAIC • 1 क; same collection data as for preceding; USNM • 1 \%; Grand Canyon Mile 32; [36 ${ }^{\circ} \mathrm{N}, 112^{\circ} \mathrm{W}$; 6 Jun. 1953; G.D. Butler leg.; UAIC • 1 \%; Mojave Co., White Hills, Lk. Mead NRA; [35.83 ${ }^{\circ} \mathrm{N}, 114.32^{\circ} \mathrm{W}$ ]; $488 \mathrm{~m}$ a.s.1.; 30 Mar. 1993; T. Griswold and V. Tepedino leg.; ex Arctomecon californica; BBSL. - California 1 q; Inyo Co., $1 \mathrm{~km} \mathrm{SE}$ of Swansea; [36.52 ${ }^{\circ} \mathrm{N}, 117.9^{\circ} \mathrm{W}$; 19 Sep. 1993; T. Griswold leg.; BBSL • 1 ; San Bernardino Co., 9 air mi. S of Baker, Zzyzx Springs; [35.14 $\left.{ }^{\circ} \mathrm{N}, 116.1^{\circ} \mathrm{W}\right] ; 20-21 \mathrm{Apr}$. 1977; M. Buegler leg.; EMEC • 1 ; ; same location as for preceding; 22 Apr. 1977; Chemsak leg.; EMEC • 1 O ; Anza Borrego, Vallecito Crk.; $32.9157^{\circ} \mathrm{N}, 116.2255^{\circ} \mathrm{W}$; 8 Jun. 2013; K.E. Borchers leg.; ex Psorothamnus spinosus; WRME • 2 우여 San Diego Co., Borrego Springs (NW side), YPT, rocky draw $\mathrm{nr}$ base of Indian head; $33.2925^{\circ} \mathrm{N}, 116.4^{\circ} \mathrm{W} ; 213 \mathrm{~m}$ a.s.1.; $12 \mathrm{Jan}$. 2011; John/Derrin Pinto leg.; UCRC • 2 q o ; Riverside Co., Corn Spring, Chuckwalla Mts, 7.5 mi. SE of Desert Center; [33.626 N, $115.325^{\circ} \mathrm{W}$ ]; $488 \mathrm{~m}$ a.s.1.; 15-17 Jun. 1973; J.P. and K.E. Donahue leg.; LACM • 1 क; San Bernardino Co., Cove Spr., Granite Mts; [34.78 N, $\left.115.65^{\circ} \mathrm{W}\right] ; 1311$ m a.s.1.; 31 May

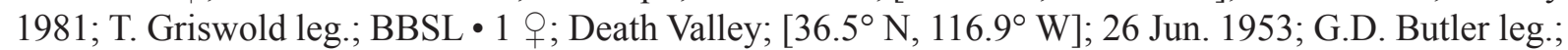
ex Stanleya; UAIC • 1 क; Death Valley National Monument; [36.5 N, $116.9^{\circ} \mathrm{W}$ ]; 31 Mar. 1976; M.A. Ivie leg.; MTEC • 1 क; Inyo Co., Death Valley NP, E of Travertine Spr.; $36.4388^{\circ} \mathrm{N}, 116.8258^{\circ} \mathrm{W} ; 16$ Mar. 1998; T. Griswold leg.; BBSL • 2 우; Riverside Co., Deep Canyon; $33.6475^{\circ} \mathrm{N}, 116.3806^{\circ} \mathrm{W}$;

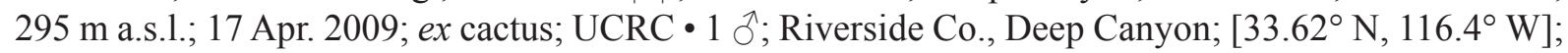
11 Jul. 1963; E.I. Schlinger leg.; UCRC • 1 đ; same location as for preceding; 13 Jun. 1963; E.I. Schlinger leg.; ex Eriogonum; UCRC • 1 q; same location as for preceding; 11 Nov. 1963; M.E. Irwin leg.; UCRC • 1 q; Inyo Co., DVNM Keane Spr.; [36.745 ${ }^{\circ}$ N, $116.902^{\circ}$ W]; 18 Apr. 1994; W.R. Bowlin leg.; ex Arctomecon merriamii; BBSL • 1 क; Inyo Co., DVNM Klare Spr.; [36.841 ${ }^{\circ} \mathrm{N}$, 117.091 ${ }^{\circ}$ W]; 15 Apr. 1994; W.R. Bowlin leg.; ex Argemone spp.; BBSL 2 o $\circ$; Inyo Co., DVNM Mes. Spr.; [36.964 N, $117.368^{\circ} \mathrm{W}$ ]; 13 Apr. 1994; W.R. Bowlin leg.; ex Arctomecon merriamii; BBSL • 1 q; Inyo Co., DVNM, Lost Burro Gap, Cottonwood Mts; $\left[36.746^{\circ} \mathrm{N}, 117.52^{\circ} \mathrm{W}\right] ; 914 \mathrm{~m}$ a.s.1.; 15 Oct. 1985; R.R. Snelling leg.; ex Ericameria nauseosa var. nauseosa; LACM • 1 क; Inyo Co., Emigrant Pass, E of Tecopa; [35.89 $\mathrm{N}, 116.08^{\circ} \mathrm{W}$ ]; 15 Sep. 1993; T. Griswold leg.; ex Eriogonum deflexum; BBSL • 2 o 우; Inyo Co., Grapevine Canyon, Death Valley N. M.; [37.005 N, $\left.117.356^{\circ} \mathrm{W}\right]$; 760 m a.s.l.; 16 Sep. 1993; T. Griswold leg.; ex Cleomella obtusifolia; BBSL • 1 क; San Bernardino 
Co., Mid Hills, SSE of Cima; [35.15 N, $115.43^{\circ}$ W]; 14 Sep. 1975; T. Griswold leg.; BBSL 1 q; San Bernardino Co., Norris Camp; 34.8089 ${ }^{\circ}$ N, $115.6281^{\circ}$ W; 1215 m a.s.1.; 13 May 2013; D. Yanega leg.; UCRC • 2 우; Riverside Co., Palm Springs; $33.6796^{\circ}$ N, $116.548^{\circ}$ W; 6-12 May 2014; F.D. Parker and T.D. McIntyre leg.; BBSL • 1 क; same location as for preceding; 13-16 May 2014; F.D. Parker and T.D. McIntyre leg.; BBSL -2 + $Q$; same location as for preceding; 14-18 Apr. 2014; F.D. Parker and T.D. McIntyre leg.; BBSL $\bullet 7$ 우; same location as for preceding; 28 Apr. 2014; F.D. Parker and T.D. McIntyre leg.; BBSL 1 1 ; Sunset Cove, Granites Res. Stn. Mojave Desert; $34.77903^{\circ}$ N, 115.66311 ${ }^{\circ}$ W; 24 Apr. 2018; J. Braun leg.; PCYU • 1 \&; same location as for preceding; 4 May 2018;

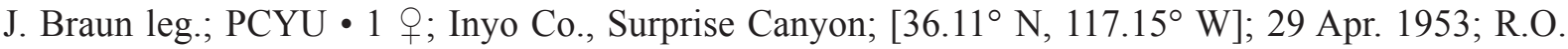
Schuster leg.; EMEC • 1 \%; same location as for preceding; 6 May 1961; D.Q. Cavagnaro leg.; UCDC •

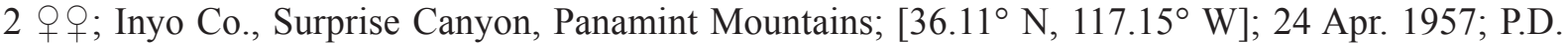
Hurd leg.; ex Eriogonum inflatum; EMEC • 1 O; San Bernardino Co., Willow Wash, Sec24 T13N R10E; $\left[35.2^{\circ} \mathrm{N}, 115.87^{\circ} \mathrm{W}\right]$; 10 May 1982; T. Griswold leg.; BBSL • 2 q $\circ$; Riverside Co., Windy Point; $33.892^{\circ} \mathrm{N}, 116.6393^{\circ} \mathrm{W}$; 24 Mar. 2019; J. Gardner leg.; ex Ferocactus cylindraceus; WRME • 1 q; San Bernardino Co., Zzyzx Springs; [35.14 N, 116.1 W]; 26 Mar. 1988; S. O'Keefe leg.; EMEC•

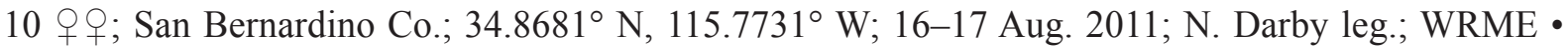
1 ㅇ ; San Bernardino Co.; $34.8794^{\circ}$ N, $115.7808^{\circ}$ W; 16-17 Aug. 2011; N. Darby leg.; WRME. Nevada - 2 q $\circ$; Clark Co., 0.2 mi. W of Hiko Spr.; 35.1675 ${ }^{\circ}$ N, $114.6813^{\circ}$ W; 609 m a.s.l.; 27 Apr. 2004; S. Higbee and E. Ahlstrom leg.; BBSL • 1 क; Clark Co., 0.5 mi. SW of Cabin Spr.; $36.5362^{\circ} \mathrm{N}$, 114.0844 ${ }^{\circ}$ W; 1368 m a.s.1.; 17 Aug. 2004; E. Ahlstrom leg.; ex Eriogonum deflexum; BBSL • 1 ㅇ; Clark Co., 2 mi. E of Sawmill Canyon; $36.7008^{\circ}$ N, 115.0492 ${ }^{\circ}$ W; 1439 m a.s.l.; 2 Sep. 2004; E. Rentz leg.; ex Eriogonum fasciculatum; BBSL 11 \%; Clark Co., 2.1 mi. $\mathrm{N}$ of Harris Spr.; $36.2707^{\circ} \mathrm{N}$, 115.5434 ${ }^{\circ}$ W; 1775 m a.s.1.; 22 Jun. 2004; L. Saul leg.; ex Argemone munita; BBSL • 4 우; same collection data as for holotype; BBSL • 1 क; Clark Co., 2.7 mi. ESE of Red Bluff Spr.; 36.6209 ${ }^{\circ} \mathrm{N}$, 114.2066 ${ }^{\circ}$ W; 15 Apr. 2005; S. Higbee leg.; PCYU • 2 oq ; Clark Co., 3.5 mi. SE of Hiko Spr.; $35.1252^{\circ} \mathrm{N}, 114.6432^{\circ} \mathrm{W} ; 235 \mathrm{~m}$ a.s.1.; 27 Apr. 2004; S. Higbee and E. Ahlstrom leg.; BBSL • 1 \%; 4 mi. E. of Glendale; [ $36.66^{\circ}$ N, $114.49^{\circ}$ W]; 20 Jun. 1939; Timberlake leg.; ex Sphaeralcea; UCRC •

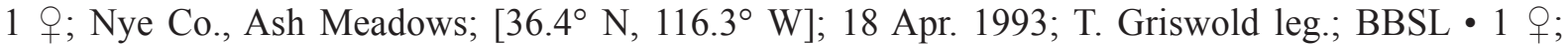

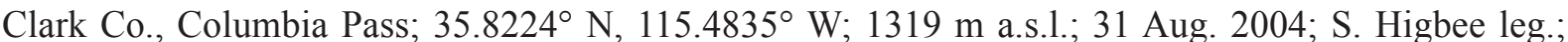
ex Eriogonum deflexum; BBSL • 1 क; Clark Co., E of Trapp Ridge; $36.3905^{\circ} \mathrm{N}, 114.1287^{\circ} \mathrm{W} ; 4$ Aug. 1998; M. Andres and C. Shultz leg.; BBSL • 4 우우 Clark Co., Gypsum Spring; $36.2093^{\circ}$ N, $114.912^{\circ}$ W; 12 Mar. 1998; T. Griswold leg.; BBSL • 1 o; Clark Co., Gypsum Wash; $36.2177^{\circ}$ N,

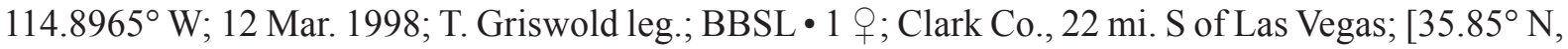
$115.25^{\circ}$ W]; 3 Apr. 1953; J.W. MacSwain leg.; EMEC • 5 o 9 ; Clark Co., Lee Canyon; $36.4476^{\circ} \mathrm{N}$, $115.5097^{\circ}$ W; $1252 \mathrm{~m}$ a.s.1.; 7 Sep. 2004; S. Higbee and T. Griswold leg.; BBSL • 3 q ; ; same location as for preceding; 7 Sep. 2004; S. Higbee and T. Griswold leg.; PCYU • 1 q; Clark Co., Mormon Well; 36.6752 ${ }^{\circ}$ N, $115.0952^{\circ}$ W; 1 Jul. 1998; M. Andres and C. Shultz leg.; BBSL 2 o $O$; Clark Co., Peek a Boo Canyon; $36.5065^{\circ} \mathrm{N}, 115.1477^{\circ} \mathrm{W} ; 16$ Jul. 1998; M. Andres and C. Shultz leg.; BBSL • 1 ; ; Clark Co., Pulsipher Wash; 36.8073 ${ }^{\circ}$ N, $114.1143^{\circ}$ W; 16 Sep. 1998; W. Bowlin leg.; BBSL • 1 \%; Clark Co., Sandy Valley; $35.852^{\circ}$ N, $115.6715^{\circ}$ W; 25 Aug. 1998; O. Messinger and C. Shultz leg.; BBSL • 1 q; Clark Co., Sheep Mountain; 35.7598 ${ }^{\circ}$ N, $115.3213^{\circ}$ W; 11 Aug. 1998; M. Andres, C. Shultz and T. Griswold leg.; BBSL • 1 q; Clark Co., Sheep Range; $36.4382^{\circ}$ N, 115.2983 W; 28 May 1998; F.D. Parker leg.; BBSL • 6 우; Clark Co., Wheeler Wash; $36.239^{\circ} \mathrm{N}, 115.8865^{\circ} \mathrm{W} ; 20$ Jul. 1998; M. Andres and C. Shultz leg.; BBSL. - Utah - 1 q; Washington Co., summit, road to Lytle Ranch Preserve; [37.14 N, 114.04 W]; 20 Aug. -9 Sep. 1987; W.J. Hanson and M. T. leg.; BBSL.

\section{Description}

\section{Female}

Colouration. Head and mesosoma blue to blue-green; clypeus apical colour orange, reddish brown, or nearly black; labrum orange; mandible orange with black basal spot or band and red tip; flagellum reddish 
brown dorsally, orange ventrally; pronotal lobe orange, reddish brown, or nearly black; metasoma red to orange with dark spiracular spots on T2-4, sometimes dark on discs of T4-5; legs reddish brown with femur-tibia joints and tarsi mostly orange; tegula orange; wing membrane hyaline, veins with subcosta dark brown, stigma pale amber, otherwise pale amber to brown.

Pubescence. Body hair colour white. Tomentum dense on paraocular area, frons, gena, pronotal collar and lobe, space between pronotal lobe and tegula, and metanotum anteromedially; sparse on preëpisternum, mesepisternum anteroventrally, metepisternum, T2-3 laterally, and T4 throughout. Scutum hair thin to moderately plumose. Wing hairs dark or light, short and dense. Acarinarial fan complete or incomplete, very sparse. T2-3 fringes very long and dense laterally, absent medially.

SURFACE SCULPTURE. Clypeus punctures irregularly sparse ( $\mathrm{i}<2 \mathrm{pd}$ ), slightly larger and sparser in apcial half, sculpture shiny; supraclypeal area punctures dense ( $i \leq 1 \mathrm{pd})$, slightly sparser medially ( $\mathrm{i}=1-2 \mathrm{pd})$, sculpture shiny; paraocular area punctures dense $(i<1 \mathrm{pd})$, sometimes becoming moderately sparse around antenna socket $(\mathrm{i}=1-2 \mathrm{pd})$, sculpture shiny; frons punctures dense $(\mathrm{i}<1 \mathrm{pd})$, sculpture shiny; vertex punctures minute, sparse ( $\mathrm{i}=1-3 \mathrm{pd})$, obscure medially, sculpture shiny; gena punctures moderately dense ( $\mathrm{i}=1-2 \mathrm{pd}$ ), sculpture shiny; postgena sculpture weakly ruguloso-lineate to imbricate; tegula punctures absent; scutum punctures dense $(\mathrm{i} \leq 1 \mathrm{pd})$, becoming sparse submedially and anteromedially ( $i=1-3$ pd), sculpture shiny, becoming tessellate anteromedially; scutellum punctures dense marginally and on median line ( $i \leq 1 \mathrm{pd})$, moderately sparse submedially ( $i=1-2 \mathrm{pd}$ ), diversopunctate, sculpture shiny; metanotum sculpture shiny and minutely reticulate-punctate medially, becoming finely rugulose laterally; metapostnotum rugae weak, anastomosing, not reaching margin, sculpture shiny on posterior margin, otherwise tessellate; preëpisternum sculpture reticulate-punctate; hypoepimeron punctures crowded ( $\mathrm{i}=0 \mathrm{pd}$ ), sculpture ruguloso-punctate; mesepisternum punctures crowded ( $\mathrm{i}=0 \mathrm{pd}$ ), becoming slightly sparser ventrally ( $\leq 1 \mathrm{pd}$ ), sculpture shiny, sometimes becoming areolate-rugulose dorsally; metepisternum sculpture lineate dorsally, rugulose medially, imbricate ventrally; propodeum lateral face sculpture tessellate; propodeum posterior face sculpture tessellate and sparsely punctate $(i=1-6 \mathrm{pd})$, becoming shiny medially; $\mathrm{T} 1$ anterior face sculpture shiny; $\mathrm{T} 1$ dorsal surface punctures fine, moderately sparse $(i=1-3 \mathrm{pd})$, becoming sparser apicolaterally $(\mathrm{i}=2-6 \mathrm{pd})$, sculpture shiny; $\mathrm{T} 2$ disc punctures fine, sparse $(i=1-3 \mathrm{pd})$, disc sculpture shiny, rim punctures fine, sparse $(i=1-3 \mathrm{pd})$, rim sculpture shiny.

Structure. Face length/width ratio 0.83 ( $\pm 0.02 \mathrm{SD}$ ). Clypeus projecting $\sim 50 \%$ below suborbital tangent; clypeal area length/width ratio $0.37( \pm 0.03 \mathrm{SD})$; apicolateral denticles rounded obtuse points; supraclypeal area length/width ratio $0.75( \pm 0.03 \mathrm{SD})$. Forewing with 3 submarginal cells; pronotal angle obtuse; tegula shape normal. Intertegular distance 0.98 ( $\pm 0.07 \mathrm{SD}) \mathrm{mm}$. Scutum length/width ratio 0.86 ( $\pm 0.03 \mathrm{SD}$ ); scutum/scutellum length ratio 2.92 ( $\pm 0.16 \mathrm{SD}$ ); scutellum/metanotum length ratio 1.52 ( $\pm 0.13 \mathrm{SD})$; metanotum/metapostnotum length ratio 0.8 ( $\pm 0.07 \mathrm{SD})$. Propodeum lateral carinae not reaching dorsal margin; oblique carina very weak or absent. T2 depressed apical rim linear. $(n=10)$

\section{Male}

Colouration. Head and mesosoma blue to blue-green; clypeus apical colour reddish brown to orange; labrum orange; mandible orange with black basal band and red tip; flagellum reddish brown, sometimes orange ventrally; pronotal lobe reddish brown to orange; metasoma black to reddish brown with downcurved lateral areas of terga becoming orange to translucent yellow; legs reddish brown with femur-tibia joints, base and apex of tibiae, and tarsi orange; tegula orange; wing membrane hyaline, veins brown to dark brown.

Pubescence. Body hair colour white. Tomentum dense on face below eye emargination, gena, pronotal angle and lobe, and space between pronotal lobe and tegula; sparse on episterna. Scutum hair thin to 


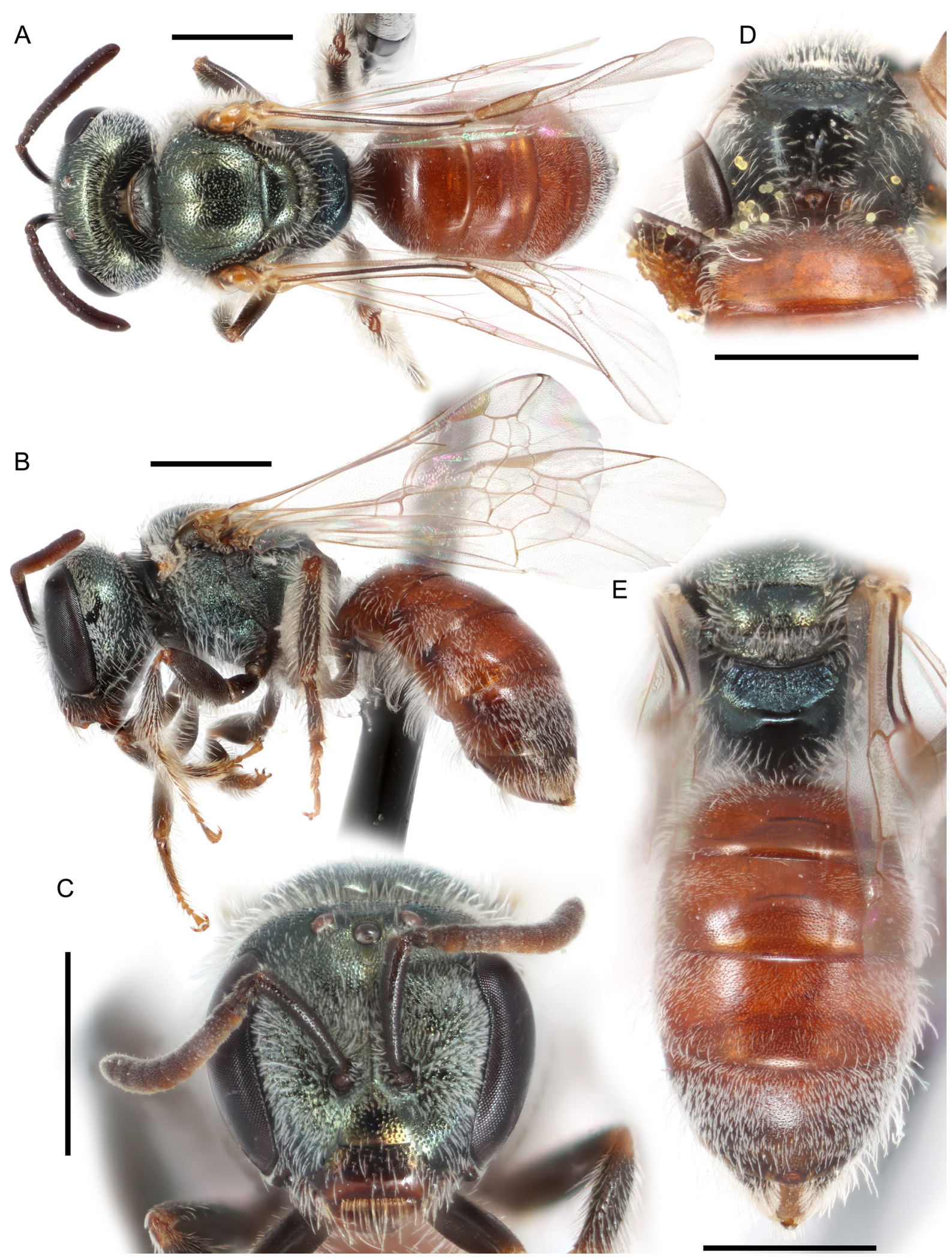

Fig. 16. Lasioglossum (D.) cactorum sp. nov., ․ A. Dorsal habitus. B. Lateral habitus. C. Face. D. Propodeum. E. Metasoma. Scale bars: $1 \mathrm{~mm}$. 
A
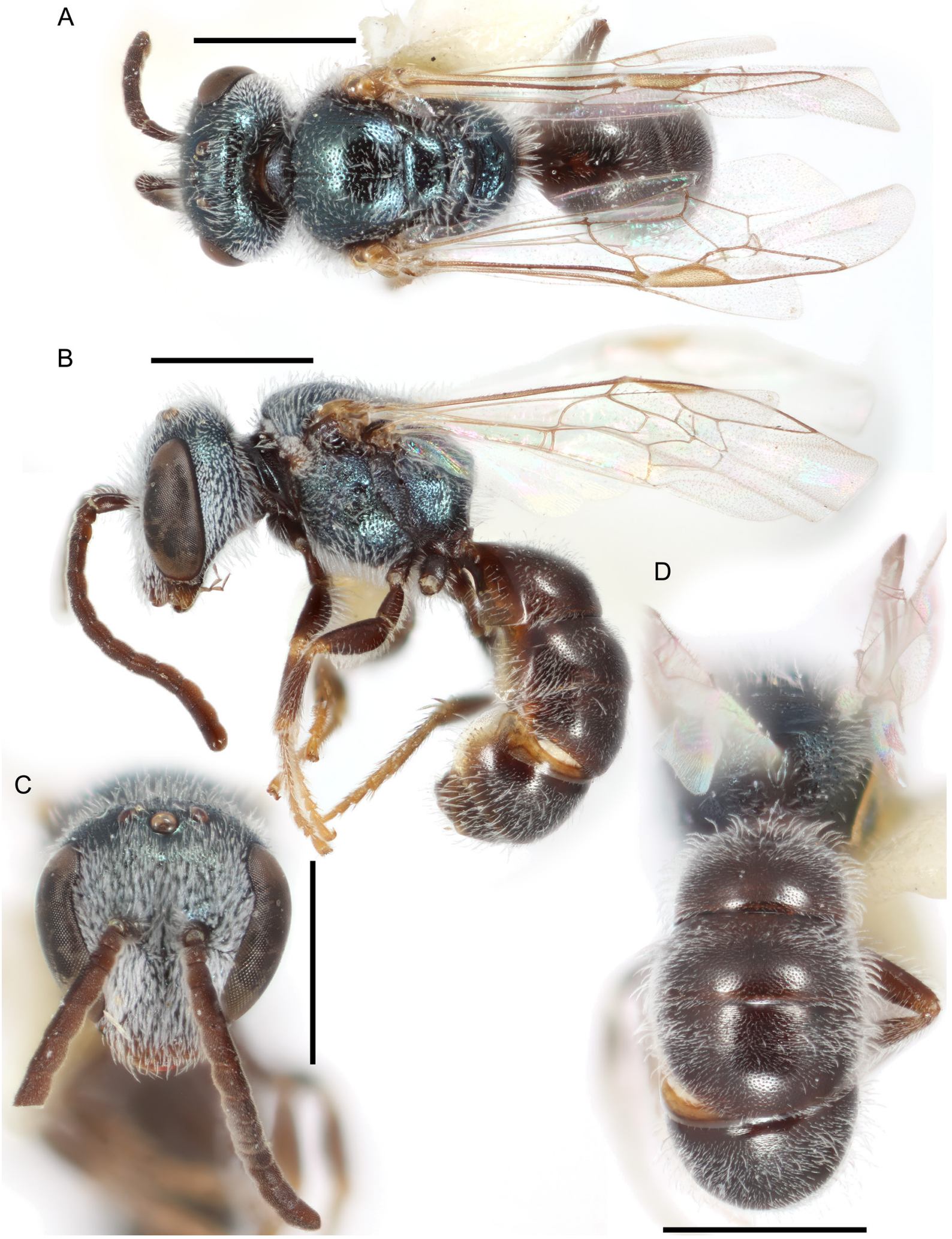

Fig. 17. Lasioglossum (D.) cactorum sp. nov., ô. A. Dorsal habitus. B. Lateral habitus. C. Face. D. Propodeum and metasoma. Scale bars: $1 \mathrm{~mm}$. 
moderately plumose. Sterna hair short (1-1.5 OD), densely plumose, dense and erect. Wing hairs dark or light, short and sparse.

SuRfaCe SCULPTURE. Clypeus punctures dense $(i \leq 1 \mathrm{pd})$, sculpture shiny; supraclypeal area punctures dense $(\mathrm{i}<1 \mathrm{pd})$, sculpture shiny; paraocular area punctures dense $(\mathrm{i}<1 \mathrm{pd})$, sculpture shiny; frons punctures dense $(\mathrm{i}<1 \mathrm{pd})$, sculpture shiny; vertex punctures moderately dense $(\mathrm{i}=1-2 \mathrm{pd})$, sculpture shiny; gena punctures moderately dense $(\mathrm{i}=1-2 \mathrm{pd})$, sculpture shiny; postgena sculpture lineate, sometimes becoming shiny anteriorly; tegula punctures absent; scutum punctures sparse $(i=1-3 \mathrm{pd})$, becoming dense marginally ( $\mathrm{i} \leq 1 \mathrm{pd})$, sculpture shiny; scutellum punctures sparse ( $\mathrm{i}=1-3 \mathrm{pd})$, becoming dense marginally ( $i \leq 1 \mathrm{pd}$ ), sculpture shiny; metanotum sculpture shiny and densely punctate $(i \leq 1 \mathrm{pd})$, becoming rugulose laterally; metapostnotum rugae strong, anastomosing, not reaching margin, sculpture shiny to weakly tessellate; preëpisternum sculpture areolate; hypoepimeron punctures crowded ( $\mathrm{i}=0 \mathrm{pd})$, sculpture areolate; mesepisternum punctures dense ( $\mathrm{i}<1 \mathrm{pd}$ ) to crowded ( $\mathrm{i}=0 \mathrm{pd})$, sculpture shiny; metepisternum sculpture lineate dorsally, weakly rugulose ventrally; propodeum lateral face punctures dense ( $\mathrm{i} \leq 1 \mathrm{pd}$ ), sculpture tessellate, becoming shiny posteriorly; propodeum posterior face sculpture shiny and densely punctate ( $i \leq 1 \mathrm{pd}$ ), becoming sparse to absent medially; T1 anterior face sculpture shiny; $\mathrm{T} 1$ dorsal surface punctures moderately sparse $(\mathrm{i}=1-3 \mathrm{pd})$, sculpture shiny; $\mathrm{T} 2$ disc punctures moderately dense ( $\mathrm{i}=1-2 \mathrm{pd})$, disc sculpture shiny, rim punctures moderately sparse $(\mathrm{i}=1-3 \mathrm{pd})$, rim sculpture shiny.

StRUCTURE. Face length/width ratio 0.85 ( $\pm 0.02 \mathrm{SD})$. F1:pedicel length ratio 1.01 ( $\pm 0.18 \mathrm{SD}) ; \mathrm{F} 2: \mathrm{F} 1$ length ratio 1.85 ( $\pm 0.03 \mathrm{SD}) ; \mathrm{F} 2$ length/width ratio $1.68( \pm 0.05 \mathrm{SD}) ; \mathrm{F} 9$ length/width ratio 1.18 $( \pm 0.01 \mathrm{SD})$. Forewing with 3 submarginal cells; pronotal angle obtuse; tegula shape normal. Intertegular distance $0.88( \pm 0.11 \mathrm{SD}) \mathrm{mm}$. Scutum length/width ratio $0.93( \pm 0.01 \mathrm{SD})$; scutum/scutellum length ratio $2.65( \pm 0.02 \mathrm{SD})$; scutellum/metanotum length ratio $1.8( \pm 0.1 \mathrm{SD}) ;$ metanotum/metapostnotum length ratio $0.62( \pm 0.03 \mathrm{SD})$. Propodeum lateral carinae not reaching dorsal margin; oblique carina absent. Metasomal terga strongly convex. $(n=2)$

Genitalia. Not examined.

\section{Range}

Mojave and Sonoran Deserts of southern Nevada and California, southwestern Utah, and Arizona (Fig. 18).

\section{Floral records}

ASTERACEAE Giseke: Ericameria:E.nauseosa (Pall. ex Pursh) G.L. Nesom \& Baird: E.n. var. nauseosa (Pall. ex Pursh) G.L. Nesom \& Baird • BRASSICACEAE Burnett: Stanleya Nutt. • CACTACEAE Juss. Ferocactus Britton \& Rose: F. cylindraceus (Engelm.) Orcutt - CLEOMACEAE Bercht. \& J. Presl: Cleomella DC. C. obtusifolia Torr. \& Frém. - FABACEAE Juss. Psorothamnus: P. spinosus (A.Gray) Barneby • MALVACEAE Juss. Sphaeralcea A. St.-Hil. PAPAVERACEAE Juss. Arctomecon Torr. \& Frém. A. californicum Torr. \& Frém. • A. merriamii Coville • Argemone L. A. munita Durand \& Hilg. • POLYGONACEAE Juss. Eriogonum Michx. E. deflexum Torr. $\bullet$ E. fasciculatum Benth. $\bullet$ E. inflatum Torr.

\section{DNA barcodes}

Six sequences available (BOLD process IDs: DLII909-07, DLII910-07, DLII1028-07, DLII1691-09, BHTT020-09, DLIII179-19; BIN: BOLD:AAE1189). Lasioglossum cactorum sp. nov. differs from all other western red-tailed L. (Dialictus) by 5 fixed substitutions: 213(C), 318(T), 379(A), 553(A), and 573(C) (Supplementary file 4). 


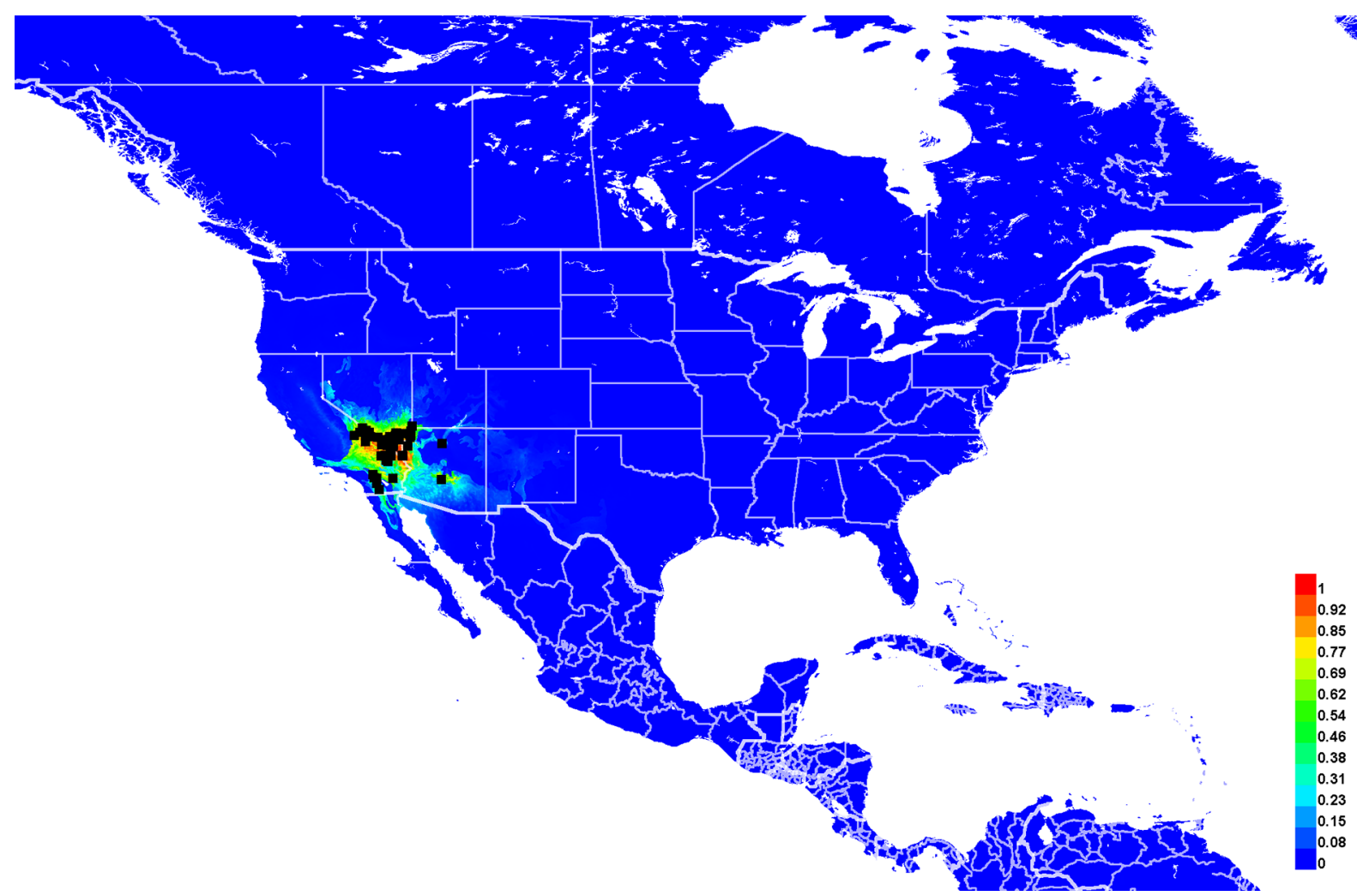

Fig. 18. Georeferenced collection records of Lasioglossum (D.) cactorum sp. nov. (black squares) and predicted distribution by maximum entropy ecological niche modeling in Maxent (colour shading). Warmer colours indicate higher cloglog probability of occurrence.

\section{Remarks}

Although Lasioglossum cactorum sp. nov. has been frequently collected from cactus flowers, it is not oligolectic on them. There is considerable variation in the size of this species, especially of the head, and in the sculpturing of the mesepisternum. This variation, combined with the year-round phenology of this species and phylogenetic data, suggests it is likely social.

An undescribed species from the Chihuahuan Desert has similarly narrow and strongly depressed rims of T1-3, but the metasoma is all black. DNA barcodes and other morphological features also distinguish these two species.

Lasioglossum (Dialictus) cembrilacus sp. nov. urn:1sid:zoobank.org:act:082AC082-F9FB-47A1-9779-9867AF24F57D

Figs 19-21, 92B

\section{Diagnosis}

Females of Lasioglossum cembrilacus sp. nov. can be easily recognized by the body mostly covered in extremely dense, long, yellowish, plumose hairs and tomentum, mesothorax shiny and densely punctate ( $\mathrm{i} \leq 1 \mathrm{pd}$ ), face relatively long (length/width ratio $\sim 0.84$ ), and metasomal terga sometimes with metallic green reflections. They are most similar to Lasioglossum albohirtum (Crawford, 1907) (see Gibbs 2010 for a redescription of this species). Females of L. albohirtum have the hair of the paraocular area shorter 
and sparser, scutum slightly more sparsely punctate $(\mathrm{i}=1-2 \mathrm{pd})$, and metasoma and clypeus apical margin never red or orange.

Males of L. cembrilacus sp. nov. can be recognized by the tomentum long and dense in the lower paraocular area and absent or very sparse over the rest of the body, red or orange colouration largely absent except on ventral surface of flagellum, propodeum dorsolateral slope shiny and distinctly punctate, flagellomeres relatively long (F2 about 2 times as long as F1), mesepisternum and metasomal terga relatively densely punctate ( $\mathrm{i} \leq 1$ and $\mathrm{i}=1-2 \mathrm{pd}$, respectively), and metasomal terga with weak metallic green reflections at least in basal half of T1. They are most similar to L. zephyrus (Smith, 1853), L. pictum, and L. decorum sp. nov. Males of L. zephyrus and L. pictum have the propodeum dorsolateral slope rugulose and face with more extensive tomentum. Males of $L$. decorum sp. nov. have the flagellomeres relatively short (F2 about 1.5 times as long as F1), face without tomentum, frons dull, and mesepisternum and metasomal terga more sparsely punctate ( $\mathrm{i}=1-2$ and $1-4 \mathrm{pd}$, respectively).

\section{Etymology}

The specific epithet cembrilacus is formed from the New Latin noun 'cembra' ('timber', especially in reference to the tree Pinus cembra L.) plus the Latin genitive noun 'lacus' (lake; spelled identically in both nominative and genitive case). It is both a dedication to Philip Hunter Timberlake, and a reference to this species' mountain habitat with its many pines and lakes. An appropriate translation would be Timberlake's sweat bee.

\section{Material examined}

\section{Holotype}

UNITED STATES - California - + ; Mariposa Co., 1.7 mi. SE by E of Ostrander Rocks; $37.679^{\circ} \mathrm{N}$, $119.567^{\circ}$ W; 2091 m a.s.1.; 3 Jul. 2005; H. Ikerd leg.; PCYU.

[Verbatim label: USA CA Mariposa Co. 1.7 mi. SEbyE Ostrander Rocks $2091 \mathrm{~m} \mathrm{11S} \mathrm{E273625}$ N4173300 3 Ju105 PnTrp fl ylw, H.W.Ikerd / BBSL613033 // 1845A10 // HOLOTYPE / Lasioglossum (Dialictus) cembrilacus Gardner and Gibbs]

\section{Paratypes}

UNITED STATES - California • 1 क ; Mariposa Co., 1.8 mi. SE by E of Ostrander Rocks; $37.6768^{\circ} \mathrm{N}$, 119.5669 ${ }^{\circ}$ W; 2096 m a.s.1.; 28-29 Jul. 2005; L. Fuerst leg.; PCYU • 2 oq ; Plumas Co., 14 mi. W of Johnsville; [39.76 N, $120.96^{\circ} \mathrm{W}$ ]; 15 Jul. 1967; J. S. Buckett leg.; UCDC • 1 क; Amador Co., 2 mi.

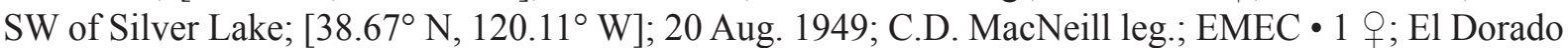
Co., 2 mi. S of Meyers; $\left[38.83^{\circ} \mathrm{N}, 120.02^{\circ} \mathrm{W}\right.$; 24 Jul. 1955; E.I. Schlinger leg.; UCDC • 1 q; Alta; $\left[39.21^{\circ} \mathrm{N}, 120.81^{\circ} \mathrm{W}\right]$; 29 Jun. 1933; BBSL 1 o ; Siskiyou Co., Ash Creek Ranger Stn, 9 mi. E of McCloud; [41.262 ${ }^{\circ} \mathrm{N}, 121.95^{\circ} \mathrm{W}$ ]; 1067 m a.s.l.; 13-16 Jun. 1974; D. Green leg.; EMEC • 1 q; same location as for preceding; 7-9 Jun. 1974; D. Green leg.; ex Ceanothus velutinus; EMEC • 1 9 ; same location as for preceding; 7-9 Jun. 1974; J. Powell leg.; ex Ceanothus cordulatus; EMEC • 1 9; same location as for preceding; 7-9 Jun. 1974; J. Powell leg.; EMEC • 1 \%; same location as for preceding; 7-9 Jun. 1974; R. Coville leg.; ex Ceanothus cordulatus; EMEC • 3 q $ᄋ$; same location as for preceding; 7-9 Jun. 1974; J. Chemsak leg.; EMEC • 1 क; Tehama Co., Battle Creek Meadows; [40.34 N, $\left.121.6^{\circ} \mathrm{W}\right]$;

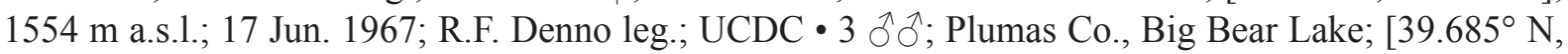
$120.67^{\circ}$ W]; 2103 m a.s.l.; 19 Aug. 1978; N.J. Smith leg.; UCDC • 2 qo ; Shasta Co., Big Spring; [40.643ํ N, $121.468^{\circ}$ W]; 23 May 1941; C.D. Michener leg.; EMEC • 4 우우 Glenn Co., Black Butte; [39.73 $\left.\mathrm{N}, 122.87^{\circ} \mathrm{W}\right]$; 1981-2134 m a.s.1.; 14 Jun. 1972; D. Veirs leg.; EMEC • 1 o; El Dorado

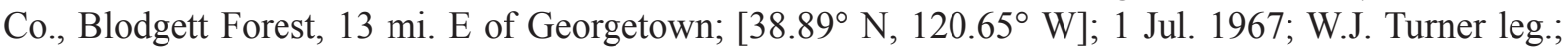

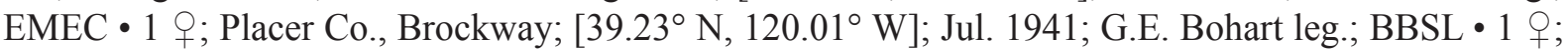
Plumas Co., Buck's Lake; [39.87 N, $121.17^{\circ}$ W]; 1 Jul. 1949; J.W. MacSwain leg.; EMEC • 1 o; same 
location as for preceding; 1 Jul. 1949; E.I. Schlinger leg.; UCDC • 2 q ; Placer Co., Carnelian Bay, Lake Tahoe; $\left[39.23^{\circ} \mathrm{N}, 120.08^{\circ} \mathrm{W}\right] ; 8$ Jul. 1964; C. Slobodchikoff leg.; CAS • 1 o; Carnelian Bay, Lake Tahoe; [39.23 N, $120.08^{\circ} \mathrm{W}$ ]; 24 Jun. 1954; R.H. Goodwin leg.; EMEC • 2 qo+; same location as for preceding; 17 Jun. 1958; R.M. Bohart leg.; UCDC • 1 क; El Dorado Co., China Flat; [38.754 $4^{\circ}$,

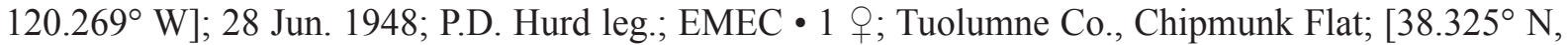
$\left.119.69^{\circ} \mathrm{W}\right] ;{ }_{1}$ Jul. 1962; J. Powell leg.; EMEC • 1 क; same location as for preceding; 13 Jun. 1962;

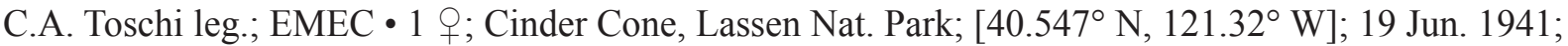
ex Ribes; EMEC • 1 q; Nevada Co., Donner Pass; [39.32 ${ }^{\circ}$ N, $\left.120.32^{\circ} \mathrm{W}\right]$; 4 Jul. 1962; J. Powell leg.; EMEC • 1 P; Trinity Co., Eagle Creek; [41.13ํ N, $\left.122.7^{\circ} \mathrm{W}\right]$; 13 Jul. 1949; A.T. McClay leg.; UCDC • 1 ; same location as for preceding; 13 Jul. 1949; A.T. McClay leg.; UCDC • 1 ; ; Calaveras Co., Garnet Hill; [38.48 N, $120.25^{\circ}$ W]; 16 Jun. 1957; H.K. Court leg.; UCDC • 3 o 9 ; Sierra Co., Gold Lake; [39.68 ${ }^{\circ}$ N, $120.64^{\circ}$ W]; 26 Jul. 1921; C.L. Fox leg.; CAS • 2 o $\circ$; Hat Lake, Lassen Nat. Park;

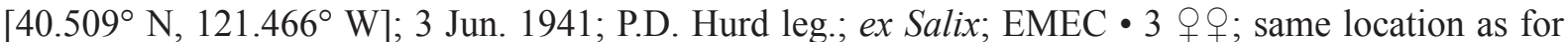
preceding; 3 Jun. 1941; EMEC • 1 क; same location as for preceding; 3 Jun. 1941; C.D. Michener leg.; EMEC • 2 우; same location as for preceding; 3 Jun. 1941; C.D. Michener leg.; ex Salix; EMEC • 5 우; same location as for preceding; 3 Jun. 1941; C.D. Michener leg.; ex Arctostaphylos; EMEC • 15 q $ᄋ$; Alpine Co., Hope Valley; [38.75 N, 119.94 W]; 22 Jul. 1955; E.E. Gilbert and N.A. Walker leg.; EMEC • 2 क $\circ$; same location as for preceding; 9 Jul. 1948; J.W. MacSwain leg.; EMEC • 1 \%; Fresno

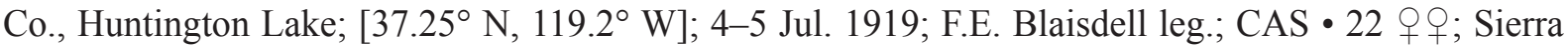

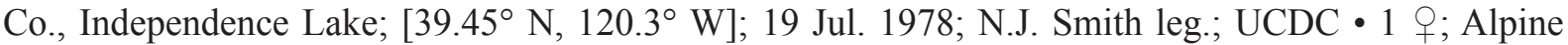

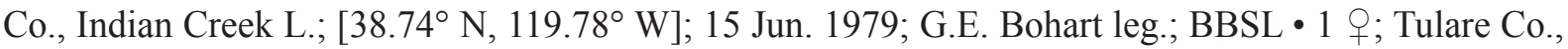

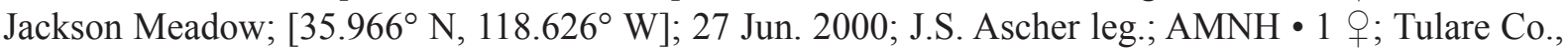
Jackson Meadow; $35.966^{\circ}$ N, $118.626^{\circ}$ W; 27 Jun. 2000; J.S. Ascher leg.; AMNH • 1 क; Lake Tahoe; [39. $\left.2^{\circ} \mathrm{N}, 120.1^{\circ} \mathrm{W}\right] ; 1897 \mathrm{~m}$ a.s.1.; 16 Jul. 1949; E.G. Linsley leg.; ex Solidago sp.; EMEC • 1 O; Inyo Co., Lone Pine Canyon; [36.59 ${ }^{\circ}$, $118.23^{\circ} \mathrm{W}$ ]; 16 Jul. 1952; BBSL • 1 क ; Mariposa Co., Merced Lake Trail, 1.9 mi. E by $\mathrm{N}$ of Bunnell Pt.; $37.744^{\circ} \mathrm{N}, 119.432^{\circ} \mathrm{W} ; 2141 \mathrm{~m}$ a.s.1.; 3 Jun. 2005; H. Briggs and E. Stephens leg.; PCYU • 1 क; Siskiyou Co., Mount Shasta; $\left[41.34^{\circ} \mathrm{N}, 122.25^{\circ} \mathrm{W}\right] ; 1829 \mathrm{~m}$ a.s.l.; 13 Jul. 1958; J. Powell leg.; EMEC • 1 क; Trinity Co., Mountain Meadow Ranch head Coffee Creek; [41.08 $\left.{ }^{\circ} \mathrm{N}, 122.94^{\circ} \mathrm{W}\right] ; 1554 \mathrm{~m}$ a.s.1.; 8-10 Jul. 1969; C. Slobodchikoff leg.; EMEC • 2 q ; ; Nevada Co., near Hobart Mills; [39.4 ${ }^{\circ}$ N, $120.18^{\circ}$ W]; 18 Jun. 1962; F.D. Parker leg.; UCDC • 1 क ; Shasta Co., Old Station; [40.675 $\mathrm{N}, 121.431^{\circ} \mathrm{W}$ ]; 16 Jun. 1941; EMEC • 1 \%; El Dorado Co., Pino Grande, SW of

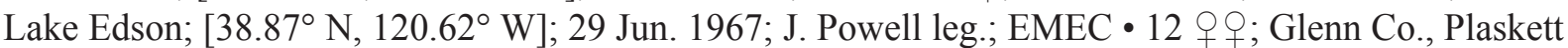
Meadows; [39.726 ${ }^{\circ} \mathrm{N}, 122.845^{\circ} \mathrm{W}$ ]; $1920 \mathrm{~m}$ a.s.1.; 14 Jun. 1972; J. Doyen leg.; ex Cistanthe umbellata;

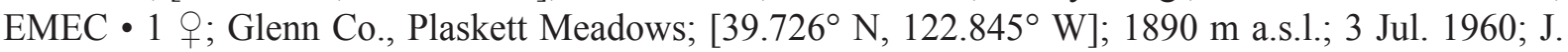
Powell leg.; EMEC • 3 q $q$; same location as for preceding; 3 Jul. 1960; J. Powell leg.; EMEC • 1 ; Nevada Co., Russell Vly; [39.43 N, $120.1^{\circ}$ W]; 26 Jun. 1964; D.F. Veirs leg.; BBSL • 1 ; ; Nevada Co., Sagehen Creek; [39.432 ${ }^{\circ}$ N, $120.241^{\circ}$ W]; 22 Jun. 1972; S.F. Casey leg.; UCDC • 3 q 9 ; Nevada Co., Sagehen Creek near Truckee; [39.432 ${ }^{\circ}$ N, $120.241^{\circ}$ W]; 2-8 Jun. 2003; Schulmeister and Keating leg.; AMNH • 1 क; Nevada Co., Sagehen Creek, near Hobart Mills; $\left[39.43^{\circ}\right.$ N, $120.2^{\circ}$ W]; 29 Jun. 1962;

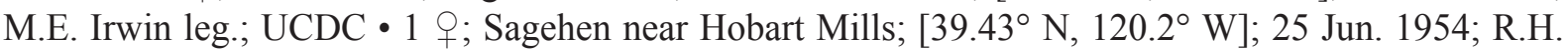
Goodwin leg.; EMEC • 1 o; Mono Co., Sardine Creek; [38.32 ${ }^{\circ}$ N, 119.61 ${ }^{\circ}$ W]; 27 Jun. 1957; John M. Burns leg.; EMEC • 2 우 ; Fresno Co., Shaver Lake; [37.1ํ N, 119.3 W]; 27 Jul. 1968; J.B. Hoy leg.;

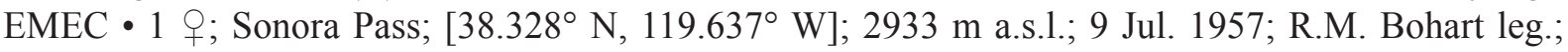
UCDC • 1 क; Tuolumne Co., Strawberry; $\left[38.2^{\circ}\right.$ N, 120.01 $\left.{ }^{\circ} \mathrm{W}\right] ; 18$ Jun. 1951; E.G. Linsley and J.W. MacSwain leg.; BBSL $\bullet 1$; same collection data as for preceding; EMEC 12 $q$; ; Placer Co., Summit; [39. $2^{\circ}$ N, $120.3^{\circ}$ W]; 2134 m a.s.l.; W.M. Giffard leg.; CAS • 1 क; Placer Co., Tahoe National Forest, Blackwood Creek, 4 km W of Kaspian Rec. site, Lake Tahoe; [39.1 $\left.{ }^{\circ} \mathrm{N}, 120.2^{\circ} \mathrm{W}\right]$; 19 Jul. 1983; Thomas W. Davies leg.; CAS • 2 o 9 ; El Dorado Co., Tallac; [38.94 N, $120.05^{\circ} \mathrm{W}$; 1829 m a.s.l.; W.M. Giffard leg.; CAS • 1 क; Siskiyou Co., Trinity Alps, Caribou Mt.; $\left[41.03^{\circ}\right.$ N, 122.97 W]; 1920-2134 m a.s.l.; 9 Jul. 1969; J. Powell leg.; EMEC • 2 q $q$; Siskiyou Co., Trout Creek 
at Forest Service Rd 43N13 (17 air mi. NE of McCloud); [41.43 N, 121.904 W]; 10 Jun. 1974;

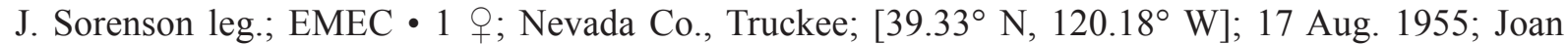
Linsley leg.; ex Chrysothamnus; EMEC • 1 ; Trinity Co.; $\left[40.5^{\circ} \mathrm{N}, 123^{\circ} \mathrm{W}\right]$; 7 Jun. 1934; R.M. and G.E. Bohart leg.; CAS. - Nevada • 6 우; Lake Tahoe, Incline Village; $\left[39.25^{\circ} \mathrm{N}, 119.95^{\circ} \mathrm{W}\right] ; 20-25$ May 1985; W.W. Middlekauff leg.; CAS • 1 क; Mount Rose; [39.34 N, $119.92^{\circ}$ W]; 12 Aug. 1936; Bryant leg.; CAS ・ 1 \%; Nevada; ANSP.

\section{Description}

\section{Female}

Colouration. Head and mesosoma blue-green to golden-green; clypeus apical colour reddish brown to orange; labrum reddish brown to orange; mandible orange with black base and red tip; flagellum reddish brown, sometimes becoming orange apically and ventrally; pronotal lobe reddish brown to orange; metasoma orange with discs of terga usually dark metallic green at base (rarely entirely orange to green) and dark spiracular spots on T3-4 and sometimes also T2; legs reddish brown with femurtibia joints, femora apices, and tarsi orange; hind femur mostly orange with brown anterior spot; tegula pale amber; wing membrane infuscated, veins with subcosta brown, otherwise pale amber.

Pubescence. Body hair colour golden to pale yellow. Tomentum dense on entire body except clypeus, postgena, pronotum anterior face, mesepisternum ventrally, propodeum dorsal and posterior face, T1 medially, and sterna. Scutum hair densely plumose. Wing hairs light, short and dense. Acarinarial fan complete, large and dense. T2 fringes dense, T3 fringes dense.

SuRfaCe SCULPTURE. Clypeus punctures dense in basal half $(\mathrm{i}<1 \mathrm{pd})$, irregularly sparse in apical half $(\mathrm{i}<2 \mathrm{pd})$, sculpture imbricate basally, shiny apically; supraclypeal area punctures dense $(\mathrm{i} \leq 1 \mathrm{pd})$, sculpture shiny; paraocular area punctures crowded ( $\mathrm{i}=0 \mathrm{pd})$, sculpture shiny; frons punctures crowded ( $i=0 \mathrm{pd})$, sculpture shiny; vertex punctures dense $(i<1 \mathrm{pd})$, sculpture shiny; gena punctures dense ( $i<1 \mathrm{pd}$ ), sculpture shiny; postgena sculpture shiny; tegula punctures absent; scutum punctures dense ( $i \leq 1 \mathrm{pd}$ ), becoming crowded laterad of parapsidal lines ( $\mathrm{i}=0 \mathrm{pd}$ ), sculpture shiny, becoming weakly tessellate anteromedially; scutellum punctures crowded marginally and on median line ( $\mathrm{i}=0 \mathrm{pd})$, dense to moderately sparse submedially ( $i \leq 2 \mathrm{pd}$ ), sculpture shiny; metanotum sculpture shiny with crowded punctures $(\mathrm{i}=0 \mathrm{pd})$; metapostnotum rugae strong, parallel or slightly anastomosing, not reaching margin, sculpture weakly imbricate; preëpisternum sculpture areolate-rugulose; hypoepimeron punctures dense $(i<1 \mathrm{pd})$, sculpture shiny; mesepisternum punctures dense $(i<1 \mathrm{pd})$, sculpture shiny, sometimes weakly lineate; metepisternum sculpture lineate dorsally, reticulate-punctate ventrally; propodeum lateral face sculpture tessellate; propodeum posterior face sculpture weakly tessellate with distinct, sparse punctures ( $\mathrm{i}=1-3 \mathrm{pd})$; $\mathrm{T} 1$ anterior face sculpture coriarious; $\mathrm{T} 1$ dorsal surface punctures moderately dense ( $i=1-2 \mathrm{pd})$, sculpture shiny; $\mathrm{T} 2$ disc punctures moderately dense $(i=1-2 \mathrm{pd})$, disc sculpture coriarious at base, shiny apically, rim punctures fine, moderately sparse $(i=1-3 \mathrm{pd})$, rim sculpture shiny.

Structure. Face length/width ratio 0.84 ( $\pm 0.01 \mathrm{SD}$ ). Clypeus projecting $\sim 75 \%$ below suborbital tangent; clypeal area length/width ratio $0.43( \pm 0.02 \mathrm{SD})$; apicolateral denticles low rounded knobs; supraclypeal area length/width ratio 0.63 ( $\pm 0.06 \mathrm{SD})$. Forewing with 3 submarginal cells; pronotal angle obtuse; tegula shape normal. Intertegular distance $1.04( \pm 0.07 \mathrm{SD}) \mathrm{mm}$. Scutum length/width ratio $0.88( \pm 0.06 \mathrm{SD}) ;$ scutum/scutellum length ratio $2.83( \pm 0.3 \mathrm{SD}) ;$ scutellum/metanotum length ratio 1.47 ( $\pm 0.14 \mathrm{SD})$; metanotum/metapostnotum length ratio $0.75( \pm 0.06 \mathrm{SD})$. Propodeum lateral carinae not reaching dorsal margin; oblique carina absent. T2 depressed apical rim length less than $50 \%$ of segment. $(n=10)$ 


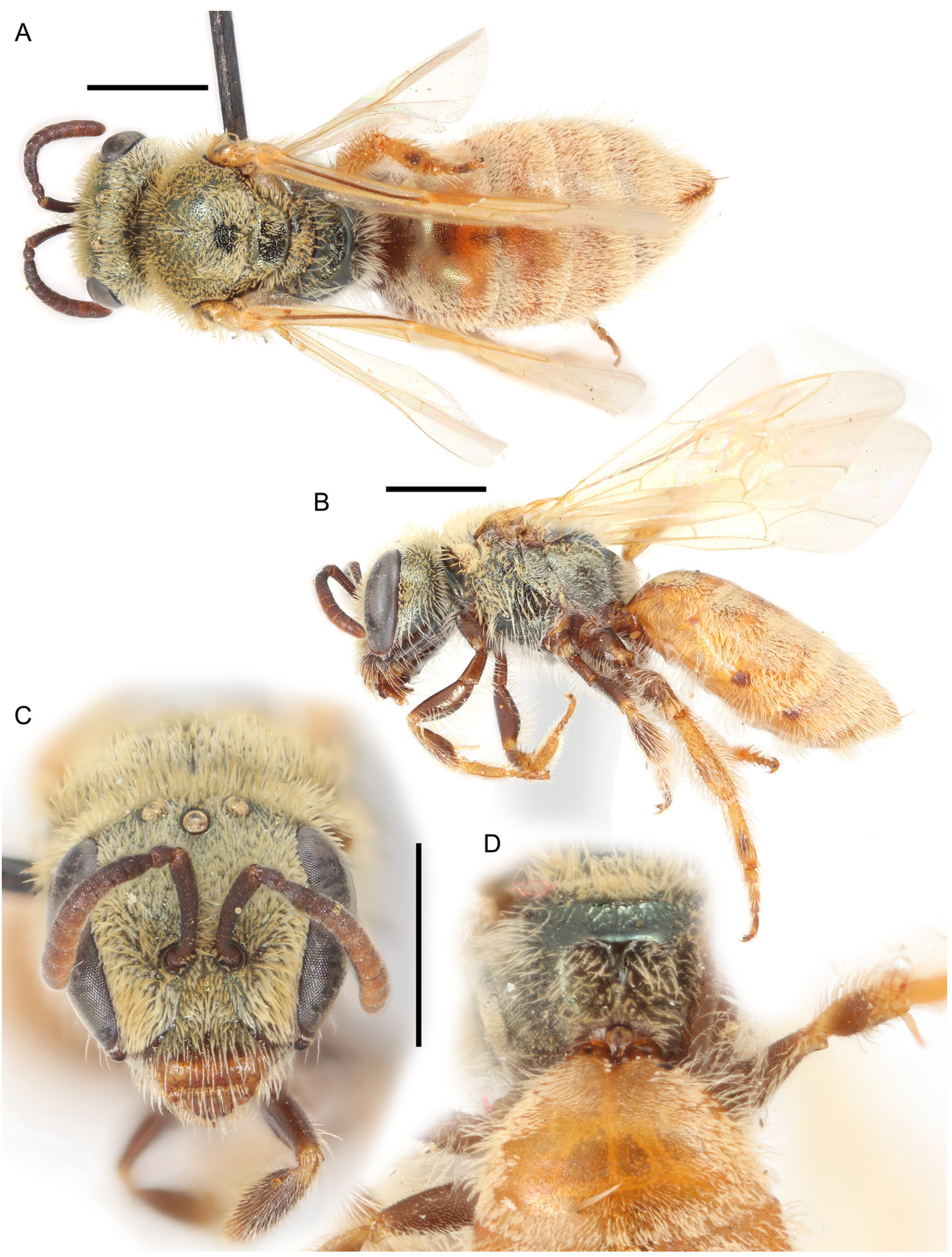

Fig. 19. Lasioglossum (D.) cembrilacus sp. nov., ․ A. Dorsal habitus. B. Lateral habitus. C. Face. D. Propodeum. Scale bars: $1 \mathrm{~mm}$. 


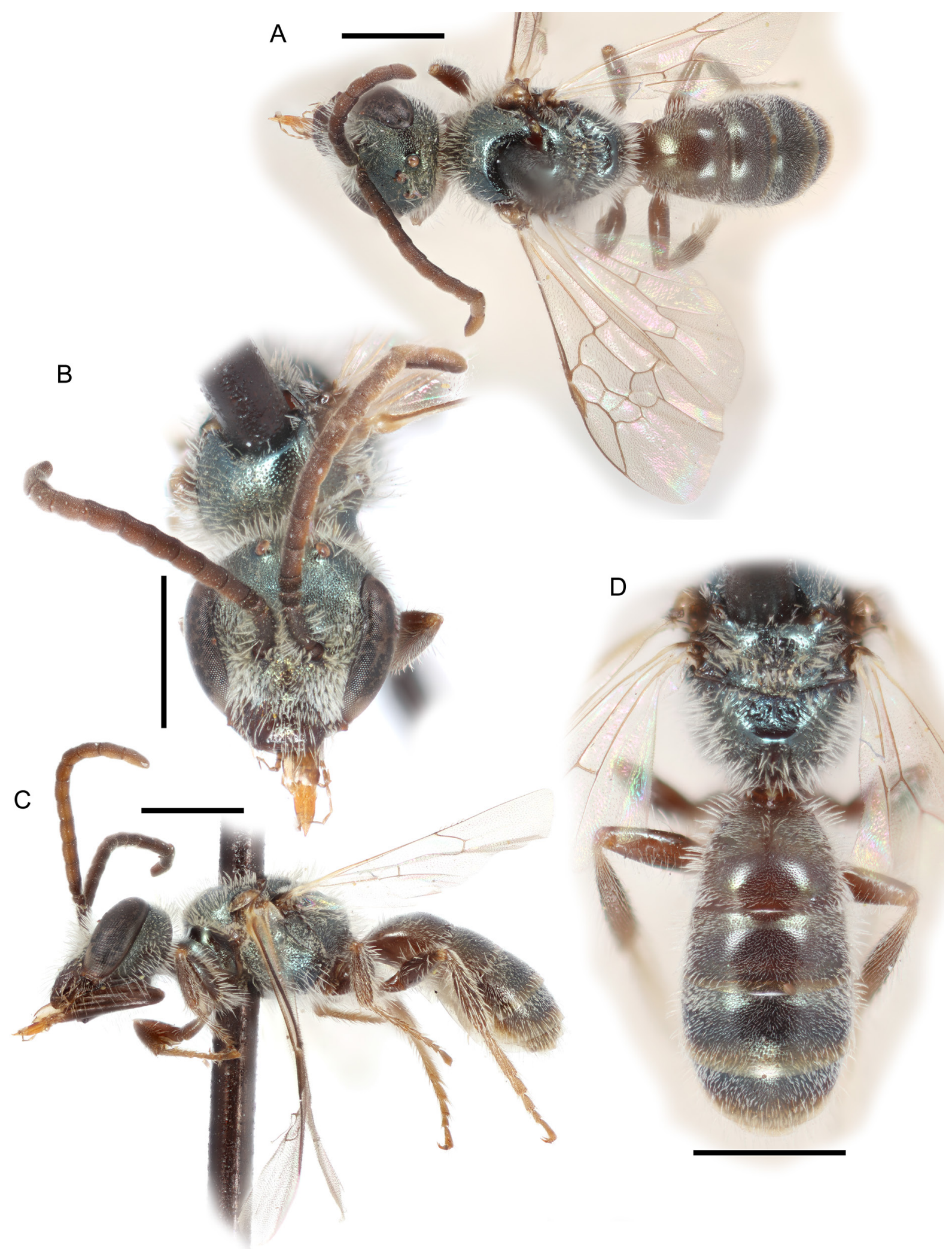

Fig. 20. Lasioglossum (D.) cembrilacus sp. nov., $\widehat{\jmath}$. A. Dorsal habitus. B. Face. C. Lateral habitus. D. Propodeum and metasoma. Scale bars: $1 \mathrm{~mm}$. 


\section{Male}

Colouration. Head and mesosoma blue-green to golden-green; clypeus apical colour black; labrum black; mandible brown with black basal band and red tip; flagellum black to reddish brown dorsally, orange to brown ventrally; pronotal lobe reddish brown; metasoma black with discs of terga metallic green marginally and rims of terga and sterna and downcurved lateral areas of terga broadly translucent brown; legs reddish brown with femur-tibia joints, base and apex of tibiae, and tarsi light brown; tegula brown; wing membrane lightly infuscated, veins dark brown.

Pubescence. Body hair colour pale yellow to white. Tomentum dense on paraocular area, pronotal angle and lobe, and space between pronotal lobe and tegula; sparse on gena, metanotum, metepisternum, T2-4 basolaterally, and T5 throughout. Scutum hair thin to moderately plumose. Sterna hair short (1-1.5 OD), thin to moderately plumose, sparse and erect. Wing hairs dark, long and dense.

SurfaCe SCULPTURE. Clypeus punctures dense in basal third ( $\mathrm{i} \leq 1 \mathrm{pd})$, irregularly sparse apically ( $i<3 \mathrm{pd}$ ), diversopunctate, sculpture shiny; supraclypeal area punctures dense ( $i \leq 1 \mathrm{pd})$, sculpture shiny; paraocular area punctures dense $(i<1 \mathrm{pd})$, sculpture shiny; frons punctures dense $(\mathrm{i}<1 \mathrm{pd})$, sculpture shiny; vertex punctures dense ( $i \leq 1 \mathrm{pd}$ ), sometimes obscure medially, sculpture shiny, sometimes rugulose medially; gena punctures fine, moderately sparse ( $i=1-3 \mathrm{pd})$, sculpture shiny; postgena sculpture shiny to weakly lineate; tegula punctures absent; scutum punctures dense $(\mathrm{i}<1 \mathrm{pd})$, becoming sparse submedially $(i=1-3 \mathrm{pd})$, sculpture shiny; scutellum punctures sparse $(i=1-3 \mathrm{pd})$, becoming dense marginally and on median line ( $i \leq 1 \mathrm{pd})$, sculpture shiny; metanotum sculpture shiny and moderately sparsely punctate $(i=1-2 \mathrm{pd})$, sometimes rugulose laterally; metapostnotum rugae strong, subparallel or anastomosing, nearly reaching margin, sculpture shiny; preëpisternum sculpture areolate; hypoepimeron punctures dense $(\mathrm{i}<1 \mathrm{pd})$, sculpture shiny; mesepisternum punctures dense (i $\leq 1 \mathrm{pd}$ ), sculpture shiny; metepisternum sculpture ruguloso-lineate; propodeum lateral face punctures dense $(i \leq 1 \mathrm{pd})$, sculpture shiny to imbricate and weakly rugulose; propodeum posterior face sculpture shiny and densely punctate ( $\mathrm{i} \leq 1 \mathrm{pd})$; $\mathrm{T} 1$ anterior face sculpture shiny; $\mathrm{T} 1$ dorsal surface punctures moderately dense ( $\mathrm{i}=1-2 \mathrm{pd})$, becoming sparse on rim ( $\mathrm{i}=1-4 \mathrm{pd})$, sculpture shiny; $\mathrm{T} 2$ disc punctures moderately dense $(i=1-2 \mathrm{pd})$, disc sculpture shiny, rim punctures sparse $(i=1-4 \mathrm{pd})$, rim sculpture shiny.

Structure. Face length/width ratio 0.85 ( $\pm 0.01 \mathrm{SD})$. F1:pedicel length ratio 1.08 ( $\pm 0.1 \mathrm{SD}) ; \mathrm{F} 2: \mathrm{F} 1$ length ratio $1.95( \pm 0.17 \mathrm{SD}) ; \mathrm{F} 2$ length/width ratio $1.37( \pm 0.13 \mathrm{SD})$; F9 length/width ratio 1.16 ( $\pm 0.17 \mathrm{SD})$. Forewing with 3 submarginal cells; pronotal angle obtuse; tegula shape normal. Intertegular distance 0.9 ( $\pm 0.03 \mathrm{SD}) \mathrm{mm}$. Scutum length/width ratio 0.78 ; scutum/scutellum length ratio 2.26; scutellum/metanotum length ratio $1.63( \pm 0.18 \mathrm{SD})$; metanotum/metapostnotum length ratio 0.69 ( \pm 0 SD). Propodeum lateral carinae not reaching dorsal margin; oblique carina absent. $(n=3)$

Genitalia. Not examined.

\section{Range}

High elevations in the Sierra Nevada Mountains of California and Nevada (Fig. 21).

\section{Floral records}

ASTERACEAE Giseke: Chrysothamnus - Solidago • ERICACEAE Juss. Arctostaphylos Adans. • GROSSUlARIACEAE DC. Ribes L. - PORTULACACEAE Juss. Cistanthe: C. umbellata (Torr.) Hershkovitz • RHAMNACEAE Juss. Ceanothus L. C. cordulatus Kellogg • C. velutinus Douglas ex Hook. $•$ SALICACEAE Mirb. Salix L. 


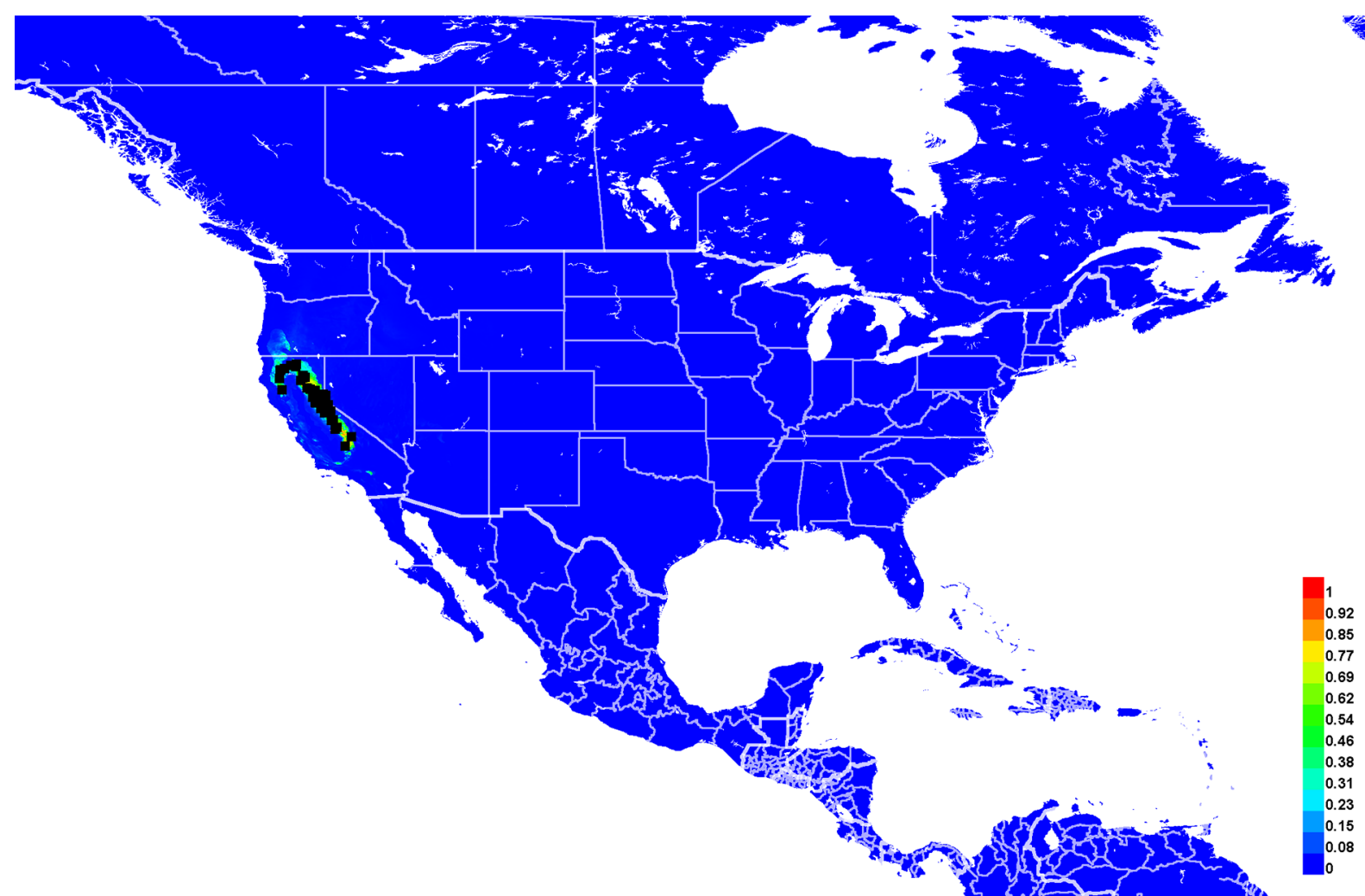

Fig. 21. Georeferenced collection records of Lasioglossum (D.) cembrilacus sp. nov. (black squares) and predicted distribution by maximum entropy ecological niche modeling in Maxent (colour shading). Warmer colours indicate higher cloglog probability of occurrence.

\section{DNA barcodes}

Three sequences available (BOLD process IDs: DLII286-07, DLII292-07, DLII298-07; BIN: BOLD:ABZ3799). Lasioglossum cembrilacus sp. nov. differs from all other western red-tailed L. (Dialictus) by 4 fixed substitutions: 336(C), 399(C), 477(T), and 595(A) (Supplementary file 4).

\section{Remarks}

Lasioglossum cembrilacus sp. nov. is a mountain habitat specialist restricted to very high elevations ( $>1000 \mathrm{~m}$ and often $>2000 \mathrm{~m}$ a.s.l.). It is infrequently collected but, when found, may occur in large numbers.

Lasioglossum (Dialictus) clastipedion sp. nov. urn:lsid:zoobank.org:act:1C13534B-7D94-4001-844A-C75CDAC05B63

Figs 22-24, 90B, 96B, 104B

\section{Diagnosis}

Females of Lasioglossum clastipedion sp. nov. can be recognized by the characters for the Lasioglossum clematisellum species complex (below), in addition to the metasomal terga with dense tomentum on T2-3 basolaterally and T4 throughout, frons and vertex usually with weak microsculpture slightly 
dulling the surface, scutum with most hairs relatively densely plumose and punctures of anteromedian margin distinct, and head and mesosoma often golden-green with more yellowish hair dorsally. They are most similar to L. clematisellum and can only be separated with great difficulty, especially if specimens are worn. Females of L. clematisellum have the metasomal terga with no tomentum; this is the most reliable character. In addition, females of $L$. clematisellum usually have the scutum with thinner and more weakly plumose hair and less distinct punctures of the anteromedian margin, and the head and mesosoma blue-green with all white hair.

Males of L. clastipedion sp. nov. can be recognized by the characters for the Lasioglossum clematisellum species complex (below) in addition to T2-3 with sparse basolateral tomentum, scutum with most hairs densely plumose, appearing thickened, and frons with dense punctures $(\mathrm{i}<1 \mathrm{pd})$. They are most similar to L. clematisellum, L. julipile sp. nov., and L. perditum sp. nov. Males of L. clematisellum have T2-3 with no tomentum and scutum with most hairs thin and weakly plumose. Males of $L$. julipile sp. nov. and L. perditum sp. nov. have the frons more sparsely punctate $(i=1-3 \mathrm{pd})$. Worn specimens are extremely difficult to distinguish from L. clematisellum.

\section{Etymology}

The specific epithet clastipedion is formed from the Latinized Greek adjective 'klastos' ('broken', 'shattered') and the Greek genitive plural noun 'pedion' ('of plains'). It refers to this species' restricted distribution in the canyonlands of southern Utah and northern Arizona, which resemble the fictional Shattered Plains of Brandon Sanderson's novel The Way of Kings. An appropriate translation would be the Shattered Plains sweat bee.

\section{Material examined}

Holotype

UNITED STATES - Utah • + ; Emery Co., Wild Horse Creek N of Goblin Valley; [38.57 ${ }^{\circ} \mathrm{N}, 110.82^{\circ} \mathrm{W}$; 1463 m a.s.1.; 26-28 Jul. 1982; Parker/Griswold leg.; BBSL.

[Verbatim label: UTAH EmeryCo 4800' Wild Horse Cr N of GoblinVly VII-26-28-82/Parkers/Griswold // HOLOTYPE / Lasioglossum (Dialictus) clastipedion Gardner and Gibbs]

\section{Paratypes}

UNITED STATES - Arizona • 7 우; Mojave Co., 11 mi. N of Mt. Trumbull; [36.56 $\left.{ }^{\circ} \mathrm{N}, 113.14^{\circ} \mathrm{W}\right]$; 9 Jun. 1994; G. Bryant leg.; ex Argemone; BBSL • 1 क ; Beaver Dam; $\left[36.91^{\circ} \mathrm{N}, 113.95^{\circ} \mathrm{W}\right]$; 20 Jun. 1939; Timberlake leg.; ex Eriogonum trichopes; UCRC • 1 q; Fredonia; [36.94 ${ }^{\circ} \mathrm{N}, 112.53^{\circ} \mathrm{W}$ ]; 20 Sep. 1956; G.F. Knowlton leg.; ex Medicago sativa; UAIC. - Utah • 1 §̊; Emery Co., Bell Canyon, San Rafael Reef; [38.589 N, 110.808 W]; 1524 m a.s.1.; 26 Jul. 1983; F.A. Parker and T. Griswold leg.; CUIC • 1 क; Garfield Co., Blind Trail Wash, SSE of Notom; [38.07 N, $111.055^{\circ} \mathrm{W}$ ]; $1615 \mathrm{~m}$ a.s.l.; 15 Sep. 1983; J. Parker leg.; BBSL 1 O'; same location as for preceding; 15 Sep. 1983; Parker and Griswold leg.; CUIC • 3 우; Cedar City; $\left[37.68^{\circ} \mathrm{N}, 113.06^{\circ} \mathrm{W}\right] ; 9$ May 1961; G.E. Bohart leg.; ex Salix; BBSL -3 우; Washington Co., Central; $\left[37.42^{\circ} \mathrm{N}, 113.62^{\circ} \mathrm{W}\right] ; 19$ Sep. 1956; Knowlton and Goodarzi leg.; ex Chrysothamnus sp.; SEMC • 1 क; Garfield Co., Escalante Riv., 1.3 mi. W of jct. Death Hollow; $37.7808^{\circ} \mathrm{N}, 111.5353^{\circ} \mathrm{W}$; 13 May 2001; C. Davidson leg.; ex Tamarix sp.; BBSL • 1 \% ; Kane Co., Glendale; [37.32 ${ }^{\circ}$ N, $112.6^{\circ}$ W]; 13 Apr. 1962; G.F. Knowlton leg.; ex Salix; SEMC • 1 ○; Garfield Co., Grand Gulch, Hall Creek; [37.6 ${ }^{\circ}$ N, $\left.110.87^{\circ} \mathrm{W}\right] ; 1372 \mathrm{~m}$ a.s.1.; 22 Aug. 1980; A.S. Menke, F.D. Parker and Kurt A. Menke leg.; CUIC • 1 क; Garfield Co., Hog Spr.; $\left[37.977^{\circ}\right.$ N, $110.52^{\circ}$ W]; $1341 \mathrm{~m}$ a.s.1.; 29 Aug. 1985; T.L. Griswold leg.; BBSL • 2 qo ; Kanab; $\left[37.05^{\circ} \mathrm{N}, 112.53^{\circ} \mathrm{W}\right] ; 6$ Aug. 1954; G.T. Knowlton and D.W. Davis leg.; SEMC • 1 क; Garfield Co., Pine Creek, 4.5 mi. N of

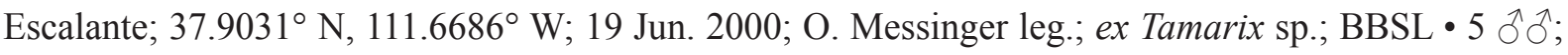


St. George; [37.1 ${ }^{\circ}$ N, $113.57^{\circ}$ W]; 9 Sep. 1954; G.F. Knowlton leg.; SEMC • 1 \%; Washington Co.,

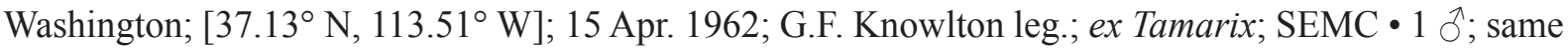
collection data as for holotype; CUIC • 1 O; Garfield Co., Wiley Creek; 20 Jun. 2000; F.D. Parker leg.;

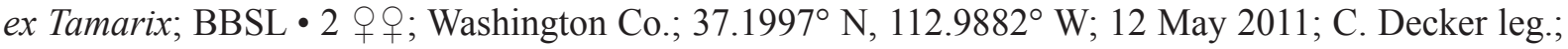
WRME.

\section{Description}

\section{Female}

Colouration. Head and mesosoma blue-green to golden-green; clypeus apical colour reddish brown to orange; labrum reddish brown to orange; mandible orange with black basal spot or band and red tip; flagellum black to reddish brown dorsally, reddish brown to orange ventrally; pronotal lobe reddish brown to orange; metasoma red to orange with dark spiracular spots on T3-4; legs reddish brown with femur-tibia joints, tibia apices, and tarsi mostly orange; tegula pale amber; wing membrane hyaline, veins with subcosta dark brown, otherwise pale amber.

PubesCEnCE. Body hair colour pale yellow to white. Tomentum dense on paraocular area, gena, pronotal collar and lobe, space between pronotal lobe and tegula, metanotum medially, metepisternum, T3 basally, and T4 throughout; sparse on supraclypeal area, preëpisternum, mesepisternum, and T2-3 basolaterally. Scutum hair densely plumose. Wing hairs light, very short and sparse. Acarinarial fan complete or nearly so, sparse. T2 fringes sparse, T3 fringes sparse.

SuRfaCe SCUlPtURE. Clypeus punctures dense in basal half ( $1<1 \mathrm{pd}$ ), large and irregularly spaced apically ( $<2 \mathrm{pd}$ ), sculpture shiny, sometimes weakly tessellate basally; supraclypeal area punctures moderately dense $(i=1-2 \mathrm{pd})$, sometimes becoming sparse medially ( $\mathrm{i}=1-4 \mathrm{pd}$ ) or dense laterally (i $<1$ pd), sculpture shiny, sometimes becoming weakly tessellate laterally; paraocular area punctures dense to moderately sparse ( $\mathrm{i} \leq 2 \mathrm{pd}$ ), sculpture shiny; frons punctures dense to moderately sparse $(i \leq 2 \mathrm{pd})$, sculpture shiny or weakly tessellate; vertex punctures dense $(i \leq 1 \mathrm{pd})$ and sometimes obscure laterally, sometimes moderately sparse $(i=1-2 \mathrm{pd})$ and distinct medially, sculpture imbricate or weakly so, sometimes shiny medially; gena punctures dense ( $\mathrm{i} \leq 1 \mathrm{pd}$ ), sculpture shiny; postgena sculpture tessellate and sometimes weakly rugulose; tegula punctures absent; scutum punctures moderately dense ( $\mathrm{i} \leq 2 \mathrm{pd}$ ), becoming sparser submedially ( $\mathrm{i}=1-3 \mathrm{pd})$ and denser posteromedially $(\mathrm{i}<1 \mathrm{pd})$, sculpture shiny to weakly tessellate, becoming more strongly tessellate anteromedially; scutellum punctures dense ( $\mathrm{i}<1 \mathrm{pd}$ ), becoming sparser submedially ( $\mathrm{i}=1-3 \mathrm{pd}$ ), diversopunctate, sculpture shiny, sometimes weakly imbricate marginally; metanotum sculpture shiny with fine, crowded punctures ( $i=0 \mathrm{pd})$, becoming rugulose laterally; metapostnotum rugae strong, highly anastomosing, reaching margin, sculpture tessellate; preëpisternum sculpture shiny or weakly imbricate with crowded punctures $(i=0 \mathrm{pd})$, becoming areolate ventrally; hypoepimeron punctures dense $(i<1 \mathrm{pd})$, sculpture shiny; mesepisternum punctures dense $(\mathrm{i}<1 \mathrm{pd})$, sculpture shiny or weakly imbricate; metepisternum sculpture rugulosolineate dorsally, tessellate and densely, obscurely punctate ventrally; propodeum lateral face sculpture rugulose anteriorly, tessellate posteriorly; propodeum posterior face sculpture tessellate; T1 anterior face sculpture shiny; T1 dorsal surface punctures fine, moderately sparse ( $i=1-4 \mathrm{pd})$, becoming finer or obscure on rim and absent in small apicolateral oval patches, sculpture shiny; T2 disc punctures fine, moderately dense $(i=1-3 \mathrm{pd})$, disc sculpture shiny, rim punctures very fine, sometimes obscure, sparse $(\mathrm{i}=2-4 \mathrm{pd})$, rim sculpture shiny to weakly coriarious.

STRUCTURE. Face length/width ratio 0.8 ( $\pm 0.03 \mathrm{SD}$ ). Clypeus projecting $\sim 50 \%$ below suborbital tangent; clypeal area length/width ratio $0.42( \pm 0.02 \mathrm{SD})$; apicolateral denticles low rounded knobs; supraclypeal area length/width ratio $0.72( \pm 0.07 \mathrm{SD})$. Forewing with 3 submarginal cells; pronotal angle obtuse; tegula shape normal. Intertegular distance 0.88 ( $\pm 0.05 \mathrm{SD}) \mathrm{mm}$. Scutum length/width ratio 0.78 


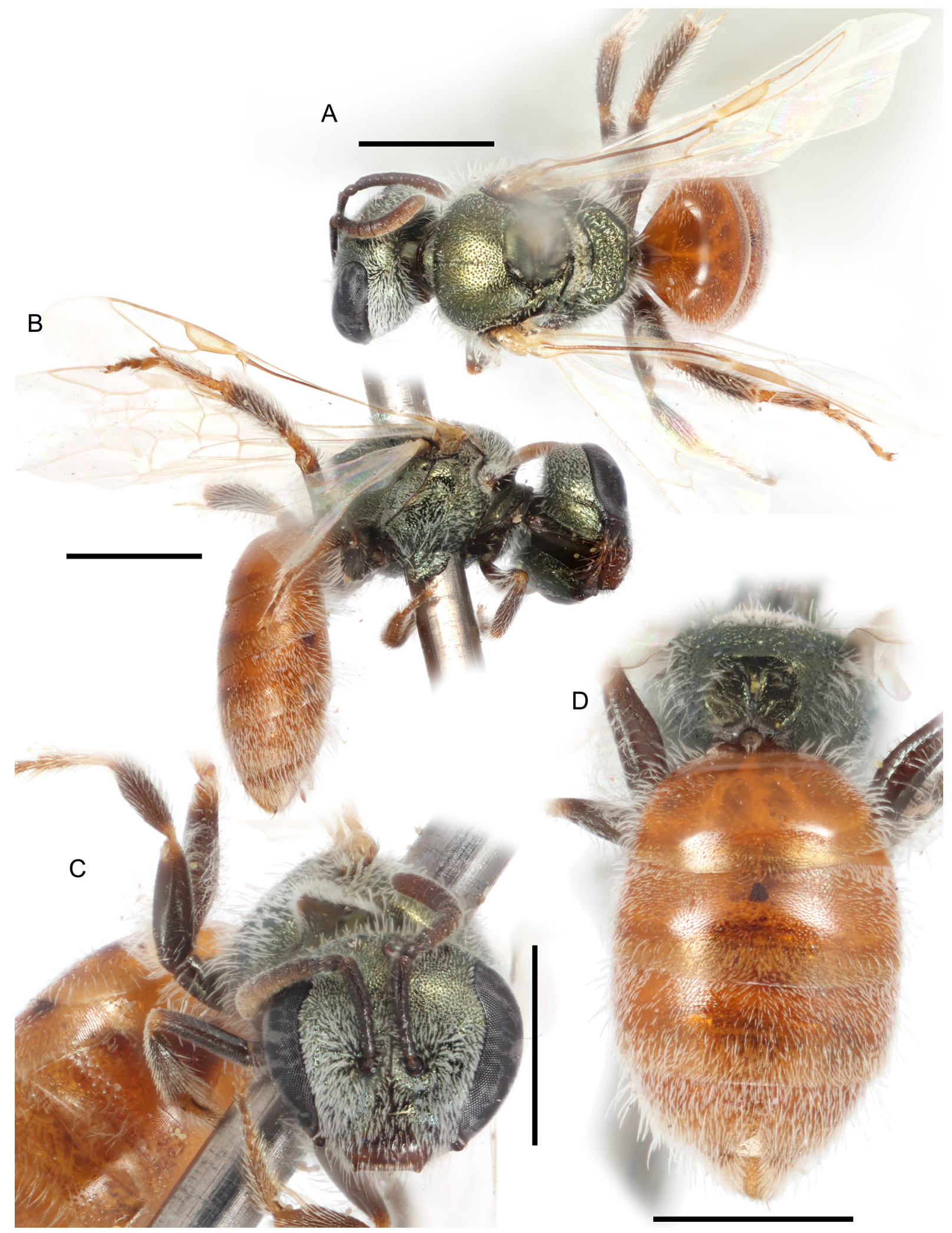

Fig. 22. Lasioglossum (D.) clastipedion sp. nov., ․ A. Dorsal habitus. B. Lateral habitus. C. Face. D. Propodeum and metasoma. Scale bars: $1 \mathrm{~mm}$. 


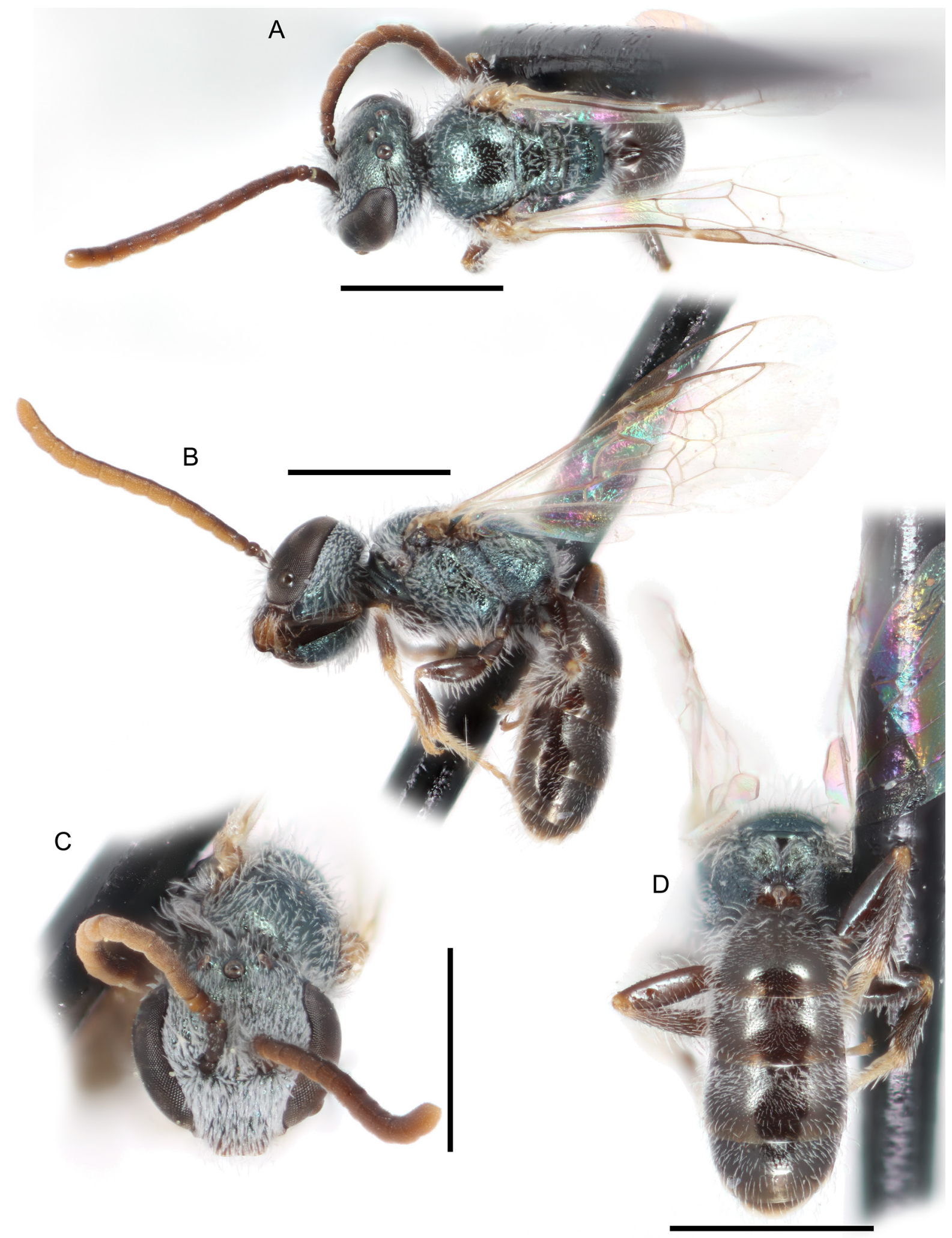

Fig. 23. Lasioglossum (D.) clastipedion sp. nov., Ô. A. Dorsal habitus. B. Lateral habitus. C. Face. D. Propodeum and metasoma. Scale bars: $1 \mathrm{~mm}$. 
( $\pm 0.02 \mathrm{SD}$ ); scutum/scutellum length ratio 3.06 ( $\pm 0.19 \mathrm{SD})$; scutellum/metanotum length ratio 1.45 ( $\pm 0.14 \mathrm{SD})$; metanotum/metapostnotum length ratio 0.71 ( $\pm 0.06 \mathrm{SD})$. Propodeum lateral carinae not reaching dorsal margin; oblique carina very weak or absent. T2 depressed apical rim length less than $50 \%$ of segment. $(n=9)$

\section{Male}

Colouration. Head and mesosoma blue to olive green; clypeus apical colour black to reddish brown; labrum reddish brown to orange; mandible orange with black basal spot and red tip; flagellum reddish brown dorsally, orange ventrally; pronotal lobe reddish brown; metasoma black to dark reddish brown with rims of terga and sterna broadly translucent reddish brown and downcurved lateral areas of terga becoming translucent orange; legs reddish brown with femur-tibia joints, base and apex of tibiae, and tarsi orange; tegula orange; wing membrane hyaline, veins dark to light brown.

Pubescence. Body hair colour white. Tomentum dense on face below eye emargination, gena, pronotal angle and lobe, and space between pronotal lobe and tegula; sparse on episterna and T2-3 basolaterally. Scutum hair moderately to densely plumose. Sterna hair short (0.75-1.25 OD), densely plumose, dense and erect. Wing hairs light, short and sparse.

SuRFACE SCULPTURE. Clypeus punctures dense to moderately dense ( $\mathrm{i} \leq 2 \mathrm{pd}$ ), sculpture shiny; supraclypeal area punctures dense to moderately dense ( $\mathrm{i} \leq 2 \mathrm{pd}$ ), sculpture shiny; paraocular area punctures dense $(\mathrm{i}<1 \mathrm{pd})$, sculpture shiny; frons punctures crowded ( $\mathrm{i}=0 \mathrm{pd})$, sculpture shiny; vertex punctures moderately dense laterally ( $\mathrm{i}=1-2 \mathrm{pd}$ ), sparse medially ( $\mathrm{i}=1-3 \mathrm{pd}$ ), sculpture shiny; gena punctures fine, sparse $(i=1-3 \mathrm{pd})$, obscure, sculpture shiny, becoming weakly ruguloso-lineate posteriorly and ventrally; postgena sculpture imbricate to ruguloso-lineate; tegula punctures absent; scutum punctures dense ( $\mathrm{i} \leq 1 \mathrm{pd}$ ), becoming moderately sparse submedially ( $\mathrm{i}=1-3 \mathrm{pd}$ ), sculpture shiny, sometimes becoming weakly tessellate anteromedially; scutellum punctures dense marginally and on median line ( $\mathrm{i} \leq 1 \mathrm{pd}$ ), sparse submedially ( $\mathrm{i}=1-3 \mathrm{pd}$ ), sculpture shiny; metanotum sculpture shiny and moderately sparsely punctate $(\mathrm{i}=1-2 \mathrm{pd})$; metapostnotum rugae strong, anastomosing, reaching margin, sculpture shiny to weakly imbricate; preëpisternum sculpture shiny with crowded punctures $(\mathrm{i}=0 \mathrm{pd})$; hypoepimeron punctures dense ( $\mathrm{i} \leq 1 \mathrm{pd})$, sculpture shiny; mesepisternum punctures dense to moderately dense $(\mathrm{i}<2 \mathrm{pd})$, sculpture shiny; metepisternum sculpture lineate dorsally, areolate-rugose ventrally; propodeum lateral face punctures obscure, sculpture imbricate to rugulose; propodeum posterior face sculpture rugulose; T1 anterior face sculpture shiny; T1 dorsal surface punctures dense to moderately dense ( $\mathrm{i} \leq 2 \mathrm{pd})$, becoming sparse towards $\operatorname{rim}(\mathrm{i}=1-3 \mathrm{pd})$ and absent or very sparse in small apicolateral oval patches, sculpture shiny; T2 disc punctures dense to moderately dense ( $\mathrm{i} \leq 2 \mathrm{pd})$, becoming sparse toward premarginal line $(i=1-3 \mathrm{pd})$, disc sculpture shiny, rim punctures sparse medially $(i=1-4 \mathrm{pd})$, absent laterally, rim sculpture shiny.

STRUCTURE. Face length/width ratio 0.82 ( $\pm 0.02 \mathrm{SD})$. F1:pedicel length ratio 0.97 ( $\pm 0.12 \mathrm{SD}) ; \mathrm{F} 2: \mathrm{F} 1$ length ratio 2.07 ( $\pm 0.15 \mathrm{SD})$; F2 length/width ratio 1.71 ( $\pm 0.1 \mathrm{SD})$; F9 length/width ratio $1.47( \pm 0.06 \mathrm{SD})$. Forewing with 3 submarginal cells; pronotal angle obtuse; tegula shape normal. Intertegular distance 0.75 ( $\pm 0.07 \mathrm{SD}) \mathrm{mm}$. Scutum length/width ratio 0.9 ( $\pm 0.05 \mathrm{SD})$; scutum/scutellum length ratio 2.77 $( \pm 0.16 \mathrm{SD})$; scutellum/metanotum length ratio $1.73( \pm 0.2 \mathrm{SD})$; metanotum/metapostnotum length ratio 0.69 ( \pm 0.12 SD). Propodeum lateral carinae not reaching dorsal margin; oblique carina absent. $(n=9)$

Genitalia. As in Fig. 90B. Gonocoxite relatively narrow, rounded. Gonostylus with a few long hairs. Retrorse lobe broad, spatulate, with very few short hairs. 


\section{Range}

Southeastern Utah and far northern Arizona (Fig. 24).

\section{Floral records}

ASTERACEAE Giseke: Chrysothamnus $\bullet$ FABACEAE Juss. Medicago: M. sativa L. •PAPAVERACEAE Juss. Argemone L. • POLYGONACEAE Juss. Eriogonum Michx. E. trichopes Torr. - SALICACEAE Mirb. Salix L. • TAMARICACEAE Link: Tamarix L.

\section{DNA barcodes}

Two sequences available (BOLD process IDs: DLII850-07, DLII1011-07; BIN: BOLD:AAJ1752). The physical specimens associated with these sequences could not be located. However, an approximately $100 \mathrm{bp}$ fragment of a failed sequence from a new specimen of Lasioglossum clastipedion sp. nov. matched these sequences at $100 \%$, and Gibbs' recollection of the specimens matches L. clastipedion sp. nov. as well. Along with the collection location in Utah, the sequences can be confidently identified.

Lasioglossum clastipedion sp. nov. differs from all other western red-tailed L. (Dialictus) by 2 fixed substitutions: 302(C) and 564(G). It differs from L. clematisellum and L.julipile sp. nov. by an additional 9 fixed substitutions: 96(C), 129(T), 219(C), 300(A), 363(C), 402(C), 531(C), 549(C), and 600(C) (Supplementary file 4).

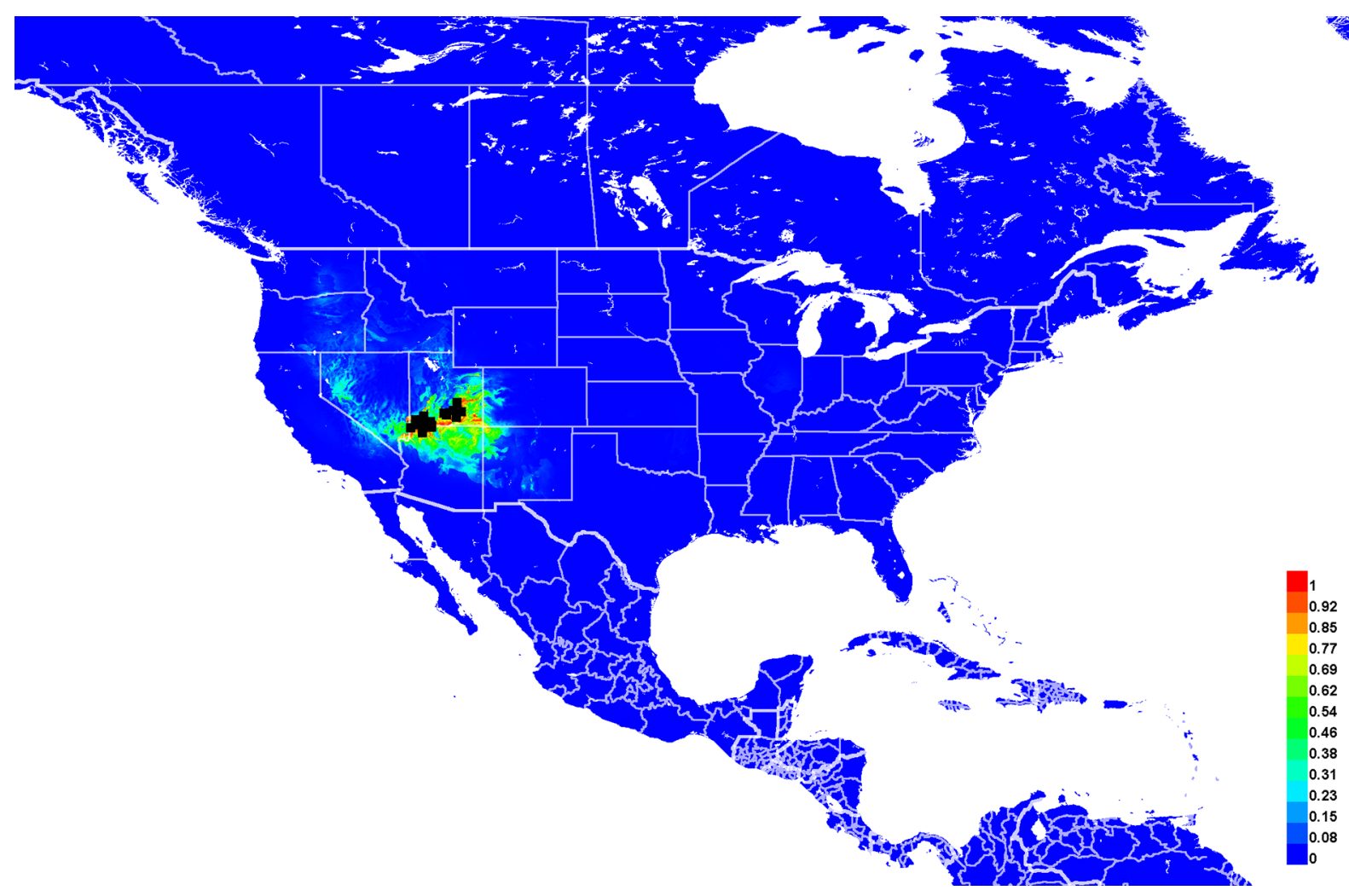

Fig. 24. Georeferenced collection records of Lasioglossum (D.) clastipedion sp. nov. (black squares) and predicted distribution by maximum entropy ecological niche modeling in Maxent (colour shading). Warmer colours indicate higher cloglog probability of occurrence. 
Lasioglossum (Dialictus) clavicorne sp. nov. urn:lsid:zoobank.org:act:4E418EEB-5C3D-4985-9546-AFB1E1B903AA

Figs 25-27, 106A

\section{Diagnosis}

Females of Lasioglossum clavicorne sp. nov. can be recognized by the characters for the L. clematisellum species complex (below), in addition to the head and mesosoma shiny with moderately sparse ( $\mathrm{i}=1-3 \mathrm{pd})$, diversopunctate punctation, scutum with numerous thin, simple hairs, metasomal terga with dense tomentum, and larger overall size than other members of the L. clematisellum group $(\sim 5 \mathrm{~mm})$. They are most similar to L. julipile sp. nov. Females of $L$. julipile sp. nov. have the head and mesosoma with finer, uniformly sized punctures, scutum with all hairs densely plumose, and smaller size $(\sim 4 \mathrm{~mm})$.

Males of L. clavicorne sp. nov. are unique among known Lasioglossum (Dialictus) in the clavate antennae (F7-11 about 1.3 times as broad as basal segments). They can also be recognized by the head and mesosoma mostly shiny, postgena lineate, propodeum shiny with strong subparallel rugae extending nearly to the apical margin and onto the dorsolateral slope, rims of T1-3 sparsely and evenly punctate ( $\mathrm{i}=1-5 \mathrm{pd}$ ), T2 -5 with abundant tomentum (especially T4-5 which have sparse tomentum throughout), mesosoma with sparse tomentum, head and mesosoma with most hairs densely plumose, and relatively large size $(\sim 5 \mathrm{~mm})$. They are most similar to L. pallidellum and L. julipile sp. nov., both of which have the antennae normal. Males of L. pallidellum have the propodeum posterior margin and dorsolateral slope smooth and rounded, and mesosoma with dense tomentum. Males of L. julipile sp. nov. have the rims of T1-3 unevenly punctate, becoming impunctate laterally, T2-5 with less extensive tomentum, and smaller size $(\sim 4 \mathrm{~mm})$.

\section{Etymology}

The specific epithet clavicorne is formed from the Latin genitive singular noun 'clavae' ('club') and the adjective 'cornis '('horned'). It refers to the clubbed antennae of the male. An appropriate translation would be the club-horned sweat bee.

\section{Material examined}

Holotype

UNITED STATES - Texas • ${ }^{\lambda}$; Culberson Co., 10 mi. W of Kent; [31.07 N, 104.39 W]; 14 Sep. 1965; Timberlake leg.; ex Pyrrhopappus; UCRC.

[Verbatim label: 10 mi. W of Kent, Tex // Culberson Co. Sept-14 65 // Sitilias // Timberlake Coll. // HOLOTYPE / Lasioglossum (Dialictus) clavicorne Gardner and Gibbs]

\section{Paratypes}

UNITED STATES - Arizona 11 ; 2.5 mi. S of Portal; $\left[31.88^{\circ} \mathrm{N}, 109.14^{\circ} \mathrm{W}\right] ; 12$ May 1940; Timberlake leg.; ex Argemone; UCRC. - New Mexico • 5 o 9 ; Carrizozo; $\left[33.64^{\circ} \mathrm{N}, 105.88^{\circ} \mathrm{W}\right] ; 10$ Jun. 1950; L.D. Beamer leg.; ex Helenium laciniatum; SEMC. - Texas • 1 +; Brewster Co., 1 mi. E. of Marathon; [30.2 ${ }^{\circ} \mathrm{N}, 103.23^{\circ} \mathrm{W}$ ]; 12 Apr. 1965; Timberlake leg.; ex Physaria; UCRC • 1 क; same location as for holotype; 14 Sep. 1965; D. Smith leg.; ex Pyrrhopappus; UCRC • 1 \&; same location as for holotype; 14 Sep. 1965; Timberlake leg.; UCRC

\section{Description}

\section{Female}

Colouration. Head and mesosoma blue-green; clypeus apical colour black to reddish brown; labrum reddish brown to orange; mandible orange with black basal spot or band and red tip; flagellum reddish brown dorsally, light brown ventrally; pronotal lobe reddish brown; metasoma red to orange with dark spiracular spots on T3-4; legs reddish brown with femur-tibia joints, tibia apices, and tarsi light brown to orange; tegula orange; wing membrane hyaline, veins with subcosta brown, otherwise pale amber. 
Pubescence. Body hair colour white. Tomentum dense on paraocular area, gena, pronotal collar and lobe, space between pronotal lobe and tegula, metanotum medially, metepisternum, T2-3 basolaterally, and T4 throughout; sparse on face below eye emargination, preëpisternum, mesepisternum, and T3 apical rim. Scutum hair densely plumose. Wing hairs light, very short and sparse. Acarinarial fan incomplete, sparse. T2 fringes sparse, T3 fringes sparse.

SuRfaCe SCUlPtURE. Clypeus punctures large and irregularly sparse ( $\mathrm{i}<4 \mathrm{pd}$ ), becoming fine and moderately dense in basal third $(i=1-2 \mathrm{pd})$, sculpture shiny; supraclypeal area punctures sparse $(\mathrm{i}=1-4 \mathrm{pd})$, sculpture shiny; paraocular area punctures dense $(\mathrm{i} \leq 1 \mathrm{pd})$, sometimes crowded around antenna socket, sculpture shiny; frons punctures dense ( $i \leq 1 \mathrm{pd})$, sculpture shiny; vertex punctures fine, sparse ( $\mathrm{i}=1-4 \mathrm{pd})$, sculpture shiny; gena punctures dense $(\mathrm{i}<1 \mathrm{pd})$, sculpture shiny, becoming tessellate and lineate posteriorly; postgena sculpture lineate, sometimes becoming smooth and tessellate to shiny anteriorly; tegula punctures absent; scutum punctures moderately sparse ( $\mathrm{i}=1-4 \mathrm{pd})$, becoming dense posteromedially ( $\mathrm{i}<1 \mathrm{pd}$ ), diversopunctate, sculpture shiny, becoming tessellate anteromedially; scutellum punctures dense marginally and on median line ( $i \leq 1 \mathrm{pd})$, sparse submedially $(i=1-4 \mathrm{pd})$, diversopunctate, sculpture shiny; metanotum sculpture shiny and finely, densely punctate $(i<1 \mathrm{pd})$, becoming rugulose laterally; metapostnotum rugae strong, highly anastomosing, reaching margin, sculpture shiny to weakly imbricate; preëpisternum sculpture areolate; hypoepimeron punctures dense $(\mathrm{i} \leq 1 \mathrm{pd})$, diversopunctate, sculpture shiny; mesepisternum punctures dense $(\mathrm{i} \leq 1 \mathrm{pd})$, diversopunctate, sculpture shiny; metepisternum sculpture lineate dorsally, finely ruguloso-punctate medially, imbricate ventrally; propodeum lateral face sculpture tessellate; propodeum posterior face sculpture tessellate; T1 anterior face sculpture shiny; T1 dorsal surface punctures fine, sparse (i=1-4 pd), absent in broad median line and large apicolateral oval patches, sculpture shiny; T2 disc punctures fine, moderately sparse ( $i=1-4 \mathrm{pd})$, disc sculpture shiny, rim punctures fine, sparse ( $i=1-5 \mathrm{pd})$, rim sculpture shiny.

SCULPTURE. Face length/width ratio 0.82 ( $\pm 0.01 \mathrm{SD}$ ). Clypeus projecting $\sim 67 \%$ below suborbital tangent; clypeal area length/width ratio $0.39( \pm 0.02 \mathrm{SD})$; apicolateral denticles low rounded knobs; supraclypeal area length/width ratio $0.74( \pm 0.08 \mathrm{SD})$. Forewing with 3 submarginal cells; pronotal angle rightangled; tegula shape normal. Intertegular distance $1.05( \pm 0.05 \mathrm{SD}) \mathrm{mm}$. Scutum length/width ratio 0.79 ( $\pm 0.01 \mathrm{SD}) ;$ scutum/scutellum length ratio 3.22 ( $\pm 0.09 \mathrm{SD})$; scutellum/metanotum length ratio 1.53 ( $\pm 0.08 \mathrm{SD})$; metanotum/metapostnotum length ratio 0.66 ( $\pm 0.07 \mathrm{SD})$. Propodeum lateral carinae not reaching dorsal margin; oblique carina weak, straight. T2 depressed apical rim length less than $50 \%$ of segment. $(n=8)$

\section{Male}

Colouration. Head and mesosoma blue to blue-green; clypeus apical colour reddish brown; labrum reddish brown; mandible orange with black basal spot and red tip; flagellum reddish brown dorsally, orange ventrally; pronotal lobe reddish brown; metasoma reddish brown with rims of terga broadly orange to translucent yellow; legs reddish brown with femur-tibia joints, base and apex of tibiae, and tarsi orange; tegula pale amber; wing membrane hyaline, veins with subcosta dark brown, otherwise pale amber to light brown.

PubesCENCE. Body hair colour white. Tomentum dense on face below ocelli, gena, pronotal angle and lobe, space between pronotal lobe and tegula, and T2-3 basolaterally; sparse on episterna and T4-5 throughout. Scutum hair densely plumose. Sterna hair short (1-1.5 OD), densely plumose, dense and erect. Wing hairs light, short and sparse.

SuRfaCE SCULPTURE. Clypeus punctures moderately dense ( $\mathrm{i}=1-2 \mathrm{pd})$, sculpture shiny; supraclypeal area punctures moderately dense $(\mathrm{i}=1-2 \mathrm{pd})$, sculpture shiny; paraocular area punctures crowded $(\mathrm{i}=0 \mathrm{pd})$, sculpture shiny; frons punctures dense $(\mathrm{i}<1 \mathrm{pd})$, sculpture shiny; vertex punctures moderately dense $(\mathrm{i}=1-2 \mathrm{pd})$, sculpture shiny; gena punctures moderately dense $(\mathrm{i}=1-2 \mathrm{pd})$, sculpture shiny; postgena sculpture weakly lineate; tegula punctures absent; scutum punctures sparse ( $\mathrm{i}=1-3 \mathrm{pd}$ ), becoming dense medially ( $i \leq 1 \mathrm{pd})$, diversopunctate, sculpture shiny; scutellum punctures sparse $(\mathrm{i}=1-3 \mathrm{pd})$, 


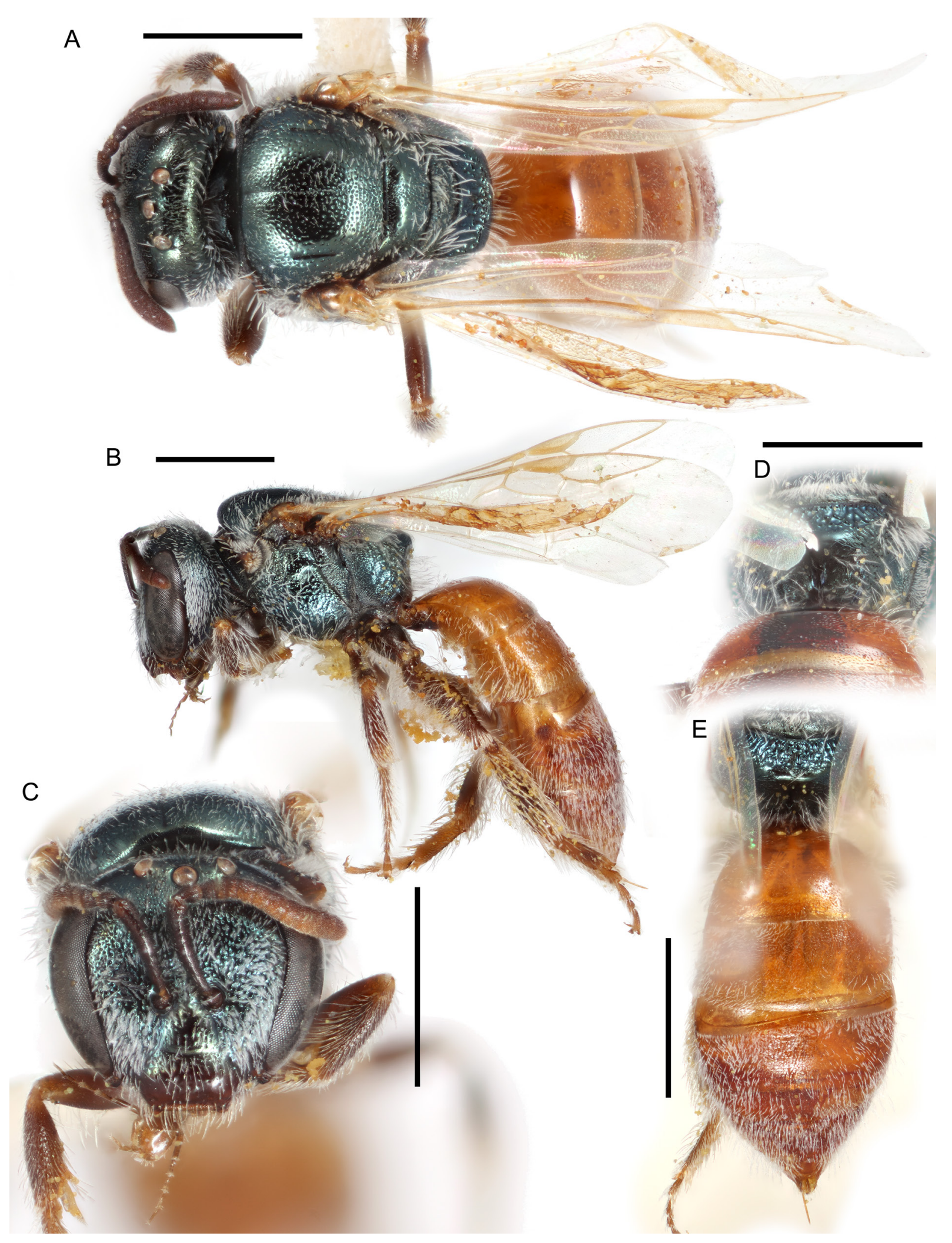

Fig. 25. Lasioglossum (D.) clavicorne sp. nov., ․ A. Dorsal habitus. B. Lateral habitus. C. Face. D. Propodeum. E. Metasoma. Scale bars: $1 \mathrm{~mm}$. 


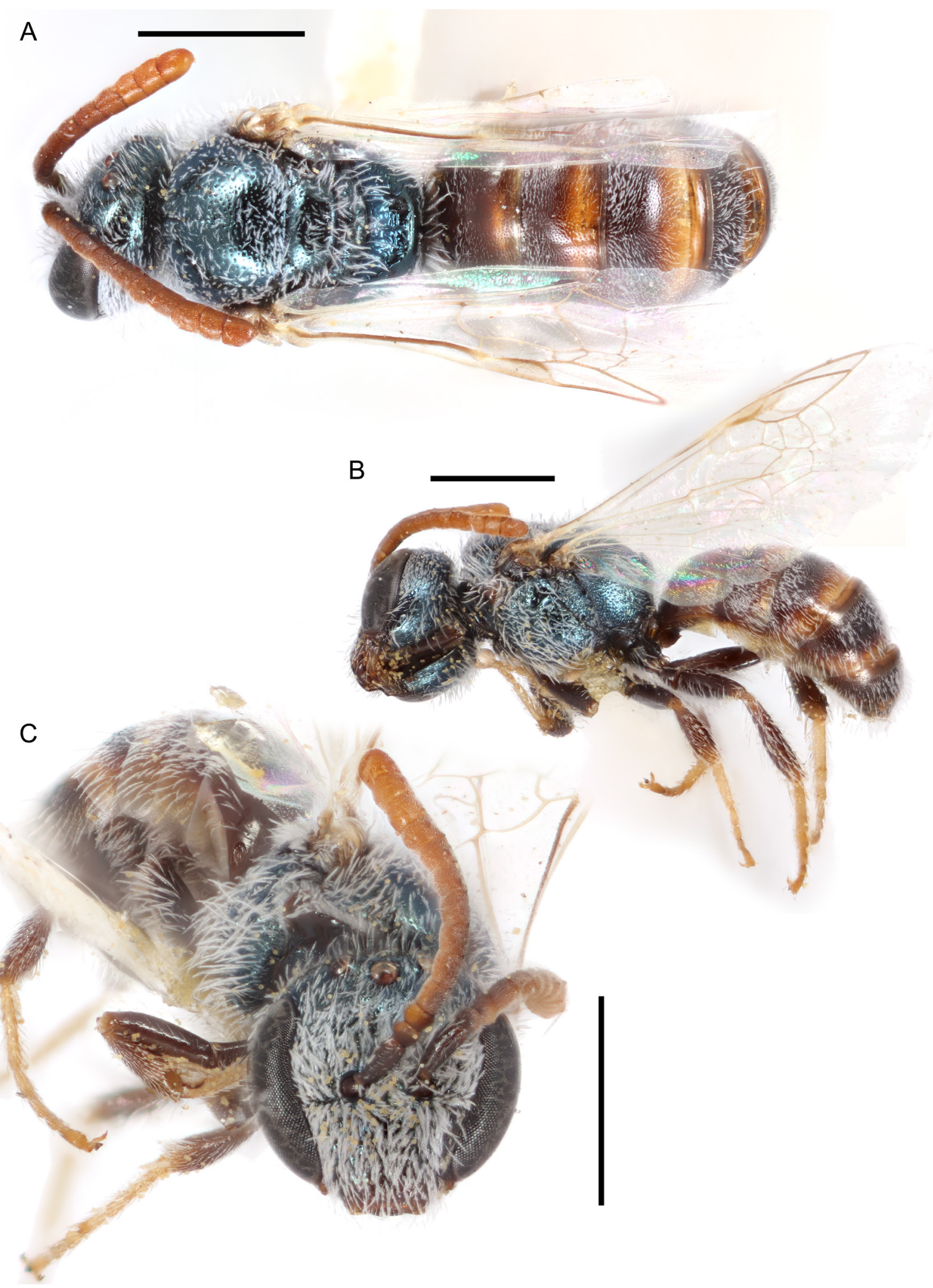

Fig. 26. Lasioglossum (D.) clavicorne sp. nov., holotype, §. A. Dorsal habitus. B. Lateral habitus. C. Face. Scale bars: $1 \mathrm{~mm}$. 
becoming dense marginally ( $\mathrm{i}<1 \mathrm{pd}$ ), diversopunctate, sculpture shiny; metanotum sculpture shiny and moderately densely punctate $(\mathrm{i}=1-2 \mathrm{pd})$, becoming rugulose laterally; metapostnotum rugae strong, anastomosing, reaching margin, sculpture shiny; preëpisternum sculpture areolate; hypoepimeron punctures dense ( $\mathrm{i} \leq 1 \mathrm{pd})$, sculpture shiny; mesepisternum punctures dense ( $\mathrm{i} \leq 1 \mathrm{pd})$, sculpture shiny; metepisternum sculpture lineate dorsally, areolate ventrally; propodeum lateral face punctures obscure, sculpture imbricate, becoming rugulose posteriorly; propodeum posterior face sculpture not visible; T1 anterior face sculpture shiny; T1 dorsal surface punctures moderately dense ( $\mathrm{i}=1-2 \mathrm{pd})$, sparse in small apicolateral oval patches $(i=1-4 \mathrm{pd})$, sculpture shiny; $\mathrm{T} 2$ disc punctures moderately dense $(\mathrm{i}=1-2 \mathrm{pd})$, disc sculpture shiny, rim punctures moderately sparse $(i=1-3 \mathrm{pd})$, rim sculpture shiny.

STRUCTURE. Face length/width ratio 0.8. F1:pedicel length ratio 0.93; F2:F1 length ratio 1.43; F2 length/ width ratio 1.18; F9 length/width ratio 0.91. F9:F2 width ratio 1.31. Forewing with 3 submarginal cells; pronotal angle obtuse; tegula shape normal. Intertegular distance $0.93 \mathrm{~mm}$. Scutum length/width ratio 0.9; scutum:scutellum length ratio 2.91; scutellum:metanotum length ratio 1.56; metanotum:metapostnotum length ratio 0.71 . Propodeum lateral carinae not reaching dorsal margin; oblique carina absent. $(n=1)$

Genitalia. Not examined.

\section{Range}

Chihuahuan Desert of southern Arizona, New Mexico, and Texas (Fig. 27).

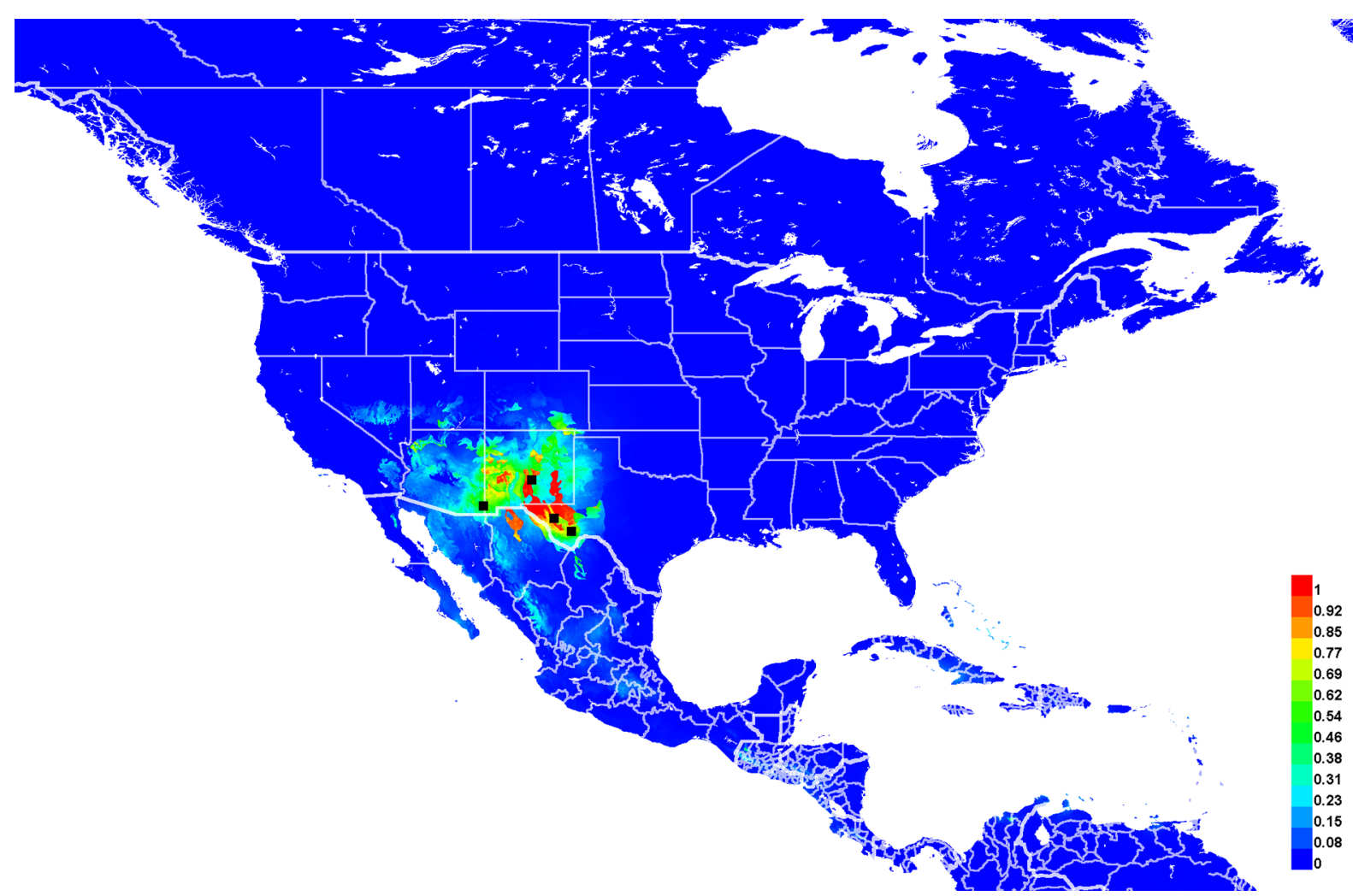

Fig. 27. Georeferenced collection records of Lasioglossum (D.) clavicorne sp. nov. (black squares) and predicted distribution by maximum entropy ecological niche modeling in Maxent (colour shading). Warmer colours indicate higher cloglog probability of occurrence. The predicted distribution was generated with a regularization multiplier of 0.5 to obtain a more conservative prediction. Due to the very small number of records, this distribution should be interpreted with caution. 


\section{Floral records}

ASTERACEAE Giseke: Helenium: H. laciniatum A.Gray • Pyrrhopappus • BRASSICACEAE Burnett: Physaria (Nutt. ex Torr. \& A.Gray) A.Gray • PAPAVERACEAE Juss. Argemone L.

\section{DNA barcodes}

Not available.

\section{Remarks}

Rare. Only 10 specimens are known.

Lasioglossum (Dialictus) clematisellum (Cockerell, 1904)

Figs 28-30, 90C, 91B, 106B, 114B, 117A

Halictus clematisellus Cockerell, 1904: 13 (holotype,, , deposited in USNM, examined).

Halictus clematisellus - Cockerell 1906: 429 (key).

Halictus (Chloralictus) clematisellus - Sandhouse 1924: 4 (key).

Lasioglossum (Chloralictus) clematisellum - Michener 1951: 1112 (catalog).

Dialictus clematisellus - Hurd 1979: 1965 (catalog). — Moure \& Hurd 1987: 95 (catalog).

Lasioglossum (Dialictus) clematisellum - Scott et al. 2011: 29 (checklist).

\section{Diagnosis}

Females of Lasioglossum clematisellum can be recognized by the characters for the L. clematisellum species complex (below), in addition to the metasomal terga lacking tomentum, the face and mesepisternum often duller than in other species in this group, scutum with most hairs relatively thin and weakly plumose and punctures of anteromedian area indistinct, and head and mesosoma blue-green with bright white hair. They are most similar to L. clastipedion sp. nov. and can only be separated with great difficulty, especially if specimens are worn. Females of L. clastipedion sp. nov. have the metasomal terga with at least some basolateral tomentum; this is the most reliable character. In addition, females of L. clastipedion sp. nov. often have the scutum with most hairs densely plumose and punctures of the anteromedian area distinct, and head and mesosoma more golden-green with slightly yellowish hair dorsally.

Males of L. clematisellum can be recognized by the characters for the Lasioglossum clematisellum species complex (below), in addition to the metasoma lacking tomentum, scutum with many hairs thin and weakly plumose, and frons densely punctate $(\mathrm{i}<1 \mathrm{pd})$. They are most similar to L. julipile sp. nov., L. perditum sp. nov., and L. clastipedion sp. nov. All of these species have the scutum with most hairs thick and densely plumose and metasomal terga with at least some basolateral tomentum. In addition, L. julipile sp. nov. and L. perditum sp. nov. have the frons more sparsely punctate ( $\mathrm{i}=1-3 \mathrm{pd})$.

\section{Etymology}

Cockerell (1904) named this species after the plant genus Clematis, plus the Latin adjectival diminutive suffix -ellus.

\section{Material examined}

Holotype

UNITED STATES - New Mexico • $q$; Pecos; [35.57 N, 105.68 W]; 14 Jul. 1903; W.P. Cockerell leg.; ex Clematis ligusticifolia; USNM 12064. 


\section{Other material}

MEXICO - Nuevo Leon • 1 क ; La Hediondilla, Galeana; $25.0694^{\circ} \mathrm{N}, 100.7186^{\circ} \mathrm{W}$; $1890 \mathrm{~m}$ a.s.1.; 29 Sep. 2013; LRF and CVM leg.; LRF. - Sonora • 1 क ; $30 \mathrm{~km} \mathrm{E} \mathrm{of} \mathrm{Agua} \mathrm{Prieta;} 31.3097^{\circ} \mathrm{N}, 109.2542^{\circ} \mathrm{W}$; 20 Apr. 2005; R.L. Minckley leg.; PCYU 1 \%; 30 km E of Agua Prieta; $31.3333^{\circ} \mathrm{N}, 109.26^{\circ} \mathrm{W} ; 3$ May 2001; A. Romero leg.; RLM・ 1 क; 30 km E of Agua Prieta; $31.3333^{\circ}$ N, $109.2597^{\circ}$ W; 20 Aug. 2003; R.L. Minckley leg.; ex Baccharis sp.; PCYU • 1 \%; $30 \mathrm{~km}$ E of Agua Prieta; $31.3133^{\circ} \mathrm{N}, 109.2503^{\circ} \mathrm{W}$; 18 Jul. 2001; R. Minckley leg.; RLM • 1 क; 30 km E of Agua Prieta; $31.3222^{\circ}$ N, $109.2772^{\circ}$ W; 22 Jul. 2001; R. Minckley leg.; RLM • 1 क; $30 \mathrm{~km}$ E of Agua Prieta; $31.3186^{\circ} \mathrm{N}, 109.2508^{\circ} \mathrm{W} ; 24$ Jul. 2001; R. Minckley leg.; RLM • 1 q; $30 \mathrm{~km}$ E of Agua Prieta; $31.31^{\circ} \mathrm{N}, 109.2536^{\circ} \mathrm{W} ; 4$ Aug. 2001; R.L. Minckley leg.; RLM • 1 ; ; Municipio de Agua Prieta, Rancho Nogalitos; $31.31177^{\circ} \mathrm{N}, 108.98622^{\circ} \mathrm{W}$; 25 May 2008; RLM - 3 우; Rancho Puerta Blanca, Pollination Site 25, Larrea, RPB, Loma Larrea; $31.31389^{\circ}$ N, $109.25028^{\circ}$ W; 3 Apr. 2007; ex Salix gooddingii; RLM • 1 \%; Rancho San Bernardino; $31.3133^{\circ}$ N, $109.2503^{\circ}$ W; 7 Aug. 2001; R.L. Minckley leg.; RLM • 2 o 9 ; Rancho San Bernardino; $31.31^{\circ} \mathrm{N}, 109.2536^{\circ} \mathrm{W} ; 25$ Aug. 2001; R. Minckley leg.; RLM • 7 우우 Rancho San Bernardino, 28 km E of Agua Prieta; [31.31 ${ }^{\circ} \mathrm{N}, 109.25^{\circ} \mathrm{W}$ ]; 6-7 Jul. 2000; R. Minckley et al. leg.; RLM • 2 o 9 ; same location as for preceding; 19 Jul. 2000; K. and R. Gyuro and S. Minckley leg.; RLM • 1 क; same location as for preceding; 22 Aug. 2000; R. Minckley and A. Romero leg.; RLM • 1 क; Rancho San Bernardino, $28 \mathrm{~km}$ E of Agua Prieta, Arroyo Grande; [31.31 $\mathrm{N}, 109.25^{\circ} \mathrm{W}$ ]; 14 Apr. 2000; R. Minckley leg.; ex Prosopis velutina; RLM - 1 क; Rancho San Bernardino, Bosque dry, Site 4, Transect 1; $31.30992^{\circ} \mathrm{N}$, 109.27783 ${ }^{\circ} \mathrm{W}$; 21 Jul. 2005; RLM • 6 우; Rancho San Bernardino, Bosque wet, Site 1; $31.33194^{\circ} \mathrm{N}$, 109.27083 ${ }^{\circ}$ W; 10 Aug. 2006; RLM • 2 우; Rancho San Bernardino, Bosque wet, Site 7; $31.30972^{\circ}$ N, 109.25417 ${ }^{\circ}$ W; 20 Apr. 2005; RLM - 1 o; Rancho San Bernardino, Bosque wet, Site 7, Transect 2; $31.31007^{\circ}$ N, $109.25348^{\circ}$ W; 22 Sep. 2001; RLM • 1 P; same location as for preceding; 12 Aug. 2002; RLM • 1 क; same location as for preceding; 1 Aug. 2004; RLM • 1 q ; same location as for preceding; 25 Jul. 2007; RLM • 1 क; Rancho San Bernardino, Bosque wet, Site $8 ; 31.33333^{\circ} \mathrm{N}, 109.26528^{\circ} \mathrm{W} ; 7$ Apr. 2006; RLM - 1 ;; Rancho San Bernardino, Cienega house; $31.33098^{\circ} \mathrm{N}, 109.2676^{\circ} \mathrm{W} ; 1$ Apr. 2006; RLM • 1 \%; Rancho San Bernardino, field, Site 1, Transect 1; $31.32422^{\circ} \mathrm{N}, 109.26663^{\circ} \mathrm{W} ; 22$ Jul. 2005; ex Convolvulus arvensis; RLM • 1 क; same location as for preceding; 24 Jul. 2005; ex Convolvulus arvensis; RLM - 1 q; Rancho San Bernardino, Los Ojitos, Lebuhn, Bee Diversity; $31.30025^{\circ} \mathrm{N}$, 109.2625 ${ }^{\circ}$ W; 19 Apr. 2002; RLM • 1 q; same location as for preceding; 3 May 2002; RLM • 1 \%; Rancho San Bernardino, main ranch house; $31.325^{\circ} \mathrm{N}, 109.265^{\circ} \mathrm{W} ; 19 \mathrm{Jul} .2001$; ex Lepidium thurberi; RLM - 2 우; Rancho San Bernardino, Pollination Site 11, Sphaeralcea, $100 \mathrm{~m} \mathrm{SW}$ of large dam on Silver Creek; $31.30972^{\circ}$ N, $109.27778^{\circ}$ W; 6 Aug. 2004; RLM • 1 o ; same location as for preceding; 8 Aug. 2004; ex Chenopodium desiccatum; RLM • 2 q ; ; same location as for preceding; 8 Aug. 2004; ex Lepidium thurberi; RLM • 1 \%; same location as for preceding; 8 Aug. 2004; ex Salsola kali subsp. tragus; RLM • 2 우; same location as for preceding; 10 Aug. 2004; ex Atriplex canescens; RLM • 2 우; same location as for preceding; 10 Aug. 2004; ex Chenopodium neomexicanum; RLM $\bullet 6$ o ; ; same location as for preceding; 10 Aug. 2004; ex Lepidium thurberi; RLM • 1 \%; Rancho San Bernardino, Pollination Site 12, Physaria, Silver Creek, upstream of Big Dam; $31.3259^{\circ} \mathrm{N}, 109.28802^{\circ} \mathrm{W} ; 13 \mathrm{Apr}$. 2006; ex Phacelia crenulata; RLM • 1 q; same location as for preceding; 11 Apr. 2008; ex Physaria gordonii; RLM • 1 q; same location as for preceding; 19 Apr. 2008; ex Descurainia sp.?; RLM • 1 \%; Rancho San Bernardino, Pollination Site 21, Sphaeralcea, Pond $100 \mathrm{~m}$ E of main gate; $31.31583^{\circ} \mathrm{N}$, 109.26861 ${ }^{\circ}$ W; 25 Jul. 2007; ex Laennecia coulteri; RLM • 2 우; Rancho San Bernardino, Pollination Site 24, Physaria, Hay Hollow; $31.31333^{\circ}$ N, 109.25028 ${ }^{\circ}$ W; 5 Apr. 2007; ex Descurainia sp.?; RLM • 1 ; ; same location as for preceding; 5 Apr. 2007; ex Physaria gordonii; RLM • 1 क ; same location as for preceding; 5 Apr. 2007; ex Salix gooddingii; RLM • 2 우; same location as for preceding; 14 Apr. 2007; ex Physaria gordonii; RLM 1 1 ; Rancho San Bernardino, Pollination Site 27, Horse Corral, $70 \mathrm{~m} \mathrm{~N}$ of main ranch house; $31.32556^{\circ} \mathrm{N}, 109.26667^{\circ} \mathrm{W} ; 8$ Aug. 2007; ex Lepidium thurberi; RLM • 1 q; Rancho

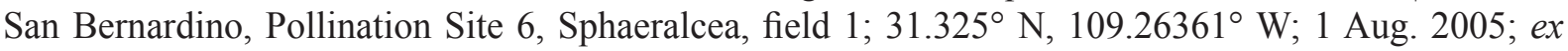
Convolvulus arvensis; RLM - 1 क; Rancho San Bernardino, quarry, at road crossing; $31.32042^{\circ} \mathrm{N}$, 109.2681 ${ }^{\circ}$ W; 2 Sep. 2003; RLM • 1 क; Rancho San Bernardino, quarry, general area; $31.3195^{\circ} \mathrm{N}$, 
109.27028 W; 8 May 2001; ex Phacelia sp.?; RLM • 2 우; Rancho San Bernardino, quarry, gravel pit; $31.31964^{\circ} \mathrm{N}, 109.26926^{\circ} \mathrm{W} ; 18$ Aug. 2003; RLM • 1 \%; Rancho San Bernardino, roadside E of quarry; $31.31996^{\circ} \mathrm{N}, 109.26848^{\circ} \mathrm{W}$; 20 Aug. 2003; RLM • 3 q ; ; Rancho San Bernardino, roadside W of quarry; $31.31948^{\circ} \mathrm{N}, 109.2701^{\circ} \mathrm{W} ; 18$ Aug. 2003; RLM • 2 우; same location as for preceding; 19 Aug. 2003; RLM • 1 क; Rancho San Bernardino; $31.32907^{\circ}$ N, $109.26553^{\circ}$ W; 1 Aug. 2000; RLM • 1 q ; same location as for preceding; $30 \mathrm{Jul}$. 2005; ex Lepidium thurberi; RLM • 1 q; Rancho San Bernardino, Riparian 3; $31.31389^{\circ} \mathrm{N}, 109.25028^{\circ} \mathrm{W} ; 20$ Aug. 2003; RLM • 1 o; same location as for preceding; 25 Aug. 2003; RLM • 1 q; Rancho San Bernardino, Riparian, Site 1; $31.33333^{\circ} \mathrm{N}, 109.25972^{\circ} \mathrm{W} ; 20$ Aug. 2003; ex Baccharis sarothroides; RLM • 1 क; same location as for preceding; 20 Aug. 2003; RLM • 1 क; same location as for preceding; 7 Apr. 2006; RLM • 2 + $O$; same location as for preceding; 13 May 2006; RLM • 1 क; Rancho San Bernardino, Riparian, Site 2; $31.33194^{\circ}$ N, 109.25833 W; 7 Apr. 2006; RLM • 1 q; Rancho San Bernardino, Riparian, Site 3, Transect 1; 31.31362 ${ }^{\circ}$ N, $109.25468^{\circ} \mathrm{W} ; 18$ Sep. 2001; RLM • 1 O; Rancho San Bernardino, Riparian, Site 3, Transect 3; [31.32 ${ }^{\circ}$ N, $\left.109.26^{\circ} \mathrm{W}\right]$; 25 Sep. 2001; RLM - 1 q; Rancho San Bernardino, Riparian, Site 4, Transect 1; $31.32965^{\circ}$ N, $109.25587^{\circ}$ W; 18 Sep. 2001; RLM • 2 우; Rancho San Bernardino, Silver Creek, Big Dam, Pol 12; $31.3259^{\circ} \mathrm{N}$, 109.28802 ${ }^{\circ}$ W; 9 May 2006; RLM • 1 9 ; Rancho San Bernardino, Tamarisk Site 3, Rio San Bernardino; 31.30743 ${ }^{\circ}$ N, $109.25687^{\circ}$ W; 16 Jun. 2004; RLM - 2 q ; ; Rancho San Bernardino, Tamarisk Site 5, Rio San Bernardino; $31.3071^{\circ} \mathrm{N}, 109.25682^{\circ} \mathrm{W} ; 12$ Jun. 2004; ex Tamarix ramosissima; RLM • 1 ᄋ; Rancho San Bernardino, Tamarisk, at Rio San Bernardino; 31.30725 ${ }^{\circ} \mathrm{N}, 109.25675^{\circ} \mathrm{W} ; 26$ May 2004; ex Tamarix ramosissima; RLM • 1 क; same location as for preceding; 29 May 2004; ex Tamarix ramosissima; RLM • 1 +; same location as for preceding; 3 Jun. 2004; ex Tamarix ramosissima; RLM • 1 q; Rancho San Bernardino, Tamarisk, at Silver Creek; $31.31611^{\circ} \mathrm{N}, 109.25806^{\circ} \mathrm{W} ; 26$ May 2004; ex Tamarix ramosissima; RLM. - Zacatecas • 1 +, 3 ô $^{\top} ; 45$ mi. SW of Saltillo; $\left[24.7^{\circ} \mathrm{N}, 101.3^{\circ} \mathrm{W}\right] ; 14$ Sep. 1977; R.W. Brooks leg.; SEMC.

UNITED STATES - Arizona • 1 q; Pinal Co., $\sim 24 \mathrm{~km} \mathrm{~S}$ of Mammoth; $32.5558^{\circ} \mathrm{N}, 110.5164^{\circ} \mathrm{W} ; 30$ Apr. 2011; R. Laport leg.; ex Larrea tridentata; RLM • 1 क; Cochise Co., $18 \mathrm{~km} \mathrm{~W}$ of Portal; [31.91 ${ }^{\circ} \mathrm{N}$, $109.33^{\circ} \mathrm{W}$ ]; 18 Jun. 1993; R.L. Minckley leg.; SEMC • 1 क ; Cochise Co., 2 mi. NE of Portal; $\left[31.94^{\circ} \mathrm{N}\right.$, $\left.109.11^{\circ} \mathrm{W}\right]$; 30 Jul. 1959; Statham leg.; AMNH • 1 \%; 21 mi. SW of Prescott; [34.32 $\left.\mathrm{N}, 112.73^{\circ} \mathrm{W}\right]$; 17 Jul. 1950; C.D. Michener leg.; ex Prosopis juliflora; SEMC 1 1 ; $23 \mathrm{mi}$. W of Winslow; [35.11 ${ }^{\circ} \mathrm{N}$,

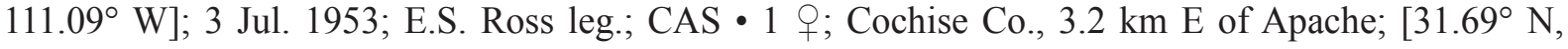
109.1 ${ }^{\circ}$ W]; 4-11 Jun. 1987; B.N. Danforth leg.; ex Senecio flaccidus; SEMC • 1 \%; Cochise Co., 4 mi. E of Willcox; [32.25 N, $\left.109.76^{\circ} \mathrm{W}\right] ; 18$ Aug. 1986; R.J. McGinley and B.M. Norden leg.; USNM • 1 क ; Graham Co., 4 mi. W of Calva; [33.19 $\left.{ }^{\circ} \mathrm{N}, 110.25^{\circ} \mathrm{W}\right]$; 17 Jun. 1956; J.W. MacSwain and D.D. Linsdale leg.; ex Prosopis sp.; EMEC • 1 q; Cochise Co., 6 mi. S of Willcox; [32.16 $\left.{ }^{\circ} \mathrm{N}, 109.83^{\circ} \mathrm{W}\right] ; 23$ Aug. 1986; R.J. McGinley and B.M. Norden leg.; USNM • 1 क; Pima Co., 7 km E of Silverbell; $32.3667^{\circ}$ N, $111.4333^{\circ}$ W; 732 m a.s.l.; 4 Apr. 1994; R.L. Minckley and S.G. Reyes leg.; ex Sphaeralcea sp.; SEMC • 1 क; Pima Co., 7 mi. N of Tucson; [32.33 ${ }^{\circ}$ N, 110.97 W]; 4 Sep. 1968; D.R. Miller and J.E. Lauck leg.; UCDC • 1 क ; Cochise Co., 8 mi. NE of Portal; [32 ${ }^{\circ}$ N, 109.48 W]; 9 May 1956; M.

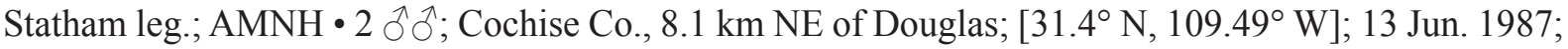
B.N. Danforth leg.; ex Lepidium; SEMC $1{ }^{\top}$; Cochise Co., Apache; $\left[31.69^{\circ} \mathrm{N}, 109.13^{\circ} \mathrm{W}\right]$; 8 Aug. 1972; G. and K. Eickwort leg.; CUIC • 5 우우, 1 ○ं; Cochise Co., Apache vicinity; [31.69 N, $\left.109.13^{\circ} \mathrm{W}\right]$;

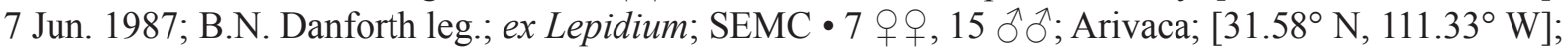
13 Jul. 1950; R.H. Beamer leg.; SEMC 1 o ; same location as for preceding; 13 Jul. 1950; J.G.

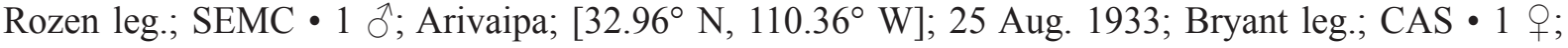
Cochise Co., Benson; [31.97 ${ }^{\circ}$ N, 110.29 ${ }^{\circ}$ W]; 14-15 Sep. 1974; E.G. and J.M. Linsley leg.; ex Helianthus

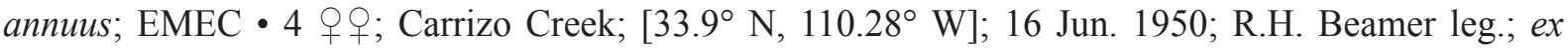
Euphorbia albomarginata; SEMC • 1 q; same location as for preceding; 16 Jun. 1950; L.D. Beamer leg.; ex Sapindus drummondii; SEMC • 1 \%; Granite Dells; [34.61 $\left.{ }^{\circ} \mathrm{N}, 112.41^{\circ} \mathrm{W}\right] ; 3$ Jul. 1950; H. Wright leg.; SEMC • 1 \%; Pima Co., Madera Canyon, $13 \mathrm{~km} \mathrm{~S}$ and $10 \mathrm{~km} \mathrm{E}$ of Continental; $31.7488^{\circ} \mathrm{N}, 110.8872^{\circ} \mathrm{W}$; 20 Jul. 2010; R.E. Wrigley leg.; WRME • 1 q; Mule Pass no. Bisbee; $\left[31.458^{\circ}\right.$ N, $109.942^{\circ}$ W]; 24 Mar. 
1946; C.D. Michener leg.; ex yellow composite; SEMC • 2 우; Cochise Co., nr Willcox Blue Sky Rd.; $32.23199^{\circ} \mathrm{N}, 109.77807^{\circ} \mathrm{W} ; 1279 \mathrm{~m}$ a.s.1.; 14 Aug. 2007; Gibbs and Sheffield leg.; PCYU • 2 q $ᄋ$; Patagonia; [31.54 ${ }^{\circ} \mathrm{N}, 110.76^{\circ} \mathrm{W}$ ]; 10 Sep. 1957; T.R. Haig leg.; UCDC 11 \%; Cochise Co., Portal

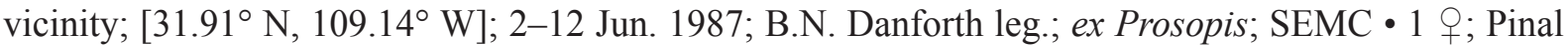
Co., 12 mi. N of Redington; [32.6 N, 110.5 W]; 22 May 1975; J.L. Neff leg.; ex Prosopis velutina; CTMI 1 क; same location as for preceding; 22 May 1975; J.L. Neff leg.; ex Larrea tridentata; CTMI • 1 q, 1 §’; Cochise Co., S of Benson; $31.935578^{\circ} \mathrm{N}, 110.28643^{\circ} \mathrm{W} ; 1273 \mathrm{~m}$ a.s.1.; 14 Aug. 2007; Gibbs and Sheffield leg.; PCYU • 1 क; Salt River Canyon; [33.7 ${ }^{\circ}$ N, $110.8^{\circ} \mathrm{W}$ ]; 16 Jun. 1950; R.H. Beamer leg.; SEMC • 1 \%; Cochise Co., San Bernardino National Wildlife Refuge; $31.3386^{\circ} \mathrm{N}, 109.2719^{\circ} \mathrm{W}$; 19 Apr. 2005; R.L. Minckley leg.; PCYU • 1 क ; Cochise Co., San Bernardino NWR; $31.3417^{\circ}$ N, $109.2583^{\circ}$ W; 30 Mar. 2002; A. Romeroleg.; RLM•1 9 ; same location as for preceding; 28 Apr. 2004; R.L. Minckley leg.;

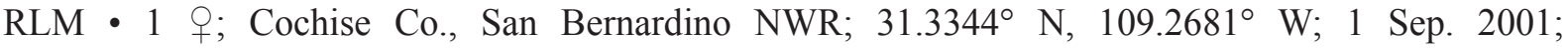
R.L. Minckley leg.; RLM • 1 \%; Cochise Co., San Bernardino NWR; $31.3406^{\circ}$ N, $109.2575^{\circ}$ W; 8 Sep. 2001; R.L. Minckley leg.; RLM • 1 क ; Cochise Co., San Bernardino NWR; $31.3417^{\circ}$ N, $109.2583^{\circ}$ W; 26 Jul. 2001; R.L. Minckley leg.; RLM • 2 우; Cochise Co., San Bernardino NWR, Bosque dry, Site 6; 31.34058 ${ }^{\circ}$ N, $109.25753^{\circ}$ W; 6 Aug. 2003; RLM • 1 q; Cochise Co., San Bernardino NWR, Bosque dry, Site 6, Transect 2; $31.34058^{\circ} \mathrm{N}, 109.25747^{\circ} \mathrm{W} ; 19 \mathrm{Jul}$. 2005; RLM • 1 क ; same location as for preceding; 16 Aug. 2005; RLM - 1 क; Cochise Co., San Bernardino NWR, Bosque dry, Site 7; $31.34118^{\circ}$ N, 109.25813 W; 6 Aug. 2003; RLM • 1 क; Cochise Co., San Bernardino NWR, Bosque dry, Site 7 , Transect 2; $31.34168^{\circ} \mathrm{N}, 109.25825^{\circ} \mathrm{W}$; 11 Oct. 2002; RLM • 1 \%; Cochise Co., San Bernardino NWR, Bosque wet, Site 2, Transect 2; $31.33627^{\circ}$ N, 109.26752 ${ }^{\circ}$ W; 15 Aug. 2005; RLM • 1 O; Cochise Co., San Bernardino NWR, Bosque wet, Site 4, Transect 1; 31.33693 ${ }^{\circ}$ N, 109.24361 ${ }^{\circ}$ W; 11 Aug. 2005; RLM • 1 O; Cochise Co., San Bernardino NWR, field, Site 5, Transect 3; $31.33417^{\circ}$ N, $109.26778^{\circ} \mathrm{W}$; 30 Jul. 2004; RLM • 4 q o ; Cochise Co., San Bernardino NWR, field, Site 6; $31.33867^{\circ}$ N, $109.2719^{\circ}$ W; 8 Aug. 2003; RLM - 1 O; Cochise Co., San Bernardino NWR, field, Site 6, Transect 4; $31.33444^{\circ}$ N, 109.26806 ${ }^{\circ}$ W; 30 Jul. 2004; RLM • 1 क; Cochise Co., San Bernardino NWR, field, Site 8; $31.33765^{\circ}$ N, 109.26068 W; 9 May 2008; RLM • 2 9 \% ; Cochise Co., San Bernardino NWR, field, Site 8, Transect 2; $31.3381^{\circ}$ N, $109.2605^{\circ}$ W; 30 Jul. 2004; RLM • 1 क; Cochise Co., San Bernardino NWR, Grassland, Site 2, Transect 3; $31.3358^{\circ} \mathrm{N}, 109.2702^{\circ} \mathrm{W}$; 30 Jul. 2004; RLM • 2 o o ; Cochise Co., San Bernardino NWR, Grassland, Site 2, Transect 4; $31.3358^{\circ} \mathrm{N}, 109.2702^{\circ} \mathrm{W}$; 11 Aug. 2005; RLM • 6 o $O$; Cochise Co., San Bernardino NWR, Grassland, Site 4; $31.33495^{\circ}$ N, 109.25314 W; 5 Aug. 2003; RLM • 1 क; same location as for preceding; 11 Aug. 2005; RLM • 2 + ; ; Cochise Co., San Bernardino NWR,

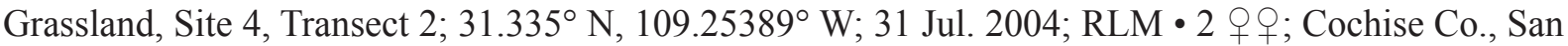
Bernardino NWR, Grassland, Site 5; $31.33683^{\circ}$ N, 109.25308 W; 5 Aug. 2003; RLM • 1 O ; Cochise Co., San Bernardino NWR, Riparian, Site 5, Transect 1; 31.33448 ${ }^{\circ}$ N, 109.25245 ${ }^{\circ}$ W; 15 Oct. 2002; RLM • 1 ; ; Cochise Co., San Bernardino NWR, Robertson cienega; $31.33417^{\circ}$ N, $109.25222^{\circ}$ W; 19 Aug. 2001; ex Xanthisma sp.?; RLM • 1 क; Cochise Co., San Bernardino NWR, Shrub, Site 5, Transect 2; 31.34258 ${ }^{\circ}$ N, 109.27013 ${ }^{\circ}$ W; 27 Jul. 2007; RLM • 1 क; Cochise Co., San Bernardino NWR, Shrub, Site 8; $31.34167^{\circ}$ N, $109.25278^{\circ}$ W; 5 Aug. 2003; RLM • 1 क; Cochise Co., San Bernardino NWR, Shrub, Site 8, Transect 3; $31.34072^{\circ}$ N, $109.253^{\circ}$ W; 11 Aug. 2005; RLM • 1 क ; Cochise Co., San Simon;

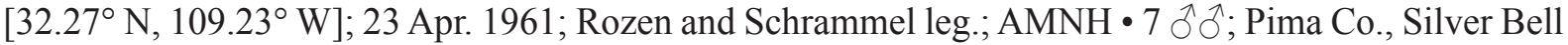

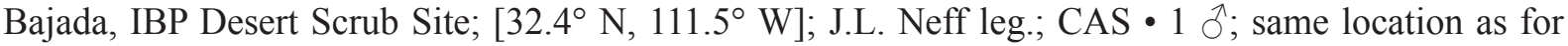
preceding; 1 Sep. 1974; J.L. Neff leg.; ex Trianthema portulacastrum; CUIC • 1 O ; Cochise Co., St David Cienega; $31.8465^{\circ}$ N, $110.2275^{\circ}$ W; 4 Apr. 2016; C.M. Delphia and J.B. Runyon leg.; MTEC • $1 \mathrm{\partial}^{\lambda}$; Cochise Co., Tombstone; [31.71 ${ }^{\circ} \mathrm{N}, 110.07^{\circ} \mathrm{W}$ ]; 3-5 Jul. 1971; M.L. Lindsey leg.; CUIC • 2 우; Tucson; [32.22 ${ }^{\circ} \mathrm{N}, 110.97^{\circ} \mathrm{W}$ ]; 4 Mar. 1934; Bryant leg.; CAS • 3 우; same location as for preceding; 6 Jul. 1950; R.H. Beamer leg.; ex Prosopis glandulosa; SEMC • 21 q + ; White Mts; [33.9 $\left.{ }^{\circ} \mathrm{N}, 109.6^{\circ} \mathrm{W}\right]$; 19 Jun. 1950; R.H. Beamer leg.; ex Amorpha fruticosa; SEMC $\bullet 1$ đ; same location as for preceding; 19 Jun. 1950; L.D. Beamer leg.; ex Penstemon superbus; SEMC • 1 क; Willcox; [32.25 N $\left.109.83^{\circ} \mathrm{W}\right]$; 2005; L. Packer leg.; PCYU • 1 क; Cochise Co., 2 mi. SE of Willcox; $32.227^{\circ}$ N, $109.781^{\circ}$ W; 24 Aug. 2016; J.L. Neff leg.; ex Eriogonum abertianum; CTMI • 1 क; Cochise Co., 6.4 km SE of Willcox on 
Blue Sky Rd; $32.2275^{\circ}$ N, $109.7814^{\circ}$ W; 1250 m a.s.1.; 21 Aug. 2001; C. Michener leg.; ex Psorothamnus scoparius; SEMC. - California 1 क; Riverside Co., 3 mi. E of Moreno Valley, Redlands Boulevard; [33.94 N, 117.16 W]; 2 Jul. 1982; N.J. Smith leg.; UCDC • 1 क ; Los Angeles Co., Frenchmans Flats; [34.617 $\left.{ }^{\circ} \mathrm{N}, 118.745^{\circ} \mathrm{W}\right] ; 8$ Jun. 1966; R.J. Hamton leg.; LACM • 6 우; Los Angeles Co., 2 mi. W of Pearblossom; [34.51 $1^{\circ} \mathrm{N}, 117.94^{\circ} \mathrm{W}$; 12 May 1956; E.G. Linsley leg.; EMEC • 1 O ; Los Angeles Co., Pico Canyon, 2 mi. W of Newhall; [34.38 N, 118.6 W]; 12 Aug. 1975; J. Lyon leg.; ex Eriogonum

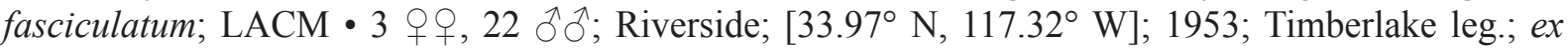
Alyssum; EMEC. - Colorado • $1 \mathrm{~J}^{\top}$; Montrose Co., $18 \mathrm{mi}$. SE of Gateway; $\left[38.49^{\circ} \mathrm{N}, 108.75^{\circ} \mathrm{W}\right] ; 19$ Aug. 1963; N. and B. Marston leg.; AMNH • 3 q $q, 5 \partial^{\lambda} \partial^{\lambda}$; Fremont Co., 6 mi. NE of Florence; $\left[38.45^{\circ} \mathrm{N}\right.$, $\left.105.04^{\circ} \mathrm{W}\right]$; 11 Aug. 1964; J.G., B.L. and K.C. Rozen leg.; AMNH • 1 क ; Boulder; [40.02 $\left.{ }^{\circ} \mathrm{N}, 105.27^{\circ} \mathrm{W}\right]$; 10 Aug. 1935; C.D. Michener leg.; ex Cleome; SEMC 11 o; Bent Co., John Martin Reservoir; $\left[38.1^{\circ} \mathrm{N}\right.$,

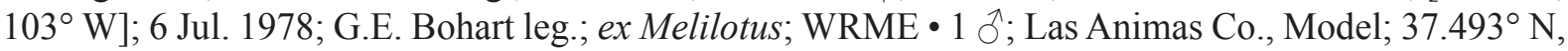
103.8345 ${ }^{\circ}$ W; 23 Jul. 2009; J. Newton leg.; PCYU • 1 क; Las Animas Co., Pergatoire Riv. Canyon, dinosaur track site, Comanche Nat. Grasslands; [37.617 ${ }^{\circ}$ N, $\left.103.598^{\circ} \mathrm{W}\right] ; 21$ Jun. 2005; H. Guarisco leg.; ex Dalea candida var. oligophylla; SEMC • 1 क; Delta Co., R. M. R. C.; [39 N, 108 W]; 3 Jul. 1996; R. Konold leg.; ex buckwheat; CSUC $\bullet 1$ q; same location as for preceding; 3 Jul. 1996; R. Konold leg.; ex Alyssum; CSUC • 1 O; same location as for preceding; 23 Jun. 1996; R. Konold leg.; ex Alyssum; CSUC $\bullet 1$ \%; White Rocks, Boulder; [40.06 ${ }^{\circ}$ N, $105.16^{\circ}$ W]; 8 Jul. 1935; C.D. Michener leg.; ex Dalea; SEMC. - Montana • 1 \%; Gallatin Co., Gallatin Grown; $45.7713^{\circ}$ N, $111.2953^{\circ}$ W; 25 Jul. 2013; C.M. Delphia leg.; MTEC • 1 क; same location as for preceding; 30 Aug. 2013; C.M. Delphia leg.; MTEC • 2 q ; s same location as for preceding; 24 Jul. 2015; C.M. Delpha and J.L. Monte leg.; MTEC • 2 우; same location as for preceding; 9 Jul. 2015; C.M. Delpha and J.L. Monte leg.; MTEC • 3 q $ᄋ$; same location as for preceding; 2 Aug. 2013; C.M. Delphia leg.; MTEC • 1 \&; same location as for preceding; 8 Aug. 2013; C.M. Delphia leg.; MTEC • 1 क; Gallatin Co., Towne's Harvest Garden, 2730 W of Garfield Avenue; [45.66 ${ }^{\circ}$ N, $111.07^{\circ}$ W]; 8 Aug. 2011; C.M. Delphia leg.; MTEC. - Nevada • 1 P ; Lincoln Co., Caliente; [37.62 ${ }^{\circ} \mathrm{N}, 114.51^{\circ} \mathrm{W}$ ]; 14 Jul. 19?8; F.D. Parker leg.; UCDC • 1 \%; Lincoln Co., Kershaw Ryan State Park; $\left[37.59^{\circ}\right.$ N, $114.52^{\circ}$ W]; 14 Jul. 1971; J. Doyen leg.; EMEC. - New Mexico • 1 ○; Hidalgo Co., 10 mi. S of Animas; [31.8 ${ }^{\circ} \mathrm{N}, 108.81^{\circ} \mathrm{W}$ ]; 22 Aug. 1972; G.C. Eickwort leg.; CUIC • 1 क ; Hidalgo Co., 13 mi. $\mathrm{N}$ of Rodeo; [32.02 ${ }^{\circ} \mathrm{N}, 109.03^{\circ} \mathrm{W}$ ]; $1372 \mathrm{~m}$ a.s.1.; 16 Aug. 1964; C.D. Michener leg.; ex Pectis; SEMC • 1 क ; same location as for preceding; 18 Aug. 1964; C.D. Michener leg.; ex Wislizenia

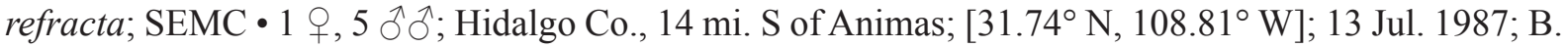
Danoff leg.; ex Lepidium; SEMC • 1 क; Hidalgo Co., $15 \mathrm{~km} \mathrm{~N}$ of Rodeo; [31.97 $\left.{ }^{\circ} \mathrm{N}, 109.03^{\circ} \mathrm{W}\right]$; 1300 m a.s.1.; 6 Jul. 1993; Robert L. Minckley leg.; ex Glandularia bipinnatifida; SEMC • 1 क; Hidalgo Co., 15 mi. S of Animas; [31.73 N, 108.81 ${ }^{\circ} \mathrm{W}$ ]; 24 Aug. 1986; R.J. McGinley and B.M. Norden leg.; USNM 1 क; Hidalgo Co., 3.5 mi. SW of Rodeo; [31.8 N, 109.07 W]; 16 Aug. 1986; R.J. McGinley and B.M. Norden leg.; USNM • 1 क; Alamogordo; [32.9 $\left.\mathrm{N}, 105.96^{\circ} \mathrm{W}\right]$; 13 May 1902; ANSP • 1 \%; same location as for preceding; 26 Jun. 1940; L.J. Lipovsky leg.; SEMC • 1 क; Hidalgo Co., Animas;

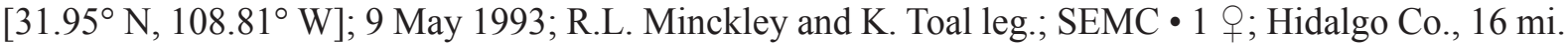
S of Animas; $31.7213^{\circ} \mathrm{N}, 108.8224^{\circ} \mathrm{W} ; 29$ Aug. 2013; T.L. Griswold leg.; ex Baccharis salicina; BBSL - 1 क; Hidalgo Co., Animas, 8.8 km E of junc. Sock N Shoe Rd; $31.9572^{\circ}$ N, $108.7144^{\circ}$ W; 1390 m a.s.l.; 15 Aug. 2000; R. Brooks leg.; ex Fallugia paradoxa; SEMC • 1 क; Hidalgo Co., Antelope Corral, 6 mi. $\mathrm{N}$ of Rodeo; 31.9167-31.9333 ${ }^{\circ}$ N, 109-109.0166 ${ }^{\circ}$ W; 1231 m a.s.1.; 25 Jun. 1984; H.A. Hespenheide leg.; ex ArtemisialAtriplex; LACM • 1 q; Hidalgo Co., 12.8 mi. W of Hachita; $31.965^{\circ} \mathrm{N}$, $108.6111^{\circ}$ W; 1440 m a.s.1.; 27 Sep. 1999; R. Brooks and C. Michener leg.; ex Heliomeris multiflora var. nevadensis; SEMC • 1 `े; Socorro Co., Bosque del Apache; [33.8 $\left.{ }^{\circ} \mathrm{N}, 106.89^{\circ} \mathrm{W}\right] ; 1375 \mathrm{~m}$ a.s.1.; 15 Jul. 1991; Ilan Yarom leg.; SEMC • 19 o ${ }^{\prime}$; Chaves Co., Bottomless Lakes, 10 mi. E of Roswell; [33.34 ${ }^{\circ}$ N, 104.34 ${ }^{\circ} \mathrm{W}$ ]; 20 May 1969; Brothers, Krueger and Michener leg.; SEMC • 1 đं; Eddy Co., Carlsbad; [32.42 $2^{\circ}$ N, 104.23 ${ }^{\circ}$ W]; 20 May 1969; Brothers, Krueger and Michener leg.; ex Lepidium integrifolium; SEMC • 2 q o ; Carrizozo; [33.64 $\left.{ }^{\circ} \mathrm{N}, 105.88^{\circ} \mathrm{W}\right]$; 10 Jun. 1950; L.D. Beamer leg.; ex

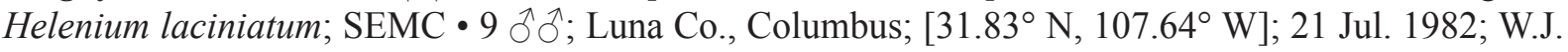
Pulawski leg.; CAS • 1 § ; same location as for preceding; 21 Jul. 1982; W.J. Pulawski leg.; CUIC • 1 क ; 


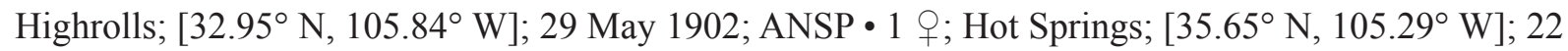

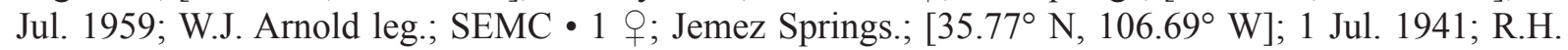
Beamer leg.; SEMC • 2 o 9 ; Nogal; [33.56 $6^{\circ}$ N, $105.71^{\circ}$ W]; 21 Jul. 1952; C. Liang, W. LaBerge, R.H. and L.D. Beamer leg.; SEMC • 5 q 9 ; Eddy Co.; $32.1095^{\circ}$ N, $104.4716^{\circ} \mathrm{W} ; 1115$ m a.s.1.; 19 Jul. 2010; J.D. Herndon leg.; ex Plantago sp.; BBSL • 1 \%; Hidalgo Co., S. of Animas; [31.9 N, $108.81^{\circ} \mathrm{W}$; 2003; L. Packer leg.; PCYU. - Texas • 5 우영 Jeff Davis Co., 0.5 mi. S of Kent; $\left[31.06^{\circ} \mathrm{N}, 104.22^{\circ} \mathrm{W}\right]$; 29 May 1999; L. Packer leg.; PCYU • 1 q; Reeves Co., 3 mi. NW of Pecos; $31.453^{\circ}$ N, $103.508^{\circ}$ W; 6 Jul. 2007; J.L. Neff and A. Hook leg.; ex Lepidium alyssoides; CTMI • 1 q; Santa Elena Canyon, Big

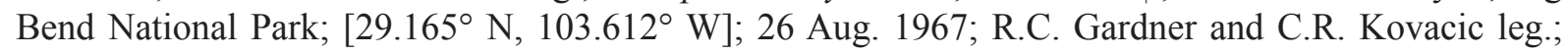
UCDC. - Utah • 1 q; Kane Co., 1.0 mi. E of Nipple Spring; 37.189 ${ }^{\circ}$ N, $111.5368^{\circ}$ W; 16 May 2002; K. Fordney leg.; ex Tamarix sp.; BBSL • 3 + $ᄋ$; Garfield Co., 10 mi. $\mathrm{N}$ of Escalante; [37.9 $\left.\mathrm{N}, 111.6^{\circ} \mathrm{W}\right]$;

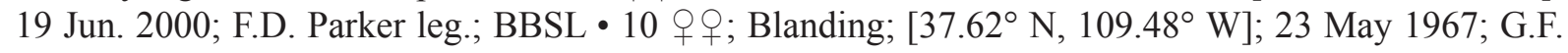

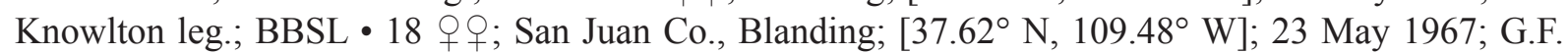
Knowlton leg.; UCDC • 1 क; Kane Co., Butler Valley Dam; $37.4693^{\circ} \mathrm{N}, 111.871^{\circ} \mathrm{W}$; 19 Jul. 2002; J. Richmond leg.; ex Helianthus petiolaris; PCYU • 2 o o ; Castle Valley; [38.64 $\left.{ }^{\circ} \mathrm{N}, 109.41^{\circ} \mathrm{W}\right]$; 10 May 1949; G.F. Knowlton leg.; SEMC • 1 क; Colorado River, 4 mi. $\mathrm{N}$ of Moab; $\left[38.62^{\circ} \mathrm{N}, 109.51^{\circ} \mathrm{W}\right] ; 1$ Aug. 1987; R.S. Beal leg.; CSUC • 1 क; Colorado River, 5 mi. N of Moab; $\left[38.63^{\circ} \mathrm{N}, 109.49^{\circ} \mathrm{W}\right] ; 1$ Aug. 1987; R.S. Beal leg.; CSUC • 2 우; Emery Co., Green River; $38.9885^{\circ}$ N, $110.162^{\circ}$ W; 9 May 2000; F.D. Parker leg.; BBSL • 1 q; Kane Spgs., 15 mi. SE of Moab; [38.392 ${ }^{\circ}$ N, $109.452^{\circ}$ W]; 9 Aug. 1957; C.D. Michener leg.; ex Cleome lutea; SEMC • 1 q, 9 ổ స̂; Grand Co., Moab Hwy 128, 2 mi marker roadside; [38.6117 ${ }^{\circ}$ N, 109.5513 W]; 15 Aug. 1992; L. Packer leg.; PCYU • 2 q ; Uintah Co., Ouray Valley; [ $40^{\circ} \mathrm{N}, 109.5^{\circ} \mathrm{W}$ ]; 12 Jul. 1956; G.F. Knowlton leg.; SEMC • 2 + ; Garfield Co., Pine Creek, 4.5 mi. N of Escalante; $37.9031^{\circ} \mathrm{N}, 111.6686^{\circ} \mathrm{W}$; 19 Jun. 2000; J. Grixti leg.; ex Tamarix sp.; BBSL • 3 q $O$; same location as for preceding; 19 Jun. 2000; R. Sellars leg.; ex Tamarix sp.; BBSL • 2 우; same location as for preceding; 19 Jun. 2000; O. Messinger leg.; ex Tamarix sp.; BBSL 4 우우 Uintah Co., Vernal; [40.46 N, $109.53^{\circ}$ W]; 18 Jul. 1961; G.F. Knowlton leg.; UCDC. - Wyoming • 1 \%; Uinta Co., Lonetree; [41.05 N, 110.15 W]; 17 Jul. 1961; G.F. Knowlton leg.; UCDC.

\section{Redescription}

\section{Female}

Colouration. Head and mesosoma blue-green to golden-green; clypeus apical colour reddish brown to orange; labrum reddish brown to orange; mandible orange with black basal spot or band and red tip; flagellum black to reddish brown dorsally, reddish brown to orange ventrally; pronotal lobe reddish brown to orange; metasoma red to orange with dark spiracular spots on T3-4; legs reddish brown with femur-tibia joints, tibia apices, and tarsi mostly orange; tegula pale amber; wing membrane hyaline, veins with subcosta dark brown, otherwise pale amber.

Pubescence. Body hair colour white. Tomentum dense on paraocular area, gena, pronotal collar and lobe, space between pronotal lobe and tegula, and metanotum anterior margin; sparse on clypeus basal half, supraclypeal area, preëpisternum, metepisternum, and T4 basally. Scutum hair thin to slightly densely plumose. Wing hairs light, very short and sparse. Acarinarial fan complete or nearly so, sparse. $\mathrm{T} 2$ fringes very sparse, $\mathrm{T} 3$ fringes very sparse.

SURFACE SCULPTURE. Clypeus punctures dense in basal half $(i<1$ pd), large and irregularly spaced apically (i $<2$ pd), sculpture shiny, sometimes weakly tessellate basally; supraclypeal area punctures sparse ( $i=1-4 \mathrm{pd})$, becoming dense laterally ( $i \leq 1 \mathrm{pd})$, sculpture shiny, sometimes becoming weakly tessellate laterally; paraocular area punctures dense ( $i \leq 1 \mathrm{pd})$, sometimes crowded around antenna socket, sculpture shiny, sometimes weakly tessellate around antenna socket; frons punctures dense ( $i<1 \mathrm{pd}$ ), sculpture tessellate; vertex punctures fine, dense laterally ( $\mathrm{i} \leq 2 \mathrm{pd}$ ), moderately sparse medially ( $i=1-2 \mathrm{pd})$, sculpture shiny or weakly tessellate; gena punctures moderately sparse $(i=1-3 \mathrm{pd})$, sculpture shiny; postgena sculpture tessellate and sometimes weakly rugulose; tegula punctures absent; 
scutum punctures moderately dense ( $i \leq 2 \mathrm{pd})$, becoming sparser submedially ( $\mathrm{i}=1-3 \mathrm{pd})$ and denser posteromedially ( $\mathrm{i}<1 \mathrm{pd}$ ), sculpture shiny to weakly tessellate, becoming more strongly tessellate anteromedially; scutellum punctures moderately dense ( $\mathrm{i} \leq 2 \mathrm{pd}$ ), becoming sparser submedially $(\mathrm{i}=1-3 \mathrm{pd})$ and denser posteromedially $(\mathrm{i}<1 \mathrm{pd})$, diversopunctate, sculpture shiny, sometimes becoming tessellate marginally and on median line; metanotum sculpture shiny and finely, densely punctate ( $<<1 \mathrm{pd}$ ), becoming rugulose laterally; metapostnotum rugae strong, highly anastomosing, reaching margin, sculpture tessellate; preëpisternum sculpture areolate; hypoepimeron punctures dense $(\mathrm{i}<1 \mathrm{pd})$, sculpture shiny or weakly imbricate; mesepisternum punctures moderately dense ( $\mathrm{i}=1-2 \mathrm{pd}$ ), becoming dense ventrally ( $\mathrm{i}<1 \mathrm{pd}$ ), sculpture shiny or weakly imbricate; metepisternum sculpture rugulosolineate dorsally, areolate or tessellate and densely punctate $(i<1 \mathrm{pd})$ ventrally; propodeum lateral face sculpture tessellate; propodeum posterior face sculpture tessellate; T1 anterior face sculpture shiny; $\mathrm{T} 1$ dorsal surface punctures moderately sparse $(\mathrm{i}=1-4 \mathrm{pd})$, becoming finer or obscure on rim and absent in large apicolateral oval patches, sculpture shiny; T2 disc punctures moderately dense ( $\mathrm{i}=1-3 \mathrm{pd})$, disc sculpture shiny, rim punctures very fine, sometimes obscure, sparse ( $i=2-4 \mathrm{pd})$, rim sculpture weakly coriarious.

StRUCTURE. Face length/width ratio 0.83 ( $\pm 0.02 \mathrm{SD}$ ). Clypeus projecting $\sim 67 \%$ below suborbital tangent; clypeal area length/width ratio $0.41( \pm 0.04 \mathrm{SD})$; apicolateral denticles low rounded knobs; supraclypeal area length/width ratio $0.67( \pm 0.08 \mathrm{SD})$. Forewing with 3 submarginal cells; pronotal angle obtuse; tegula shape normal. Intertegular distance 0.86 ( $\pm 0.03 \mathrm{SD}) \mathrm{mm}$. Scutum length/width ratio 0.78 ( $\pm 0.02 \mathrm{SD}) ;$ scutum/scutellum length ratio 2.99 ( $\pm 0.16 \mathrm{SD})$; scutellum/metanotum length ratio 1.51 ( $\pm 0.13 \mathrm{SD})$; metanotum/metapostnotum length ratio 0.65 ( $\pm 0.06 \mathrm{SD})$. Propodeum lateral carinae not reaching dorsal margin; oblique carina very weak or absent. T2 depressed apical rim length less than $50 \%$ of segment. $(n=11)$

\section{Male}

Colouration. Head and mesosoma blue to olive green; clypeus apical colour black to reddish brown; labrum reddish brown to orange; mandible orange with black basal spot and red tip; flagellum reddish brown dorsally, orange ventrally; pronotal lobe reddish brown; metasoma black to dark reddish brown with rims of terga and sterna broadly translucent reddish brown and downcurved lateral areas of terga becoming translucent orange; legs reddish brown with femur-tibia joints, base and apex of tibiae, and tarsi orange; tegula orange; wing membrane hyaline, veins dark to light brown.

Pubescence. Body hair colour white. Tomentum dense on face below eye emargination, gena, pronotal angle and lobe, and space between pronotal lobe and tegula; sparse on episterna. Scutum hair thin to moderately plumose. Sterna hair short (0.75-1.25 OD), densely plumose, dense and erect. Wing hairs dark or light, short and sparse.

SuRfaCe SCULPTURE. Clypeus punctures dense to moderately dense ( $\mathrm{i} \leq 2 \mathrm{pd})$, sculpture shiny; supraclypeal area punctures dense to moderately dense ( $\mathrm{i} \leq 2 \mathrm{pd}$ ), sculpture shiny; paraocular area punctures dense $(\mathrm{i}<1 \mathrm{pd})$, sculpture shiny; frons punctures crowded $(\mathrm{i}=0 \mathrm{pd})$, sculpture shiny to weakly imbricate; vertex punctures moderately dense laterally $(i=1-2 \mathrm{pd})$, sparse medially $(i=1-3 \mathrm{pd})$, sculpture shiny to imbricate; gena punctures fine, sparse ( $i=1-3 \mathrm{pd}$ ), obscure, sculpture shiny, becoming weakly ruguloso-lineate posteriorly and ventrally; postgena sculpture imbricate to ruguloso-lineate; tegula punctures absent; scutum punctures dense to moderately sparse ( $\mathrm{i} \leq 2 \mathrm{pd})$, becoming sparse submedially ( $\mathrm{i}=1-3 \mathrm{pd}$ ), sculpture shiny to weakly tessellate, becoming more strongly tessellate anteromedially; scutellum punctures dense marginally and on median line ( $\mathrm{i} \leq 1 \mathrm{pd})$, sparse submedially $(\mathrm{i}=1-3 \mathrm{pd})$, sculpture shiny; metanotum sculpture shiny and densely to moderately sparsely punctate ( $\mathrm{i} \leq 2 \mathrm{pd}$ ); metapostnotum rugae strong, anastomosing, reaching margin, sculpture shiny to weakly imbricate; preëpisternum sculpture shiny with crowded punctures $(\mathrm{i}=0 \mathrm{pd})$; hypoepimeron punctures dense $(\mathrm{i} \leq 1 \mathrm{pd})$, sculpture shiny; mesepisternum punctures dense $(\mathrm{i} \leq 1 \mathrm{pd})$, sculpture shiny; metepisternum 


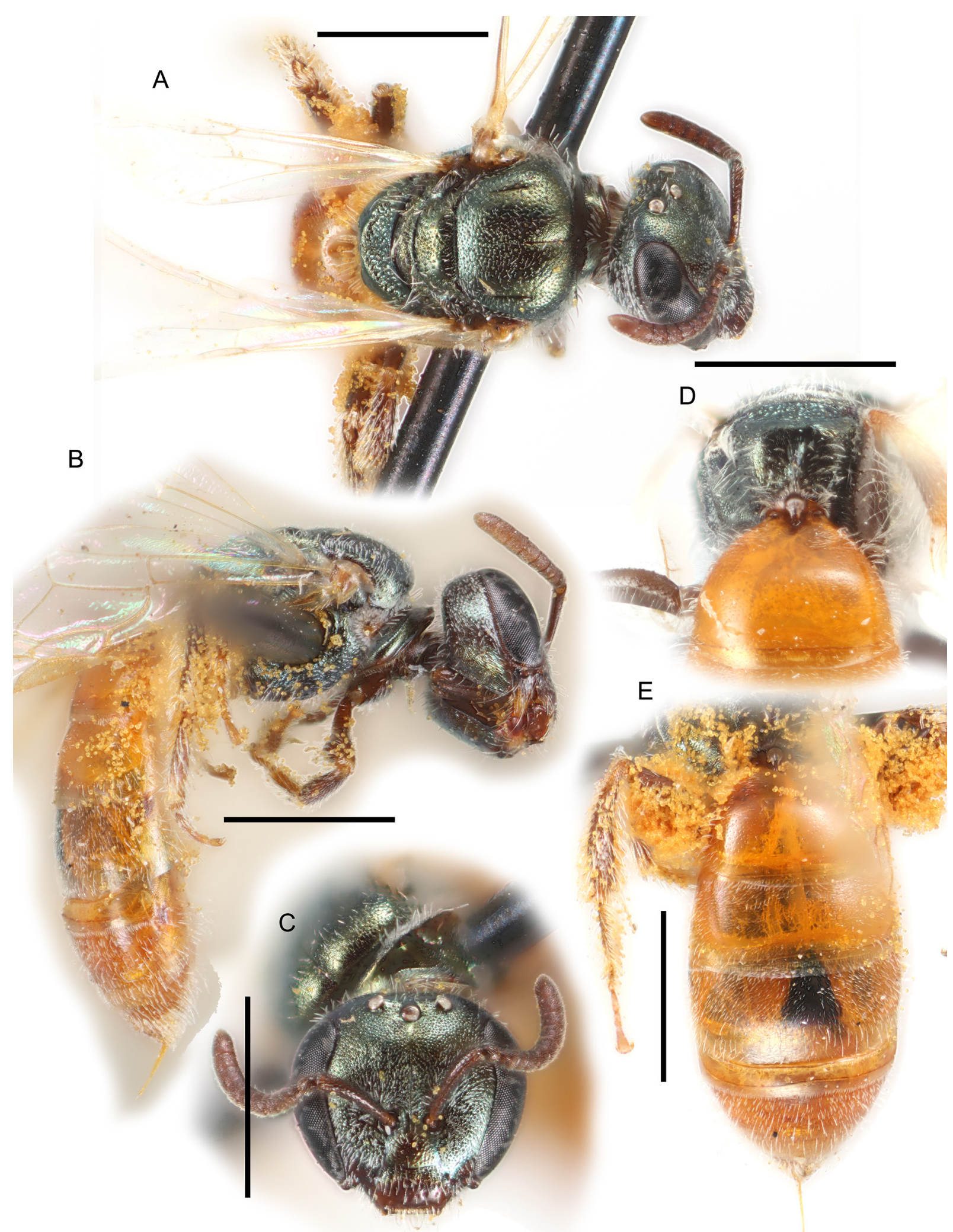

Fig. 28. Lasioglossum (D.) clematisellum (Cockerell, 1904), holotype, $q$ (USNM 12064). A. Dorsal habitus. B. Lateral habitus. C. Face. D. Propodeum (not holotype). E. Metasoma. Scale bars: $1 \mathrm{~mm}$. 


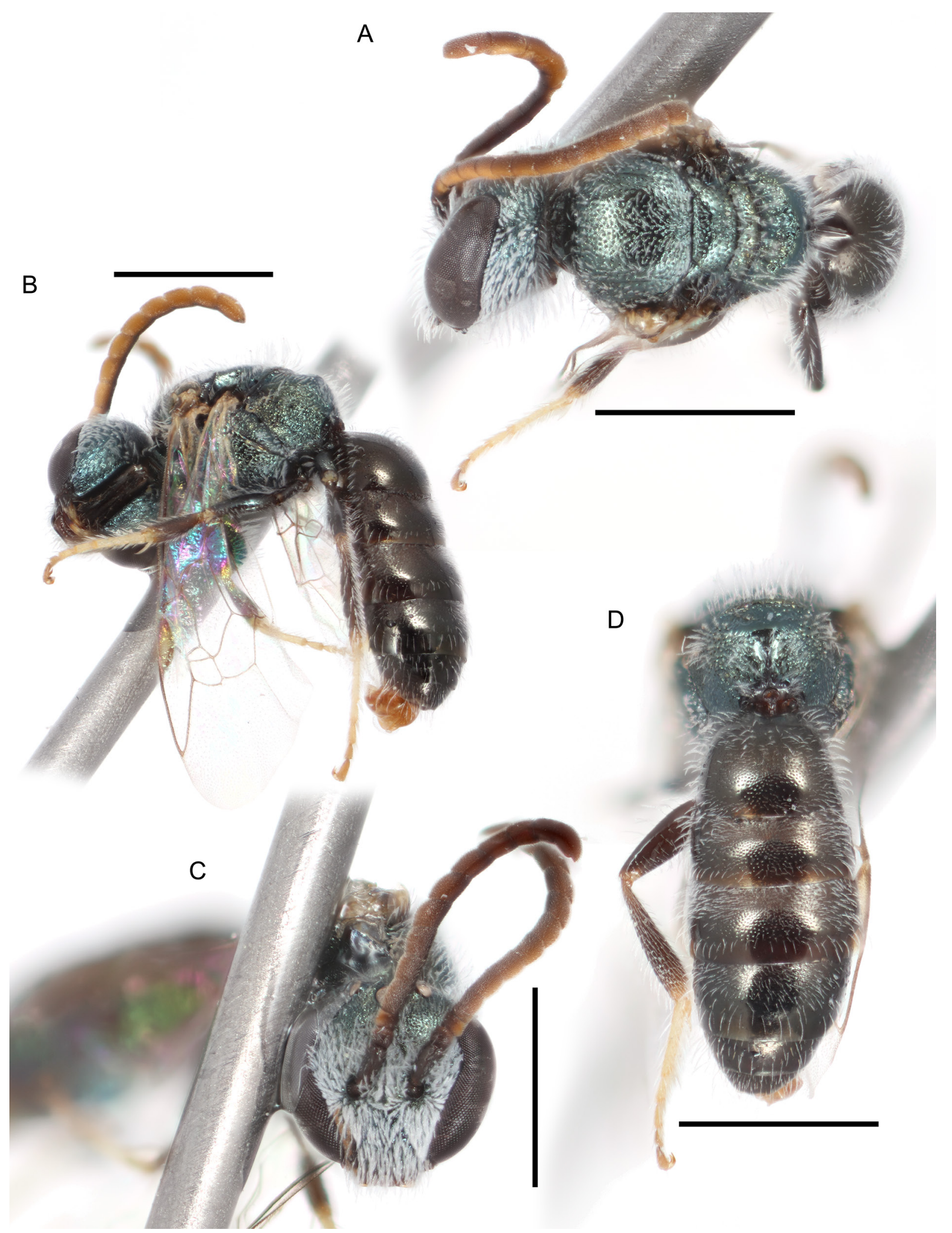

Fig. 29. Lasioglossum (D.) clematisellum (Cockerell, 1904), đ̂. A. Dorsal habitus. B. Lateral habitus. C. Face. D. Propodeum and metasoma. Scale bars: $1 \mathrm{~mm}$. 
sculpture lineate dorsally, areolate-rugose ventrally; propodeum lateral face punctures obscure, sculpture imbricate to rugulose; propodeum posterior face sculpture rugulose; T1 anterior face sculpture shiny; T1 dorsal surface punctures dense $(i \leq 1 \mathrm{pd})$, becoming sparse towards rim $(\mathrm{i}=1-3 \mathrm{pd})$ and absent or very sparse in small apicolateral oval patches, sculpture shiny; T2 disc punctures dense ( $i \leq 1 \mathrm{pd})$, becoming sparse toward premarginal line $(i=1-3 \mathrm{pd})$, disc sculpture shiny, rim punctures sparse medially $(i=1-4 \mathrm{pd})$, absent laterally, rim sculpture shiny.

Structure. Face length/width ratio 0.83 ( $\pm 0.02 \mathrm{SD})$. F1:pedicel length ratio 0.89 ( $\pm 0.13 \mathrm{SD}) ; \mathrm{F} 2: \mathrm{F} 1$ length ratio $1.97( \pm 0.23 \mathrm{SD}) ; \mathrm{F} 2$ length/width ratio $1.56( \pm 0.12 \mathrm{SD}) ; \mathrm{F} 9$ length/width ratio 1.39 ( $\pm 0.09 \mathrm{SD}$ ). Forewing with 2 or 3 submarginal cells; pronotal angle obtuse; tegula shape normal. Intertegular distance $0.75( \pm 0.05 \mathrm{SD}) \mathrm{mm}$. Scutum length/width ratio $0.89( \pm 0.05 \mathrm{SD})$; scutum/ scutellum length ratio 2.77 ( $\pm 0.14 \mathrm{SD})$; scutellum/metanotum length ratio $1.68( \pm 0.17 \mathrm{SD})$; metanotum/ metapostnotum length ratio $0.66( \pm 0.03 \mathrm{SD})$. Propodeum lateral carinae not reaching dorsal margin; oblique carina absent. $(n=10)$

Genitalia. As in Fig. 90C. Gonocoxite relatively narrow, rounded. Gonostylus with a few long hairs. Retrorse lobe relatively narrow, ovoid, covered in sparse short hairs.

\section{Range}

Southwestern Montana south to Zacatecas and west to California (Fig. 30).

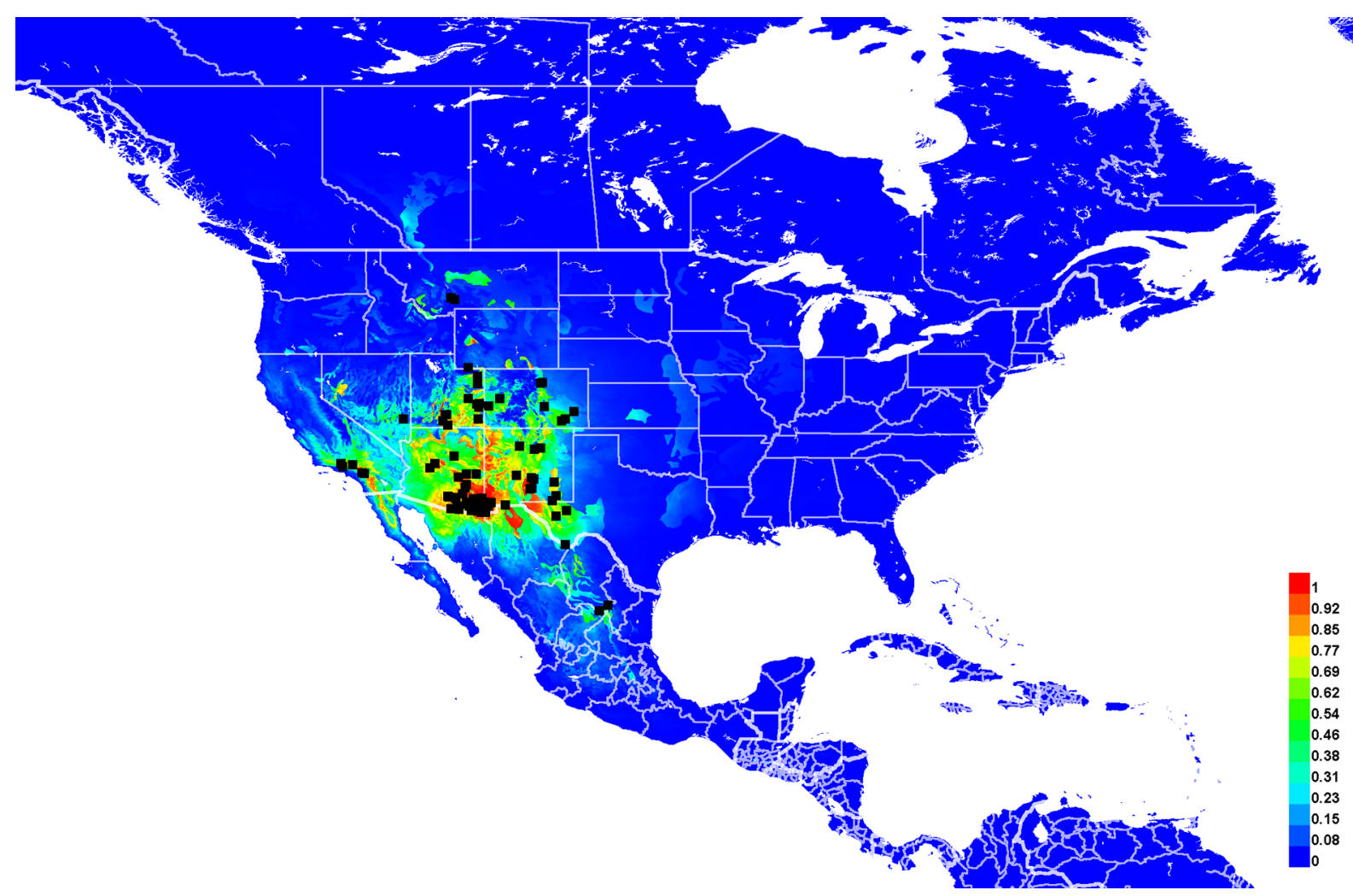

Fig. 30. Georeferenced collection records of Lasioglossum (D.) clematisellum (Cockerell, 1904) (black squares) and predicted distribution by maximum entropy ecological niche modeling in Maxent (colour shading). Warmer colours indicate higher cloglog probability of occurrence. 


\section{Floral records}

AIZOACEAE Martinov: Trianthema L. T. portulacastrum L. - AMARANTHACEAE Juss. Atriplex L. A. canescens (Pursh) Nutt. $・$ Chenopodium L. C. desiccatum A. Nelson • C. neomexicanum Standl. - Salsola L. S. kali L. S. $k$. subsp. tragus (L.) Čelak. - ANACARDIACEAE R.Br. Schinus L. S. molle L. (MH87) • APIACEAE Lindl. Apium L. A. graveolens L. (MH87) • ASPARAGACEAE Juss. Nolina Michx. (MH87) - ASTERACEAE Giseke: Baccharis: B. salicina Torr. \& A.Gray • B. sarothroides A. Gray - Chrysothamnus (MH87) - Ericameria: E. palmeri (A.Gray) H. M. Hall $(\mathrm{MH} 87) \cdot$ Erigeron: E. bonariensis L. $(\mathrm{MH} 87) \cdot$ E. canadensis L. (MH87) $\bullet$ Euthamia: E. occidentalis Nutt. (MH87) - Gutierrezia: G. californica (DC.) Torr. \& A.Gray (MH87) • G. sarothrae (Pursh) Britton \& Rusby (MH87) • Helenium: H. laciniatum A.Gray $\bullet$ Helianthus: H. annuus L. $\bullet$ H. petiolaris Nutt. - Heliomeris: H. multiflora Nutt. H. m. var. nevadensis (A. Nelson) W. F. Yates - Laennecia: L. coulteri (A.Gray) G.L. Nesom • Lepidospartum: L. squamatum (A.Gray) A.Gray (MH87) • Pectis • Senecio: S. flaccidus Less. - Solidago (MH87) - BORAGINACEAE Juss. Cryptantha Lehm. ex G.Don: C. intermedia (A. Gray) Greene (MH87) - Phacelia Juss. P. crenulata Torr. ex S. Watson • P. distans Benth. (MH87) • P. heterophylla Pursh (MH87) • BRASSICACEAE Burnett: Alyssum L. • Hirschfeldia: H. incana (L.) Lagr.-Foss. (MH87) • Lepidium L. L. alyssoides A.Gray • L. integrifolium Nutt. $\bullet$ L. thurberi Wooton $\bullet$ Lobularia Desv. L. maritima (L.) Desv. (MH87) • Physaria (Nutt. ex Torr. \& A.Gray) A.Gray: P. gordonii (A.Gray) O'Kane \& Al-Shehbaz • Sisymbrium L. S. irio L. (MH87) • CLEOMACEAE Bercht. \& J. Presl: Cleome L. C. lutea Hook. - Wislizenia Engelm. W. refracta Engelm. - CONVOLVUlACEAE Juss. Convolvulus L. C. arvensis L. - CRASSULACEAE J. St.Hil. Sedum L. (MH87) • EUPHORBIACEAE Juss. Croton L. C. californicus Müll. Arg. (MH87) • C. setigerus Hook. (MH87) • Euphorbia L. E. albomarginata Torr. \& A.Gray • FABACEAE Juss. Amorpha: A. fruticosa L. - Dalea: D. candida Willd. D. c. var. oligophylla (Torr.) Shinners $\bullet$ Melilotus $\bullet$ Prosopis: P. glandulosa Torr. P. g. var. torreyana (L.D. Benson) M.C. Johnst. $\bullet$ P. juliflora (Sw.) DC. $・$ P. velutina Wooton - Psorothamnus: P. scoparius (A.Gray) Rydb. LAMIACEAE Martinov: Mentha L. M. spicata L. (MH87) • LOASACEAE Juss. Mentzelia L. M. laevicaulis (Douglas) Torr. \& A. Gray (MH87) • MALVACEAE Juss. Sphaeralcea A. St.-Hil. •PAPAVERACEAE Juss. Eschscholzia Cham. E. californica Cham. (MH87) • PLANTAGINACEAE Juss. Penstemon Schmidel: P. superbus A. Nelson - Plantago L. POLEMONIACEAE Juss. Eriastrum Wooton \& Standl. E. virgatum (Benth.) H. Mason (MH87) • POLYGONACEAE Juss. Eriogonum Michx. E. abertianum Torr. $\bullet$ E. fasciculatum Benth. $\bullet$ E. gracile Benth. (MH87) • Persicaria Mill. P. lapathifolia (L.) Delarbe (MH87) • RANUNCULACEAE Juss. Clematis L. C. ligusticifolia Nutt. - ROSACEAE Juss. Fallugia Endl. F. paradoxa (D. Don) Endl. ex Torr. • Prunus L. (MH87) • SALICACEAE Mirb. Salix L. S. exigua Nutt. (MH87) • S. lasiolepis Benth. (MH87) • S. nigra Marshall (MH87) • SAPINDACEAE Juss. Sapindus L. S. drummondii Hook. \& Arn. • TAMARICACEAE Link: Tamarix L. T. ramosissima Ledeb. • VERBENACEAE J. St.-Hil. Glandularia J.F. Gmel. G. bipinnatifida (Schauer) Nutt. • Lantana L. (MH87) • ZYGOPHYLLACEAE R.Br. Larrea Cav. L. tridentata (Sessé \& Moc. ex DC.) Coville.

\section{DNA barcodes}

Eight sequences available (BOLD process IDs: DIAL1098-07, DIAL1108-07, DLII755-07, DLII78307, DLII1085-07, BOWGF2050-10, BWTWO1316-10, BWTWO1318-10; BIN: BOLD:AAE5948). There is some shallow within-species divergence (maximum p-distance $1.33 \%$ ), but this is much less than the difference between species in the L. clematisellum complex (minimum p-distance $2.78 \%$ ). Lasioglossum clematisellum differs from L. clastipedion sp. nov. and L. julipile sp. nov. by 11 fixed substitutions: 18(C), 80(T), 123(C), 141(C), 286(C), 312(T), 354(T), 390(C), 426(C), 474(C), and 579(C) (Supplementary file 4). No unique fixed substitutions distinguish L. clematisellum from all other western red-tailed L. (Dialictus). 


\section{Remarks}

Common. The male is described here for the first time. Lasioglossum clematisellum has an extremely wide distribution, but unlike the similarly-distributed L. hudsoniellum, L. clematisellum is not found at very high elevations and is more common in deserts.

Lasioglossum (Dialictus) decorum sp. nov. urn:1sid:zoobank.org:act:6516BD4E-BBFE-48CB-82D0-6420F8C2748A

Figs $31-33,100 \mathrm{~B}$

\section{Diagnosis}

Females of Lasioglossum decorum sp. nov. can be recognized by the head and mesosoma with strong tessellate microsculpture, acarinarial fan dense but incomplete with a wide central gap, T1 anterior surface coriarious, T2 -3 with small but distinct basolateral patches of tomentum and T4 with sparse tomentum, pronotal angle obtuse, ocelli somewhat enlarged (separated by $\sim 0.5$ OD), and T2 lacking dark spiracular spots. They are most similar to L. austerum sp. nov., L. torrens sp. nov., and L. meteorum sp. nov. All of these species have a weak acarinarial fan and normal ocelli. In addition, females of L. austerum sp. nov. have the metasomal terga without tomentum and T2 with dark spiracular spots. Females of $L$. torrens sp. nov. have the postgena and $\mathrm{T} 1$ anterior surface shiny, pronotal angle nearly 90 degrees, and T2 with dark spiracular spots. Females of L. meteorum sp. nov. have the scutum duller and more sparsely punctate $(i=1-4 \mathrm{pd})$ and metasoma with less extensive tomentum.

Males of $L$. decorum sp. nov. can be recognized by the frons dull with crowded punctures ( $\mathrm{i}=0 \mathrm{pd})$ becoming reticulate above antenna sockets, rims of T1-3 impunctate, tomentum largely absent except on paraocular area and pronotum, mesepisternum shiny and sparsely punctate $(\mathrm{i}=1-2 \mathrm{pd})$, propodeum smooth with metapostnotal rugae not reaching the posterior margin, and clypeus distinctly projecting below the eyes. They are most similar to L. austerum sp. nov. and L. arenisaltans sp. nov. Males of L. austerum sp. nov. have the frons punctures distinctly separated, clypeus not strongly projecting below the eyes, and rims of T1 -3 sparsely punctate medially $(i=1-4 \mathrm{pd})$. Males of $L$. arenisaltans $\mathrm{sp}$. nov. have the flagellomeres relatively longer (F2 about 1.75 times as long as F1), metapostnotum with strong rugae reaching the posterior margin, mesepisternum densely punctate $(\mathrm{i} \leq 1 \mathrm{pd})$ and rims of T1-3 moderately densely punctate $(\mathrm{i}=1-3 \mathrm{pd})$.

\section{Etymology}

The specific epithet decorum is formed from the Latin adjective 'decorus' ('decorated', 'beautiful', 'proper'). It refers to the similarity of this species with L. austerum sp. nov., except adorned with more hair and inhabiting the tropical regions of southern Mexico. An appropriate translation would be the decorated sweat bee.

\section{Material examined}

\section{Holotype}

MEXICO - Jalisco • +; Puerto Vallarta; 20.6214 N, $105.226^{\circ}$ W; 8 Dec. 1984; G.E. Bohart leg.; BBSL.

[Verbatim label: Mexico: Jalisco Puerto Vallarta N20.6214 W105.2260 8 Dec 1984 G.E. Bohart / BBSL1100700 // MEXICO Jalisco / Puerto Vallarta / 8 Dec 1984 / G. E. Bohart // HOLOTYPE / Lasioglossum (Dialictus) decorum Gardner and Gibbs] 
Paratypes

MEXICO - Jalisco • 1 क; Careyes; $19.4289^{\circ}$ N, 105.0274 W; 12 Feb.-19 Mar. 1997; F.D. Parker leg.;

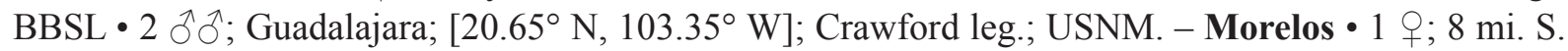
of Yautepec; [18.76 N, 99.06 ${ }^{\circ}$ W]; 16 Aug. 1962; Roberts and Marston leg.; SEMC.

\section{Description}

Female

Colouration. Head and mesosoma blue-green to olive green; clypeus apical colour orange; labrum reddish brown to orange; mandible orange with black basal spot and red tip; flagellum reddish brown; pronotal lobe orange; metasoma orange to reddish brown with dark spiracular spots on T3-4; legs reddish brown with femur-tibia joints, base and apex of tibiae, inner surface of fore tibia, and tarsi orange; tegula orange; wing membrane infuscated, veins dark brown.

PubesCence. Body hair colour white to pale yellow. Tomentum dense on pronotal collar and lobe, space between pronotal lobe and tegula, and T2-3 basolaterally; sparse on paraocular area, gena, and T4 throughout. Scutum hair thin. Wing hairs dark, long and dense. Acarinarial fan incomplete, dense. T2 fringes sparse, $\mathrm{T} 3$ fringes sparse.

SuRfaCe SCULPTURE. Clypeus punctures sparse ( $\mathrm{i}=1-3 \mathrm{pd}$ ), sculpture shiny, becoming weakly imbricate basally; supraclypeal area punctures sparse $(i=1-3 \mathrm{pd})$, sculpture tessellate, becoming shiny medially; paraocular area punctures dense to moderately sparse $(i \leq 2 \mathrm{pd})$, sculpture tessellate to imbricate around antenna socket, otherwise shiny; frons punctures dense $(\mathrm{i}<1 \mathrm{pd})$ to crowded $(\mathrm{i}=0 \mathrm{pd})$, sculpture finely reticulate to rugulose, becoming smooth and shiny to imbricate dorsally; vertex punctures moderately dense laterally ( $\mathrm{i}=1-2 \mathrm{pd}$ ), sparse medially $(\mathrm{i}=1-3 \mathrm{pd}$ ), sculpture shiny, sometimes weakly rugulose laterally; gena punctures fine, moderately dense ( $\mathrm{i}=1-2 \mathrm{pd})$, sculpture shiny, becoming lineolate posteriorly and ventrally; postgena sculpture lineolate to tessellate; tegula punctures absent; scutum punctures dense ( $\mathrm{i} \leq 1 \mathrm{pd})$, becoming moderately sparse submedially and anteromedially ( $\mathrm{i}=1-3 \mathrm{pd})$, sculpture shiny to weakly tessellate, becoming more strongly tessellate anteromedially; scutellum punctures moderately sparse marginally and on median line $(i=1-2 \mathrm{pd})$, sparse submedially $(i=1-4 \mathrm{pd})$, diversopunctate, sculpture shiny, becoming weakly tessellate marginally; metanotum sculpture imbricate, sometimes finely and densely punctate medially ( $<1 \mathrm{pd}$ ); metapostnotum rugae strong, subparallel or anastomosing, not reaching margin, sculpture tessellate to finely reticulate; preëpisternum sculpture imbricate and weakly rugulose; hypoepimeron punctures moderately sparse $(\mathrm{i}=1-2 \mathrm{pd})$, obscure, sculpture tessellate to finely reticulate; mesepisternum punctures moderately sparse ( $\mathrm{i}=1-2 \mathrm{pd})$, sculpture tessellate to finely reticulate; metepisternum sculpture lineate dorsally, imbricate ventrally; propodeum lateral face sculpture tessellate; propodeum posterior face sculpture tessellate; T1 anterior face sculpture coriarious; $\mathrm{T} 1$ dorsal surface punctures fine, sparse $(\mathrm{i}=1-4 \mathrm{pd})$, absent in narrow median line, large apicolateral patches, and on rim, sculpture shiny; T2 disc punctures fine, sparse ( $\mathrm{i}=1-3 \mathrm{pd})$, disc sculpture shiny, rim punctures minute, sparse $(i=2-4 \mathrm{pd})$, hardly visible, rim sculpture weakly coriarious.

STRUCTURE. Face length/width ratio 0.87 ( $\pm 0.02 \mathrm{SD}$ ). Clypeus projecting $\sim 67 \%$ below suborbital tangent; clypeal area length/width ratio $0.48( \pm 0.06 \mathrm{SD})$; apicolateral denticles rounded knobs; supraclypeal area length/width ratio 0.79 ( $\pm 0.02 \mathrm{SD})$. Ocelli separated by about $0.5 \mathrm{OD}$. Forewing with 3 submarginal cells; pronotal angle obtuse; tegula shape normal. Intertegular distance $0.9( \pm 0.03 \mathrm{SD}) \mathrm{mm}$. Scutum length/ width ratio $0.77( \pm 0.01 \mathrm{SD})$; scutum/scutellum length ratio 2.67 ( $\pm 0.01 \mathrm{SD})$; scutellum/metanotum length ratio $1.62( \pm 0.12 \mathrm{SD})$; metanotum/metapostnotum length ratio $0.71( \pm 0.1 \mathrm{SD})$. Propodeum lateral carinae not reaching dorsal margin; oblique carina absent. T2 depressed apical rim length less than $50 \%$ of segment. $(n=3)$ 


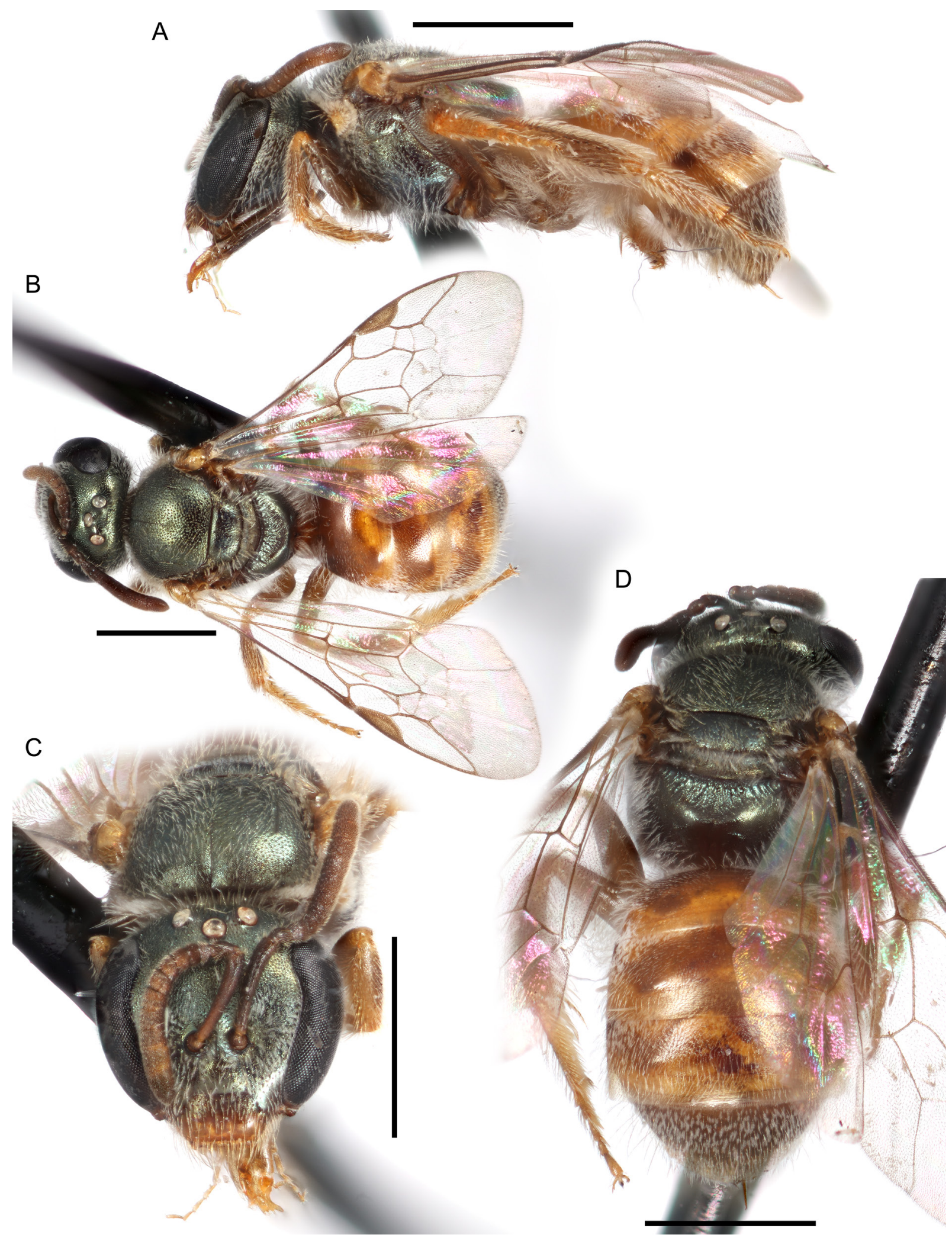

Fig. 31. Lasioglossum (D.) decorum sp. nov., ${ }^{+}$. A. Lateral habitus. B. Dorsal habitus. C. Face. D. Propodeum and metasoma. Scale bars: $1 \mathrm{~mm}$. 


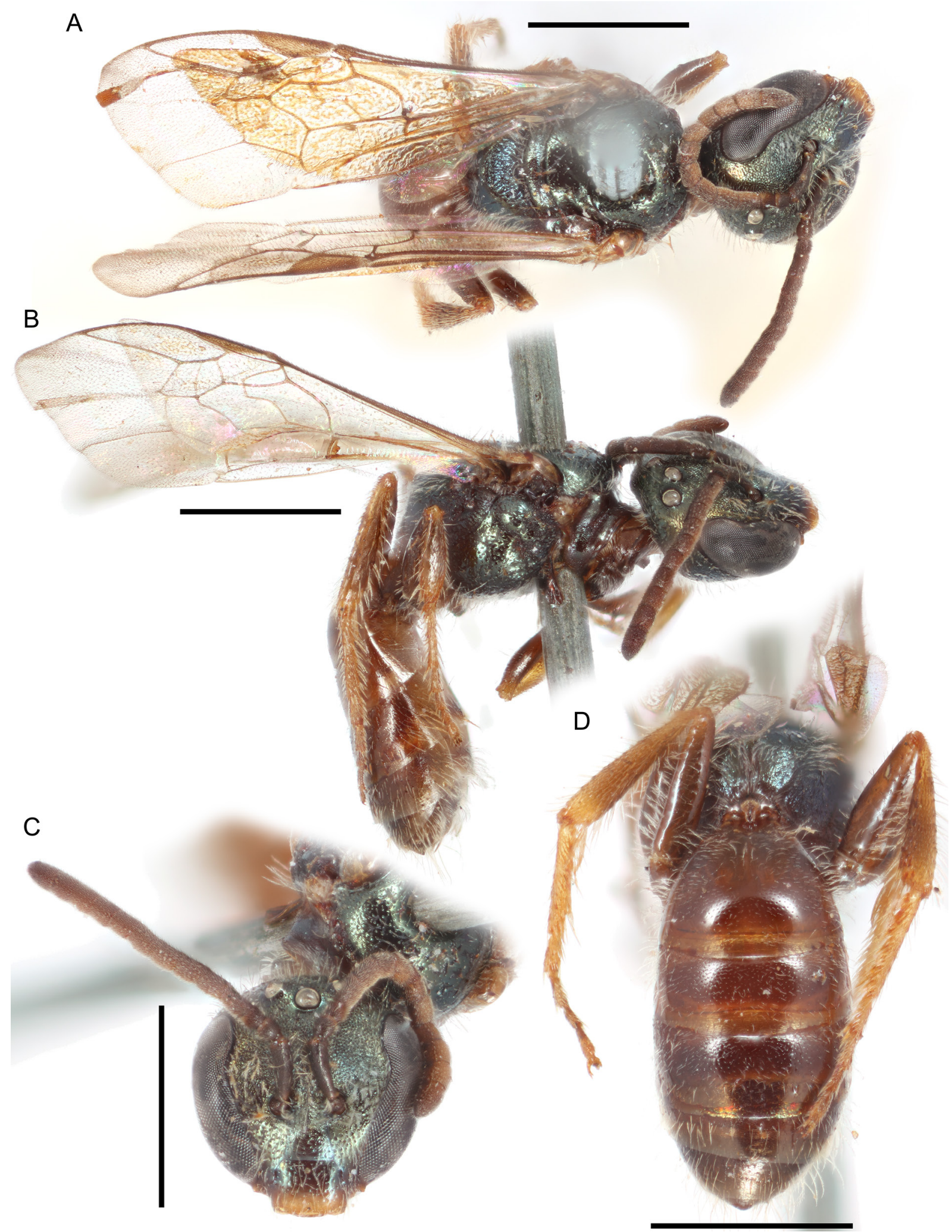

Fig. 32. Lasioglossum (D.) decorum sp. nov., §̂. A. Dorsal habitus. B. Lateral habitus. C. Face. D. Propodeum and metasoma. Scale bars: $1 \mathrm{~mm}$. 


\section{Male}

Colouration. Head and mesosoma blue-green to golden-green; clypeus apical colour orange; labrum orange; mandible orange with black basal spot and red tip; flagellum black to reddish brown, F2-5 becoming light brown ventrally; pronotal lobe orange; metasoma black to reddish brown with rims of terga and sterna and downcurved lateral areas of terga broadly translucent brown; legs reddish brown with femur-tibia joints, tarsi, and sometimes tibiae orange; tegula orange; wing membrane infuscated, veins dark brown.

PubesCEnCE. Body hair colour pale yellow to white. Tomentum dense on pronotal angle and lobe, and space between pronotal lobe and tegula; sparse on paraocular area. Scutum hair thin. Sterna hair moderately long (1-2 OD), densely plumose, dense and erect. Wing hairs dark, long and dense.

SuRfaCE SCULPTURE. Clypeus punctures sparse ( $\mathrm{i}=1-3 \mathrm{pd}$ ), sculpture shiny; supraclypeal area punctures sparse ( $\mathrm{i}=1-3 \mathrm{pd})$, sculpture shiny, becoming tessellate dorsally; paraocular area punctures moderately dense $(i=1-2 \mathrm{pd})$, sculpture shiny; frons punctures crowded $(\mathrm{i}=0 \mathrm{pd})$, sculpture shiny to imbricate, becoming reticulate above antenna; vertex punctures moderately dense laterally $(i=1-2 \mathrm{pd})$, sparse medially ( $\mathrm{i}=1-3 \mathrm{pd})$, sculpture shiny to weakly imbricate; gena punctures moderately dense $(\mathrm{i}=1-2 \mathrm{pd})$, sculpture shiny, becoming imbricate posteriorly and ventrally; postgena sculpture shiny, becoming imbricate laterad of hypostomal carina; tegula punctures absent; scutum punctures moderately dense ( $i=1-2$ pd), becoming sparse submedially ( $i=1-3$ pd), sculpture shiny, becoming tessellate anteromedially; scutellum punctures sparse $(i=1-5 \mathrm{pd})$, sculpture shiny; metanotum sculpture shiny and moderately densely punctate $(i=1-2 \mathrm{pd})$, becoming imbricate laterally; metapostnotum rugae strong or weak, anastomosing, not reaching margin, sculpture imbricate; preëpisternum sculpture shiny and densely punctate $(\mathrm{i}<1 \mathrm{pd})$; hypoepimeron punctures dense $(\mathrm{i} \leq 1 \mathrm{pd})$, sculpture shiny to weakly imbricate; mesepisternum punctures moderately sparse ( $\mathrm{i}=1-2 \mathrm{pd})$, sculpture shiny; metepisternum sculpture lineate dorsally, imbricate ventrally; propodeum lateral face punctures obscure, sculpture tessellate to weakly rugulose; propodeum posterior face sculpture tessellate; T1 anterior face sculpture coriarious; $\mathrm{T} 1$ dorsal surface punctures sparse $(\mathrm{i}=1-4 \mathrm{pd})$, absent in large apicolateral oval patches, $\mathrm{rim}$, and sometimes median line, sculpture shiny; T2 disc punctures sparse ( $\mathrm{i}=1-4 \mathrm{pd})$, disc sculpture shiny, rim punctures absent, rim sculpture shiny.

StRUCtuRE. Face length/width ratio $0.86( \pm 0.02 \mathrm{SD})$. F1:pedicel length ratio $0.81( \pm 0.07 \mathrm{SD}) ; \mathrm{F} 2: \mathrm{F} 1$ length ratio $1.52( \pm 0.09 \mathrm{SD}) ; \mathrm{F} 2$ length/width ratio $1.52( \pm 0.09 \mathrm{SD}) ; \mathrm{F} 9$ length/width ratio 1.26 $( \pm 0.01 \mathrm{SD})$. Forewing with 3 submarginal cells; pronotal angle obtuse; tegula shape normal. Intertegular distance $0.71( \pm 0.06 \mathrm{SD}) \mathrm{mm}$. Scutum length/width ratio 0.91 ( $\pm 0.05 \mathrm{SD})$; scutum/scutellum length ratio $2.43( \pm 0.33 \mathrm{SD}$ ); scutellum/metanotum length ratio 1.78 ( $\pm 0 \mathrm{SD}$ ); metanotum/metapostnotum length ratio $0.64( \pm 0.08 \mathrm{SD})$. Propodeum lateral carinae not reaching dorsal margin; oblique carina absent. $(n=2)$

Genitalia. Not examined.

\section{Range}

Jalisco and Morelos (Fig. 33).

\section{DNA barcodes}

Not available.

\section{Remarks}

Rare. Only known from five specimens from four locations. The enlarged ocelli (similar to those of L. eophilus) suggest that L. decorum sp. nov. may have low-light activity. 


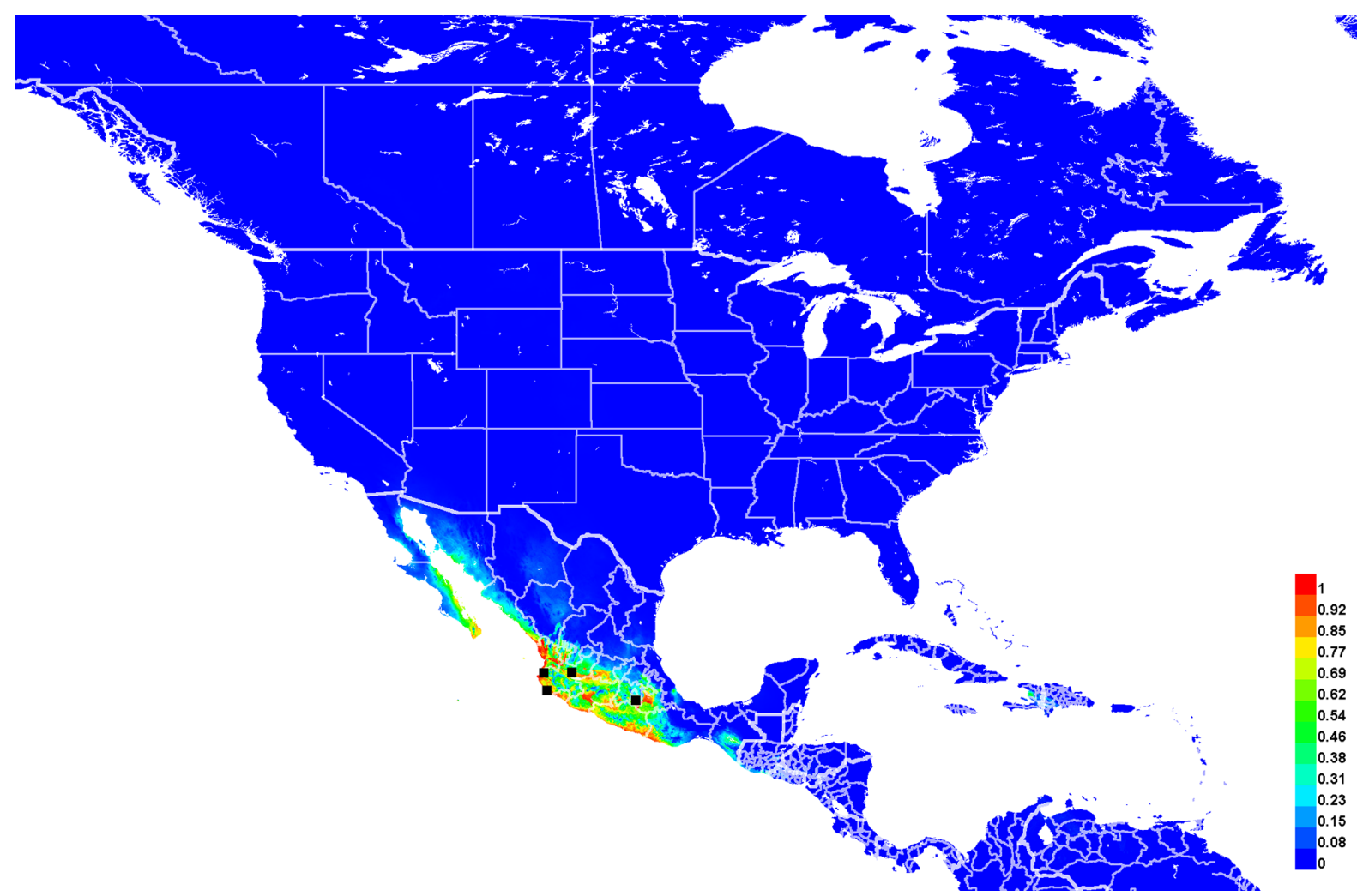

Fig. 33. Georeferenced collection records of Lasioglossum (D.) decorum sp. nov. (black squares) and predicted distribution by maximum entropy ecological niche modeling in Maxent (colour shading). Warmer colours indicate higher cloglog probability of occurrence. Due to the very small number of records, this distribution should be interpreted with caution.

Lasioglossum (Dialictus) droegei Gibbs, 2009

Fig. 34-35, 93B, 111B, 112B

Lasioglossum (Dialictus) droegei Gibbs, 2009b: 387, figs 14a-b (holotype, ㅇ, deposited at PCYU, examined).

\section{Diagnosis}

Both sexes of Lasioglossum droegei can be recognized by the characters for the Lasioglossum petrellum species complex (below), in addition to the characters given in the keys. They are most similar to L. petrellum (Cockerell, 1903). Females of L. petrellum have T2-4 with less extensive tomentum, and usually metasoma with larger brown to black patches laterally. Males of $L$. petrellum have the T1-3 apical areas slightly shinier and distinctly punctate. In addition, the distributions of these two species are not known to overlap: L. droegei is only known from the Chihuahuan Desert, while L. petrellum is known from the Sonoran Desert and west coast of California and Baja California.

\section{Material examined}

\section{Holotype}

UNITED STATES - Texas • + ; Brewster Co.; $29.1466^{\circ}$ N, $103.1876^{\circ}$ W; 1 May [2002]; H.W. Ikerd leg.; PCYU. 


\section{Other material}

MEXICO - Nuevo Leon 1 क; 3 km SW of Galeana; [24.81 ${ }^{\circ} \mathrm{N}, 100.1^{\circ} \mathrm{W}$ ]; $1550 \mathrm{~m}$ a.s.1.; 19 Mar. 1991; R. Brooks and R. Leschen leg.; SEMC.

UNITED STATES - New Mexico• 1 đ̊; Eddy Co.; $32.178^{\circ}$ N, $104.4877^{\circ}$ W; 8 Jun. 2010; A. Druk leg.; BBSL. - Texas • 1 ; Brewster Co., Big Bend Natl. Park, Oak Canyon; $\left[29.28^{\circ} \mathrm{N}, 103.33^{\circ} \mathrm{W}\right] ; 1400$ 1520 m a.s.l.; 11 Apr. 1986; T. Griswold leg.; ex Penstemon; BBSL • 1 o; Brewster Co., Big Bend Natl. Park, Oak Spring; [29.282 N, 103.332 ${ }^{\circ}$ W]; 18 Jun. 1999; S. Droege leg.; BBSL.

See also material in Gibbs (2009b).

\section{Description}

\section{Female}

See Gibbs (2009b) for a complete description and figures of the female.

\section{Male}

COLOURATION. Head and mesosoma blue-green to golden-green; clypeus apical colour black with narrow translucent orange flange; labrum reddish brown to orange; mandible orange with black base and red tip; flagellum black dorsally, orange ventrally; pronotal lobe reddish brown; metasoma black to reddish brown with rims of terga and sterna and downcurved lateral areas of terga narrowly translucent brown to orange; legs metallic green with tibiae and basitarsi black and distitarsi reddish brown; tegula orange; wing membrane hyaline, veins dark brown.

Pubescence. Body hair colour white. Tomentum dense on paraocular area, supraclypeal area between antennae, pronotal angle and lobe, and space between pronotal lobe and tegula; sparse on clypeus, gena anteriorly, and metanotum anteriorly. Scutum hair thin. Sterna hair short (0.5-1 OD), moderately plumose, sparse and erect. Wing hairs dark, long and dense.

SuRfaCe SCULPTURE. Clypeus punctures moderately dense $(i=1-2 \mathrm{pd})$, sculpture finely reticulate in basal $3 / 4$, shiny apically; supraclypeal area punctures sparse $(i=1-4 \mathrm{pd})$, sculpture imbricate; paraocular area punctures dense $(\mathrm{i}<1 \mathrm{pd})$, sculpture imbricate; frons punctures crowded $(\mathrm{i}=0 \mathrm{pd})$, indistinct, sculpture reticulate; vertex punctures crowded laterally ( $i=0 \mathrm{pd})$, absent medially, sculpture imbricate laterally, rugulose medially; gena punctures moderately dense ( $\mathrm{i}=1-2 \mathrm{pd})$, sculpture shiny, becoming imbricate and weakly lineate posteriorly and ventrally; postgena sculpture finely reticulate, becoming weakly lineate posteriorly; tegula punctures absent; scutum punctures dense $(i<1 \mathrm{pd})$, sculpture finely reticulate; scutellum punctures dense marginally and on median line ( $\mathrm{i}<1 \mathrm{pd}$ ), moderately sparse submedially ( $\mathrm{i}=1-2 \mathrm{pd}$ ), sculpture finely reticulate; metanotum sculpture imbricate and densely punctate $(\mathrm{i}<1 \mathrm{pd})$; metapostnotum rugae strong, anastomosing, not reaching margin, sculpture finely reticulate; preëpisternum sculpture rugulose; hypoepimeron punctures absent, sculpture finely reticulate; mesepisternum punctures dense $(\mathrm{i}<1 \mathrm{pd})$, indistinct, sculpture finely reticulate; metepisternum sculpture lineate dorsally, rugulose ventrally; propodeum lateral face punctures obscure, sculpture finely reticulate; propodeum posterior face sculpture imbricate and densely punctate laterally $(\mathrm{i}<1 \mathrm{pd})$, becoming shiny and sparsely punctate medially $(\mathrm{i}=1-4 \mathrm{pd}) ; \mathrm{T} 1$ anterior face sculpture weakly coriarious; $\mathrm{T} 1$ dorsal surface punctures crowded ( $\mathrm{i}=0 \mathrm{pd})$, becoming dense laterally $(\mathrm{i} \leq 1 \mathrm{pd})$, sculpture weakly coriarious, becoming finely reticulate apically; $\mathrm{T} 2$ disc punctures crowded ( $\mathrm{i}=0 \mathrm{pd})$, becoming dense laterally ( $\mathrm{i} \leq 1 \mathrm{pd}$ ), disc sculpture weakly coriarious, becoming finely reticulate apically, rim punctures dense $(\mathrm{i}<1 \mathrm{pd})$, rim sculpture finely reticulate.

StRUCTURe. Face length/width ratio 0.91. F1:pedicel length ratio 1.29; F2:F1 length ratio 1.55; F2 length/ width ratio 1.7; F9 length/width ratio 1.37. Forewing with 3 submarginal cells; pronotal angle obtuse; tegula shape normal. Intertegular distance $1.08 \mathrm{~mm}$. Scutum length/width ratio 0.88 ; scutum:scutellum 


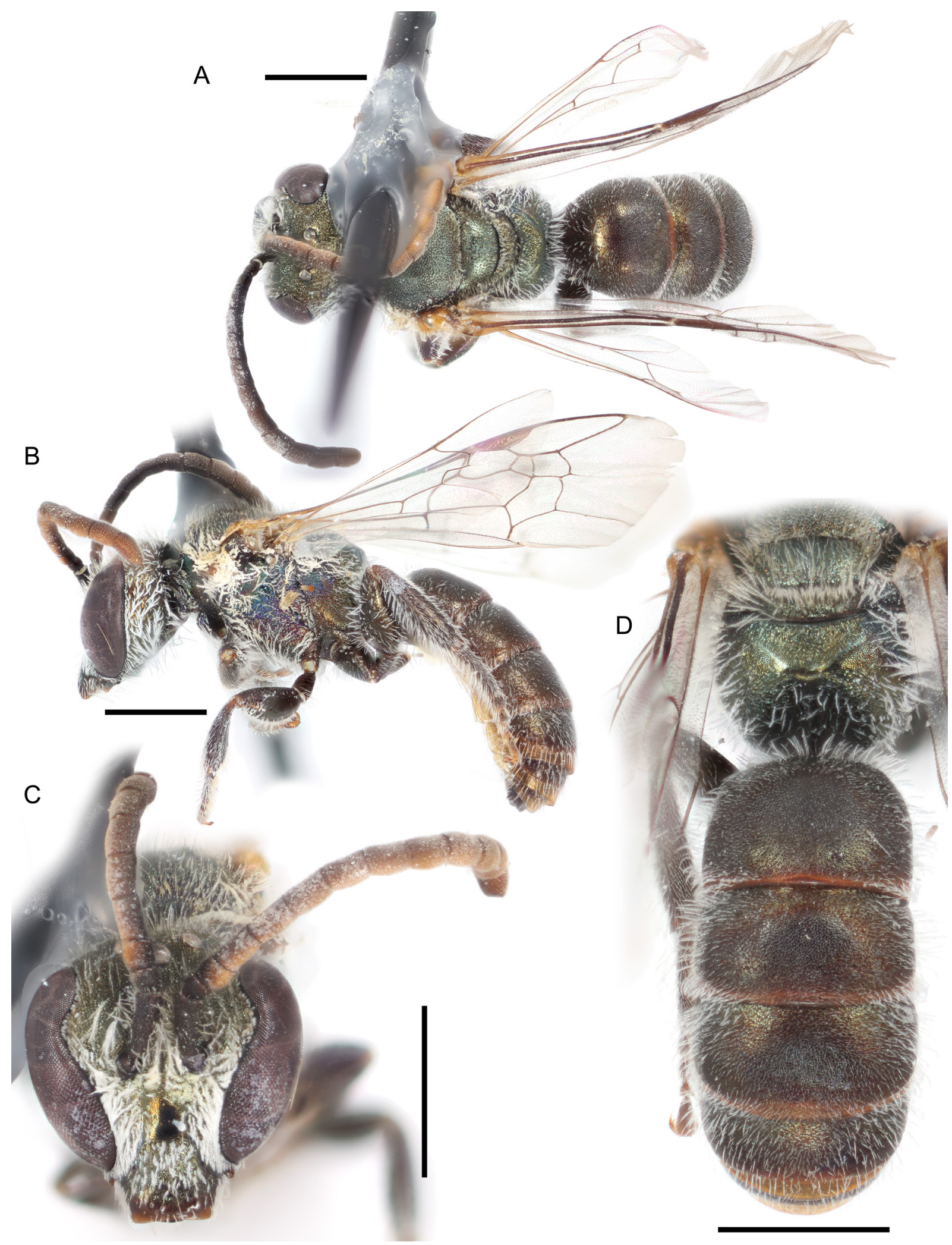

Fig. 34. Lasioglossum (D.) droegei Gibbs, 2009, §ิ. A. Dorsal habitus. B. Lateral habitus. C. Face. D. Propodeum and metasoma. Scale bars: $1 \mathrm{~mm}$. 
length ratio 2.57; scutellum:metanotum length ratio 1.72; metanotum:metapostnotum length ratio 0.74 . Propodeum lateral carinae not reaching dorsal margin; oblique carina absent. $(\mathrm{n}=1)$

Genitalia. Not examined.

\section{Range}

Chihuahuan Desert from New Mexico and Texas to Nuevo Leon (Fig. 35).

\section{Floral records}

LAMIACEAE Martinov: Hedeoma Pers. H. apiculata W.S. Stewart (G09) • PLANTAGINACEAE Juss. Penstemon Schmidel.

\section{DNA barcodes}

Five sequences available (BOLD process IDs: DLII034-06, DLII035-06, DLII1302-08, DLII153809, LASNA009-08; BIN: BOLD:AAZ1858). Lasioglossum droegei differs from all other western red-tailed L. (Dialictus) by 1 fixed substitution: 303(C). It differs from L. griswoldi, L. petrellum, and L. tuolumnense by an additional 2 fixed substitutions: 117(C) and 228(G) (Supplementary file 4).

\section{Remarks}

Gibbs (2009b) recorded L. droegei from only a single location in Texas. Additional specimens are now known from New Mexico and Nuevo Leon. The male is described here for the first time.

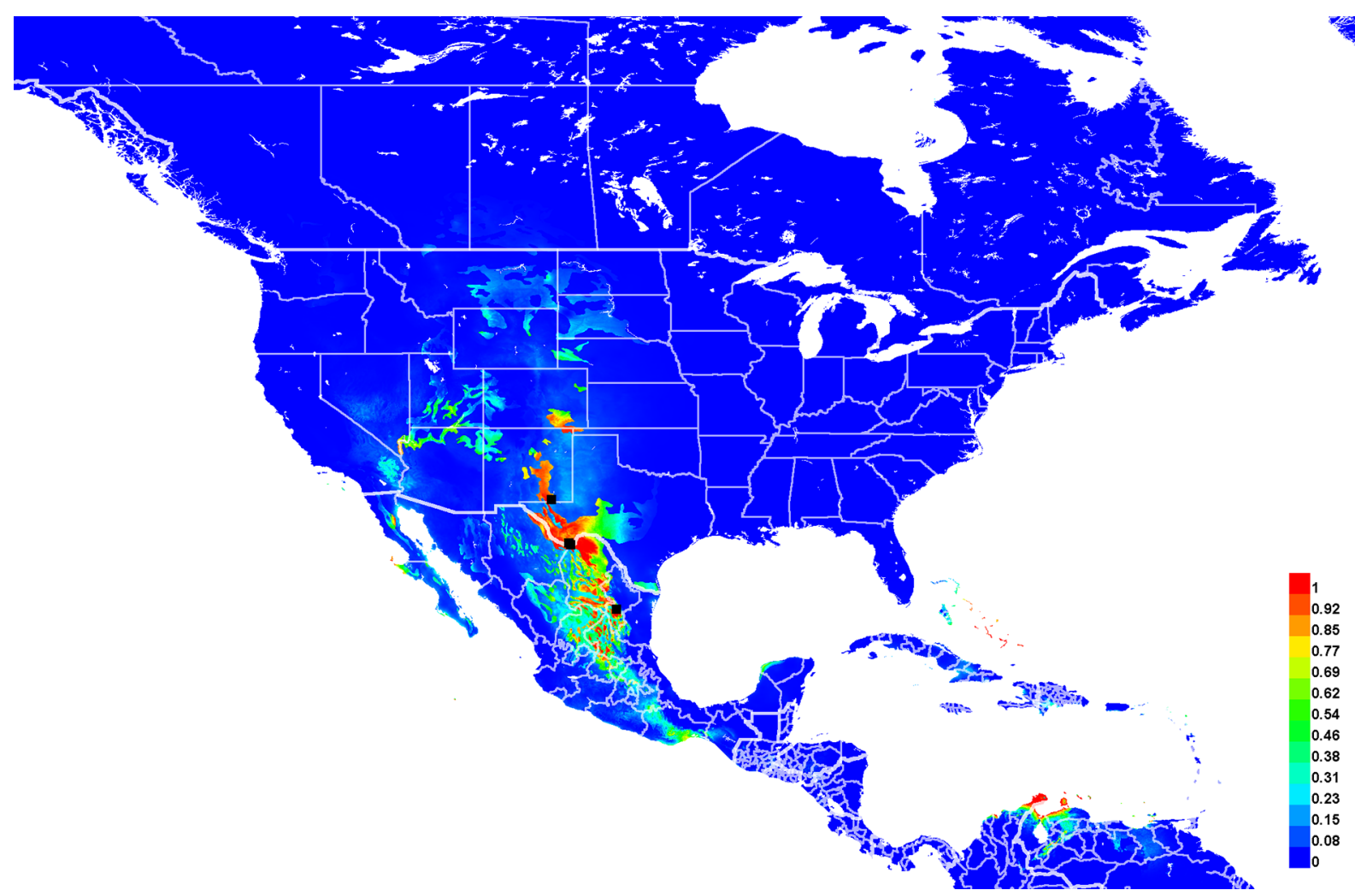

Fig. 35. Georeferenced collection records of Lasioglossum (D.) droegei Gibbs, 2009 (black squares) and predicted distribution by maximum entropy ecological niche modeling in Maxent (colour shading). Warmer colours indicate higher cloglog probability of occurrence. The predicted distribution was generated with a regularization multiplier of 0.5 to obtain a more conservative prediction. Due to the very small number of records, this distribution should be interpreted with caution. 
Lasioglossum (Dialictus) eophilus (Ellis, 1914)

Figs 36-37, 103A, 108A, 109B

Halictus eophilus Ellis, 1914a: 153 (holotype,, , deposited in CAS, examined).

Halictus (Chloralictus) eophilus - Sandhouse 1924: 3 (key).

Lasioglossum (Chloralictus) eophilium - Michener 1951: 1113 (catalog, lapsus calami).

Dialictus eophilius - Hurd 1979: 1966 (catalog, lapsus calami).

Dialictus eophilus - Moure \& Hurd 1987: 100 (catalog).

\section{Diagnosis}

Females of Lasioglossum eophilus can be recognized by the mesepisternum very coarsely sculptured and mostly rugose, ocelli slightly enlarged (separated by $\sim 0.5 \mathrm{OD}$ ), metapostnotal rugae strong and reaching the posterior margin, $\mathrm{T} 1$ with wedge-shaped median impunctate area, and metasoma mostly brown, only sometimes becoming orange on the sterna and rims of the terga. They are most similar to L. miltolepoides sp. nov. and L. lilianae sp. nov. Females of L. miltolepoides sp. nov. have the acarinarial fan absent, ocelli normal (separated by $\sim 0.8$ OD), mesepisternum often rugulose but not strongly rugose, and metasomal terga with less extensive tomentum. Females of L. lilianae sp. nov. have the mesepisternum shiny and distinctly (although densely) punctate, tomentum more extensive (especially on the mesosoma), and clypeus apical margin and metasoma usually red-orange.

\section{Etymology}

Ellis (1914a) named this species by Latinizing the Greek noun 'eos' ('dawn') and the word 'philos'. 'Philos' can be interpreted as either a noun ('friend') or an adjective ('loving', 'beloved'). According to Article 31.2.2 of the International Code of Zoological Nomenclature (International Commission of Zoological Nomenclature 1999), if the original author does not specify the intended grammar of an ambiguous name (Ellis 1914a did not), it is treated as a noun by default. The specific epithet is therefore not declinable and should remain eophilus.

\section{Material examined}

\section{Holotype}

UNITED STATES - New Mexico • $\%$; La Cueva, Organ Mountains; $\left[32.32^{\circ} \mathrm{N}, 106.61^{\circ} \mathrm{W}\right] ; 1615 \mathrm{~m}$ a.s.1.; 5 Sep. year unknown; Townsend leg.; ex Datura inoxia; CAS 15604.

\section{Other material}

MEXICO - Sonora - 1우 Rancho San Bernardino, Cafe Transect 1, Elia Thesis Study; $\left[31.32^{\circ} \mathrm{N}\right.$, 109.26 W]; 1 Aug. 2001; RLM • 1q; Rancho San Bernardino, Cafe Transect 5, Elia Thesis Study; [31.32 ${ }^{\circ}$ N, $\left.109.26^{\circ} \mathrm{W}\right] ; 3$ Aug. 2001; RLM - 2q 9 ; Rancho San Bernardino, quarry, gravel pit; $31.31964^{\circ} \mathrm{N}, 109.26926^{\circ} \mathrm{W}$; 18 Aug. 2003; RLM

UNITED STATES - Arizona • 1\%; Cochise Co., San Bernardino NWR, Bosque dry, Site 8, Transect 2; $31.33735^{\circ} \mathrm{N}, 109.231^{\circ} \mathrm{W}$; 15 Oct. 2002; RLM • 1 \%; Cochise Co., San Bernardino NWR, Grassland, Site 5, Transect 3; $31.33861^{\circ}$ N, $109.25306^{\circ}$ W; 18 Sep. 2001; RLM • 1 q; Cochise Co., San Bernardino NWR, Shrub, Site 1, Transect 4; $31.33423^{\circ}$ N, 109.23325 W; 22 Jul. 2007; RLM. - New Mexico • 1 ; ;

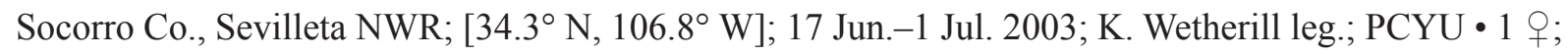
same location as for preceding; 26 Jul. 2001; K. Wetherill leg.; PCYU. 


\section{Redescription}

\section{Female}

Colouration. Head and mesosoma blue-green; clypeus apical colour black to reddish brown; labrum reddish brown to orange; mandible orange with black base and red tip; flagellum black to reddish brown dorsally, reddish brown to orange ventrally; pronotal lobe reddish brown to orange; metasoma reddish brown to orange with discs of terga sometimes black, rims of terga and sterna broadly translucent yellow, and dark spiracular spots on T3-4; legs reddish brown with femur-tibia joints, base and apex of tibiae, and tarsi reddish brown to orange; tegula orange; wing membrane hyaline, veins with subcosta dark brown, otherwise orange.

Pubescence. Body hair colour white. Tomentum dense on gena, pronotal collar and lobe, space between pronotal lobe and tegula, T2 basolaterally, and T3-4 throughout; sparse on face below eye emargination and metepisternum. Scutum hair thin. Wing hairs dark or light, short and dense. Acarinarial fan complete, sparse. T2 fringes sparse, T3 fringes sparse.

SURFACE SCULPTURE. Clypeus punctures moderately dense in basal half $(\mathrm{i}=1-2 \mathrm{pd})$, large and irregularly sparse apically ( $\mathrm{i}<3 \mathrm{pd}$ ), sculpture shiny, sometimes weakly imbricate basally; supraclypeal area punctures moderately dense ( $\mathrm{i}=1-2 \mathrm{pd})$, sculpture shiny; paraocular area punctures crowded $(\mathrm{i}=0 \mathrm{pd})$, sculpture shiny, becoming weakly imbricate around antenna socket; frons punctures crowded $(\mathrm{i}=0 \mathrm{pd})$, sculpture shiny to imbricate; vertex punctures dense laterally ( $i \leq 1 \mathrm{pd})$, sparse medially $(\mathrm{i}=1-3 \mathrm{pd})$, sculpture shiny, sometimes imbricate laterally; gena punctures moderately dense ( $i=1-2 \mathrm{pd})$, sculpture shiny, sometimes becoming weakly lineate posteriorly and ventrally; postgena sculpture shiny, becoming tessellate and weakly lineate posteriorly and laterad of hypostomal carina; tegula punctures absent; scutum punctures dense $(i<1 \mathrm{pd})$, slightly sparser submedially ( $i \leq 1 \mathrm{pd})$, sculpture shiny, becoming tessellate anteromedially; scutellum punctures dense marginally and on median line ( $\mathrm{i} \leq 1 \mathrm{pd}$ ), slightly sparser submedially ( $i=1-2 \mathrm{pd}$ ), sculpture shiny, sometimes tessellate marginally; metanotum sculpture finely ruguloso-punctate; metapostnotum rugae strong, anastomosing, reaching margin, sculpture shiny to weakly tessellate; preëpisternum sculpture areolate-rugose; hypoepimeron punctures absent, sculpture finely areolate-rugose; mesepisternum punctures dense ( $\mathrm{i} \leq 1 \mathrm{pd})$, becoming crowded $(\mathrm{i}=0 \mathrm{pd})$ and indistinct dorsally, sculpture areolate-rugose dorsally, becoming smooth and shiny or imbricate ventrally; metepisternum sculpture lineate dorsally, finely areolate-rugose medially, imbricate ventrally; propodeum lateral face sculpture rugulose anteriorly, tessellate posteriorly; propodeum posterior face sculpture tessellate with weak vertical striae; T1 anterior face sculpture shiny; T1 dorsal surface punctures moderately dense $(i=1-2 \mathrm{pd})$, becoming fine and sparse medially $(\mathrm{i}=2-6 \mathrm{pd})$, impunctate in large apicolateral oval patches and median wedge, sculpture shiny; T2 disc punctures moderately sparse ( $\mathrm{i}=1-2 \mathrm{pd})$, becoming finer and sparser medially $(\mathrm{i}=2-4 \mathrm{pd})$, disc sculpture shiny, rim punctures moderately dense $(i=1-2 \mathrm{pd})$, sometimes obscure, rim sculpture shiny to weakly coriarious.

Structure. Face length/width ratio 0.84 . Clypeus projecting $~ 50 \%$ below suborbital tangent; clypeal area length/width ratio 0.47; apicolateral denticles rounded knobs; supraclypeal area length/width ratio 0.89. Ocelli separated by about 0.5 OD. Forewing with 3 submarginal cells; pronotal angle obtuse; tegula shape normal. Intertegular distance $1.03 \mathrm{~mm}$. Scutum length/width ratio 0.76 ; scutum/scutellum length ratio 2.35; scutellum/metanotum length ratio 1.74; metanotum/metapostnotum length ratio 0.64 . Propodeum lateral carinae not reaching dorsal margin; oblique carina absent. T2 depressed apical rim length less than $50 \%$ of segment. $(n=1)$

\section{Male}

Unknown. 


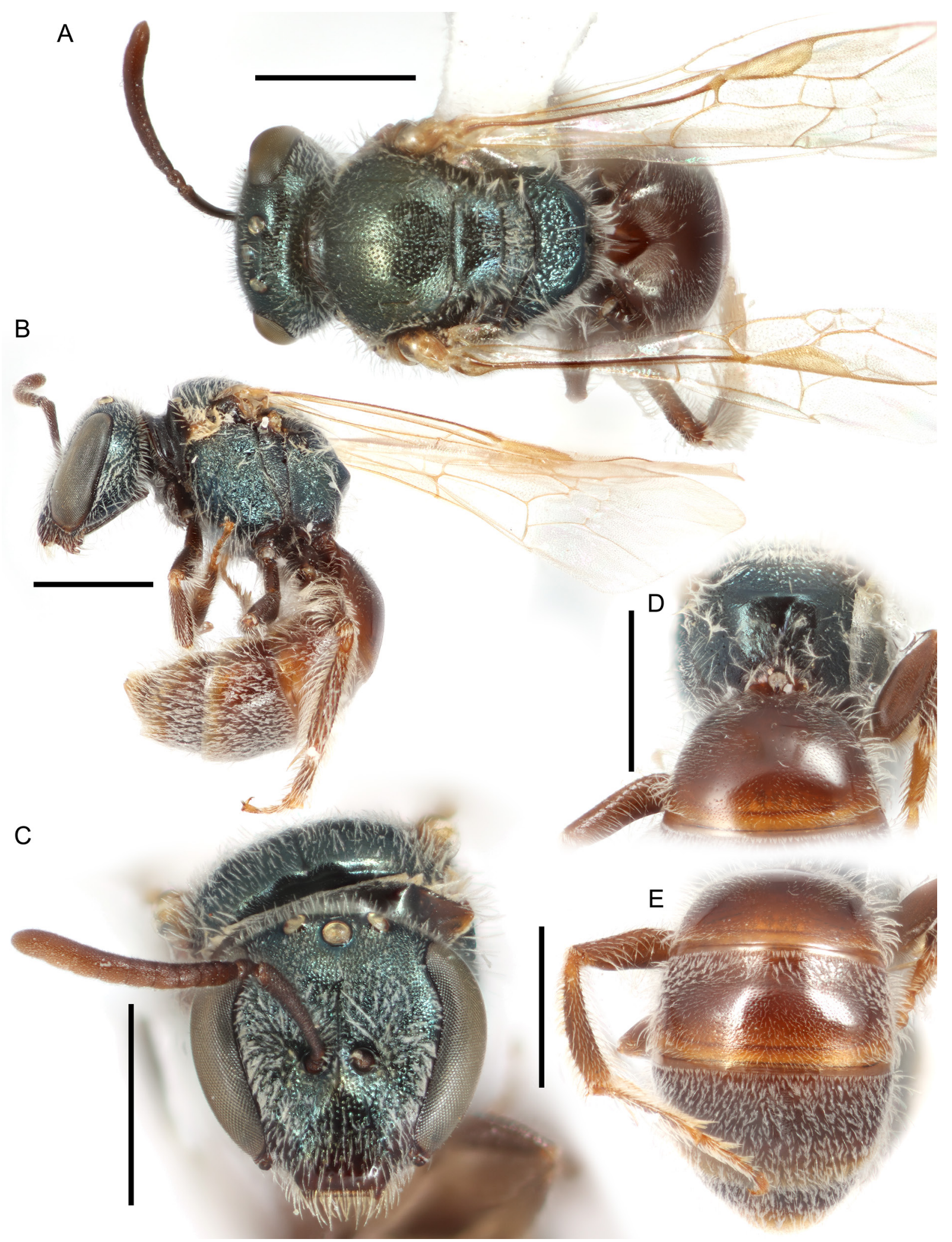

Fig. 36. Lasioglossum (D.) eophilus (Ellis, 1914), +. A. Dorsal habitus. B. Lateral habitus. C. Face. D. Propodeum. E. Metasoma. Scale bars: $1 \mathrm{~mm}$. 


\section{Range}

Southern Arizona, New Mexico, and northern Sonora (Fig. 37).

\section{Floral records}

ASTERACEAE Giseke: Helianthus (MH87) - FABACEAE Juss. Dalea: D. lanata Spreng. D. L. var. terminalis (M.E. Jones) Barneby (MH87) - Psorothamnus: P. scoparius (A.Gray) Rydb. (MH87) • LOASACEAE Juss. Mentzelia L. M. multiflora (Nutt.) A.Gray (MH87) - SOLANACEAE Adans. Datura L. D. inoxia Mill.

\section{DNA barcodes}

Two sequences available (BOLD process IDs: DLII1271-08, DLII1272-08; BIN: BOLD:AAF3976). Lasioglossum eophilus differs from all other western red-tailed L. (Dialictus) by 1 fixed substitution: 102(C) (Supplementary file 4).

\section{Remarks}

Rare. Ellis (1914a) reports that the type specimen was collected before sunrise, and notes the relatively large ocelli. Lasioglossum eophilus may be specialized for matinal activity; additional early morning collecting may reveal it to be more common than current records indicate.

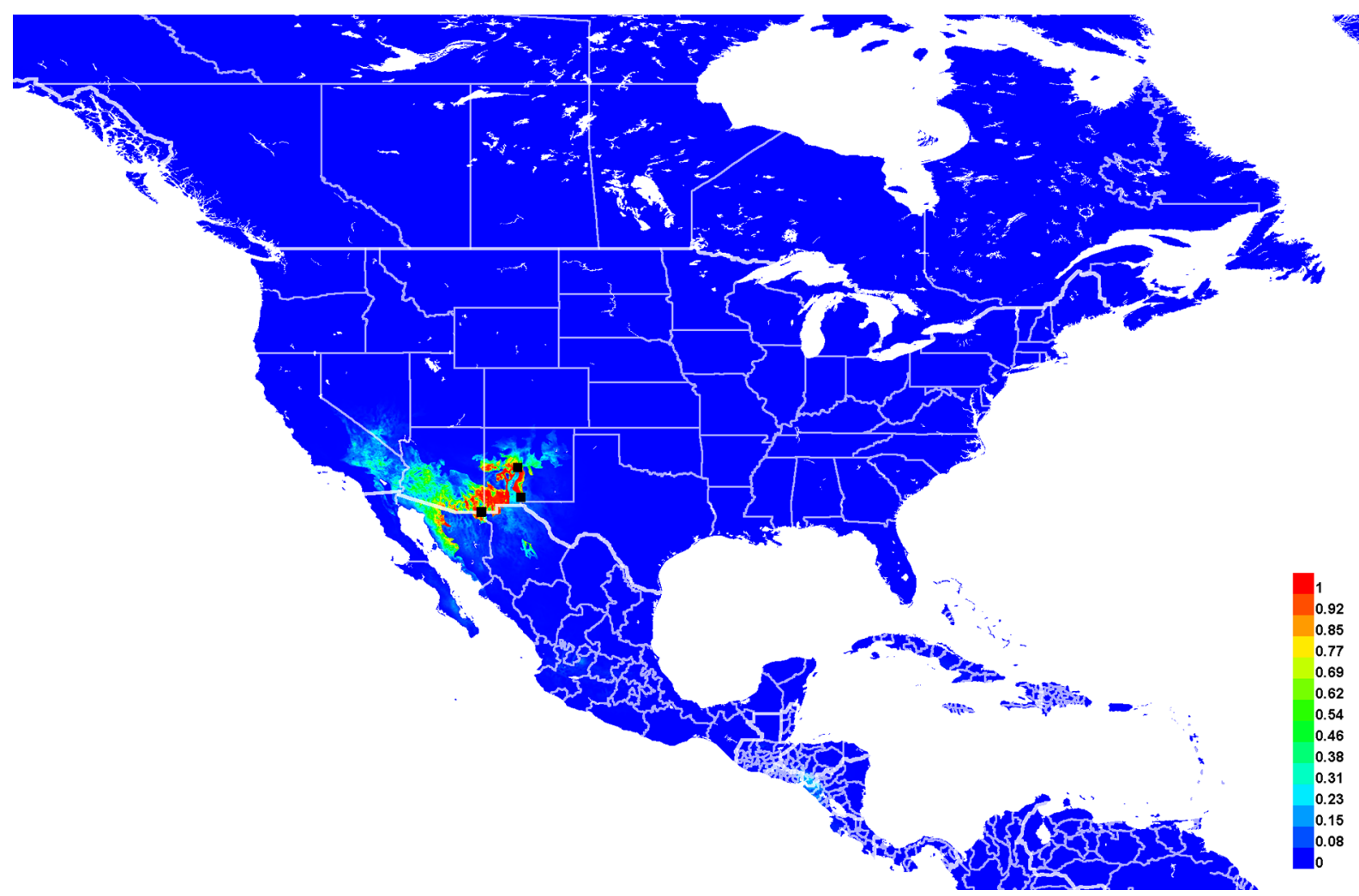

Fig. 37. Georeferenced collection records of Lasioglossum (D.) eophilus (Ellis, 1914) (black squares) and predicted distribution by maximum entropy ecological niche modeling in Maxent (colour shading). Warmer colours indicate higher cloglog probability of occurrence. Due to the very small number of records, this distribution should be interpreted with caution. 
Lasioglossum (Dialictus) festinum sp. nov. urn:Isid:zoobank.org:act:AAAFC1FC-074D-4A87-80DC-3E47F9563E99

Figs $38-40115 \mathrm{~A}$

\section{Diagnosis}

Females of Lasioglossum festinum sp. nov. can be recognized by the mesepisternum rugulose, metasomal terga with very sparse tomentum, head wide with flat clypeus, ocelli slightly enlarged (separated by $\sim 0.6$ OD), T1-3 rims punctate to the apical margin without change in sculpture across the premarginal line, T2 without dark spiracular spots, and legs often mostly or entirely orange, even in specimens with a dark metasoma. They are most similar to L. mesillense and L. minckleyi sp. nov. Both of these species have the ocelli normal (separated by $\sim 0.75 \mathrm{OD}$ ), $\mathrm{T} 2$ with dark spiracular spots, and T2-3 rims slightly duller and less distinctly punctate than the discs.

Males of $L$. festinum sp. nov. can be recognized by the face short (length/width ratio $\sim 0.86$ ), flagellomeres relatively very long (F2 more than 2 times as long as F1 and nearly 2 times as long as broad), mesepisternum densely punctate $(\mathrm{i} \leq 1 \mathrm{pd}), \mathrm{T} 1-3 \mathrm{rims}$ punctate to the apical margin without change in sculpture across the premarginal line, face with sparse tomentum, and legs, metasoma, clypeus apical margin, and sometimes pronotum and metepisternum extensively bright orange (which is unique among known Lasioglossum (Dialictus)). They are most similar to L. mesillense, L. minckleyi sp. nov., and L. pictum. All of these species have T1-3 rims less distinctly and densely punctate than the discs. In addition, males of $L$. mesillense have the face below ocelli covered in dense tomentum and metasomal terga often with basolateral tomentum. Males of L. minckleyi sp. nov. have the flagellomeres relatively short (F2 about 1.6 times as long as F1 and 1.25 times as long as broad). Males of L. pictum have the clypeus apical margin, legs, and metasoma dark, and clypeus and supraclypeal area more convex.

\section{Etymology}

The specific epithet festinum is a Latin adjective meaning 'swift', 'quick', 'impatient', or 'early'. It was first coined by Timberlake in an unpublished work, probably in reference to early morning activity. An appropriate translation would be the early sweat bee.

\section{Material examined}

\section{Holotype}

UNITED STATES - Utah • + ; Garfield Co., Alvey Wash; $37.7295^{\circ}$ N, $111.6332^{\circ}$ W; 27 Jul. 2000; F.D. Parker leg.; PCYU.

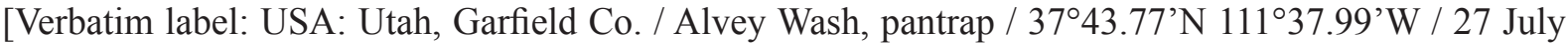
2000, F.D. Parker // 2388E09 // NativeBeeSurvey / USDA,Logan,Utah / BBSL389322 // HOLOTYPE / Lasioglossum (Dialictus) festinum Gardner and Gibbs]

\section{Paratypes}

UNITED STATES - California • 1 \%; Riverside Co., Deep Canyon; $\left[33.62^{\circ} \mathrm{N}, 116.4^{\circ} \mathrm{W}\right]$; 6 Mar. 1963; E.I. Schlinger leg.; ex Beloperone californica; UCRC • 1 क; Riverside Co., Palm Springs

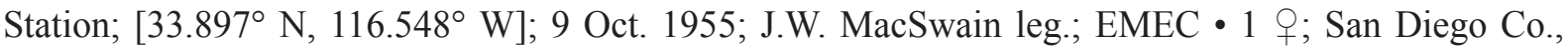

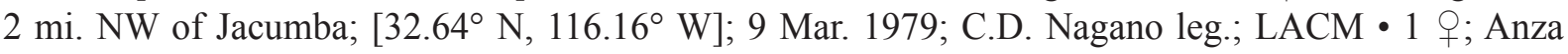

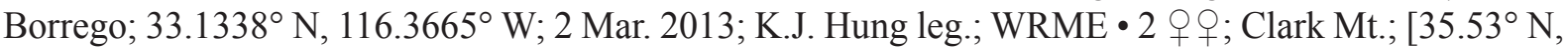
$115.59^{\circ} \mathrm{W}$ ]; 8 Jul. 1938; Timberlake leg.; ex Agave utahensis var. nevadensis; UCRC. - Colorado • 2 우; Mesa Co., 4.3 air mi. W of De Beque; $39.329^{\circ} \mathrm{N}, 108.2948^{\circ} \mathrm{W} ; 1634 \mathrm{~m}$ a.s.l.; 9 May 2012; J. Florez leg.; BBSL • 1 q; Mesa Co.; 39.1019 ${ }^{\circ}$ N, $108.7376^{\circ}$ W; 24-25 Aug. 2011; WRME. - Utah • 2 우; Emery Co., Calf Canyon, San Rafael Swell; [39.1 ${ }^{\circ}$ N, $110.67^{\circ}$ W]; 7 Jun. 1982; T.L. and R.T.

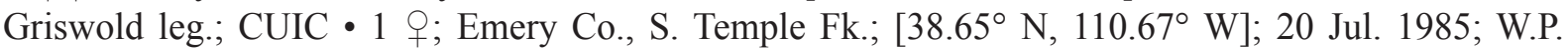


Nye leg.; ex Dalea compacta; CUIC • 1 क; Emery Co., Temple Mt. Wash; $38.6572^{\circ} \mathrm{N}, 110.6627^{\circ} \mathrm{W} ; 10$ May 2000; F.D. Parker leg.; BBSL • 1 क; Emery Co., Wild Horse Creek N of Goblin Valley; [38.57 $\mathrm{N}$, $110.82^{\circ}$ W]; 1494 m a.s.l.; 21-23 Jul. 1981; Veirs, Parker and Griswold leg.; BBSL • 1 \%; Garfield Co., Alvey Wash; $37.7005^{\circ}$ N, $111.6275^{\circ}$ W; 27 Jul. 2000; F.D. Parker leg.; BBSL • 1 क; same location as for preceding; 27 Jul. 2000; F.D. Parker leg.; PCYU • 1 q; Garfield Co., east slope Mount Hillers;

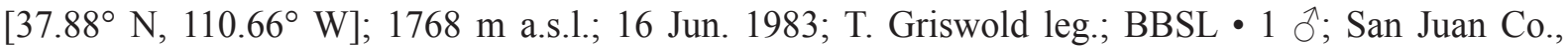
10 mi. NNW of Mexican Hat, Moki Dugway nr base; $37.27817^{\circ} \mathrm{N}, 109.94357^{\circ} \mathrm{W} ; 1637 \mathrm{~m}$ a.s.1.; 20 Jun. 2000; A.L. Hicks and V.L. Scott leg.; ex Stanleya; UCMC • 1 đ̊; San Juan Co., Moki Dugway, ca. 10 mi. NNW of Mexican Hat; $37.27817^{\circ}$ N, $109.94357^{\circ}$ W; 1637 m a.s.l.; 20 Jun. 2000; A.L. Hicks and V.L. Scott leg.; ex Stanleya sp.; UCMC • 1 ; N Navajo Mt. (Beaver Creek); $\left[37.1^{\circ} \mathrm{N}, 110.82^{\circ} \mathrm{W}\right] ; 6$ Aug. 1936; D.D. Jensen leg.; BBSL 3 우여 W Washington Co.; 37.1988 N, $112.9812^{\circ}$ W; 9 Aug. 2011; C. Decker leg.; WRME • 2 우; Washington Co.; $37.2033^{\circ}$ N, $112.9876^{\circ}$ W; 9 Aug. 2011; C. Decker leg.;

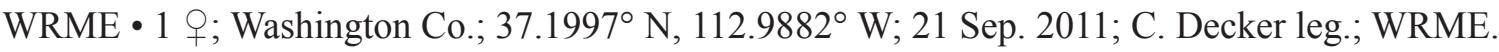

\section{Description}

\section{Female}

Colouration. Head and mesosoma blue-green to olive green; clypeus apical colour black, reddish brown, or orange; labrum black, reddish brown, or orange; mandible orange with black basal spot or band and red tip; flagellum black to reddish brown dorsally, reddish brown to orange ventrally; pronotal lobe reddish brown to orange; metasoma orange to reddish brown with dark spiracular spots on T3-4, usually with dark transverse subbasal bands on T1-2; legs orange to reddish brown; tegula orange; wing membrane hyaline, veins with subcosta dark brown, otherwise brown to orange.

PubesCENCE. Body hair colour pale yellow to white. Tomentum dense on pronotal collar and lobe, space between pronotal lobe and tegula, and gena; sparse on paraocular area, T2-3 laterally, and T4 throughout. Scutum hair thin. Wing hairs dark, long and dense. Acarinarial fan complete, very sparse. $\mathrm{T} 2$ fringes sparse, $\mathrm{T} 3$ fringes sparse.

SuRfaCe SCUlPtURE. Clypeus punctures irregularly sparse $(\mathrm{i}<2 \mathrm{pd}$ ), sometimes dense in basal half ( $\mathrm{i} \leq 1 \mathrm{pd}$ ), diversopunctate, sculpture shiny, becoming weakly tessellate basally; supraclypeal area punctures dense ( $i \leq 1 \mathrm{pd})$, sometimes sparser medially ( $\mathrm{i}=1-2 \mathrm{pd}$ ), sculpture shiny, becoming weakly tessellate laterally; paraocular area punctures dense $(i<1 \mathrm{pd})$, becoming crowded medially $(i=0 \mathrm{pd})$ and irregularly sparse around antenna socket $(\mathrm{i}<2 \mathrm{pd})$, sculpture shiny, becoming imbricate and sometimes weakly rugulose around antenna socket; frons punctures crowded ( $\mathrm{i}=0 \mathrm{pd})$, sculpture shiny; vertex punctures dense $(i \leq 1 \mathrm{pd})$ and diversopunctate laterally, sparse medially $(\mathrm{i}=1-6 \mathrm{pd})$, sculpture shiny; gena punctures moderately dense $(i=1-2 \mathrm{pd})$, sculpture shiny; postgena sculpture shiny, becoming imbricate posteriorly and laterad of hypostomal carina; tegula punctures absent; scutum punctures dense $(\mathrm{i}<1 \mathrm{pd})$ or moderately dense $(\mathrm{i}=1-2 \mathrm{pd})$, becoming sparser $(\mathrm{i}=1-3 \mathrm{pd})$ submedially and anteromedially, sculpture shiny, becoming tessellate medially; scutellum punctures dense ( $\mathrm{i} \leq 1 \mathrm{pd})$, sometimes sparser submedially ( $\mathrm{i}=1-3 \mathrm{pd})$, sculpture shiny to tessellate; metanotum sculpture tessellate and finely, densely punctate $(\mathrm{i}<1 \mathrm{pd})$, becoming weakly rugulose laterally; metapostnotum rugae strong or weak, subparallel or anastomosing, not reaching margin, sculpture tessellate; preëpisternum sculpture areolate-rugulose; hypoepimeron punctures crowded $(\mathrm{i}=0 \mathrm{pd})$, indistinct, sculpture areolate-rugulose to ruguloso-lineate; mesepisternum punctures crowded ( $\mathrm{i}=0 \mathrm{pd})$, becoming sparser ventrally $(\mathrm{i} \leq 1 \mathrm{pd})$, indistinct, sculpture imbricate, becoming ruguloso-punctate dorsally; metepisternum sculpture lineate dorsally, becoming tessellate and obscurely, densely punctate ventrally $(\mathrm{i}<1 \mathrm{pd})$; propodeum lateral face sculpture tessellate; propodeum posterior face sculpture tessellate and sparsely punctate $(\mathrm{i}=2-6 \mathrm{pd}) ; \mathrm{T} 1$ anterior face sculpture shiny to weakly coriarious; T1 dorsal surface punctures fine, sparse ( $\mathrm{i}=1-4 \mathrm{pd})$, moderately dense on rim (i=1-2 pd), absent in large apicolateral oval patches and on median line basally, 


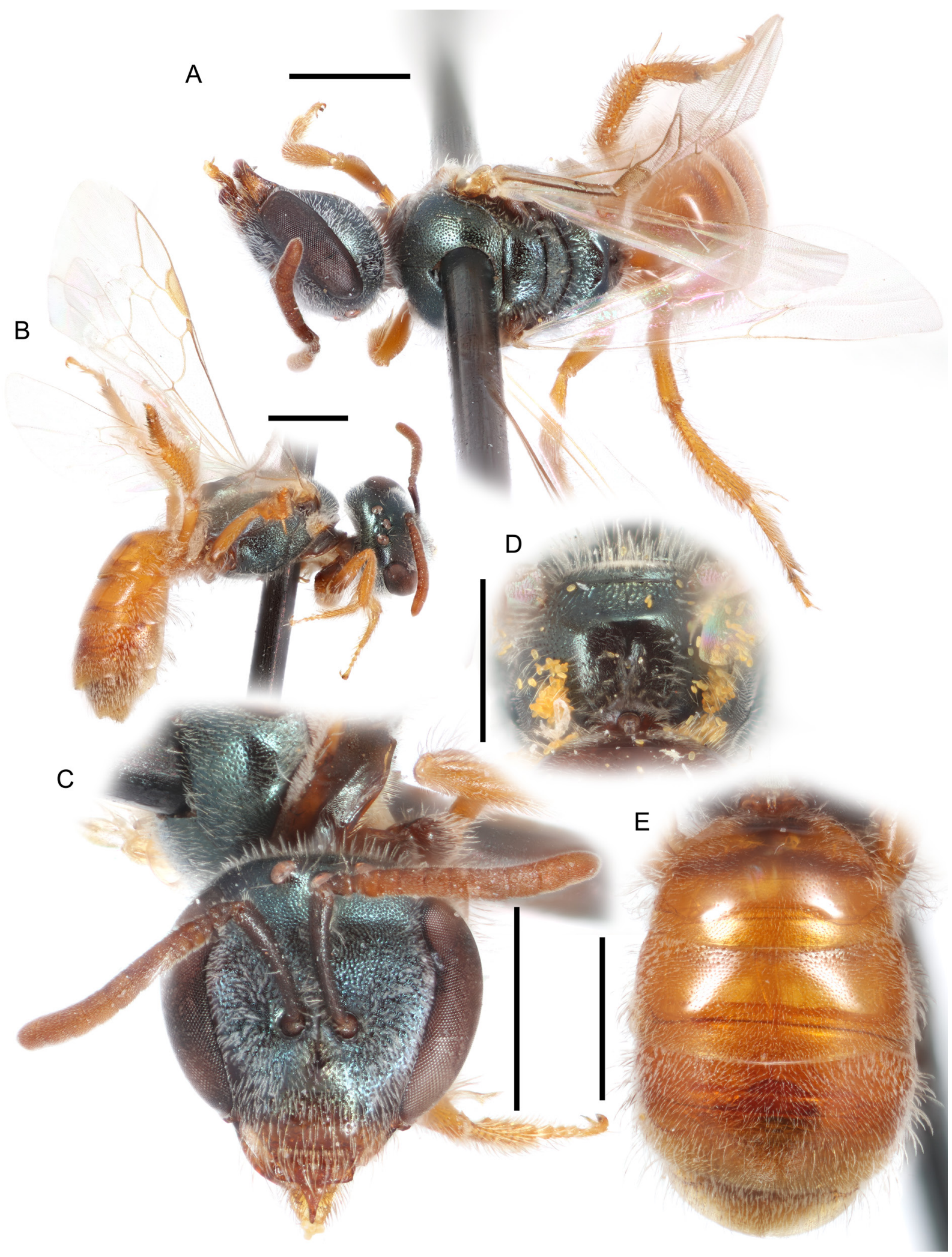

Fig. 38. Lasioglossum (D.) festinum sp. nov., ․ A. Dorsal habitus. B. Lateral habitus. C. Face. D. Propodeum. E. Metasoma. Scale bars: $1 \mathrm{~mm}$. 


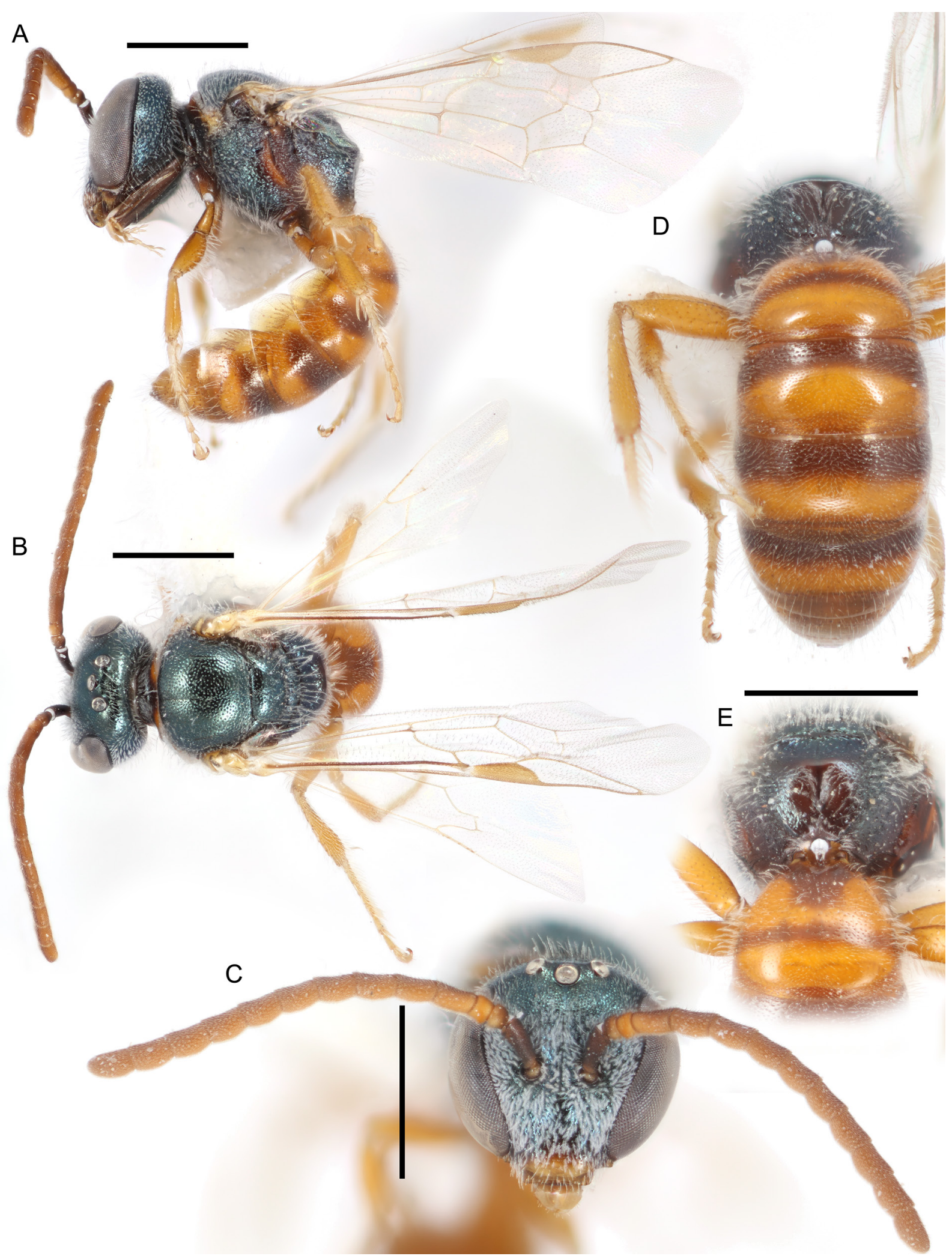

Fig. 39. Lasioglossum (D.) festinum sp. nov., §ิ. A. Lateral habitus. B. Dorsal habitus. C. Face. D. Metasoma. E. Propodeum. Scale bars: $1 \mathrm{~mm}$. 
sculpture shiny; T2 disc punctures fine, sparse $(i=1-4 \mathrm{pd})$, becoming denser basolaterally $(\mathrm{i} \leq 1 \mathrm{pd})$, disc sculpture shiny, rim punctures moderately dense $(i=1-3 \mathrm{pd})$, rim sculpture shiny.

Structure. Face length/width ratio 0.79 ( $\pm 0.03 \mathrm{SD}$ ). Clypeus projecting $\sim 50 \%$ below suborbital tangent; clypeal area length/width ratio 0.35 ( $\pm 0.04 \mathrm{SD})$; apicolateral denticles rounded acute points; supraclypeal area length/width ratio $0.9( \pm 0.1 \mathrm{SD})$. Ocelli separated by about $0.6 \mathrm{OD}$. Forewing with 3 submarginal cells; pronotal angle obtuse; tegula shape normal. Intertegular distance $1.11( \pm 0.08 \mathrm{SD}) \mathrm{mm}$. Scutum length/width ratio $0.79( \pm 0.03 \mathrm{SD})$; scutum/scutellum length ratio $2.9( \pm 0.17 \mathrm{SD})$; scutellum/ metanotum length ratio $1.58( \pm 0.2 \mathrm{SD})$; metanotum/metapostnotum length ratio 0.63 ( $\pm 0.07 \mathrm{SD})$. Propodeum lateral carinae not reaching dorsal margin; oblique carina absent. T2 depressed apical rim length less than $50 \%$ of segment. $(n=10)$

\section{Male}

Colouration. Head and mesosoma blue-green to olive green, except pronotal collar, metepisternum, and mesepisternum posterior margin sometimes orange; clypeus apical colour orange to yellow; labrum orange to yellow; mandible orange to yellow with brown basal spot and red tip; flagellum reddish brown, sometimes orange ventrally; pronotal lobe orange to yellow; metasoma orange with brown basal bands on T1-5; legs orange to yellow; tegula orange to pale amber; wing membrane hyaline, veins with subcosta dark brown, otherwise brown to orange.

PubesCENCE. Body hair colour white. Tomentum dense on paraocular area; sparse on face below eye emargination, gena, pronotal angle and lobe, and space between pronotal lobe and tegula. Scutum hair thin. Sterna hair short (0.75-1.25 OD), densely plumose, dense and erect. Wing hairs dark, long and sparse.

SuRfaCE SCULPTURE. Clypeus punctures dense to moderately dense ( $\mathrm{i} \leq 2 \mathrm{pd}$ ), sculpture shiny; supraclypeal area punctures dense to moderately dense $(\mathrm{i} \leq 2 \mathrm{pd}$ ), sculpture shiny; paraocular area punctures crowded $(\mathrm{i}=0 \mathrm{pd})$, sculpture shiny; frons punctures crowded ( $\mathrm{i}=0 \mathrm{pd})$, sculpture shiny, becoming rugulose above antenna; vertex punctures fine, sparse $(i=1-3 \mathrm{pd})$, sculpture shiny; gena punctures fine, sparse ( $i=1-3 \mathrm{pd})$, sculpture shiny, becoming imbricate and weakly lineate posteriorly and ventrally; postgena sculpture imbricate and weakly lineate; tegula punctures absent; scutum punctures dense to moderately dense ( $\mathrm{i} \leq 2 \mathrm{pd}$ ), sculpture shiny, becoming tessellate anteromedially; scutellum punctures dense marginally and on median line ( $\mathrm{i} \leq 1 \mathrm{pd})$, moderately sparse submedially ( $\mathrm{i}=1-2 \mathrm{pd}$ ), sculpture shiny; metanotum sculpture ruguloso-punctate; metapostnotum rugae strong, anastomosing, reaching or nearly reaching margin, sculpture shiny to weakly imbricate; preëpisternum sculpture areolate-rugulose; hypoepimeron punctures crowded $(\mathrm{i}=0 \mathrm{pd})$, indistinct, sculpture rugulose; mesepisternum punctures dense ( $\mathrm{i} \leq 1 \mathrm{pd}$ ), sculpture shiny to weakly imbricate; metepisternum sculpture lineate dorsally, areolate ventrally; propodeum lateral face punctures obscure, sculpture rugulose; propodeum posterior face sculpture shiny, sometimes becoming densely punctate dorsolaterally ( $\leq 1 \mathrm{pd}$ ); T1 anterior face sculpture shiny; $\mathrm{T} 1$ dorsal surface punctures sparse $(\mathrm{i}=1-4 \mathrm{pd})$, becoming moderately dense on rim $(\mathrm{i}=1-2 \mathrm{pd})$, sculpture shiny; $\mathrm{T} 2$ disc punctures sparse $(\mathrm{i}=1-3 \mathrm{pd})$, disc sculpture shiny, rim punctures moderately dense $(i=1-2 \mathrm{pd})$, rim sculpture shiny.

StRUCTURE. Face length/width ratio 0.86 ( \pm 0 SD). F1:pedicel length ratio 1.02 ( $\pm 0.01 \mathrm{SD}) ;$ F2:F1 length ratio $2.29( \pm 0.03 \mathrm{SD}) ; \mathrm{F} 2$ length/width ratio $1.85( \pm 0.31 \mathrm{SD}) ; \mathrm{F} 9$ length/width ratio 1.4 ( $\pm 0.05 \mathrm{SD})$. Forewing with 3 submarginal cells; pronotal angle obtuse; tegula shape normal. Intertegular distance 1.06 ( $\pm 0 \mathrm{SD}) \mathrm{mm}$. Scutum length/width ratio 0.71 ( $\pm 0.01 \mathrm{SD})$; scutum/scutellum length ratio 2.43 $( \pm 0.31 \mathrm{SD})$; scutellum/metanotum length ratio $1.85( \pm 0.16 \mathrm{SD})$; metanotum/metapostnotum length ratio 0.61 ( \pm 0.05 SD). Propodeum lateral carinae not reaching dorsal margin; oblique carina absent. $(n=2)$

Genitalia. Not examined. 


\section{Range}

Western Colorado to southern California (Fig. 40).

\section{Floral records}

ACANTHACEAE Juss. Beloperone Nees: B. californica Benth. - ASPARAGACEAE Juss. Agave L. A. utahensis Engelm. A. u. var. nevadensis Engelm. ex Greenm. \& Roush • BRASSICACEAE Burnett: Stanleya Nutt. FABACEAE Juss. Dalea: D. compacta Spreng.

\section{DNA barcodes}

Two sequences available (BOLD process IDs: DLII868-07, DLII1089-07; BIN: BOLD:AAJ1601). Lasioglossum festinum sp. nov. differs from all other western red-tailed L. (Dialictus) by 4 fixed substitutions: 204(C), 240(T), 507(C), and 561(T) (Supplementary file 4).

\section{Remarks}

Rare. The slightly enlarged ocelli of the female (resembling those of L. eophilus) suggest that L.festinum sp. nov. has low-light activity, which could explain why it is infrequently collected. The close genetic relationship of L. festinum sp. nov. and L. eophilus (Fig. 7) supports this hypothesis.

Lasioglossum festinum sp. nov. is the species previously reported as "Lasioglossum (Dialictus) sp. E7" by Tepedino et al. (2008), Messinger-Carril et al. (2018), and Wilson et al. (2018).

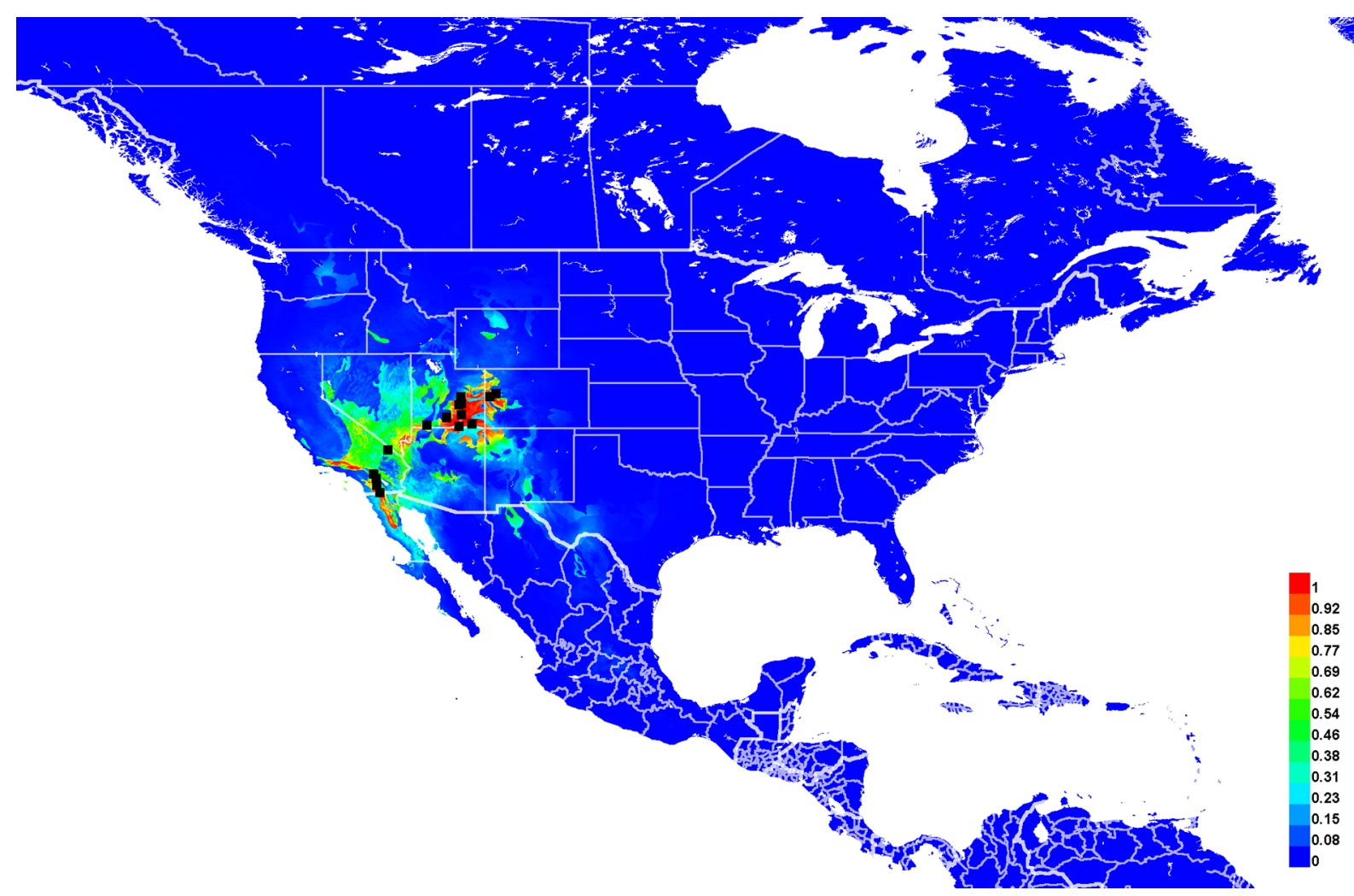

Fig. 40. Georeferenced collection records of Lasioglossum (D.) festinum sp. nov. (black squares) and predicted distribution by maximum entropy ecological niche modeling in Maxent (colour shading). Warmer colours indicate higher cloglog probability of occurrence. 
Lasioglossum (Dialictus) griswoldi Gibbs, 2009

Fig. 41

Lasioglossum (Dialictus) griswoldi Gibbs, 2009b: 385, figs 13a-b (holotype, + , deposited in BBSL, examined).

\section{Diagnosis}

Females of Lasioglossum griswoldi can be recognized by the characters for the Lasioglossum petrellum species complex (below), in addition to the characters given in the key and the metasomal terga with very narrow rims with long, dense apicolateral fringes (resembling L. cactorum sp. nov. in this respect). They are most similar to L. tuolumnense. Females of L. tuolumnense have the malar space and supraclypeal area shorter, and scutum slightly duller and more densely punctate. In addition, the geographic ranges of these two species are not known to overlap: L. griswoldi is only known from the mountains of Utah and Colorado, while L. tuolumnense is only known from the mountains of California north to British Columbia.

\section{Material examined}

\section{Holotype}

UNITED STATES - Utah • + ; Kane Co., No Mans Mesa; $37.34555^{\circ}$ N, 112.12921 W; 31 May 2002; C. Davidson leg.; ex Packera multilobata; BBSL.

\section{Other material}

UNITED STATES - Colorado • 1 क; White Rocks, near Boulder; $\left[40.06^{\circ} \mathrm{N}, 105.16^{\circ} \mathrm{W}\right] ; 1676 \mathrm{~m}$ a.s.l.; 5 Jun. 1922; AMNH. - Utah • 1 क; Davis Co., Farmington Canyon; [41 $\left.{ }^{\circ} \mathrm{N}, 111.86^{\circ} \mathrm{W}\right] ; 24$ Jul. 1970; G.F. Knowlton and G.E. Bohart leg.; BBSL 1 क; Salt Lake Co., N of Mill B trail, Big Cottonwood Canyon; [40.64 $\left.{ }^{\circ} \mathrm{N}, 111.72^{\circ} \mathrm{W}\right]$; 6 Jun. 2010; J S. Ascher leg.; AMNH.

See also material in Gibbs (2009b).

\section{Description}

See Gibbs (2009b) for a complete description and figures of the female. The male has not been described. Some male specimens are located at BBSL, but were unavailable for study.

\section{Range}

Utah and Colorado (Fig. 41).

\section{Floral records}

APIACEAE Lindl. Cymopterus Raf. C. beckii S. L. Welsh \& Goodrich (G09) • ASTERACEAE Giseke: Cirsium (G09) - Erigeron (G09) - Heterotheca: H. villosa (Pursh) Shinners (G09) • Machaeranthera: M. canescens (Pursh) A.Gray (G09) • Packera: P. multilobata (Torr. \& A.Gray ex A.Gray) W.A. Weber \& Á. Löve • Psilostrophe: P. sparsiflora (A.Gray) A. Nelson (G09) • Senecio: S. spartioides Torr. \& A.Gray (G09) - Symphyotrichum: S. chilense (Nees) G.L. Nesom (G09) • Townsendia: T. incana Nutt. (G09) • BRASSICACEAE Burnett: Erysimum L. E. capitatum (Douglas) Greene (G09) - Stanleya Nutt. S. pinnata (Pursh) Britton (G09) • CACTACEAE Juss. Opuntia Mill. O. erinacea Engelm. \& J.M. Bigelow (G09) • FABACEAE Juss. Melilotus: M. officinalis (L.) Pall. (G09) • MALVACEAE Juss. Sphaeralcea A. St.-Hil. S. coccinea (Nutt.) Rydb. (G09) • PLANTAGINACEAE Juss. Penstemon Schmidel: P. eatonii A.Gray (G09) • POLEMONIACEAE Juss. Aliciella Brand: A. caespitosa (A. Gray) J.M. Porter (G09) • 
Gilia Ruiz \& Pav. (G09) • RANUNCULACEAE Juss. Aquilegia L. (G09) • ROSACEAE Juss. Purshia DC. ex Poir. P. mexicana (D. Don) Henr. (G09) • TAMARICACEAE Link: Tamarix L. (G09).

\section{DNA barcodes}

Five sequences available (BOLD process IDs: DLII860-07, DLII861-07, DLII1532-09, DLII1533-09, DLII1535-09; BIN: BOLD:AAD3535). Lasioglossum griswoldi differs from all other western red-tailed L. (Dialictus) by 2 fixed substitutions: 198(G) and 327(G). It differs from L. droegei, L. petrellum, and L. tuolumnense by an additional 2 fixed substitutions: 81(T) and 387(C) (Supplementary file 4).

\section{Remarks}

Gibbs (2009b) recorded this species only from Utah. It was recorded from Boulder County, Colorado, as L. aff. griswoldi in Scott et al. (2011). A Boulder County specimen has been examined by the authors and seems identical to the Utah specimens.

A few specimens not examined by Gibbs (2009b) from Utah have the metasoma completely black, but are otherwise morphologically identical. These might be a distinct species, but without additional evidence, it seems best to consider them no more than a dark variant.

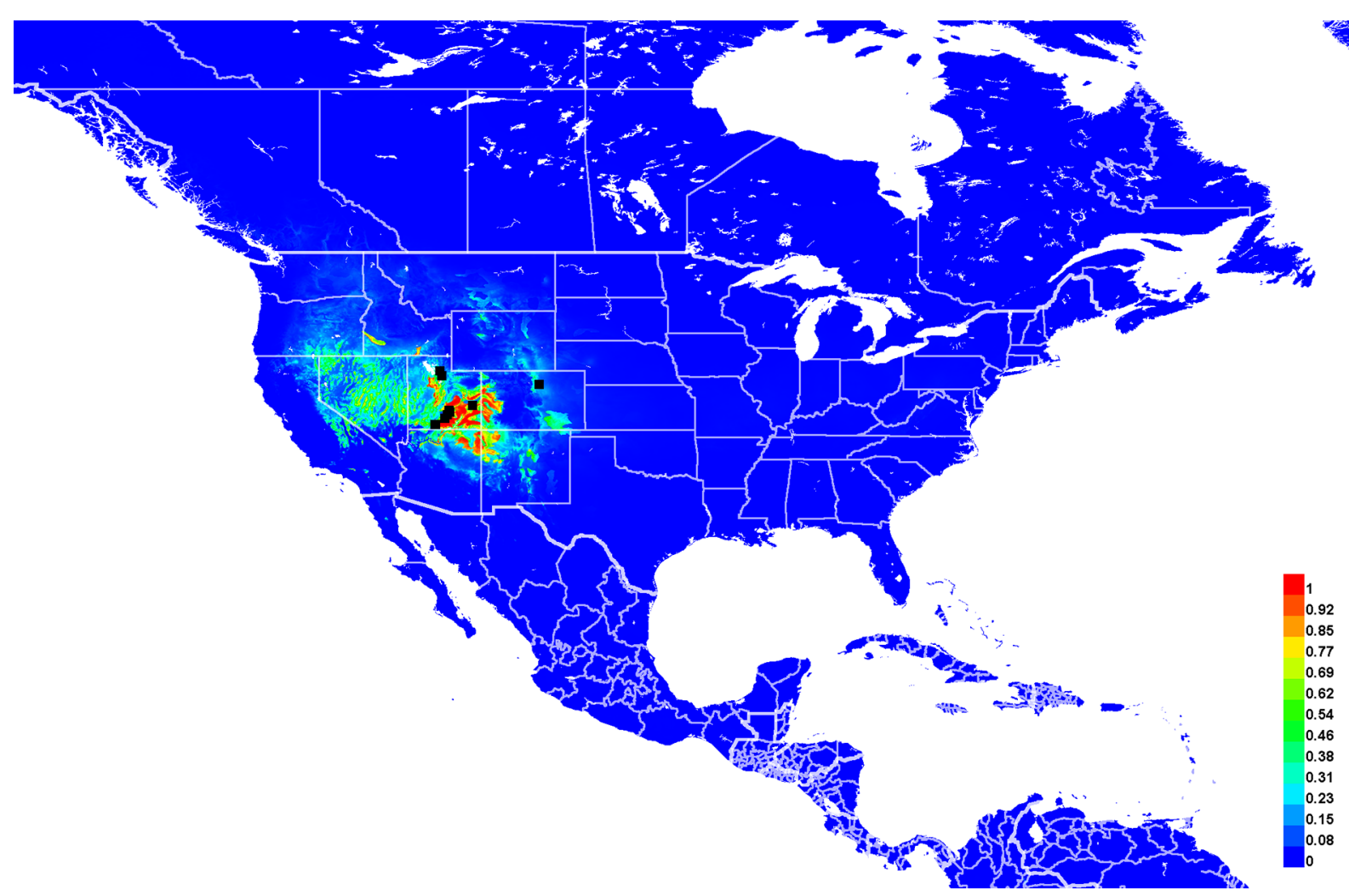

Fig. 41. Georeferenced collection records of Lasioglossum (D.) griswoldi Gibbs, 2009 (black squares) and predicted distribution by maximum entropy ecological niche modeling in Maxent (colour shading). Warmer colours indicate higher cloglog probability of occurrence. 
Lasioglossum (Dialictus) hudsoniellum (Cockerell, 1919)

Figs 1, 42, 90D, 107A, 116C

Halictus hudsoniellus Cockerell, 1919: 290 (holotype,, , deposited in USNM, examined).

Halictus (Chloralictus) hudsoniellus - Sandhouse 1924: 4 (key).

Lasioglossum (Chloralictus) hudsoniellum - Michener 1951: 1114 (catalog).

Dialictus hudsoniellus - Hurd 1979: 1966 (catalog). — Moure \& Hurd 1987: 104 (catalog).

Lasioglossum (Dialictus) hudsoniellum - Gibbs 2010: 140, figs 116a-c, 117a-g (key, redescription). Scott et al. 2011: 30 (checklist).

\section{Diagnosis}

Females of Lasioglossum hudsoniellum can be recognized by the face shiny with dense but distinctly separated and similarly-sized punctures over the frons and paraocular area, mesepisternum with crowded punctures $(\mathrm{i}=0 \mathrm{pd})$, scutum usually moderately densely and evenly punctate ( $\mathrm{i} \leq 1-2 \mathrm{pd}), \mathrm{T} 1$ usually with moderately dense punctures $(\mathrm{i}=1-2 \mathrm{pd})$ outlining distinct impunctate apicolateral ovals (present but less distinct in most other species), pronotal angle usually about 90 degrees, postgena shiny, and T2 without dark spiracular spots. They are most similar to L. spivakae sp. nov., L. arenisaltans sp. nov., and $L$. lilianae sp. nov. Females of $L$. spivakae sp. nov. have the scutum punctures with interspaces 2-3 pd wide immediately mesad of the parapsidal line, metasoma with tomentum mostly restricted to T3-4, pronotal angle obtuse, and metasomal terga usually with sharply delineated brown to black bands. Females of L. arenisaltans sp. nov. have the frons punctures crowded and indistinct, scutum punctures very dense $(\mathrm{i}<1 \mathrm{pd})$, postgena lineate, and T2 with dark spiracular spots. Females of $L$. lilianae sp. nov. have the scutum and T1 more finely and sparsely punctate ( $i=1-4 \mathrm{pd}$ in part).

Males of $L$. hudsoniellum can be recognized by S5 apical rim strongly concave, flagellomeres relatively short (F2 about 1.25 times as long as F1 and about as long as broad), face below eye emargination covered in dense tomentum, T1-3 rims usually moderately densely punctate ( $\mathrm{i}=1-2 \mathrm{pd})$, and $\mathrm{T} 1-5$ with abundant tomentum. They are most similar to L. arenisaltans sp. nov. and L. imbriumbrae sp. nov. Both of these species have S5 apical rim straight and metasoma with tomentum sparse or absent. In addition, males of $L$. arenisaltans sp. nov. have the flagellomeres relatively longer (F2 about 1.75 times as long as F1 and 1.5 times as long as broad), and males of L. imbriumbrae sp. nov. have T1-3 rims impunctate.

\section{Etymology}

Cockerell (1919) named this species after the Hudsonian life zone in the Rocky Mountains, where the type specimen was collected, plus the Latin adjectival diminutive suffix -ellus.

\section{Material examined}

\section{Holotype}

UNITED STATES - Colorado • $q$; Longs Peak Trail, Hudsonian Zone; $\left[40.274^{\circ} \mathrm{N}, 105.579^{\circ} \mathrm{W}\right]$; 3200 m a.s.l.; 22 Jul.; Cockerell leg.; USNM 27765.

\section{Other material}

CANADA - Alberta • 3 우; $11.8 \mathrm{~km} \mathrm{SSW}$ of Empress (stabilizing dune); $50.8504^{\circ} \mathrm{N}, 110.0489^{\circ} \mathrm{W}$; 24 May 2019; T.M. Onuferko leg.; CMNC • 1 q; Writing-On-Stone Prov. Pk., Sand-North; [49.09 N, 111.62 W]; 1 Aug. 1990; M. Klassen leg.; PCYU. - Saskatchewan • 2 우; N Bitter Lake, Tunstall (stabilizing dune); $50.1456^{\circ} \mathrm{N}, 109.8062^{\circ} \mathrm{W} ; 2$ Jun. 2019; T.M. Onuferko leg.; CMNC

MEXICO - Baja California Sur • 7 $q$; 17 mi. NE of Santa Rita; [24.76 N, $\left.111.27^{\circ} \mathrm{W}\right]$; 18 Sep. 1983; R.R. Snelling leg.; ex Euphorbia; LACM • 1 q; Constitución, 18 km N; [25.18 N, $\left.111.75^{\circ} \mathrm{W}\right]$; 
40 m a.s.l.; 13 Apr. 1994; A. Rodríquez leg.; ex Brassica nigra; SEMC • 1 P; Playa Buenaventura, nr San Buenaventura; [26.64 N, 111.83 W]; 0 m a.s.l.; 14 Apr. 1997; A. Rodríquez leg.; ex Brassica nigra; SEMC. - Chihuahua 1 i ; 3 mi. W of Jimenez; $\left[27.14^{\circ} \mathrm{N}, 104.97^{\circ} \mathrm{W}\right] ; 1250 \mathrm{~m}$ a.s.l.; 26 Jul. 1952; SEMC 1 1 ; Hwy 45, 5 rd mi. NE of Salaices (15 mi. SW of jct. Hwy 49); [27.08 ${ }^{\circ}$ N, $\left.105.13^{\circ} \mathrm{W}\right]$; 1585 m a.s.l.; 24 Aug. 1976; J.P. and K.E. Donahue leg.; LACM. - Coahuila 1 q; 2 km N of San Lorenzo; 25.5167 ${ }^{\circ}$ N, 102.1833 ${ }^{\circ}$ W; 1430 m a.s.1.; 24 Mar. 1992; R. Brooks leg.; ex Varilla mexicana; SEMC • 1 क; same location as for preceding; 24 Mar. 1992; B. Alexander leg.; ex Bahia absinthifolia;

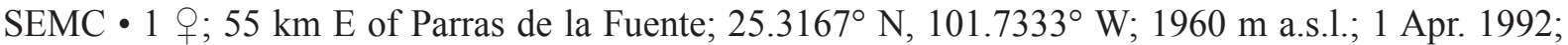
D. Yanega leg.; ex Dalea eriophylla; SEMC • 4 q + ; Arteaga, San Antonio de las Alazanas; $\left[25.2847^{\circ} \mathrm{N}\right.$, 100.6131 ${ }^{\circ} \mathrm{W}$ ]; $2015 \mathrm{~m}$ a.s.1.; 21 Jul. 2013; LRF and CVM leg.; LRF • 1 ; San Antonio de las Alazanas, Arteaga; $25.2847^{\circ} \mathrm{N}, 100.6131^{\circ} \mathrm{W} ; 2015 \mathrm{~m}$ a.s.1.; 9 Mar. 2014; LRF and CVM leg.; LRF. - Durango • 2 우; 36 mi. SW of Cd. Lerdo; [25.17 $\mathrm{N}, 103.93^{\circ} \mathrm{W}$ ]; $1113 \mathrm{~m}$ a.s.l.; 16 Aug. 1963; Naumann and Willis leg.; SEMC • 1 o ; Nombre de Dios; $\left[23.85^{\circ} \mathrm{N}, 104.24^{\circ} \mathrm{W}\right]$; 13 May 1952; F.D. Parker leg.; UCDC. - Hidalgo • 1 \%; Lagunilla; $\left[20.34^{\circ}\right.$ N, $99.02^{\circ}$ W]; 14 Jun. 1951; H.E. Evans leg.; SEMC • 1 \%;

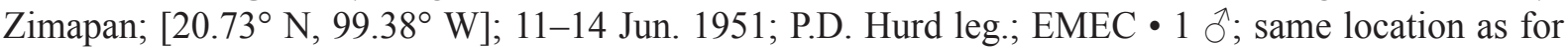
preceding; 8 Jul. 1968; M.S. Wasbauer and J.E. Slansky leg.; UCDC. - Nuevo Leon • 2 o $O ; 3$ km SW of Galeana; [24.81 ${ }^{\circ} \mathrm{N}, 100.1^{\circ} \mathrm{W}$ ]; $1550 \mathrm{~m}$ a.s.1.; 19 Mar. 1991; R. Brooks and R. Leschen leg.; SEMC • 1 ; ; $37 \mathrm{~km} \mathrm{SW}$ of Linares; [24.6 ${ }^{\circ} \mathrm{N}, 99.8^{\circ} \mathrm{W}$ ]; $310 \mathrm{~m}$ a.s.1.; 20 Mar. 1991; R. Brooks and R. Leschen leg.; SEMC • 6 우; Galeana, La Hediondilla; $\left[25.0694^{\circ} \mathrm{N}, 100.7186^{\circ} \mathrm{W}\right] ; 1890$ m a.s.1.; 28 Jul. 2013; LRF and CVM leg.; LRF • 1 क; same location as for preceding; 2 Jun. 2013; LRF and CVM leg.; LRF • 1 क; same location as for preceding; 3 Apr. 2013; LRF and CVM leg.; LRF • 1 ; Galeana, Los Llanos; [24.8089 ${ }^{\circ} \mathrm{N}, 100.7119^{\circ} \mathrm{W}$ ]; $1865 \mathrm{~m}$ a.s.1.; $27 \mathrm{Jul}$. 2013; LRF and CVM leg.; LRF • 5 o $\circ$; same location as for preceding; 1 Jun. 2013; LRF and CVM leg.; LRF • 5 o $\circ$; Galeana, Pablillo; [24.6119 ${ }^{\circ}$, 100.0014 ${ }^{\circ} \mathrm{W}$ ]; $2085 \mathrm{~m}$ a.s.1.; 5 Apr. 2013; LRF and CVM leg.; LRF • 2 o $\circ$; La Hediondilla, Galeana; 25.0694 ${ }^{\circ} \mathrm{N}, 100.7186^{\circ} \mathrm{W}$; $1890 \mathrm{~m}$ a.s.1.; 29 Sep. 2013; LRF and CVM leg.; LRF • 1 q; Los Llanos, Galeana; $24.8089^{\circ} \mathrm{N}, 100.7119^{\circ} \mathrm{W}$; $1865 \mathrm{~m}$ a.s.1.; 29 Mar. 2014; LRF and CVM leg.; LRF 1 1 ; Pablillo, Galeana; $24.6119^{\circ} \mathrm{N}, 100.0014^{\circ} \mathrm{W}$; $2083 \mathrm{~m}$ a.s.1.; 13 Apr. 2014; LRF and CVM leg.; LRF 1 q; San Antonio de las Alazanas; $25.2847^{\circ} \mathrm{N}, 100.6131^{\circ} \mathrm{W}$; $2015 \mathrm{~m}$ a.s.1.; 1 Sep. 2013; LRF and CVM leg.; LRF. - San Luis Potosi • 1 ; El Huizache; $\left[22.92^{\circ}\right.$ N, $100.46^{\circ}$ W]; 22 Aug. 1954; ex Larrea tridentata; SEMC • 6 9 ; same location as for preceding; 22 Aug. 1954; SEMC. - Sonora • 1 \%; $30 \mathrm{~km} \mathrm{E} \mathrm{of} \mathrm{Agua}$ Prieta; $31.3294^{\circ} \mathrm{N}, 109.2547^{\circ} \mathrm{W} ; 24$ Jul. 2001; R.L. Minckley leg.; RLM • 1 9 ; $30 \mathrm{~km}$ E of Agua Prieta; $31.3322^{\circ} \mathrm{N}, 109.2703^{\circ} \mathrm{W} ; 31$ Mar. 2002; R.L. Minckley leg.; RLM • 4 q $\circ$; Municipio de Agua Prieta, Rancho Las Anitas, Grassland Site 1; 31.31389 ${ }^{\circ}$ N, $109.31417^{\circ}$ W; 21 May 2008; RLM • 1 क ; Rancho San Bernardino, Bosque dry, Site 4; $31.30972^{\circ}$ N, $109.27778^{\circ}$ W; 8 May 2008; RLM • 1 O ; Rancho

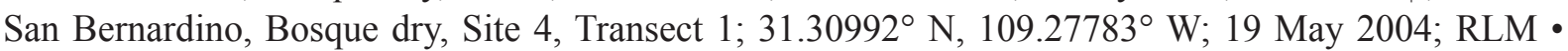
1 q; Rancho San Bernardino, Bosque wet, Site 1, Transect 2; $31.33215^{\circ} \mathrm{N}, 109.27015^{\circ} \mathrm{W} ; 3$ Jul. 2002; RLM • 1 क; same location as for preceding; 13 Aug. 2002; RLM • 1 \%; Rancho San Bernardino, field, Site 1, Transect 2; $31.32422^{\circ} \mathrm{N}, 109.26663^{\circ} \mathrm{W} ; 11$ Oct. 2002; RLM • 1 \%; Rancho San Bernardino, Grassland, Site 1; $31.3187^{\circ} \mathrm{N}, 109.28023^{\circ} \mathrm{W}$; 19 Aug. 2003; ex Kallstroemia californica; RLM • 1 \%; same location as for preceding; 19 Aug. 2003; RLM • 1 q; Rancho San Bernardino, Grassland, Site 6, Transect 1; $31.3112^{\circ} \mathrm{N}, 109.26625^{\circ} \mathrm{W} ; 11$ Aug. 2007; RLM • 1 \% ; Rancho San Bernardino, Grassland, Site 7; $31.30833^{\circ} \mathrm{N}, 109.27361^{\circ} \mathrm{W} ; 12$ Aug. 2006; RLM • 1 o ; Rancho San Bernardino, Grassland, Site 7, Transect 3; $31.30865^{\circ} \mathrm{N}, 109.27308^{\circ} \mathrm{W} ; 21$ Jul. 2005; RLM • 1 q; Rancho San Bernardino, Little dam site, $\mathrm{nr}$ main houses; $31.32083^{\circ} \mathrm{N}, 109.27601^{\circ} \mathrm{W} ; 16$ Jul. 2005; ex Salsola kali subsp. tragus; RLM • 1 \%; Rancho San Bernardino, quarry, at road crossing; $31.32042^{\circ} \mathrm{N}, 109.2681^{\circ} \mathrm{W}$; 2 Sep. 2003; RLM - 1 \%; Rancho San Bernardino, quarry, gravel pit; $31.31964^{\circ} \mathrm{N}, 109.26926^{\circ} \mathrm{W}$; 19 Aug. 2003; RLM - 1 O; Rancho San Bernardino, Rancho San Bernardino; $31.32907^{\circ} \mathrm{N}, 109.26553^{\circ} \mathrm{W}$; 1 Aug. 2000; RLM - 1 क; Rancho San Bernardino, Riparian, Site 4; $31.32972^{\circ} \mathrm{N}, 109.25583^{\circ} \mathrm{W}$; 20 Aug. 2003; RLM • 2 우; Rancho San Bernardino, Shrub 3; $31.32222^{\circ}$ N, $109.29306^{\circ}$ W; 7 Apr. 2006; RLM • 1 क; same location as for preceding; 23 Apr. 2008; RLM • 1 क; Rancho San Bernardino, 
Shrub, Site 4, Transect 2; $31.333^{\circ}$ N, 109.24035 ${ }^{\circ}$ W; 6 Jul. 2002; RLM • 1 o; Sonora Rancho, San Bernardino; $31.33215^{\circ}$ N, 109.27015 W; 5 Jul. 2001; R. Minckley leg.; RLM • 1 क; Sonora Rancho, San Bernardino; $31.3322^{\circ}$ N, $109.2703^{\circ}$ W; 1 Sep. 2001; R. Minckley leg.; RLM • 1 q; Sonora Rancho, San Bernardino; $31.3222^{\circ}$ N, $109.2769^{\circ}$ W; 11 Sep. 2001; R.L. Minckley leg.; RLM • 1 q; Sonora Rancho, San Bernardino; $31.3222^{\circ}$ N, 109.2772 ${ }^{\circ}$ W; 22 Aug. 2001; R.L. Minckley leg.; RLM • 1 \%; Sonora Rancho, San Bernardino; $31.3189^{\circ}$ N, $109.2511^{\circ}$ W; 31 Aug. 2001; R. Minckley leg.; RLM. -

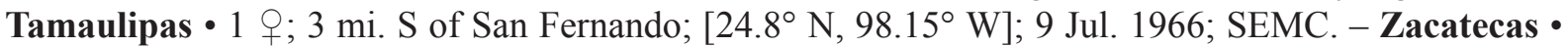
$1 \partial^{\lambda} ; 45$ mi. SW of Saltillo; [24.7 N, 101.3 W]; 14 Sep. 1977; R.W. Brooks leg.; SEMC.

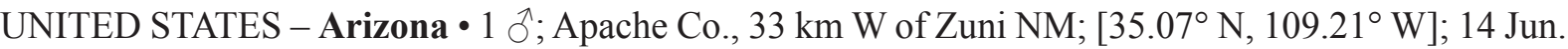
1999; L. Packer leg.; PCYU • 1 + ; Cochise Co., 1 mi. S of Willcox; [32.24 N, 109.83 W]; 7 Aug. 1978; R.W. Brooks leg.; ex Wislizenia refracta; SEMC • 1 क; Cochise Co., 4 mi. E of Willcox; $\left[32.25^{\circ} \mathrm{N}\right.$, $109.76^{\circ} \mathrm{W}$ ]; 17 Aug. 1994; J.G. Rozen and J.S. Ascher leg.; AMNH • 1 क; same location as for preceding; 11-18 Aug. 1987; K.V. Krombein leg.; USNM • 2 o $o$; Cochise Co., 40 km S I-10 on Portal Rd;

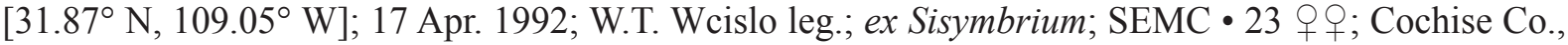

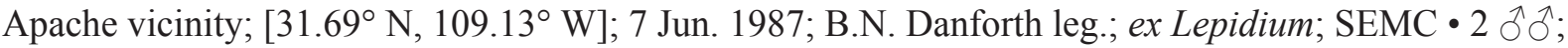
Cochise Co., Chiricahua Mts, Desert Scrub Site; [31.9 N, 109.3 W]; J.L. Neff and A.R. Moldenke leg.;

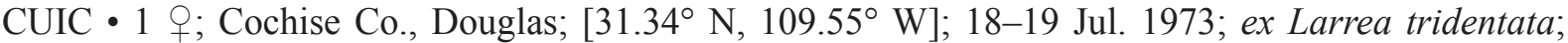
EMEC • 1 क; same location as for preceding; 18-19 Jul. 1973; P.D. Hurd, E.G. and J.M. Linsley, A.E. and M.M. Michelbacher leg.; ex Larrea tridentata; EMEC • 1 \%; same location as for preceding; 27 Jul. 1975; E.G. and J.M. Linsley leg.; ex Tamarix chinensis; EMEC • 3 우우 Cochise Co., Dry Lake, 4 mi. SE of Willcox, Sand dunes; $32.2271^{\circ}$ N, $109.7812^{\circ}$ W; 27 Aug. 2013; T.L. Griswold leg.; BBSL • 1 क; Cochise Co., Portal Creek Lodge; $31.9138^{\circ}$ N, $109.1408^{\circ}$ W; 1448 m a.s.l.; 5-6 Apr. 2016; C.M. Delphia leg.; MTEC • 1 क; Cochise Co., roadside, Out Side of Willcox; $32.2392^{\circ} \mathrm{N}, 109.7716^{\circ} \mathrm{W} ; 10$ May 2017; T. McMahon leg.; WRME - 1 q; Cochise Co., San Bernardino NWR; $31.33627^{\circ}$ N, 109.26752 ${ }^{\circ}$ W; 5 Jul. 2001; R. Minckley leg.; PCYU • 1 q; Cochise Co., San Bernardino NWR; 31.3406 ${ }^{\circ}$ N, $109.2575^{\circ}$ W; 31 Aug. 2001; R. Minckley leg.; RLM • 1 o; Cochise Co., San Bernardino NWR; $31.3358^{\circ}$ N, $109.2703^{\circ}$ W; 13 Aug. 2001; R.L. Minckley leg.; RLM • 1 q; same location as for preceding; 31 Mar. 2002; R.L. Minckley leg.; RLM • 1 q; Cochise Co., San Bernardino NWR, Bosque dry, Site 8; $31.33728^{\circ}$ N, 109.23105 W; 4 Aug. 2003; RLM • 2 o of; Cochise Co., San Bernardino NWR, Bosque dry, Site 8, Transect 2; $31.33735^{\circ}$ N, 109.231 ${ }^{\circ}$ W; 18 Jul. 2005; RLM • 1 ; ; Cochise Co., San Bernardino NWR, Bosque wet, Site 2; $31.33633^{\circ} \mathrm{N}, 109.2673^{\circ} \mathrm{W} ; 22$ Apr. 2007; RLM • 1 \%; Cochise Co., San Bernardino NWR, field, Site 6, Transect 4; $31.33444^{\circ}$ N, 109.26806 ${ }^{\circ}$ W; 5 Jul. 2001; RLM • 1 \%; Cochise Co., San Bernardino NWR, field, Site 8, Transect 2; $31.3381^{\circ} \mathrm{N}, 109.2605^{\circ} \mathrm{W} ; 19$ Sep. 2001; RLM • 1 q; Cochise Co., San Bernardino NWR, Grassland, Site 2, Transect 3; $31.3358^{\circ}$ N, 109.2702 ${ }^{\circ}$ W; 3 Jul. 2002; RLM • 1 9 ; Cochise Co., San Bernardino NWR, Shrub, Site 5, Transect 2; $31.34258^{\circ} \mathrm{N}, 109.27013^{\circ} \mathrm{W}$; 30 Jul. 2004; RLM • 1 \%; Cochise Co., Willcox; [32.25 $\left.\mathrm{N}, 109.83^{\circ} \mathrm{W}\right]$; 18 Aug. 1958; P.D. Hurd leg.; ex Asclepias subverticillata; EMEC • 1 \% ; same location as for preceding; 14 Aug. 1958; C.G. Moore leg.; UCDC • 1 क; Cochise Co., 6.4 km SE of Willcox on Blue Sky Rd; $32.2275^{\circ} \mathrm{N}$, $109.7814^{\circ} \mathrm{W}$; $1250 \mathrm{~m}$ a.s.1.; 25 Aug. 2001; R. Brooks leg.; SEMC • 1 \%; same location as for preceding; 21 Aug. 2001; C. Michener leg.; ex Psorothamnus scoparius; SEMC • 1 ठ̊; Pima Co., Silver Bell Bajada, IBP Desert Scrub Site; $\left[32.4^{\circ}\right.$ N, 111.5 W]; 17 Jul. 1974; J.L. Neff leg.; ex Heliotropium curassavicum; CUIC • 1 क; Yavapai Co., 8 mi. NW of Wickenburg (Maricopa Co.); [34.05 $\left.{ }^{\circ} \mathrm{N}, 112.83^{\circ} \mathrm{W}\right] ; 15$ May 1992; J.G. and B.L. Rozen leg.; AMNH • 1 \%; 20 mi. N of Flagstaff; $\left[35.51^{\circ} \mathrm{N}, 111.55^{\circ} \mathrm{W}\right] ; 2$ Jul. 1952; C. Liang and W. LaBerge, R.H. and L.D. Beamer leg.; SEMC •

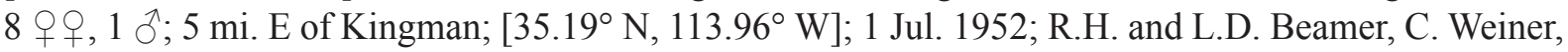
A. Wolf, C. Liang and W. LaBerge leg.; SEMC $\bullet 1$ q; 5 mi. W of Kingman; $\left[35.19^{\circ} \mathrm{N}, 114.14^{\circ} \mathrm{W}\right] ; 1$ Jul. 1952; R.W. Brooks leg.; R.H. and L.D. Beamer, C. Weiner, A. Wolf, C. Liang and W. LaBerge leg.; SEMC -3 우; 8 mi. SW of Willcox; [32.17 $\mathrm{N}, 109.93^{\circ} \mathrm{W}$ ]; 12 Jul. 1952; R.H. and L.D. Beamer, Walley LaBerge and Cheng Liang leg.; SEMC • 1 क ; Rustlers Pk.; $\left[31.905^{\circ} \mathrm{N}, 109.28^{\circ} \mathrm{W}\right] ; 25$ Jul. 1959; 
K.V. Krombein leg.; USNM. - California - 1 \%; Riverside Co., Anza; $\left[33.56^{\circ}\right.$ N, $116.67^{\circ}$ W]; 5 Jul. 1956; H.R. Moffitt leg.; UCDC • 1 क ; Riverside Co., San Timoteo Canyon; [33.98 N, $\left.117.11^{\circ} \mathrm{W}\right]$; 14 Aug. 1993; L. Packer and T. Pare leg.; PCYU • 1 o; San Bernardino Co., Cima Rd 6 mi. S I-15; $35.3667^{\circ}$ N, $115.6^{\circ}$ W; 1402 m a.s.l.; 1 Sep. 1994; R.R. Snelling leg.; ex Asclepias erosa; LACM • 1 क ; San Bernardino Co., Cima, 5.1 air mi. S of Sec. 31 T13N R14E; [35.17 N, 115.52 ${ }^{\circ}$ W]; 10 Jun. 1980; T. Griswold leg.; ex Eriogonum fasciculatum var. polifolium; BBSL 1 9; San Bernardino Co., Cotton Wash, Sec. 3 T9N R12E; [34.9 ${ }^{\circ} \mathrm{N}, 115.71^{\circ} \mathrm{W}$ ]; 16 Jun. 1980; T. Griswold leg.; ex Acacia greggii; BBSL • 1 \%; San Bernardino Co., Granite Mts, T8N R13E Sec. 16; $\left[34.78^{\circ} \mathrm{N}, 115.62^{\circ} \mathrm{W}\right] ; 29$ May 1981; Griswold leg.; BBSL • 1 क; San Bernardino Co., NE of Granite Mts, T9N R13E Sc33; [34.83 N, $115.62^{\circ} \mathrm{W}$ ]; $1160 \mathrm{~m}$ a.s.1.; 21 Sep. 1993; T. Griswold leg.; ex Eriogonum deflexum; BBSL • 1 q; San Bernardino Co., Valley Wells; [35.79 N, $117.34^{\circ}$ W]; 23 Apr. 1993; J.G. Rozen leg.; AMNH • 1 \%; San Bernardino Co., Wild Horse Canyon, T11N R14E Sec. 1; [35.06 N, 115.44 W]; 23 Sep. 1993; T. Griswold leg.; ex Eriogonum deflexum; BBSL • 1 क; San Bernardino Co., Winston Wash, Sec. 3 T10N

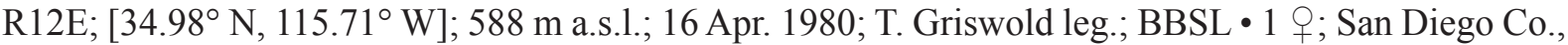
Borrego Valley; [33.26 $6^{\circ}$, $116.38^{\circ} \mathrm{W}$ ]; 26 May 1954; F.X. Williams leg.; CAS • 5 o $q$; Anza Borrego; $33.108^{\circ} \mathrm{N}, 116.4976^{\circ} \mathrm{W}$; 20 Apr. 2013; Hung, Borchers and Nabor leg.; WRME • 8 q ; ; same location as for preceding; 23 Mar. 2012; K.J. Hung leg.; WRME • 1 क ; Anza-Borrego; $33.05713^{\circ} \mathrm{N}, 116.4197^{\circ} \mathrm{W}$; 13 Oct. 2014; K.J. Hung leg.; ex Isocoma acradenia; WRME • 1 क; San Diego; [32.7 N, $\left.117.1^{\circ} \mathrm{W}\right] ; 25$ Jun. 2012; K. Hung leg.; WRME • 2 우; same location as for preceding; 3 May 2016; K.J. Hung leg.; WRME • 1 क; same location as for preceding; 31 Mar. 2016; Hung and Lee leg.; WRME • 1 +; same location as for preceding; 29 Jun. 2016; Hung and Lee leg.; WRME 1 1 ; ; same location as for preceding; 28 Apr. 2015; Hung and Cen leg.; WRME • 1 q; Sunset Cove, Granites Res. Stn. Mojave Desert; $34.77903^{\circ} \mathrm{N}, 115.66311^{\circ} \mathrm{W} ; 12 \mathrm{Apr}$. 2017; J. Braun leg.; PCYU • 1 q; same location as for preceding; 15 Apr. 2017; J. Braun leg.; PCYU. - Colorado • 1 क; Boulder Co., Niwot Ridge Alpine Zone; [ $40.06^{\circ}$ N, 105.6 ${ }^{\circ} \mathrm{W}$ ]; 3 Jul. 1962; M.E. Smith leg.; UCMC • 1 q; Boulder Co., Open Space: Beech; $40.0961^{\circ}$ N,

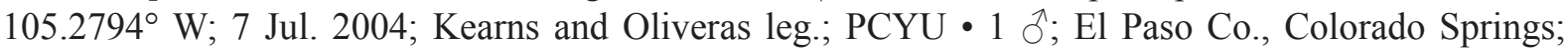
$38.82539^{\circ} \mathrm{N}, 104.85979^{\circ} \mathrm{W} ; 1886 \mathrm{~m}$ a.s.1.; 30 Jul. 2007; Gibbs and Sheffield leg.; PCYU • 1 ○; Fremont

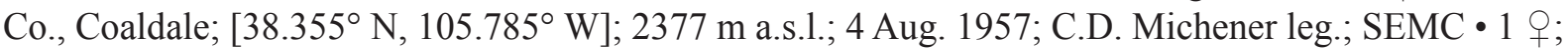
Huerfano Co., W of Walsenburg, Hwy 160; 37.6101 ${ }^{\circ}$ N, $104.81438^{\circ}$ W; 1929 m a.s.l.; 4 Aug. 2007; Gibbs and Sheffield leg.; PCYU • 1 \%; Jefferson Co.; $39.7476^{\circ}$ N, $105.2453^{\circ}$ W; 22 Aug. 2001; S.W.

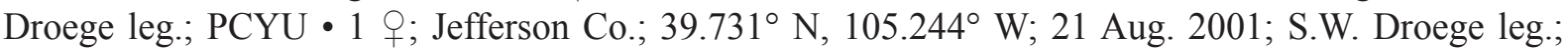
PCYU • 1 9; Lincoln Co., 17 mi. S of Limon; [39.02 ${ }^{\circ} \mathrm{N}, 103.64^{\circ} \mathrm{W}$ ]; $1585 \mathrm{~m}$ a.s.1.; 25 Jul. 1968; R.R. and R.A. Snelling leg.; ex Helianthus; LACM • 18 o o ; Moffat Co., Artesia; [40.24 N, $109.01^{\circ}$ W]; 22 Jul. 1950; C.D. Michener leg.; ex Helianthus petiolaris; SEMC • 3 o $o$; Otero Co., Vogel Canyon; $\left[37.7^{\circ} \mathrm{N}, 103.5^{\circ} \mathrm{W}\right]$; 12 Jun. 2008; W. Cranshaw leg.; CSUC • 1 q; Prowers Co., 1 mi. S of Granada;

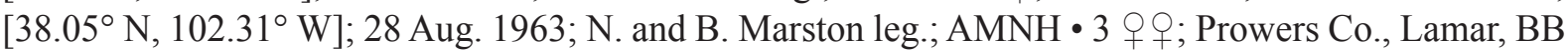
Road; [37.998 $\mathrm{N}, 102.7^{\circ} \mathrm{W}$ ]; 13 Apr. 2002; H. Miller leg.; CSUC • 1 \%; Weld Co., 10 mi. NE of Nunn Rd 45 and Rd 114; $40.81176^{\circ}$ N, $104.6537^{\circ}$ W; $1622 \mathrm{~m}$ a.s.1.; 11 Jun. 2001; V.L. Scott and S. Armstead leg.; ex Cleome serrulata; WRME $\bullet 3$ + 9 ; Weld Co., 10 mi. NE of Nunn Road 45 and Road 114; $40.81176^{\circ}$ N, 104.6537 ${ }^{\circ}$ W; 1622 m a.s.1.; 11 Jun. 2001; Virginia Scott and S. Armstead leg.; ex Cleome serrulata; UCMC • 1 万; Weld Co., Owl Creek, 12 mi. NE of Nunn; [40.86 N, $104.7^{\circ}$ W]; 4 Aug. 1983; H.E. Evans leg.; CSUC $\bullet 1$ क ; same collection data as for preceding; CSUC $\bullet 1$ त ; Yuma Co., 4 mi. N. E. Idalia; [39.75 $\mathrm{N}, 102.24^{\circ} \mathrm{W}$ ]; 10 Aug. 1964; J.G. and B.L. Rozen leg.; AMNH • 1 \%; Boulder; [ $40.01^{\circ} \mathrm{N}$, 105.27 W]; 23 May 1913; M.D. Ellis leg.; ex Capsella bursa-pastoris; AMNH • 1 \%; Julesburg;

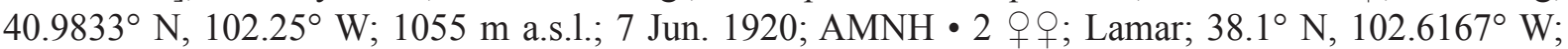

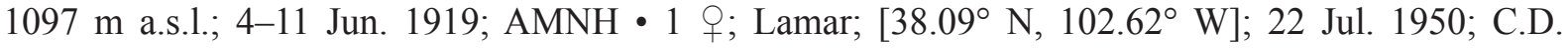
Michener leg.; ex Melilotus officinalis; SEMC. - Idaho - 4 우; Canyon Co., Deer Flat NWR, DFO; $43.562^{\circ} \mathrm{N}, 116.66801^{\circ} \mathrm{W}$; 11 Aug. 2011; Paul Backus leg.; FWSE • 3 q ; ; same location as for preceding; 29 Jul. 2011; Addison Mohler leg.; FWSE $\bullet 5$ 우; same location as for preceding; 20 Apr. 2012; Addison Mohler leg.; FWSE • 4 우; same location as for preceding; 5 Jul. 2012; Sabrina 
Seidel leg.; FWSE • 4 q 9 ; Canyon Co., Deer Flat NWR, DFS; $43.55281^{\circ} \mathrm{N}, 116.64137^{\circ} \mathrm{W} ; 5$ Jul. 2012;

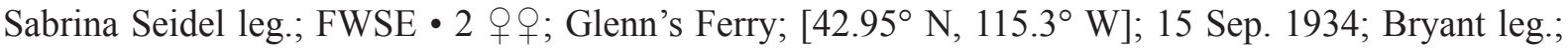
CAS • 1 q; Wendell, Pure Stand; [42.77 N, $114.7^{\circ}$ W]; 9 Jun. 1933; ex Sisymbrium/Salsola; USNM.

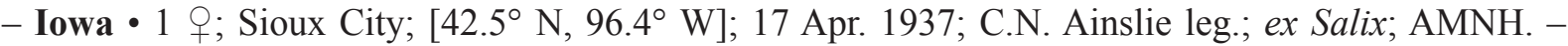
Kansas • 17 우; Barber Co.; 37.015374 N, 98.371489 W; 27 Apr. 2013; S. Griffin leg.; WRME • 2 우; same location as for preceding; 28 Apr. 2013; S. Griffin leg.; WRME • 4 + $q$; same location as for preceding; 29 Apr. 2013; S. Griffin leg.; WRME • 11 o $\circ$; same location as for preceding; 30 Apr. 2013; S. Griffin leg.; WRME • 4 qo+; Barber Co.; 37.087305 N, 98.370901 W; 27 Apr. 2013; S. Griffin leg.; WRME 1 1 ; same location as for preceding; 28 Apr. 2013; S. Griffin leg.; WRME 2 우 same location as for preceding; 29 Apr. 2013; S. Griffin leg.; WRME • 4 q $q$; same location as for preceding; 30 Apr. 2013; S. Griffin leg.; WRME • 1 क; Barber Co.; $37.034554^{\circ}$ N, $98.366604^{\circ}$ W; 27 Apr. 2013; S. Griffin leg.; WRME 11 \%; Reno Co., 5 mi. SE of Hutchinson; [38.01 $\left.{ }^{\circ} \mathrm{N}, 98^{\circ} \mathrm{W}\right]$; 2 Sep. 1951; C.D. Michener and W.E. LaBerge leg.; ex Heterotheca; UNSM. - Montana • 1 \% ; Choteau Co., Big Sandy, C1; $48.0443^{\circ}$ N, $110.0695^{\circ}$ W; 19 Jun. 2013; S. Adhikari leg.; MTEC • 3 q ; ; same location as for preceding; 25 Jun. 2014; S. Adhikari leg.; MTEC • 1 क; Choteau Co., Big Sandy, C2; $48.0428^{\circ}$ N, $110.0593^{\circ}$ W; 25 Jun. 2014; S. Adhikari leg.; MTEC • 1 क; Choteau Co., Big Sandy, C3; $48.0383^{\circ}$ N, $110.0593^{\circ}$ W; 25 Jun. 2014; S. Adhikari leg.; MTEC • 1 क; Choteau Co., Big Sandy, O1; $48.0407^{\circ}$ N, 110.0488 ${ }^{\circ}$ W; 8 Aug. 2014; S. Adhikari leg.; MTEC • 1 q; same location as for preceding; 5 Aug. 2015; S. Adhikari leg.; MTEC • 10 o 9 ; Choteau Co., Big Sandy, O2; $48.0363^{\circ}$ N, $110.0383^{\circ}$ W; 8 Aug. 2014; S. Adhikari leg.; MTEC • 8 우; same location as for preceding; 25 Jun. 2014; S. Adhikari leg.; MTEC • 1 q; same location as for preceding; 18 Jun. 2015; S. Adhikari leg.; MTEC • 1 \%; same location as for preceding; 25 Jun. 2013; S. Adhikari leg.; MTEC • 1 \%; Choteau Co., Big Sandy, O3; $48.0297^{\circ}$ N, $110.0545^{\circ} \mathrm{W} ; 8$ Aug. 2014; S. Adhikari leg.; MTEC • 1 क; same location as for preceding; 25 Jun. 2014; S. Adhikari leg.; MTEC • 1 क; Gallatin Co., Gallatin Grown; $45.7713^{\circ} \mathrm{N}, 111.2953^{\circ}$ W; 9 Jul. 2015; C.M. Delphia and J.L. Monte leg.; MTEC • 2 q $\circ$; same location as for preceding; 13 Aug. 2015; C.M. Delphia and J.L. Monte leg.; MTEC • 1 क; same location as for preceding; 24 Jul. 2015; C.M. Delphia and J.L. Monte leg.; MTEC • 1 क; same location as for preceding; 25 Jul. 2013; C.M. Delphia leg.; MTEC • 1 क; same location as for preceding; 8 Aug. 2013; C.M. Delphia leg.; MTEC • 1 क; same location as for preceding; 22 Aug. 2013; C.M. Delphia leg.; MTEC • 1 \% ; same location as for preceding; 19 Jul. 2013; C.M. Delphia leg.; MTEC • 1 ㅇ; Gallatin Co., MSU BART Farm; $45.6675^{\circ} \mathrm{N}, 111.0663^{\circ} \mathrm{W}$; 29 Jul. 2010; C.M. Delphia leg.; ex Campanula rotundifolia; MTEC • 1 ㅇ; Gallatin Co., Rocky Creek

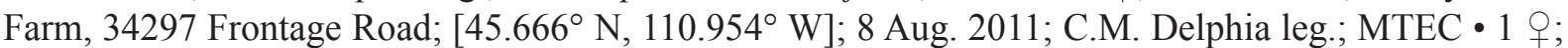
Rosebud Co., Four Wing Saltbush, 3 mi. S of Colstrip; [45.84 N, $106.62^{\circ}$ W]; 10 Aug. 1983; MTEC • 1 \%; same location as for preceding; 13 Sep. 1983; MTEC • 1 q; Rosebud Co., Rosebud Rest Stop; [46.265 $\left.{ }^{\circ} \mathrm{N}, 106.3^{\circ} \mathrm{W}\right]$; 22 Jun. 1987; T.A. Ebert leg.; CSUC. - Nebraska • 1 क; Lincoln Co., 8-14 mi. NNW of North Platte; [41.3 $3^{\circ}$, $100.9^{\circ}$ W]; 8 Aug. 1971; J.G., B.L. and K.C. Rozen leg.; WRME •

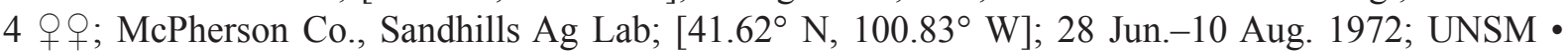
2 우; same location as for preceding; $15 \mathrm{Jul}$. 1972; UNSM • 4 + $O$; same location as for preceding; 20

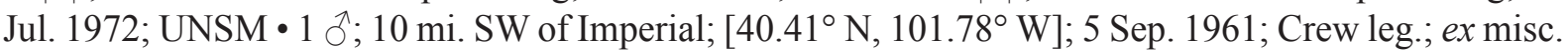
vegetation; UNSM • 1 o; 3 mi. E of Thedford; [ $\left.41.98^{\circ} \mathrm{N}, 100.52^{\circ} \mathrm{W}\right]$; 19 Aug. 1960; W.E. LaBerge leg.;

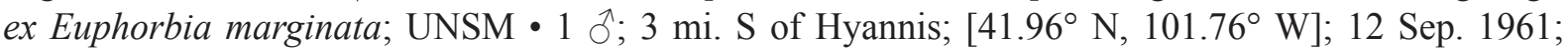
Crew leg.; ex misc. vegetation; UNSM. - Nevada • 1 क ; Clark Co., 0.3 mi. SE of Corn Creek Spr.; $36.4365^{\circ} \mathrm{N}, 115.3525^{\circ} \mathrm{W} ; 901 \mathrm{~m}$ a.s.1.; 24 Aug. 2004; S. Higbee and E. Ahlstrom leg.; BBSL • 1 ; Clark Co., 1.4 mi. WSW of Bonanza Hill; $35.7623^{\circ} \mathrm{N}, 115.5796^{\circ} \mathrm{W} ; 792 \mathrm{~m}$ a.s.1.; 4 May 2004; Higbee, Ahlstrom, Saul leg.; BBSL 11 q; Clark Co., 2.5 mi. SE of Rainbow Mtn; $36.0896^{\circ} \mathrm{N}, 115.4493^{\circ} \mathrm{W}$; 1138 m a.s.l.; 9 Sep. 2004; S. Higbee and T. Griswold leg.; BBSL • 1 q; Clark Co., 0.69 mi. N of Beehive Rock; $36.4196^{\circ} \mathrm{N}, 114.5509^{\circ} \mathrm{W} ; 23$ Aug. 2005; E. North leg.; ex Croton wigginsii; WRME • 1 O; Clark Co., Peek-A-Boo Canyon; $36.5326^{\circ}$ N, $115.1117^{\circ}$ W; 1760 m a.s.1.; 16 Aug. 2004; S. Higbee leg.; ex Eriogonum cernuum; BBSL • 1 q; Clark Co., 2.85 mi. SE of Rainbow Mtn; $36.0804^{\circ} \mathrm{N}$, 115.4587 W; 24 Jun. 2005; S. Higbee leg.; PCYU • 1 क; Clark Co., Wheeler Wash; $36.2748^{\circ} \mathrm{N}$, 
115.8425 ${ }^{\circ}$ W; 1591 m a.s.1.; 9 Jun. 2004; E. Ahlstrom leg.; ex Baileya multiradiata; BBSL 3 우; Elko Co., Elko; [40.83 N, $115.76^{\circ}$ W]; 7 Jun. 1982; R.W. Brooks leg.; ex Sphaeralcea ambigua; SEMC. New Mexico - 2 qo ; Bernalillo Co., Cibola NF, Sandia Crest, Kiwanis Meadow; $35.2058^{\circ} \mathrm{N}$, 106.4423 ${ }^{\circ}$ W; 3057 m a.s.1.; 21 Jun. 2017; J.A. Fowler leg.; UCRC • 1 क; Bernalillo Co., Cibola NF, Sandia Crest, Kiwanis Meadow; 35.2059 ${ }^{\circ}$ N, $106.4431^{\circ}$ W; 3057 m a.s.1.; 21 Jun. 2017; J.A. Fowler leg.; UCRC • 1 क; Bernalillo Co., Cibola NF, Sandia Crest, Kiwanis Meadow; $35.2052^{\circ}$ N, $106.4424^{\circ}$ W; 3057 m a.s.1.; 21 Jun. 2017; J.A. Fowler leg.; UCRC • 2 우; same location as for preceding; 22 Jun. 2017; J.A. Fowler leg.; UCRC • 1 क; Bernalillo Co., Cibola NF, Sandia Crest, Kiwanis Meadow;

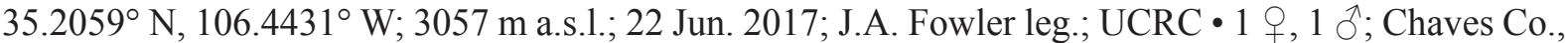
$5 \mathrm{~km} \mathrm{~S}$ of Roswell, Hwy 285; 33.36177 ${ }^{\circ}$ N, 104.51238 ${ }^{\circ}$ W; 1108 m a.s.1.; 10 Aug. 2007; Gibbs and

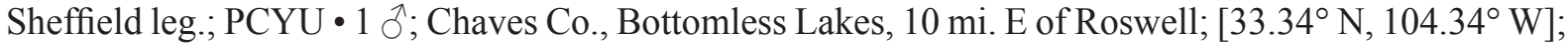
20 May 1969; Brothers, Krueger and Michener leg.; ex Tamarix gallica; SEMC • 1 ; Colfax Co., 7.6 mi. NW of Kiowa; $36.73724^{\circ} \mathrm{N}, 104.22277^{\circ} \mathrm{W} ; 2195 \mathrm{~m}$ a.s.l.; 18 Sep. 1999; A.L. Hicks and Virginia Scott leg.; ex Schizachyrium scoparium; UCMC - 2 ofo; Colfax Co., Hwy 64; 36.63692 ${ }^{\circ} \mathrm{N}$, 104.67754 W; 1932 m a.s.1.; 6 Aug. 2007; Gibbs and Sheffield leg.; PCYU • 1 q; DeBaca Co., 10 mi. S of Ft Sumner; [34.32 ${ }^{\circ}$ N, 104.25 W]; 19 May 1969; Brothers, Krueger and Michener leg.; ex Sphaeralcea; SEMC • 1 o ; same location as for preceding; 19 May 1969; Brothers, Krueger and

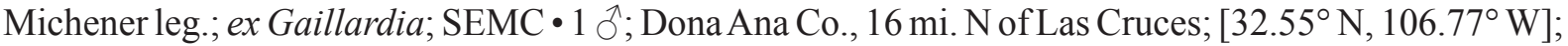
18 Aug. 1993; J.W. Van Zee leg.; ex Gutierrezia; NMSU • 1 गे; same location as for preceding; 1 Sep. 1993; J.W. Van Zee leg.; NMSU • 1 क; Dona Ana Co., jct. West Afton Rd and Black Mt. Rd; $32.0908^{\circ}$ N, 106.7794 W; 1272 m a.s.1.; 14 Sep. 2008; G.R. Ballmer leg.; ex Gutierrezia; UCRC • 1 क; Dona Ana Co., Jornada Range $26 \mathrm{~km} \mathrm{~N}$ of Las Cruces, stressor Site $8 \mathrm{~km} \mathrm{~W}$ of USDA HQ, Plot 17; [32.61 ${ }^{\circ} \mathrm{N}$, $\left.106.83^{\circ} \mathrm{W}\right]$; 1200 m a.s.1.; 4 Sep. 1996; G. Forbes leg.; NMSU • 1 क; Dona Ana Co., Jornada Range $26 \mathrm{~km} \mathrm{~N}$ of Las Cruces, stressor Site $8 \mathrm{~km} \mathrm{~W}$ of USDA HQ, Plot 7; [32.61 $\left.{ }^{\circ} \mathrm{N}, 106.83^{\circ} \mathrm{W}\right] ; 1200 \mathrm{~m}$ a.s.1.; 11 Aug. 1997; G. Forbes leg.; NMSU • 1 क; Dona Ana Co., Las Cruces; $\left[32.32^{\circ} \mathrm{N}, 106.76^{\circ} \mathrm{W}\right]$; 15 Aug. 1967; R.M. Bohart leg.; UCDC • 2 우, 1 `े; Eddy Co., 22 mi. SW of Carlsbad; [32. $\left.2^{\circ} \mathrm{N}, 104.5^{\circ} \mathrm{W}\right] ; 21$ May 1969; ex Lepidium integrifolium; SEMC • 1 +, 1 त); same location as for preceding; 21 May 1969; Brothers, Krueger and Michener leg.; ex Chamaesaracha; SEMC • 1 \%; Eddy Co., 3 mi. S of Artesia; [32. $\left.8^{\circ} \mathrm{N}, 104.4^{\circ} \mathrm{W}\right]$; 20 May 1969; Brothers, Krueger and Michener leg.; ex Chamaesaracha coniodes;

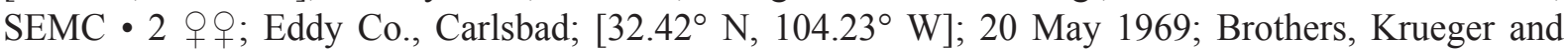
Michener leg.; ex Lepidium integrifolium; SEMC • 3 o $\circ$; Eddy Co.; $32.1^{\circ} \mathrm{N}, 104.5^{\circ} \mathrm{W} ; 1109 \mathrm{~m}$ a.s.1.; 10 Jun. 2010; J.D. Herndon and A. Druk leg.; BBSL • 1 क; Eddy Co.; $32.1^{\circ}$ N, $104.6^{\circ}$ W; 1551 m a.s.l.; 9 Jun. 2010; J.D. Herndon and A. Druk leg.; BBSL • 1 q; same location as for preceding; 1 Sep. 2010; R. Krauss and J.D. Herndon leg.; BBSL • 1 क; Eddy Co.; $32.1638^{\circ} \mathrm{N}, 104.4443^{\circ} \mathrm{W} ; 1152 \mathrm{~m}$ a.s.1.; 31 Aug. 2010; J.D. Herndon and R. Krauss leg.; BBSL • 2 qo ; Eddy Co.; $32.1768^{\circ}$ N, $104.4407^{\circ}$ W; 30 Aug. 2010; R. Krauss leg.; BBSL・ 1 q; Eddy Co.; $32.1117^{\circ}$ N, $104.5631^{\circ} \mathrm{W} ; 1324$ m a.s.1.; 12 Jul. 2010; J.D. Herndon and A. Druk leg.; BBSL • 1 \%; Eddy Co.; $32.1856^{\circ}$ N, $104.4326^{\circ}$ W; 7 Apr. 2010; H. Ikerd and T.L. Griswold leg.; BBSL -2 + ; Hidalgo Co., $1.6 \mathrm{~km} \mathrm{~S}$ of Rodeo; $\left[31.82^{\circ} \mathrm{N}, 109.03^{\circ} \mathrm{W}\right] ; 1$ Aug. 1987; B.N. Danforth leg.; ex Eriogonum; SEMC • 2 o 9 ; Hidalgo Co., 12.3 mi. N of Rodeo; [ $32.01^{\circ} \mathrm{N}$, $109.03^{\circ}$ W]; Aug. 1986; R.J. McGinley and B.M. Norden leg.; USNM • 1 क; Hidalgo Co., 13 mi. S of Animas; $31.7656^{\circ} \mathrm{N}, 108.8069^{\circ} \mathrm{W}$; 1219 m a.s.1.; 19 Aug. 2001; SEMC • 2 ðð; Hidalgo Co., 20 mi. S of Animas; [31.66 N, $108.81^{\circ} \mathrm{W}$ ]; 23 Aug. 2008; J.S. Ascher, J.G. Rozen and M.A. Rozen leg.; AMNH • 1 ऊ; Hidalgo Co., 23.5 mi. S of Animas; $31.61838^{\circ} \mathrm{N}, 108.86737^{\circ} \mathrm{W} ; 23$ Aug. 2007; J.J. Gibbs leg.;

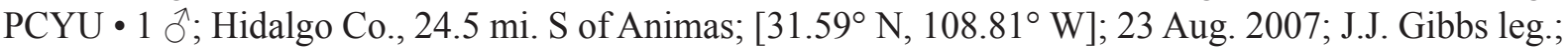
PCYU • 12 우, 1 ô; Hidalgo Co., 9 mi. N of Cotton City; [32.21 ${ }^{\circ}$ N, $108.88^{\circ}$ W]; 11 Aug. 1978; R.W. Brooks leg.; ex Wislizenia refracta; SEMC $\bullet 2$ + ; Hidalgo Co., Animas, 8.8 km E of junc. Sock N Shoe Rd.; $31.9572^{\circ} \mathrm{N}, 108.7144^{\circ} \mathrm{W} ; 1390 \mathrm{~m}$ a.s.1; 15 Aug. 2000; R. Brooks leg.; ex Fallugia paradoxa;

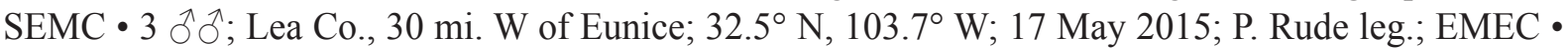
4 우; Lincoln Co., N Carrizozo on Rte 54; [33.8 N, 105.84 W]; 15 May 1987; B.N. Danforth leg.; ex Physaria; SEMC • 1 q; Otero Co., Lincoln NF, 2 mi. E of Sunspot, meadow; $32.7886^{\circ}$ N, $105.786^{\circ}$ W; 
11 Jul. 2017; J.A. Fowler leg.; UCRC • 1 क; Roosevelt Co., nr Boone Draw, 14 mi. SW of Portales; [34.05 N, 103.52 ${ }^{\circ}$ W]; 26 May 1972; Saul Frommer and N. Jorgensen leg.; UCRC・ 1 q; Santa Fe Co., Santa Fe NF, Jack's Creek Campground, meadow; $35.8555^{\circ}$ N, $105.6398^{\circ}$ W; 3042 m a.s.1.; 24 Jul. 2017; J.A. Fowler leg.; UCRC • 1 क; Socorro Co., Bosque del Apache; [33.8 $\left.{ }^{\circ} \mathrm{N}, 106.89^{\circ} \mathrm{W}\right] ; 1375 \mathrm{~m}$ a.s.l.; 15 Jul. 1991; Ilan Yarom leg.; SEMC • 3 ㅇ $ᄋ$; Socorro Co., Cibola NF, South Baldy meadow; $33.9901^{\circ}$ N, 107.1841 ${ }^{\circ}$ W; 3178 m a.s.1.; 16 Jun. 2017; K.C. Tom leg.; UCRC • 2 o o ; Socorro Co., Cibola NF, South Baldy meadow; $33.9919^{\circ} \mathrm{N}, 107.1836^{\circ} \mathrm{W} ; 3178 \mathrm{~m}$ a.s.1.; 16 Jun. 2017; M P. Argueta leg.; UCRC • 2 우; Socorro Co., Cibola NF, South Baldy meadow; $33.9911^{\circ}$ N, $107.1837^{\circ}$ W; 3178 m a.s.1.; 16 Jun. 2017; J.A. Fowler leg.; UCRC • 1 q; Socorro Co., Sevilleta NWR; [34.3 N, $\left.106.8^{\circ} \mathrm{W}\right]$; 18 Apr.-2 May 2006; K. Wetherill leg.; PCYU • 1 क; same location as for preceding; 17-31 May 2005; K. Wetherill leg.; PCYU • 1 o; same location as for preceding; 5-19 Oct. 2004; K. Wetherill leg.; PCYU • 1 क; Torrance Co., 5 mi. NW of Cedarvale; [34.42 ${ }^{\circ}$ N, $105.76^{\circ} \mathrm{W}$ ]; 6 Aug. 1966; C.R. Kovacic leg.; UCDC • 1 q; Valencia Co., ASC Los Lunas; [34.81 ${ }^{\circ} \mathrm{N}, 106.73^{\circ} \mathrm{W}$ ]; 25 Apr. 1988; C.R. Ward leg.; NMSU • 1 ; ; Valencia Co., Los Lunas; [34.81 ${ }^{\circ} \mathrm{N}, 106.73^{\circ} \mathrm{W}$ ]; 10 May 1988; C.R. Ward leg.; NMSU • 1 क; 25 mi. W of Tularosa; [33.07 $\left.{ }^{\circ} \mathrm{N}, 106.45^{\circ} \mathrm{W}\right] ; 1$ Jul. 1940; L.J. Lipovsky leg.; SEMC • 4 9 우 ; same location as for preceding; 1 Jul. 1940; D.E. Hardy leg.; SEMC • 1 क ; Alamogordo; [32.9 N, $\left.105.96^{\circ} \mathrm{W}\right] ; 26$ Jun. 1940; D.E. Hardy leg.; SEMC • 1 $\%$; same location as for preceding; 26 Jun. 1940; L.J. Lipovsky leg.; SEMC • 1 o; Bernalillo; [35.3 N, 106.55 W]; 23 Jul. 1950; L.D. Beamer leg.; SEMC • 1 q; Carrizozo; $\left[33.64^{\circ} \mathrm{N}, 105.88^{\circ} \mathrm{W}\right]$; 9 Jun. 1950; L.D. Beamer leg.; ex Mertensia franciscana; SEMC • 1 क; same location as for preceding; 9 Jun. 1950; L.D. Beamer leg.; ex Asclepias verticillata; SEMC • 2 o $\circ$; same location as for preceding; 9 Jun. 1950; L.D. Beamer leg.; SEMC • 1 \% ; Cloudcroft; [32.96 $\left.{ }^{\circ} \mathrm{N}, 105.74^{\circ} \mathrm{W}\right]$; 14 Jul. 1936; J.D. Beamer leg.; SEMC • 1 क; Cuervo; [35.03 N, 104.41ํ W]; 23 Jun. 1940; E.E. Kenaga leg.; SEMC • 1 क; Las Cruces; [32.32 ${ }^{\circ}$ N, $106.76^{\circ} \mathrm{W}$ ]; 12 Jun. 1950; R.H. Beamer leg.; SEMC •

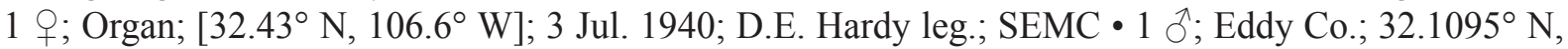

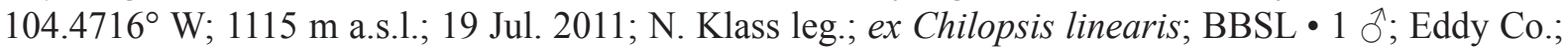
$32.1669^{\circ} \mathrm{N}, 104.5028^{\circ} \mathrm{W} ; 1373 \mathrm{~m}$ a.s.1.; 27 Jun. 2011; J.D. Herndon and N. Klass leg.; BBSL. - North

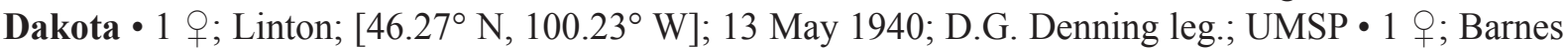
Co., $6.6 \mathrm{~km} \mathrm{NW}$ of Sandborn; $46.9794^{\circ}$ N, $98.2916^{\circ} \mathrm{W}$; 14 Jun. 2011; E. Evans leg.; UMSP. Oklahoma • 1 o; Cleveland Co.; [36.31 ${ }^{\circ} \mathrm{N}, 96.46^{\circ} \mathrm{W}$ ]; 6 Jul. 1981; Pat Terry leg.; ex Aphanostephus skirrhobasis; UNSM • 2 9 9 ; same location as for preceding; 7 Jul. 1981; Pat Terry leg.; ex Aphanostephus

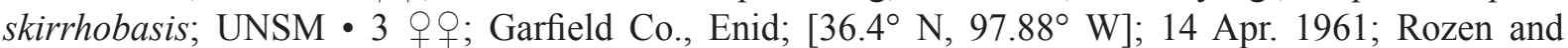

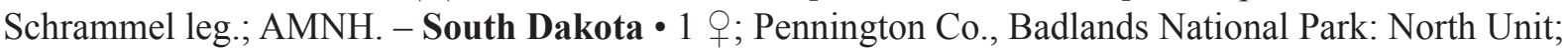

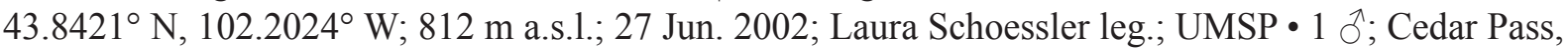

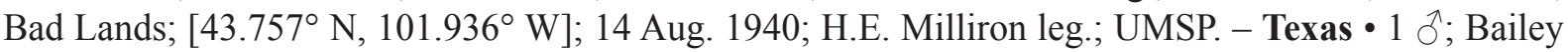
Co., Muleshoe Wildlife Refuge, Grassland 1; $33.963^{\circ}$ N, $102.7282^{\circ}$ W; 14 May 2013; S.J. Galdek leg.;

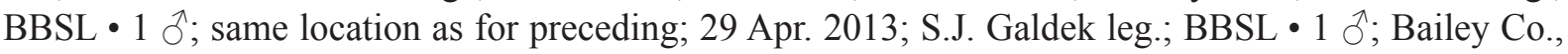
Muleshoe Wildlife Refuge, Prairie Dog 2; $33.9302^{\circ}$ N, $102.7584^{\circ}$ W; 10 Jun. 2013; S.J. Galdek leg.; BBSL 1 đ; Bailey Co., Muleshoe Wildlife Refuge, Prairie Dog 3; $33.963^{\circ} \mathrm{N}, 102.7749^{\circ} \mathrm{W} ; 30$ Apr. 2013; S.J. Galdek leg.; BBSL • 1 q; Brewster Co., Big Bend Nat'l. Park, Cattail Falls; [29.273 N, 103.336 W]; 9 Jun. 1999; S. Droege leg.; BBSL • 1 +; Brewster Co., Calamity Creek Rd., 25 km S of Alpine; [30.14 $\left.{ }^{\circ} \mathrm{N}, 103.63^{\circ} \mathrm{W}\right]$; 29 Apr. 2003; Packer and Fraser leg.; PCYU • 2 우; Brewster Co., Marathon; [30.21 ${ }^{\circ} \mathrm{N}, 103.24^{\circ} \mathrm{W}$ ]; 30 May 1993; R.L. Minckley and K. Toal leg.; ex Sphaeralcea leptophylla; SEMC • 1 क ; Colorado Co.; $\left[29.6^{\circ} \mathrm{N}, 96.5^{\circ} \mathrm{W}\right]$; 30 Mar. 1922; Mrs. Grace Wiley leg.; SEMC • 1 \%; same location as for preceding; 7 Apr. 1922; Mrs Grace Wiley leg.; SEMC • 1 \%; Culberson Co., Hurds Draw Rd and Hwy 10 intersection; [31.07 N, 104.28 ${ }^{\circ}$ W]; 30 May 1999; L. Packer leg.;

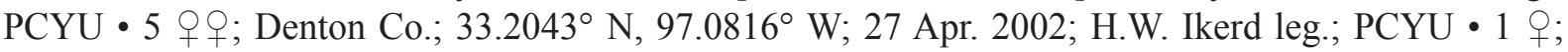
Dimmit Co., Chaparral Wildlife Management Area; [28.33 N, 99.41 ${ }^{\circ}$ W]; 20 Aug. 1994; J.L. Neff and A. Hook leg.; ex Amphiachyris dracunculoides; CTMI - 2 우; Ellis Co., Waxahachie; [32.39 $\mathrm{N}$,

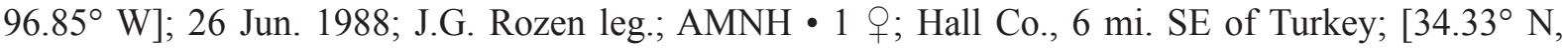
$100.97^{\circ} \mathrm{W}$ ]; 28 May 1970; C.W. O’Brien leg.; LACM • 1 + ; Hall Co., 16 mi. S of Memphis; $\left[34.49^{\circ} \mathrm{N}\right.$, 
100.54 ${ }^{\circ}$ W]; 22 Jun. 1959; R.R. Snelling and M.D. Snelling leg.; ex Tamarix gallica; LACM • 1 क; Hidalgo Co., Bentsen Rio Grande SP; $26.186^{\circ}$ N, $98.374^{\circ}$ W; 16 Mar. 2007; J.L. Neff leg.; ex Nama sp.;

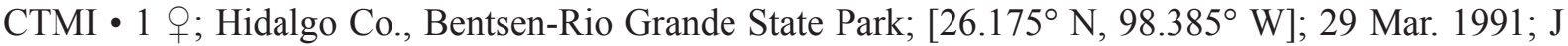
L. Neff leg.; CTMI • 1 o; Hudspeth Co., Fort Hancock; $31.3028^{\circ}$ N, $105.8395^{\circ}$ W; 17 Aug. 2000; J.L. Neff leg.; CTMI • 1 क , 3 ô $^{\wedge}$; Jeff Davis Co., 0.5 mi. S of Kent; [31.06 ${ }^{\circ} \mathrm{N}, 104.22^{\circ} \mathrm{W}$ ]; 29 May

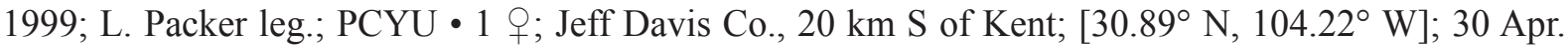
2003; Packer and Fraser leg.; PCYU • 3 q 9 ; Jeff Davis Co., 9 mi. S of Kent; [30.94 N, $104.22^{\circ}$ W]; 29 May 1999; L. Packer leg.; PCYU • 1 q; Jeff Davis Co., 9 mi. SE of Fort Davis; [30.5 $\left.{ }^{\circ} \mathrm{N}, 103.79^{\circ} \mathrm{W}\right]$; 23 Aug. 1967; R.C. Gardner and C.R. Kovacic leg.; UCDC • 2 우; Jeff Davis Co., Davis Mts TNC Res.; $30.708^{\circ} \mathrm{N}, 104.099^{\circ} \mathrm{W} ; 1800 \mathrm{~m}$ a.s.1.; 16 May 2005; J L. Neff and A. Hook leg.; CTMI • 1 \%; Kimble Co., 19 mi. NNW of Harper; $30.483^{\circ}$ N, $99.302^{\circ}$ W; 28 May 2006; J.L. Neff and A. Hook leg.; ex Thelesperma filifolium; CTMI • 1 क ; Kimble Co., RM385 and Salt Branch Rd; $30.478^{\circ} \mathrm{N}, 99.402^{\circ} \mathrm{W}$; 2 Jul. 2005; A.W. Hook and J.L. Neff leg.; CTMI • 1 क; Kimble Co., Salt Branch Rd.; 30.478 ${ }^{\circ}$ N, 99.402 ${ }^{\circ}$ W; 6 Jun. 2004; A.W. Hook and J.L. Neff leg.; CTMI • 1 क; La Salle Co., Chaparral Wildlife

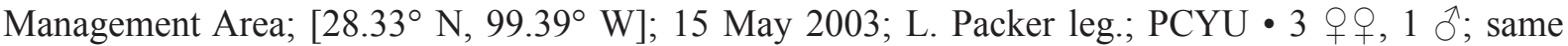
location as for preceding; 15-16 May 2003; L. Packer leg.; PCYU • 1 J'; La Salle Co.; 15 May 2003;

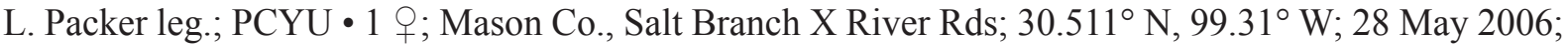
J.L. Neff and A. Hook leg.; ex Erigeron modestus; CTMI • 1 今̄; Randall Co., Buffalo Lake Wildlife Refuge, Grassland 2; $34.8892^{\circ}$ N, $102.1084^{\circ}$ W; 7 May 2013; S.J. Galdek leg.; BBSL・ 1 ơ; Randall Co., Buffalo Lake Wildlife Refuge, Prairie Dog 1; $34.9028^{\circ}$ N, $102.0989^{\circ}$ W; 3 Jun. 2013; S.J. Galdek leg.; BBSL 1 ô; Randall Co., Buffalo Lake Wildlife Refuge, Prairie Dog 2; $34.8896^{\circ} \mathrm{N}$, 102.1084 W; 4 Jun. 2013; S.J. Galdek leg.; BBSL・ 1 के; same location as for preceding; 3 Jun. 2013; S.J. Galdek leg.; BBSL 1 o ; Randall Co., Buffalo Lake Wildlife Refuge, Prairie Dog 3; $34.8713^{\circ} \mathrm{N}$, $102.1492^{\circ} \mathrm{W} ; 6$ Jun. 2013; S.J. Galdek leg.; BBSL $\bullet 2$ q ; Reeves Co., 3 mi. NW of Pecos; $31.453^{\circ} \mathrm{N}$, 103.508 ${ }^{\circ}$ W; 6 Jul. 2007; J.L. Neff and A. Hook leg.; ex Lepidium alyssoides; CTMI • 1 \%; San Patricio Co., Welder Refuge; $28.1122^{\circ}$ N, $97.4167^{\circ}$ W; Mar. 2004; Sty Reed leg.; PCYU • 1 क; 10 mi. N of Mason; [30.89 $\mathrm{N}, 99.23^{\circ} \mathrm{W}$; 13 Apr. 1952; Michener, Beamers, Wille and LaBerge leg.; ex Physaria; LACM • 1 क; Lubbock; [33.58 ${ }^{\circ}$ N, 101.86 ${ }^{\circ}$ W]; 19 Feb. 1967; V. Barber leg.; LACM. - Utah • 1 क; Emery Co., $9 \mathrm{~km} \mathrm{~N}$ of Goblin Valley St. Pk.; $\left[38.65^{\circ} \mathrm{N}, 110.66^{\circ} \mathrm{W}\right] ; 1360 \mathrm{~m}$ a.s.l.; 14 Aug. 1992; D. Yanega leg.; SEMC • 1 क ; same location as for preceding; 14 Aug. 1992; R. Minckley leg.; ex Lathrocasis tenerrima?; SEMC • 11 우; Emery Co., Green River; $38.9885^{\circ} \mathrm{N}, 110.162^{\circ} \mathrm{W}$; 9 May 2000; F.D. Parker leg.; BBSL・ 1 \%; Emery Co., San Rafael Desert, 1 mi. E of Flat Top Pass; $38.5417^{\circ} \mathrm{N}, 110.465^{\circ} \mathrm{W}$; 23 May 2000; F.D. Parker leg.; BBSL • 1 क; Emery Co., San Rafael Desert, 3 mi. E of Flat Top Pass; $38.51^{\circ} \mathrm{N}, 110.4255^{\circ} \mathrm{W} ; 24$ May 2000; F.D. Parker leg.; BBSL • 1 q; Garfield Co., 2.3 mi. S of Pioneer Mesa; 37.7622 ${ }^{\circ} \mathrm{N}, 111.1293^{\circ} \mathrm{W} ; 20$ May 2002; C. Davidson leg.; ex Fallugia paradoxa; BBSL • 1 q; Grand Co., 8 mi. SE of Emery; [38.84 N, 111.14 W]; 19 May 1980; D.B. Wahl leg.; SEMC • 1 q; same location as for preceding; 22 May 1979; D.B. Wahl leg.; SEMC • 1 क; Kane Co., 1.32 mi. SSW of Cougar Knoll; $37.2911^{\circ} \mathrm{N}, 111.1414^{\circ} \mathrm{W} ; 7$ Jul. 2000; O. Messinger leg.; ex Potentilla gracilis; BBSL • 1 \&; Kane Co., 1.33 mi. SSW of Cougar Knoll; $37.2931^{\circ} \mathrm{N}, 111.1505^{\circ} \mathrm{W} ; 7$ Jul. 2000; C. Davidson leg.; ex yellow composite; PCYU 1 क; Kane Co., 2.3 mi. NNW of White Point; $37.5452^{\circ} \mathrm{N}, 111.322^{\circ} \mathrm{W}$; 17 Sep. 2001; K. Moredock leg.; ex Ericameria nauseosa var. nauseosa; PCYU • 1 \&; Kane Co., 2.7 mi. SE of Left Hand Collet Canyon; $37.5085^{\circ} \mathrm{N}, 111.4069^{\circ} \mathrm{W} ; 13$ May 2002; K. Fordney leg.; ex Amelanchier utahensis; BBSL • 1 क; Kane Co., Tibbet Canyon; 37.1606 N, $111.5392^{\circ} \mathrm{W} ; 16$ May 2002; S. Higbee leg.; ex Sphaeralcea grossulariifolia; BBSL • 2 o $\circ$; Millard Co., Kanosh; [38.8 ${ }^{\circ} \mathrm{N}, 112.44^{\circ} \mathrm{W}$ ]; 24 May 1963; G.F. Knowlton leg.; SEMC • 1 + ; Millard Co., Oak Creek Canyon; [39.35 ${ }^{\circ}$ N, 112.26 W]; 27 May 1958; G.F. Knowlton leg.; ex Salix sp.; SEMC • 1 ; Washington Co., 0.72 mi. E of Firepit Knoll; 37.3494 ${ }^{\circ}$ N, $113.0912^{\circ}$ W; 19 Jun. 2006; B. Hays and F. Nicklen leg.; PCYU • 1 क; Washington Co., 1.1 mi. N of Rockville; $37.1773^{\circ}$ N, $113.0368^{\circ}$ W; 11 May 2006; B. Hays leg.; PCYU • 1 q ; Cedar

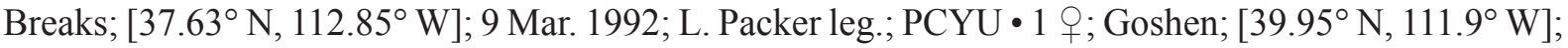
12 Aug. 1949; Geo. E. Bohart leg.; BBSL・ 1 q; Kane Spgs., 15 mi. SE of Moab; [38.392 N, $109.452^{\circ}$ W]; 
9 Aug. 1957; C.D. Michener leg.; ex Cleome lutea; SEMC • 1 क; Roosevelt; [40.3 N, $\left.109.99^{\circ} \mathrm{W}\right] ; 29$ Jun. 1954; G.F. Knowlton leg.; SEMC • 1 \%; Tremonton; [41.71 ${ }^{\circ} \mathrm{N}, 112.17^{\circ} \mathrm{W}$ ]; 5 Jul. 1931; J.O. Nottingham leg.; SEMC. - Wyoming • 1 क; Big Horn Co., 4 mi. southwest Lane 13, Lovell Local Training Area; $\left[44.77^{\circ} \mathrm{N}, 108.3^{\circ} \mathrm{W}\right] ; 1$ Jun. 2004; B. Kondratieff and J. Owens leg.; CSUC • 1 q; Hot Springs Co., $10 \mathrm{mi}$. N of Thermopolis; [43.79 $\mathrm{N}, 108.21^{\circ} \mathrm{W}$ ]; 12 Aug. 1975; C.D. Michener leg.; ex Cleome lutea; SEMC • 1 o; Natrona Co., 25 mi. SW of Casper; [42.59 $\left.{ }^{\circ} \mathrm{N}, 106.67^{\circ} \mathrm{W}\right] ; 12$ Aug. 1972; J.G. Rozen and R. McGinley leg.; AMNH • 1 क; Natrona Co., 9 mi. E. Casper; [42.85 N, $106.14^{\circ} \mathrm{W}$; 12 Aug. 1962; J.G. and B.L. Rozen leg.; AMNH • 4 우; Platte Co., Warm Spring, Camp Guernsey; [42.26 N, 104.76 W]; 1372 m a.s.l.; 6 Jun. 2000; P.M. Pineda and J. Schmidt leg.; CSUC • 1 क ; Sheridan Co., 5 mi. N of Sheridan; [44.87 ${ }^{\circ}$ N, $106.96^{\circ} \mathrm{W}$ ]; 6 Aug. 1963; G. Bohart and P. Torchio leg.; ex Solidago; BBSL・ 1 क; Grand Teton Nat. Pk.; [43.8 N, 110.7 W]; Jul. 1937; BBSL.

\section{Description}

See Gibbs (2010) for a complete redescription and figures.

\section{Range}

Alberta east to North Dakota, south to Baja California Sur and San Luis Potosi (Fig. 42).

\section{Floral records}

AMARANTHACEAE Juss. Salsola L. S. kali L. S. $k$. subsp. tragus (L.) Čelak. • APOCYNACEAE Juss. Asclepias L. A. erosa Torr. $・$ A. subverticillata (A.Gray) Vail $・$ A. verticillata L. • ASTERACEAE Giseke: Amphiachyris: A. dracunculoides (DC.) Nutt. Aphanostephus: A. skirrhobasis (DC.) Trel. ex Coville \&

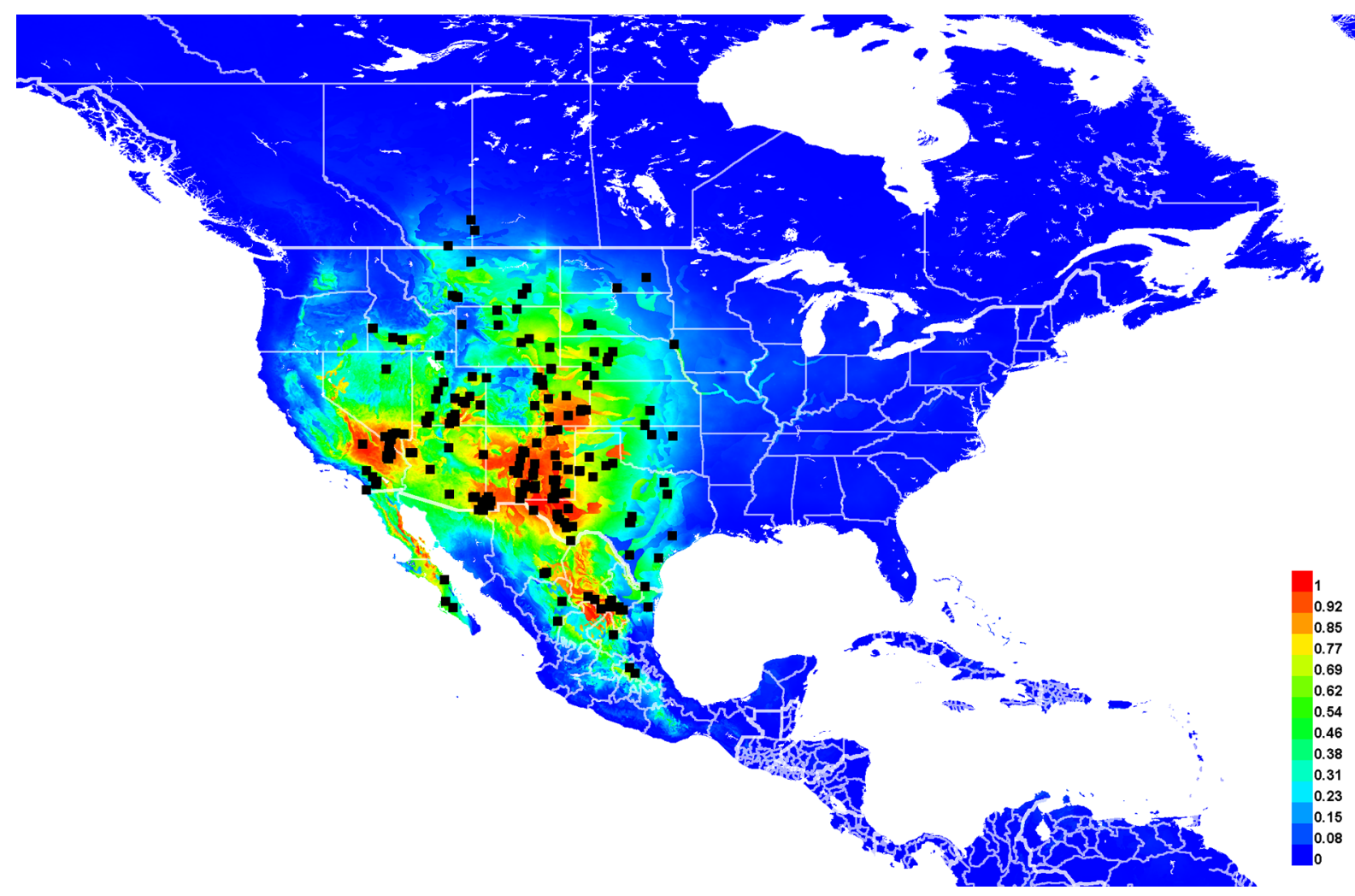

Fig. 42. Georeferenced collection records of Lasioglossum (D.) hudsoniellum (Cockerell, 1919) (black squares) and predicted distribution by maximum entropy ecological niche modeling in Maxent (colour shading). Warmer colours indicate higher cloglog probability of occurrence. 
Branner $\bullet$ Bahia: B. absinthifolia Benth. Baileya: B. multiradiata Harv. \& A.Gray ex Torr. Ericameria: E. nauseosa (Pall. ex Pursh) G.L. Nesom \& Baird: E.n. var. nauseosa (Pall. ex Pursh) G.L. Nesom \& Baird - Erigeron: E. modestus A.Gray • Gaillardia • Gutierrezia • Helianthus: H. petiolaris Nutt. • Heterotheca Isocoma: I. acradenia (Greene) Greene • Solidago • Thelesperma: T. filifolium (Hook.) A.Gray - Varilla: V. mexicana A.Gray • BIGONIACEAE Juss. Chilopsis D. Don: C. linearis (Cav.) Sweet • BORAGINACEAE Juss. Heliotropium L. H. curassavicum L. • Mertensia Roth: M. franciscana A. Heller - Nama L. - BRASSICACEAE Burnett: Brassica L. B. nigra (L.) K. Koch - Capsella Medik. C. bursa-pastoris (L.) Medik. - Lepidium L. L. alyssoides A.Gray • L. integrifolium Nutt. • Physaria (Nutt. ex Torr. \& A.Gray) A.Gray • Sisymbrium L. $\bullet$ CAMPANULACEAE Juss. Campanula L. C. rotundifolia L. CLEOMACEAE Bercht. \& J. Presl: Cleome L. C. lutea Hook. - C. serrulata Pursh • Wislizenia Engelm. W. refracta Engelm. EUPHORBIACEAE Juss. Croton L. C. wigginsii L.C. Wheeler - Euphorbia L. E. marginata Pursh - FABACEAE Juss. Acacia: A. greggii A.Gray - Dalea: D. eriophylla S. Watson - Melilotus: M. officinalis (L.) Pall. P Psorothamnus: P. scoparius (A.Gray) Rydb. MALVACEAE Juss. Sphaeralcea A. St.-Hil. S. ambigua A.Gray • S. grossulariifolia (Hook. \& Arn.) Rydb. • S. leptophylla (A.Gray) Rydb. • POACEAE Barnhart: Schizachyrium Nees: S. scoparium (Michx.) Nash • POLYGONACEAE Juss. Eriogonum Michx. E. cernuum Nutt. • E. deflexum Torr. • E. fasciculatum Benth. E. f. var. polifolium (Benth.) Torr. \& A.Gray • ROSACEAE Juss. Amelanchier Medik. A. utahensis Koehne $\bullet$ Fallugia Endl. F. paradoxa (D. Don) Endl. ex Torr. Potentilla L. P. gracilis Douglas ex Hook. • SALICACEAE Mirb. Salix L. SOLANACEAE Adans. Chamaesaracha Franch. \& Sav. ex A.Gray: C. coniodes (Dunal) Britton - TAMARICACEAE Link: Tamarix L. T. chinensis Lour. T. gallica L. ZZYGOPHYLLACEAE R.Br. Kallstroemia Scop. K. californica (S. Watson) Vail $\bullet$ Larrea Cav. L. tridentata (Sessé \& Moc. ex DC.) Coville.

\section{DNA barcodes}

Twnety-four sequences available (BOLD process IDs: DLII021-06, DLII052-06, DLII785-07, DLII801-07, DLII851-07, DLII852-07, DLII874-07, DLII912-07, DLII913-07, DLII983-07, DLII99907, DLII1032-07, DLII1079-07, DLII1083-07, DLII1125-07, DLII1127-07, DLII1266-08, DLII127308, DLII1472-08, DLIII103-18, DIAL1111-07, DIAL1128-07, DIAL1130-07, DIAL1138-07; BINs: BOLD:AAB5530, BOLD:ABZ0512, BOLD:ACE7697). There is a deep divergence between some of these sequences (maximum p-distance 3.21\%) compared to the divergence between L. hudsoniellum and its nearest neighbour, L. nymphale (minimum p-distance 1.68\%). No unique fixed substitutions distinguish L. hudsoniellum from all other western red-tailed L. (Dialictus).

\section{Remarks}

Lasioglossum hudsoniellum is undoubtedly the most common and widespread of the red-tailed L. (Dialictus). It occurs in all habitats from prairies to mountains to deserts. There is considerable variation in several characters over its range, which has led to much confusion with identification. Specimens from the easternmost part of its range in the Great Plains often have a black metasoma (even in the female), specimens from the western deserts often have pale wings with white hairs, and specimens from Texas and Mexico often have sparser punctures of the mesosoma and T1. In addition, there is significant variation in the length of the face, but this does not seem to be correlated with any other characters.

Given such extreme morphological, genetic, and geographic variation, L. hudsoniellum seems likely to include some cryptic species. However, clear consistencies between these sources of variation cannot be found to support a species hypothesis. 
Lasioglossum (Dialictus) imbriumbrae sp. nov. urn:Isid:zoobank.org:act:FA28AC7F-5BB1-4AD7-9FDB-2C3506701720

Figs 43-45, 108C, 115B

\section{Diagnosis}

Females of Lasioglossum imbriumbrae sp. nov. can be recognized by the head and mesepisternum shiny with large, dense ( $i<1 \mathrm{pd}$ ) but distinctly separated punctures (except on the frons medially), face short (length/width ratio $\sim 0.8$ ), and $\mathrm{T} 2-4$ with dark spiracular spots. They are most similar to L. mesillense. Females of $L$. mesillense have the frons with finer and slightly but distinctly separated punctures, and hypoepimeron and upper mesepisternum with slightly smaller and crowded, indistinct punctures $(\mathrm{i}=0 \mathrm{pd})$.

Males of $L$. imbriumbrae sp. nov. can be recognized by the propodeum smooth with very strong dull microsculpture, mesepisternum densely punctate $(\mathrm{i}<1 \mathrm{pd}), \mathrm{T} 1-3$ rims almost completely impunctate, wings lightly infuscated with dark veins and dark hairs, scutum and metasoma with somewhat densely plumose hair, and clypeus apical margin and labrum reddish brown. They are most similar to L. minckleyi sp. nov., L. mesillense, L. austerum sp. nov., and L. hudsoniellum. All of these species have T1-3 rims punctate. In addition, males of $L$. mesillense have the flagellomeres relatively long (F2 about 2 times as long as F1 and 1.6 times as long as broad), propodeum shiny, and clypeus apical margin and labrum usually orange. Males of $L$. austerum sp. nov. have the mesepisternum slightly more sparsely punctate $(i \leq 1 \mathrm{pd})$ and scutum and metasoma with mostly thin and weakly plumose hair. Males of $L$. hudsoniellum have S5 apical margin straight, mesosoma and metasoma with more extensive tomentum, and clypeus apical margin and labrum usually orange.

\section{Etymology}

The specific epithet imbriumbrae is formed from the Latin noun 'imber' ('rain') and the genitive singular noun 'umbrae' ('shadow'). It refers to this species' narrow distribution in the rain shadow of the Sierra Nevada Mountains. An appropriate translation would be the rain shadow sweat bee.

\section{Material examined}

\section{Holotype}

UNITED STATES - California • ${ }_{+}$; Olancha; $\left[36.28^{\circ} \mathrm{N}, 118.01^{\circ} \mathrm{W}\right] ; 2$ May 1927; Timberlake leg.; ex Salix exigua; UCRC.

[Verbatim label: on Salix exigua // Olancha / Cal. May 2, 27 // Timberlake Coll. // HOLOTYPE / Lasioglossum (Dialictus) imbriumbrae Gardner and Gibbs]

\section{Paratypes}

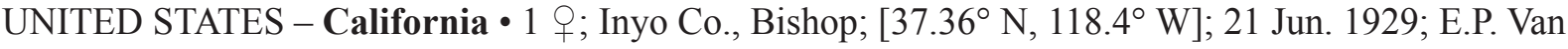
Duzee leg.; CAS • 1 क; Mono Co., Tom's Place, Rock Creek; $\left[37.56^{\circ} \mathrm{N}, 118.68^{\circ} \mathrm{W}\right] ; 1888 \mathrm{~m}$ a.s.1.; 6 Oct. 2002; E.F. Drake leg.; ex Chrysothamnus sp.; UCRC • 2 +q ; same collection data as for holotype; UCRC. - Nevada • 1 +, 1 \%; Washoe Co., Pyramid Lake; [40 N, 119.5 W]; 29 Jun. 1968; D.S. Horning

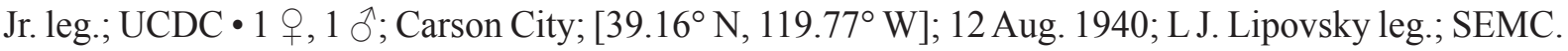

\section{Description}

Female

Colouration. Head and mesosoma blue to blue-green; clypeus apical colour black to reddish brown; labrum black, reddish brown, or orange; mandible orange with black basal band and red tip; flagellum black to reddish brown dorsally, reddish brown to orange ventrally; pronotal lobe black to reddish brown; 
metasoma red to orange with dark spiracular spots on T2-4 (sometimes faint), sometimes dark on T1 and T3 laterally and T4-5 throughout; legs black to reddish brown with femur-tibia joints, base and apex of tibiae, and tarsi sometimes orange; tegula reddish brown; wing membrane lightly infuscated, veins with subcosta dark brown, otherwise pale amber to brown.

Pubescence. Body hair colour white. Tomentum dense on gena, pronotal collar and lobe, space between pronotal lobe and tegula, T2 narrow basolateral patches, T3 narrow basal band, and T4 throughout; sparse on paraocular area and episterna. Scutum hair thin to moderately plumose. Wing hairs dark, short and dense. Acarinarial fan complete, very sparse. T2 fringes sparse, T3 fringes sparse.

Surface sculpture. Clypeus punctures dense in basal third ( $\mathrm{i}<1 \mathrm{pd}$ ), large and irregularly spaced apically (i $<2$ pd), sculpture shiny, sometimes weakly imbricate basally; supraclypeal area punctures dense $(i \leq 1 \mathrm{pd})$, sometimes sparse medially $(\mathrm{i}=1-3 \mathrm{pd})$, sculpture shiny or weakly imbricate; paraocular area punctures dense $(\mathrm{i}<1 \mathrm{pd})$, irregularly sparser around antenna socket $(\mathrm{i}<2 \mathrm{pd})$, sculpture weakly imbricate around antenna socket, otherwise shiny; frons punctures crowded ( $\mathrm{i}=0 \mathrm{pd})$, sculpture weakly imbricate; vertex punctures dense laterally ( $i<1 \mathrm{pd}$ ), sparse medially ( $\mathrm{i}=1-3 \mathrm{pd}$ ), sculpture shiny, sometimes weakly imbricate laterally; gena punctures moderately sparse $(i=1-3 \mathrm{pd})$, sculpture shiny; postgena sculpture shiny; tegula punctures absent; scutum punctures dense ( $\mathrm{i}<1 \mathrm{pd}$ ), becoming moderately sparse submedially and anteromedially $(i=1-3 \mathrm{pd})$, sculpture shiny, becoming tessellate anteromedially; scutellum punctures dense marginally and on median line ( $\mathrm{i} \leq 1 \mathrm{pd})$, sparse submedially ( $i=1-4 \mathrm{pd}$ ), diversopunctate, sculpture shiny; metanotum sculpture shiny and finely, densely punctate ( $i<1 \mathrm{pd}$ ), becoming rugulose laterally; metapostnotum rugae strong, anastomosing or subparallel, not reaching margin, sculpture imbricate; preëpisternum sculpture shiny with crowded punctures $(\mathrm{i}=0 \mathrm{pd})$ or areolate; hypoepimeron punctures dense $(\mathrm{i}<1 \mathrm{pd})$, sculpture shiny or weakly imbricate; mesepisternum punctures dense $(i<1 \mathrm{pd})$, sometimes becoming crowded dorsally ( $i=0 \mathrm{pd})$, sculpture shiny or weakly imbricate; metepisternum sculpture ruguloso-lineate dorsally, reticulate-punctate medially, imbricate ventrally; propodeum lateral face sculpture tessellate; propodeum posterior face sculpture tessellate; $\mathrm{T} 1$ anterior face sculpture shiny; $\mathrm{T} 1$ dorsal surface punctures moderately dense laterally ( $\mathrm{i}=1-3 \mathrm{pd}$ ), becoming fine and very sparse medially ( $\mathrm{i}=1-6 \mathrm{pd})$, absent in narrow median line, large apicolateral oval patches, and on rim, sculpture shiny, becoming weakly coriarious on rim; T2 disc punctures moderately dense ( $i=1-3 \mathrm{pd})$, disc sculpture shiny, rim punctures fine, sparse $(i=1-4 \mathrm{pd})$, sometimes obscure, rim sculpture coriarious.

STRUCTURE. Face length/width ratio 0.8 ( $\pm 0.02 \mathrm{SD}$ ). Clypeus projecting $\sim 67 \%$ below suborbital tangent; clypeal area length/width ratio 0.38 ( $\pm 0.03 \mathrm{SD})$; apicolateral denticles low rounded knobs; supraclypeal area length/width ratio $0.82( \pm 0.08 \mathrm{SD})$. Forewing with 3 submarginal cells; pronotal angle obtuse; tegula shape normal. Intertegular distance $0.95( \pm 0.06 \mathrm{SD}) \mathrm{mm}$. Scutum length/width ratio $0.79( \pm 0.04 \mathrm{SD})$; scutum/scutellum length ratio $2.79( \pm 0.16 \mathrm{SD})$; scutellum/metanotum length ratio $1.53( \pm 0.16 \mathrm{SD})$; metanotum/metapostnotum length ratio $0.73( \pm 0.07 \mathrm{SD})$. Propodeum lateral carinae not reaching dorsal margin; oblique carina weak, straight. T2 depressed apical rim length less than $50 \%$ of segment. $(n=7)$

\section{Male}

Colouration. Head and mesosoma blue to blue-green; clypeus apical colour black to reddish brown; labrum reddish brown; mandible orange with black basal spot and red tip; flagellum reddish brown dorsally, orange ventrally; pronotal lobe reddish brown; metasoma black to reddish brown with rims of terga and sterna and downcurved lateral areas of terga becoming orange to translucent yellow, dark spiracular spots on T2-4; legs reddish brown with femur-tibia joints, base and apex of tibiae, and tarsi orange; tegula brown; wing membrane lightly infuscated, veins with subcosta dark brown, otherwise brown. 


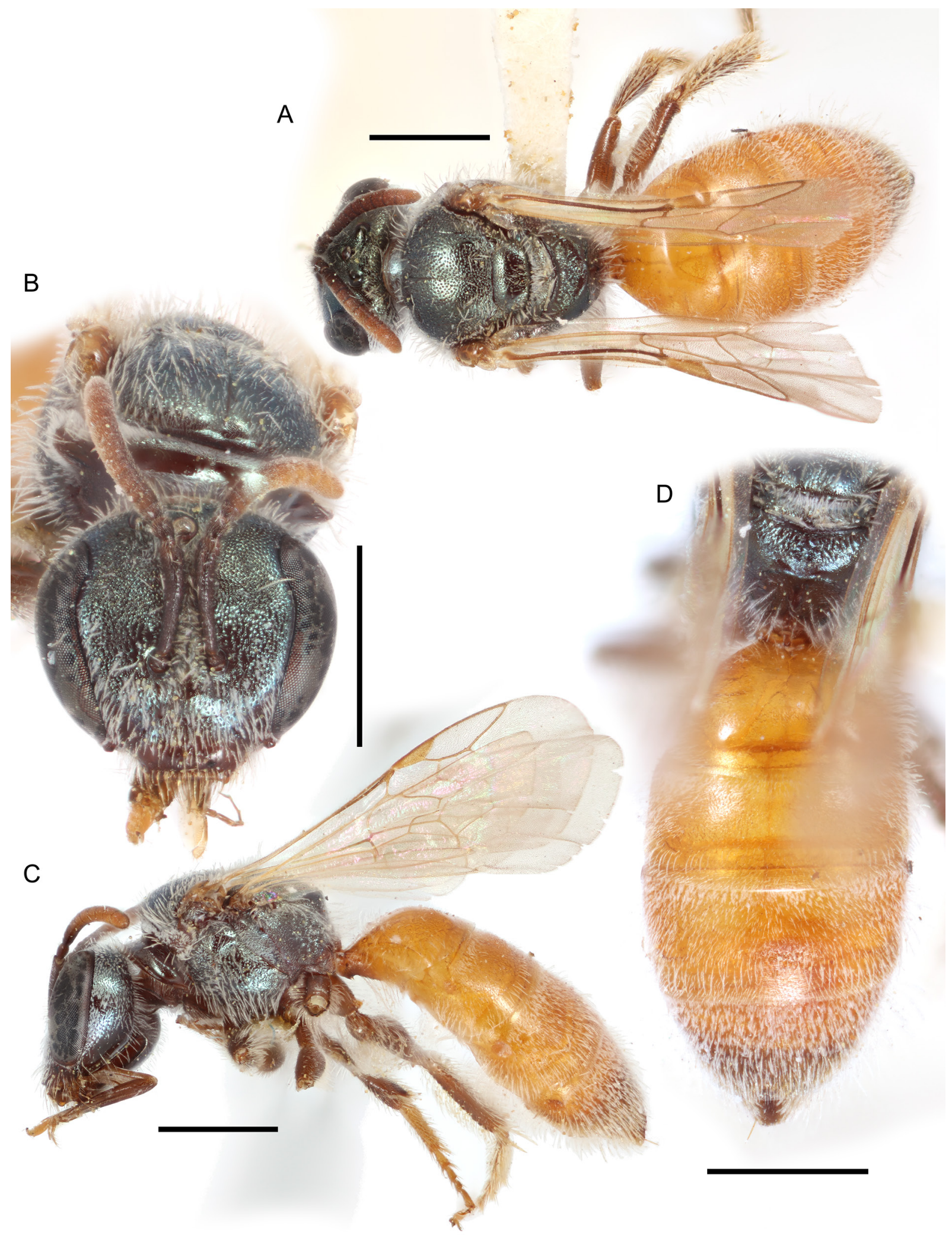

Fig. 43. Lasioglossum (D.) imbriumbrae sp. nov., ${ }^{\circ}$. A. Dorsal habitus. B. Face. C. Lateral habitus. D. Propodeum and metasoma. Scale bars: $1 \mathrm{~mm}$. 


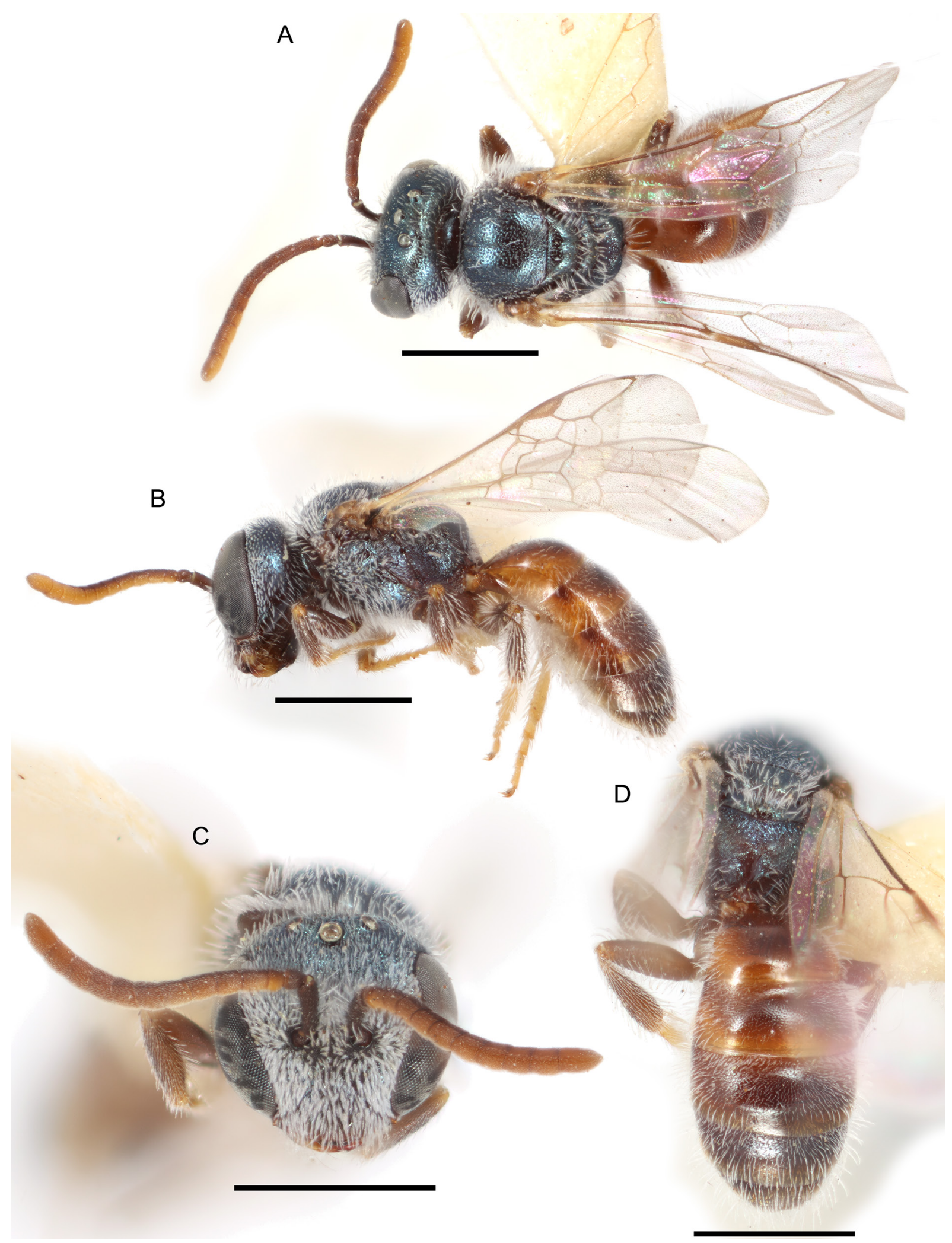

Fig. 44. Lasioglossum (D.) imbriumbrae sp. nov., วิ. A. Dorsal habitus. B. Lateral habitus. C. Face. D. Propodeum and metasoma. Scale bars: $1 \mathrm{~mm}$. 
Pubescence. Body hair colour white. Tomentum dense on face below eye emargination, pronotal angle and lobe, and space between pronotal lobe and tegula; sparse on gena, scutum lateral margin, episterna, and T3-5 basolaterally. Scutum hair thin to densely plumose. Sterna hair moderately long (1-2 OD), densely plumose, dense and erect. Wing hairs dark or light, short and dense.

SURFACE SCULPTURE. Clypeus punctures moderately dense ( $\mathrm{i}=1-2 \mathrm{pd})$, sculpture shiny; supraclypeal area punctures moderately dense $(i=1-2 \mathrm{pd})$, sculpture shiny; paraocular area punctures dense $(\mathrm{i}<1 \mathrm{pd})$, sculpture shiny; frons punctures dense $(\mathrm{i}<1 \mathrm{pd})$, sculpture shiny; vertex punctures dense laterally ( $\mathrm{i} \leq 1 \mathrm{pd}$ ), moderately sparse medially ( $\mathrm{i}=1-2 \mathrm{pd}$ ), sculpture shiny; gena punctures sparse ( $i=1-3$ pd), sculpture shiny; postgena sculpture shiny; tegula punctures absent; scutum punctures dense ( $i \leq 1 \mathrm{pd}$ ), becoming moderately sparse submedially ( $\mathrm{i}=1-2 \mathrm{pd}$ ), sculpture shiny, becoming weakly tessellate anteromedially; scutellum punctures dense ( $\mathrm{i} \leq 1 \mathrm{pd})$, sometimes becoming moderately sparse submedially ( $\mathrm{i}=1-2 \mathrm{pd}$ ), sculpture shiny; metanotum sculpture shiny and moderately densely punctate ( $i=1-2 \mathrm{pd}$ ), becoming rugulose laterally; metapostnotum rugae strong, anastomosing, nearly reaching margin, sculpture imbricate; preëpisternum sculpture areolate; hypoepimeron punctures dense $(\mathrm{i}<1 \mathrm{pd})$, sculpture shiny; mesepisternum punctures dense $(i<1 \mathrm{pd})$, sculpture shiny; metepisternum sculpture lineate dorsally, areolate ventrally; propodeum lateral face punctures obscure, sculpture imbricate and weakly rugulose; propodeum posterior face sculpture imbricate; T1 anterior face sculpture shiny; T1 dorsal surface punctures moderately dense $(\mathrm{i}=1-2 \mathrm{pd})$, sparse in large apicolateral oval patches $(i=2-4 \mathrm{pd})$ and absent on rim, sculpture shiny; $\mathrm{T} 2$ disc punctures dense basally ( $\mathrm{i} \leq 1 \mathrm{pd})$, becoming moderately sparse toward premarginal line $(i=1-2 \mathrm{pd})$, disc sculpture shiny, rim punctures absent, rim sculpture shiny.

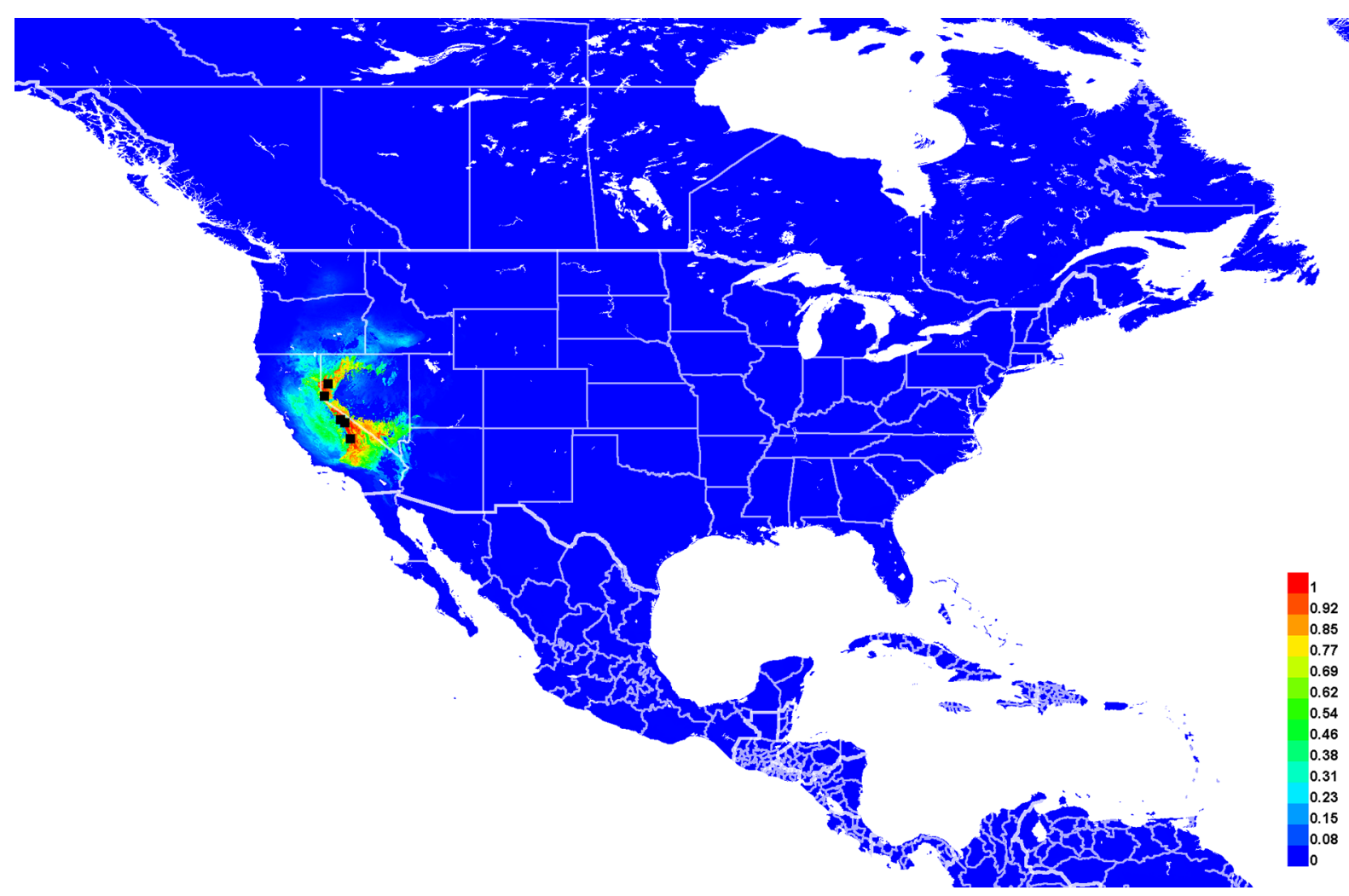

Fig. 45. Georeferenced collection records of Lasioglossum (D.) imbriumbrae sp. nov. (black squares) and predicted distribution by maximum entropy ecological niche modeling in Maxent (colour shading). Warmer colours indicate higher cloglog probability of occurrence. 
Structure. Face length/width ratio 0.78 ( $\pm 0 \mathrm{SD})$. F1:pedicel length ratio $0.73( \pm 0 \mathrm{SD}) ; \mathrm{F} 2: \mathrm{F} 1$ length ratio $1.64( \pm 0.13 \mathrm{SD}) ; \mathrm{F} 2$ length/width ratio $1.24( \pm 0.04 \mathrm{SD}) ; \mathrm{F} 9$ length/width ratio $1.08( \pm 0.04 \mathrm{SD})$. Forewing with 3 submarginal cells; pronotal angle obtuse; tegula shape normal. Intertegular distance $0.71( \pm 0.04 \mathrm{SD}) \mathrm{mm}$. Scutum length/width ratio $0.88( \pm 0.01 \mathrm{SD})$; scutum/scutellum length ratio 2.63 $( \pm 0.1 \mathrm{SD})$; scutellum/metanotum length ratio $1.56( \pm 0.19 \mathrm{SD})$; metanotum/metapostnotum length ratio 0.8 ( \pm 0.05 SD). Propodeum lateral carinae not reaching dorsal margin; oblique carina absent. $(n=2)$

Genitalia. Not examined.

\section{Range}

Eastern California and western Nevada, just east of the Sierra Nevada Mountains (Fig. 45).

\section{Floral records}

ASTERACEAE Giseke: Chrysothamnus • SALICACEAE Mirb. Salix L. S. exigua Nutt.

\section{DNA barcodes}

Not available.

\section{Remarks}

Rare. Only 9 specimens are known.

Lasioglossum (Dialictus) julipile sp. nov. urn:lsid:zoobank.org:act:A8597CBD-1211-418D-99EE-1741EDB814E9

Figs 46-48, 90E, 94C, 117B

\section{Diagnosis}

Females of Lasioglossum julipile sp. nov. can be recognized by the characters for the Lasioglossum clematisellum species complex (below), in addition to most of the body with very extensive, dense tomentum, and scutum with densely plumose hair. They are most similar to L. pallidellum, L. clavicorne sp. nov., and L. clastipedion sp. nov. Females of L. pallidellum have the metapostnotum rounded with rugae not reaching the posterior margin. Females of L. clavicorne sp. nov. have the scutum with numerous thin and weakly plumose hairs and $\operatorname{are} \operatorname{larger}(5 \mathrm{~mm})$. Females of L. clastipedion sp. nov. have the head and mesosoma slightly duller and more densely punctate $(i \leq 2 \mathrm{pd})$.

Males of L. julipile sp. nov. can be recognized by the characters for the Lasioglossum clematisellum species complex (below), in addition to the frons with distinctly separated punctures $(i \leq 1 \mathrm{pd})$, propodeum lateral face rugulose, T2-4 with basolateral tomentum, and mesosoma with all hair densely plumose, appearing thickened. They are most similar to L. clematisellum, L. perditum sp. nov., and L. clastipedion sp. nov. Males of L. clematisellum and L. clastipedion sp. nov. have the frons slightly duller and more densely punctate $(\mathrm{i}<1 \mathrm{pd})$. Males of L. clematisellum also have the scutum with most hairs weakly plumose and T2-4 without basolateral tomentum. Males of $L$. perditum sp. nov. have the frons more sparsely punctate $(\mathrm{i}=1-3 \mathrm{pd})$ and propodeum lateral face smooth and tessellate.

\section{Etymology}

The specific epithet julipile is formed from the Latin noun 'iulus' ('catkin') and the adjective 'pile' ('haired'). It refers to the densely plumose hair of this species, which resembles willow catkins. An appropriate translation would be the catkin-haired sweat bee. 


\section{Material examined}

Holotype

UNITED STATES - Arizona • + ; Cochise Co., Junction of Moonlight and Blue Sky Roads; $32.2376^{\circ}$ N, $109.731^{\circ}$ W; 16 Sep. 2012; T.L. Griswold leg.; ex Wislizenia refracta; BBSL.

[Verbatim label: USA:AZ Cochise Co. Junction of Moonlight \& Blue Sky Roads S109.7310 E32.2376 16 Sep 2012 T.L. Griswold Wislizenia refracta / BBSL1028260 // BOLD / gard0113-AZ / DLIII157-19 // HOLOTYPE / Lasioglossum (Dialictus) julipile Gardner and Gibbs]

\section{Paratypes}

MEXICO - Chihuahua • 1 q; 12 mi. S of Samalayuca; [31.11 N, $106.48^{\circ} \mathrm{W}$ ]; 10 Jul. 1964; J. Powell leg.; ex Yucca; EMEC • 3 우; Villa Ahumada; [30.6 $\left.{ }^{\circ} \mathrm{N}, 106.5^{\circ} \mathrm{W}\right] ; 1128$ m a.s.1.; 28 Jul. 1953; SEMC.

UNITED STATES - Arizona $・ 7$ + $\circ$; Cochise Co., 1 mi. S of Willcox; $\left[32.23^{\circ} \mathrm{N}, 109.83^{\circ} \mathrm{W}\right] ; 7$ Aug. 1978; R.W. Brooks leg.; ex Wislizenia refracta; SEMC 1 क ; Cochise Co., 6 mi. S of Willcox; $\left[32.16^{\circ} \mathrm{N}\right.$, 109.83 ${ }^{\circ}$ W]; 23 Aug. 1986; R.J. McGinley and B.M. Norden leg.; USNM • 3 qo+; same collection data as for holotype; BBSL 3 우; Cochise Co., Willcox; $\left[32.25^{\circ} \mathrm{N}, 109.83^{\circ} \mathrm{W}\right]$; 19 Jun. 1969; R.M. Bohart leg.; UCDC • 6 우우 ; Cochise Co., 3.45 mi. SEbE of Willcox; $32.2262^{\circ} \mathrm{N}, 109.7822^{\circ} \mathrm{W}$; 1273 m a.s.l.; 1 Sep. 2009; T.L. Griswold leg.; BBSL 11 क; Snowflake; [34.51 ${ }^{\circ}$ N, $110.08^{\circ}$ W]; 25 Jul. 1956; Butler and Gerhardt leg.; UAIC 1 q; same location as for preceding; 25 Jul. 1956; Butler and Gerhardt leg.; ex Medicago sativa; UAIC. - New Mexico 2 o $o$; Hidalgo Co., 2 mi. N of Rodeo;

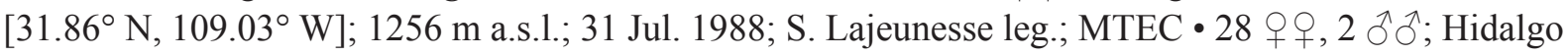
Co., 9 mi. N of Cotton City; [32.21 ${ }^{\circ} \mathrm{N}, 108.88^{\circ} \mathrm{W}$ ]; 11 Aug. 1978; R.W. Brooks leg.; ex Wislizenia refracta; SEMC - 1 +; Hidalgo Co., Antelope Corral, 6 mi. N of Rodeo; 31.9167-31.9333 N, 109109.0166 ${ }^{\circ}$ W; 1231 m a.s.1.; 25 Jun. 1984; H.A. Hespenheide leg.; ex Artemisia/Atriplex; LACM •

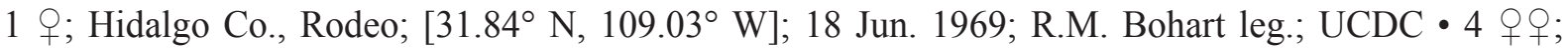
Hidalgo Co., Rodeo vicinity; [31.84 N, 109.03 W]; 11 Jun. 1987; B.N. Danforth leg.; ex Cirsium;

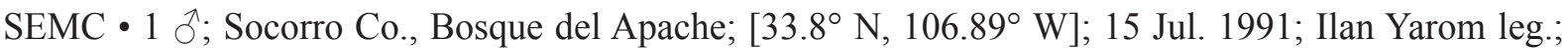
SEMC • 1 क; Socorro Co., Rio Salado, 5 mi. W of Interstate 25; [33.6 $\left.{ }^{\circ} \mathrm{N}, 107.1^{\circ} \mathrm{W}\right]$; $12 \mathrm{Jul} .1977$; W.

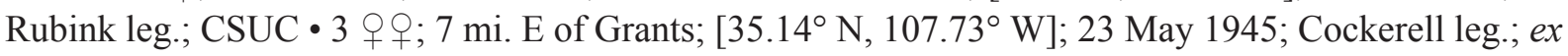
Fallugia paradoxa; UCRC $\bullet 1$ + ; same location as for preceding; 23 May 1945; W.P. Cockerell leg.; ex Fallugia paradoxa; UCRC • 2 + ; B Bernalillo; $\left[35.3^{\circ} \mathrm{N}, 106.55^{\circ} \mathrm{W}\right] ; 23$ Jul. 1950; R.H. Beamer leg.; ex Solanum; SEMC • 1 q; Clovis; [34.41 ${ }^{\circ}$ N, 103.21 ${ }^{\circ} \mathrm{W}$ ]; 24 Jun. 1964; Gerald and L. Nielsen leg.;

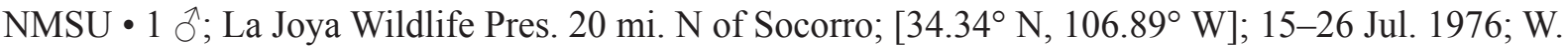

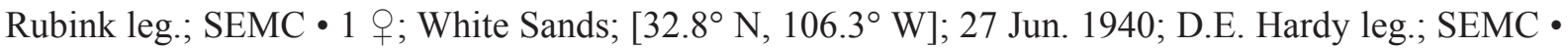
1 ; ; same location as for preceding; 30 Jun. 1932; R.H. Beamer leg.; SEMC • 1 q; White Sands National Monument; [32.8 $8^{\circ}$, $106.3^{\circ} \mathrm{W}$ ]; 16 Aug. 1967; R.M. Bohart leg.; UCDC • 1 क; same location as for preceding; 5 Aug. 1966; D.R. Miller leg.; UCDC • 1 क; same location as for preceding; 23 Aug. 1974; R.M. Bohart leg.; UCDC.

\section{Description}

\section{Female}

Colouration. Head and mesosoma blue-green to olive green; clypeus apical colour black, reddish brown, or orange; labrum orange; mandible orange with black basal spot and red tip; flagellum reddish brown dorsally, orange ventrally; pronotal lobe reddish brown to orange; metasoma orange with dark spiracular spots on T3-4; legs reddish brown with femur-tibia joints, tibia apices, and tarsi mostly orange; tegula pale amber; wing membrane hyaline, veins with subcosta dark brown, otherwise pale amber to light brown. 
Pubescence. Body hair colour white. Tomentum dense on paraocular area and gena, pronotal collar and lobe, space between pronotal lobe and tegula, metanotum anteriorly, metepisternum, T2 basolaterally, T3 throughout except medially, and T4 throughout; sparse on face below ocelli, preëpisternum, and mesepisternum. Scutum hair densely plumose. Wing hairs light, very short and sparse. Acarinarial fan complete or nearly so, sparse. T2 fringes sparse, T3 fringes sparse.

SuRfaCe SCULPTURE. Clypeus punctures large and irregularly sparse $(\mathrm{i}<2 \mathrm{pd})$, becoming smaller and denser in basal half ( $i \leq 1 \mathrm{pd})$, sculpture shiny, sometimes weakly imbricate basally; supraclypeal area punctures sparse ( $i=1-4 \mathrm{pd})$, sometimes becoming dense laterally $(i \leq 1 \mathrm{pd})$, diversopunctate, sculpture shiny; paraocular area punctures dense to moderately sparse $(i \leq 2 \mathrm{pd})$, sculpture shiny; frons punctures dense to moderately sparse ( $\mathrm{i} \leq 2 \mathrm{pd})$, sculpture shiny or weakly tessellate; vertex punctures moderately sparse ( $i=1-3 \mathrm{pd})$, sculpture shiny; gena punctures moderately sparse $(i=1-3 \mathrm{pd})$, sculpture shiny; postgena sculpture tessellate to weakly so; tegula punctures absent; scutum punctures moderately sparse ( $i=1-2 \mathrm{pd})$, becoming sparser submedially $(\mathrm{i}=1-3 \mathrm{pd})$ and denser posteromedially $(\mathrm{i} \leq 1 \mathrm{pd})$, sculpture shiny, becoming tessellate anteromedially; scutellum punctures moderately dense ( $\mathrm{i} \leq 2 \mathrm{pd})$, becoming sparser submedially ( $\mathrm{i}=1-3 \mathrm{pd}$ ) and denser posteromedially $(\mathrm{i}<1 \mathrm{pd})$, diversopunctate, sculpture shiny; metanotum sculpture shiny and finely, densely punctate $(\mathrm{i}<1 \mathrm{pd})$, becoming rugulose laterally; metapostnotum rugae strong, highly anastomosing, reaching margin, sculpture shiny to weakly imbricate; preëpisternum sculpture shiny with crowded punctures $(\mathrm{i}=0 \mathrm{pd})$ or areolate; hypoepimeron punctures dense ( $\mathrm{i} \leq 1 \mathrm{pd})$, sculpture shiny; mesepisternum punctures dense ( $\mathrm{i} \leq 1 \mathrm{pd})$, sculpture shiny; metepisternum sculpture ruguloso-lineate dorsally, tessellate and densely, obscurely punctate ventrally; propodeum lateral face sculpture tessellate; propodeum posterior face sculpture tessellate; T1 anterior face sculpture shiny; T1 dorsal surface punctures fine, sparse ( $i=1-4 \mathrm{pd}$ ), becoming finer or obscure on rim and absent in large apicolateral oval patches, sculpture shiny; T2 disc punctures moderately dense $(i=1-2 \mathrm{pd})$, disc sculpture shiny, rim punctures fine, sparse $(i=1-4 \mathrm{pd})$, rim sculpture shiny.

Structure. Face length/width ratio 0.81 ( $\pm 0.02 \mathrm{SD}$ ). Clypeus projecting $\sim 50 \%$ below suborbital tangent; clypeal area length/width ratio 0.42 ( $\pm 0.02 \mathrm{SD})$; apicolateral denticles low rounded knobs; supraclypeal area length/width ratio $0.68( \pm 0.06 \mathrm{SD})$. Forewing with 3 submarginal cells; pronotal angle obtuse; tegula shape normal. Intertegular distance 0.89 ( $\pm 0.06 \mathrm{SD}) \mathrm{mm}$. Scutum length/width ratio $0.75( \pm 0.06 \mathrm{SD}) ;$ scutum/scutellum length ratio $2.95( \pm 0.22 \mathrm{SD})$; scutellum/metanotum length ratio 1.49 ( $\pm 0.12 \mathrm{SD})$; metanotum/metapostnotum length ratio $0.63( \pm 0.04 \mathrm{SD})$. Propodeum lateral carinae not reaching dorsal margin; oblique carina very weak or absent. T2 depressed apical rim length about $50 \%$ of segment. $(n=11)$

\section{Male}

Colouration. Head and mesosoma blue to blue-green; clypeus apical colour reddish brown; labrum orange; mandible orange to yellow with brown basal spot and red tip; flagellum reddish brown dorsally, red to orange ventrally; pronotal lobe black, reddish brown, or orange; metasoma black to dark reddish brown with rims of terga and sterna broadly translucent reddish brown and downcurved lateral areas of terga becoming translucent orange; legs black to reddish brown with femur-tibia joints, base and apex of tibiae, and tarsi orange to yellow; tegula pale amber; wing membrane hyaline, veins with subcosta dark brown, otherwise brown to light brown.

Pubescence. Body hair colour white. Tomentum dense on face below ocelli, gena, pronotal angle and lobe, space between pronotal lobe and tegula, mesepisternum ventrally, and metepisternum; sparse on preëpisternum, mesepisternum dorsally, and T2-4 basolaterally. Scutum hair densely plumose. Sterna hair short (1-1.5 OD), densely plumose, dense and erect. Wing hairs light, short and sparse. 


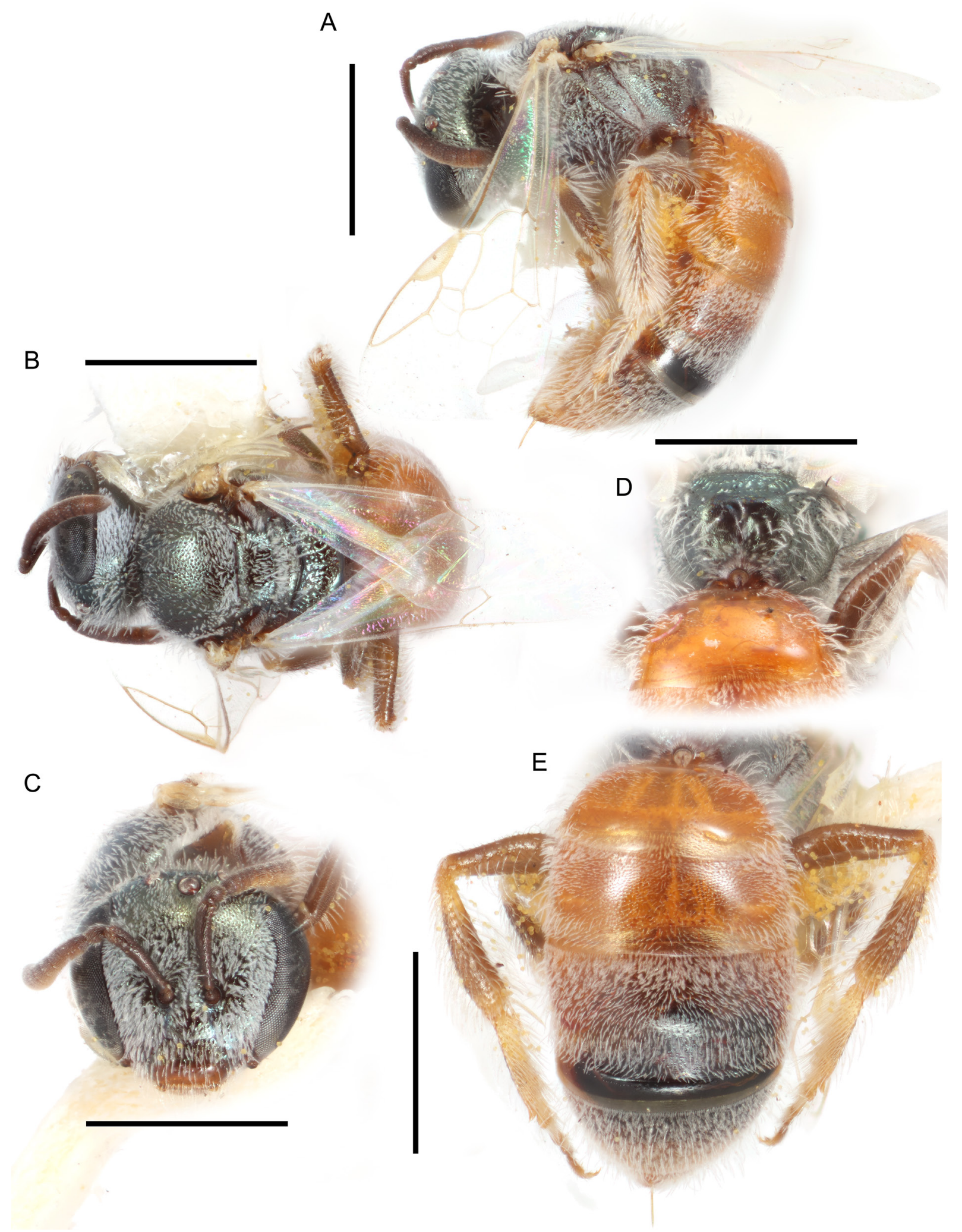

Fig. 46. Lasioglossum (D.) julipile sp. nov., + . A. Lateral habitus. B. Dorsal habitus. C. Face. D. Propodeum. E. Metasoma. Scale bars: $1 \mathrm{~mm}$. 


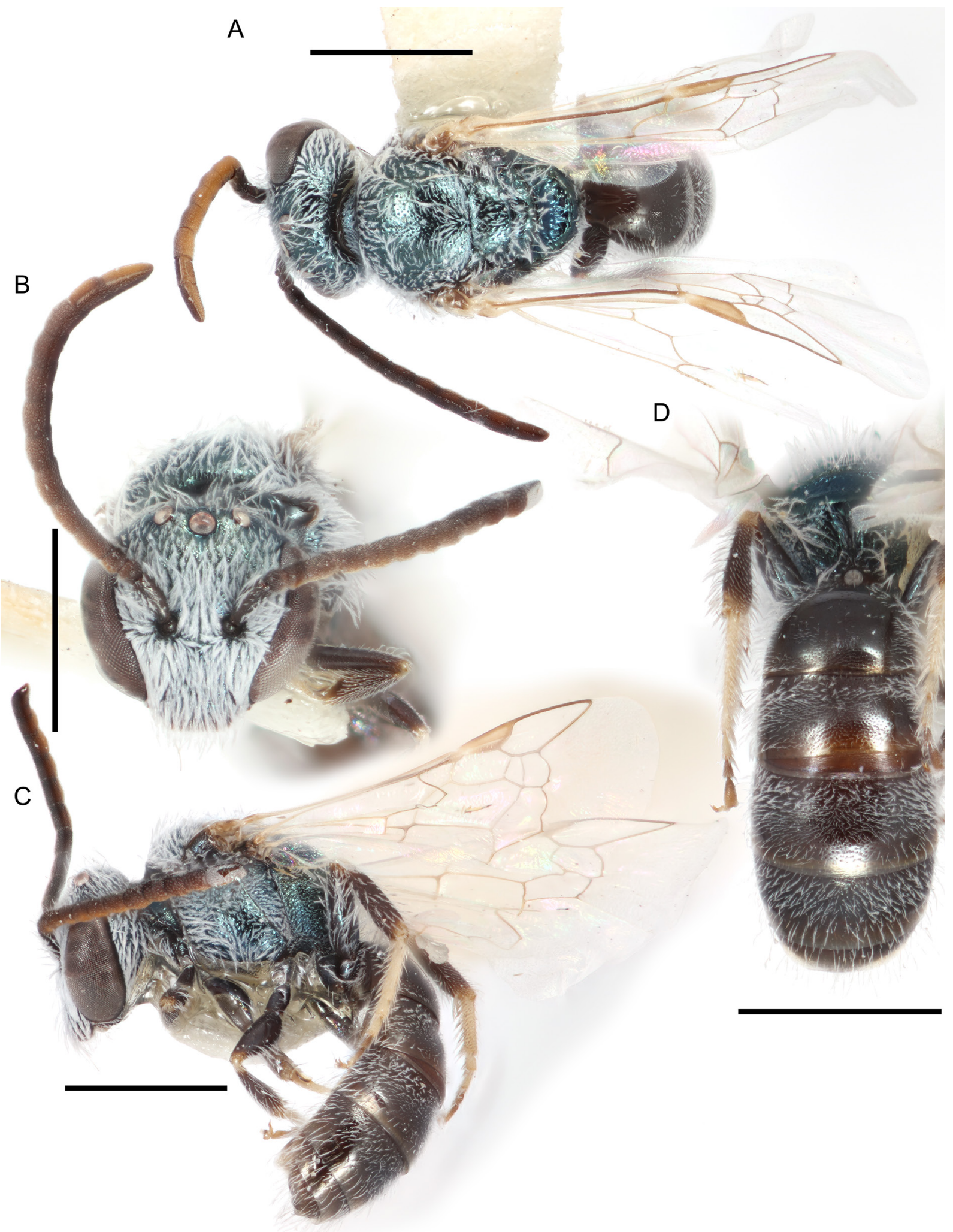

Fig. 47. Lasioglossum (D.) julipile sp. nov., ふૈ. A. Dorsal habitus. B. Face. C. Lateral habitus. D. Propodeum and metasoma. Scale bars: $1 \mathrm{~mm}$. 
SuRface SCUlPTURE. Clypeus punctures dense ( $i \leq 1 \mathrm{pd}$ ), sculpture shiny; supraclypeal area punctures dense ( $\mathrm{i} \leq 1 \mathrm{pd})$, sculpture shiny; paraocular area punctures dense $(\mathrm{i} \leq 1 \mathrm{pd})$, sculpture shiny; frons punctures dense $(\mathrm{i} \leq 1 \mathrm{pd})$, sculpture shiny; vertex punctures moderately dense laterally $(\mathrm{i}=1-2 \mathrm{pd})$, sparse medially ( $\mathrm{i}=1-3 \mathrm{pd}$ ), sculpture shiny; gena punctures moderately dense $(\mathrm{i}=1-2 \mathrm{pd})$, sculpture shiny; postgena sculpture shiny to weakly lineate; tegula punctures absent; scutum punctures moderately dense ( $i=1-2 \mathrm{pd}$ ), becoming sparse submedially ( $\mathrm{i}=1-3 \mathrm{pd})$, sculpture shiny; scutellum punctures moderately dense marginally and on median line $(i=1-2 \mathrm{pd})$, sparse submedially $(i=1-3 \mathrm{pd})$, sculpture shiny; metanotum sculpture shiny and moderately densely punctate $(\mathrm{i}=1-2 \mathrm{pd})$, becoming rugulose laterally; metapostnotum rugae strong, anastomosing, reaching margin, sculpture shiny; preëpisternum sculpture areolate; hypoepimeron punctures crowded ( $\mathrm{i}=0 \mathrm{pd}$ ), sculpture shiny; mesepisternum punctures dense $(\mathrm{i}<1 \mathrm{pd})$, diversopunctate, sculpture shiny; metepisternum sculpture lineate dorsally, areolate ventrally; propodeum lateral face punctures obscure, sculpture rugulose; propodeum posterior face sculpture shiny and sparsely punctate $(\mathrm{i}=1-3 \mathrm{pd})$; $\mathrm{T} 1$ anterior face sculpture shiny; $\mathrm{T} 1 \mathrm{dorsal}$ surface punctures dense $(\mathrm{i} \leq 1 \mathrm{pd})$, becoming sparse laterally and in small apicolateral oval patches $(\mathrm{i}=1-4 \mathrm{pd})$, sculpture shiny; $\mathrm{T} 2$ disc punctures dense ( $\mathrm{i} \leq 1 \mathrm{pd})$, becoming sparse laterally and in small apicolateral oval patches $(i=1-4 \mathrm{pd})$, disc sculpture shiny, rim punctures sparse medially $(i=1-4 \mathrm{pd})$, absent laterally, rim sculpture shiny.

StRUCture. Face length/width ratio 0.8 ( $\pm 0.04 \mathrm{SD})$. F1:pedicel length ratio $1.05( \pm 0.12 \mathrm{SD}) ; \mathrm{F} 2: \mathrm{F} 1$ length ratio $1.56( \pm 0.21 \mathrm{SD}) ; \mathrm{F} 2$ length/width ratio $1.2( \pm 0.18 \mathrm{SD}) ; \mathrm{F} 9$ length/width ratio $1.1( \pm 0.2 \mathrm{SD})$. Forewing with 2 or 3 submarginal cells; pronotal angle obtuse; tegula shape normal. Intertegular distance $0.84( \pm 0.04 \mathrm{SD}) \mathrm{mm}$. Scutum length/width ratio 0.79 ( $\pm 0.05 \mathrm{SD})$; scutum/scutellum length ratio 2.95

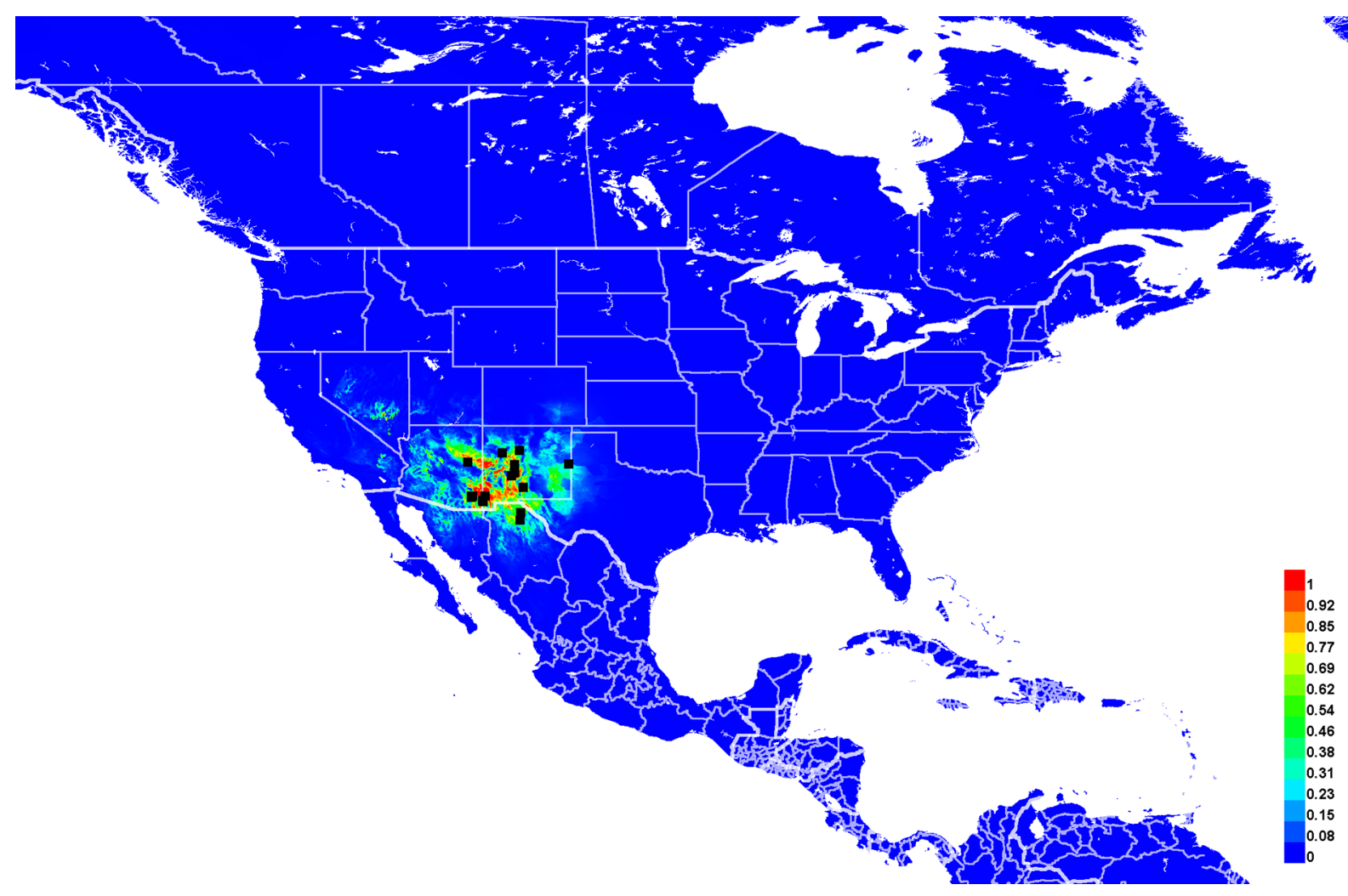

Fig. 48. Georeferenced collection records of Lasioglossum (D.) julipile sp. nov. (black squares) and predicted distribution by maximum entropy ecological niche modeling in Maxent (colour shading). Warmer colours indicate higher cloglog probability of occurrence. 
$( \pm 0.26 \mathrm{SD})$; scutellum/metanotum length ratio $1.51( \pm 0.36 \mathrm{SD})$; metanotum/metapostnotum length ratio $0.71( \pm 0.06 \mathrm{SD})$. Propodeum lateral carinae not reaching dorsal margin; oblique carina absent. $(n=4)$

Genitalia. As in Fig. 90E. Gonocoxite relatively narrow, rounded. Gonostylus broad, with a few long hairs. Retrorse lobe broad, spatulate, covered in sparse short hairs.

\section{Range}

Chihuahuan Desert of Arizona, New Mexico, and Chihuahua, north to Albuquerque (Fig. 48).

\section{Floral records}

ASPARAGACEAE Juss. Yucca L. • ASTERACEAE Giseke: Cirsium • CLEOMACEAE Bercht. \& J. Presl: Wislizenia Engelm. W. refracta Engelm. - FABACEAE Juss. Medicago: M. sativa L. • ROSACEAE Juss. Fallugia Endl. F. paradoxa (D. Don) Endl. ex Torr. - SOLANACEAE Adans. Solanum L.

\section{DNA barcodes}

Two sequences available (BOLD process IDs: DLIII157-19, DLIII158-19; BIN: BOLD:AEA0802). Lasioglossum julipile sp. nov. differs from L. clastipedion sp. nov. and L. clematisellum by 6 fixed substitutions: 54(T), 270(C), 304(A), 387(C), 495(C), and 541(C) (Supplementary file 4). No unique fixed substitutions distinguish L. julipile sp. nov. from all other western red-tailed L. (Dialictus).

Lasioglossum (Dialictus) kunzei (Cockerell, 1898)

Figs 49-50, 51(left), 95A, 109A

Halictus Kunzei Cockerell, 1898a: 238 (holotype,, , depository unknown).

Halictus (Chloralictus) kunzei - Sandhouse 1924: 4 (key).

Lasioglossum (Chloralictus) kunzei - Michener 1951: 1114 (catalog).

Dialictus kunzei - Hurd 1979: 1967 (catalog). — Moure \& Hurd 1987: 109 (catalog).

\section{Diagnosis}

Females of Lasioglossum kunzei are easily recognized by the head and mesosoma shiny and very finely, sparsely punctate $(i=1-6 \mathrm{pd})$, metapostnotum smooth and shiny, pronotal ridge strongly carinate, and head often large with mandibles elongate and gena with a ventral tubercle. They resemble many socially parasitic $L$. (Dialictus) in these characters, but the well-developed scopa on the hind legs readily identifies L. kunzei as a pollen-collecting species. They are extremely similar to Lasioglossum semibrunneum (Cockerell, 1895) and may be indistinguishable in some cases. Lasioglossum kunzei has a red, nonmetallic metasoma (black with metallic green reflections in L. semibrunneum), and the postgena tends to be duller and more extensively lineate (shiny with no microsculpture in L. semibrunneum).

Males of $L$. kunzei can be recognized by the same characters as for females, except the characters of the head. The head of male L. kunzei is unremarkable except that the compound eyes are only weakly convergent below. They are most similar to L. semibrunneum and may be indistinguishable in some cases. Males of $L$. kunzei have the discs of the metasomal terga black to brown without metallic reflections, and the rest of the metasoma orange (all brown to black with metallic green reflections in L. semibrunneum).

\section{Etymology}

Cockerell (1898a) dedicated this species to R.E. Kunzé, who collected the type specimen. 


\section{Material examined}

MEXICO - Baja California • 1 क; Mexicali; $\left[32.62^{\circ}\right.$ N, $115.45^{\circ}$ W]; 4 Oct. 1941; ex Sinapis alba; USNM.

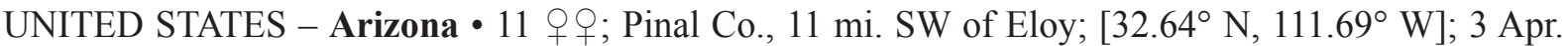
1954; T.R. Haig leg.; UCDC • 3 क $ᄋ$; same location as for preceding; 3 Apr. 1934; T.R. Haig leg.;

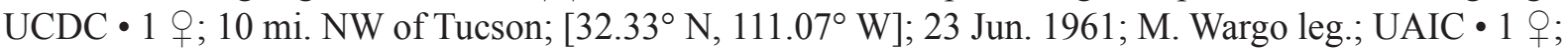

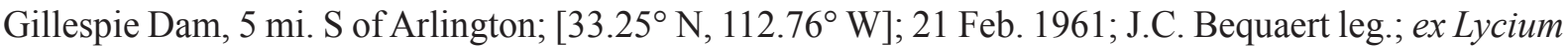

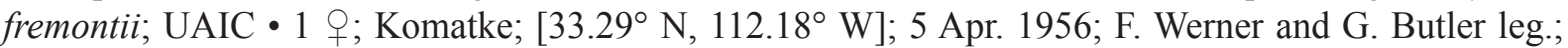
ex Medicago sativa; BBSL • 1 क; Phoenix; $\left[33.45^{\circ} \mathrm{N}, 112.07^{\circ} \mathrm{W}\right] ; 10$ Oct.; Cockerell leg.; ex pink oleander; USNM • 1 क; Tucson; [32.22 $\left.{ }^{\circ} \mathrm{N}, 110.97^{\circ} \mathrm{W}\right]$; 17 Jun. 1957; G.D. Butler leg.; ex Zea mays; UAIC • 3 우; Wellton; [32.67 ${ }^{\circ}$ N, $114.15^{\circ} \mathrm{W}$ ]; 4 Apr. 1956; F. Werner and G. Butler leg.; ex Medicago sativa; UAIC • 3 우; same location as for preceding; 4 Apr. 1956; F. Werner and G. Butler leg.; ex yellow composite; UAIC • 8 क $\circ$; USNM. - California - 1 q; Fresno Co., 20 mi. W of Fireburgh; $\left[36.85^{\circ} \mathrm{N}, 120.82^{\circ} \mathrm{W}\right]$; 6 Jun. 1972; P. Torchio leg.; BBSL • 10 오; Imperial Co., $10 \mathrm{mi} . \mathrm{NW}$ of

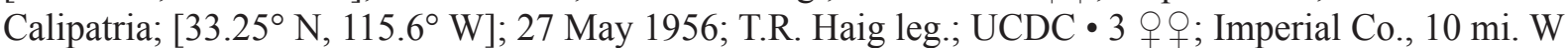
of Calipatria; [33.12 $2^{\circ} \mathrm{N}, 115.69^{\circ} \mathrm{W}$; 27 May 1956; T.R. Haig leg.; UCDC • 1 ते; same location as for preceding; 23 May 1956; T. R. Haig leg.; UCDC • 3 o ; Imperial Co., 12 mi. E of Heber; $\left[32.73^{\circ} \mathrm{N}\right.$, $115.32^{\circ}$ W]; 15 May 1956; T.R. Haig leg.; UCDC • 6 우; Imperial Co., 5 mi. E of Brawley; [32.98 ${ }^{\circ}$, $\left.115.44^{\circ} \mathrm{W}\right] ; 24$ May 1956; T.R. Haig leg.; UCDC • 2 우; same location as for preceding; 24 May 1956; T.R. Haig leg.; UCDC • 1 q ; Imperial Co., 8 mi. E of Brawley; $\left[32.98^{\circ} \mathrm{N}, 115.39^{\circ} \mathrm{W}\right] ; 24$ May 1956; T.R. Haig leg.; UCDC • 1 \%; same location as for preceding; 26 May 1956; T.R. Haig leg.; UCDC • 1 क; Imperial Co., Coachella Canal Road $11.6 \mathrm{~km}$ NW of CA Hwy 78, then $1.0 \mathrm{~km} \mathrm{NE} ; 33.063^{\circ} \mathrm{N}$, 115.25 W; 30 May-3 Jun. 2008; UCDC • 1 क; Imperial Co., Grays Well Road 3.0 km W of Buttercup Ranger Station; $32.72303^{\circ} \mathrm{N}, 114.90918^{\circ} \mathrm{W}$; $18-22$ Sep. 2008; UCDC • 1 क; Imperial Co., Niland; [33.24 $\left.{ }^{\circ} \mathrm{N}, 115.52^{\circ} \mathrm{W}\right]$; 18 Mar. 1956; T.R. Haig leg.; UCDC • 1 \%; same location as for preceding; 22 May 1956; T.R. Haig leg.; UCDC • 1 क; Imperial Co., Palo Verde; $\left[33.43^{\circ} \mathrm{N}, 114.73^{\circ} \mathrm{W}\right] ; 1$ Apr. 1968 ; R.O. Schuster leg.; UCDC • 2 $q+$; same location as for preceding; 11 Oct. 1972; C. Goodpasture leg.; $\mathrm{UCDC} \bullet 1$ q; same location as for preceding; 10 Oct. 1971; C. Goodpasture leg.; UCDC • 1 q; same location as for preceding; 21 Mar. 1967; R.M. Bohart leg.; UCDC • 1 q; Imperial Co., Roadrunner Campground, Gecko Road 9.8 km SE of CA Hwy 78; $32.9079^{\circ} \mathrm{N}, 115.11664^{\circ} \mathrm{W} ; 31$ May-3 Jun. 2008; UCDC • 1 क; same location as for preceding; 31 May-3 Jun. 2008; UCDC • 1 q; Imperial Co., Wash Road $11.3 \mathrm{~km} \mathrm{SE}$ of CA Hwy 78, then $1.0 \mathrm{~km} \mathrm{SW}$; $32.92347^{\circ} \mathrm{N}, 114.99039^{\circ} \mathrm{W}$; 9-18 Oct. 2014;

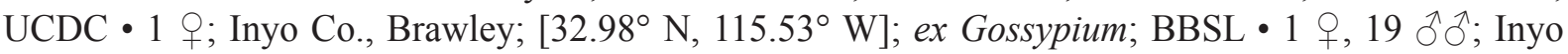
Co., China Ranch, 5 mi. SE of Tecopa; $35.79994^{\circ}$ N, 116.19547 W; 8 Nov. 2016; Paul A. Rude leg.;

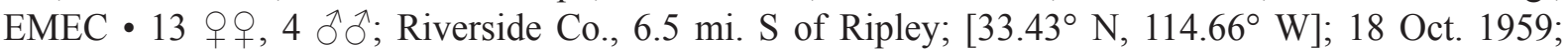
J.W. MacSwain leg.; ex Sesuvium; EMEC • 1 đ; Riverside Co., E Salton Sea, Hwy 111, 28 km NW of Calipatria; [33.34 ${ }^{\circ} \mathrm{N}, 115.64^{\circ} \mathrm{W}$ ]; 17 Jul. 2010; R.E. Wrigley leg.; WRME • 1 क; Riverside Co., Indio; [33.72 $\mathrm{N}, 116.22^{\circ} \mathrm{W}$; 11 Mar. 1957; E.I. Schlinger leg.; UCDC • 1 o; San Bernardino Co., 9 air mi. S of Baker, Zzyzx Springs; [35.14 N, 116.1 ${ }^{\circ} \mathrm{W}$ ]; 22 Apr. 1977; Powell leg.; ex Cleomella obtusifolia; EMEC • 1 क; San Bernardino Co., Salt Springs, Death Valley; [36.5 N, 116.9 W]; 24 Mar. 1959; C.L. Hogue leg.; LACM • 1 क ; San Bernardino Co., Zzyzx Springs, 9 mi. S of Baker; [35.14 N,

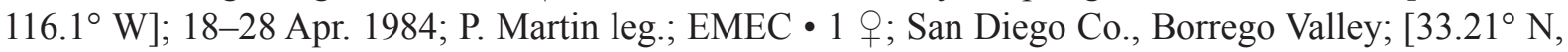
116.23 W]; 11 Apr. 1969; T.R. Haig leg.; UCDC. - Nevada • 3 + 9 ; Clark Co., 0.5 mi. SW of Glendale; $36.6602^{\circ} \mathrm{N}, 114.5781^{\circ} \mathrm{W} ; 464 \mathrm{~m}$ a.s.1.; 22 Apr. 2004; E. Ahlstrom leg.; ex Prosopis glandulosa var. torreyana; BBSL - 1 क; same location as for preceding; 22 Apr. 2004; S. Higbee leg.; ex Prosopis glandulosa var. torreyana; BBSL • 1 क; Clark Co., 1 mi. $\mathrm{N}$ of Warm Springs Rd., $8.5 \mathrm{mi} . \mathrm{N}$ of Glendale on HWY 168; $36.7182^{\circ} \mathrm{N}, 114.6913^{\circ} \mathrm{W} ; 2$ May 1997; F. Parker and V. Tepedino leg.; ex Prosopis; BBSL 1 क ; Carson Sink, 30 mi. NE of Fallen; [39.8 N, 118.42 ${ }^{\circ}$ W]; 24 Aug. 1969; R.M. Peterman leg.; $\mathrm{UCDC} \bullet 1$ \&; same location as for preceding; 27 Aug. 1969; R.M. Peterman leg.; UCDC. - Utah • 1 क; 


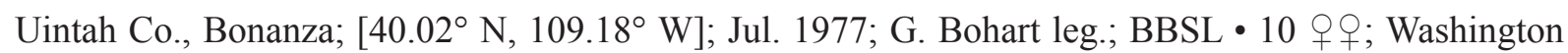
Co., Santa Clara; $\left[37.13^{\circ}\right.$ N, $113.65^{\circ}$ W]; 15 Apr. 1962; G.F. Knowlton leg.; SEMC • 30 q ; Washington Co., St. George; [37. $1^{\circ}$ N, $113.57^{\circ} \mathrm{W}$ ]; 14 Apr. 1962; G.F. Knowlton leg.; ex Tamarix; SEMC • 14 우; Washington Co., Washington; [37.13 N, 113.51 ${ }^{\circ} \mathrm{W}$ ]; 15 Apr. 1962; G.F. Knowlton leg.; ex Tamarix sp.; SEMC • 1 क ; Myton; [40.19 ${ }^{\circ}$ N, $110.06^{\circ}$ W]; 19 Sep. 1958; Geo. E. Bohart and Wm. P. Nye leg.; BBSL • 1 †; Myton, Nomia melanderi Cockerell, 1906 nesting site; [40.19 $\left.{ }^{\circ} \mathrm{N}, 110.06^{\circ} \mathrm{W}\right] ; 18$ May 1962; G.E.

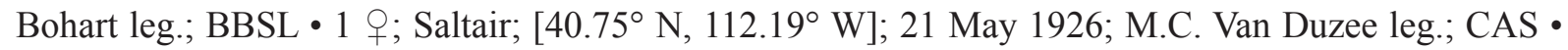

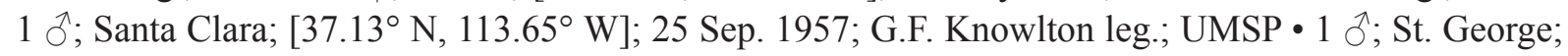
$\left[37.1^{\circ} \mathrm{N}, 113.57^{\circ} \mathrm{W}\right] ; 9$ Sep. 1954; G.F. Knowlton leg.; SEMC.

\section{Redescription}

\section{Female}

Colouration. Head and mesosoma blue-green; clypeus apical colour reddish brown to orange; labrum reddish brown to orange; mandible orange with black basal spot and red tip; flagellum reddish brown dorsally, orange ventrally; pronotal lobe reddish brown to orange; metasoma orange to reddish brown with basal half of T1 often reddish brown and discs of T4-5 often black, sometimes weakly metallic green, and dark spiracular spots on T2-4; legs mostly dark reddish brown to mostly orange; tegula pale amber to brown; wing membrane hyaline, veins with subcosta dark brown, otherwise light brown.

Pubescence. Body hair colour white. Tomentum dense on pronotal collar and lobe, space between pronotal lobe and tegula, axilla, scutum anterolateral margin, metanotum, preëpisternum, metepisternum, T2-3 basolaterally, and T4 throughout; sparse on paraocular area and gena. Scutum hair thin. Wing hairs dark, short and dense. Acarinarial fan absent or sparse and incomplete with narrow gap. T2 fringes dense, T3 fringes dense.

SURFACE SCULPTURE. Clypeus punctures sparse ( $\mathrm{i}=1-4 \mathrm{pd}$ ), sculpture shiny; supraclypeal area punctures dense marginally $(i \leq 1 \mathrm{pd})$, sparse medially $(\mathrm{i}=1-3 \mathrm{pd})$, sculpture shiny; paraocular area punctures dense dorsally $(\mathrm{i}<1 \mathrm{pd})$, becoming moderately sparse ventrally $(\mathrm{i}=1-2 \mathrm{pd}$ ), sculpture shiny; frons punctures dense ( $\mathrm{i}<1 \mathrm{pd})$, sculpture shiny; vertex punctures moderately dense ( $\mathrm{i}=1-2 \mathrm{pd})$, sculpture shiny; gena punctures moderately dense ( $\mathrm{i}=1-2 \mathrm{pd}$ ), often obscure, sculpture ruguloso-lineate; postgena sculpture ruguloso-lineate to tessellate; tegula punctures absent; scutum punctures sparse ( $\mathrm{i}=1-6 \mathrm{pd}$ ), sculpture shiny; scutellum punctures dense in narrow marginal band and median line $(\mathrm{i}<1 \mathrm{pd})$, otherwise impunctate, sculpture shiny; metanotum sculpture minutely, densely punctate ( $i \leq 1 \mathrm{pd})$; metapostnotum rugae absent or very short, not reaching halfway to margin, sculpture tessellate; preëpisternum sculpture shiny with crowded punctures $(i=0 \mathrm{pd})$; hypoepimeron punctures moderately dense to sparse ( $\mathrm{i}=1-2 \mathrm{pd})$, sculpture shiny; mesepisternum punctures moderately dense to sparse ( $\mathrm{i}=1-2 \mathrm{pd})$, sculpture shiny; metepisternum sculpture ruguloso-lineate dorsally, imbricate ventrally; propodeum lateral face sculpture imbricate on anterior margin, otherwise tessellate; propodeum posterior face sculpture tessellate; T1 anterior face sculpture shiny; $\mathrm{T} 1$ dorsal surface punctures minute, sparse $(\mathrm{i}=1-3 \mathrm{pd})$, becoming impunctate medially and apically, sculpture shiny; T2 disc punctures dense and distinct ( $\mathrm{i} \leq 1 \mathrm{pd}$ ) to minute and sparse $(i=1-4 \mathrm{pd})$, disc sculpture shiny, rim punctures minute and sparse $(i=1-3 \mathrm{pd})$ or absent, rim sculpture shiny.

StRUCtuRe. Face length/width ratio $0.78( \pm 0.04 \mathrm{SD})$. Clypeus projecting weakly or strongly below suborbital tangent; clypeal area length/width ratio 0.32 ( $\pm 0.04 \mathrm{SD})$; apicolateral denticles normal; supraclypeal area length/width ratio $0.65( \pm 0.07 \mathrm{SD})$. Gena often with ventral tubercle. Forewing with 3 submarginal cells; pronotal angle acute, forming strong dorsoventral ridge; tegula shape normal. Intertegular distance $0.93( \pm 0.06 \mathrm{SD}) \mathrm{mm}$. Scutum length/width ratio 0.77 ( $\pm 0.04 \mathrm{SD})$; scutum/ scutellum length ratio 2.7 ( $\pm 0.18 \mathrm{SD})$; scutellum/metanotum length ratio 1.61 ( $\pm 0.14 \mathrm{SD})$; metanotum/ 


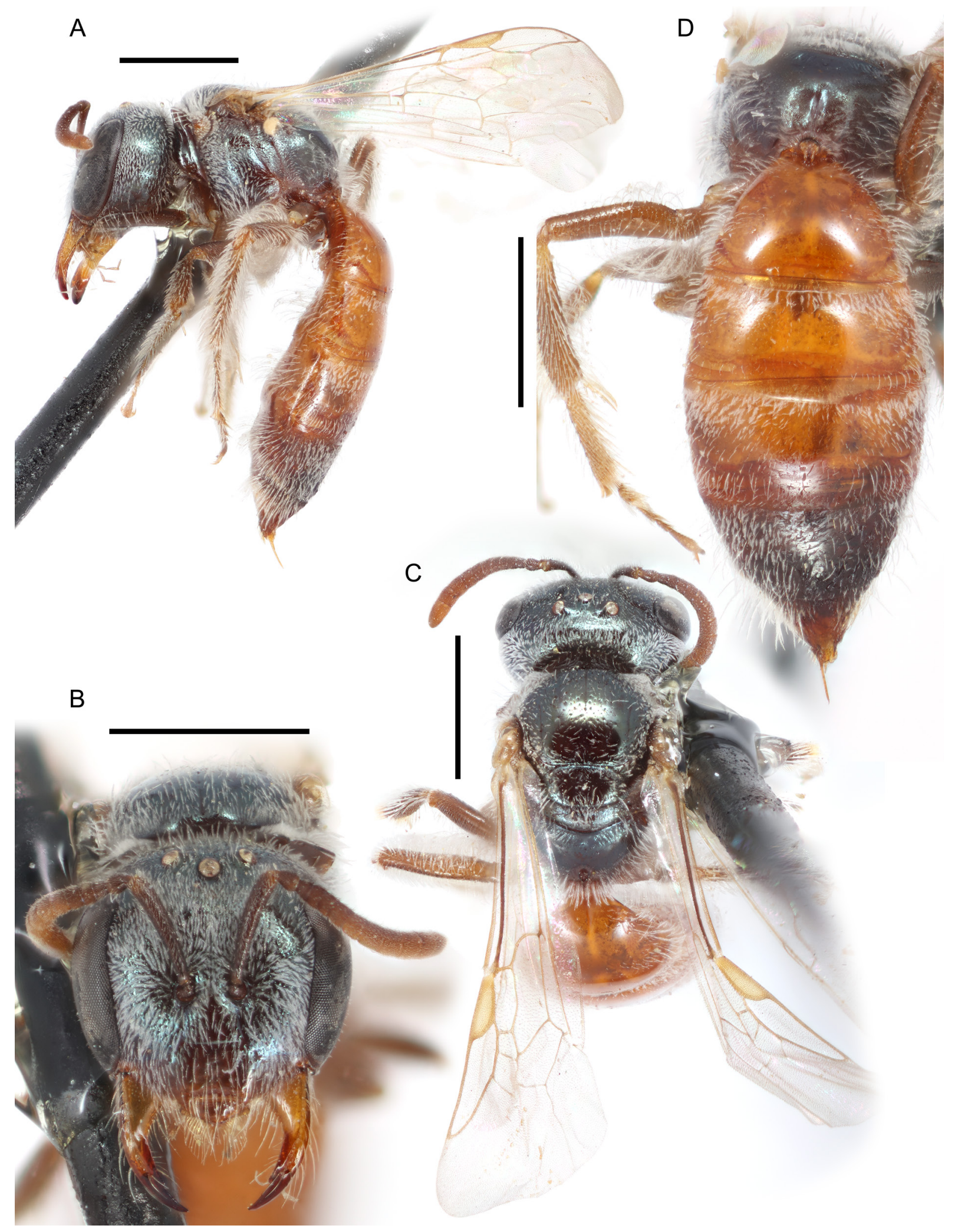

Fig. 49. Lasioglossum (D.) kunzei (Cockerell, 1898), ․ A. Lateral habitus. B. Face. C. Dorsal habitus. D. Propodeum and metasoma. Scale bars: $1 \mathrm{~mm}$. 


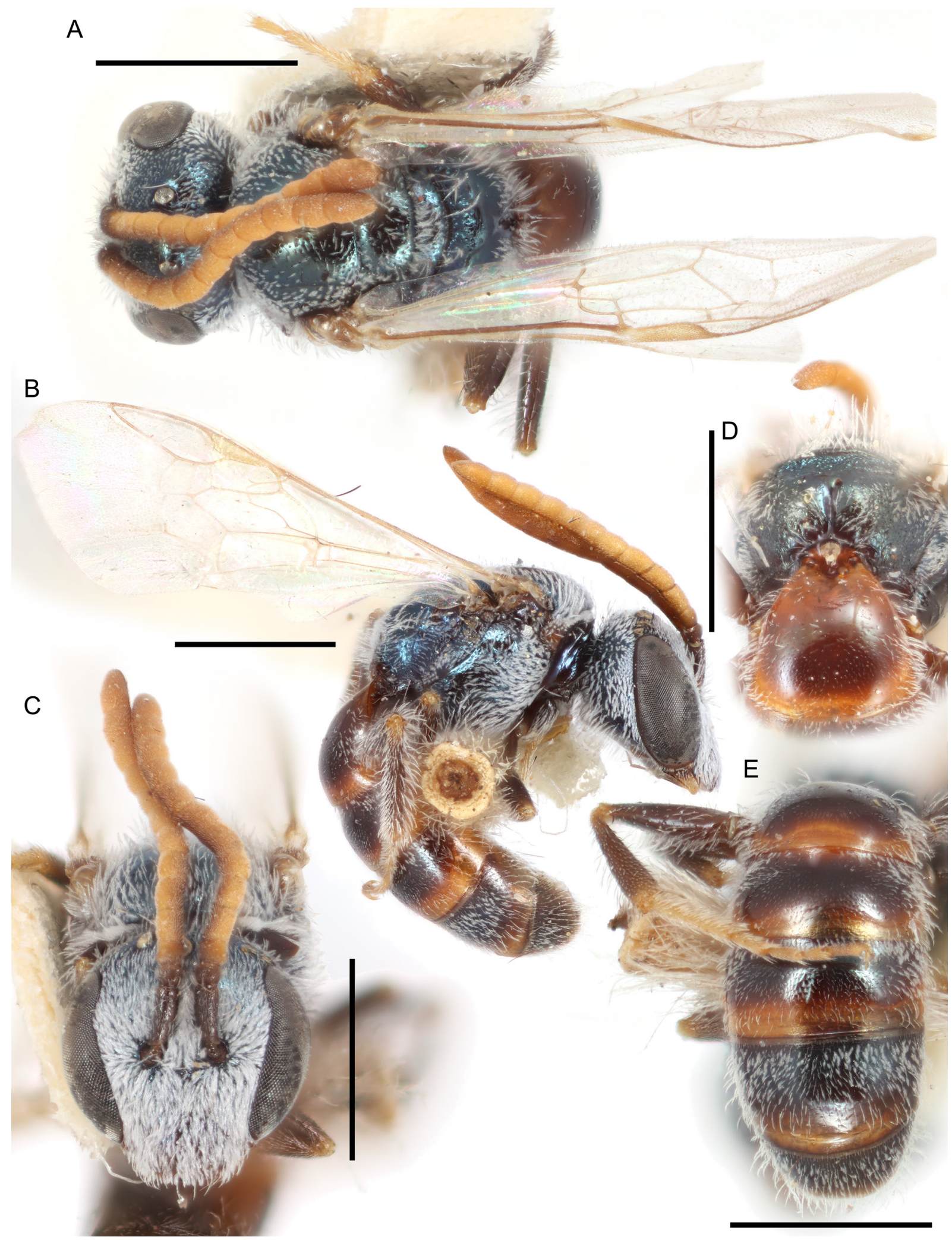

Fig. 50. Lasioglossum (D.) kunzei (Cockerell, 1898), đ̃. A. Dorsal habitus. B. Lateral habitus. C. Face. D. Propodeum. E. Metasoma. Scale bars: $1 \mathrm{~mm}$. 
metapostnotum length ratio $0.72( \pm 0.11 \mathrm{SD})$. Propodeum lateral carinae not reaching dorsal margin; oblique carina absent. T2 depressed apical rim length $50 \%$ or less of segment. $(n=10)$

\section{Male}

Colouration. Head and mesosoma blue to olive green; clypeus apical colour black to reddish brown; labrum reddish brown; mandible yellow with black basal spot and red tip; flagellum reddish brown dorsally, yellow-orange ventrally; pronotal lobe reddish brown; metasoma black to orange, sometimes weakly metallic green, with dark spiracular spots on T2-4; legs reddish brown with femur-tibia joints, base and apex of tibiae, and tarsi orange; tegula orange; wing membrane hyaline, veins with subcosta dark brown, otherwise pale amber to brown.

Pubescence. Body hair colour white. Tomentum dense on face below ocelli, gena anteriorly, pronotal angle, preëpisternum, and metepisternum; sparse on gena posteriorly, pronotal lobe, mesepisternum, T1 laterally, T2-4 basolaterally, and T5 throughout. Scutum hair thin to densely plumose. Sterna hair short (0.5-1.25 OD), moderately plumose, sparse and erect. Wing hairs dark or light, short and dense.

SuRFACE SCULPTURE. Clypeus punctures irregularly sparse ( $\mathrm{i} \leq 2 \mathrm{pd}$ ), sculpture shiny; supraclypeal area punctures dense $(i<1 \mathrm{pd})$, sculpture shiny; paraocular area punctures dense $(\mathrm{i}<1 \mathrm{pd})$, sculpture shiny; frons punctures moderately dense $(\mathrm{i}=1-2 \mathrm{pd})$, sculpture shiny; vertex punctures moderately sparse ( $i=1-2 \mathrm{pd})$, sculpture shiny; gena punctures sparse $(i=1-3 \mathrm{pd})$, sculpture shiny; postgena sculpture shiny, becoming weakly tessellate to weakly lineate laterad of hypostomal carina; tegula punctures absent; scutum punctures sparse ( $\mathrm{i}=1-6 \mathrm{pd}$ ), sculpture shiny; scutellum punctures dense marginally and on median line ( $\mathrm{i} \leq 1 \mathrm{pd}$ ), sparse submedially ( $\mathrm{i}=1-6 \mathrm{pd}$ ), sculpture shiny; metanotum sculpture shiny and sparsely punctate ( $\mathrm{i}=1-3 \mathrm{pd})$; metapostnotum rugae weak, anastomosing, not reaching margin, sculpture shiny to imbricate; preëpisternum sculpture areolate-rugulose; hypoepimeron punctures moderately dense ( $\mathrm{i}=1-2 \mathrm{pd})$, sculpture shiny; mesepisternum punctures sparse $(\mathrm{i}=1-3 \mathrm{pd})$, sculpture shiny; metepisternum sculpture ruguloso-lineate dorsally, smooth and shiny ventrally; propodeum lateral face punctures sparse $(\mathrm{i}=1-3 \mathrm{pd})$, sculpture shiny to weakly rugulose; propodeum posterior face sculpture shiny and sparsely punctate $(\mathrm{i}=1-3 \mathrm{pd})$, becoming ruguloso-punctate laterally; $\mathrm{T} 1$ anterior face sculpture shiny; T1 dorsal surface punctures minute, sparse $(i=2-4 \mathrm{pd})$, very sparse or absent in large apicolateral oval patches and on rim, sculpture shiny; T2 disc punctures minute, sparse $(i=2-4 \mathrm{pd})$, disc sculpture shiny, rim punctures minute, very sparse $(i=2-6 \mathrm{pd})$, absent laterally, rim sculpture shiny.

Structure. Face length/width ratio 0.87 ( $\pm 0.01 \mathrm{SD})$. F1:pedicel length ratio 1.27 ( $\pm 0.05 \mathrm{SD}) ; \mathrm{F} 2: \mathrm{F} 1$ length ratio $1.31( \pm 0.1 \mathrm{SD}) ; \mathrm{F} 2$ length/width ratio $1.23( \pm 0.09 \mathrm{SD}) ; \mathrm{F} 9$ length/width ratio 1.27 $( \pm 0.11 \mathrm{SD})$. Forewing with 3 submarginal cells; pronotal angle acute, forming strong dorsoventral ridge; tegula shape normal. Intertegular distance 0.8 ( $\pm 0.03 \mathrm{SD}) \mathrm{mm}$. Scutum length/width ratio 0.91 ( $\pm 0.04 \mathrm{SD}) ;$ scutum/scutellum length ratio 2.53 ( $\pm 0.19 \mathrm{SD}) ;$ scutellum/metanotum length ratio 1.92 $( \pm 0.39 \mathrm{SD}) ;$ metanotum/metapostnotum length ratio 0.74 ( $\pm 0.16 \mathrm{SD})$. Propodeum lateral carinae not reaching dorsal margin; oblique carina absent. $(n=3)$

Genitalia. Not examined.

\section{Range}

Baja California north to Nevada and east to Utah (Fig. 51A).

\section{Floral records}

AIZOACEAE Martinov: Sesuvium L. S. portulacastrum (L.) L. (MH87) • S. verrucosum Raf. (MH87) • AMARANTHACEAE Juss. Salsola L. S. kali L. (MH87) - Suaeda Forssk. ex J.F. Gmel. (MH87) • APOCYNACEAE Juss. Nerium L. N. oleander L. A ASTERACEAE Giseke: Baccharis: B. salicina Torr. \& 
A.Gray (MH87) - Dieteria: D. asteroides Torr. D. a. var. asteroides Torr. (MH87) - Pectis: P. papposa Harv. \& A.Gray (MH87) • BORAGINACEAE Juss. Heliotropium L. H. curassavicum L. (MH87) • BRASSICACEAE Burnett: Lepidium L. L. virginicum L. (MH87) • Sinapis L. S. alba L. CLEOMACEAE Bercht. \& J. Presl: Cleomella DC. C. obtusifolia Torr. \& Frém. - CONVOLVULACEAE Juss. Cressa L. C. truxillensis Kunth (MH87) - FABACEAE Juss. Medicago: M. sativa L. • Prosopis: P. glandulosa Torr. P. g. var. torreyana (L.D. Benson) M.C. Johnst. - MALVACEAE Juss. Gossypium L. • Malvella Jaub. \& Spach: M. leprosa (Ortega) Krapov. (MH87) - POACEAE Barnhart: Zea L. Z. mays L. • POLYGONACEAE Juss. Rumex L. (MH87) - SALICACEAE Mirb. Salix L. S. exigua Nutt. S. e. var. hindsiana (Benth.) Dorn (MH87) - SOLANACEAE Adans. Lycium L. L. brevipes Benth. (MH87) • L. fremontii A.Gray • TAMARICACEAE Link: Tamarix L.

\section{DNA barcodes}

One sequence available (BOLD process ID: DLIII121-18; BIN: BOLD:AAD0757). This sequence is unique, but poorly distinguished from L semibrunneum sequences (minimum p-distance $1.11 \%$, compared to maximum p-distance of $2.2 \%$ within L. semibrunneum). Lasioglossum kunzei differs from

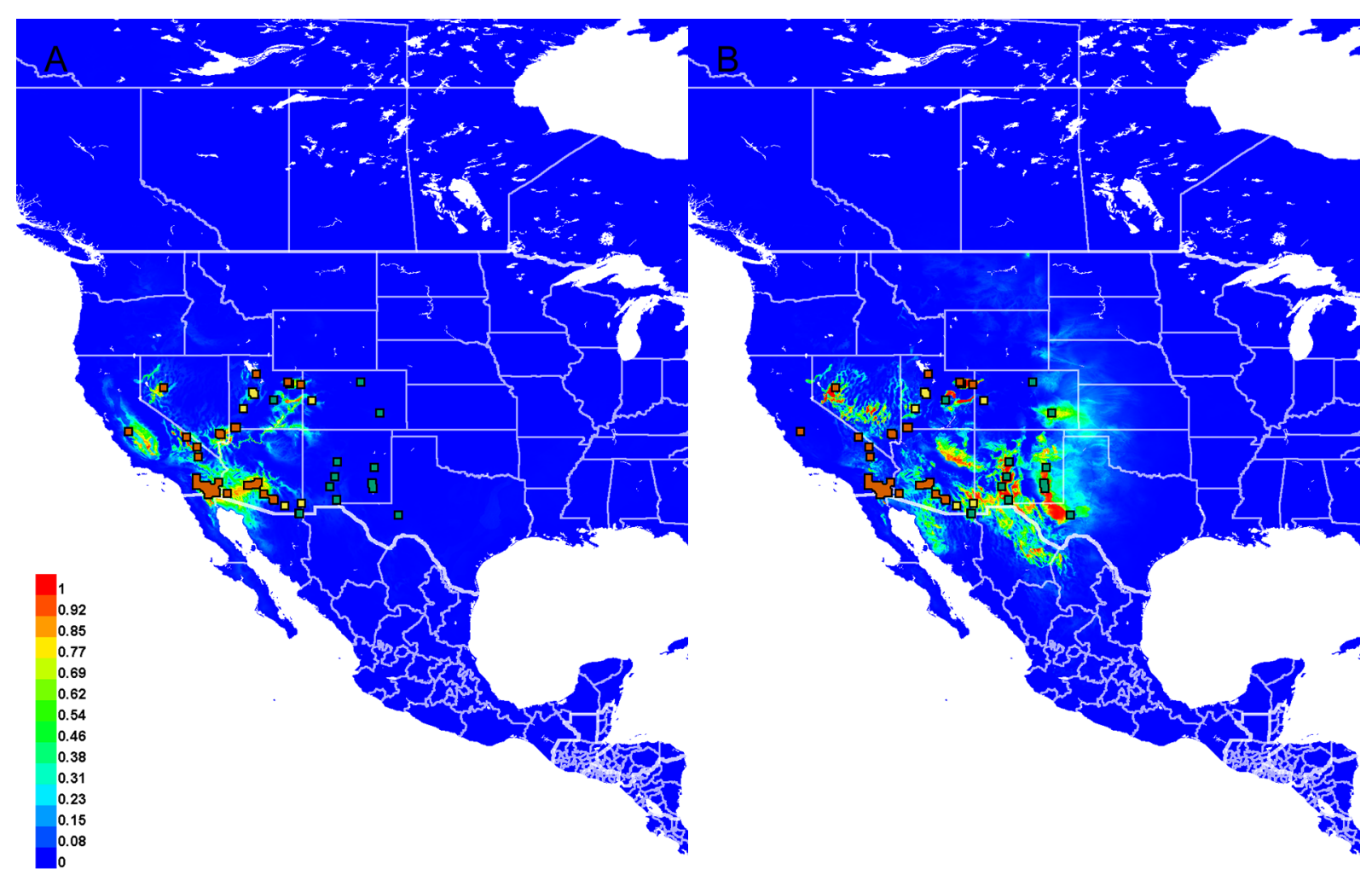

Fig. 51. Georeferenced collection records of Lasioglossum (D.) kunzei (Cockerell, 1898) (vermilion squares), L. (D.) semibrunneum (Cockerell, 1895) (blue-green squares) and intermediate specimens of uncertain identity (yellow squares) as well as predicted distribution by maximum entropy ecological niche modeling in Maxent (colour shading). Warmer colours indicate higher cloglog probability of occurrence. Maxent analysis was run separately for $L$. (D.) kunzei (left) and $L$. (D.) semibrunneum (right), but all georeferenced records for both species are shown on both maps to highlight distributional differences. Lasioglossum (D.) kunzei and L. (D.) semibrunneum have largely non-overlapping distributions, but are predicted to intermix in northern Utah, southern Arizona/northern Sonora, and northern Nevada (although no L. (D.) semibrunneum records are known from Nevada). 
all other western red-tailed L. (Dialictus) by 1 fixed substitution: 369(C). It differs from L. semibrunneum by an additional 4 fixed substitutions: 219(C), 222(A), 633(C), and 645(T) (Supplementary file 4).

\section{Remarks}

The location of the type specimen is unknown (Moure \& Hurd 1987), and was not found despite extensive searching by the authors. Cockerell (1898a) described L. kunzei from a single specimen given to him by C.F. Baker. The type was collected in Phoenix, Arizona on 27 May 1897. A series of specimens in USNM, with labels reading only "Ariz / 2340 // Collection CF Baker" (one specimen with 2572 instead of 2340) might constitute material from which the type was taken. However, none of these are likely to be the type itself, as Cockerell explicitly stated that he only had one specimen. Despite this difficulty, the numerous unique characters possessed by L. kunzei and referenced in the original description allow the name to be quite unambiguously associated.

Lasioglossum kunzei had been considered a likely junior synonym of L. semibrunneum. However, in light of the fairly distinct distributions of the two species west and east of the Rocky Mountains, respectively (Fig. 51A-B), it seems advisable to keep them separated. This may be a case of vicariant speciation in action.

The male is described here for the first time.

Lasioglossum (Dialictus) lilianae sp. nov. urn:1sid:zoobank.org:act:D94D4513-8282-4448-9849-3DECFF4F1384

Figs 52-54, 90F, 102B

\section{Diagnosis}

Females of Lasioglossum lilianae sp. nov. can be recognized by the face long (length/width ratio $\sim 0.84$ ), scutum very shiny and finely, sparsely punctate, especially laterally ( $\mathrm{i}=1-4 \mathrm{pd})$, mesepisternum densely and distinctly punctate $(\mathrm{i}<1 \mathrm{pd}), \mathrm{T} 1$ anterior surface shiny, tomentum very dense and abundant over most of the body, T2-4 with dark spiracular spots, ocelli slightly enlarged (separated by about 0.6 OD), and of relatively large size (ITD usually $>1 \mathrm{~mm}$ ). They are most similar to L. argammon sp. nov. and $L$. eophilus. Females of $L$. argammon sp. nov. have the scutum more densely punctate laterally ( $\mathrm{i} \leq 1 \mathrm{pd}$ ), T1 anterior surface coriarious, and are smaller (ITD usually $<1 \mathrm{~mm}$ ). Females of L. eophilus have the mesepisternum rugose, scutum densely punctate ( $i \leq 1 \mathrm{pd})$, and mesosoma with less extensive tomentum.

Males of L. lilianae sp. nov. can be recognized by the same characters as for females, in addition to the clypeus usually sparsely punctate $(\mathrm{i}=1-4 \mathrm{pd})$, flagellomeres relatively short (F2 about 1.5 times as long as F1 and 1.25 times as long as broad), and S5 apical margin straight. They are most similar to L. hudsoniellum and L. arenisaltans sp. nov. Males of L. hudsoniellum have S5 apical margin straight and scutum more densely punctate $(\mathrm{i}=1-2 \mathrm{pd})$. Males of $L$. arenisaltans $\mathrm{sp}$. nov. have the flagellomeres relatively longer (F2 about 1.75 times as long as F1 and 1.5 times as long as broad), scutum more densely punctate ( $\mathrm{i} \leq 3 \mathrm{pd}$ ), and mesosoma and metasoma with less extensive tomentum.

\section{Etymology}

The specific epithet lilianae is a dedication to Liliana Ramírez Freire, who collected many specimens from Nuevo Leon, and went above and beyond the call of duty to get them shipped to us. An appropriate translation would be Liliana's sweat bee. 


\section{Material examined}

Holotype

MEXICO - Sonora • + ; $30 \mathrm{~km}$ E of Agua Prieta; $31.3206^{\circ} \mathrm{N}, 109.2681^{\circ} \mathrm{W} ; 2$ Sep. 2003; A. Romero leg.; PCYU.

[Verbatim label: Mexico: Sonora / 30km E Agua Prieta / 31 ${ }^{\circ} 19^{\prime} 14^{\prime \prime} \mathrm{N} 109^{\circ} 16^{\prime} 05^{\prime \prime} \mathrm{W} / 02 \mathrm{Sept} 2003$, A Romero / ex. Flyellow bowl / Quarry; road cross, SBV036702 // 1849A10 // HOLOTYPE / Lasioglossum (Dialictus) lilianae Gardner and Gibbs]

\section{Paratypes}

MEXICO -Chihuahua • 1 + ; $6 \mathrm{~km} \mathrm{~N}$ of Samalayuca; [31.39 N, $106.48^{\circ} \mathrm{W}$ ]; 29 Aug. 1991; J.L. Neff leg.; ex Mentzelia multiflora; SEMC 11 क; $8 \mathrm{~km} \mathrm{~N}$ of Samalayuca; [31.41 $\left.{ }^{\circ} \mathrm{N}, 106.48^{\circ} \mathrm{W}\right]$; 29 Aug. 1991; T. Griswold leg.; ex Mentzelia multiflora; SEMC • 1 क ; $8 \mathrm{~km} \mathrm{~N}$ of Samalayuca; [31.41 $\left.{ }^{\circ} \mathrm{N}, 106.48^{\circ} \mathrm{W}\right] ; 29$ Aug. 1991; T. Griswold leg.; ex Mentzelia multiflora; SEMC • 1 क; $6 \mathrm{~km} \mathrm{~N}$ of Samalayuca; [31.39 N, $106.48^{\circ}$ W]; 29 Aug. 1991; J.L. Neff leg.; ex Mentzelia multiflora; SEMC. - Coahuila • 1 q; Gypsum dunes, $20 \mathrm{~km} \mathrm{~S}$ of Cuatrocienégas; [26.82 $\left.{ }^{\circ} \mathrm{N}, 102.15^{\circ} \mathrm{W}\right]$; 16 Feb. 2000; R.L. Minckley leg.; RLM. Nuevo Leon - 1 ; ; Camio al Espinazo, Mina; [26.2 ${ }^{\circ}$ N, 101.1 $\left.{ }^{\circ} \mathrm{W}\right]$; 703 m a.s.1.; 4 Oct. 2009; L. RamírezFreire leg.; LRF • 13 o of; Exhacienda del Muerto, Mina; [25.966 $\left.{ }^{\circ} \mathrm{N}, 100.647^{\circ} \mathrm{W}\right] ; 632 \mathrm{~m}$ a.s.1.; 25 Jul. 2009; L. Ramírez-Freire leg.; LRF. - Sonora • 1 \%; $30 \mathrm{~km}$ E of Agua Prieta; $31.32^{\circ} \mathrm{N}, 109.2717^{\circ} \mathrm{W}$; 4 Sep. 2000; R. Minckley leg.; ex Mentzelia aspera; PCYU • 1 § ; Municipio de Agua Prieta, Rancho Las Anitas, Shrub Site 4; $31.31389^{\circ}$ N, $109.31417^{\circ}$ W; 11 Aug. 2007; RLM • 1 O; Rancho Puerta Blanca, along road after main gate; $\left[31.31^{\circ} \mathrm{N}, 109.1^{\circ} \mathrm{W}\right] ; 14 \mathrm{Apr} .2007$; ex Mentzelia pumila; RLM • 1 ; Rancho San Bernardino, Agua Verde; $31.33068^{\circ}$ N, 109.21 ${ }^{\circ}$ W; 28 Jul. 2004; ex Malvaceae sp.?; RLM • 1 \&; Rancho San Bernardino, quarry, along Arroyo Grande; $31.3196^{\circ} \mathrm{N}, 109.27028^{\circ} \mathrm{W} ; 27$ May 2004; RLM • 1 q; Rancho San Bernardino, quarry, at road crossing; $31.32042^{\circ} \mathrm{N}, 109.2681^{\circ} \mathrm{W}$; 2 Sep. 2003; RLM - 1 क; Rancho San Bernardino, quarry, general area; $31.3195^{\circ} \mathrm{N}, 109.27028^{\circ} \mathrm{W}$; 17 Aug. 2001; ex Mentzelia aspera; RLM • 2 ㅇ; ; same location as for preceding; 14 Apr. 2002; ex Mentzelia pumila; RLM • 1 क; same location as for preceding; 27 May 2004; RLM 4 우; same location as for preceding; 13 Aug. 2007; ex Mentzelia pumila; RLM • 5 o ; ; Rancho San Bernardino, quarry, Tai Site $1 ; 31.3194^{\circ} \mathrm{N}, 109.27142^{\circ} \mathrm{W} ; 2$ Sep. 2003; RLM.

UNITED STATES - Arizona • 9 q $q$; Cochise Co., $3.2 \mathrm{~km}$ E of Apache; [31.69 N, 109.1 ${ }^{\circ} \mathrm{W}$ ]; $26 \mathrm{Jun}$. 1987; B.N. Danforth leg.; ex Mentzelia; SEMC • 1 \%; Cochise Co., Railroad Ave., 2 mi. SW of Willcox; $32.2172^{\circ} \mathrm{N}, 109.8544^{\circ} \mathrm{W} ; 1275 \mathrm{~m}$ a.s.l.; 31 Jul. 2005; D. Yanega leg.; ex ?Lepidium; UCRC • 1 \%; Cochise Co., San Bernardino NWR; [31.34 N, 109.26 W]; 9 Jun. 2002; R.L. Minckley leg.; RLM • 1 क; Cochise Co., San Bernardino NWR, field, nr Bathroom, Bee Diversity; $31.3411^{\circ}$ N, $109.27048^{\circ}$ W; 19 Jun. 2002; RLM • 2 q ; Cochise Co., San Bernardino NWR, Mesa de la Avanzada, Bee diversity; 31.34147 N, 109.27278 W; 19 Jun. 2002; RLM • 1 क; Cochise Co., San Bernardino NWR, Shrub, nr Bathroom, Bee Diversity; $31.34052^{\circ} \mathrm{N}, 109.27165^{\circ} \mathrm{W}$; 19 Jun. 2002; RLM. - California • 1 q; Tulare Co., Jackson Meadow; [37.495 ${ }^{\circ}$ N, $118.97^{\circ}$ W]; 27 Jun. 2000; J.S. Ascher leg.; AMNH. - Colorado •

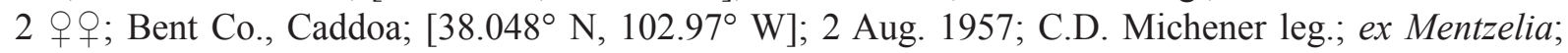
SEMC • 1 đ’; Las Animas Co., Model; $37.4702^{\circ}$ N, $103.9712^{\circ}$ W; 9 Jul. 2009; J. Newton leg.; PCYU. New Mexico 1 đ; ; Lincoln Co., 22 mi. NNE of Carrizozo; $33.8461^{\circ} \mathrm{N}, 105.7825^{\circ} \mathrm{W} ; 1730 \mathrm{~m}$ a.s.1.; 16 Sep. 1999; R. Brooks and C. Michener leg.; ex Grindelia squarrosa; SEMC • 2 우, 1 đo; Socorro Co., Sevilleta NWR; [34.3 N, 106.8 W]; 17 Jun.-1 Jul. 2003; K. Wetherill leg.; PCYU • 1 q; same location as for preceding; 12-26 Jul. 2005; K. Wetherill leg.; PCYU • 1 क; same location as for preceding; 10-24 Sep. 2002; K. Wetherill leg.; PCYU • 1 q; same location as for preceding; 26 Jul. 2001; K. Wetherill leg.; PCYU • 1 क; same location as for preceding; 5-19 Oct. 2004; K. Wetherill leg.; PCYU •

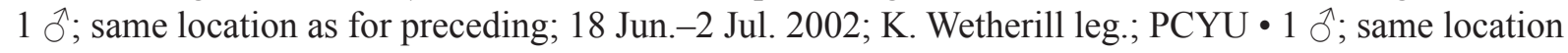
as for preceding; 15-29 Jul. 2003; K. Wetherill leg.; PCYU • 1 \&; Torrance Co., Town of Gran Quivira; 
[34.27 $\left.\mathrm{N}, 106.1^{\circ} \mathrm{W}\right]$; 9 Aug. 1965; Hugh B. Leech leg.; CAS • 2 q o ; Santa Fe; [35.69 $\left.{ }^{\circ} \mathrm{N}, 105.94^{\circ} \mathrm{W}\right]$; 19 Jul. 1952; R.H. and L.D. Beamer, LaBerge and C. Liang leg.; SEMC. - Texas - 1 q; Mitchell Co., Lake Colorado St. Park; [32.33 N, 100.93 W]; 26 Jul. 1976; ex Mentzelia nuda; LACM - 2 우; Mitchell Co., Tex. Rd. 670; [32.3 N, 101.0075 W]; 27 Jul. 1976; ex Mentzelia nuda; LACM • 1 +; Ward Co., 5.5 mi. E of Monahans; $31.605^{\circ} \mathrm{N}, 102.845^{\circ} \mathrm{W}$; 15 Jun. 2005; J. Neff and A. Hook leg.; ex Mentzelia strictissima; PCYU - 1 ô; same location as for preceding; 15 Jun. 2005; J. Neff and A. Hook leg.; ex Mentzelia strictissima; PCYU. - Utah • 1 q; Emery Co., South Temple Wash, San Rafael Reef; [38.65 ${ }^{\circ}$ N, $110.67^{\circ} \mathrm{W}$ ]; 1615 m a.s.l.; 4 Jun. 1984; F.D. and A. Parker leg.; BBSL • 1 क; same location as for preceding; 4 Jun. 1984; F.D. and A. Parker leg.; CUIC • 1 \%; Emery Co., Wild Horse Creek N of Goblin Valley; [38.57 ${ }^{\circ}$ N, 110.82 ${ }^{\circ}$ W]; 1494 m a.s.1.; 25-28 Jul. 1983; Parker and Griswold leg.; BBSL.

\section{Description}

Female

Colouration. Head and mesosoma blue-green; clypeus apical colour reddish brown to orange; labrum orange; mandible orange with black basal spot and red tip; flagellum reddish brown dorsally, orange ventrally; pronotal lobe orange; metasoma orange with discs of T3-4 red to dark brown and dark spiracular spots on T2-4; legs orange with coxae, trochanters, and femora reddish brown; tegula pale amber; wing membrane hyaline, veins pale amber to brown.

PuBESCENCE. Body hair colour white. Tomentum dense on paraocular area, frons, vertex, gena, pronotal collar and lobe, space between pronotal lobe and tegula, scutum lateral and posterior margins, metanotum medially, hypoepimeron, metepisternum, T1 laterally, T2-3 basolaterally, and T4 throughout; sparse on preëpisternum and mesepisternum. Scutum hair thin to moderately plumose. Wing hairs light, long or short, and dense. Acarinarial fan complete, sparse. T2 fringes sparse, T3 fringes sparse.

SuRfaCE SCULPTURE. Clypeus punctures large, irregularly sparse ( $\mathrm{i}<3 \mathrm{pd}$ ), interspersed with scattered minute punctures, sculpture shiny; supraclypeal area punctures dense $(i \leq 1 \mathrm{pd})$, somewhat sparser medially ( $\mathrm{i}=1-2 \mathrm{pd})$, sculpture shiny; paraocular area punctures dense $(\mathrm{i}<1 \mathrm{pd})$, sculpture imbricate around antenna socket, otherwise shiny; frons punctures dense ( $i<1 \mathrm{pd})$, sculpture shiny; vertex punctures fine, moderately dense laterally $(i=1-2 \mathrm{pd})$, sparse medially $(i=1-4 \mathrm{pd})$, sculpture shiny; gena punctures fine, moderately dense $(i=1-2 \mathrm{pd})$, sculpture shiny; postgena sculpture shiny, weakly imbricate laterad of hypostomal carina; tegula punctures absent; scutum punctures moderately dense $(\mathrm{i}=1-2 \mathrm{pd})$, becoming sparse submedially and anteriorly $(\mathrm{i}=1-4 \mathrm{pd})$, sometimes minute, sculpture shiny, sometimes becoming weakly tessellate anteromedially; scutellum punctures dense marginally and on median line $(\mathrm{i}<1 \mathrm{pd})$, sometimes finer and sparser anteriorly $(\mathrm{i}=1-2 \mathrm{pd})$, sparse submedially ( $\mathrm{i}=1-3 \mathrm{pd}$ ), diversopunctate, sculpture shiny; metanotum sculpture shiny and finely, densely punctate ( $<<1 \mathrm{pd}$ ), becoming weakly rugulose laterally; metapostnotum rugae strong or weak, anastomosing, not reaching margin, sculpture tessellate; preëpisternum sculpture areolate-rugulose; hypoepimeron punctures dense $(i \leq 1 \mathrm{pd})$ to crowded $(\mathrm{i}=0 \mathrm{pd})$, sculpture shiny; mesepisternum punctures dense ( $i<1 \mathrm{pd})$ to crowded ( $\mathrm{i}=0 \mathrm{pd}$ ), sculpture shiny; metepisternum sculpture ruguloso-lineate dorsally, imbricate ventrally; propodeum lateral face sculpture tessellate; propodeum posterior face sculpture tessellate; T1 anterior face sculpture shiny; T1 dorsal surface punctures moderately sparse (i=1-3 pd), absent or very sparse in large apicolateral oval patches, sculpture shiny; T2 disc punctures moderately sparse $(i=1-3 \mathrm{pd})$, disc sculpture shiny, rim punctures sparse $(i=1-4 \mathrm{pd})$, rim sculpture shiny.

Structure. Face length/width ratio 0.84 ( $\pm 0.03 \mathrm{SD}$ ). Clypeus projecting $\sim 67 \%$ below suborbital tangent; clypeal area length/width ratio 0.44 ( $\pm 0.03 \mathrm{SD})$; apicolateral denticles rounded knobs; supraclypeal area length/width ratio 0.78 ( $\pm 0.04 \mathrm{SD})$. Ocelli separated by about $0.6 \mathrm{OD}$. Forewing with 3 submarginal cells; pronotal angle obtuse; tegula shape normal. Intertegular distance 1.09 ( $\pm 0.07 \mathrm{SD}) \mathrm{mm}$. Scutum 


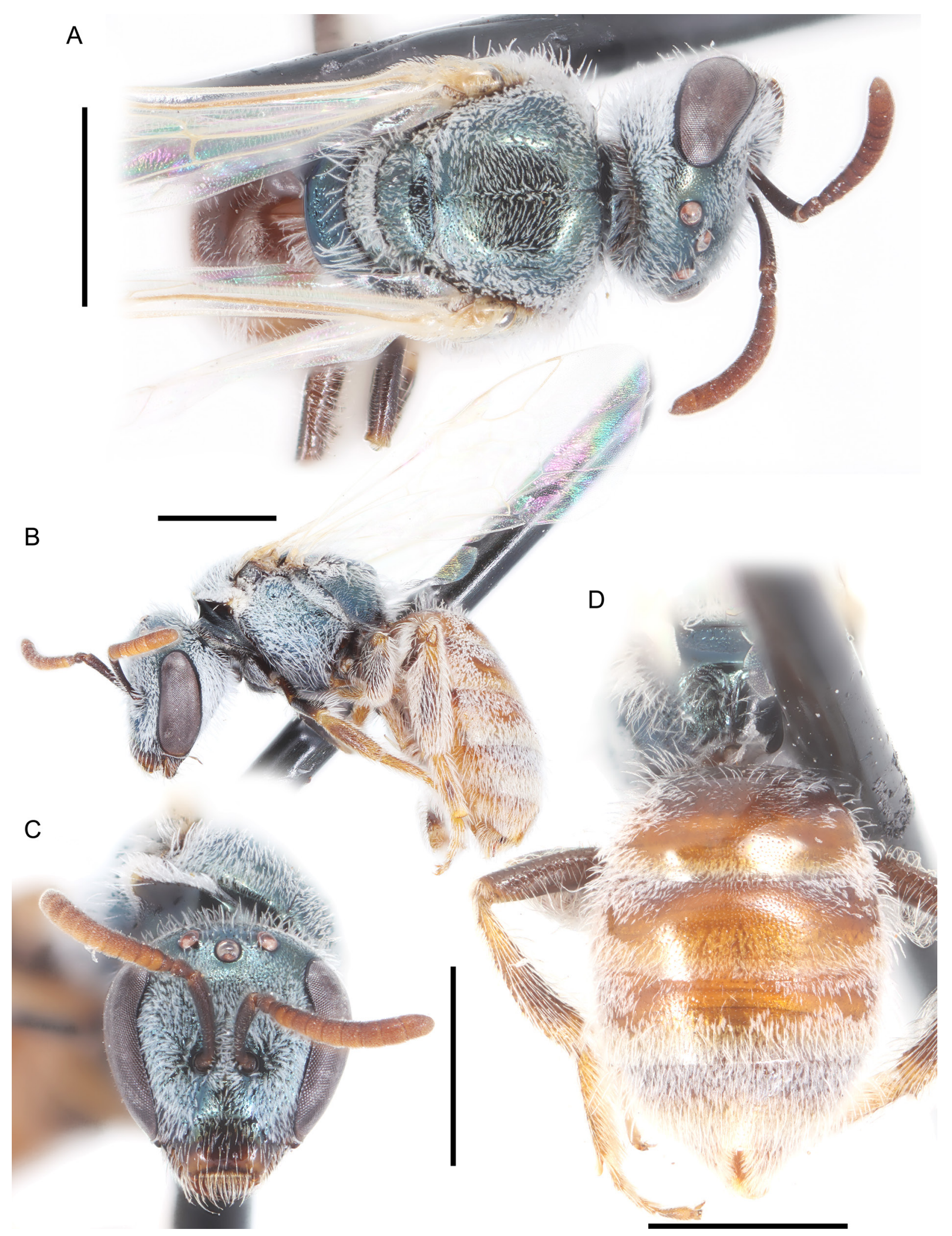

Fig. 52. Lasioglossum (D.) lilianae sp. nov., †. A. Dorsal habitus. B. Lateral habitus. C. Face. D. Propodeum and metasoma. Scale bars: $1 \mathrm{~mm}$. 


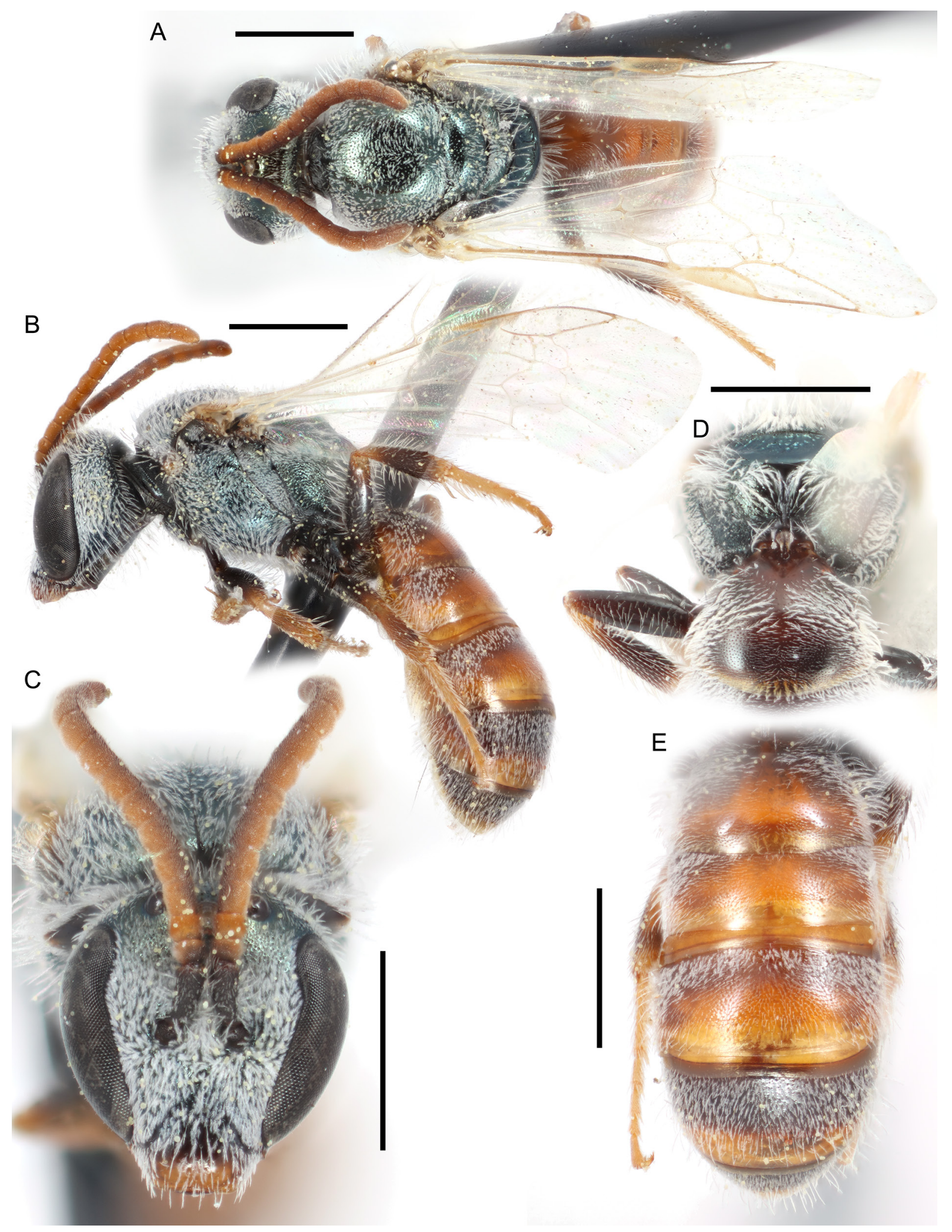

Fig. 53. Lasioglossum (D.) lilianae sp. nov., Ô. A. Dorsal habitus. B. Lateral habitus. C. Face. D. Propodeum. E. Metasoma. Scale bars: $1 \mathrm{~mm}$. 
length/width ratio $0.83( \pm 0.03 \mathrm{SD})$; scutum/scutellum length ratio $2.95( \pm 0.16 \mathrm{SD})$; scutellum/ metanotum length ratio $1.56( \pm 0.12 \mathrm{SD})$; metanotum/metapostnotum length ratio $0.71( \pm 0.06 \mathrm{SD})$. Propodeum lateral carinae not reaching dorsal margin; oblique carina absent. T2 depressed apical rim length less than $50 \%$ of segment. $(n=10)$

\section{Male}

Colouration. Head and mesosoma blue-green to golden-green; clypeus apical colour reddish brown to orange; labrum reddish brown to orange; mandible orange with black basal spot and red tip; flagellum reddish brown dorsally, orange ventrally; pronotal lobe reddish brown to orange; metasoma orange with dark spiracular spots on T2-4, to black with rims of terga and sterna and downcurved lateral areas of terga broadly translucent yellow; legs black to reddish brown with femur-tibia joints, tarsi, and sometimes tibiae orange; tegula orange; wing membrane hyaline, veins with subcosta dark brown, otherwise brown to pale amber.

Pubescence. Body hair colour white. Tomentum dense on face below eye emargination except clypeus, gena, pronotal angle and lobe, space between pronotal lobe and tegula, metanotum, episterna, T1 laterally, T2-3 basolaterally, and T4-5 throughout; sparse on clypeus, and mesonotum. Scutum hair densely plumose. Sterna hair short (0.75-1.5 OD), densely plumose, dense and erect. Wing hairs light, short and dense.

SURFACE SCULPTURE. Clypeus punctures sparse ( $\mathrm{i}=1-4 \mathrm{pd}$ ), sculpture shiny; supraclypeal area punctures dense to moderately dense ( $\mathrm{i} \leq 2 \mathrm{pd}$ ), sculpture shiny; paraocular area punctures dense $(\mathrm{i}<1 \mathrm{pd})$, sculpture shiny; frons punctures dense $(i<1 \mathrm{pd})$, sculpture shiny; vertex punctures moderately dense $(i=1-2 \mathrm{pd})$, sometimes fine and sparse medially $(\mathrm{i}=1-4 \mathrm{pd})$, sculpture shiny; gena punctures moderately dense ( $\mathrm{i}=1-2 \mathrm{pd})$, sculpture shiny; postgena sculpture shiny to weakly imbricate; tegula punctures absent; scutum punctures sparse $(\mathrm{i}=1-4 \mathrm{pd}$ ), sometimes becoming moderately dense marginally and on median line ( $\mathrm{i}=1-2 \mathrm{pd}$ ), sculpture shiny; scutellum punctures sparse ( $\mathrm{i}=1-3 \mathrm{pd})$, sometimes becoming dense marginally and on median line ( $i \leq 1 \mathrm{pd})$, sculpture shiny; metanotum sculpture shiny and moderately densely punctate $(\mathrm{i}=1-2 \mathrm{pd})$; metapostnotum rugae strong or weak, anastomosing or subparallel, not reaching margin, sculpture shiny to weakly imbricate; preëpisternum sculpture areolaterugulose; hypoepimeron punctures crowded $(i=0 \mathrm{pd})$, sculpture shiny; mesepisternum punctures dense ( $\leq 1 \mathrm{pd}$ ), sculpture shiny; metepisternum sculpture lineate dorsally, areolate ventrally; propodeum lateral face punctures moderately dense $(i=1-2$ pd), often obscure, sculpture shiny to imbricate and weakly rugulose; propodeum posterior face sculpture shiny; T1 anterior face sculpture shiny; T1 dorsal surface punctures dense to moderately sparse ( $\mathrm{i} \leq 3 \mathrm{pd}$ ), sculpture shiny; $\mathrm{T} 2$ disc punctures dense to moderately dense $(i \leq 2 \mathrm{pd})$, disc sculpture shiny, rim punctures sparse $(\mathrm{i}=1-3 \mathrm{pd})$, sometimes absent apically, rim sculpture shiny.

StRUCTURE. Face length/width ratio 0.89 ( $\pm 0.03 \mathrm{SD})$. F1:pedicel length ratio $1.01( \pm 0.09 \mathrm{SD}) ; \mathrm{F} 2: \mathrm{F} 1$ length ratio $1.52( \pm 0.13 \mathrm{SD}) ; \mathrm{F} 2$ length/width ratio $1.25( \pm 0.07 \mathrm{SD}) ; \mathrm{F} 9$ length/width ratio 1.16 ( $\pm 0.13 \mathrm{SD})$. Forewing with 3 submarginal cells; pronotal angle obtuse; tegula shape normal. Intertegular distance $1.02( \pm 0.14 \mathrm{SD}) \mathrm{mm}$. Scutum length/width ratio $0.82( \pm 0.06 \mathrm{SD})$; scutum/scutellum length ratio $2.71( \pm 0.11 \mathrm{SD})$; scutellum/metanotum length ratio $1.66( \pm 0.12 \mathrm{SD})$; metanotum/metapostnotum length ratio $0.71( \pm 0.05 \mathrm{SD})$. Propodeum lateral carinae not reaching dorsal margin; oblique carina absent. $(n=8)$

Genitalia. As in Fig. 90F. Gonocoxite broad, rounded. Gonostylus long, distinctly narrowed basally, with a few long hairs. Retrorse lobe very broad, ovoid, covered in sparse short hairs. 


\section{Range}

Chihuahuan Desert of Arizona, New Mexico, Texas, Chihuahua, Coahuila, and Nuevo Leon, north to Colorado and Utah (Fig. 54).

\section{Floral records}

ASTERACEAE Giseke: Grindelia: G. squarrosa (Pursh) Dunal - LOASACEAE Juss. Mentzelia L. M. aspera L. • M. multiflora (Nutt.) A.Gray • M. nuda (Pursh) Torr. \& A.Gray • M. pumila Torr. \& A. Gray $\bullet$ M. strictissima (Wooton \& Standl.) J. Darl.

\section{DNA barcodes}

Five sequences available (BOLD process IDs: BOWGF2054-10, DIAL1429-09, DIAL1435-09, DLII762-07, DLII1468-08; BINs: BOLD:AAF3975, BOLD:ABY5037). There is a moderate amount of divergence within these sequences (maximum p-distance 2.29\%). No unique fixed substitutions distinguish L. lilianae sp. nov. from all other western red-tailed L. (Dialictus).

\section{Remarks}

Lasioglossum lilianae sp. nov. is most commonly found in the Chihuahuan Desert, but has a fairly wide distribution beyond it. An extreme outlier specimen from Jackson Meadow, Tulare County, California, however, may be a labeling error. All other known specimens are from much lower elevation deserts or arid grasslands.

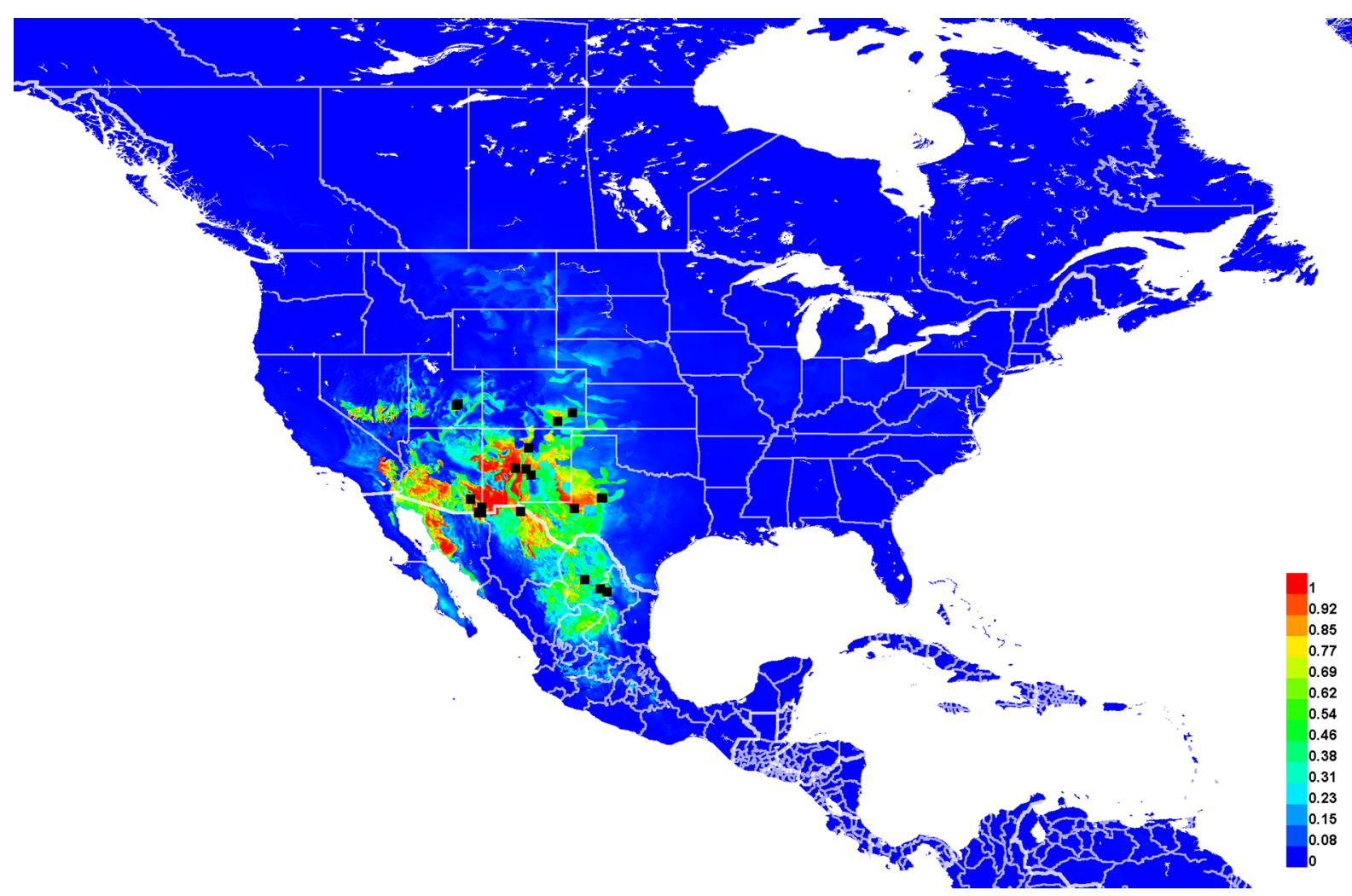

Fig. 54. Georeferenced collection records of Lasioglossum (D.) lilianae sp. nov. (black squares) and predicted distribution by maximum entropy ecological niche modeling in Maxent (colour shading). Warmer colours indicate higher cloglog probability of occurrence. One doubtful record from Tulare County, California was left out of the analysis. 
Out of 32 specimens from 13 distinct collection events for which the host plant was recorded, all were collected from Mentzelia species except for three (two of which are males). Out of 13 specimens with full pollen loads, 12 appeared to be pure Mentzelia based on visual examination, and assuming that the host plant recorded on the label is an accurate indicator of the identity of the pollen. The thirteenth specimen appeared to have a pure load of large-grained Cactaceae or Malvaceae; the host plant of this specimen (collected from South Temple Wash, Utah, and deposited in CUIC) was not recorded. This species may be oligolectic on Mentzelia; if so, it would be the first known instance of pollen specialization in L. (Dialictus).

Lasioglossum lilianae sp. nov. may also be active at dawn or dusk, as indicated by the slightly enlarged ocelli. Several species of Mentzelia, including some which L. lilianae sp. nov. has been recorded visiting, have blooms which open in late afternoon or evening and stay open into the night (Smyth 1897; Glad 1975; Brown \& Kaul 1981; Keeler 1981; Holmgren \& Holmgren 2002). An alternative explanation is that L. lilianae sp. nov. is not oligolectic, but is most active in late afternoon, and incidentally collects Mentzelia pollen because it is the most abundant food source at that time.

Lasioglossum (Dialictus) mesillense (Cockerell, 1898)

Figs 55-57, 90G, 101B, 108B

Halictus nymphalis race mesillensis Cockerell, 1898b: 47 (lectotype designated herein, $\uparrow$, deposited in EMEC, examined).

Halictus clarissimus Ellis, 1914b: 222 (holotype, $q$, deposited in USNM, examined) syn. nov.

Halictus (Chloralictus) perexiguus Sandhouse, 1924: 26 (holotype, ô, deposited in USNM, examined) syn. nov.

Halictus mesillensis - Cockerell 1906: 429 (key).

Halictus (Chloralictus) clarissimus - Sandhouse 1924: 4 (key).

Halictus (Chloralictus) mesillensis - Sandhouse 1924: 4 (key).

Lasioglossum (Chloralictus) clarissimum - Michener 1951: 1112 (catalog).

Lasioglossum (Chloralictus) mesillense - Michener 1951: 1115 (catalog).

Lasioglossum (Chloralictus) perexiguum - Michener 1951: 1116 (catalog).

Dialictus clarissimus - Hurd 1979: 1965 (catalog). — Moure \& Hurd 1987: 95 (catalog).

Dialictus mesillensis - Hurd 1979: 1968 (catalog). — Moure \& Hurd 1987: 112 (catalog).

Dialictus perexiguus - Hurd 1979: 1969 (catalog). — Moure \& Hurd 1987: 120 (catalog).

\section{Diagnosis}

Females of Lasioglossum mesillense can be recognized by the face short (length/width ratio $\sim 0.8$ ), frons with very fine and dense but distinctly separated punctures, clypeus with distinct apicolateral denticles, mesepisternum with dense punctures ( $\mathrm{i} \leq 1 \mathrm{pd}$ ) becoming crowded and weakly rugulose on the dorsal margin, T2-4 with dark spiracular spots, T1 anterior surface usually shiny, metasomal terga usually with some dense basolateral tomentum, and mesoscutum long (length/width ratio usually $\geq 0.84$ ). They are most similar to L. minckleyi sp. nov., L. austerum sp. nov., and L. imbriumbrae sp. nov. Females of L. minckleyi sp. nov. have the frons with crowded and indistinct punctures, mesepisternum more densely punctate ventrally $(\mathrm{i}<1 \mathrm{pd}), \mathrm{T} 1$ anterior surface coriarious, metasomal terga without tomentum, and mesoscutum broad (length/width ratio usually $\leq 0.8$ ). Females of $L$. austerum $\mathrm{sp}$. nov. have the head and mesosoma with more extensive dull microsculpture, mesepisternum more sparsely and distinctly punctate dorsally ( $\mathrm{i} \leq 1 \mathrm{pd}$ ), $\mathrm{T} 1$ anterior surface coriarious, and metasomal terga without tomentum. Females of L. imbriumbrae sp. nov. have the frons with crowded indistinct punctures (nearly rugulose) 
medially, and mesepisternum and hypoepimeron usually with dense but distinctly separated punctures throughout $(\mathrm{i}<1 \mathrm{pd})$.

Males of $L$. mesillense can be recognized by the flagellomeres relatively long (F2 about 2 times as long as F1 and 1.6 times as long as broad), face short (length/width ratio $\sim 0.84$ ) and covered in dense tomentum below the ocelli, mesepisternum shiny and moderately densely punctate $(\mathrm{i}=1-2 \mathrm{pd})$, metapostnotum with relatively weak rugae not reaching the posterior margin or extending onto the dorsolateral slope, T2-3 often with basolateral tomentum, and clypeus apical margin and labrum orange to yellow. They are most similar to L. minckleyi sp. nov., L. austerum sp. nov., and L. clematisellum. Males of L. minckleyi sp. nov. have the flagellomeres relatively short (F2 about 1.6 times as long as F1 and 1.25 times as long as broad), frons with crowded and indistinct punctures, and T2-3 never with basolateral tomentum. Males of L. austerum sp. nov. have the flagellomeres relatively short (F2 about 1.75 times as long as F1 and 1.4 times as long as broad) and face with sparse tomentum. Males of L. clematisellum have the clypeus apical margin, labrum, and metasoma black, metapostnotum with strong rugae reaching the posterior margin and extending onto the dorsolateral slope, and T2-3 never with basolateral tomentum.

\section{Etymology}

Cockerell (1898b) named this species after the Mesilla Valley in New Mexico, where the type series was collected, plus the Latin adjectival suffix '-ensis', indicating association with a place.

\section{Material examined}

\section{Lectotype}

UNITED STATES - New Mexico • + ; Las Cruces; [32.32 ${ }^{\circ}$ N, $106.76^{\circ}$ W]; 5 Aug. 1893; Cockerell leg.; EMEC 530953.

[Verbatim label: Las Cruces, NM / Aug. 51893 (Cockerell) // stultus? // Halictus mesillensis Cockerell // Stanford University // Halictus mesillensis Ckll / Ckll // UC Berkeley / EMEC 530,953]

\section{Other material}

MEXICO - Baja California 11 ' $; 12$ mi. S of Palacio; [30 N, $115^{\circ} \mathrm{W}$ ]; Apr. 1939; C.D. Michener leg.; ex Prosopis; CAS. - Chihuahua • 1 क ; Hidalgo del Parral; [26.93 ${ }^{\circ}$ N, $\left.105.67^{\circ} \mathrm{W}\right]$; 31 Jul. 1967; R.C. Gardner, C.R. Kovacic and K. Lorenzen leg.; UCDC. - Sonora - 2 + $\odot ; 30$ km E of Agua Prieta; 31.3294 ${ }^{\circ}$ N, $109.2547^{\circ}$ W; 24 Jul. 2001; R.L. Minckley leg.; RLM • 1 क; same location as for preceding; 18 Jul. 2001; R.L. Minckley leg.; RLM • 1 q; $30 \mathrm{~km}$ E of Agua Prieta; $31.3097^{\circ}$ N, $109.2778^{\circ} \mathrm{W} ; 6$ Aug. 2004; R.L. Minckley leg.; ex Chenopodium neomexicanum; RLM • 1 \%; same location as for preceding; 8 Aug. 2004; R.L. Minckley leg.; ex Salsola iberica; RLM • 1 ;; $30 \mathrm{~km}$ E of Agua Prieta; $31.3297^{\circ}$ N,

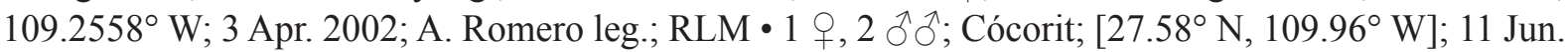
1961; F.D. Parker leg.; UCDC • 1 क; Municipio de Agua Prieta, Rancho Nogalitos; $31.31177^{\circ} \mathrm{N}$, 108.98622 ${ }^{\circ} \mathrm{W}$; 25 May 2008; RLM • 2 우; Rancho San Bernardino; $31.3294^{\circ} \mathrm{N}, 109.2547^{\circ} \mathrm{W}$; 5 Sep. 2001; R.L. Minckley leg.; RLM • 1 क; Rancho San Bernardino; $31.3186^{\circ}$ N, $109.2508^{\circ}$ W; 5 Sep. 2001; R.L. Minckley leg.; RLM • 1 q; Rancho San Bernardino; $31.3303^{\circ}$ N, $109.26^{\circ}$ W; 8 Sep. 2001; R.L. Minckley leg.; RLM • 1 \%; Rancho San Bernardino, 28 km E of Agua Prieta, cienega transect; $\left[31.32^{\circ} \mathrm{N}, 109.26^{\circ} \mathrm{W}\right] ; 27$ Jul. 2000; K. Toal leg.; RLM • 2 q ; ; Rancho San Bernardino, Bosque wet, Site 1; $31.33194^{\circ} \mathrm{N}, 109.27083^{\circ} \mathrm{W} ; 10$ Aug. 2006; RLM • 1 O; Rancho San Bernardino, Bosque wet, Site 6, Transect 3; $31.32957^{\circ} \mathrm{N}, 109.25462^{\circ} \mathrm{W} ; 18$ Sep. 2001; RLM • 1 q; Rancho San Bernardino, Bosque wet, Site 7, Transect 2; $31.31007^{\circ}$ N, $109.25348^{\circ}$ W; 16 Aug. 2005; RLM • 1 q; Rancho San Bernardino, Cafe Transect 5, Elia Thesis Study; [31.32 ${ }^{\circ}$ N, $109.26^{\circ}$ W]; 3 Aug. 2001; RLM • 1 q; Rancho San Bernardino, Grassland, Site 3; $31.30222^{\circ} \mathrm{N}, 109.26694^{\circ} \mathrm{W} ; 12$ Aug. 2006; RLM • 1 \%; Rancho San Bernardino, Loma Transect 2, Elia Thesis Study; [31.32 ${ }^{\circ}$ N, $\left.109.26^{\circ} \mathrm{W}\right]$; 2 Aug. 2001; RLM 1 क ; Rancho San Bernardino, Loma Transect 3, Elia Thesis Study; [31.32 ${ }^{\circ}$ N, $109.26^{\circ}$ W]; 2 Aug. 2001; RLM• 
2 ㅇ; Rancho San Bernardino, Los Ojitos, Lebuhn, Bee Diversity; $31.30025^{\circ}$ N, $109.2625^{\circ}$ W; 3 May 2002; RLM • 1 \%; Rancho San Bernardino, Pollination Site 12, Lesquerella, Silver Creek, upstream of Big Dam; $31.3259^{\circ} \mathrm{N}, 109.28802^{\circ} \mathrm{W} ; 13$ Apr. 2006; ex Physaria gordonii; RLM • 1 \%; Rancho San Bernardino, Pollination Site 22, Sphaeralcea, field 3; 31.32222 ${ }^{\circ}$ N, 109.27778 ${ }^{\circ}$ W; 6 Aug. 2006; ex Laennecia coulteri; RLM • 1 \&; Rancho San Bernardino, Pollination Site 24, Lesquerella, Hay Hollow; $31.31333^{\circ} \mathrm{N}, 109.25028^{\circ} \mathrm{W} ; 5$ Apr. 2007; ex Salix gooddingii; RLM • 1 o; Rancho San Bernardino, Pollination Site 27, Horse Corral, $70 \mathrm{~m}$ N of main ranch house; $31.32556^{\circ} \mathrm{N}, 109.26667^{\circ} \mathrm{W}$; 9 Sep. 2007; ex Antennaria sp.?; RLM • 2 q 9 ; same location as for preceding; 9 Sep. 2007; ex Gaura mollis; RLM • 3 P 9 ; Rancho San Bernardino, quarry, at road crossing; $31.32042^{\circ} \mathrm{N}, 109.2681^{\circ} \mathrm{W}$; 2 Sep. 2003 ; RLM - 1 क; Rancho San Bernardino, quarry, general area; $31.3195^{\circ} \mathrm{N}, 109.27028^{\circ} \mathrm{W}$; 18 Aug. 2003; ex Mentzelia pumila; RLM • 1 \%; same location as for preceding; 27 May 2004; ex Mentzelia pumila; RLM • 1 \%; Rancho San Bernardino, quarry, gravel pit; $31.31964^{\circ} \mathrm{N}, 109.26926^{\circ} \mathrm{W} ; 18$ Aug. 2003; RLM - 1 क; Rancho San Bernardino, quarry, W roadside; $31.31948^{\circ} \mathrm{N}, 109.2701^{\circ} \mathrm{W}$; 18 Aug. 2003; RLM - 2 우; same location as for preceding; 19 Aug. 2003; RLM • 2 q 9 ; Rancho San Bernardino, Riparian 3; $31.31389^{\circ} \mathrm{N}, 109.25028^{\circ} \mathrm{W}$; 25 Aug. 2003; RLM • 1 क ; Rancho San Bernardino, Riparian, Site 2; $31.33194^{\circ} \mathrm{N}, 109.25833^{\circ} \mathrm{W} ; 20$ Aug. 2003; ex Baccharis sarothroides; RLM • 2 우; Rancho

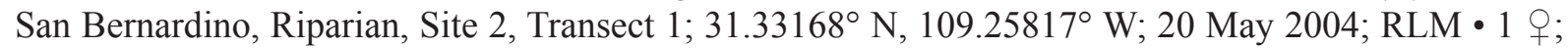
Rancho San Bernardino, Riparian, Site 4, Transect 1; $31.32965^{\circ}$ N, $109.25587^{\circ}$ W; 8 May 2002; RLM • 1 क; same location as for preceding; 30 Apr. 2004; RLM.

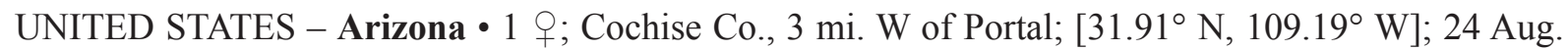
1973; R. Coville leg.; EMEC • 1 क; Cochise Co., 4 mi. E. Willcox; $\left[32.25^{\circ} \mathrm{N}, 109.76^{\circ} \mathrm{W}\right] ; 17$ Aug. 1994; J.G. Rozen and J.S. Ascher leg.; AMNH 1 q ; Cochise Co., 4 mi. ESE of Willcox; $\left[32.23^{\circ} \mathrm{N}\right.$,

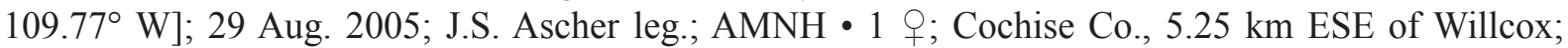
$32.2366^{\circ} \mathrm{N}, 109.7782^{\circ} \mathrm{W}$; 11 Apr. 2019; J. Gardner leg.; ex yellow mustard; WRME 1 क; Cochise Co., 6 mi. S of Willcox; [32.16 N, 109.83 ${ }^{\circ}$ W]; 23 Aug. 1986; R.J. McGinley and B.M. Norden leg.; USNM •

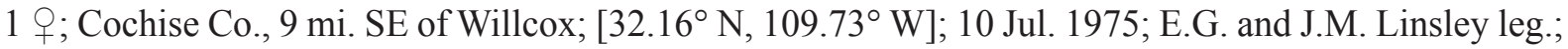

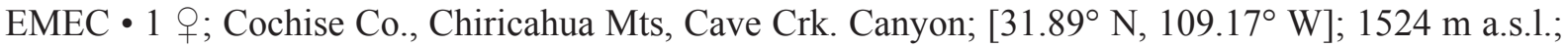
4 Aug. 1986; D.R. Corr leg.; MTEC • 1 q; Cochise Co., Chiricahua Mts, Portal to Ranger Stn; [31.91 ${ }^{\circ}$, 109.15 W]; 4 Aug. 1986; D.R. Corr leg.; MTEC • 1 क; Cochise Co., Coronado NF, Barfoot Meadow; $31.9176^{\circ} \mathrm{N}, 109.2793^{\circ} \mathrm{W} ; 2536 \mathrm{~m}$ a.s.1.; 1 Jul. 2017; M.P. Argueta leg.; ex Erigeron divergens; UCRC • $1 \mathrm{O}^{\mathrm{N}}$; Cochise Co., Douglas; [31.34 ${ }^{\circ} \mathrm{N}, 109.51^{\circ} \mathrm{W}$ ]; 27 Jul. 1975; E.G. and J.M. Linsley leg.; ex Tamarix chinensis; CUIC • 1 क; Cochise Co., 12.5 mi. N of Portal; 32.0649 ${ }^{\circ} \mathrm{N}, 109.1804^{\circ} \mathrm{W}$; 1 Sep. 2009; T.L. Griswold leg.; BBSL 1 क; Cochise Co., Southwestern Research Station 5 mi. W of Portal; [31.884 $\left.{ }^{\circ} \mathrm{N}, 109.206^{\circ} \mathrm{W}\right] ; 1646 \mathrm{~m}$ a.s.1.; 4 Jul. 1963; A.R. Moldenke and J.A. Woods leg.; AMNH 1 क ; same location as for preceding; 7 Oct. 1962; Vincent Roth leg.; AMNH • 1 क; same location as for preceding; 18 Apr. 1963; Vincent Roth leg.; AMNH - 1 o; Cochise Co., San Bernardino NWR; $31.335^{\circ}$ N, $109.2528^{\circ}$ W; 18 Jul. 2001; R. Minckley leg.; PCYU • 1 क; Cochise Co., San Bernardino NWR; $31.3353^{\circ}$ N, 109.2514 W; 30 Mar. 2002; R.L. Minckley leg.; PCYU • 1 क; Cochise Co., San Bernardino NWR; $31.3381^{\circ} \mathrm{N}, 109.2606^{\circ} \mathrm{W} ; 8$ Sep. 2001; R.L. Minckley leg.; PCYU • 2 q + ; same location as for preceding; 8 Sep. 2001; R.L. Minckley leg.; RLM • 1 q; same location as for preceding; 31 Aug. 2001; R.L. Minckley leg.; RLM • 2 o + ; Cochise Co., San Bernardino NWR; 31.3344 N, 109.2681 ${ }^{\circ}$ W; 22 Jul. 2001; R. Minckley leg.; RLM • 1 क; Cochise Co., San Bernardino NWR; $31.3417^{\circ} \mathrm{N}, 109.2583^{\circ} \mathrm{W} ; 17$ Jul. 2001; R. Minckley leg.; RLM • 1 q; same location as for preceding; 26 Jul. 2001; R.L. Minckley leg.; RLM • 1 क; same location as for preceding; 8 Sep. 2001; R.L. Minckley leg.; RLM • 1 \%; Cochise Co., San Bernardino NWR; $31.3394^{\circ}$ N, $109.2631^{\circ}$ W; 11 Jul. 2001; R. Minckley leg.; RLM • 1 क; Cochise Co., San Bernardino NWR; $31.3353^{\circ}$ N, $109.2667^{\circ}$ W; 31 Jun. 2004; R.L. Minckley leg.; RLM • 1 क; Cochise Co., San Bernardino NWR; $31.3342^{\circ}$ N, 109.2678 W; 31 Mar. 2002; R.L. Minckley leg.; RLM • 1 o; Cochise Co., San Bernardino NWR; $31.3353^{\circ}$ N, $109.2514^{\circ}$ W; 30 Mar. 2002; R.L. Minckley leg.; RLM • 1 क ; Cochise Co., San Bernardino 
NWR; $31.3408^{\circ}$ N, $109.2531^{\circ}$ W; 3 Apr. 2002; A. Romero leg.; RLM • 1 क; Cochise Co., San Bernardino NWR; $31.3417^{\circ}$ N, $109.2583^{\circ}$ W; 9 Jun. 2002; R.L. Minckley leg.; RLM • 1 q; Cochise Co., San Bernardino NWR; $31.3364^{\circ} \mathrm{N}, 109.2675^{\circ} \mathrm{W} ; 31$ Mar. 2002; A. Romero leg.; RLM • 1 क; Cochise Co., San Bernardino NWR; $31.3353^{\circ}$ N, $109.2667^{\circ}$ W; 31 Jun. 2004; R.L. Minckley leg.; RLM • 1 क ; Cochise

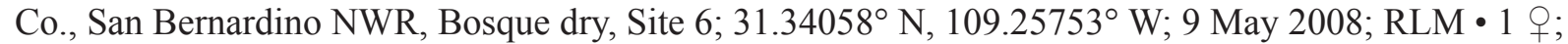
Cochise Co., San Bernardino NWR, Bosque dry, Site 6, Transect 2; $31.34058^{\circ}$ N, $109.25747^{\circ}$ W; 11 Oct. 2002; RLM • 6 우; same location as for preceding; 30 Jul. 2004; RLM • 2 o ㅇ; Cochise Co., San Bernardino NWR, Bosque dry, Site 7; $31.34118^{\circ} \mathrm{N}, 109.25813^{\circ} \mathrm{W} ; 3$ Jul. 2002; RLM • 1 o ; same location as for preceding; 6 Aug. 2003; RLM • 1 क; same location as for preceding; 6 Apr. 2006; RLM • 1 q; Cochise Co., San Bernardino NWR, Bosque dry, Site 7, Transect 2; $31.34168^{\circ}$ N, $109.25825^{\circ}$ W; 17 Apr. 2001; RLM • 7 우; same location as for preceding; 30 Jul. 2004; RLM • 1 q; Cochise Co., San Bernardino NWR, Bosque wet, Site 2, Transect 2; $31.33627^{\circ}$ N, 109.26752 ${ }^{\circ}$ W; 31 Jul. 2004; RLM • 7 우; Cochise Co., San Bernardino NWR, Bosque wet, Site 3, Transect 2; $31.33527^{\circ}$ N, $109.2668^{\circ}$ W; 15 Aug. 2005; RLM - 3 우; Cochise Co., San Bernardino NWR, Bosque wet, Site 4; $31.334^{\circ}$ N, 109.2372 ${ }^{\circ}$ W; 5 Aug. 2003; RLM • 3 ㅇ; Cochise Co., San Bernardino NWR, Bosque wet, Site 4, Transect 1; $31.33693^{\circ} \mathrm{N}, 109.24361^{\circ} \mathrm{W} ; 11$ Aug. 2005; RLM • 1 ; Cochise Co., San Bernardino NWR, field, Site 5, Transect 2; $31.33528^{\circ}$ N, 109.26806 W; 11 Aug. 2005; RLM • 2 q $O$; Cochise Co., San Bernardino NWR, field, Site 5, Transect 3; $31.33417^{\circ}$ N, 109.26778 W; 27 Jul. 2007; RLM • 2 우; Cochise Co., San Bernardino NWR, field, Site 6; $31.33867^{\circ}$ N, 109.2719 ${ }^{\circ}$ W; 8 Aug. 2003; RLM • 1 क; Cochise Co., San Bernardino NWR, field, Site 6, Transect 1; 31.33417 ${ }^{\circ}$ N, $109.26833^{\circ}$ W; 11 Aug. 2005; RLM - 1 क; Cochise Co., San Bernardino NWR, field, Site 6, Transect 4; $31.33444^{\circ}$ N, 109.26806 ${ }^{\circ}$ W; 30 Jul. 2004; RLM • 1 क; Cochise Co., San Bernardino NWR, field, Site 7; $31.33583^{\circ}$ N, 109.2629 W; 6 Aug. 2003; RLM • 1 क; Cochise Co., San Bernardino NWR, field, Site 7, Transect 2; $31.33528^{\circ} \mathrm{N}, 109.25139^{\circ} \mathrm{W} ; 28 \mathrm{Apr} .2004$; RLM 1 q; same location as for preceding; 27 Jul. 2007; RLM • 1 क; Cochise Co., San Bernardino NWR, field, Site 8; $31.33765^{\circ}$ N, $109.26068^{\circ}$ W; 3 Jul. 2002; RLM • 2 O 9 ; same location as for preceding; 22 Apr. 2007; RLM • 1 ㅇ; Cochise Co., San Bernardino NWR, field, Site 8, Transect 2; $31.3381^{\circ} \mathrm{N}, 109.2605^{\circ} \mathrm{W} ; 19$ Sep. 2001; RLM • 1 \%; same location as for preceding; 11 Oct. 2002; RLM • 1 q; same location as for preceding; 28 Apr. 2004; RLM • 1 q; same location as for preceding; 16 May 2004; RLM • 3 우; same location as for preceding; 30 Jul. 2004; RLM • 4 우; Cochise Co., San Bernardino NWR, Grassland, Site 4; $31.33495^{\circ}$ N, $109.25314^{\circ}$ W; 5 Aug. 2003; RLM • 2 우; same location as for preceding; 11 Aug. 2005; RLM • 1 ㅇ; Cochise Co., San Bernardino NWR, Grassland, Site 4, Transect 1; $31.33487^{\circ}$ N, $109.2529^{\circ}$ W; 18 Sep. 2001; RLM • 3 qo ; same location as for preceding; 26 Jul. 2007; RLM • 1 क; Cochise Co., San Bernardino NWR, Grassland, Site 4, Transect 2; $31.335^{\circ} \mathrm{N}, 109.25389^{\circ} \mathrm{W}$; 14 May 2004; RLM • 1 +; Cochise Co., San Bernardino NWR, Riparian, Site 7, Transect 1; $31.33955^{\circ}$ N, $109.26312^{\circ}$ W; 18 Sep. 2001; RLM • 1 q; Cochise Co., San Bernardino NWR, Shrub, nr Bathroom, Bee Diversity; $31.34052^{\circ}$ N, $109.27165^{\circ}$ W; 15 Oct. 2002; RLM • 2 q ; Cochise Co., Southwestern Research Station, 5 mi. W of Portal; [31.883 ${ }^{\circ}$ N, 109.206 ${ }^{\circ}$ W]; 1646 m a.s.1.; 3 Oct. 1966; P.H. Arnaud Jr. leg.; CAS • 1 o; Cochise Co., Southwestern

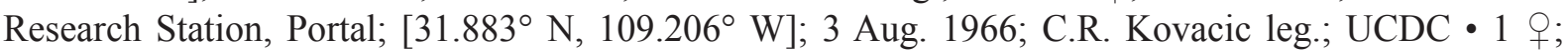
Cochise Co., St David Cienega; $31.8465^{\circ}$ N, $110.2275^{\circ}$ W; 4 Apr. 2016; C.M. Delphia and J.B. Runyon leg.; MTEC • 1 ; ; Cochise Co., Stewart Camp, 2 mi. SW of Portal; [31.89 N, $\left.109.17^{\circ} \mathrm{W}\right]$; 1463 m a.s.l.; 2-3 Aug. 1973; R. Coville leg.; EMEC • 1 क ; Cochise Co., Willcox; [32.25 ${ }^{\circ} \mathrm{N}, 109.83^{\circ} \mathrm{W}$;; 19 Jun. 1969; R.M. Bohart leg.; UCDC 13 우; Cochise Co., 3.45 mi. SEbE of Willcox; $32.2262^{\circ} \mathrm{N}$, $109.7822^{\circ}$ W; 1273 m a.s.1.; 1 Sep. 2009; T.L. Griswold leg.; BBSL • 1 q; La Paz Co., Bill Williams NWR; $34.2683^{\circ} \mathrm{N}, 114.0461^{\circ} \mathrm{W} ; 26$ Mar. 2001; R.L. Minckley leg.; RLM • 2 q $O$; Maricopa Co.,

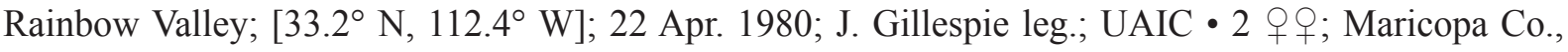
Wickenburg, $7 \mathrm{~km} \mathrm{SE}$ of Hassayampa River Preserve; $33.9097^{\circ} \mathrm{N}, 112.6752^{\circ} \mathrm{W} ; 550 \mathrm{~m}$ a.s.1.; $11-13$

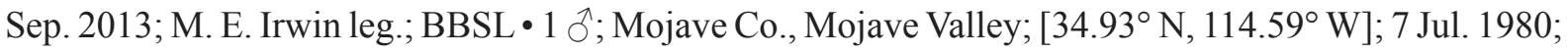
Paul Artz leg.; CUIC • 1 q ; Pima Co., 22 km S of Robles Jct.; [31.88 ${ }^{\circ}$ N, $111.31^{\circ}$ W]; 14 Aug. 1998; R. Minckley and K. Toal leg.; ex Kallstroemia grandiflora; SEMC • 1 क ; Pima Co., 8 km E of Robles Jct.; 
32.0667 N, 111.25 W; 6 Sep. 1997; R.L. Minckley leg.; ex Mentzelia sp.; SEMC • 1 o; Pima Co., Sandario Rd $7 \mathrm{~km} \mathrm{~N}$ of Hwy 86; $32.1833^{\circ} \mathrm{N}, 111.2167^{\circ}$ W; 740 ma.s.l.; 12 Aug. 1998; R.L. Minckley leg.; ex Pectis sp.; SEMC • 1 क; Pima Co., Tucson; [32.22 ${ }^{\circ} \mathrm{N}, 110.97^{\circ} \mathrm{W}$ ]; 15 Apr. 1997; P.F. Torchio leg.; ex Nama; BBSL • 1 q; Pima Co., Tucson, $68.3 \mathrm{~km} \mathrm{SW}$ of Hwy 286; $31.8647^{\circ} \mathrm{N}, 111.3978^{\circ} \mathrm{W} ; 6$ Apr. 2014; M.C. Orr leg.; ex Phacelia sp.; BBSL • 1 q; Pinal Co., 11 mi. SW of Eloy; [32.64 N, $111.69^{\circ} \mathrm{W}$ ]; 3 Apr. 1934; T.R. Haig leg.; UCDC • 1 क; Pinal Co., 8 km SE of San Manuel; $32.5974^{\circ}$ N, $110.5388^{\circ}$ W; 15 Apr. 2014; R.G. Laport leg.; ex Larrea tridentata; RLM • 1 ô; Pinal Co., 10 mi. SE of Florence; [32.91 $\left.{ }^{\circ} \mathrm{N}, 111.29^{\circ} \mathrm{W}\right] ; 29$ May 1976; P.D. Hurd, E.G. and J.M. Linsley leg.; ex Parkinsonia aculeata; CUIC - 1 क; Pinal Co., 10 mi. SE of Florence; [32.91 ${ }^{\circ}$ N, $111.28^{\circ}$ W]; 25 May 1976; E.G. and J.M. Linsley leg.; ex Parkinsonia aculeata; EMEC • 1 क; Pinal Co., Page Ranch 7 mi. W of Oracle; $\left[32.61^{\circ} \mathrm{N}\right.$, $110.89^{\circ}$ W]; 3 Aug. 1992; L. Packer leg.; PCYU • 1 q; Yavapai Co., Beaver Creek R.S.; [34.6 ${ }^{\circ}$ N, $111.8^{\circ}$ W]; 1219 m a.s.1.; 27 Apr. 1996; R.S. Beal leg.; CSUC • 1 đ̊; Yavapai Co., Indian Springs Wash, T18N R4W Sc32; [34.9 $\left.{ }^{\circ} \mathrm{N}, 112.7^{\circ} \mathrm{W}\right] ; 1524 \mathrm{~m}$ a.s.1.; 2 Jun. 2000; R.S. Beal and E. Tilgner leg.; CSUC • 1 J ; Yavapai Co., Skull Valley; [34.5 N, $112.69^{\circ} \mathrm{W}$ ]; $1311 \mathrm{~m}$ a.s.1.; 14 Sep. 2001; J. Schafer and R.S. Beal leg.; CSUC • 5 o ; ; Yuma Co., Imperial NWR; $32.9^{\circ} \mathrm{N}, 114.45^{\circ} \mathrm{W} ; 20$ Mar. 2002; A. Romero leg.; RLM 11 ; 10 mi. E of Cottonwood; [34.74 $\left.{ }^{\circ} \mathrm{N}, 111.83^{\circ} \mathrm{W}\right]$; 30 Mar. 1956; F. Werner and G. Butler leg.;

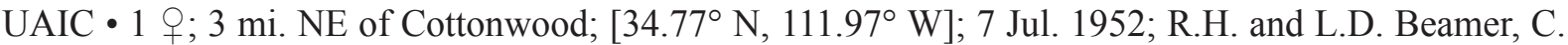
Liang and W. LaBerge leg.; SEMC • 2 우; Baboquivari Mts; [31.8 $8^{\circ}$, 111.6 ${ }^{\circ} \mathrm{W}$ ]; 24 Jul. 1941; R.H. Beamer leg.; SEMC • 1 क; Campe Verde; $\left[34.56^{\circ} \mathrm{N}, 111.85^{\circ} \mathrm{W}\right]$; 30 Mar. 1956; F. Werner and

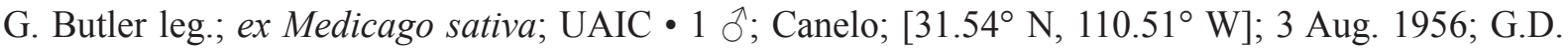
Butler leg.; CUIC • 1 q; Duncan; [32.72 ${ }^{\circ}$ N, 109.11 ${ }^{\circ} \mathrm{W}$ ]; 20 Mar. 1956; F. Werner and G. Butler leg.;

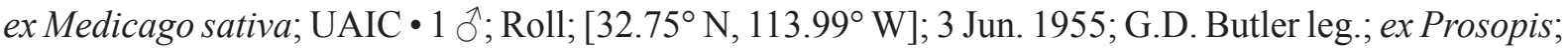

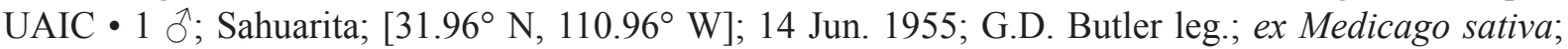
UAIC • 1 क; Sedona; [34.87 N, $111.76^{\circ}$ W]; 6 Jul. 1952; R.H. and L.D. Beamer, C. Weiner, A. Wolf, C. Liang and W. LaBerge leg.; SEMC 11 今; Tucson; [32.22 ${ }^{\circ}$ N, $110.97^{\circ}$ W]; 14 Aug. 1961; G.D.

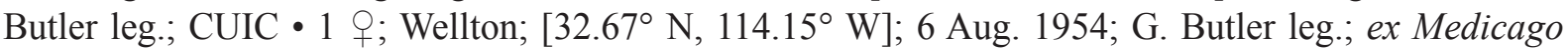
sativa; UAIC. - California • 1 क; Imperial Co., 17 mi. NW of Glamis; $\left[33.15^{\circ} \mathrm{N}, 115.3^{\circ} \mathrm{W}\right]$; 27 Jun. 1978; J. Doyen leg.; ex Croton; EMEC • 1 क ; Imperial Co., 3.5 mi. W of Gordons Wells; [32.72 ${ }^{\circ} \mathrm{N}$, $\left.115.01^{\circ} \mathrm{W}\right] ; 10$ Oct. 1957; E.I. Schlinger leg.; UCDC • 2 q 9 ; Imperial Co., Brock Agric. Res. Stn; $32.7^{\circ} \mathrm{N}, 115.0367^{\circ} \mathrm{W} ; 12$ Mar. 1994; R.R. Snelling leg.; ex Larrea tridentata; LACM • 1 q; Imperial Co., Coachella Canal Road 11.6 km NW of CA Hwy 78, then $1.0 \mathrm{~km} \mathrm{NE} ; 33.063^{\circ} \mathrm{N}, 115.25^{\circ} \mathrm{W} ; 30$ May-3 Jun. 2008; UCDC - 1 क; Imperial Co., Niland-Glamis Road 28.0 km NW of CA Hwy 78; $33.15201^{\circ} \mathrm{N}, 115.3085^{\circ} \mathrm{W} ; 10$ Feb. 2008; UCDC • 2 क 9 ; Imperial Co., Regina Siding, Niland-Glamis

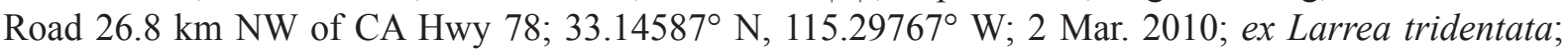
$\mathrm{UCDC} \bullet 4$ 우; same location as for preceding; 2 Mar. 2010; ex Tamarix ramosissima; UCDC • 1 ; Imperial Co., Winterhaven; [32.74 N, 114.63 W]; 25 Mar. 1956; R.R. Snelling leg.; ex Larrea tridentata; LACM • 1 क; Inyo Co., Amargosa R./Shoshone; $35.9713^{\circ} \mathrm{N}, 116.2618^{\circ} \mathrm{W} ; 457 \mathrm{~m}$ a.s.1.; 24 Apr. 2014; J.B. Runyon leg.; MTEC • 1 क; Inyo Co., China Ranch, 5 mi. SE of Tecopa; 35.79994 N,

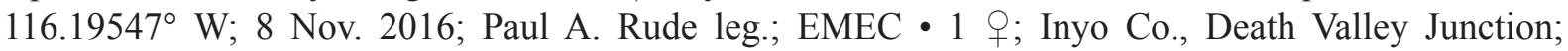
$36.3142^{\circ} \mathrm{N}, 116.4567^{\circ} \mathrm{W} ; 671 \mathrm{~m}$ a.s.1.; 19 Sep. 2013; M.C. Orr leg.; ex Cleomella obtusifolia; BBSL • 1 o; Riverside Co., Blythe; [33.62 ${ }^{\circ}$ N, $114.59^{\circ}$ W]; 5-6 Jun. 1977; E.G. and J.M. Linsley leg.; ex Parkinsonia aculeata; EMEC • 3 q ; Riverside Co., Corn Spring, Chuckwalla Mts, 7.5 mi. SE of

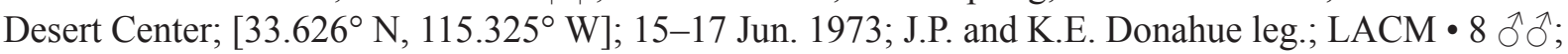
Riverside Co., Hunter's Spring; [33.49 ${ }^{\circ}$ N $115.8^{\circ} \mathrm{W}$ ]; 10 May 1952; LACM • 1 q; Riverside Co., Indio; $\left[33.72^{\circ}\right.$ N, $116.22^{\circ}$ W]; 11 Mar. 1957; E.I. Schlinger leg.; UCDC • 5 qo ; San Bernardino Co., Salt Springs, Death Valley; [36.5 N, 116.9 W]; 24 Mar. 1957; C.L. Hogue leg.; LACM • 1 q; Coachella;

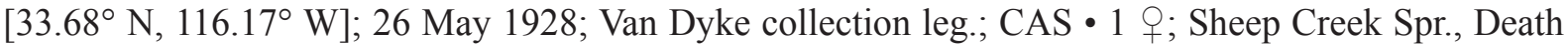
Valley; [35.589 ${ }^{\circ} \mathrm{N}, 116.36^{\circ} \mathrm{W}$; 31 May 1953; LACM. - Colorado • 1 क; Fremont Co., $0.5 \mathrm{mi}$. W of

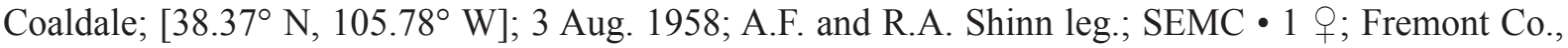
Coaldale; [38.37 $\mathrm{N}, 105.76^{\circ} \mathrm{W}$ ]; $1981 \mathrm{~m}$ a.s.l.; 11 Aug. 1964; C.D. Michener leg.; ex Cleome serrulata; 
SEMC. - Nevada - 2 + $\circ$; Clark Co., 0.1 mi. N of Corn Creek Sprs; $36.4403^{\circ} \mathrm{N}, 115.3577^{\circ} \mathrm{W}$; 896 m a.s.l.; 5 Apr. 2004; T. Griswold leg.; ex Lepidium fremontii; BBSL • 11 q ; ; Clark Co., 0.3 mi. SE of Corn Creek Spr.; $36.4365^{\circ}$ N, 115.3525 ${ }^{\circ}$ W; 901 m a.s.1.; 8 Sep. 2004; T. Griswold and S. Higbee leg.; BBSL 10 우 ; same location as for preceding; 24 Aug. 2004; S. Higbee and E. Ahlstrom leg.; BBSL • 1 क; Clark Co., 1.1 mi. NNW of Stump Spr.; $35.9991^{\circ}$ N, $115.8317^{\circ}$ W; 895 m a.s.1.; 12 May 2004; L. Saul leg.; ex Prosopis glandulosa var. torreyana; BBSL・ 1 ; ; Clark Co., 1.5 mi. NW of Corn Creek Sprs.; $36.4497^{\circ} \mathrm{N}, 115.3812^{\circ} \mathrm{W} ; 27$ May 2004; Higbee leg.; ex Prosopis glandulosa var. torreyana; BBSL • 1 ; Clark Co., Las Vegas Valley Water District; $36.1756^{\circ} \mathrm{N}, 115.1904^{\circ} \mathrm{W} ; 651 \mathrm{~m}$ a.s.1.; $10 \mathrm{Jun}$. 2004; S. Higbee and L. Saul leg.; BBSL • 1 O; Clark Co., Mesquite Valley, E.; 35.76 ${ }^{\circ}$ N, $115.5832^{\circ}$ W; 10 Aug. 1998; M. Andres, C. Shultz and T. Griswold leg.; BBSL 4 q 9 ; Lincoln Co., 15 mi. S of Alamo; $37.1937^{\circ} \mathrm{N}, 115.0379^{\circ} \mathrm{W} ; 5$ Sep. 2013; T.L. Griswold leg.; ex Cleome sp.; BBSL • 1 q; Lincoln Co., Lower Pahranagat Lake North; 37.2806 N, 115.1191 W; 5 Sep. 2013; T.L. Griswold leg.; ex

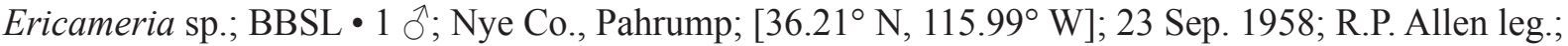
LACM. - New Mexico • 1 q; Dona Ana Co., 3 km W of Hatch; $32.6458^{\circ} \mathrm{N}, 107.192^{\circ}$ W; $1240 \mathrm{~m}$ a.s.l.;

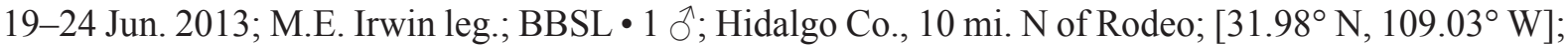
24 May 1973; P.D. Hurd, E.G. and J.M. Linsley, A.E. and M.M. Michelbacher leg.; ex Larrea tridentata; EMEC • 1 क; Hidalgo Co., 12.8 mi. N of Rodeo; $\left[32.02^{\circ} \mathrm{N}, 109.03^{\circ} \mathrm{W}\right]$; Aug. 1986; R.J. McGinley and B.M. Norden leg.; USNM • 1 ơ; Hidalgo Co., 15 km N of Rodeo; [31.97 N, 109.03 W]; 18 Jun. 1993; R.L. Minckley leg.; ex Glandularia bipinnatifida; SEMC • 1 \%; Hidalgo Co., 9 mi. N of Cotton City; $\left[32.21^{\circ} \mathrm{N}, 108.88^{\circ} \mathrm{W}\right] ; 11$ Aug. 1978; R.W. Brooks leg.; ex Wislizenia refracta; SEMC • 1 \%; Hidalgo Co., Cienega Lake; [32.3 N, 108.9 W]; 8 Aug. 1972; J. Doyen leg.; EMEC • 1 o; Hidalgo Co., Cienega Lake, N of Rodeo; [32.3 ${ }^{\circ}$ N, 108.9 ${ }^{\circ}$ W]; 3 Sep. 1967; J.G. Rozen and G. Krueger leg.; AMNH • 1 क; Hidalgo Co., Hwy 80; $32.09351^{\circ} \mathrm{N}, 108.96841^{\circ}$ W; 1367 m a.s.1.; 16 Aug. 2007; Gibbs and Sheffield leg.; PCYU • 1 क; Hidalgo Co., Rodeo vicinity; [31.84 N, $109.03^{\circ} \mathrm{W}$ ]; 11-12 Jun. 1987; B.N. Danforth leg.;

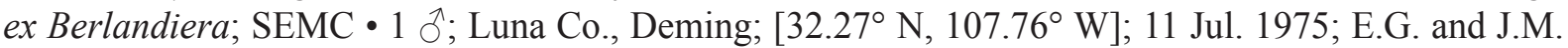
Linsley leg.; ex Chilopsis linearis; CUIC - 1 o; Sierra Co., Percha Dam State Park; $\left[32.867^{\circ} \mathrm{N}\right.$, 107.306 W]; 16 May 1986; W.J. Pulawski leg.; CAS • 2 o of; Socorro Co., Sevilleta Wildlife Refuge; $34.4162^{\circ} \mathrm{N}, 106.8027^{\circ} \mathrm{W} ; 1450 \mathrm{~m}$ a.s.1.; 19-24 Jun. 2013; M.E. Irwin leg.; BBSL • 1 \%; $10 \mathrm{mi}$. E of Portal; [31.91 $\left.{ }^{\circ} \mathrm{N}, 108.97^{\circ} \mathrm{W}\right]$; 16 Sep. 1955 ; G.E. Bohart leg.; BBSL • 1 q ; Eddy; [32.42 ${ }^{\circ} \mathrm{N}, 104.23^{\circ} \mathrm{W}$ ]; 13 Apr. year unknown; Cockerell leg.; ex Malvastrum; USNM • 1 \%; Hot Springs; [35. $\left.65^{\circ} \mathrm{N}, 105.29^{\circ} \mathrm{W}\right]$;

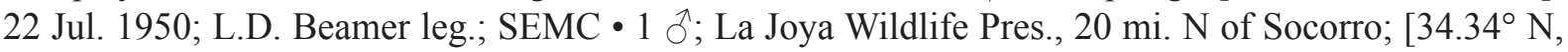
106.89 ${ }^{\circ}$ W]; 15-26 Jul. 1976; W. Rubink leg.; CUIC • 1 क; Las Cruces; [32.32 ${ }^{\circ}$ N, $106.76^{\circ}$ W]; 7 Aug. 1972; J. Ellington leg.; NMSU • 1 §ं; same location as for preceding; 3 Jul. 1940; D.E. Hardy leg.; SEMC • 1 \} \text { ; same location as for preceding; } 1 2 \text { Oct. year unknown; Cockerell leg.; USNM • } 1 \text { q; same } location as for preceding; Cockerell leg.; USNM • 1 क; Las Valles; [35.4 $\left.4^{\circ} \mathrm{N}, 105.2^{\circ} \mathrm{W}\right] ; 6$ Aug. year

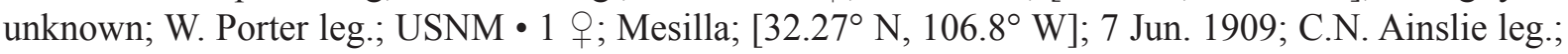
LACM • 1 q; Mesilla Park; $\left[32.27^{\circ} \mathrm{N}, 106.76^{\circ} \mathrm{W}\right] ; 6$ May year unknown; Cockerell leg.; ex Mentzelia; USNM • 1 O ; White Sands; [32.8 N, 106.3 W]; 27 Jun. 1940; R.H. Beamer leg.; SEMC. - Texas • 1 q; Brewster Co., Big Bend National Park, Rio Grande River, Santa Elena Canyon Trail; [29.165 N, $103.612^{\circ} \mathrm{W}$ ]; 2 Oct. 2001; Paul H. Arnaud Jr. and Madeline M. Arnaud leg.; CAS • 1 q, 1 §ै; Big Bend N. P., Santa Elena Can.; [29.165 N ${ }^{\circ} 103.612^{\circ}$ W]; 640 m a.s.1.; 5 May 1959; W.R.M. Mason leg.; CNC • 1 क; Santa Elena Canyon, Big Bend National Park; $\left[29.165^{\circ}\right.$ N, $103.612^{\circ}$ W]; 26 Aug. 1967; R.C. Gardner and C.R. Kovacic leg.; UCDC. - Utah - 1 万ै; Emery Co., Wild Horse Creek N of Goblin Valley; [38.57 ${ }^{\circ}$ N $\left.110.82^{\circ} \mathrm{W}\right]$; $1494 \mathrm{~m}$ a.s.1.; 21-23 Jul. 1981; Veir, Parker and Griswold leg.; CUIC • 1 ; Garfield Co., 3.9 mi. S of Horse Pasture Mesa; $37.6429^{\circ}$ N, $111.0702^{\circ}$ W; 12 Aug. 2002; C. Davidson leg.; ex Tamarix sp.; BBSL • 1 क; Garfield Co., Capitol Reef NP, Sulfur Creek; [38.3 N, 111.3 $\left.{ }^{\circ} \mathrm{W}\right] ; 26$ Jul. 1978; Lindahl leg.; BBSL・ 10 o $\circ$; Garfield Co., Star Springs Rec. Area; [37.85 ${ }^{\circ}$ N, $\left.110.66^{\circ} \mathrm{W}\right] ; 25$ May 2000; F.D. Parker leg.; BBSL • 1 क; Kane Co., Tibbet Canyon; $37.1606^{\circ}$ N, $111.5392^{\circ}$ W; 13 Jun. 2002; C. Davidson leg.; ex Stanleya pinnata; BBSL • 1 क; San Juan Co., 10 mi. SW of Blanding; [37.52 $\left.{ }^{\circ} \mathrm{N}, 109.61^{\circ} \mathrm{W}\right]$; 22 Aug. 1979; F.D. Parker leg.; BBSL • 1 q; Uintah Co., Bonanza; [40.02 ${ }^{\circ}$, 


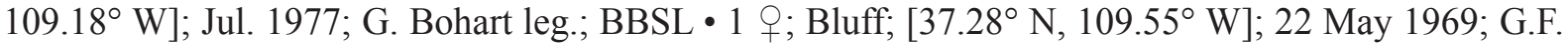
Knowlton leg.; BBSL.

\section{Redescription}

\section{Female}

Colouration. Head and mesosoma blue to blue-green; clypeus apical colour black, reddish brown, or orange; labrum black, reddish brown, or orange; mandible orange with black basal spot and red tip; flagellum black to reddish brown dorsally, reddish brown to orange ventrally; pronotal lobe reddish brown to orange; metasoma orange with dark spiracular spots on T3-4 and usually also T2; T4-5 usually darker than T1-3; legs reddish brown with femur-tibia joints, tarsi, and sometimes tibiae mostly orange; tegula pale amber; wing membrane hyaline, veins with subcosta brown, otherwise light brown to pale amber.

Pubescence. Body hair colour white. Tomentum dense on paraocular area, lower frons, gena, pronotal collar and lobe, space between pronotal lobe and tegula, scutum anterolateral margin, metepisternum, T2-3 basolaterally, and T4 throughout; sparse on supraclypeal area, scutum posterolateral margin, preëpisternum, and mesepisternum. Scutum hair thin to moderately plumose. Wing hairs dark or light, short and dense. Acarinarial fan complete, sparse. T2 fringes sparse, T3 fringes sparse.

SuRface SCUlPtURE. Clypeus punctures fine and moderately sparse in basal half ( $\mathrm{i}=1-2 \mathrm{pd})$, larger and irregular apically ( $\leq 2 \mathrm{pd}$ ), diversopunctate, sculpture shiny, sometimes weakly tessellate basally; supraclypeal area punctures sparse to moderately dense $(i=1-4 \mathrm{pd})$, sculpture shiny or tessellate; paraocular area punctures dense $(\mathrm{i}<1 \mathrm{pd})$, becoming crowded and indistinct around antenna socket, sculpture shiny, becoming imbricate and ruguloso-punctate around antenna socket; frons punctures dense $(\mathrm{i}<1 \mathrm{pd})$, sculpture shiny or weakly imbricate; vertex punctures dense laterally $(\mathrm{i}<1 \mathrm{pd})$, sparser between ocelli $(i=1-2 \mathrm{pd})$, sculpture shiny or weakly imbricate; gena punctures moderately sparse ( $\mathrm{i}=1-2 \mathrm{pd}$ ), sculpture shiny; postgena sculpture shiny to tessellate, sometimes becoming weakly rugulose posteriorly; tegula punctures absent; scutum punctures moderately sparse ( $\mathrm{i}=1-3 \mathrm{pd}$ ), sometimes becoming dense laterad of parapsidal lines and posteriorly $(i<1 \mathrm{pd})$, sculpture shiny to tessellate; scutellum punctures dense marginally and on median line ( $i \leq 1 \mathrm{pd})$, sparse submedially $(i=1-5 \mathrm{pd})$, diversopunctate, sculpture shiny, sometimes becoming tessellate marginally and on median line; metanotum sculpture shiny and finely, densely punctate $(\mathrm{i}<1 \mathrm{pd})$, becoming weakly ruguloso-punctate laterally and anteriorly; metapostnotum rugae strong, anastomosing, not reaching margin, sculpture tessellate; preëpisternum sculpture areolate-rugulose; hypoepimeron punctures dense ( $\mathrm{i} \leq 1 \mathrm{pd}$ ), obscure, sculpture weakly ruguloso-punctate; mesepisternum punctures crowded and indistinct dorsally $(\mathrm{i}=0 \mathrm{pd})$, becoming sparser ventrally $(\mathrm{i} \leq 1 \mathrm{pd})$, sculpture imbricate and ruguloso-punctate dorsally, becoming smooth and shiny ventrally; metepisternum sculpture ruguloso-lineate dorsally, finely reticulate ventrally; propodeum lateral face sculpture tessellate; propodeum posterior face sculpture tessellate with sparse, obscure punctures $(\mathrm{i}=1-3 \mathrm{pd})$; $\mathrm{T} 1$ anterior face sculpture shiny to coriarious; $\mathrm{T} 1$ dorsal surface punctures fine, sparse $(i=1-3 \mathrm{pd})$, becoming sparser apicomedially ( $i=3-6 \mathrm{pd}$ ), nearly impunctate in large apicolateral oval patches, sculpture shiny; T2 disc punctures fine, sparse ( $\mathrm{i}=1-3 \mathrm{pd})$, disc sculpture shiny, rim punctures fine, sparse $(i=1-4 \mathrm{pd})$, rim sculpture weakly coriarious.

StRUCTURE. Face length/width ratio 0.8 ( $\pm 0.03 \mathrm{SD}$ ). Clypeus projecting $\sim 50 \%$ below suborbital tangent; clypeal area length/width ratio $0.45( \pm 0.04 \mathrm{SD})$; apicolateral denticles rounded acute points; supraclypeal area length/width ratio 0.67 ( $\pm 0.04 \mathrm{SD})$. Forewing with 3 submarginal cells; pronotal angle obtuse, sometimes nearly right-angled; tegula shape normal. Intertegular distance $0.87( \pm 0.08 \mathrm{SD}) \mathrm{mm}$. Scutum length/width ratio 0.86 ( $\pm 0.03 \mathrm{SD}) ;$ scutum/scutellum length ratio $2.79( \pm 0.15 \mathrm{SD})$; scutellum/ metanotum length ratio $1.69( \pm 0.14 \mathrm{SD})$; metanotum/metapostnotum length ratio 0.61 ( $\pm 0.07 \mathrm{SD})$. 


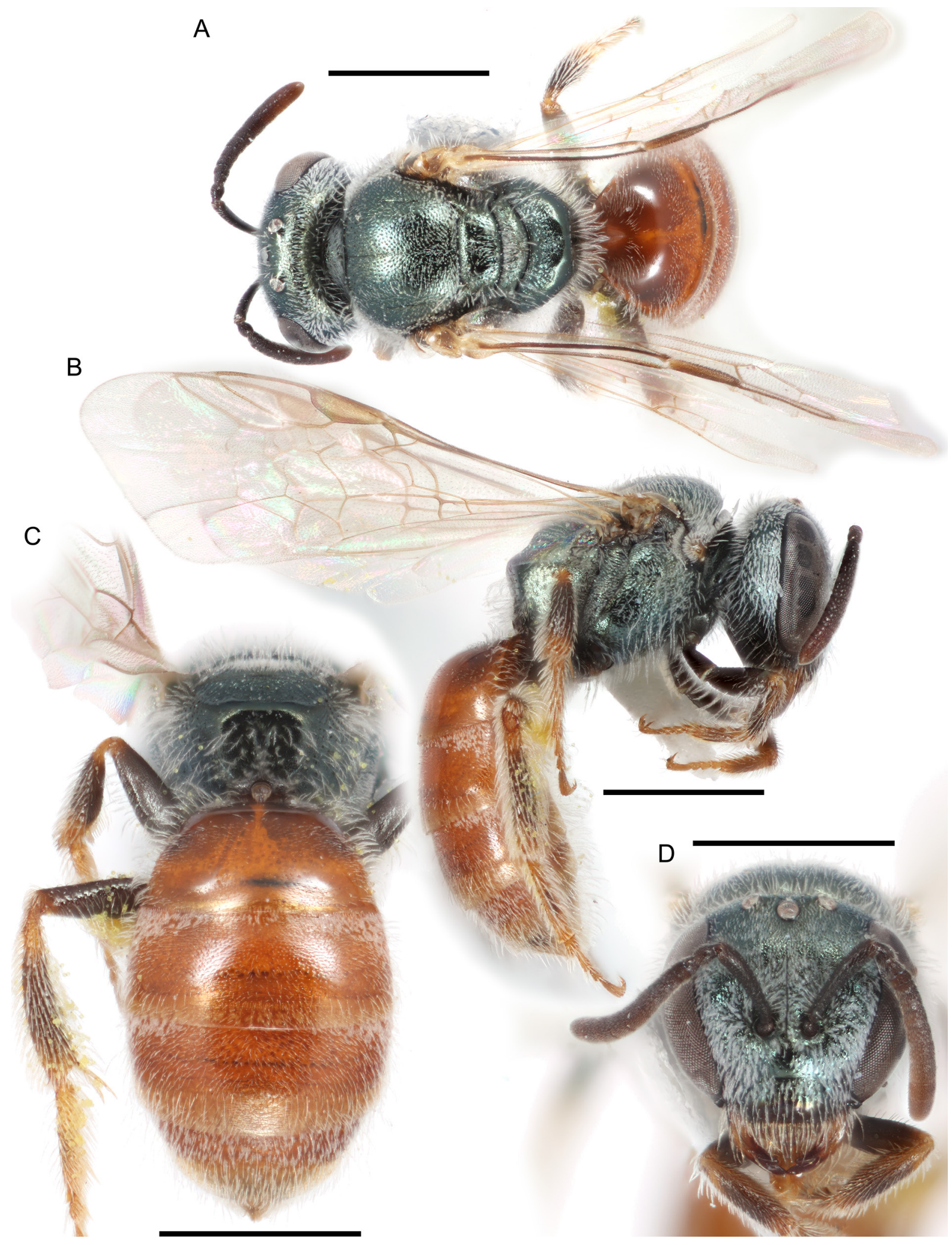

Fig. 55. Lasioglossum (D.) mesillense (Cockerell, 1898), + . A. Dorsal habitus. B. Lateral habitus. C. Propodeum and metasoma. D. Face. Scale bars: $1 \mathrm{~mm}$. 


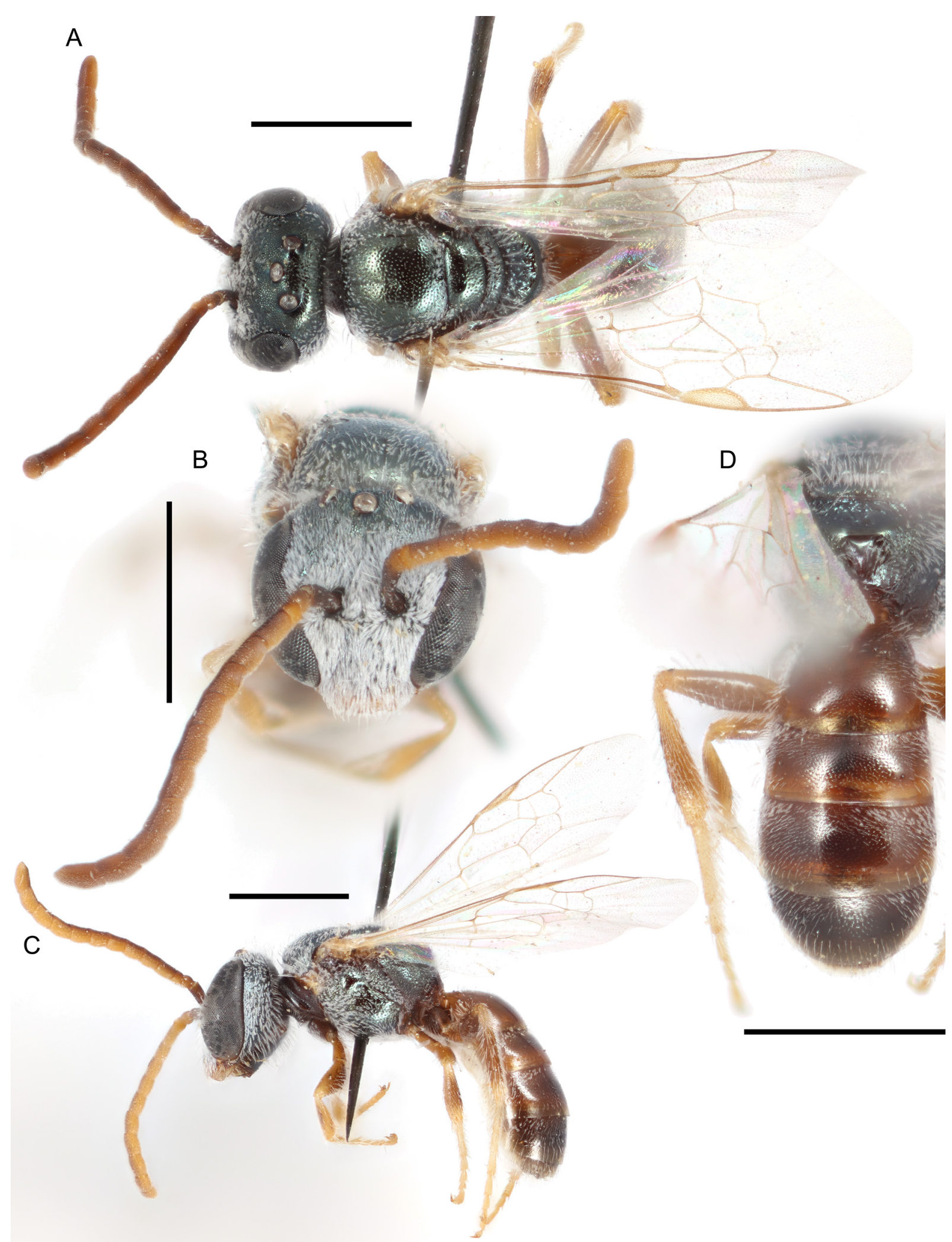

Fig. 56. Lasioglossum (D.) mesillense (Cockerell, 1898), ô. A. Dorsal habitus. B. Face. C. Lateral habitus. D. Propodeum and metasoma. Scale bars: $1 \mathrm{~mm}$. 
Propodeum lateral carinae not reaching dorsal margin; oblique carina absent. T2 depressed apical rim length less than $50 \%$ of segment. $(n=12)$

\section{Male}

Colouration. Head and mesosoma blue-green to olive green; clypeus apical colour orange to yellow; labrum orange to yellow; mandible orange to yellow with brown basal spot and red tip; flagellum reddish brown, sometimes orange ventrally; pronotal lobe orange; metasoma black to reddish brown with rims of terga and sterna and downcurved lateral areas of terga becoming orange to translucent yellow to brown, dark spiracular spots on T2-4; legs orange to yellow with coxae, trochanters, femora, and tibiae sometimes reddish brown; tegula orange; wing membrane hyaline, veins with subcosta dark brown, otherwise orange to dark brown.

Pubescence. Body hair colour white. Tomentum dense on face below ocelli, gena, pronotal collar and lobe, space between pronotal lobe and tegula, and sometimes T2-4 basolaterally; sparse on episterna and sometimes T5 basally. Scutum hair thin to densely plumose. Sterna hair short (0.75-1.25 OD), moderately plumose, dense and erect. Wing hairs dark or light, short and sparse.

SuRfaCE SCULPTURE. Clypeus punctures fine, moderately sparse ( $\mathrm{i}=1-3 \mathrm{pd})$, sculpture shiny; supraclypeal area punctures dense to moderately dense ( $\mathrm{i} \leq 2 \mathrm{pd}$ ), sculpture shiny; paraocular area punctures dense $(\mathrm{i}<1 \mathrm{pd})$, sculpture shiny; frons punctures dense $(\mathrm{i}<1 \mathrm{pd})$, sculpture shiny; vertex punctures moderately dense laterally ( $i=1-2 \mathrm{pd})$, sparse medially $(\mathrm{i}=1-4 \mathrm{pd})$, sculpture shiny; gena punctures sparse ( $i=1-3 \mathrm{pd}$ ), sculpture shiny; postgena sculpture shiny to imbricate, sometimes lineolate; tegula punctures absent; scutum punctures moderately sparse (i=1-2 pd), becoming sparse submedially ( $\mathrm{i}=1-3 \mathrm{pd}$ ), sculpture shiny, becoming tessellate anteromedially; scutellum punctures dense marginally and on median line ( $\mathrm{i} \leq 1 \mathrm{pd}$ ), sparse submedially ( $\mathrm{i}=1-4 \mathrm{pd}$ ), sculpture shiny; metanotum sculpture shiny and finely, sparsely punctate $(\mathrm{i}=1-4 \mathrm{pd})$; metapostnotum rugae strong or weak, anastomosing or subparallel, reaching or not reaching margin, sculpture shiny to imbricate; preëpisternum sculpture areolate-rugulose; hypoepimeron punctures dense ( $\mathrm{i}<1 \mathrm{pd})$, sculpture shiny; mesepisternum punctures moderately dense $(\mathrm{i}=1-2 \mathrm{pd})$, sculpture shiny; metepisternum sculpture lineate dorsally, areolate ventrally; propodeum lateral face punctures obscure, sculpture rugulose; propodeum posterior face sculpture shiny and finely, sparsely punctate $(\mathrm{i}=1-4 \mathrm{pd})$; T1 anterior face sculpture shiny; T1 dorsal surface punctures sparse ( $\mathrm{i}=1-4 \mathrm{pd})$, sculpture shiny; $\mathrm{T} 2$ disc punctures moderately sparse $(\mathrm{i}=1-3 \mathrm{pd})$, disc sculpture shiny, rim punctures sparse medially $(i=1-4 \mathrm{pd})$, absent laterally, rim sculpture shiny.

STRUCtURE. Face length/width ratio $0.84( \pm 0.03 \mathrm{SD})$. F1:pedicel length ratio $1.02( \pm 0.13 \mathrm{SD}) ; \mathrm{F} 2: \mathrm{F} 1$ length ratio $1.99( \pm 0.24 \mathrm{SD}) ; \mathrm{F} 2$ length/width ratio $1.57( \pm 0.17 \mathrm{SD}) ; \mathrm{F} 9$ length/width ratio 1.32 $( \pm 0.13 \mathrm{SD})$. Forewing with 3 submarginal cells; pronotal angle obtuse; tegula shape normal. Intertegular distance 0.88 ( $\pm 0.05 \mathrm{SD}) \mathrm{mm}$. Scutum length/width ratio 0.79 ( $\pm 0.05 \mathrm{SD})$; scutum/scutellum length ratio $3.18( \pm 0.28 \mathrm{SD})$; scutellum/metanotum length ratio 1.48 ( $\pm 0.21 \mathrm{SD})$; metanotum/metapostnotum length ratio 0.68 ( $\pm 0.07 \mathrm{SD})$. Propodeum lateral carinae not reaching dorsal margin; oblique carina absent. $(n=10)$

Genitalia. As in Fig. 90G. Gonocoxite broad, weakly truncate. Gonostylus broad, weakly concave apically, with a few long hairs. Retrorse lobe relatively small, ovoid, covered in sparse short hairs.

\section{Range}

Colorado south to Chihuahua, west to California (Fig. 57).

\section{Floral records}

AMARANTHACEAE Juss. Chenopodium L. C. neomexicanum Standl. • Salsola L. S. iberica Sennen \& Pau $\bullet$ ASTERACEAE Giseke: Baccharis: B. sarothroides A.Gray $\bullet$ Berlandiera $\bullet$ Ericameria $\bullet$ Erigeron: 
E. divergens Torr. \& A.Gray $\bullet$ Laennecia: L. coulteri (A.Gray) G.L. Nesom $\bullet$ Pectis $\bullet$ BIGONIACEAE Juss. Chilopsis D. Don: C. linearis (Cav.) Sweet • BORAGINACEAE Juss. Nama L. • Phacelia Juss. • BRASSICACEAE Burnett: Lepidium L. L. fremontii S. Watson - Physaria (Nutt. ex Torr. \& A.Gray) A.Gray: P. gordonii (A.Gray) O'Kane \& Al-Shehbaz - Stanleya Nutt. S. pinnata (Pursh) Britton • CLEOMACEAE Bercht. \& J. Presl: Cleome L. C. serrulata Pursh • Cleomella DC. C. obtusifolia Torr. \& Frém. - Wislizenia Engelm. W. refracta Engelm. EUPHORBIACEAE Juss. Croton L. • FABACEAE Juss. Medicago: M. sativa L. Melilotus: M. indicus (L.) All. (MH87) • Parkinsonia: P. aculeata L. • Prosopis: P. glandulosa Torr. P. g. var. torreyana (L.D. Benson) M.C. Johnst. • LOASACEAE Juss. Mentzelia L. M. pumila Torr. \& A.Gray • MALVACEAE Juss. Malvastrum A.Gray • ONAGRACEAE Juss. Gaura L. G. mollis E. James - SALICACEAE Mirb. Salix L. S. gooddingii C.R. Ball • TAMARICACEAE Link: Tamarix L. T. chinensis Lour. $・$ T. ramosissima Ledeb. • VERBENACEAE J. St.-Hil. Glandularia J. F. Gmel. G. bipinnatifida (Schauer) Nutt. - ZYGOPHYLLACEAE R.Br. Kallstroemia Scop. K. grandiflora Torr. ex A.Gray $\bullet$ Larrea Cav. L. tridentata (Sessé \& Moc. ex DC.) Coville.

\section{DNA barcodes}

Six sequences available (BOLD process IDs: DLII1074-07, DLIII118-18, DLIII119-18, DLIII120-18, DLIII161-19, DLIII162-19; BIN: BOLD:AAB5532). These sequences are all identical. No unique fixed substitutions distinguish $L$. mesillense from all other western red-tailed L. (Dialictus).

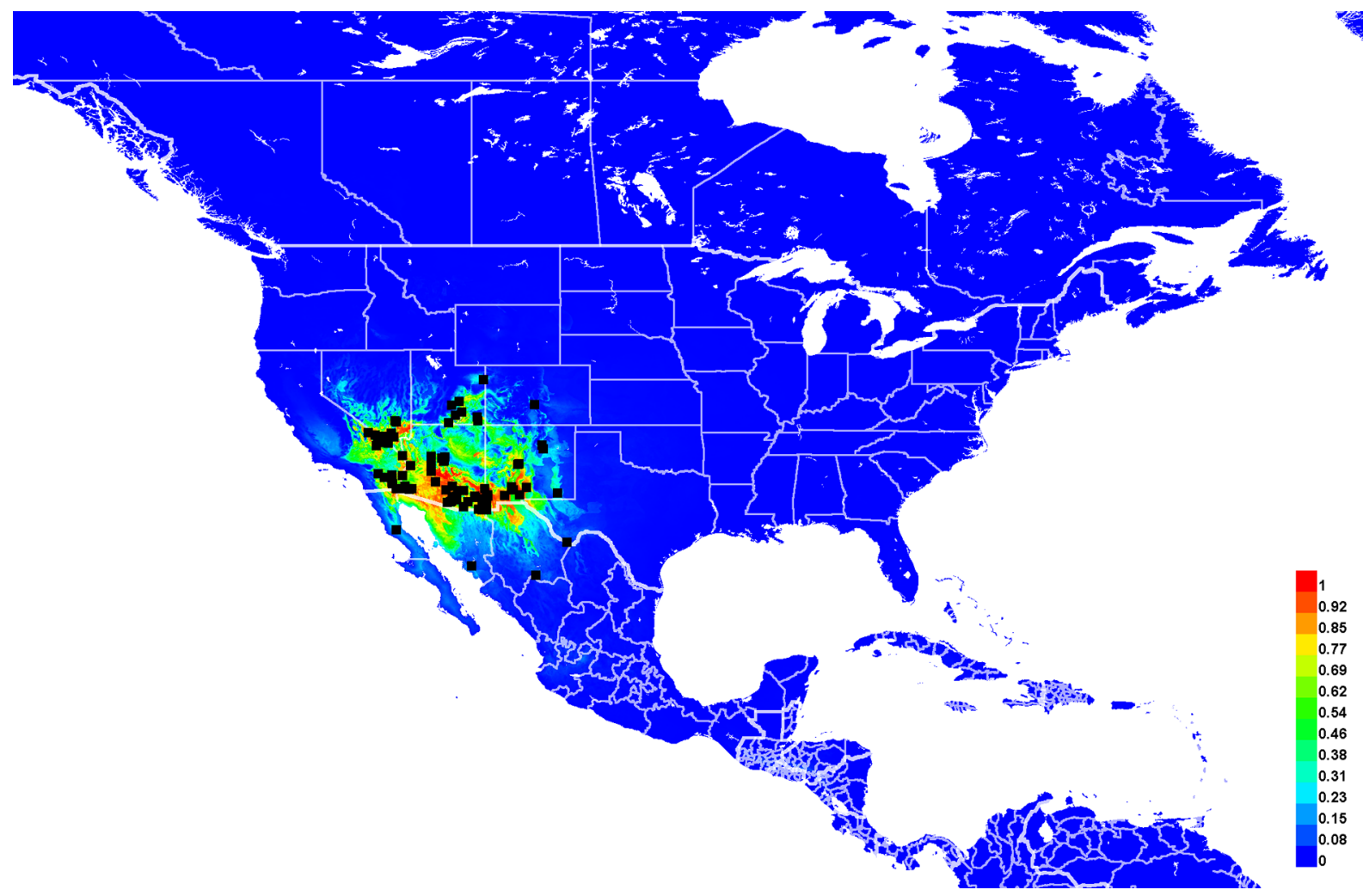

Fig. 57. Georeferenced collection records of Lasioglossum (D.) mesillense (Cockerell, 1898) (black squares) and predicted distribution by maximum entropy ecological niche modeling in Maxent (colour shading). Warmer colours indicate higher cloglog probability of occurrence. 


\section{Remarks}

There has been a great deal of confusion over the identity of L. mesillense. Neither Cockerell (1898b) nor Moure \& Hurd (1987) reported the location or number of the type specimen(s), and the original description is vague and could fit almost any red-tailed L. (Dialictus). Keys in Cockerell (1906) and Sandhouse (1924) are more helpful, but still highly prone to user error. Specimens determined as L. mesillense in various collections were a mixed series including L. hudsoniellum, L. clematisellum, and L. julipile sp. nov. in addition to the true L. mesillense.

Two specimens possibly belonging to the type series were located, one in EMEC and one in SEMC. The EMEC specimen can be assigned to the type series with greater confidence, given the collection date before 1898, the small label reading "stultus?" (Cockerell 1898b writes that L. mesillense has "formerly been recorded as H. stultus"), and the location label and second identification label with red borders. This specimen appears identical to the holotype of L. clarissimum. The SEMC specimen has labels reading "Ck1l. 2876. / on Melilotus // Las Cruces / NM // H. mesillensis Ck1l.", and is a typical L. hudsoniellum. In order to stabilize the application of the name Lasioglossum mesillense, the EMEC specimen is herein designated the lectotype.

Lasioglossum mesillense, as defined here, is morphologically variable. Most specimens have distinct punctures on the lower half of the mesepisternum, a shiny anterior surface of $\mathrm{T} 1$, and abundant tomentum on the metasomal terga. However, a few specimens can have any combination of the mesepisternum mostly ruguloso-punctate, $\mathrm{T} 1$ anterior surface coriarious, and metasomal terga with no tomentum. It is possible that there are additional cryptic species awaiting discovery, but so far there is no supporting genetic or geographic variation.

Lasioglossum (Dialictus) meteorum sp. nov. urn:lsid:zoobank.org:act:FD56C6BC-5A3F-436B-B3F2-A3DD6B50C9F1

Figs 58-60, 90H, 94B, 105B

\section{Diagnosis}

Females of Lasioglossum meteorum sp. nov. can be recognized by the head and mesosoma with very strong microsculpture, scutum with sparse punctures $(i=1-4 \mathrm{pd})$ becoming abruptly denser laterad of the parapsidal lines ( $\mathrm{i}=1-2 \mathrm{pd}$ ), frons with fine and dense but distinctly separated punctures, pronotal angle nearly 90 degrees, T1 anterior surface weakly coriarious, acarinarial fan weakly developed, and metasoma with very sparse or absent tomentum. They are most similar to L. tessellatosum sp. nov., L. decorum sp. nov., and L. austerum sp. nov. Females of L. tessellatosum sp. nov. have the frons with crowded and indistinct punctures, scutum with even stronger microsculpture obscuring the punctures, and T1 anterior surface shiny. Females of $L$. decorum sp. nov. have the scutum shinier with more uniformly sparse punctures ( $\mathrm{i}=1-3 \mathrm{pd}$ ), acarinarial fan dense, and metasomal terga with more extensive tomentum. Females of $L$. austerum sp. nov. have the scutum more densely punctate in part (i=1-3 pd), mesosoma with weaker microsculpture, and pronotal angle obtuse.

Males of L. meteorum sp. nov. can be recognized by the clypeus, supraclypeal area, and mesepisternum sparsely punctate $(i=1-4 \mathrm{pd})$, scutum very sparsely punctate and weakly tessellate $(i=1-6 \mathrm{pd})$, metasomal terga with broad impunctate rims, and clypeus lacking a non-metallic apical band. They are most similar to L. tessellatosum sp. nov. Males of L. tessellatosum sp. nov. have the clypeus and mesepisternum densely punctate ( $\mathrm{i} \leq 1 \mathrm{pd})$, scutum with strong dull microsculpture, and clypeus with a very narrow non-metallic black apical band. 


\section{Etymology}

The specific epithet meteorum is formed from the Latinized Greek adjective 'meteoros' ('lifted up', 'in air'). It refers to the fiery red colouration of the metasoma and this species' proximity to the Chicxulub meteor impact crater. An appropriate translation would be the meteor sweat bee.

\section{Material examined}

\section{Holotype}

MEXICO - Yucatan • 9 ; Merida, $15 \mathrm{~km}$ S of University of Yucatan; $\left[20.83^{\circ} \mathrm{N}, 89.62^{\circ} \mathrm{W}\right] ; 7$ Apr. 1997; R. Brooks leg.; ex Piscidia piscipula; SEMC.

[Verbatim label: MEXICO: Yucatan / Merida, 15 km S / University of Yucatan / 7 APR 1997; R.Brooks / MEX 1B 01 / ex: Piscidia piscipula // SM0105926 / KUNHM-ENT // HOLOTYPE / Lasioglossum (Dialictus) meteorum Gardner and Gibbs]

\section{Paratypes}

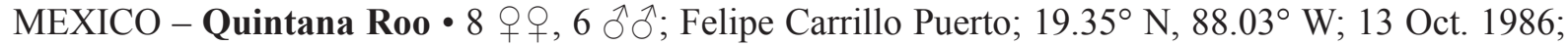
T.L. Griswold and F.D. Parker leg.; ex Mentha sp.; BBSL 15 우영 same location as for preceding; 10-14 Oct. 1986; BBSL 1 क ; 25 km W of Felipe Carrillo Puerto; $19.35^{\circ}$ N, 88.17 W; 15 Oct. 1986;

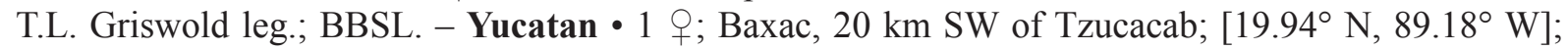
12 Feb. 1987; C. Michener leg.; ex Viguiera dentata; SEMC.

\section{Description}

\section{Female}

Colouration. Head and mesosoma blue-green to golden-green; clypeus apical colour black, reddish brown, or orange; labrum reddish brown to orange; mandible orange with black basal spot or band and red tip; flagellum black to dark reddish brown dorsally, slightly lighter ventrally; pronotal lobe reddish brown to orange; metasoma orange to reddish brown with dark spiracular spots on T3-4 and sometimes T2; legs reddish brown with femur-tibia joints, tarsi, and sometimes tibiae mostly orange; tegula orange; wing membrane infuscated, veins brown to dark brown.

Pubescence. Body hair colour golden to white. Tomentum dense on pronotal angle and lobe and space between pronotal lobe and tegula; sparse on paraocular area, gena, metepisternum, T2-3 basolaterally, and T4 throughout. Scutum hair thin. Wing hairs dark, long and dense. Acarinarial fan incomplete, sparse. T2 fringes sparse, T3 fringes sparse.

SURFACE SCULPTURE. Clypeus punctures sparse $(\mathrm{i}=1-4 \mathrm{pd})$, sculpture shiny in apical half or more, tessellate basally; supraclypeal area punctures sparse $(i=1-4 \mathrm{pd})$, sculpture tessellate, sometimes shiny medially; paraocular area punctures dense $(i \leq 1 \mathrm{pd})$, sculpture shiny ventrally and in narrow line adjacent to compound eye, otherwise tessellate; frons punctures crowded ( $\mathrm{i}=0 \mathrm{pd})$, sometimes indistinct above antenna, sculpture imbricate, sometimes becoming weakly rugulose above antenna; vertex punctures fine, dense to moderately dense laterally ( $i \leq 2 \mathrm{pd}$ ), sparse medially ( $\mathrm{i}=1-3 \mathrm{pd})$, often obscure, sculpture imbricate, sometimes shiny medially; gena punctures fine, sparse ( $i=1-3 \mathrm{pd})$, sculpture shiny, becoming tessellate and sometimes ruguloso-lineate posteriorly and ventrally; postgena sculpture tessellate and sometimes ruguloso-lineate; tegula punctures absent; scutum punctures sparse ( $\mathrm{i}=1-4 \mathrm{pd}$ ), abruptly becoming moderately dense laterad of parapsidal line $(i=1-2 \mathrm{pd})$, sculpture tessellate to finely reticulate; scutellum punctures sparse $(i=1-5 \mathrm{pd})$, sculpture tessellate to finely reticulate; metanotum sculpture tessellate and finely rugulose; metapostnotum rugae strong, anastomosing, not reaching margin, sculpture tessellate to finely reticulate; preëpisternum sculpture finely reticulate and densely but obscurely punctate $(i \leq 1 \mathrm{pd})$ or rugulose; hypoepimeron punctures dense $(\mathrm{i}<1 \mathrm{pd})$, obscure, sculpture finely reticulate; mesepisternum punctures moderately dense $(i=1-2 \mathrm{pd})$, sculpture finely reticulate; metepisternum sculpture lineate dorsally, ruguloso-punctate ventrally; propodeum lateral face sculpture 
tessellate; propodeum posterior face sculpture tessellate; T1 anterior face sculpture weakly coriarious; T1 dorsal surface punctures fine, sparse $(i=2-4 \mathrm{pd})$, absent in large apicolateral oval patches, rim medially, and sometimes median line, sculpture shiny; T2 disc punctures fine, sparse ( $i=2-4 \mathrm{pd})$, disc sculpture shiny to weakly coriarious, rim punctures absent, rim sculpture coriarious.

STRUCTURE. Face length/width ratio $0.82( \pm 0.03 \mathrm{SD})$. Clypeus projecting $\sim 50 \%$ below suborbital tangent; clypeal area length/width ratio 0.47 ( $\pm 0.02 \mathrm{SD})$; apicolateral denticles rounded knobs; supraclypeal area length/width ratio 0.66 ( $\pm 0.05 \mathrm{SD})$. Forewing with 3 submarginal cells; pronotal angle slightly obtuse, nearly right-angled; tegula shape normal. Intertegular distance $0.88( \pm 0.07 \mathrm{SD}) \mathrm{mm}$. Scutum length/ width ratio $0.77( \pm 0.02 \mathrm{SD})$; scutum/scutellum length ratio $3.14( \pm 0.24 \mathrm{SD})$; scutellum/metanotum length ratio 1.5 ( $\pm 0.11 \mathrm{SD})$; metanotum/metapostnotum length ratio 0.7 ( $\pm 0.06 \mathrm{SD})$. Propodeum lateral carinae not reaching dorsal margin; oblique carina absent. T2 depressed apical rim length less than $50 \%$ of segment. $(n=10)$

\section{Male}

Colouration. Head and mesosoma blue-green to golden-green; clypeus apical colour concolourous with base; labrum black to reddish brown; mandible orange with black basal band and red tip; flagellum black to reddish brown dorsally, orange to brown ventrally; pronotal lobe reddish brown; metasoma black to reddish brown; legs black to reddish brown with femur-tibia joints, base and apex of tibiae, and tarsi orange to brown; tegula orange to brown; wing membrane hyaline to infuscated, veins brown to dark brown.

PuBESCEnCE. Body hair colour pale yellow to white. Tomentum dense on paraocular area, lower half of frons, and pronotal angle and lobe; sparse on clypeus, supraclypeal area, and gena. Scutum hair thin to moderately plumose. Sterna hair moderately long (1-2 OD), densely plumose, sparse and erect. Wing hairs dark, short and dense.

SuRfaCe SCULPTURE. Clypeus punctures sparse ( $\mathrm{i}=1-4 \mathrm{pd})$, sculpture shiny; supraclypeal area punctures sparse ( $\mathrm{i}=1-4 \mathrm{pd})$, sculpture shiny to tessellate; paraocular area punctures moderately dense $(\mathrm{i}=1-2 \mathrm{pd})$, sculpture shiny; frons punctures crowded $(\mathrm{i}=0 \mathrm{pd})$, becoming shallow and indistinct above antenna, sculpture reticulate-rugulose, becoming shiny to imbricate laterally; vertex punctures dense laterally ( $\mathrm{i} \leq 1 \mathrm{pd}$ ), sparse medially ( $\mathrm{i}=1-3 \mathrm{pd}$ ), sculpture imbricate, sometimes shiny medially; gena punctures sparse ( $\mathrm{i}=1-3 \mathrm{pd})$, sculpture shiny, sometimes becoming weakly imbricate and weakly lineate posteriorly and ventrally; postgena sculpture shiny, becoming weakly imbricate and weakly lineate posteriorly and laterad of hypostomal carina; tegula punctures absent; scutum punctures sparse ( $\mathrm{i}=1-6 \mathrm{pd}$ ), abruptly becoming moderately dense laterad of parapsidal line ( $\mathrm{i}=1-2 \mathrm{pd})$, sculpture shiny to weakly tessellate, becoming more strongly tessellate anteromedially; scutellum punctures sparse ( $i=1-6 \mathrm{pd})$, sculpture shiny to weakly tessellate; metanotum sculpture shiny to weakly imbricate and weakly rugulosopunctate; metapostnotum rugae strong or weak, anastomosing or subparallel, nearly reaching margin, sculpture shiny to imbricate; preëpisternum sculpture areolate-rugulose to rugose; hypoepimeron punctures moderately dense $(\mathrm{i}=1-2 \mathrm{pd})$, sculpture shiny to finely reticulate; mesepisternum punctures moderately sparse ( $\mathrm{i}=1-3 \mathrm{pd}$ ), sculpture shiny to finely reticulate; metepisternum sculpture lineate dorsally, weakly rugulose ventrally; propodeum lateral face punctures obscure, sculpture tessellate to weakly rugulose; propodeum posterior face sculpture shiny to imbricate; T1 anterior face sculpture shiny; T1 dorsal surface punctures sparse $(\mathrm{i}=1-4 \mathrm{pd})$, absent or very sparse in large apicolateral oval patches and on rim, sculpture shiny; $\mathrm{T} 2$ disc punctures sparse $(\mathrm{i}=1-3 \mathrm{pd})$, disc sculpture shiny, rim punctures absent, rim sculpture shiny.

STRUCTURE. Face length/width ratio 0.87 ( $\pm 0.03 \mathrm{SD})$. F1:pedicel length ratio 1.14 ( $\pm 0.33 \mathrm{SD}) ; \mathrm{F} 2: \mathrm{F} 1$ length ratio $1.86( \pm 0.39 \mathrm{SD}) ; \mathrm{F} 2$ length/width ratio 1.53 ( $\pm 0.3 \mathrm{SD}) ; \mathrm{F} 9$ length/width ratio 1.21 $( \pm 0.07 \mathrm{SD})$. Forewing with 3 submarginal cells; pronotal angle slightly obtuse, nearly right-angled; tegula shape normal. Intertegular distance $0.72( \pm 0.03 \mathrm{SD}) \mathrm{mm}$. Scutum length/width ratio 0.79 


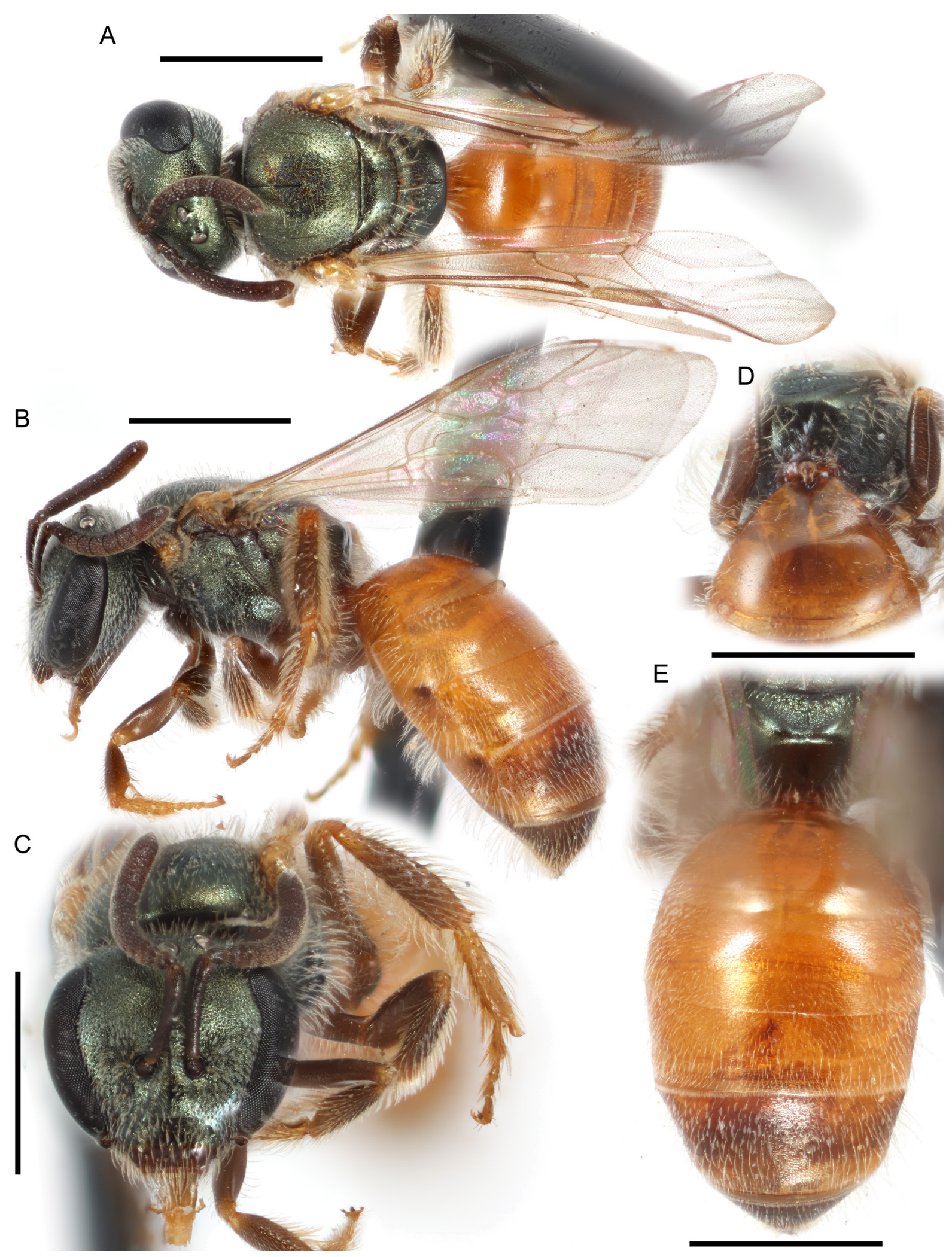

Fig. 58. Lasioglossum (D.) meteorum sp. nov., ․ A. Dorsal habitus. B. Lateral habitus. C. Face. D. Propodeum. E. Metasoma. Scale bars: $1 \mathrm{~mm}$. 


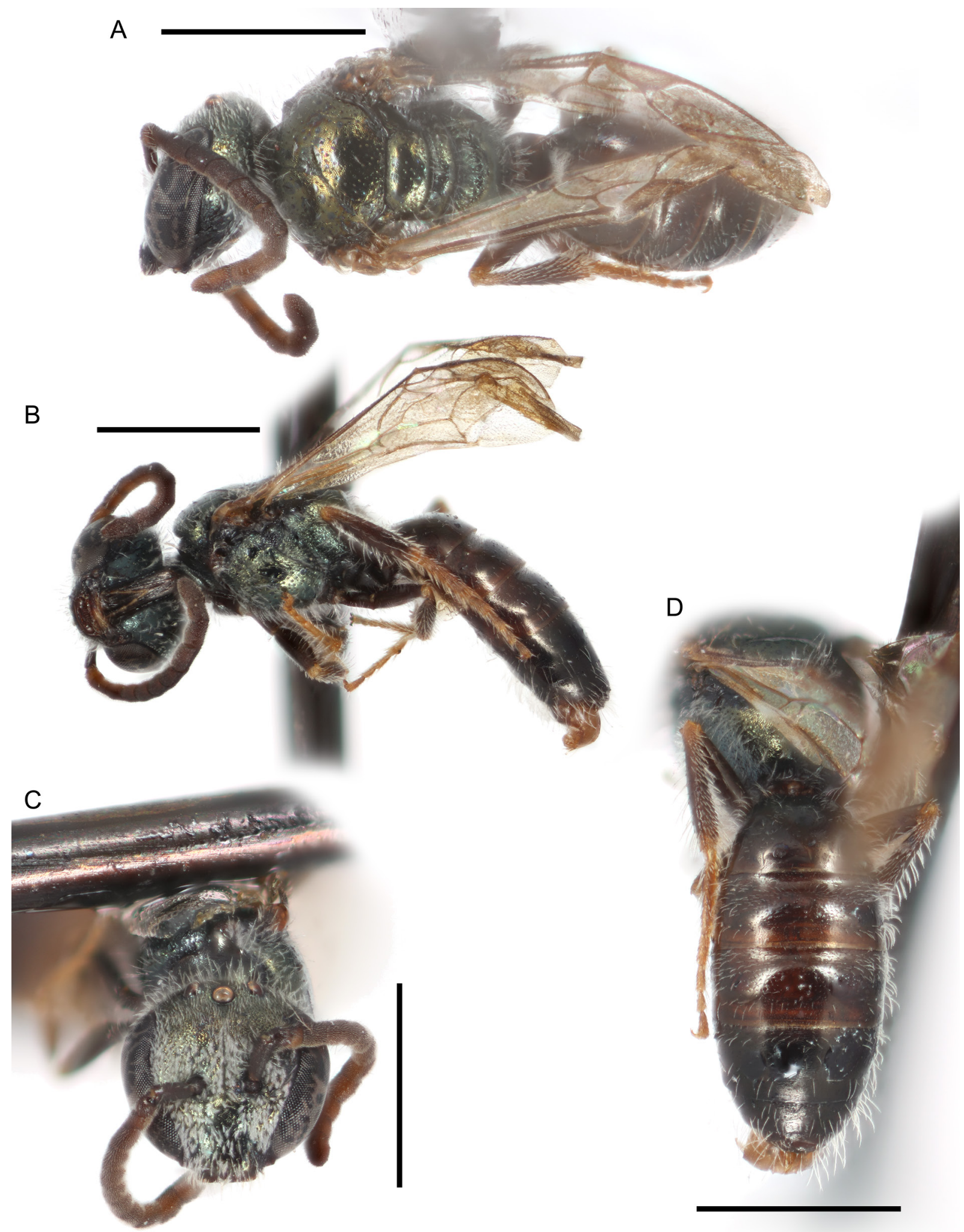

Fig. 59. Lasioglossum (D.) meteorum sp. nov., Ô. A. Dorsal habitus. B. Lateral habitus. C. Face. D. Metasoma. Scale bars: $1 \mathrm{~mm}$. 
( $\pm 0.03 \mathrm{SD}) ;$ scutum/scutellum length ratio 2.96 ( $\pm 0.23 \mathrm{SD})$; scutellum/metanotum length ratio 1.41 ( $\pm 0.11 \mathrm{SD})$; metanotum/metapostnotum length ratio 0.71 ( $\pm 0.07 \mathrm{SD})$. Propodeum lateral carinae not reaching dorsal margin; oblique carina absent. $(n=6)$

Genitalia. As in Fig. 90H. Gonocoxite broad, weakly truncate. Gonostylus broad, with a few long hairs. Retrorse lobe very broad, ovoid, covered in sparse short hairs.

\section{Range}

Yucatan and Quintana Roo (Fig. 60).

\section{Floral records}

ASTERACEAE Giseke: Viguiera: V. dentata (Cav.) Spreng. - FABACEAE Juss. Piscidia: P. piscipula (L.) Sarg. • LAMIACEAE Martinov: Mentha L.

\section{DNA barcodes}

Not available.

\section{Remarks}

Lasioglossum meteorum sp. nov. is apparently an exclusively Neotropical species endemic to the Yucatan Peninsula. Due to its close similarity to the much more widely distributed L. tessellatosum sp. nov., it seems advisable to describe it here to avoid future confusion between the two.

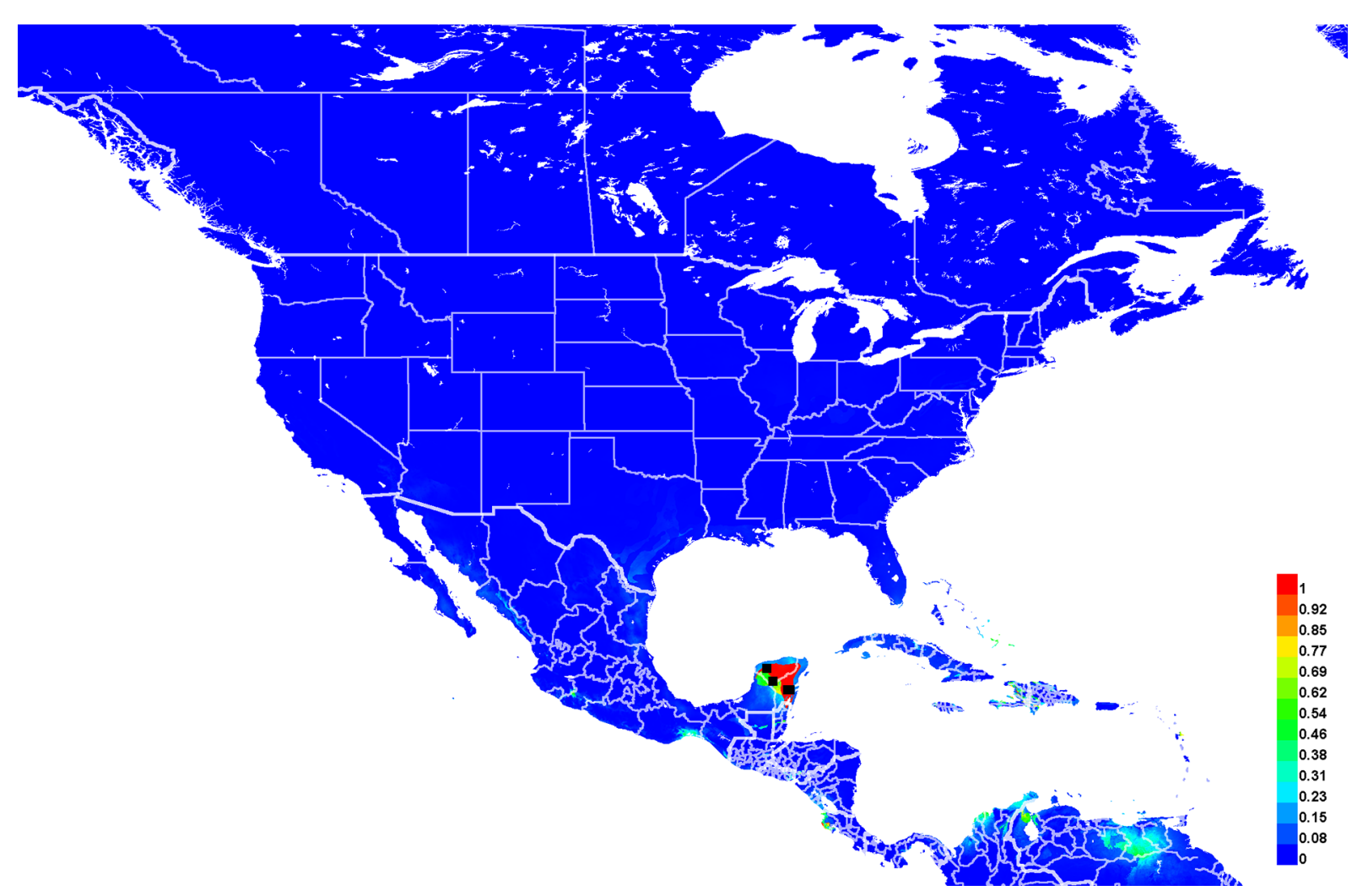

Fig. 60. Georeferenced collection records of Lasioglossum (D.) meteorum sp. nov. (black squares) and predicted distribution by maximum entropy ecological niche modeling in Maxent (colour shading). Warmer colours indicate higher cloglog probability of occurrence. 
Lasioglossum (Dialictus) miltolepoides sp. nov. urn:lsid:zoobank.org:act:93B5EE64-174D-455C-B8C2-10F6BA154B6A

Figs 61-63, 90I, 97B, 103B, 113A

\section{Diagnosis}

Females of Lasioglossum miltolepoides sp. nov. can be recognized by the acarinarial fan absent, T1 anterior surface with sparse erect hairs, mesepisternum rugulose, metasoma partially or entirely reddish brown, and ocelli normal. They are most similar to Lasioglossum microlepoides (Ellis, 1914) and L. eophilus. Females of L. microlepoides have the metasoma metallic green, scutum with stronger dull microsculpture, and metasomal terga with less extensive tomentum. Females of L. eophilus have the ocelli enlarged (separated by about $0.5 \mathrm{OD}$ ), mesepisternum strongly rugose, acarinarial fan sparse but present, and metasomal terga with more extensive tomentum.

Males of L. miltolepoides sp. nov. can be recognized by the clypeus shiny and sparsely punctate $(\mathrm{i}=1-4 \mathrm{pd})$ and without a non-metallic apical band, face entirely lacking tomentum, mesepisternum often dull or rugulose, $\mathrm{T} 1$ anterior surface with abundant erect hair and sparse appressed hair, metasomal sterna with relatively short hair (1-1.5 OD), and metasoma mostly dark. They are most similar to L. microlepoides, L. rufornatum sp. nov., and L. minckleyi sp. nov. Males of L. microlepoides have the metasoma metallic green. Males of L. rufornatum sp. nov. have the metasomal sterna with long hair (2-4 OD), face and metasomal terga with dense tomentum, T1 anterior surface with erect hair limited to lateral margins, and metasomal terga metallic at least in part. Males of L. minckleyi sp. nov. have the face below eye emargination covered in dense tomentum, clypeus apical margin reddish brown to orange and not metallic, and $\mathrm{T} 1$ anterior surface with erect hair limited to lateral margins.

\section{Etymology}

The specific epithet miltolepoides is formed from the Greek adjective 'miltos' ('red earth'), the noun 'lepas' ('rock'), and the suffix '-oides' (indicating resemblance). The first part of the name is a reference to the earthy-red colour of the metasoma and the rocks typical of this species' habitat. The latter part of the name is a reference to the very similar species, Lasioglossum microlepoides (Ellis, 1914), of unknown etymology but probably similar to that given here. An appropriate translation would be the red rock sweat bee.

\section{Material examined}

\section{Holotype}

UNITED STATES - Utah • +; Kane Co., Tibbet Canyon, nr JCS; $37.1606^{\circ} \mathrm{N}, 111.5392^{\circ} \mathrm{W} ; 26$ Jun. 2002; J. Wilson leg.; ex Tamarix sp.; PCYU.

[Verbatim label: USA UT Kane Co. / Tibbet Cyn 12S E452127 N4112825 nr JCS // Tamarix sp. // 26 June 2002 / J. Wilson // 2374A09 // NativeBeeSurvey / USA,Logan,Utah / BBSL439722 // HOLOTYPE / Lasioglossum (Dialictus) miltolepoides Gardner and Gibbs]

\section{Paratypes}

UNITED STATES - Arizona - 1 क; Grand Canyon Mile 0; $\left[36^{\circ} \mathrm{N}, 112^{\circ} \mathrm{W}\right] ; 5$ Jun. 1953; G.D.

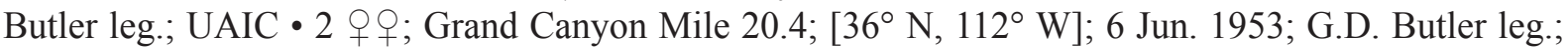
UAIC. - California • 1 क; Inyo Co., 5 mi. W of Big Pine; [37.16 N, $118.38^{\circ} \mathrm{W}$ ]; 15 Jun. 1999; J.S.

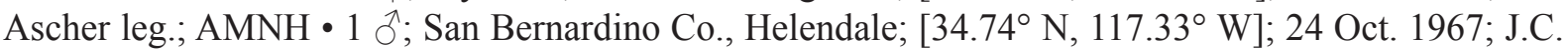
Hall leg.; ex Chrysothamnus; UCRC • 1 क; Twentynine Palms; [34.14 $\left.{ }^{\circ} \mathrm{N}, 116.05^{\circ} \mathrm{W}\right]$; 4 Aug. 1933;

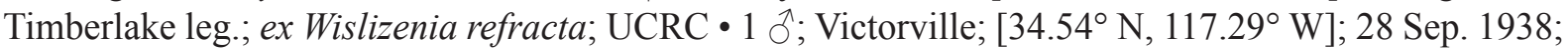
Timberlake leg.; ex Baccharis salicina; UCRC. - Nevada • 4 우; Clark Co., Lee Canyon; $36.4476^{\circ} \mathrm{N}$, $115.5097^{\circ}$ W; 1252 m a.s.l.; 7 Sep. 2004; S. Higbee and T. Griswold leg.; BBSL • 1 \%; 4 mi. E of 
Glendale; [36.66 ${ }^{\circ}$ N, $114.49^{\circ} \mathrm{W}$; 20 Jun. 1939; Timberlake leg.; ex Baileya pauciradiata; UCRC. -

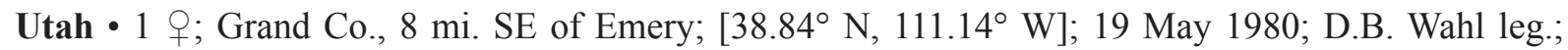
SEMC • 1 q ; Kane Co., Tibbet Canyon, nr JCS; $37.1606^{\circ}$ N, $111.5392^{\circ}$ W; 26 Jun. 2002; S. Higbee leg.;

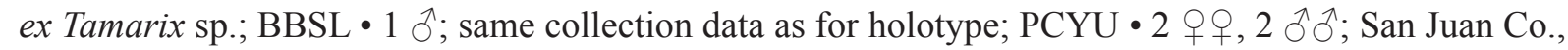
10 mi. NNW of Mexican Hat, Moki Dugway, nr base; $37.27817^{\circ}$ N, $109.94357^{\circ}$ W; 1637 m a.s.1.; 20

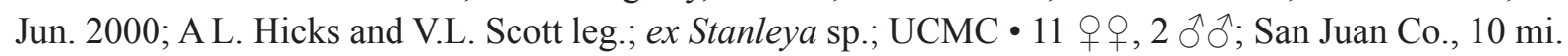
NNW of Mexican Hat, Moki Dugway near base; 37.2781 ${ }^{\circ} \mathrm{N}, 109.94357^{\circ} \mathrm{W} ; 1637 \mathrm{~m}$ a.s.1.; 20 Jun. 2000; A.L. Hicks and Virginia Scott leg.; ex Stanleya; UCMC • 1 क; San Juan Co., Moki Dugway, ca 10 mi. NNW of Mexican Hat; $37.27817^{\circ} \mathrm{N}, 109.94357^{\circ} \mathrm{W} ; 1637 \mathrm{~m}$ a.s.1.; 20 Jun. 2000; A.L. Hicks and V.L. Scott leg.; ex Stanleya sp.; WRME • 1 क; Wayne Co., 2 mi. W of Caineville; [38.33 N, $111.06^{\circ} \mathrm{W}$ ]; 28 Jun. 1999; L. Packer leg.; PCYU.

\section{Description}

\section{Female}

Colouration. Head and mesosoma blue-green to golden-green; clypeus apical colour black to reddish brown; labrum reddish brown to orange; mandible orange with black basal spot or band and red tip; flagellum reddish brown dorsally, reddish brown to orange ventrally; pronotal lobe black to reddish brown; metasoma orange to black with rims of terga and sterna broadly translucent yellow, dark spiracular spots on T2-4; legs reddish brown with femur-tibia joints, base and apex of tibiae, and tarsi orange; tegula orange to brown; wing membrane hyaline or lightly infuscated, veins with subcosta dark brown, otherwise pale amber to brown.

Pubescence. Body hair colour pale yellow to white. Tomentum dense on gena, pronotal collar and lobe, space between pronotal lobe and tegula, metanotum medially, T2-3 basolaterally, and T4 throughout; sparse on paraocular area. Scutum hair thin to moderately plumose. Wing hairs dark or light, short and dense. Acarinarial fan absent. T2 fringes sparse, T3 fringes sparse.

Surface sculpture. Clypeus punctures sparse $(i=1-3 \mathrm{pd})$, sculpture shiny, sometimes becoming tessellate basally; supraclypeal area punctures sparse $(i=1-3 \mathrm{pd})$, sculpture tessellate, usually becoming shiny medially; paraocular area punctures dense $(i \leq 1 \mathrm{pd})$, sculpture tessellate to imbricate around antenna socket, otherwise shiny; frons punctures crowded ( $\mathrm{i}=0 \mathrm{pd})$, often indistinct, sculpture areolaterugulose, sometimes becoming smooth and shiny dorsally; vertex punctures dense to moderately sparse laterally ( $\mathrm{i}<2 \mathrm{pd}$ ), often indistinct, fine and sparse medially ( $\mathrm{i}=1-4 \mathrm{pd}$ ), sculpture shiny, usually imbricate to rugulose laterally; gena punctures moderately dense ( $i=1-2 \mathrm{pd})$, sculpture shiny, sometimes becoming weakly rugulose posteriorly and ventrally; postgena sculpture shiny, becoming tessellate to imbricate posteriorly; tegula punctures absent; scutum punctures dense ( $\mathrm{i} \leq 1 \mathrm{pd})$, becoming moderately sparse submedially and anteromedially $(i=1-3 \mathrm{pd})$, sculpture tessellate, sometimes becoming shiny posterolaterally; scutellum punctures dense marginally and on median line $(\mathrm{i}<1 \mathrm{pd})$, moderately sparse submedially ( $\mathrm{i}=1-2 \mathrm{pd}$ ), diversopunctate, sculpture shiny to imbricate; metanotum sculpture imbricate to weakly rugulose, becoming finely, densely punctate and sometimes shiny medially; metapostnotum rugae strong or weak, anastomosing to subparallel, reaching or nearly reaching margin, sculpture imbricate to weakly tessellate; preëpisternum sculpture imbricate to rugulose; hypoepimeron punctures crowded ( $\mathrm{i}=0 \mathrm{pd}$ ), indistinct, sculpture imbricate to rugulose; mesepisternum punctures moderately sparse ( $\mathrm{i}=1-2 \mathrm{pd})$, indistinct, sculpture imbricate to rugulose; metepisternum sculpture ruguloso-lineate dorsally, imbricate ventrally; propodeum lateral face sculpture tessellate; propodeum posterior face sculpture tessellate; T1 anterior face sculpture shiny; T1 dorsal surface punctures fine, sparse ( $\mathrm{i}=1-4 \mathrm{pd})$, absent or very sparse in large apicolateral oval patches, sculpture shiny; T2 disc punctures moderately dense $(i=1-3 \mathrm{pd})$, disc sculpture shiny, rim punctures moderately dense $(i=1-3 \mathrm{pd})$, rim sculpture shiny. 
Structure. Face length/width ratio 0.85 ( $\pm 0.01 \mathrm{SD})$. Clypeus projecting $\sim 67 \%$ below suborbital tangent; clypeal area length/width ratio $0.44( \pm 0.03 \mathrm{SD})$; apicolateral denticles low rounded knobs; supraclypeal area length/width ratio $0.86( \pm 0.09 \mathrm{SD})$. Forewing with 3 submarginal cells; pronotal angle obtuse; tegula shape normal. Intertegular distance $0.96( \pm 0.06 \mathrm{SD}) \mathrm{mm}$. Scutum length/width ratio $0.76( \pm 0.04 \mathrm{SD}) ;$ scutum/scutellum length ratio 2.89 ( $\pm 0.1 \mathrm{SD}) ;$ scutellum/metanotum length ratio $1.58( \pm 0.13 \mathrm{SD})$; metanotum/metapostnotum length ratio 0.64 ( $\pm 0.04 \mathrm{SD})$. Propodeum lateral carinae not reaching dorsal margin; oblique carina absent. T2 depressed apical rim length less than 50\% of segment. $(\mathrm{n}=10)$

\section{Male}

Colouration. Head and mesosoma blue to olive green; clypeus apical colour concolourous with base; labrum black, reddish brown, to orange; mandible orange with black basal band and red tip; flagellum reddish brown dorsally, red to orange ventrally; pronotal lobe reddish brown; metasoma black to reddish brown with rims of terga and sterna and downcurved lateral areas of terga becoming orange to translucent yellow; legs reddish brown with femur-tibia joints, base and apex of tibiae, and tarsi orange; tegula orange; wing membrane hyaline, veins with subcosta dark brown, otherwise brown to light brown.

PubesCence. Body hair colour white. Tomentum sparse on gena, pronotal angle and lobe, space between pronotal lobe and tegula, and T2-4 basolaterally. Scutum hair densely plumose. Sterna hair short (1-1.5 OD), moderately plumose, sparse and erect. Wing hairs dark or light, long and sparse.

SuRFACE SCULPTURE. Clypeus punctures sparse ( $\mathrm{i}=1-4 \mathrm{pd})$, sculpture shiny; supraclypeal area punctures moderately sparse ( $\mathrm{i}=1-2 \mathrm{pd}$ ), sculpture shiny to imbricate; paraocular area punctures moderately dense $(\mathrm{i}=1-2 \mathrm{pd})$, sculpture imbricate around antenna socket, otherwise shiny; frons punctures crowded $(\mathrm{i}=0 \mathrm{pd})$, sculpture shiny to imbricate, becoming rugulose above antenna; vertex punctures dense laterally ( $i \leq 1 \mathrm{pd}$ ), slightly sparser medially ( $\mathrm{i}=1-2 \mathrm{pd}$ ), sculpture shiny, sometimes imbricate laterally; gena punctures sparse $(i=1-3 \mathrm{pd})$, sculpture shiny, becoming imbricate and weakly ruguloso-lineate posteriorly and ventrally; postgena sculpture imbricate and weakly ruguloso-lineate, sometimes becoming shiny anteriorly; tegula punctures absent; scutum punctures sparse $(i=1-4 \mathrm{pd})$, becoming moderately dense marginally ( $i=1-2 \mathrm{pd}$ ), sculpture shiny, sometimes becoming tessellate anteromedially; scutellum punctures sparse $(i=1-4 \mathrm{pd})$, sometimes dense marginally and on median line ( $\mathrm{i}<1 \mathrm{pd}$ ), sculpture shiny; metanotum sculpture shiny and moderately sparsely punctate $(i=1-2 \mathrm{pd})$; metapostnotum rugae strong, anastomosing, reaching margin, sculpture shiny; preëpisternum sculpture areolate to rugulose; hypoepimeron punctures dense $(\mathrm{i}<1 \mathrm{pd})$, sometimes indistinct, sculpture shiny to imbricate or rugulose; mesepisternum punctures dense ( $\mathrm{i} \leq 1 \mathrm{pd}$ ), sculpture shiny to imbricate to rugulose; metepisternum sculpture lineate dorsally, becoming smooth and shiny ventrally; propodeum lateral face punctures dense (i $<1 \mathrm{pd}$ ), often obscure, sculpture imbricate, becoming rugulose posteriorly; propodeum posterior face sculpture shiny and sparsely punctate $(\mathrm{i}=1-3 \mathrm{pd})$; $\mathrm{T} 1$ anterior face sculpture shiny; $\mathrm{T} 1 \mathrm{dorsal}$ surface punctures moderately dense $(\mathrm{i}=1-3 \mathrm{pd})$, sculpture shiny; $\mathrm{T} 2$ disc punctures moderately dense $(\mathrm{i}=1-3 \mathrm{pd})$, disc sculpture shiny, rim punctures sparse $(\mathrm{i}=1-4 \mathrm{pd})$, rim sculpture shiny.

StRUCTURE. Face length/width ratio 0.87 ( $\pm 0.02 \mathrm{SD}$ ). F1:pedicel length ratio 1.15 ( $\pm 0.08 \mathrm{SD}) ; \mathrm{F} 2: \mathrm{F} 1$ length ratio $1.75( \pm 0.17 \mathrm{SD}) ; \mathrm{F} 2$ length/width ratio $1.36( \pm 0.16 \mathrm{SD}) ; \mathrm{F} 9$ length/width ratio 1.2 $( \pm 0.13 \mathrm{SD})$. Forewing with 3 submarginal cells; pronotal angle obtuse; tegula shape normal. Intertegular distance $0.94( \pm 0.07 \mathrm{SD}) \mathrm{mm}$. Scutum length/width ratio 0.78 ( $\pm 0.05 \mathrm{SD})$; scutum/scutellum length ratio $2.78( \pm 0.18 \mathrm{SD})$; scutellum/metanotum length ratio $1.43( \pm 0.13 \mathrm{SD})$; metanotum/metapostnotum length ratio $0.72( \pm 0.08 \mathrm{SD})$. Propodeum lateral carinae not reaching dorsal margin; oblique carina absent. $(n=5)$ 


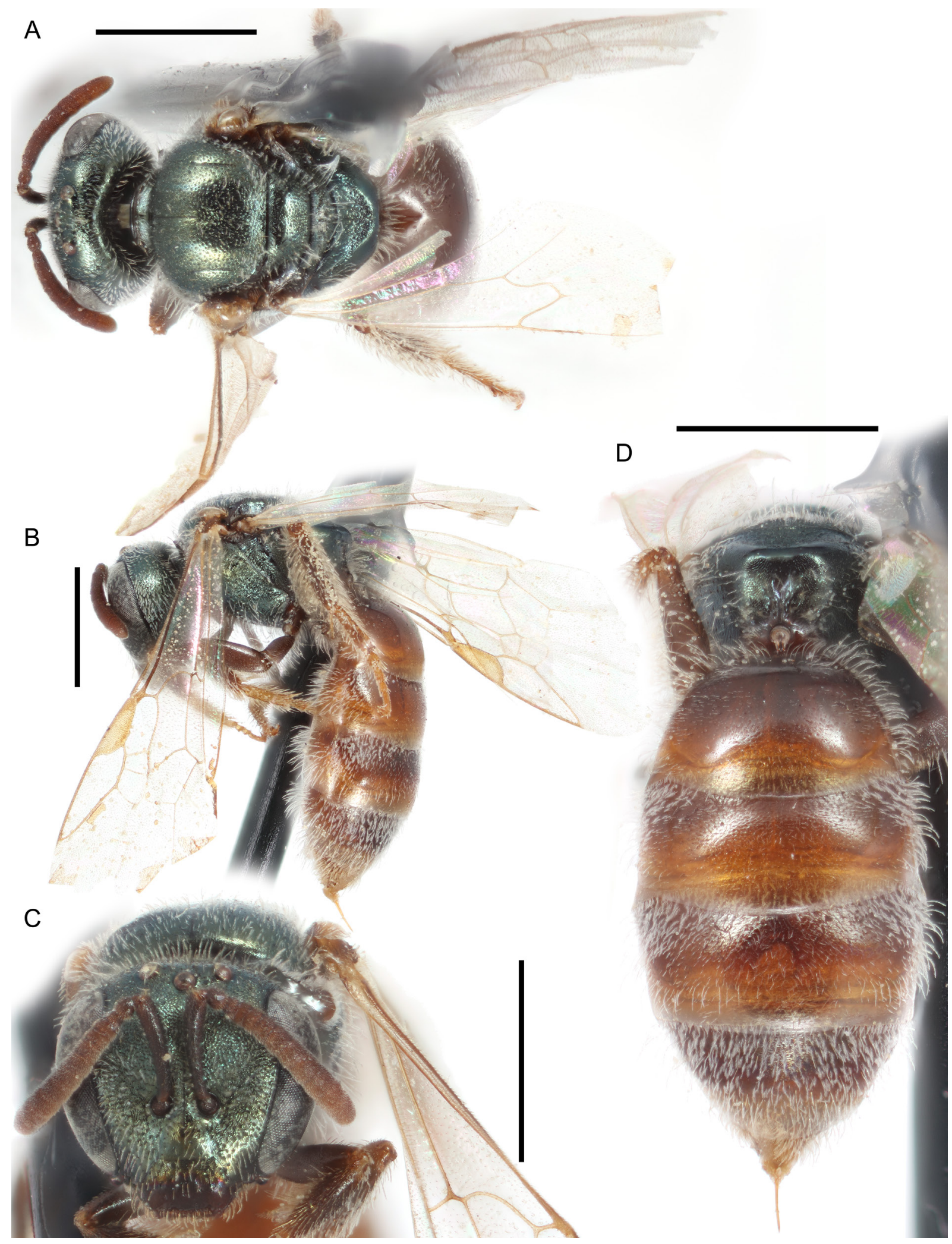

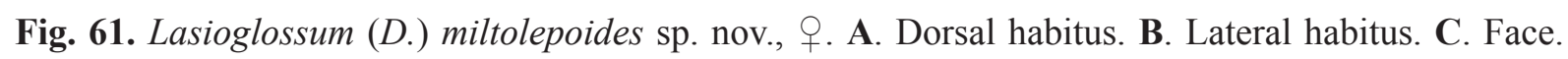
D. Propodeum and metasoma. Scale bars: $1 \mathrm{~mm}$. 


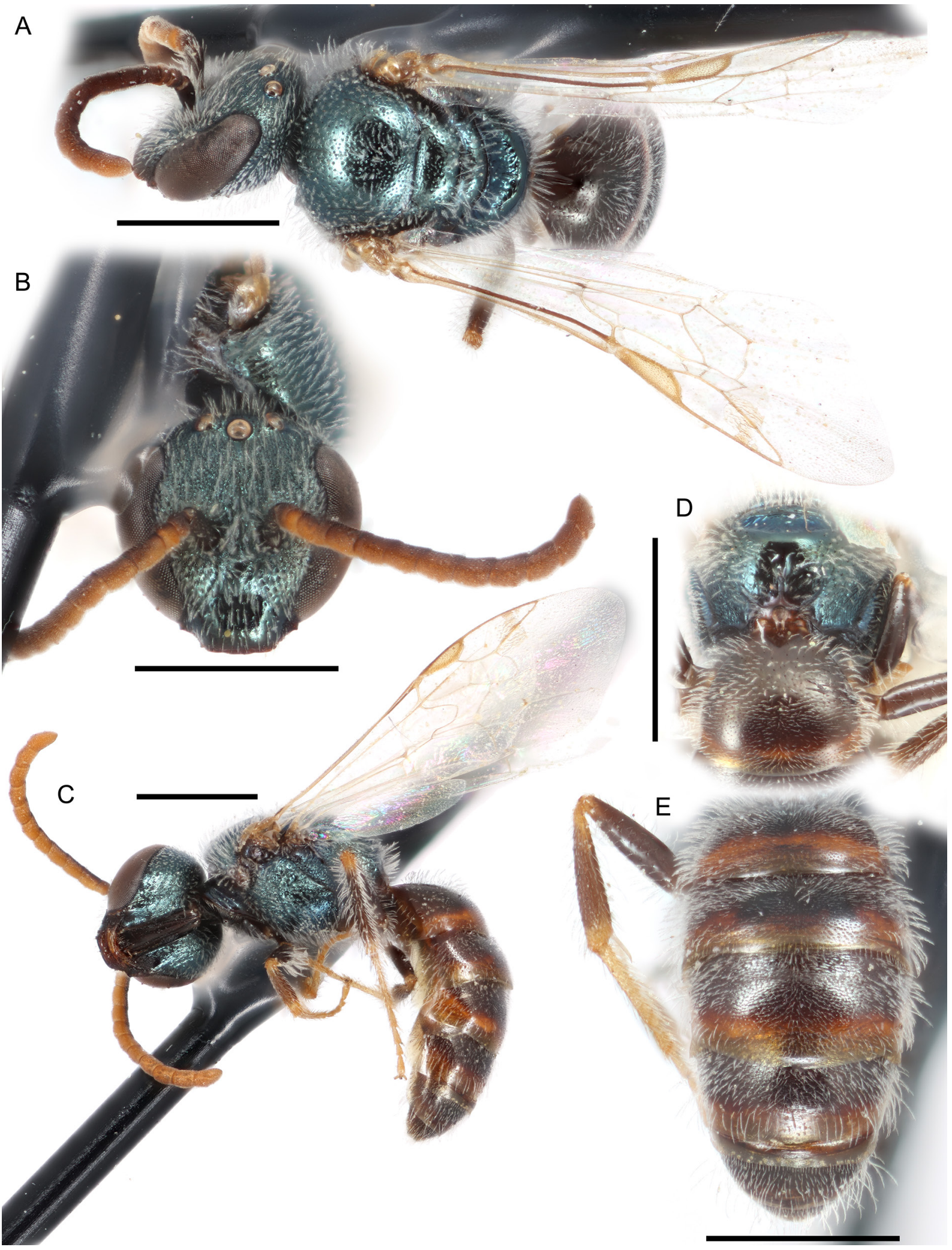

Fig. 62. Lasioglossum (D.) miltolepoides sp. nov., $\widehat{~}$. A. Dorsal habitus. B. Face. C. Lateral habitus. D. Propodeum. E. Metasoma. Scale bars: $1 \mathrm{~mm}$. 
Genitalia. As in Fig. 90I. Gonocoxite broad, weakly truncate. Gonostylus large, triangular, with a few long hairs. Retrorse lobe relatively narrow, ovoid, covered with sparse short hairs.

\section{Range}

Mojave Desert of California and Nevada, east through Utah and northern Arizona (Fig. 63).

\section{Floral records}

ASTERACEAE Giseke: Baccharis: B. salicina Torr. \& A.Gray - Baileya: B. pauciradiata A.Gray • Chrysothamnus - BRASSICACEAE Burnett: Stanleya Nutt. - CLEOMACEAE Bercht. \& J. Presl: Wislizenia Engelm. W. refracta Engelm. •TAMARICACEAE Link: Tamarix L.

\section{DNA barcodes}

Two sequences available (BOLD process IDs: DLII855-07, DLII1102-07; BIN: BOLD:AAJ1685). Lasioglossum miltolepoides sp. nov. differs from all other western red-tailed L. (Dialictus) by 4 fixed substitutions: 174(G), 186(C), 462(C), and 540(C) (Supplementary file 4).

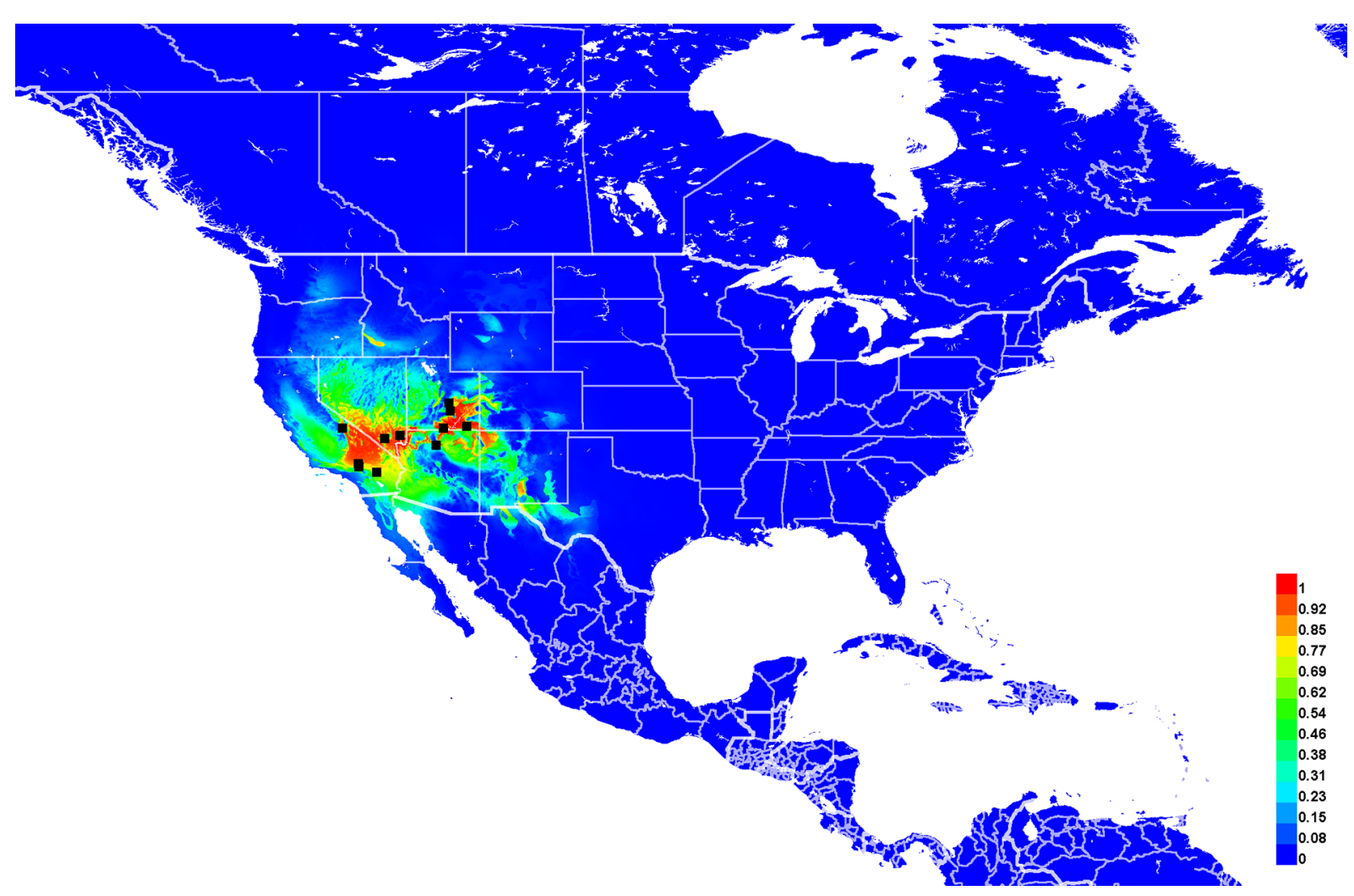

Fig. 63. Georeferenced collection records of Lasioglossum (D.) miltolepoides sp. nov. (black squares) and predicted distribution by maximum entropy ecological niche modeling in Maxent (colour shading). Warmer colours indicate higher cloglog probability of occurrence. 
Lasioglossum (Dialictus) minckleyi sp. nov. urn:1sid:zoobank.org:act:3197BF55-611C-4EE4-BC8B-4E133355826C

Figs 3A, 64-66, 90J, 98B, 107B

\section{Diagnosis}

Females of Lasioglossum minckleyi sp. nov. can be recognized by the face short (length/width ratio $\sim 0.8$ ), frons and mesepisternum with coarse and crowded punctures ( $\mathrm{i}=0 \mathrm{pd}$ ), clypeus with distinct apicolateral denticles, T1 anterior surface corarious, T2-4 with dark spiracular spots, metasomal terga lacking tomentum, and mesoscutum broad (length/width ratio usually $\leq 0.8$ ). They are most similar to L. mesillense and L. imbriumbrae sp. nov. Both of these species usually have the frons and mesepisternum with finer or distinctly separated punctures at least in part, T1 anterior surface shiny, and metasomal terga with basolateral tomentum. Occasional specimens of L. mesillense lack some of these diagnostic characters, and are very difficult to distinguish from L. minckleyi sp. nov. Females of L. mesillense also have the mesoscutum longer (length/width ratio usually $\geq 0.84$ ); this and the frons punctation seem to be the most reliable diagnostic characters.

Males of $L$. minckleyi sp. nov. can be recognized by the face short (length/width ratio $\sim 0.83$ ) and covered in moderately dense tomentum below the ocelli, flagellomeres relatively short (F2 about 1.6 times as long as $\mathrm{F} 1$ and 1.25 times as long as broad), mesepisternum punctures dense ( $\mathrm{i}<1 \mathrm{pd})$, metasomal terga lacking tomentum, T1-3 rims punctate basally, and clypeus apical margin and labrum usually orange. They are most similar to L. mesillense, L. austerum sp. nov., and L. imbriumbrae sp. nov. Males of L. mesillense have the flagellomeres relatively long (F2 about 2 times as long as F1 and 1.6 times as long as broad), mesepisternum more sparsely punctate ( $\mathrm{i}=1-2 \mathrm{pd}$ ), and metasomal terga sometimes with basolateral tomentum. Males of $L$. austerum sp. nov. have the face below ocelli covered in sparser tomentum and mesepisternum more sparsely punctate ( $\mathrm{i} \leq 1 \mathrm{pd}$ ). Males of $L$. imbriumbrae sp. nov. have T1-3 rims impunctate and clypeus apical margin and labrum reddish brown to black.

\section{Etymology}

The specific epithet minckleyi is a dedication to Robert L. Minckley (University of Rochester), who collected the vast majority of currently known specimens. An appropriate translation would be Minckley's sweat bee.

\section{Material examined}

Holotype

MEXICO - Sonora - 9 ; Rancho San Bernardino, $28 \mathrm{~km}$ E of Agua Prieta, Hay Hollow riparian site; [31.31 $\left.{ }^{\circ} \mathrm{N}, 109.25^{\circ} \mathrm{W}\right] ; 25$ Aug. 2000; R. Minckley and A. Romero leg.; PCYU.

[Verbatim label: MEXICO:Sonora, Rancho San Bernardino,28kmE Agua Prieta 25Aug2000 Hay Hollow riparian Site / R Minckley, A Romero / ex. blue pan trap 003025 // 1849C01 // HOLOTYPE / Lasioglossum (Dialictus) minckleyi Gardner and Gibbs]

\section{Paratypes}

MEXICO - Sonora • 1 \%; $30 \mathrm{~km}$ E of Agua Prieta; $31.3217^{\circ} \mathrm{N}, 109.2583^{\circ} \mathrm{W} ; 25 \mathrm{Jul}$. 2000; R. Minckley leg.; PCYU • 2 우; $30 \mathrm{~km}$ E of Agua Prieta; $31.3097^{\circ} \mathrm{N}, 109.2542^{\circ} \mathrm{W} ; 19$ Aug. 2003; A. Nelson leg.; PCYU • 1 क ; $30 \mathrm{~km} \mathrm{E}$ of Agua Prieta; $31.3322^{\circ} \mathrm{N}, 109.2703^{\circ} \mathrm{W} ; 3$ Aug. 2001;

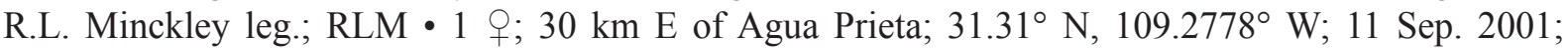
R.L. Minckley leg.; RLM • 1 q; Rancho Puerta Blanca, Pollination Site 26, Opuntia, Bulldozer, Rancho Puerta Blanca; $31.32222^{\circ} \mathrm{N}, 109.07972^{\circ} \mathrm{W}$; 24 May 2008; ex Opuntia engelmannii; RLM • 1 q; Rancho San Bernardino; $31.31^{\circ}$ N, 109.2536 W; 25 Aug. 2001; R. Minckley leg.; RLM • 1 क; Rancho San Bernardino, $28 \mathrm{~km}$ E of Agua Prieta, cienega transect; [31.31 ${ }^{\circ} \mathrm{N}, 109.25^{\circ} \mathrm{W}$; $27 \mathrm{Jul} .2000$; K. Toal leg.; 
RLM • 1 \%; same collection data as for holotype; PCYU • 2 $q$; same collection data as for holotype; RLM - 1 क; Rancho San Bernardino, Bosque dry, Site 4; 31.30972 ${ }^{\circ}$ N, 109.27778 ${ }^{\circ}$ W; 19 Aug. 2003; RLM - 3 우; Rancho San Bernardino, Bosque wet, Site 1; $31.33194^{\circ}$ N, $109.27083^{\circ}$ W; 10 Aug. 2006; RLM • 1 o; Rancho San Bernardino, Bosque wet, Site 6, Transect 3; $31.32957^{\circ} \mathrm{N}, 109.25462^{\circ} \mathrm{W} ; 27$ Jul. 2007; RLM • 3 o $ᄋ$; Rancho San Bernardino, Bosque wet, Site 7; $31.30972^{\circ} \mathrm{N}, 109.25417^{\circ} \mathrm{W}$; 19 Aug. 2003; RLM - 2 우; Rancho San Bernardino, Bosque wet, Site 7, Transect 2; $31.31007^{\circ}$ N, 109.25348 ${ }^{\circ}$ W; 26 Jul. 2000; RLM • 1 क; same location as for preceding; 12 Aug. 2002; RLM • 1 \%; Rancho San Bernardino, Cienega house; $31.33098^{\circ}$ N, $109.2676^{\circ}$ W; 1 Apr. 2006; RLM • 1 क; Rancho San Bernardino, field, Site 3, Transect 3; $31.32222^{\circ} \mathrm{N}, 109.27722^{\circ} \mathrm{W} ; 12$ Aug. 2002; RLM • 1 \%; Rancho San Bernardino, grassland, Site 3; $31.30222^{\circ}$ N, 109.26694 ${ }^{\circ}$ W; 12 Aug. 2006; RLM • 1 \%; Rancho San Bernardino, Pollination Site 21, Sphaeralcea, Pond $100 \mathrm{~m}$ E of main gate; $31.31583^{\circ} \mathrm{N}, 109.26861^{\circ} \mathrm{W}$; 8 Aug. 2006; ex Lycium andersonii; RLM • 1 क; same location as for preceding; 18 Aug. 2006; ex Larrea tridentata; RLM • 3 우, 1 万; same location as for preceding; 10 Aug. 2007; ex Sphaeralcea laxa; RLM • 1 P; Rancho San Bernardino, Pollination Site 22, Sphaeralcea, field 3; $31.32222^{\circ} \mathrm{N}, 109.27778^{\circ} \mathrm{W} ; 6$ Aug. 2006; ex Argemone pleiacantha; RLM • 1 \%; Rancho San Bernardino, Pollination Site 27, Horse Corral, $70 \mathrm{~m}$ N of main ranch house; $31.32556^{\circ} \mathrm{N}, 109.26667^{\circ} \mathrm{W}$; 9 Sep. 2007; ex Sphaeralcea laxa; RLM • 8 우; Rancho San Bernardino, quarry, gravel pit; $31.31964^{\circ} \mathrm{N}, 109.26926^{\circ} \mathrm{W}$; 19 Aug. 2003; RLM • 2 O ㅇ; Rancho San Bernardino, roadside E of quarry; $31.31996^{\circ} \mathrm{N}, 109.26848^{\circ} \mathrm{W} ; 19$ Aug. 2003; RLM • 1 q; Rancho San Bernardino, roadside W of quarry; $31.31948^{\circ} \mathrm{N}, 109.2701^{\circ} \mathrm{W} ; 18$ Aug. 2003; RLM 3 क 9 ; same location as for preceding; 19 Aug. 2003; RLM • 3 + $ᄋ$; Rancho San Bernardino, Riparian, Site 1, Transect 1; $31.33347^{\circ} \mathrm{N}, 109.2601^{\circ} \mathrm{W} ; 16$ Aug. 2000; RLM • 1 \%; same location as for preceding; 10 Aug. 2006; RLM • 1 क; same location as for preceding; 22 Jul. 2007; RLM • 1 \%;

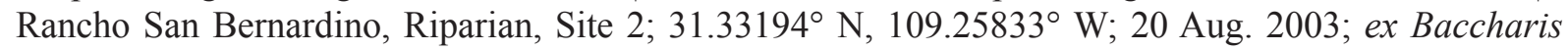
sarothroides; RLM • 1 q; same location as for preceding; 20 Aug. 2003; RLM • 4 o 0 ; Rancho San Bernardino, Riparian, Site 4; $31.32972^{\circ} \mathrm{N}, 109.25583^{\circ} \mathrm{W} ; 20$ Aug. 2003; RLM.

UNITED STATES - Arizona • 1 ôं Cochise Co., Benson; $\left[31.97^{\circ} \mathrm{N}, 110.29^{\circ} \mathrm{W}\right]$; $1091 \mathrm{~m}$ a.s.1.; $14-$ 15 Sep. 1974; E.G. and J.M. Linsley leg.; ex Helianthus annuus; CUIC $\bullet 2$ q 9 ; Cochise Co., San Bernardino National Wildlife Refuge, Black Draw, Riparian Site 2; [31.34 N, $\left.109.26^{\circ} \mathrm{W}\right]$; 16 Aug. 2000; K. Toal leg.; RLM • 1 क; Cochise Co., San Bernardino NWR; $31.3344^{\circ}$ N, $109.2681^{\circ}$ W; 3 Aug. 2001; R.L. Minckley leg.; RLM • 1 q; Cochise Co., San Bernardino NWR; [31.34 N, 109.26 W]; 27 Aug. 2001; R. Minckley leg.; RLM • 4 q 9 ; Cochise Co., San Bernardino NWR, Bosque dry, Site 7;

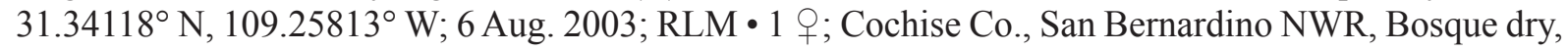
Site 7, Transect 2; $31.34168^{\circ} \mathrm{N}, 109.25825^{\circ} \mathrm{W} ; 30$ Jul. 2004; RLM • 5 q ; Cochise Co., San Bernardino

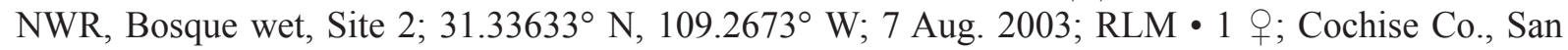
Bernardino NWR, Bosque wet, Site 2, Transect 2; $31.33627^{\circ}$ N, $109.26752^{\circ}$ W; 31 Jul. 2004; RLM • 4 우; Cochise Co., San Bernardino NWR, Bosque wet, Site 3; $31.33517^{\circ}$ N, 109.26653 W; 7 Aug. 2003; RLM • 1 क; Cochise Co., San Bernardino NWR, Bosque wet, Site 3, Transect 2; $31.33527^{\circ}$ N, 109.2668 W; 11 Oct. 2002; RLM • 1 q; same location as for preceding; 15 Aug. 2005; RLM • 8 q $\circ$; Cochise Co., San Bernardino NWR, Bosque wet, Site 4; $31.334^{\circ}$ N, $109.2372^{\circ}$ W; 5 Aug. 2003; RLM • 1 क; Cochise Co., San Bernardino NWR, Bosque wet, Site 4, Transect 1; $31.33693^{\circ}$ N, $109.24361^{\circ}$ W; 26 Jul. 2007; RLM - 1 क; Cochise Co., San Bernardino NWR, field, nr Bathroom, Bee Diversity; $31.3411^{\circ}$ N, $109.27048^{\circ}$ W; 15 Oct. 2002; RLM • 1 q; Cochise Co., San Bernardino NWR, field, Site 5, Transect 3; $31.33417^{\circ}$ N, 109.26778 ${ }^{\circ}$ W; 27 Jul. 2007; RLM • 2 o $\circ$; Cochise Co., San Bernardino NWR, field, Site 6; $31.33867^{\circ} \mathrm{N}, 109.2719^{\circ} \mathrm{W} ; 8$ Aug. 2003; RLM • 1 \%; Cochise Co., San Bernardino NWR, field, Site 6, Transect 1; $31.33417^{\circ} \mathrm{N}, 109.26833^{\circ} \mathrm{W}$; 11 Aug. 2005; RLM • 2 q 9 ; Cochise Co., San Bernardino NWR, field, Site 6, Transect 4; $31.33444^{\circ}$ N, $109.26806^{\circ}$ W; 3 Jul. 2002; RLM • 2 q ; ; Cochise Co., San Bernardino NWR, field, Site 8; $31.33765^{\circ}$ N, 109.26068 ${ }^{\circ}$ W; 6 Aug. 2003; RLM • 1 O; same location as for preceding; 6 Apr. 2006; RLM • 1 \%; Cochise Co., San Bernardino NWR, Grassland, Site 2, Transect 3; $31.3358^{\circ}$ N, 109.2702 ${ }^{\circ}$ W; 27 Jul. 2007; RLM • 3 우; Cochise Co., San Bernardino NWR, Grassland, Site 4; $31.33495^{\circ}$ N, 109.25314 W; 5 Aug. 2003; RLM • 2 q ; C Cochise Co., San 
Bernardino NWR, Grassland, Site 4, Transect 1; $31.33487^{\circ}$ N, $109.2529^{\circ}$ W; 26 Jul. 2007; RLM • 2 우우; Cochise Co., San Bernardino NWR, Grassland, Site 5; 31.33683 ${ }^{\circ}$ N, $109.25308^{\circ}$ W; 5 Aug. 2003; RLM • 2 우; Cochise Co., San Bernardino NWR, Grassland, Site 5, Transect 4; $31.3385^{\circ}$ N, 109.25308 ${ }^{\circ}$ W; 26 Jul. 2007; RLM - 1 क; Cochise Co., San Bernardino NWR, Grassland, Site 8;

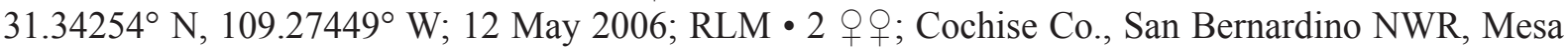
de la Avanzada, Bee diversity; $31.34147^{\circ} \mathrm{N}, 109.27278^{\circ} \mathrm{W}$; 15 Aug. 2002; RLM • 1 क; same location as for preceding; 15 Oct. 2002; RLM • 1 今ं; Pima Co., Greaterville Rd.; $31.7864^{\circ} \mathrm{N}, 110.8231^{\circ} \mathrm{W}$; 1320 m a.s.l.; 8 Jul. 2010; Yanega leg.; ex Condalia; UCRC • 1 क; Pinal Co., 8 km SE of San Manuel; $32.5974^{\circ} \mathrm{N}, 110.5388^{\circ} \mathrm{W} ; 15$ Apr. 2014; R.G. Laport leg.; ex Larrea tridentata; RLM • 1 ; ; Yavapai Co., Montezuma Castle; [34.61 ${ }^{\circ} \mathrm{N}, 111.84^{\circ} \mathrm{W}$ ]; 25 Jun. 1973; H. Nagase leg.; LACM・ 1 क; $30 \mathrm{~km} \mathrm{E} \mathrm{of}$ Douglas; $31.3383^{\circ}$ N, $109.2683^{\circ}$ W; 3 Sep. 2000; R. Minckley leg.; ex Baccharis sarothroides; RLM • $10^{\top} ; 6$ mi. SE of Patagonia; [31.48 $\mathrm{N}, 110.83^{\circ} \mathrm{W}$ ]; 26 Jul. 1962; Werner, Bequaert and Noller leg.; ex Condalia lycioides and Baccharis salicina; CUIC 11 \% ; Arivaca; $\left[31.58^{\circ} \mathrm{N}, 111.33^{\circ} \mathrm{W}\right] ; 12 \mathrm{Jul} .1940$;

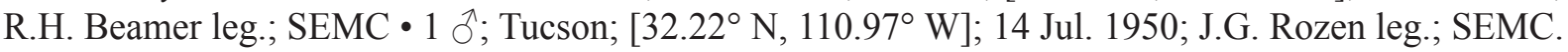

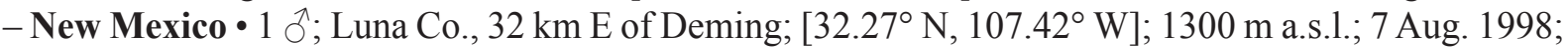
R. Minckley and K. Toal leg.; RLM.

\section{Description}

\section{Female}

Colouration. Head and mesosoma blue-green to golden-green; clypeus apical colour black, reddish brown, or orange; labrum reddish brown to orange; mandible orange with black basal spot or band and red tip; flagellum reddish brown dorsally, brown to orange ventrally; pronotal lobe reddish brown to orange; metasoma red to orange with dark spiracular spots on T2-4; legs reddish brown with femur-tibia joints, base and apex of tibiae, and tarsi orange; tegula orange; wing membrane hyaline, veins brown to dark brown.

PuBESCENCE. Body hair colour white. Tomentum dense on paraocular area, gena, pronotal collar and lobe, and space between pronotal lobe and tegula; sparse on face below eye emargination, episterna, very narrow basal bands on T2-3, and basal half of T4. Scutum hair thin. Wing hairs dark or light, short and dense. Acarinarial fan complete, sparse. T2 fringes sparse, T3 fringes sparse.

Surface SCUlPtURE. Clypeus punctures large and irregularly sparse $(\mathrm{i}<2 \mathrm{pd})$, becoming fine and moderately dense in basal third $(i=1-2 \mathrm{pd})$, sculpture shiny, becoming weakly tessellate basally; supraclypeal area punctures sparse $(i=1-3 \mathrm{pd})$, sculpture weakly tessellate, sometimes shiny medially; paraocular area punctures crowded $(\mathrm{i}=0 \mathrm{pd})$, slightly sparser around antenna socket $(\mathrm{i} \leq 1 \mathrm{pd})$, sculpture weakly tessellate around antenna socket, otherwise shiny; frons punctures crowded $(\mathrm{i}=0 \mathrm{pd})$, sculpture shiny or imbricate; vertex punctures dense laterally ( $i \leq 1 \mathrm{pd})$, sparse medially $(i=1-3 \mathrm{pd})$, sometimes obscure, sculpture shiny, sometimes imbricate laterally; gena punctures fine, moderately sparse ( $i=1-3 \mathrm{pd})$, sculpture shiny; postgena sculpture shiny, becoming tessellate posteriorly; tegula punctures absent; scutum punctures dense $(\mathrm{i}<1 \mathrm{pd})$, becoming moderately sparse submedially and anteromedially ( $\mathrm{i}=1-2 \mathrm{pd})$, sculpture shiny to weakly tessellate, becoming more strongly tessellate anteromedially; scutellum punctures dense marginally and on median line $(\mathrm{i}<1 \mathrm{pd})$, sparse submedially $(i=1-4 \mathrm{pd})$, diversopunctate, sculpture shiny to tessellate; metanotum sculpture tessellate or imbricate, sometimes becoming shiny and finely, densely punctate medially $(\mathrm{i}<1 \mathrm{pd})$; metapostnotum rugae strong, anastomosing, not reaching margin, sculpture tessellate; preëpisternum sculpture areolaterugulose; hypoepimeron punctures crowded $(\mathrm{i}=0 \mathrm{pd})$, sculpture shiny to weakly imbricate, sometimes weakly ruguloso-punctate; mesepisternum punctures crowded ( $\mathrm{i}=0 \mathrm{pd})$, sculpture weakly imbricate and ruguloso-punctate; metepisternum sculpture ruguloso-lineate dorsally, imbricate ventrally; propodeum lateral face sculpture tessellate; propodeum posterior face sculpture tessellate; T1 anterior face sculpture coriarious; $\mathrm{T} 1$ dorsal surface punctures fine, sparse $(\mathrm{i}=2-5 \mathrm{pd})$, absent or very sparse in large apicolateral patches, sculpture shiny, becoming weakly coriarious on rim; T2 disc punctures fine, moderately sparse 
$(i=1-4 \mathrm{pd})$, disc sculpture shiny to weakly coriarious, rim punctures fine, sparse ( $\mathrm{i}=1-4 \mathrm{pd})$, obscure, rim sculpture coriarious.

StRUCTURE. Face length/width ratio 0.8 ( $\pm 0.02 \mathrm{SD})$. Clypeus projecting $\sim 50 \%$ below suborbital tangent; clypeal area length/width ratio $0.43( \pm 0.02 \mathrm{SD})$; apicolateral denticles rounded acute points; supraclypeal area length/width ratio $0.74( \pm 0.09 \mathrm{SD})$. Forewing with 3 submarginal cells; pronotal angle obtuse; tegula shape normal. Intertegular distance $0.95( \pm 0.05 \mathrm{SD}) \mathrm{mm}$. Scutum length/width ratio 0.77 ( $\pm 0.05 \mathrm{SD})$; scutum/scutellum length ratio 2.87 ( $\pm 0.2 \mathrm{SD})$; scutellum/metanotum length ratio 1.61 ( $\pm 0.22 \mathrm{SD})$; metanotum/metapostnotum length ratio 0.69 ( $\pm 0.08 \mathrm{SD})$. Propodeum lateral carinae not reaching dorsal margin; oblique carina very weak or absent. T2 depressed apical rim length about $50 \%$ of segment. $(n=10)$

\section{Male}

Colouration. Head and mesosoma blue to olive green; clypeus apical colour reddish brown to orange; labrum orange; mandible orange with black basal spot and red tip; flagellum reddish brown dorsally, orange to brown ventrally; pronotal lobe orange; metasoma orange to reddish brown with dark spiracular spots on T2-4; legs orange with coxae, trochanters, femora, and tibiae sometimes reddish brown; tegula orange; wing membrane hyaline, veins brown to dark brown.

PubESCEnCE. Body hair colour white. Tomentum dense on face below eye emargination, gena, pronotal angle and lobe, and space between pronotal lobe and tegula; sparse on episterna. Scutum hair thin to moderately plumose. Sterna hair short (0.5-1.5 OD), moderately plumose, dense and erect. Wing hairs dark or light, short and sparse.

SuRfaCE SCULPTURE. Clypeus punctures moderately dense ( $\mathrm{i}=1-2 \mathrm{pd})$, sculpture shiny; supraclypeal area punctures moderately dense $(i=1-2 \mathrm{pd})$, sculpture shiny; paraocular area punctures dense $(\mathrm{i}<1 \mathrm{pd})$, sculpture shiny; frons punctures dense $(\mathrm{i}<1 \mathrm{pd})$, becoming crowded above antenna $(\mathrm{i}=0 \mathrm{pd})$, sculpture shiny, becoming rugulose above antenna; vertex punctures dense laterally ( $i \leq 1 \mathrm{pd}$ ), sparse medially ( $i=1-4 \mathrm{pd})$, sculpture shiny; gena punctures sparse ( $i=1-3 \mathrm{pd})$, sculpture shiny; postgena sculpture imbricate; tegula punctures absent; scutum punctures moderately sparse ( $i=1-2 \mathrm{pd})$, becoming sparse submedially ( $\mathrm{i}=1-3 \mathrm{pd})$, sculpture shiny, sometimes becoming tessellate anteromedially; scutellum punctures dense to moderately sparse marginally and on median line ( $i \leq 2 \mathrm{pd})$, sparse submedially ( $i=1-4 \mathrm{pd})$, sculpture shiny; metanotum sculpture shiny and densely punctate $(\mathrm{i} \leq 1 \mathrm{pd})$; metapostnotum rugae strong, anastomosing, not reaching margin, sculpture shiny to tessellate; preëpisternum sculpture areolate-rugulose; hypoepimeron punctures crowded ( $i=0 \mathrm{pd})$, sculpture shiny to imbricate; mesepisternum punctures dense $(i<1 \mathrm{pd})$, sculpture shiny to imbricate; metepisternum sculpture ruguloso-lineate dorsally, areolate ventrally; propodeum lateral face punctures obscure, sculpture imbricate to rugulose; propodeum posterior face sculpture shiny; T1 anterior face sculpture shiny; T1 dorsal surface punctures moderately dense $(i=1-3 \mathrm{pd})$, sculpture shiny; $\mathrm{T} 2$ disc punctures moderately dense $(i=1-2 \mathrm{pd})$, disc sculpture shiny, rim punctures sparse medially ( $\mathrm{i}=1-4 \mathrm{pd})$, absent laterally, rim sculpture shiny.

StRucture. Face length/width ratio 0.83 ( $\pm 0.01 \mathrm{SD})$. F1:pedicel length ratio 0.87 ( $\pm 0.06 \mathrm{SD}) ; \mathrm{F} 2: \mathrm{F} 1$ length ratio $1.58( \pm 0.11 \mathrm{SD}) ; \mathrm{F} 2$ length/width ratio $1.25( \pm 0.09 \mathrm{SD}) ; \mathrm{F} 9$ length/width ratio 1.18 $( \pm 0.12 \mathrm{SD})$. Forewing with 3 submarginal cells; pronotal angle obtuse; tegula shape normal. Intertegular distance $0.87( \pm 0.05 \mathrm{SD}) \mathrm{mm}$. Scutum length/width ratio $0.76( \pm 0.02 \mathrm{SD})$; scutum/scutellum length ratio $3.16( \pm 0.32 \mathrm{SD})$; scutellum/metanotum length ratio $1.33( \pm 0.15 \mathrm{SD})$; metanotum/metapostnotum length ratio 0.71 ( $\pm 0.08 \mathrm{SD})$. Propodeum lateral carinae not reaching dorsal margin; oblique carina absent. $(n=7)$ 


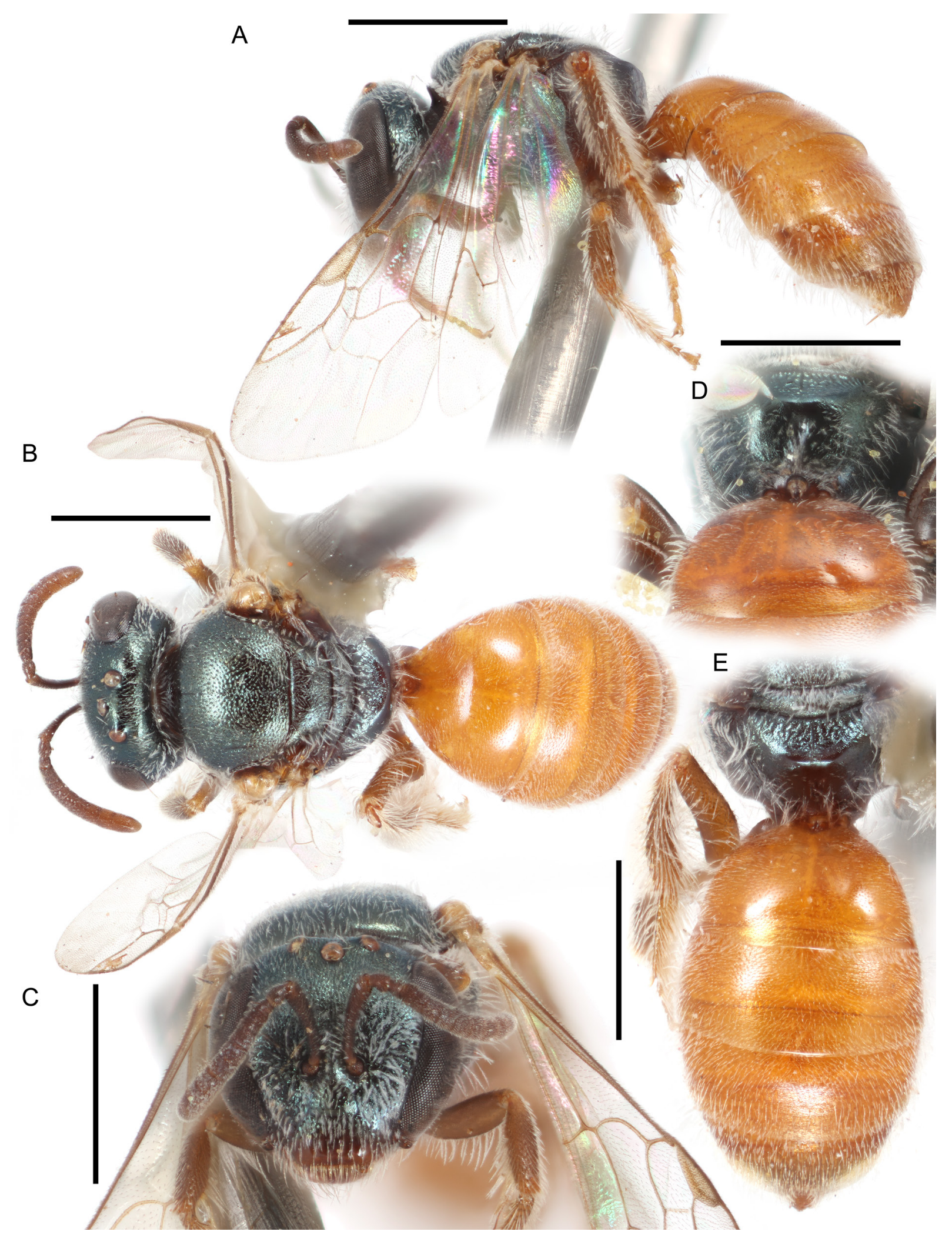

Fig. 64. Lasioglossum (D.) minckleyi sp. nov., ․ A. Lateral habitus. B. Dorsal habitus. C. Face. D. Propodeum. E. Metasoma. Scale bars: $1 \mathrm{~mm}$. 


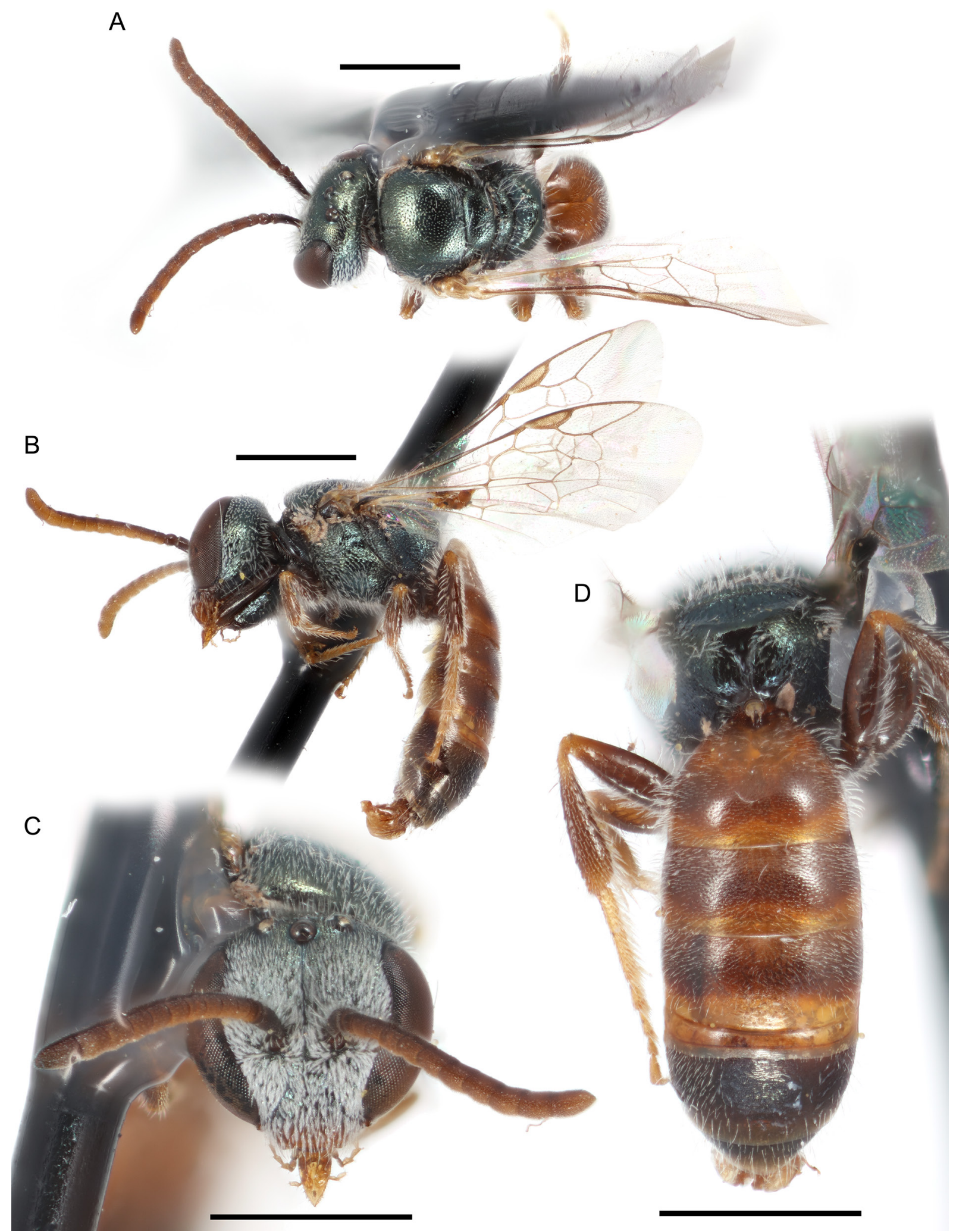

Fig. 65. Lasioglossum (D.) minckleyi sp. nov., ô. A. Dorsal habitus. B. Lateral habitus. C. Face. D. Propodeum and metasoma. Scale bars: $1 \mathrm{~mm}$. 
Genitalia. As in Fig. 90J. Gonocoxite relatively narrow, rounded. Gonostylus with very few long hairs. Retrorse lobe very broad, ovoid, with very sparse short hairs.

\section{Range}

Southern Arizona and New Mexico and northern Sonora (Fig. 66).

\section{Floral records}

ASTERACEAE Giseke: Baccharis: B. sarothroides A.Gray • Helianthus: H. annuus L. • CACTACEAE Juss. Opuntia Mill. O. engelmannii Salm-Dyck ex Engelm. - MALVACEAE Juss. Sphaeralcea A. St.-Hil. S. laxa Wooton \& Standl. P PAPAVERACEAE Juss. Argemone L. A. pleiacantha Greene • RHAMNACEAE Juss. Condalia Cav. - SOLANACEAE Adans. Lycium L. L. andersonii A.Gray • ZYGOPHYLLACEAE R.Br. Larrea Cav. L. tridentata (Sessé \& Moc. ex DC.) Coville.

\section{DNA barcodes}

Three sequences available (BOLD process IDs: DLII775-07, DLII1555-09, DLII1572-09; BIN: BOLD:AAJ1796). Lasioglossum minckleyi sp. nov. differs from all other western red-tailed L. (Dialictus) by 2 fixed substitutions: 348(C) and 510(G) (Supplementary file 4).

\section{Remarks}

The vast majority of known specimens are from Minckley's collections in the San Bernardino National Wildlife Refuge and Rancho San Bernardino (summarized in Minckley 2008). Lasioglossum minckleyi sp. nov. seems to be uncommon outside of this area.

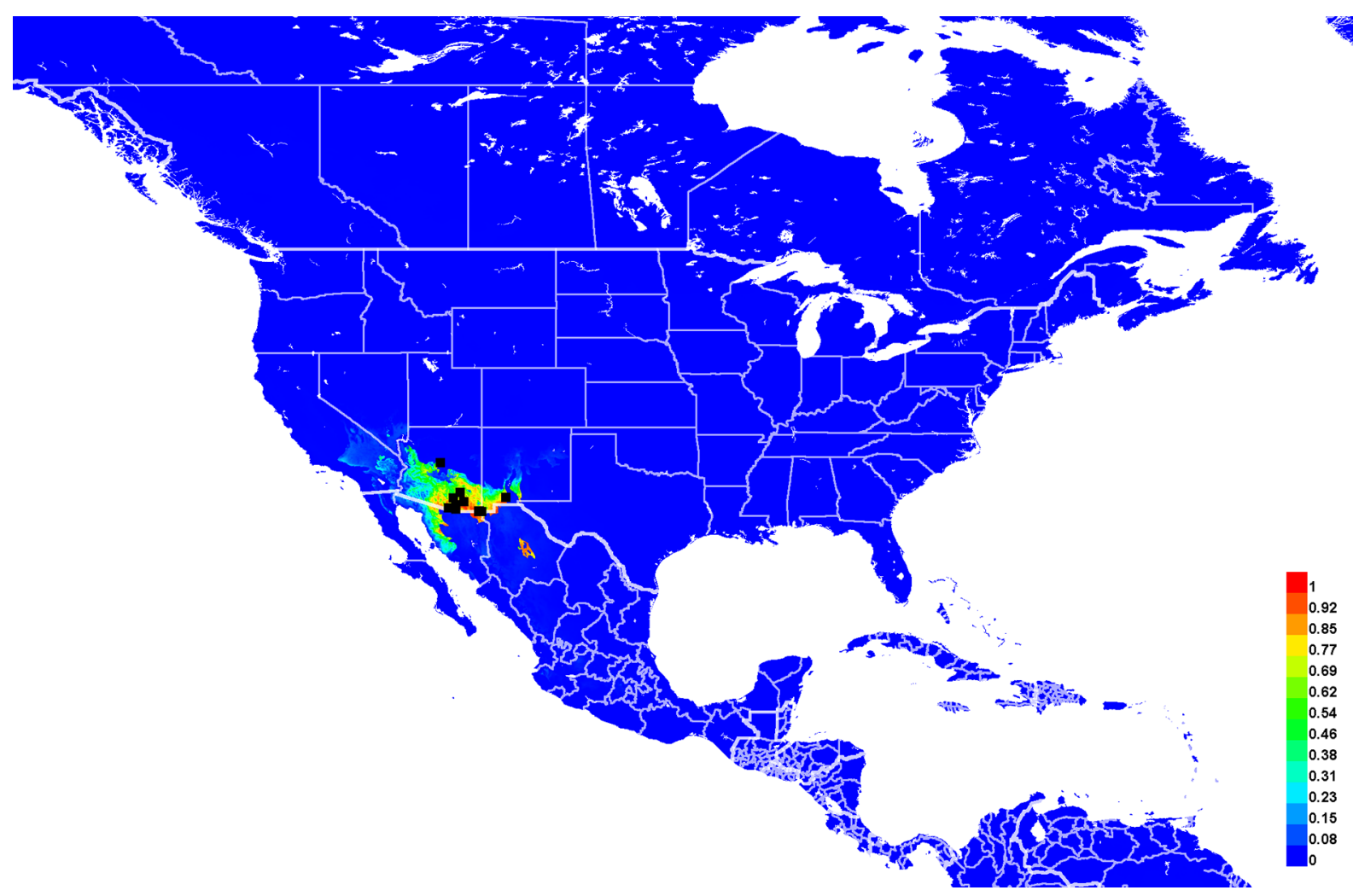

Fig. 66. Georeferenced collection records of Lasioglossum (D.) minckleyi sp. nov. (black squares) and predicted distribution by maximum entropy ecological niche modeling in Maxent (colour shading). Warmer colours indicate higher cloglog probability of occurrence. 
Lasioglossum (Dialictus) pallidellum (Ellis, 1914)

Figs $67-69$, 90K, 100A, 104A

Halictus pallidellus Ellis, 1914a: 151 (holotype,, , deposited in UCMC, examined).

Halictus (Chloralictus) pallidellus - Sandhouse 1924: 4 (key).

Lasioglossum (Chloralictus) pallidellum - Michener 1951: 1116 (catalog).

Dialictus pallidellus - Hurd 1979: 1969 (catalog). - Moure \& Hurd 1987: 119 (catalog).

Lasioglossum (Dialictus) pallidellum - Gibbs 2010: 234, fig. 169a-c (key, redescription). — Scott et al. 2011: 30 (checklist).

\section{Diagnosis}

Females of Lasioglossum pallidellum can be recognized by most of the body covered in very dense white tomentum, head and mesosoma shiny and sparsely punctate ( $\mathrm{i}=1-3 \mathrm{pd})$, metapostnotum shiny with weak rugae not reaching the rounded posterior margin, face short (length/width ratio $\sim 0.78$ ), and T2-4 with dark spiracular spots. They are most similar to L. julipile sp. nov. Females of $L$. julipile sp. nov. have the metapostnotum with strong rugae reaching the posterior margin and T2 without dark spiracular spots.

Males of L. pallidellum can be recognized by the same characters as for females, although the metapostnotal rugae are sometimes stronger and nearly reaching the posterior margin, and the dark spiracular spots are only visible in red-tailed forms. In addition, they have the flagellomeres relatively short (F2 about 1.75 times as long as F1 and 1.25 times as long as broad), gonocoxite very broad, and gonostylus large and boot-shaped. They are most similar to L. julipile sp. nov. Males of L. julipile sp. nov. have the mesosoma and metasoma with less extensive tomentum (especially noticeable on T2-4 basolaterally), metasoma always completely black, and gonocoxite and gonostylus narrower with no unusual modifications.

\section{Etymology}

Ellis (1914a) named this species from the Latin adjective 'pallidus' ('pale', 'ashen') and the diminutive suffix '-ellus'.

\section{Material examined}

\section{Holotype}

UNITED STATES - New Mexico • ${ }_{+}$; Roswell; $\left[33.39^{\circ}\right.$ N, $104.52^{\circ}$ W]; 14 Apr.; Cockerell leg.; ex Prunus; UCMC.

\section{Other material}

MEXICO - Coahuila • 1 \%; $18 \mathrm{~km} \mathrm{~S}$ of Cuatrociénegas; $26.8667^{\circ} \mathrm{N}, 102.1333^{\circ} \mathrm{W} ; 920 \mathrm{~m}$ a.s.l.; 27 Mar. 1992; D. Yanega leg.; ex Prosopis glandulosa; SEMC • 4 q $\circ$; Churince Spring, 18 km S of Cuatrociénegas; [26.84 ${ }^{\circ}$ N, $102.134^{\circ} \mathrm{W}$ ]; 15 Feb. 2000; R.L. Minckley leg.; ex Acacia greggii; RLM. Nuevo Leon • 1 \%; $2.7 \mathrm{~km} \mathrm{~S}$ of El Refugio de Cedilla; $23.9825^{\circ} \mathrm{N}, 100.294583^{\circ} \mathrm{W} ; 1973 \mathrm{~m}$ a.s.1.; 18 Sep. 2008; G.R. Ballmer leg.; SEMC.

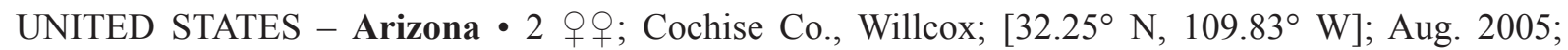
L. Packer leg.; PCYU • 1 क; Willcox; [32.25 N, $109.83^{\circ}$ W]; 2005; L. Packer leg.; PCYU. - California • 1 क; Fresno Co., Mendota; [36.75 N, $120.38^{\circ}$ W]; 23 Apr. 1949; J.W. MacSwain and R.F. Smith leg.; ex Melilotus; EMEC • 2 ㅇ; Inyo Co., 2 km NW of Swansea; [36.54 N, 117.93 W]; 19 Sep. 1993; T. Griswold leg.; ex Ericameria nauseosa var. nauseosa; BBSL $\bullet 3$ 우; same location as for preceding; 19 Sep. 1993; T. Griswold leg.; ex Oxystylis lutea; BBSL • 2 qo ; Inyo Co., 2.9 mi. S of Shoshone; 
[35.929 $\left.\mathrm{N}, 116.269^{\circ} \mathrm{W}\right]$; 18 Oct. 1956; A.C. Dickson leg.; ex Aplopappus; UCRC • 1 q; Inyo Co., 3.5 mi. W of Lone Pine; [36.6 $\left.{ }^{\circ} \mathrm{N}, 118.125^{\circ} \mathrm{W}\right]$; 8 Jul. 1961; H.V. Daly leg.; EMEC • 3 o 9 ; Inyo Co., Death Valley Junction; $36.3142^{\circ} \mathrm{N}, 116.4567^{\circ} \mathrm{W} ; 671 \mathrm{~m}$ a.s.1.; 19 Sep. 2013; M.C. Orr leg.; ex Cleomella obtusifolia; BBSL • 1 क; Inyo Co., Eureka Dunes, North inner; $37.0986^{\circ} \mathrm{N}, 117.6776^{\circ} \mathrm{W}$; 20 Apr. 1999; R. Andrus and T. Griswold leg.; ex Astragalus lentiginosus; BBSL • 1 q; Inyo Co., Keeler; $\left[36.49^{\circ} \mathrm{N}, 117.87^{\circ} \mathrm{W}\right] ; 28$ Aug. 1977; E.G. and J.M. Linsley leg.; ex Tamarix aralensis; EMEC • 1 \%; Inyo Co., Lone Pine Creek; [36.6 $\mathrm{N}, 118.085^{\circ} \mathrm{W}$ ]; $1219 \mathrm{~m}$ a.s.1.; 31 Aug. 1969; R.M. Bohart leg.; UCDC • 1 q; Inyo Co., Saline Valley, Salt Lake; [36. $7^{\circ}$ N, 117.82 ${ }^{\circ}$ W]; 323 m a.s.1.; 23 May 1976; D. Giuliani leg.; LACM • 1 o; same location as for preceding; 7 Jun. 1976; D. Giuliani leg.; LACM • 1 q; Inyo Co., Saline Valley, Upper Warm Spring; [36.813 ${ }^{\circ}$ N, $\left.117.766^{\circ} \mathrm{W}\right]$; 579 m a.s.1.; 6 Apr. 1976; D. Giuliani leg.; LACM • 2 q 9 ; Inyo Co., Shoshone; $\left[35.97^{\circ}\right.$ N, $116.27^{\circ}$ W]; 22 Sep. 1966; J.C. Hall leg.; ex Oxystylis lutea; UCRC • 1 q; Lassen Co., Amedee; [40.3 N, 120.2 ${ }^{\circ}$ W]; 4 Jul. 1947; T.F. Leigh leg.; EMEC • 1 q; San Bernardino Co., 9 air mi. S of Baker, sand dunes S of Zzyzx Springs;

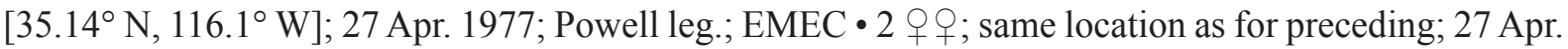
1977; J. Doyen leg.; ex Cleomella obtusifolia; EMEC • 3 o ; ; San Bernardino Co., 9 air mi. S of Baker, Zzyzx Springs; [35.14 N, 116.1ํ W]; 22 Apr. 1977; Powell leg.; ex Cleomella obtusifolia; EMEC • 2 우; same location as for preceding; 23 Apr. 1977; Powell leg.; ex Cleomella obtusifolia; EMEC • 2 우; same location as for preceding; 20 Apr. 1977; R. Cave leg.; EMEC • 4 + $O$; same location as for preceding; 20 Apr. 1977; J. Doyen leg.; ex Tamarix; EMEC • 1 क ; same location as for preceding; 20-21 Apr. 1977; M. Buegler leg.; EMEC • 1 q; San Bernardino Co., Zzyzx Spring; $35.1333^{\circ}$ N, $116.1^{\circ}$ W; 22-24 May 1982; J.P. and K.E.S. Donahue leg.; AMNH • 28 q $q$; same location as for preceding; $22-24$ May 1982; J.P. and K.E.S. Donahue leg.; LACM • 1 क; San Bernardino Co., Zzyzx Springs; [35.14 N, 116. $1^{\circ} \mathrm{W}$; 22 Apr. 1984; Dan Sandri leg.; EMEC • 4 우; San Bernardino Co., Zzyzx Springs, 9 mi. S of Baker; [35.14 N, 116.1 ${ }^{\circ} \mathrm{W}$ ]; 18-28 Apr. 1984; P. Martin leg.; EMEC • 3 우; same location as for preceding; 21-23 Apr. 1984; EMEC • 1 q; Tulare Co., Wood Lake; $\left[36.41^{\circ} \mathrm{N}, 119.1^{\circ} \mathrm{W}\right]$; 1 May 1947; Norman W. Frazier leg.; EMEC • 1 क ; 1.3 mi. E of Little Rock; $\left[34.52^{\circ} \mathrm{N}, 117.96^{\circ} \mathrm{W}\right] ; 13 \mathrm{Sep} .1950$; Timberlake leg.; ex Gutierrezia microcephala; UCRC • 1 क; 12 mi. SE of Palm Springs; $\left[33.71^{\circ} \mathrm{N}\right.$, $\left.116.39^{\circ} \mathrm{W}\right] ; 12$ Nov. 1952; Timberlake leg.; ex Eriogonum trichopes; UCRC • 2 o 9 ; Twentynine Palms; [34.13ㅇ N, $116.06^{\circ} \mathrm{W}$ ]; 9 Aug. 1946; Timberlake leg.; ex Wislizenia refracta; UCRC • 3 qo ; same location as for preceding; 4 Aug. 1933; Timberlake leg.; ex Wislizenia refracta; UCRC • 1 9; same location as for preceding; 1946; A.L. Melander leg.; UCRC • 1 क ; 5 mi. N of Lancaster; $\left[34.75^{\circ} \mathrm{N}\right.$, $118.15^{\circ} \mathrm{W}$; 29 Sep. 1956; Timberlake leg.; ex Isocoma acradenia; UCRC 11 \%; Lancaster; $\left[34.69^{\circ} \mathrm{N}\right.$, $\left.118.15^{\circ} \mathrm{W}\right] ; 10$ Oct. 1936; BBSL • 1 \%; Olancha; [36.28 $\left.\mathrm{N}, 118.01^{\circ} \mathrm{W}\right] ; 2$ May 1927; Timberlake leg.; ex Salix exigua; UCRC • 1 क; Saratoga Springs, Death Valley; [35.681 $\left.{ }^{\circ} \mathrm{N}, 116.423^{\circ} \mathrm{W}\right]$; $16-19$ Jun. 1954; Belkin and McDonald leg.; LACM • 1 क ; same location as for preceding; 23-24 Apr. 1955; LACM. - Idaho • 1 क; Twin Falls; $\left[42.56^{\circ}\right.$ N, $114.47^{\circ}$ W]; 25 Aug. 1927; V.E. Romney leg.; MTEC. Nevada • 1 क; Humboldt Co., Valmy; [40.79 ${ }^{\circ}$ N, $117.13^{\circ}$ W]; 25 May 1957; J.C. Hall leg.; UCDC • 1 \%;

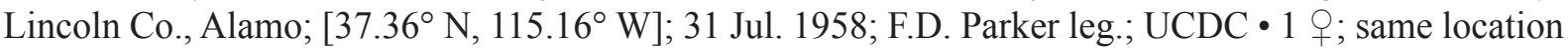
as for preceding; 30 Jul. 1968; F.D. Parker leg.; UCDC • 2 q $\circ$; Nye Co., Beatty; [36.91 $\left.{ }^{\circ} \mathrm{N}, 116.76^{\circ} \mathrm{W}\right]$; 4 Aug. 1950; J.W. MacSwain leg.; ex Melilotus; EMEC. - New Mexico • 1 \%; Hidalgo Co., 9 mi. $\mathrm{N}$ of

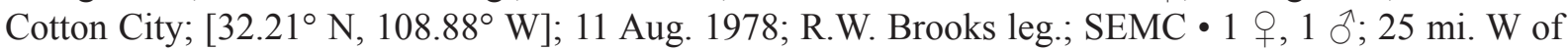

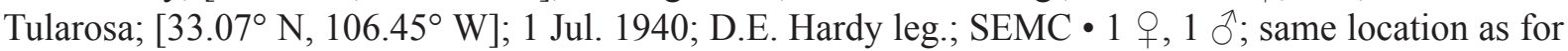
preceding; 1 Jul. 1940; L.C. Kuitert leg.; SEMC • 1 \%; same location as for preceding; 1 Jul. 1940; R H. Beamer leg.; SEMC • $1 \delta^{\top}$; Estancia; [34.76 N, 106.06 W]; 24 Jun. 1940; R.H. Beamer leg.; SEMC •

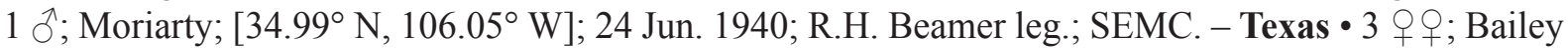
Co., Muleshoe Wildlife Refuge, Grassland 2; $33.9302^{\circ}$ N, $102.4861^{\circ}$ W; 15 Apr. 2013; S.J. Galdek leg.; BBSL 1 1 ; ; Bailey Co., Muleshoe Wildlife Refuge, Prairie Dog 2; $33.9302^{\circ} \mathrm{N}, 102.7584^{\circ} \mathrm{W} ; 15$ Apr. 2013; S.J. Galdek leg.; BBSL. - Utah • 2 우; Emery Co., 1.5 mi. NE of Little Gilson Butte; [38.6 N, $\left.110.58^{\circ} \mathrm{W}\right]$; $1539 \mathrm{~m}$ a.s.1.; 23 Jul. 1981; Parker, Veirs and Griswold leg.; BBSL • 1 q; Emery Co., 2 mi. E of Little Gilson Butte; [38.59 $\mathrm{N}, 110.57^{\circ} \mathrm{W}$ ]; $1554 \mathrm{~m}$ a.s.1.; 23 Jul. 1981; Parker, Veirs and 
Griswold leg.; BBSL • 1 q; Emery Co., above Little Wild Horse Canyon SW of Goblin Vly; [38.55 ${ }^{\circ}$, $\left.110.82^{\circ} \mathrm{W}\right] ; 1463 \mathrm{~m}$ a.s.l.; 26 Jul. 1983; Parker and Griswold leg.; BBSL • 4 q ; ; Emery Co., Wild Horse Creek N of Goblin Vly; 38.5962 ${ }^{\circ} \mathrm{N}, 110.7071^{\circ} \mathrm{W}$; $1494 \mathrm{~m}$ a.s.1.; 25-28 Jul. 1983; Parker and Griswold leg.; BBSL・1 q, 1 đ; same location as for preceding; 21-23 Jul. 1981; Veirs, Parker and Griswold leg.; BBSL • 1 \%; Kane Co., 1 mi. N of Little White Rock Canyon; 37.14 N, $111.8354^{\circ} \mathrm{W}$; 9 Aug. 2002; S. Messinger leg.; ex Tamarix sp.; PCYU • 1 q; Kane Co., Tibbet Canyon; $37.1606^{\circ}$ N, $111.5392^{\circ} \mathrm{W}$; ex Salsola paulsenii; PCYU • 1 क ; Tooele Co., 4.17 mi. SE of Wig Mt.; $40.276^{\circ} \mathrm{N}$, 113.0068 W; 26 Sep. 2005; T. Griswold and K. Huntzinger leg.; PCYU • 1 q; Tooele Co., 4.6 mi. WSW

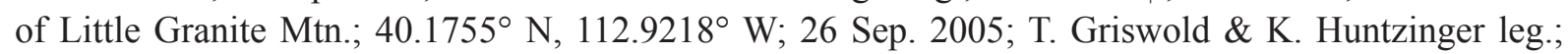

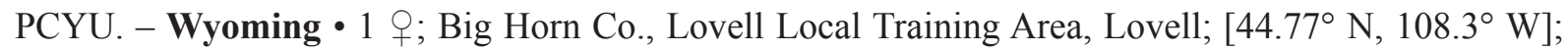
$1158 \mathrm{~m}$ a.s.l.; 11 Jul. 2000; B.C. Kondratieff, P.M. Pineda and H. Al-Dhafer leg.; CSUC • $1{ }^{\Uparrow}$; Carbon Co., 8 mi. N of Baggs; [41.15 N, 107.66 W]; 16 Aug. 1963; N. and B. Marston leg.; AMNH.

\section{Redescription}

\section{Female}

See Gibbs (2010) for a complete redescription of the female (but note that the metasoma colour can vary from black to orange).

\section{Male}

Colouration. Head and mesosoma blue to blue-green; clypeus apical colour reddish brown; labrum reddish brown to orange; mandible orange with black basal spot and red tip; flagellum reddish brown dorsally, yellow-orange ventrally; pronotal lobe reddish brown; metasoma black or reddish brown with rims of terga and sterna narrowly translucent reddish brown and downcurved lateral areas of terga becoming narrowly transparent; legs reddish brown with femur-tibia joints, base and apex of tibiae, and tarsi yellow; tegula pale amber; wing membrane hyaline, veins with subcosta and costa apically brown, otherwise pale amber.

Pubescence. Body hair colour white. Tomentum dense on head except postgena and between ocelli, mesosoma except pronotum anterior face, scutum and scutellum medially, and propodeum, T1-3 basally and laterally, and T4-6 throughout; sparse on propodeum posterolateral slope. Scutum hair densely plumose. Sterna hair short (1-1.5 OD), densely plumose, dense and erect. Wing hairs light, short and dense.

SuRfACE SCUlPTURE. Clypeus punctures moderately dense ( $\mathrm{i}=1-2 \mathrm{pd})$, sculpture shiny; supraclypeal area punctures dense ( $\mathrm{i} \leq 1 \mathrm{pd})$, sculpture shiny; paraocular area punctures dense ( $\mathrm{i} \leq 1 \mathrm{pd})$, sculpture shiny; frons punctures dense $(\mathrm{i}<1 \mathrm{pd})$, sculpture shiny; vertex punctures dense laterally $(\mathrm{i}<1 \mathrm{pd})$, moderately sparse medially ( $i=1-2 \mathrm{pd}$ ), sculpture shiny; gena punctures moderately dense ( $\mathrm{i}=1-2 \mathrm{pd})$, sculpture shiny; postgena sculpture shiny, becoming tessellate laterad of hypostomal carina; tegula punctures absent; scutum punctures sparse ( $\mathrm{i}=1-4 \mathrm{pd}$ ), becoming dense marginally ( $\mathrm{i} \leq 1 \mathrm{pd}$ ), sculpture shiny; scutellum punctures sparse ( $\mathrm{i}=1-3 \mathrm{pd})$, sculpture shiny; metanotum sculpture shiny and finely, sparsely punctate ( $\mathrm{i}=1-3 \mathrm{pd})$; metapostnotum rugae strong, subparallel, not reaching margin, sculpture weakly imbricate; preëpisternum sculpture areolate; hypoepimeron punctures crowded ( $\mathrm{i}=0 \mathrm{pd})$, sculpture shiny; mesepisternum punctures dense $(\mathrm{i}<1 \mathrm{pd})$, sculpture shiny; metepisternum sculpture lineate dorsally, areolate ventrally; propodeum lateral face punctures dense $(\mathrm{i}<1 \mathrm{pd})$, obscure, sculpture weakly imbricate; propodeum posterior face sculpture shiny and sparsely punctate ( $\mathrm{i}=1-4 \mathrm{pd})$; $\mathrm{T} 1$ anterior face sculpture shiny; $\mathrm{T} 1$ dorsal surface punctures sparse $(\mathrm{i}=1-3 \mathrm{pd})$, sculpture shiny; $\mathrm{T} 2$ disc punctures moderately dense $(i=1-2 \mathrm{pd})$, becoming sparse apicomedially ( $\mathrm{i}=1-3 \mathrm{pd})$, disc sculpture shiny, rim punctures minute, very sparse $(i=3-6 \mathrm{pd})$, rim sculpture shiny. 


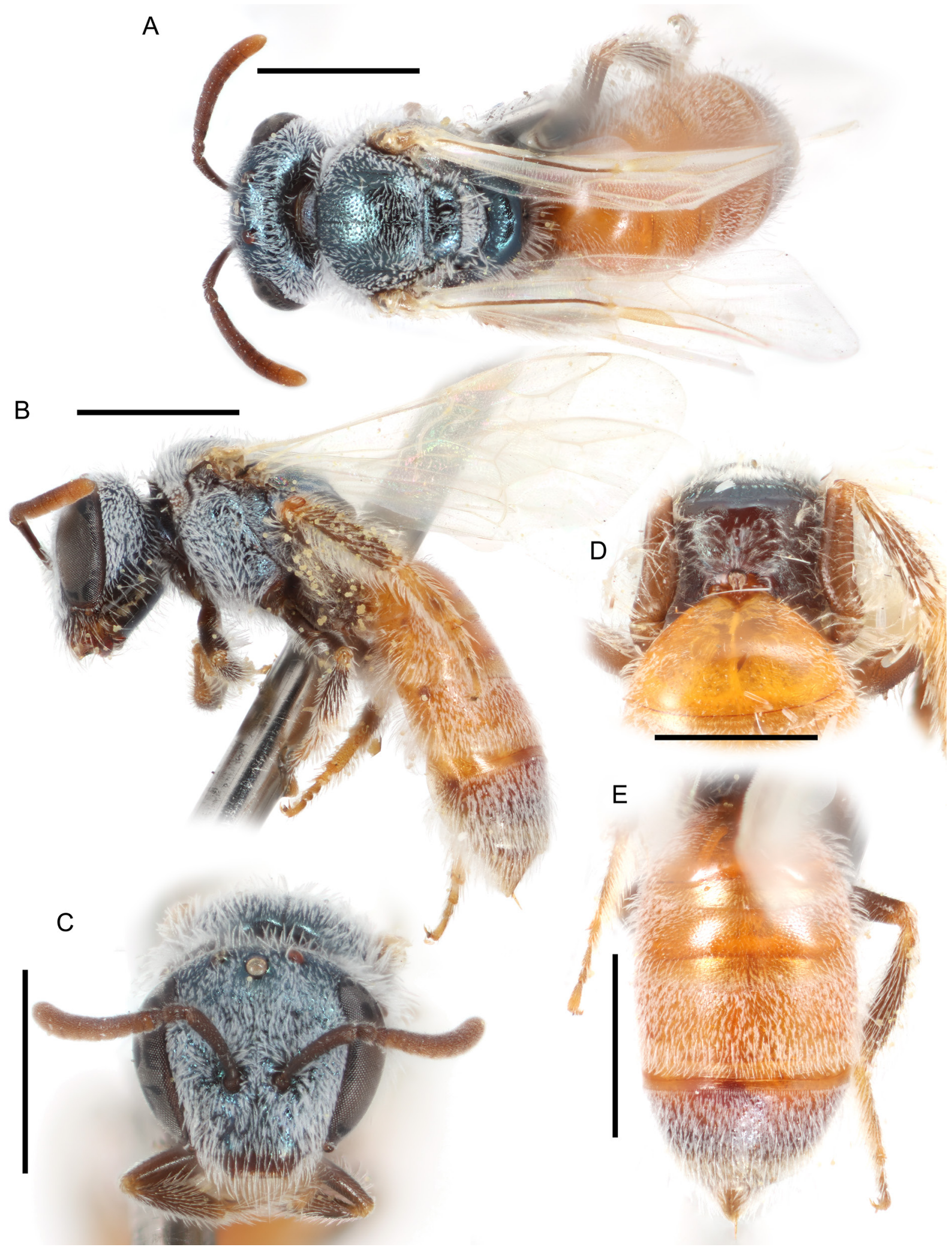

Fig. 67. Lasioglossum (D.) pallidellum (Ellis, 1914), red morph, ㅇ. A. Dorsal habitus. B. Lateral habitus. C. Face. D. Propodeum. E. Metasoma. Scale bars: $1 \mathrm{~mm}$. 


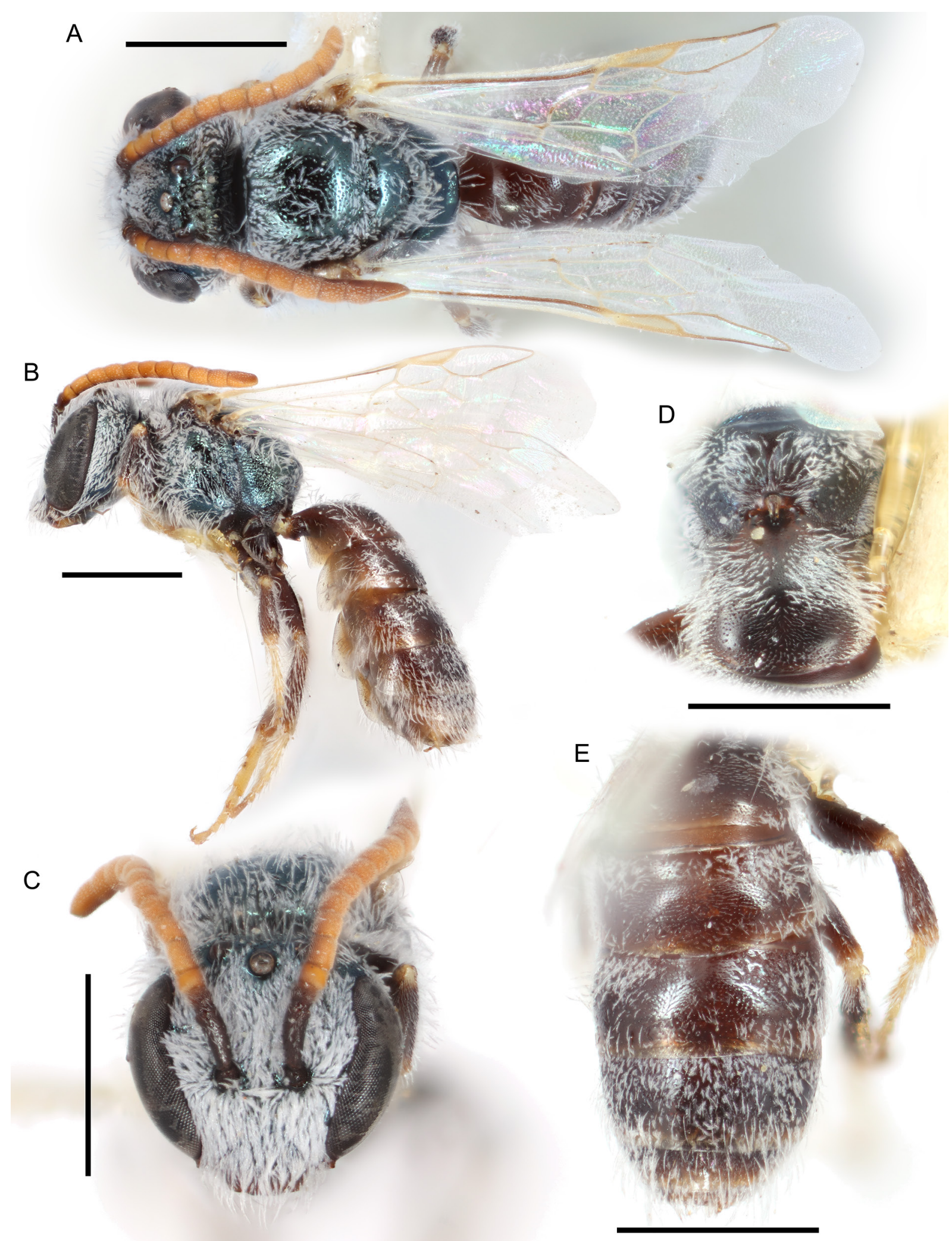

Fig. 68. Lasioglossum (D.) pallidellum (Ellis, 1914), dark morph, đ̂. A. Dorsal habitus. B. Lateral habitus. C. Face. D. Propodeum. E. Metasoma. Scale bars: $1 \mathrm{~mm}$. 
STRUCTURE. Face length/width ratio $0.82( \pm 0.02 \mathrm{SD})$. F1:pedicel length ratio 1.08 ( $\pm 0.21 \mathrm{SD}) ; \mathrm{F} 2: \mathrm{F} 1$ length ratio $1.75( \pm 0.27 \mathrm{SD}) ; \mathrm{F} 2$ length/width ratio $1.26( \pm 0.09 \mathrm{SD}) ; \mathrm{F} 9$ length/width ratio 1.11 $( \pm 0.12 \mathrm{SD})$. Forewing with 3 submarginal cells; pronotal angle obtuse; tegula shape normal. Intertegular distance $0.9( \pm 0.03 \mathrm{SD}) \mathrm{mm}$. Scutum length/width ratio $0.77( \pm 0.05 \mathrm{SD})$; scutum/scutellum length ratio 2.54 ( $\pm 0.16 \mathrm{SD})$; scutellum/metanotum length ratio 1.52 ( $\pm 0.11 \mathrm{SD}) ;$ metanotum/metapostnotum length ratio $0.76( \pm 0.08 \mathrm{SD})$. Propodeum lateral carinae not reaching dorsal margin; oblique carina absent. $(n=5)$

GenitALIA. As in Fig. 90K. Gonocoxite very broad, cashew-shaped. Gonostylus very large, boot-shaped, with a few long hairs. Retrorse lobe broad, ovoid, with dense short hairs concentrated at apex.

\section{Range}

Alberta south to Nuevo Leon and west to California (Fig. 69).

\section{Floral records}

AMARANTHACEAE Juss. Salsola L. S. paulsenii Litv. • ASTERACEAE Giseke: Aplopappus • Ericameria: E. nauseosa (Pall. ex Pursh) G.L. Nesom \& Baird: E. n. var. nauseosa (Pall. ex Pursh) G.L. Nesom \& Baird - Gutierrezia: G. microcephala (DC.) A.Gray - Isocoma: I. acradenia (Greene) Greene - CLEOMACEAE Bercht. \& J. Presl: Cleomella DC. C. obtusifolia Torr. \& Frém. • Oxystylis Torr. \& Frém. O. lutea Torr. \& Frém. - Wislizenia Engelm. W. refracta Engelm. • FABACEAE Juss. Acacia: A. greggii A.Gray - Astragalus: A. lentiginosus Hook. - Melilotus $・$ Prosopis: P. glandulosa

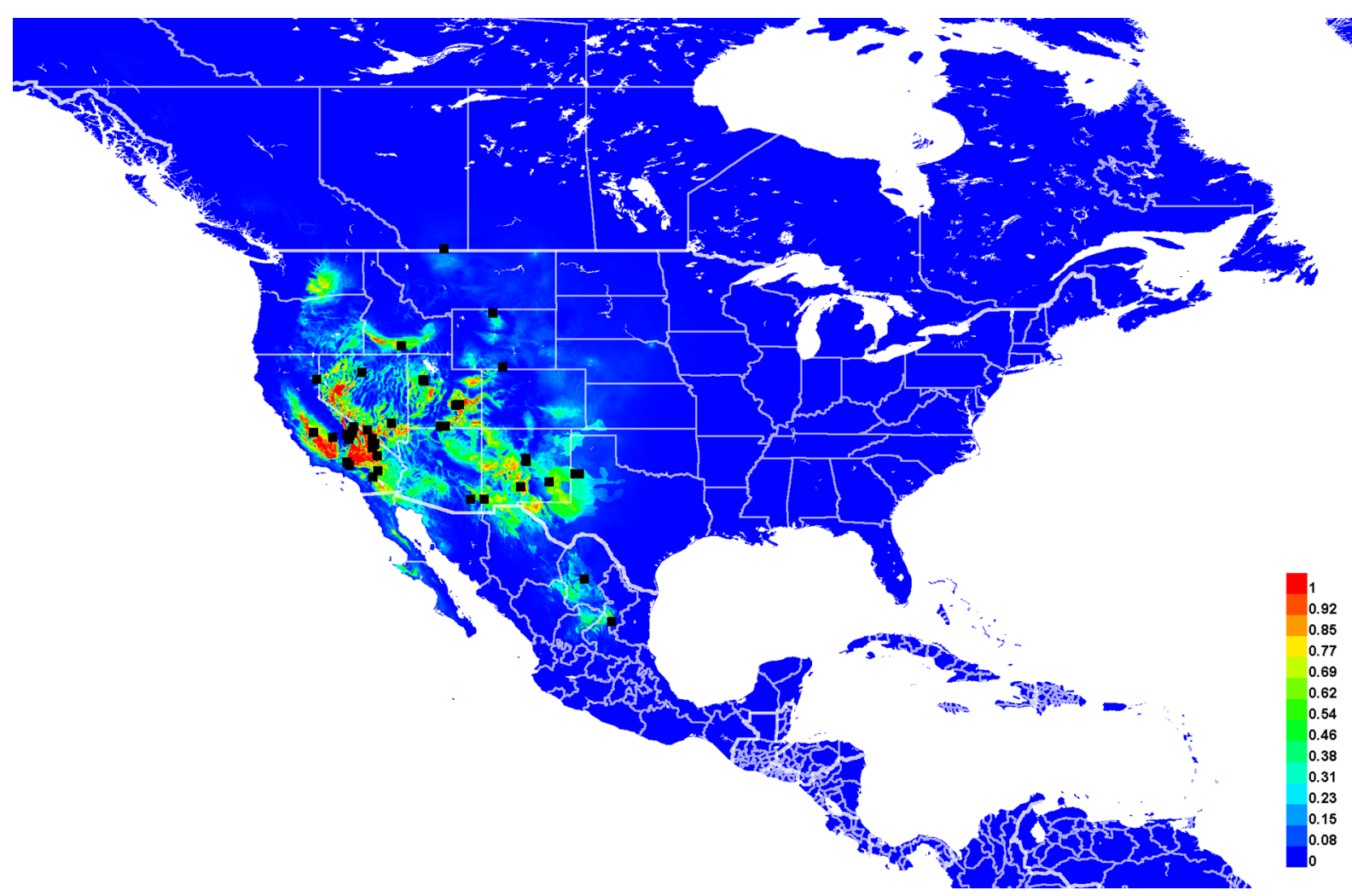

Fig. 69. Georeferenced collection records of Lasioglossum (D.) pallidellum (Ellis, 1914) (black squares) and predicted distribution by maximum entropy ecological niche modeling in Maxent (colour shading). Warmer colours indicate higher cloglog probability of occurrence. 
Torr. • POLYGONACEAE Juss. Eriogonum Michx. E. trichopes Torr. • ROSACEAE Juss. Prunus L. • SALICACEAE Mirb. Salix L. S. exigua Nutt. -TAMARICACEAE Link: Tamarix L. T. aralensis Bunge.

\section{DNA barcodes}

Six sequences available (BOLD process IDs: DLII676-07, DLII1090-07, DIAL1137-07, DLII1229-08, DLII1234-08, DLII1236-08). However, all are incomplete ( $<400 \mathrm{bp})$ and do not have an assigned BIN. Lasioglossum pallidellum differs from all other western red-tailed $L$. (Dialictus) by 2 fixed substitutions: 426(A) and 543(T) (Supplementary file 4).

\section{Remarks}

Lasioglossum pallidellum was redescribed by Gibbs (2010); however, neither the male nor the red-tailed form was known to him at that time. The male is described here for the first time. In addition, the known range of this species is expanded to Nuevo Leon and California.

Almost all specimens from the Mojave Desert are of the red-tailed form, and most of the rest are dark. The red-tailed form is especially abundant in and around Death Valley National Park. It is possible that there is a geographic barrier between the Mojave Desert and the rest of L. pallidellum's range, and the red and dark forms are distinct species. However, there are no other obvious morphological features distinguishing the two forms, and the geographic separation is not perfect. There are several red-tailed specimens and intermediate forms from eastern Utah, Coahuila, and Nuevo Leon, and a single dark female and two males from the Death Valley area. Along with the lack of high-quality DNA barcodes for both forms, the two-species hypothesis is difficult to test. Until more evidence to the contrary is found, it seems best to consider these a single species.

Lasioglossum (Dialictus) perditum sp. nov.

urn:1sid:zoobank.org:act:71D0A259-8327-4C5B-8615-A7DF4C2F373B

Figs 6B, 70-72, 94D

\section{Diagnosis}

Females of Lasioglossum perditum sp. nov. can be recognized by the characters for the Lasioglossum clematisellum species complex (below), in addition to their very small size $(3.5 \mathrm{~mm})$, forewing almost always with only two submarginal cells (a single specimen has three submarginal cells in one wing), and head and mesosoma very shiny and sparsely punctate (up to $\mathrm{i}=6 \mathrm{pd}$ ). They are most similar to L. julipile sp. nov. and L. spivakae sp. nov. Both of these species have the head and mesosoma more densely punctate (up to $\mathrm{i}=3 \mathrm{pd}$ ) and forewing rarely with two submarginal cells. In addition, females of L. julipile sp. nov. are larger $(4-4.5 \mathrm{~mm})$ and L. spivakae sp. nov. have the face slightly longer (length/ width ratio $\sim 0.87$ ).

Males of L. perditum sp. nov. can be recognized by the same characters as for females. In addition, the propodeum lateral face is usually smooth and tessellate. They are most similar to L. julipile sp. nov., L. clematisellum, L. clastipedion sp. nov., and L. spivakae sp. nov. All of these species have the frons and vertex more densely punctate (up to $\mathrm{i}=2 \mathrm{pd}$ ) and propodeum lateral face usually rugulose. In addition, males of $L$. spivakae sp. nov. have T1-3 rims completely punctate to the apex with no change in sculpture across the premarginal line.

\section{Etymology}

The specific epithet 'perditum' is a Latin perfect participle meaning 'lost'. It refers to this species' extremely small size and two submarginal cells, which is reminiscent of the bee genus Perdita Smith, 1853. An appropriate translation would be the lost sweat bee. 


\section{Material examined}

Holotype

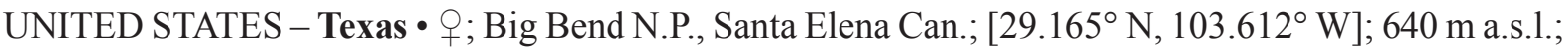
5 May 1959; W.R.M. Mason leg.; CNC.

[Verbatim label: Big Bend N.P.TEX. / Santa Elena Can. / 2100 ft. May 5 / W.R.M. Mason 1959 // HOLOTYPE / Lasioglossum (Dialictus) perditum Gardner and Gibbs]

\section{Paratypes}

UNITED STATES - New Mexico • 1 đ̊; Eddy Co.; 32.1095 N, $104.4716^{\circ}$ W; 1115 m a.s.1.; 2 Aug. 2010; J.D. Herndon and A. Druk leg.; ex Asclepias subverticillata; BBSL. - Texas • 1 ○े; same collection data as for holotype; BBSL・1,+ 1 ; ; same location as for holotype; 9 May 1959; W.R.M. Mason leg.;

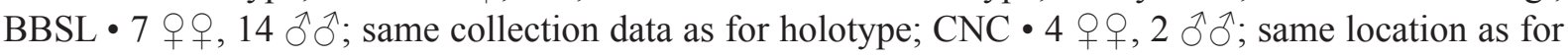
holotype; 9 May 1959; W.R.M. Mason leg.; CNC.

\section{Description}

\section{Female}

Colouration. Head and mesosoma blue-green to golden-green; clypeus apical colour black to reddish brown; labrum reddish brown to orange; mandible orange with black basal spot and red tip; flagellum reddish brown dorsally, light brown to orange ventrally; pronotal lobe orange; metasoma orange with dark spiracular spots on T3-4; legs reddish brown with femur-tibia joints, tibia apices, and tarsi mostly orange; tegula pale amber; wing membrane hyaline, veins with subcosta brown, otherwise pale amber to light brown.

PubesCENCE. Body hair colour white. Tomentum dense on paraocular area, gena, pronotal collar and lobe, space between pronotal lobe and tegula, metanotum medially, metepisternum, narrow basal band on T2-3, and T4 throughout; sparse on face below eye emargination, preëpisternum, mesepisternum, and T3 apical rim. Scutum hair densely plumose. Wing hairs light, very short and sparse. Acarinarial fan complete, very sparse. T2 fringes sparse, T3 fringes sparse.

SuRfACE SCULPTURE. Clypeus punctures irregularly sparse ( $\mathrm{i} \leq 2 \mathrm{pd}$ ), sometimes becoming dense in basal half $(\mathrm{i}<1 \mathrm{pd})$, sculpture shiny, sometimes weakly imbricate basally; supraclypeal area punctures sparse ( $i=1-6 \mathrm{pd}$ ), sometimes becoming dense laterally $(\mathrm{i} \leq 1 \mathrm{pd})$, diversopunctate, sculpture shiny; paraocular area punctures large and moderately sparse $(i=1-2 \mathrm{pd})$ to extremely minute and sparse $(\mathrm{i}=2-4 \mathrm{pd})$, sculpture shiny; frons punctures fine and moderately sparse $(i=1-2 \mathrm{pd})$ to extremely minute and sparse $(\mathrm{i}=2-6 \mathrm{pd})$, sculpture shiny; vertex punctures minute, sparse $(\mathrm{i}=2-6 \mathrm{pd})$, sculpture shiny; gena punctures minute, sparse ( $i=2-4 \mathrm{pd})$, sculpture shiny; postgena sculpture tessellate to weakly so; tegula punctures absent; scutum punctures moderately dense $(i=1-2 \mathrm{pd})$, becoming sparse $(\mathrm{i}=1-5 \mathrm{pd})$ and sometimes minute laterally and anteromedially, sculpture shiny, becoming tessellate anteromedially; scutellum punctures sparse ( $\mathrm{i}=1-5 \mathrm{pd}$ ), sometimes becoming dense posteriorly ( $i<1 \mathrm{pd}$ ), diversopunctate, sculpture shiny to tessellate; metanotum sculpture shiny and minutely, sparsely punctate $(i=1-4 \mathrm{pd})$, sometimes weakly rugulose; metapostnotum rugae weak, highly anastomosing, reaching margin, or sometimes absent, sculpture tessellate; preëpisternum sculpture shiny with crowded punctures $(i=0 \mathrm{pd})$, sometimes slightly sparser $(\mathrm{i} \leq 1 \mathrm{pd})$; hypoepimeron punctures large and crowded $(\mathrm{i}=0 \mathrm{pd})$ to minute and sparse ( $\mathrm{i}=2-6 \mathrm{pd})$, sculpture shiny; mesepisternum punctures large and crowded ( $\mathrm{i}=0 \mathrm{pd}$ ) to minute and sparse ( $i=2-6 \mathrm{pd})$, sculpture shiny; metepisternum sculpture ruguloso-lineate dorsally, weakly rugulose ventrally; propodeum lateral face sculpture tessellate; propodeum posterior face sculpture tessellate; $\mathrm{T} 1$ anterior face sculpture shiny; T1 dorsal surface punctures sparse $(\mathrm{i}=1-5 \mathrm{pd})$, becoming finer or obscure on rim and absent in large apicolateral oval patches, sculpture shiny; T2 disc punctures sparse 
$(i=1-4 \mathrm{pd})$, disc sculpture shiny, rim punctures sparse $(\mathrm{i}=1-4 \mathrm{pd})$, sometimes minute and obscure, rim sculpture shiny to weakly coriarious.

Structure. Face length/width ratio 0.82 ( $\pm 0.03 \mathrm{SD}$ ). Clypeus projecting $\sim 50 \%$ below suborbital tangent; clypeal area length/width ratio 0.38 ( $\pm 0.03 \mathrm{SD})$; apicolateral denticles very low rounded knobs; supraclypeal area length/width ratio $0.76( \pm 0.12 \mathrm{SD})$. Forewing with 2 submarginal cells; pronotal angle obtuse; tegula shape normal. Intertegular distance $0.67( \pm 0.03 \mathrm{SD}) \mathrm{mm}$. Scutum length/width ratio $0.82( \pm 0.05 \mathrm{SD})$; scutum/scutellum length ratio $3.31( \pm 0.19 \mathrm{SD})$; scutellum/metanotum length ratio 1.39 ( $\pm 0.11 \mathrm{SD})$; metanotum/metapostnotum length ratio $0.65( \pm 0.07 \mathrm{SD})$. Propodeum lateral carinae not reaching dorsal margin; oblique carina absent. T2 depressed apical rim length less than 50\% of segment. $(n=10)$

\section{Male}

Colouration. Head and mesosoma blue to olive green; clypeus apical colour black to reddish brown; labrum reddish brown to orange; mandible orange with black basal spot and red tip; flagellum reddish brown dorsally, orange to brown ventrally; pronotal lobe reddish brown; metasoma black to dark reddish brown with rims of terga and sterna broadly translucent reddish brown and downcurved lateral areas of terga becoming translucent orange; legs black to reddish brown with femur-tibia joints, base and apex of tibiae, and tarsi orange to yellow; tegula orange to pale amber; wing membrane hyaline, veins with subcosta dark brown, otherwise brown to light brown.

Pubescence. Body hair colour white. Tomentum dense on face below ocelli, gena, pronotal angle and lobe, space between pronotal lobe and tegula, metepisternum, and mesosterna; sparse on preëpisternum, mesepisternum, and T2-3 basolaterally. Scutum hair densely plumose. Sterna hair short (0.75-1.25 OD), densely plumose, dense and erect. Wing hairs light, short and sparse.

SURFACE SCULPTURE. Clypeus punctures moderately dense ( $\mathrm{i}=1-2 \mathrm{pd})$, sculpture shiny; supraclypeal area punctures moderately dense ( $\mathrm{i}=1-2 \mathrm{pd})$, sculpture shiny; paraocular area punctures moderately dense $(i=1-2 \mathrm{pd})$, sculpture shiny; frons punctures sparse $(i=1-3 \mathrm{pd})$, sculpture shiny; vertex punctures fine, sparse $(i=1-4 \mathrm{pd})$, sometimes larger and denser laterally $(\mathrm{i}=1-2 \mathrm{pd})$, sculpture shiny; gena punctures moderately dense ( $\mathrm{i}=1-2 \mathrm{pd})$, sculpture shiny; postgena sculpture shiny to imbricate, sometimes lineolate; tegula punctures absent; scutum punctures sparse ( $i=1-5 \mathrm{pd})$, becoming moderately dense posteriorly and on median line ( $\mathrm{i}=1-2 \mathrm{pd}$ ), sculpture shiny, sometimes becoming weakly tessellate anteromedially; scutellum punctures sparse $(i=1-5 \mathrm{pd})$, sometimes becoming moderately dense posteriorly $(\mathrm{i}=1-2 \mathrm{pd})$, sculpture shiny; metanotum sculpture shiny and moderately densely punctate ( $i=1-2 \mathrm{pd}$ ), becoming rugulose laterally; metapostnotum rugae strong or weak, anastomosing, nearly reaching margin, sculpture shiny to imbricate; preëpisternum sculpture areolate; hypoepimeron punctures moderately dense ( $i=1-2 \mathrm{pd})$, sculpture shiny; mesepisternum punctures moderately dense $(i=1-2 \mathrm{pd})$, sculpture shiny; metepisternum sculpture lineate dorsally, areolate ventrally; propodeum lateral face punctures dense ( $i \leq 1 \mathrm{pd}$ ), often obscure, sculpture tessellate to rugulose; propodeum posterior face sculpture shiny and sparsely punctate $(\mathrm{i}=1-3 \mathrm{pd})$; $\mathrm{T} 1$ anterior face sculpture shiny; $\mathrm{T} 1$ dorsal surface punctures moderately dense $(i=1-2 \mathrm{pd})$, becoming sparse laterally and in small apicolateral oval patches $(i=1-4 \mathrm{pd})$, sculpture shiny; $\mathrm{T} 2$ disc punctures moderately dense $(i=1-2 \mathrm{pd})$, becoming sparse laterally and in small apicolateral oval patches $(i=1-4 \mathrm{pd})$, disc sculpture shiny, rim punctures sparse medially $(i=1-4 \mathrm{pd})$, absent laterally, rim sculpture shiny.

StRUCTURE. Face length/width ratio 0.84 ( $\pm 0.04 \mathrm{SD})$. F1:pedicel length ratio $0.9( \pm 0.14 \mathrm{SD}) ; \mathrm{F} 2$ :F1 length ratio $1.75( \pm 0.17 \mathrm{SD}) ; \mathrm{F} 2$ length/width ratio $1.39( \pm 0.23 \mathrm{SD}) ; \mathrm{F} 9$ length/width ratio $1.26( \pm 0.14 \mathrm{SD})$. Forewing with 2 or 3 submarginal cells; pronotal angle obtuse; tegula shape normal. Intertegular distance $0.65( \pm 0.06 \mathrm{SD}) \mathrm{mm}$. Scutum length/width ratio 0.84 ( $\pm 0.04 \mathrm{SD})$; scutum/scutellum length ratio 3.13 


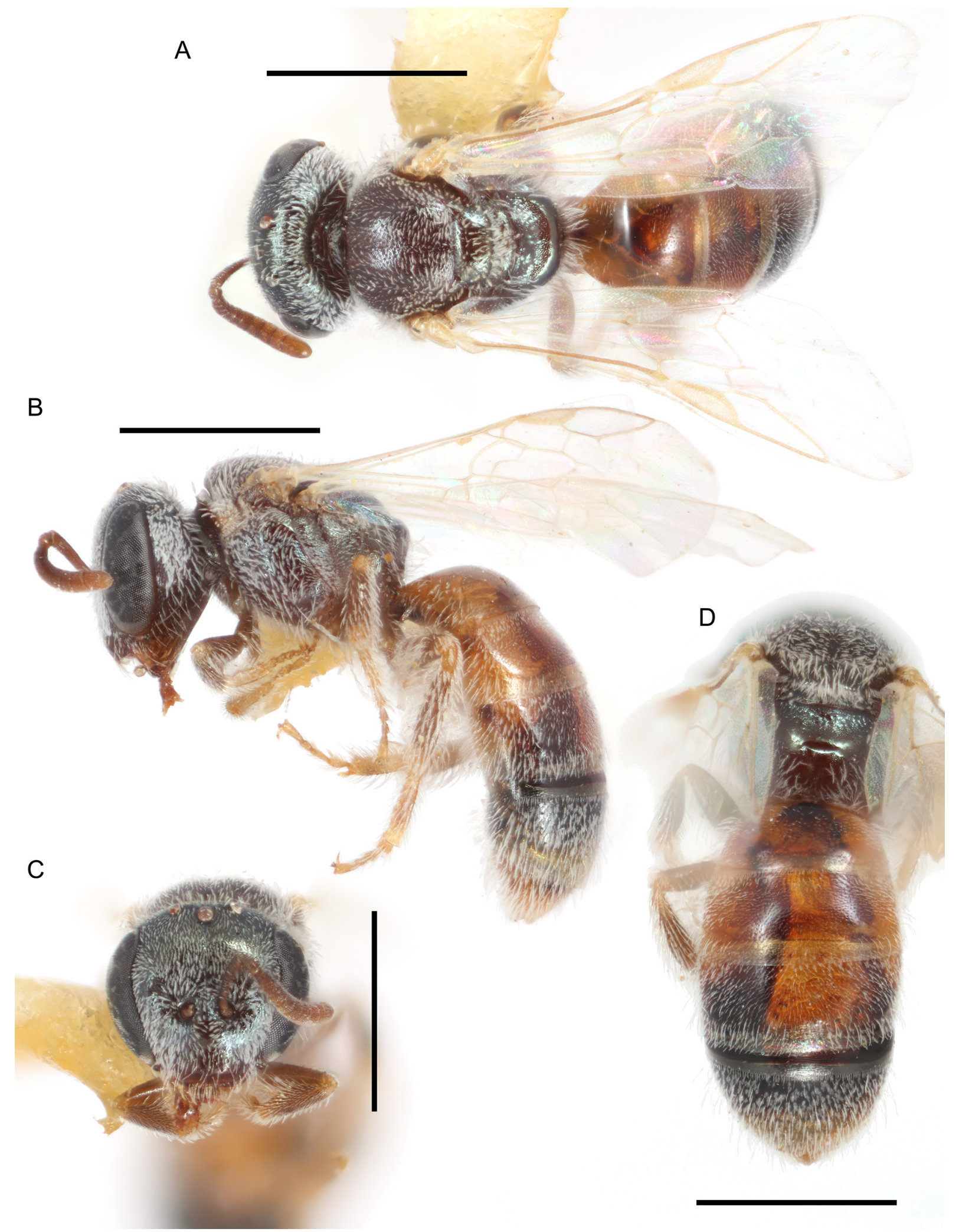

Fig. 70. Lasioglossum (D.) perditum sp. nov., + . A. Dorsal habitus. B. Lateral habitus. C. Face. D. Propodeum and metasoma. Scale bars: $1 \mathrm{~mm}$. 


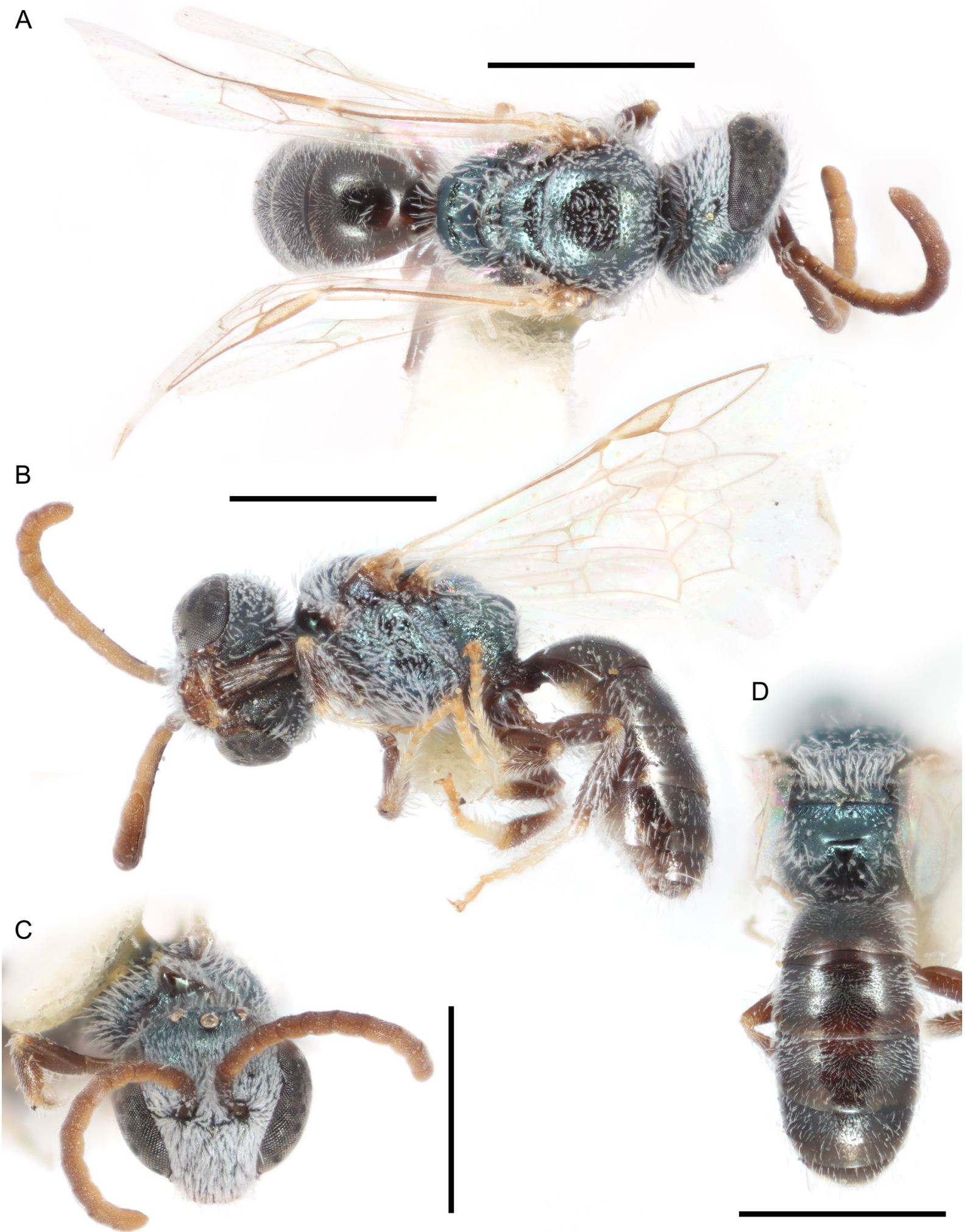

Fig. 71. Lasioglossum (D.) perditum sp. nov., §. A. Dorsal habitus. B. Lateral habitus. C. Face. D. Propodeum and metasoma. Scale bars: $1 \mathrm{~mm}$. 
$( \pm 0.31 \mathrm{SD}) ;$ scutellum/metanotum length ratio 1.55 ( $\pm 0.19 \mathrm{SD})$; metanotum/metapostnotum length ratio $0.64( \pm 0.06 \mathrm{SD})$. Propodeum lateral carinae not reaching dorsal margin; oblique carina absent. $(\mathrm{n}=10)$

Genitalia. Not examined.

\section{Range}

Chihuahuan Desert of southeastern New Mexico and western Texas (Fig. 72).

\section{Floral records}

APOCYNACEAE Juss. Asclepias L. A. subverticillata (A.Gray) Vail.

\section{DNA barcodes}

Not available.

\section{Remarks}

Rare, but may be locally common in some areas. Lasioglossum perditum sp. nov. is only known from a single specimen from Carlsbad Caverns National Park and a relatively long series from Big Bend National Park.

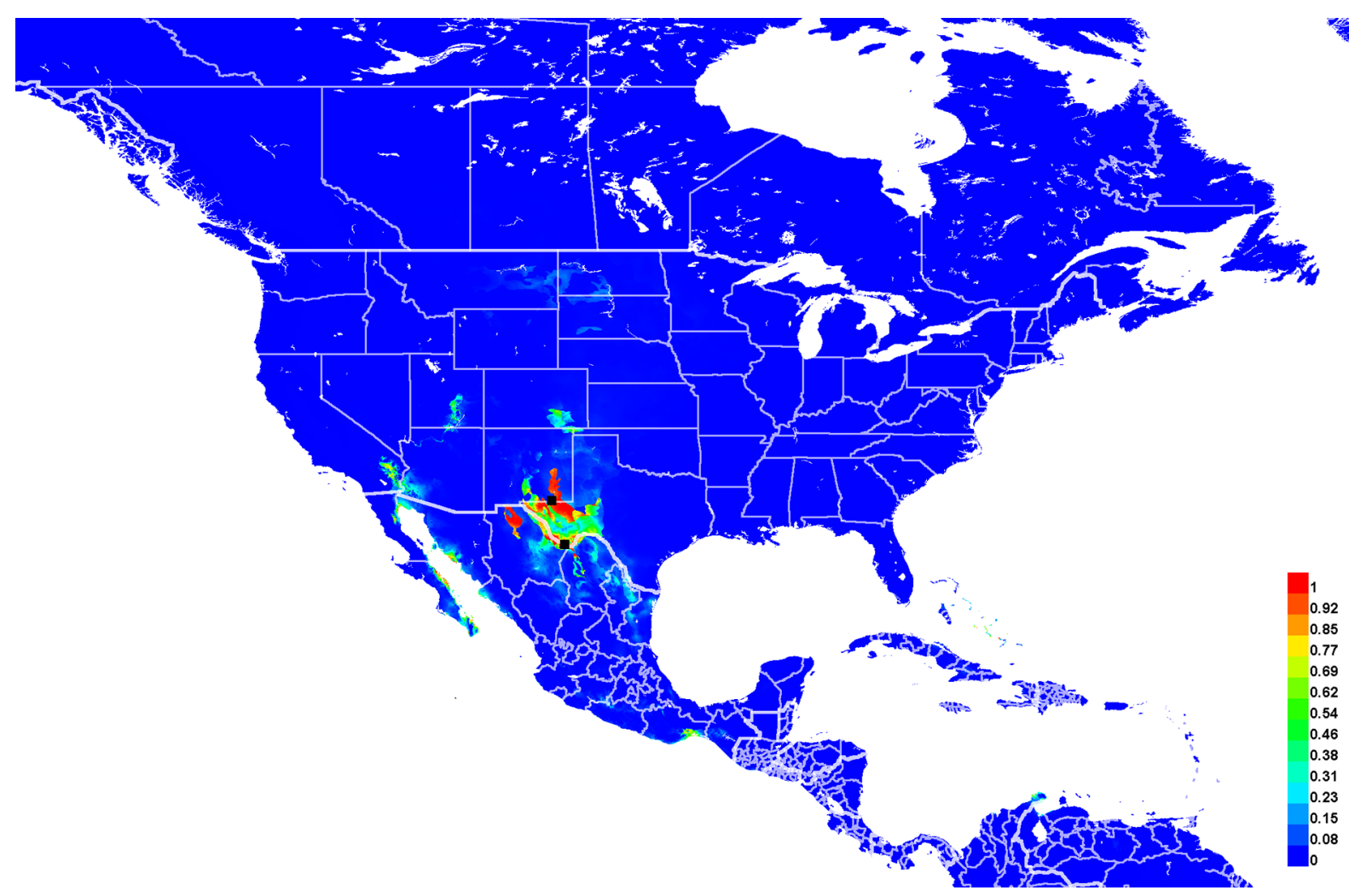

Fig. 72. Georeferenced collection records of Lasioglossum (D.) perditum sp. nov. (black squares) and predicted distribution by maximum entropy ecological niche modeling in Maxent (colour shading). Warmer colours indicate higher cloglog probability of occurrence. The predicted distribution was generated with a regularization multiplier of 0.5 to obtain a more conservative prediction. Due to the very small number of records, this distribution should be interpreted with caution. 
Lasioglossum (Dialictus) petrellum (Cockerell, 1903)

Fig. 73

Halictus petrellus Cockerell, 1903: 84 (holotype,, , deposited in USNM, examined).

Halictus coronadensis Cockerell, 1916: 56 (holotype, ô, deposited in USNM, examined; synonymy by Timberlake in Michener 1951).

Halictus (Chloralictus) petrellus - Sandhouse 1924: 3 (key).

Lasioglossum (Chloralictus) petrellum - Michener 1951: 1116 (catalog).

Dialictus petrellus - Hurd 1979: 1970 (catalog). — Moure \& Hurd 1987: 121 (catalog).

Lasioglossum (Dialictus) petrellum - Gibbs 2009: 376, figs 7a-b, 8a-b, 9a-d (key, redescription).

\section{Diagnosis}

Both sexes of Lasioglossum petrellum can be recognized by the characters for the Lasioglossum petrellum species complex (below), in addition to the characters given in the keys. They are most similar to $L$. droegei. Females of $L$. droegei have T2 -4 with more extensive tomentum, and metasoma usually mostly red-orange. Males of $L$. droegei have T1-3 apical areas with very strong microsculpture (similar to scutum) and indistinct punctures. In addition, the distributions of these two species are not known to overlap: L. petrellum is known from the Sonoran Desert and west coast of California and Baja California, while L. droegei is only known from the Chihuahuan Desert.

\section{Etymology}

Cockerell (1903) named this species after the type locality of San Pedro, California, after Latinizing the name Pedro to Petra and adding the adjectival diminutive suffix '-ellus'.

\section{Material examined}

\section{Holotype}

UNITED STATES - California • + ; San Pedro; $\left[33.74^{\circ}\right.$ N, $\left.118.29^{\circ} \mathrm{W}\right]$; Jul. 11 year unknown; Cockerell leg.; USNM 27771.

\section{Other material}

MEXICO - Baja California • 1 \% ; Coronado Islands; [32.4 $\mathrm{N}, 117.244^{\circ} \mathrm{W}$ ]; Aug. 21 year unknown; T. and W. Cockerell leg.; USNM • 3 우; Los Frailles; 18 Mar. 1953; P.H. Arnaud leg.; CAS. - Baja

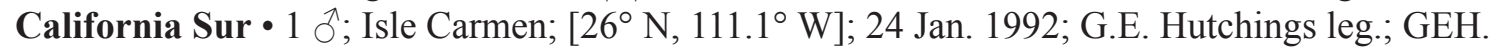

UNITED STATES - Arizona • 1 q ; Yuma Co., Palm Canyon, Kofa Mountains; $\left[33.36^{\circ} \mathrm{N}, 114.1^{\circ} \mathrm{W}\right]$; 8 Apr. 1963; W.A. Steffan leg.; EMEC • 1 q; Sabino Canyon; $\left[32.33^{\circ}\right.$ N, $110.8^{\circ}$ W]; 9 Jul. 1952; R.H. and L.D. Beamer, W. LaBerge, A. Well, C. Liang and C. Weiner leg.; SEMC. - California •

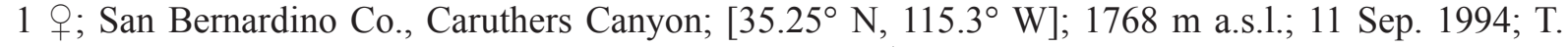
Griswold leg.; ex Gutierrezia microcephala; BBSL 1 d ; San Bernardino Co., Cima Dome, Sec 12 T14N R13E; [35.31 N, 115.54 W]; 1539 m a.s.l.; 16 Jun. 1980; T. Griswold leg.; BBSL • 2 q ; San Bernardino Co., Granite Mts, Sc17T8NR13E; [34.78 N, 115.64 W]; 1234 m a.s.1.; 22-25 May 1983; T L. Griswold leg.; ex Cylindropuntia echinocarpa; BBSL • 1 +; San Bernardino Co., Granite Mts, Sec 17 T8N R13E; [34.78 N, 115.64 W]; 1234 m a.s.l.; 9 Jun. 1980; T. Griswold leg.; BBSL • 1 영 San Bernardino Co., Sheep Corral; $34.7348^{\circ}$ N, $115.6815^{\circ}$ W; 24 May 1995; T. Griswold leg.; ex Sphaeralcea ambigua; BBSL • 1 q; San Bernardino Co., Willow Wash, Sec24 T13N R10E; [35.2 ${ }^{\circ}$ N, $115.87^{\circ} \mathrm{W}$ ]; $671 \mathrm{~m}$ a.s.l.; 6 Apr. 1983; T. and R. Griswold leg.; BBSL • 4 q + ; San Diego Co., La Jolla; $\left[32.83^{\circ} \mathrm{N}, 117.27^{\circ} \mathrm{W}\right] ;$ J Jul. 1963; P.D. Hurd leg.; ex Mesembryanthemum crystallinum; EMEC • 1 क ;

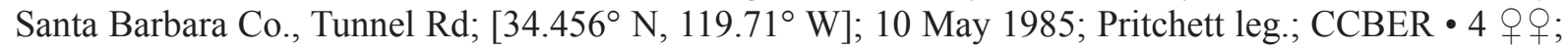
Anza-Borrego, Lost Vlly. Rd.; 33.3746 ${ }^{\circ} \mathrm{N}$, 116.6141 ${ }^{\circ} \mathrm{W}$; 2 May 2015; Hung, Cen and Davids leg.;

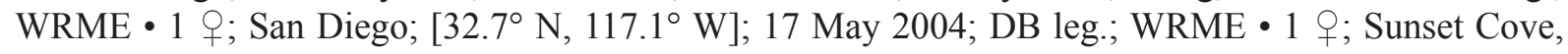


Granites Res. Stn Mojave Desert; $34.779^{\circ}$ N, $115.6631^{\circ}$ W; 13 Apr. 2017; J. Braun leg.; PCYU • 1 q; same location as for preceding; 25 Apr. 2018; J. Braun leg.; PCYU • 1 \&; same location as for preceding; 28 Apr. 2018; J. Braun leg.; PCYU.

\section{Description}

See Gibbs (2009b) for a complete redescription and figures.

\section{Range}

Coasts and deserts of southern California and Baja California, east through southern Arizona (Fig. 73).

\section{Floral records}

AIZOACEAE Martinov: Mesembryanthemum L. M. crystallinum L. - AMARANTHACEAE Juss. Atriplex L. A. semibaccata R.Br. (G09) - ASTERACEAE Giseke: Encelia: E. farinosa A.Gray ex Torr. (G09) - Ericameria: E. nauseosa (Pall. ex Pursh) G.L. Nesom \& Baird: E. n. var. nauseosa (Pall. ex Pursh) G.L. Nesom \& Baird (G09) • E. palmeri (A.Gray) H.M. Hall (G09) • E. parishii (Greene) H.M. Hall (G09) • Gutierrezia: G. microcephala (DC.) A.Gray • Hypochaeris: H. glabra L. (G09) • Lepidospartum: L. squamatum (A.Gray) A.Gray (G09) - BORAGINACEAE Juss. Phacelia Juss. P. distans Benth. (G09) • P. minor (Harv.) Thell. ex F. Zimm. (G09) • CACTACEAE Juss. Cylindropuntia (Engelm.) F.M. Knuth: C. echinocarpa (Engelm. \& J. M. Bigelow) F.M. Knuth • Ferocactus Britton \& Rose: F. cylindraceus (Engelm.) Orcutt (G09) - CRASSULACEAE J. St.-Hil. Dudleya Britton \& Rose (G09) • MALVACEAE Juss. Sphaeralcea A. St.-Hil. S. ambigua A.Gray • PLANTAGINACEAE Juss.

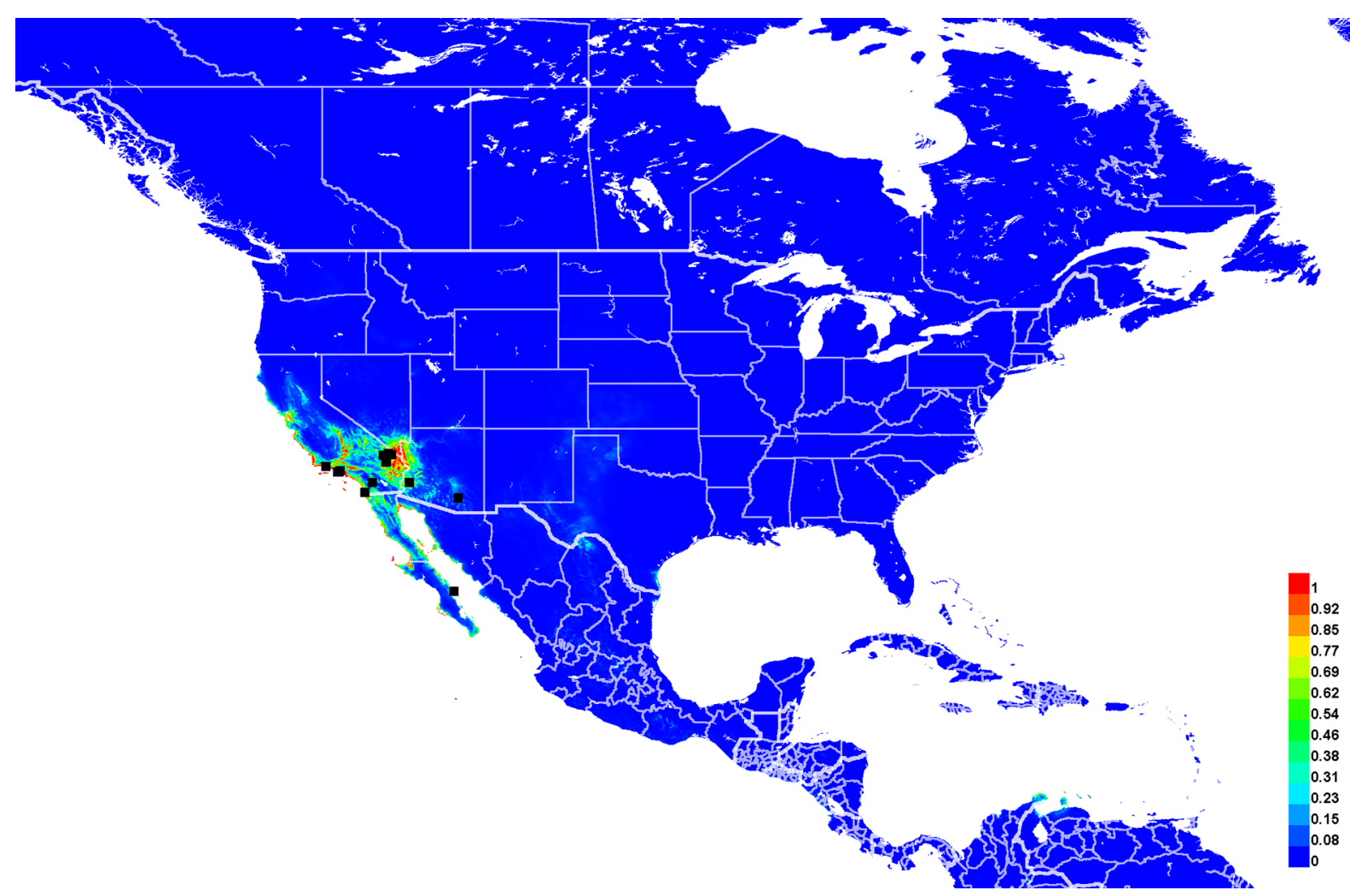

Fig. 73. Georeferenced collection records of Lasioglossum (D.) petrellum (Cockerell, 1903) (black squares) and predicted distribution by maximum entropy ecological niche modeling in Maxent (colour shading). Warmer colours indicate higher cloglog probability of occurrence. 
Penstemon Schmidel (G09) - SCROPHULARIACEAE Juss. Scrophularia L. S. californica Cham. \& Schltdl. (G09) • SOLANACEAE Adans. Datura L. D. inoxia Mill. (G09).

\section{DNA barcodes}

Three sequences available (BOLD process IDs: DLII1372-08, DLII1415-08, DLII1418-08; BIN: BOLD:AAF4030). Lasioglossum petrellum differs from all other western red-tailed L. (Dialictus) by 4 fixed substitutions: 4(T), 373(G), 402(G), and 432(C). It differs from L. droegei, L. griswoldi, and L. tuolumnense by an additional 8 fixed substitutions: 78(T), 105(T), 132(C), 216(G), 334(G), 363(C), 456(C), and 624(T) (Supplementary file 4).

\section{Remarks}

Gibbs (2009b) recorded this species only from California and Baja California. Additional specimens are now known from Arizona.

Lasioglossum (Dialictus) pictum (Crawford, 1902)

Figs $74,90 \mathrm{~L}, 102 \mathrm{~A}, 105 \mathrm{~A}$

Halictus pictus Crawford, 1902: 236 (syntypes, $\rightarrow+$, deposited in UNSM, examined).

Halictus graenicheri Ellis, 1914b: 221 (holotype, + , deposited in USNM, examined; synonymy by Mitchell 1960).

Dialictus muskegoensis Mitchell, 1960: 439 (holotype, ô, deposited in NCSU, examined by second author; synonymy by Mitchell 1962).

Halictus (Chloralictus) pictus - Sandhouse 1924: 3 (key).

Halictus (Chloralictus) graenicheri - Sandhouse 1924: 4 (key).

Lasioglossum (Chloralictus) graenicheri - Michener 1951: 1113 (catalog).

Lasioglossum (Chloralictus) pictum - Michener 1951: 1116 (catalog).

Dialictus pictus - Mitchell 1960: 412 (key, redescription). - Knerer \& Atwood 1962: 170 (checklist, biology). - Hurd 1979: 1970 (catalog). — Moure \& Hurd 1987: 122 (catalog).

Lasioglossum (Dialictus) graenicheri - Krombein 1967: 463 (catalog).

Lasioglossum (Dialictus) muskegoense - Krombein 1967: 464 (catalog).

Lasioglossum (Dialictus) pictum - Krombein 1967: 465 (catalog). — Wolf \& Ascher 2009: 144 (checklist). - Gibbs 2010: 249, figs 178a-d, 179a-f (key, redescription); 2011 (key). — Scott et al. 2011: 31 (checklist). — Gibbs et al. 2017: 84 (checklist).

\section{Diagnosis}

Females of Lasioglossum pictum can be recognized by the face short (length/width ratio $\sim 0.82$ ), supraclypeal area dull, frons and mesepisternum with crowded punctures, scutum with dense but distinctly separated punctures $(\mathrm{i} \leq 1 \mathrm{pd}$ ), postgena lineate, clypeus apical margin reddish brown to black, and T2-4 with dark spiracular spots. They are most similar to L. arenisaltans sp. nov. Females of $L$. arenisaltans sp. nov. have the face slightly longer (length/width ratio $\sim 0.85$ ), scutum very densely punctate $(\mathrm{i}<1 \mathrm{pd})$, and clypeus apical margin sometimes orange.

Males of L. pictum can be recognized by the face short (length/width ratio $\sim 0.83$ ), propodeum dorsolateral slope rugose, postgena lineate, face covered in relatively sparse tomentum not hiding the surface underneath, mesepisternum densely punctate $(i \leq 1 \mathrm{pd})$, S3-5 with uniform short hair $(0.75-$ $1.25 \mathrm{OD}$ ), and legs and metasoma mostly dark reddish brown to black, becoming red to orange on the extremities. They are most similar to L. arenisaltans sp. nov. and L. zephyrus. Males of L. arenisaltans sp. nov. have a longer face (length/width ratio $\sim 0.89$ ) and legs and metasoma often entirely orange. 
Males of L. zephyrus have the mesosoma more finely and sparsely punctate ( $\mathrm{i}=1-3 \mathrm{pd})$ and $\mathrm{S} 3-5$ with shorter, densely plumose hair concentrated laterally (Fig. 116A).

\section{Etymology}

Crawford (1902) named this species with the Latin perfect participle 'pictus' ('painted').

\section{Material examined}

\section{Syntypes}

UNITED STATES - Nebraska • 4 우; Sioux Co., War Bonnet Canyon; $\left[42.8^{\circ} \mathrm{N}, 103.6^{\circ} \mathrm{W}\right]$; 23 Jun. 1901; M.A. Carriker Jr. leg.; ex wild rose; UNSM • 1 क ; same location as for preceding; 3 Jun. 1901; M. Cary leg.; ex Symphoricarpos; UNSM • 6 우; same location as for preceding; 23 Jun. 1901; J.C. Crawford leg.; ex wild rose; UNSM • 3 o $\circ$; Crawford; [42.68 N, 103.41 ${ }^{\circ}$ W]; 29 Jul. 1901; M.A. Carriker leg.; ex Mentzelia; UNSM.

\section{Other material}

CANADA - Alberta 6 \% 9 ; $11.8 \mathrm{~km} \mathrm{SSW}$ of Empress (stabilizing dune); $50.8504^{\circ} \mathrm{N}, 110.0489^{\circ} \mathrm{W}$; 24 May 2019; T.M. Onuferko leg.; CMNC. - Manitoba 2 q $\circ$; Spirit Sands, SWPP (stabilized dune); 49.664 N, 99.2951 ${ }^{\circ}$ W; 30 May 2019; T.M. Onuferko leg.; CMNC. - Ontario • 1 P; Norfolk Co., South Walsingham, Pterophylla Farm; [42.66 ${ }^{\circ}$ N, 80.52 ${ }^{\circ}$ W]; P.J. Carson leg.; WRME. - Saskatchewan • 2 ㅇ; $6.4 \mathrm{~km} \mathrm{NNW}$ of Webb (active dune); $50.2341^{\circ} \mathrm{N}, 108.2403^{\circ} \mathrm{W} ; 1$ Jun. 2019; T.M. Onuferko leg.; CMNC • 1 ; $7.6 \mathrm{~km} \mathrm{NNW}$ of Webb (active dune); $50.2431^{\circ} \mathrm{N}, 108.2503^{\circ} \mathrm{W} ; 1$ Jun. 2019; T.M. Onuferko leg.; CMNC • 5 우; $\mathrm{N}$ Bitter Lake, Tunstall (stabilizing dune); $50.1489^{\circ} \mathrm{N}, 109.8244^{\circ} \mathrm{W} ; 2$ Jun. 2019; T.M. Onuferko leg.; CMNC.

UNITED STATES - Arizona $・ 2$ 우; 20 mi. N of Flagstaff; $\left[35.51^{\circ} \mathrm{N}, 111.55^{\circ} \mathrm{W}\right] ; 3 \mathrm{Jul} .1952$; C. Liang, W. LaBerge, R.H. and L.D. Beamer leg.; SEMC. - Colorado 2 우, 1 \; Alamosa Co., Great Sand Dunes National Monument; $\left[37.8^{\circ} \mathrm{N}, 105.6^{\circ} \mathrm{W}\right]$; 22 Jul.-8 Aug. 1978; H.E. Evans leg.; CSUC • 1 क; Alamosa Co., Sand Dunes National Monument; $\left[37.8^{\circ} \mathrm{N}, 105.6^{\circ} \mathrm{W}\right]$; 21 Jun. 1955; Hugo G. Rodeck leg.; UCMC • 1 q; White Rocks, near Boulder; $\left[40.06^{\circ} \mathrm{N}, 105.16^{\circ} \mathrm{W}\right] ; 1676 \mathrm{~m}$ a.s.l.; 5 Jun. 1922; AMNH 1 क; Wray; $40^{\circ}$ N, $102.1667^{\circ}$ W; 1128 m a.s.1.; 17-19 Aug. 1919; AMNH. Kansas 1 1 ; 9 mi S of Garden City; $\left[37.84^{\circ} \mathrm{N}, 100.87^{\circ} \mathrm{W}\right.$ ]; 12 Jun. 1952; Wille and Michener leg.; ex Melilotus officinalis; SEMC. - Michigan - 2 ๙ิ $^{\lambda}$; Berrien Co., Warren Dunes S.P.; $41.903^{\circ}$ N,

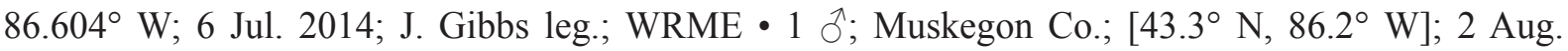
1944; Dreisbach leg.; NCSU. - Minnesota • 7 o 9 ; Anoka Co., Bunker Hills Reg. Park; $45.2037^{\circ}$ N, 93.2792 ${ }^{\circ}$ W; $278 \mathrm{~m}$ a.s.1.; 8 Jun. 2015; J. Gardner leg.; UMSP • 1 क ; same location as for preceding; 20

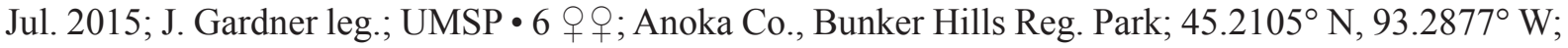
274 m a.s.l.; 8 Jun. 2015; J. Gardner leg.; UMSP • 1 q; Anoka Co., Bunker Hills Reg. Park; $45.2109^{\circ}$ N,

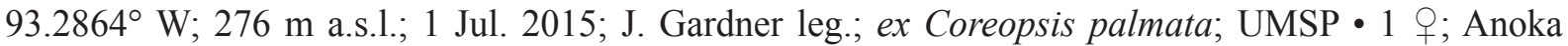

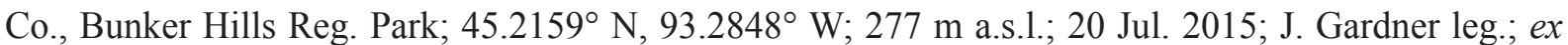
Amorpha canescens; UMSP • 2 우우 Anoka Co., Bunker Hills Reg. Park; 45.2176 N $93.2899^{\circ} \mathrm{W}$; $274 \mathrm{~m}$ a.s.l.; 20 Jul. 2015; J. Gardner leg.; UMSP • 2 + ; same location as for preceding; 10 Aug. 2015; J. Gardner leg.; UMSP • 2 q ; same location as for preceding; 19 May 2016; J. Gardner leg.; UMSP • 7 q $ᄋ$; same location as for preceding; 8 Jun. 2016; E. Evans leg.; UMSP • 1 ㅇ; Anoka Co., Bunker Hills Reg. Park; $45.2176^{\circ}$ N, $93.2898^{\circ}$ W; 8 Jun. 2015; J. Gardner leg.; ex Tradescantia occidentalis; UMSP • 1 o; Anoka Co., Cedar Creek Ecosystem Science Reserve; $45.388^{\circ} \mathrm{N}, 93.1831^{\circ} \mathrm{W} ; 283 \mathrm{~m}$ a.s.1.; 3 Jul. 2015; J. Gardner leg.; ex Oenothera clelandii; UMSP • 8 우; Anoka Co., Cedar Creek Ecosystem

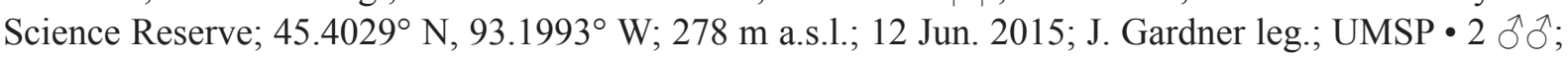
Anoka Co., Cedar Creek Ecosystem Science Reserve; $45.4037^{\circ}$ N, 93.1834 W; 280 m a.s.1.; 12 Aug. 2015; J. Gardner leg.; ex Dalea villosa; UMSP • 3 우; Anoka Co., Cedar Creek Ecosystem Science 


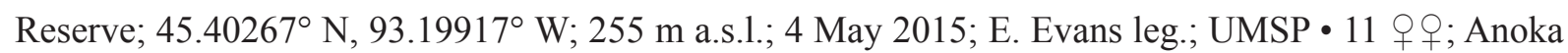
Co., Cedar Creek Ecosystem Science Reserve; $45.43096^{\circ}$ N, $93.18936^{\circ}$ W; 247 m a.s.1.; 4 May 2015; E. Evans leg.; UMSP - 5 ㅇ; Isanti Co., Cedar Creek Ecosystem Science Reserve; $45.4323^{\circ} \mathrm{N}$, 93.1894 ${ }^{\circ}$ W; $281 \mathrm{~m}$ a.s.1.; 22 Jul. 2015; J. Gardner leg.; UMSP • 1 q; same location as for preceding; 12 Aug. 2015; J. Gardner leg.; UMSP • 1 q; same location as for preceding; 31 Aug. 2015; J. Gardner leg.; UMSP • 1 \%; Isanti Co., Irving \& John Anderson Co. Park; $45.4617^{\circ} \mathrm{N}$, 93.0536 ${ }^{\circ} \mathrm{W} ; 255 \mathrm{~m}$ a.s.1.; 21 May 2015; E. Evans leg.; ex Prunus virginiana; UMSP • 1 q; Isanti Co., Irving \& John Anderson Co. Park; $45.462^{\circ} \mathrm{N}, 93.061^{\circ} \mathrm{W} ; 261 \mathrm{~m}$ a.s.l.; 10 Jun. 2015; E. Evans leg.; UMSP • 1 q; Isanti Co., Irving \& John Anderson Co. Park; 45.4641 ${ }^{\circ}$ N, $93.0598^{\circ}$ W; 20 Jul. 2015; E. Evans leg.; ex Melilotus officinalis subsp. alba; UMSP • 1 ; ; Isanti Co., Irving \& John Anderson Co. Park; $45.4663^{\circ} \mathrm{N}, 93.0542^{\circ} \mathrm{W}$; 248 m a.s.l.; 10 Jun. 2015; E. Evans leg.; UMSP • 7 q ; Isanti Co., Irving \& John Anderson Co. Park; 45.4662 $7^{\circ} \mathrm{N}, 93.05422^{\circ} \mathrm{W} ; 248$ m a.s.1.; 20 Jul. 2015; E. Evans leg.; UMSP • 1 +; Washington Co., Belwin Conservancy; $44.9248^{\circ} \mathrm{N}, 92.7894^{\circ} \mathrm{W} ; 230 \mathrm{~m}$ a.s.1.; 9 Jun. 2015; E. Evans leg.; ex Penstemon gracilis; UMSP • 1 J ; Washington Co., St. Croix Savanna Scientific and Natural Area; $44.9955^{\circ} \mathrm{N}$, 92.7788 W; 192 m a.s.1.; 21 Jul. 2015; E. Evans leg.; ex Verbena stricta; UMSP. - Nebraska • 4 q $\bigcirc$; McPherson Co., Sandhills Ag Lab; [41.62 ${ }^{\circ}$ N, $\left.100.83^{\circ} \mathrm{W}\right] ; 15$ Jul. 1972; UNSM • 4 q ; ; same location as for preceding; 28 Jun.-10 Aug. 1972; UNSM - 2 우; McPherson Co.; 28 Jun.-10 Aug. 1972;

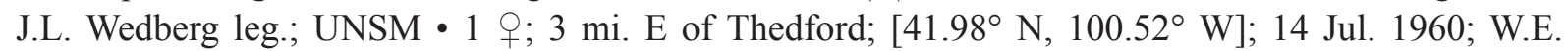

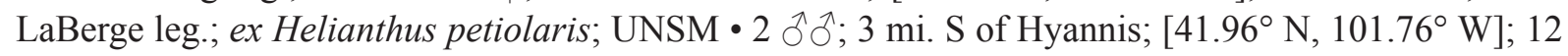
Sep. 1961; Crew leg.; ex misc. vegetation; UNSM • 1 ô; same location as for preceding; 10 Sep. 1961; Atyeo and Isakson leg.; ex dry prairie; UNSM • 1 क ; $3.5 \mathrm{mi}$. W of Marquette; [41.01 $\left.{ }^{\circ} \mathrm{N}, 98.08^{\circ} \mathrm{W}\right] ; 31$ Jul. 1958; Atyeo and Andresen leg.; UNSM • 2 우; F. Crow Ranch, 25 mi. SW of Valentine; [42.7 N, $100.9^{\circ}$ W]; 3 Sep. 1959; W.E. LaBerge leg.; ex Helianthus petiolaris; UNSM • 1 क; same location as for preceding; 3 Sep. 1959; W.E. LaBerge leg.; ex Solidago; UNSM • 1 q ; Mitchell; [41.94 N, $103.81^{\circ} \mathrm{W}$ ]; 2 Aug. 1917; E.J. Yates leg.; ex Melilotus officinalis subsp. alba; UNSM・ 1 + ; Smith Lake, 20 mi. S of Rushville; [42.42 ${ }^{\circ} \mathrm{N}, 102.45^{\circ} \mathrm{W}$ ]; 31 Aug. 1959; ex Helianthus petiolaris; UNSM • 1 क ; same location as for preceding; 31 Aug. 1959; W.E. LaBerge leg.; ex Erigeron; UNSM • 3 q $O$; West Point; $\left[41.84^{\circ} \mathrm{N}\right.$, 96.71 ${ }^{\circ} \mathrm{W}$ ]; Jun. 1900; UNSM. - North Dakota • 22 q $\circ$; Ransom Co., 7 mi. SE of Sheldon; [46.52 ${ }^{\circ}$ N, 97.39 ${ }^{\circ}$ W]; 24 Sep. 1983; J.R. Powers leg.; EMEC • 11 o $\%$; Richland Co., 1 mi. SE of McLeod;

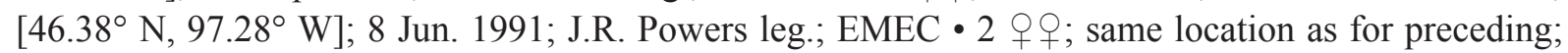
2 Aug. 1995; J.R. Powers leg.; EMEC. - Wisconsin • 1 क; Vernon Co., Genoa; [43.58 N, $\left.91.22^{\circ} \mathrm{W}\right]$; 13 Jul. 1911; Graenicher leg.; USNM.

\section{Description}

See Gibbs (2010) for a complete redescription and figures.

\section{Range}

Ontario west to Alberta and south to northern Arizona (Fig. 74).

\section{Floral records}

ASTERACEAE Giseke: Coreopsis: C.palmata Nutt. Erigeron: E. philadelphicus L. (G10) • Helianthus: H. petiolaris Nutt. - Solidago • BORAGINACEAE Juss. Phacelia Juss. P. congesta Hook. (G10) • BRASSICACEAE Burnett: Lepidium L. (G10) • CAPRIFOLIACEAE Juss. Symphoricarpos Duhamel • COMMELINACEAE Mirb. Tradescantia L. T. occidentalis (Britton) Smyth - FABACEAE Juss. Amorpha: A. canescens Pursh • Dalea:D. villosa (Nutt.) Spreng. Melilotus: M. officinalis (L.) Pall. M.o. subsp. alba (Medik.) H. Ohashi \& Tateishi - LAMIACEAE Martinov: Salvia L. S. texana (Scheele) Torr. (G10) • LOASACEAE Juss. Mentzelia L. • ONAGRACEAE Juss. Oenothera L. O. clelandii W. Dietr., P.H. Raven \& W.L. Wagner • PLANTAGINACEAE Juss. Penstemon Schmidel: P. gracilis 
Nutt. • ROSACEAE Juss. Prunus L. P. virginiana L.・Rosa L. • SALICACEAE Mirb. Salix L. (G10) • VERBENACEAE J. St.-Hil. Verbena L. V. stricta Vent.

\section{DNA barcodes}

Nine sequences available (BOLD process IDs: DIAL020-06, DIAL021-06, DIAL102-06, DIAL103-06, DIAL104-06, DIAL343-06, DIAL434-06, DIAL435-06, LASNA022-08; BIN: BOLD:AAC1104). No unique fixed substitutions distinguish L. pictum from all other western red-tailed L. (Dialictus).

\section{Remarks}

Gibbs (2011) recorded Lasioglossum pictum as far southwest as Alberta and Nebraska. Additional specimens are now known from Colorado and Arizona. Lasioglossum arenisaltans sp. nov. is also known from these same localities. Both species are sand dune specialists and seem to occur sympatrically throughout much of their range, although $L$. arenisaltans sp. nov. is more common in the southwest and L. pictum is more common in the northeast.

The type locality of $L$. pictum is within the known range of $L$. arenisaltans sp. nov., but all 14 syntype specimens were examined and found to represent $L$. pictum as traditionally understood.

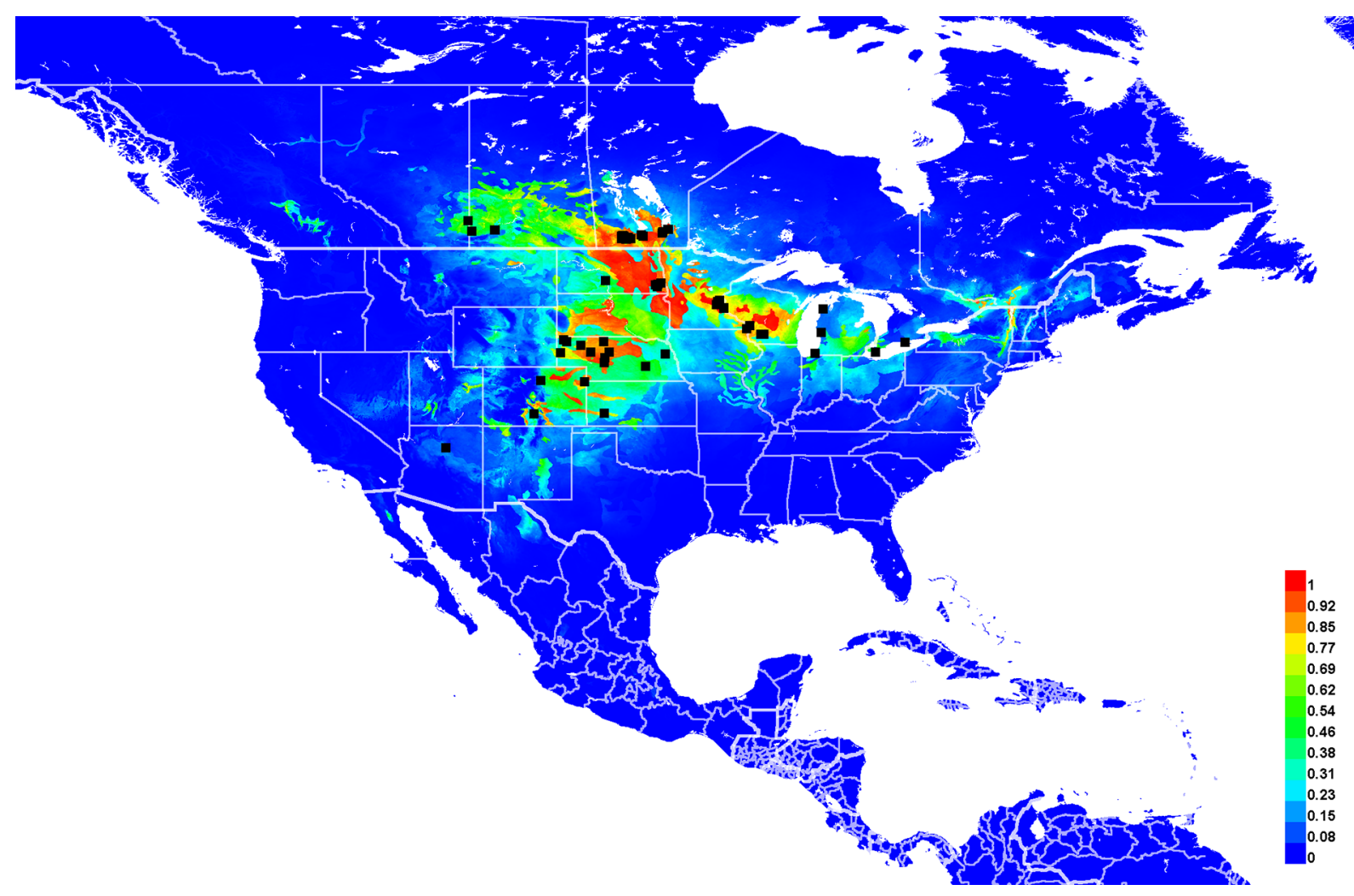

Fig. 74. Georeferenced collection records of Lasioglossum (D.) pictum (Crawford, 1902) (black squares) and predicted distribution by maximum entropy ecological niche modeling in Maxent (colour shading). Warmer colours indicate higher cloglog probability of occurrence. 


\section{Lasioglossum (Dialictus) rufornatum sp. nov. urn:1sid:zoobank.org:act:5B74FC3D-BFF2-49CB-87D1-06002ACF1565}

Figs $75-77,90 \mathrm{M}, 95 \mathrm{C}$

\section{Diagnosis}

Females of Lasioglossum rufornatum sp. nov. can be recognized by the metasoma mostly dark with terga often metallic green and sterna and apical rims of the terga becoming orange to pale yellow, wings very pale with white hairs and sometimes nearly white veins, frons and mesepisternum very finely and densely punctate ( $\mathrm{i} \leq 1 \mathrm{pd}$ ), face moderately long (length/width ratio $\sim 0.85$ ), scutum shiny and moderately densely punctate $(\mathrm{i}=1-2 \mathrm{pd})$, and $\mathrm{T} 1$ anterior surface coriarious. They are most similar to an undescribed species widespread across most of the western United States with a longer face and no red or yellow colouration. They are also somewhat similar to L. eophilus. Females of L. eophilus have the mesepisternum rugose, metasomal terga not metallic, T1 anterior surface shiny, and ocelli slightly enlarged (separated by about 0.5 OD).

Males of $L$. rufornatum sp. nov. can be recognized by the clypeus sparsely punctate (i=1-5 pd) and entirely metallic blue-green, metasoma mostly dark reddish brown often with metallic green terga, with the sterna and apical rims of the terga becoming orange to pale yellow, S4-5 with very long and dense erect hair (2-4 OD), face between the eye emargination and clypeus and metasomal terga with dense tomentum, and gonostylus large, with very long, dense hair, and projecting laterally beyond the gonocoxite. They are most similar to an undescribed species widespread across most of the western United States with a longer face and no red or yellow colouration. They are also somewhat similar to L. miltolepoides sp. nov. Males of L. miltolepoides sp. nov. have tomentum absent on the face and sparse on the metasoma, S4-5 with relatively short hair (1-1.5 OD), and metasomal terga not metallic.

Specimens of both sexes from the southernmost part of their range (in the Algodones Dunes of Imperial County, California) often have extensive pale yellow to nearly white colouration rather than orange or red, which is unique.

\section{Etymology}

The specific epithet rufornatum is formed from the Latin adjective 'rufus' ('red') and the perfect participle 'ornatum' ('adorned'). It refers to this species' mostly dark metasoma which is adorned with red to yellow bands. An appropriate translation would be the ornate red sweat bee.

\section{Material examined}

\section{Holotype}

UNITED STATES - California • ; ; Imperial Co., Algodones Dunes, $6 \mathrm{~km} \mathrm{~W}$ of Glamis; $32.9861^{\circ} \mathrm{N}$, 115.1346 ${ }^{\circ}$ W; 118 m a.s.1.; 3 Apr. 2013; T.L. Griswold leg.; ex Psorothamnus sp.; BBSL.

\section{Paratypes}

UNITED STATES - California $・ 1$ +, $2 \hat{\jmath}$; Imperial Co., $0.5 \mathrm{~km}$ NNE of jct. CA Hwy 78 and Osborne Park Road; $32.99^{\circ} \mathrm{N}, 115.132^{\circ} \mathrm{W}$; $25-28$ Mar. 2008; UCDC • 6 q $\odot$; Imperial Co., $0.7 \mathrm{~km} \mathrm{NE}$ of jct.

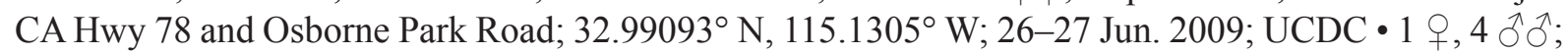
same location as for preceding; 24-29 Jun. 2009; UCDC・42 웅, $4 \hat{\delta} \widehat{\jmath}$; same location as for preceding;

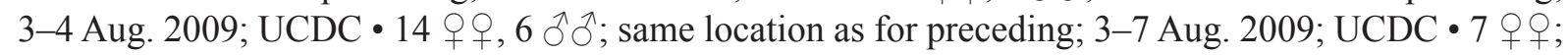

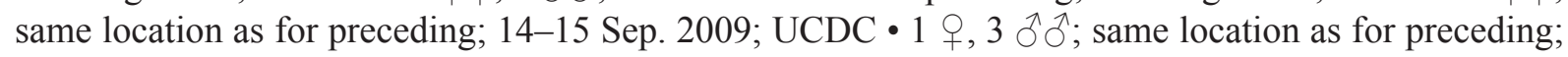
13-18 Sep. 2009; UCDC • 1 +; Imperial Co., 0.7 km NW of jct. CA Hwy 78 and Gecko Rd; $32.9812^{\circ}$ N,

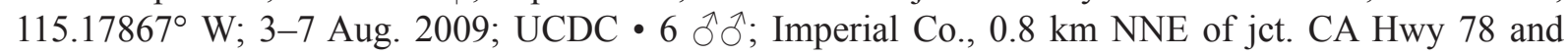
Osborne Park Road; $32.99183^{\circ} \mathrm{N}, 115.13043^{\circ} \mathrm{W}$; 19-22 Sep. 2008; UCDC • 1 q; Imperial Co., $1.9 \mathrm{~km}$ ESE of Roadrunner Campground; $32.90178^{\circ} \mathrm{N}, 115.09807^{\circ} \mathrm{W} ; 10$ Mar. 2009; ex Mentzelia longiloba; 
$\mathrm{UCDC} \bullet 4$ q 9 ; Imperial Co., 15 mi. NW of Glamis; $33.1167^{\circ} \mathrm{N}, 115.2667^{\circ} \mathrm{W} ; 84 \mathrm{~m}$ a.s.1.; $13-15$ Nov. 1994; R.R. Snelling leg.; LACM • 4 우; same location as for holotype; 3 Apr. 2013; T.L. Griswold leg.; ex Petalonyx sp.; BBSL 3 우; same collection data as for holotype; BBSL $\bullet 2$ 우; same location as for holotype; 3 Apr. 2013; T.L. Griswold leg.; ex Palafoxia sp.; BBSL・ 1 क; Imperial Co., All American Canal Water Drop One, $1.3 \mathrm{~km}$ ENE of Gordons Well exit Hwy I-8; $32.71289^{\circ} \mathrm{N}, 114.9441^{\circ} \mathrm{W} ; 16$ Oct.

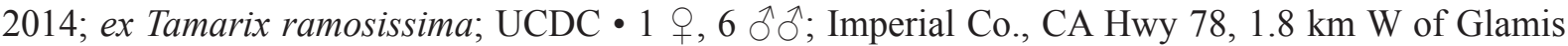
Store, then $0.2 \mathrm{~km} \mathrm{~N} ; 32.997^{\circ} \mathrm{N}, 115.092^{\circ} \mathrm{W} ; 24$ Apr. 2009; ex Palafoxia arida; UCDC・ 1 \%; Imperial Co., CA Hwy 78, $1.8 \mathrm{~km} \mathrm{~W}$ of Glamis Store, then $0.3 \mathrm{~km} \mathrm{~N} ; 32.99742^{\circ} \mathrm{N}, 115.09212^{\circ} \mathrm{W}$; $24-29 \mathrm{Jun}$. 2009; UCDC • 2 우; same location as for preceding; 3-4 Aug. 2009; UCDC • 5 q 9 ; same location as for preceding; 3-7 Aug. 2009; UCDC • 3 우; Imperial Co., CA Hwy 78, $1.8 \mathrm{~km} \mathrm{~W}$ of Glamis Store, then $0.4 \mathrm{~km} \mathrm{~S} ; 32.99113^{\circ} \mathrm{N}, 115.09195^{\circ} \mathrm{W} ; 3-4$ Aug. 2009; UCDC • 1 ' ; same location as for preceding; 3-7 Aug. 2009; UCDC • 1 क; Imperial Co., Coachella Canal Road 0.4 km NW of CA Hwy $78 ; 32.97535^{\circ} \mathrm{N}, 115.18722^{\circ} \mathrm{W} ; 20$ Sep. 2008 ; ex Psorothamnus emoryi; UCDC • 1 \%; Imperial Co., Coachella Canal Road 11.6 km NW of CA Hwy 78; 33.05738 $8^{\circ}$, $115.25752^{\circ}$ W; 1 Mar. 2010;

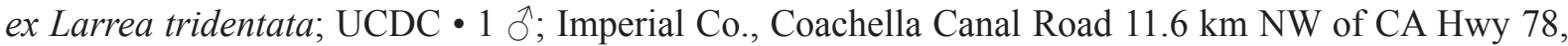
then $1.0 \mathrm{~km} \mathrm{NE} ; 33.063^{\circ} \mathrm{N}, 115.25^{\circ} \mathrm{W} ; 28-29 \mathrm{Apr}$. 2008; UCDC • 1 \%; same location as for preceding;

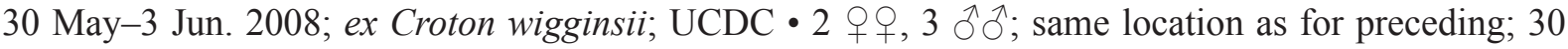
May-3 Jun. 2008; UCDC • 40 웅, 1 ㅅ; Imperial Co., Coachella Canal Road 11.6 km NW of CA Hwy 78, then $1.3 \mathrm{~km} \mathrm{E} ; 33.05827^{\circ} \mathrm{N}, 115.24338^{\circ} \mathrm{W} ; 12$ Mar. 2009; ex Mentzelia longiloba; UCDC • 8 우, 1 ô; same location as for preceding; 9-14 Mar. 2009; ex Palafoxia arida; UCDC・ 1 +; Imperial Co., Coachella Canal Road $11.6 \mathrm{~km} \mathrm{NW}$ of CA Hwy 78, then $1.7 \mathrm{~km}$ E; $33.05763^{\circ} \mathrm{N}, 115.2397^{\circ} \mathrm{W} ; 14$ Mar. 2009; ex Croton wigginsii; UCDC • 1 क; Imperial Co., Coachella Canal Road $6.5 \mathrm{~km} \mathrm{NW}$ of CA Hwy $78 ; 33.02037^{\circ} \mathrm{N}, 115.22538^{\circ} \mathrm{W} ; 29$ Apr.-2 May 2008; UCDC • 1 क; same location as for preceding; 30 May-1 Jun. 2008; UCDC • 1 क, 1 §; Imperial Co., End of Ogilby Road, 0.4 km S of Hwy I-8; $32.75567^{\circ} \mathrm{N}, 114.83617^{\circ} \mathrm{W}$; 19 Mar. 2016; ex Palafoxia arida; UCDC • 2 우영 Imperial Co., end of Ogilby Road, $0.5 \mathrm{~km} \mathrm{~S}$ of Hwy I-8; $32.75462^{\circ} \mathrm{N}, 114.83667^{\circ} \mathrm{W} ; 10-11$ Mar. 2009; ex Larrea tridentata; $\mathrm{UCDC} \bullet 13$ 우; same location as for preceding; $10-11$ Mar. 2009; ex Mentzelia longiloba; UCDC • 1 万; Imperial Co., Evan Hewes Hwy, 1.5 mi. E of Brock Res. Stn Rd.; $32.7092^{\circ} \mathrm{N}, 115.0139^{\circ} \mathrm{W} ; 41 \mathrm{~m}$ a.s.1.; 19 Apr. 2008; G.R. Ballmer leg.; ex Palafoxia linearis; UCRC• 1 q; Imperial Co., Gecko Road 0.4 km SSE of CA Hwy 78; $32.9725^{\circ} \mathrm{N}, 115.17322^{\circ} \mathrm{W}$; 26-27 Jun. 2009; UCDC • 1 \%; same location as for

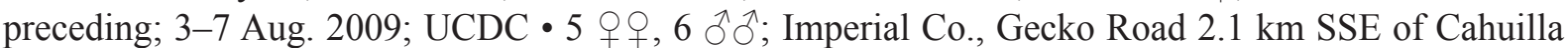
Ranger Station; $32.95737^{\circ} \mathrm{N}, 115.16328^{\circ} \mathrm{W} ; 7-10$ Jul. 2008; ex Eriogonum deserticola; UCDC • 2 우웅,

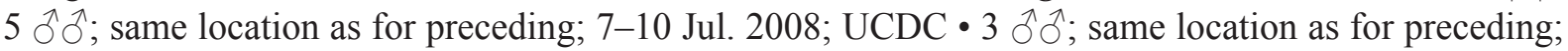

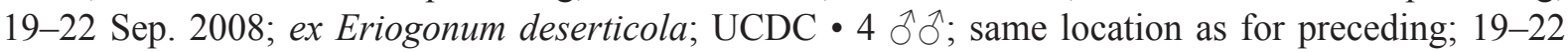

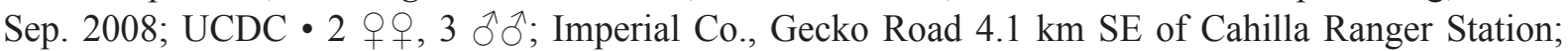

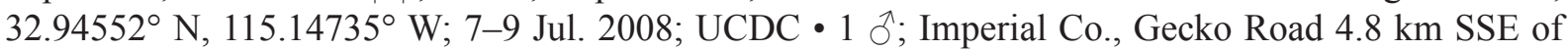
Cahilla Ranger Station; $32.938^{\circ} \mathrm{N}, 115.145^{\circ} \mathrm{W}$; 23-24 Apr. 2009; ex Psorothamnus emoryi; UCDC • 2 우; Imperial Co., Glamis; [ $33^{\circ} \mathrm{N}, 115.07^{\circ} \mathrm{W}$ ]; 24 Jan. 1964; UCDC • 3 우; same location as for preceding; 23 Mar. 1967; UCDC • 1 क ; same location as for preceding; 11 Oct. 1972; UCDC • 2 우;

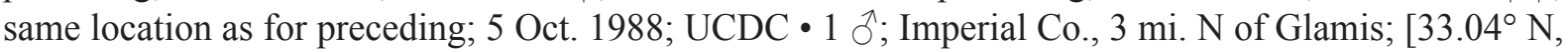

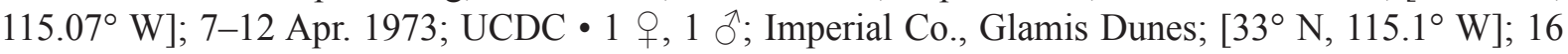
Dec. 1982; UCDC • 1 + ; Imperial Co., Glamis Dunes, W Glamis; $32.9941^{\circ} \mathrm{N}, 115.1013^{\circ} \mathrm{W}$; $91 \mathrm{~m}$ a.s.l.; 29 Sep. 1987; T.L. Griswold leg.; BBSL 1 क; Imperial Co., Niland-Glamis Road 23.4 km NW of CA Hwy 78, then $0.8 \mathrm{~km}$ WSW; $33.1237^{\circ} \mathrm{N}, 115.27735^{\circ} \mathrm{W} ; 25$ Mar. 2013; ex Sphaeralcea sp.; UCDC • 1 क; Imperial Co., Niland-Glamis Road $3.0 \mathrm{~km} \mathrm{NW}$ of CA Hwy 78, then $0.8 \mathrm{~km} \mathrm{SW} ; 33.00773^{\circ} \mathrm{N}$, 115.10257 ${ }^{\circ}$ W; 6-9 Feb. 2008; UCDC • 1 \% ; same location as for preceding; 6-9 Feb. 2008; UCDC • 1 क; Imperial Co., Niland-Glamis Road $7.4 \mathrm{~km} \mathrm{NW}$ of CA Hwy $78 ; 33.03727^{\circ} \mathrm{N}, 115.13487^{\circ} \mathrm{W} ; 30$ May-3 Jun. 2008; UCDC • 3 우; same location as for preceding; 23-24 Apr. 2009; ex Stephanomeria pauciflora; UCDC • 3 우; Imperial Co., Niland-Glamis Road $9.8 \mathrm{~km} \mathrm{NW}$ of CA Hwy 78, then $1.9 \mathrm{~km}$ SSW; $33.03778^{\circ} \mathrm{N}, 115.16474^{\circ} \mathrm{W}$; 18 Oct. 2014; ex Palafoxia arida; UCDC • 1 क; Imperial Co., 
Niland-Glamis Road $9.8 \mathrm{~km} \mathrm{NW}$ of CA Hwy 78, then $3.2 \mathrm{~km} \mathrm{SW} ; 33.02868^{\circ} \mathrm{N}, 115.17459^{\circ} \mathrm{W} ; 18$ Nov. 2014; ex Baccharis sarothroides; UCDC • 1 ते; Imperial Co., Roadrunner Campground, Gecko Road

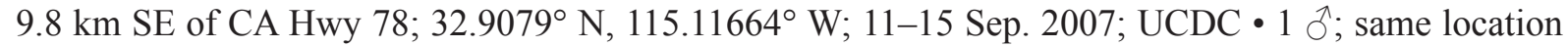

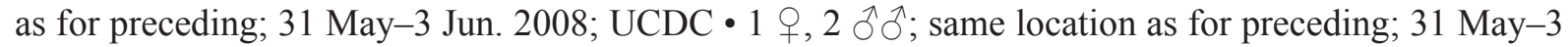
Jun. 2008; UCDC • 1 क; same location as for preceding; 6-8 Jul. 2008; UCDC • 1 ऊं; Imperial Co., Wash Road 0.6-1.1 km SE of CA Hwy 78; 32.99399-32.99138 ${ }^{\circ} \mathrm{N}, 115.06573-115.0617^{\circ} \mathrm{W}$; 30 May-3

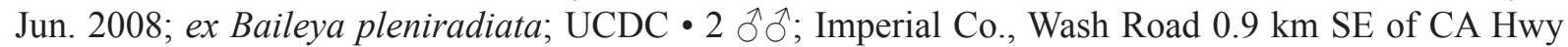
78; $32.99243^{\circ} \mathrm{N}, 115.06378^{\circ} \mathrm{W} ; 24$ Apr. 2009; ex Asclepias subulata; UCDC • 1 ㅇ; Imperial Co., Wash Road 1.6 km SE of CA Hwy 78, then $0.3 \mathrm{~km} \mathrm{SW}$; $32.98677^{\circ} \mathrm{N}, 115.05927^{\circ} \mathrm{W}$; 26-27 Jun. 2009; UCDC - 1 क ; Imperial Co., Wash Road $11.3 \mathrm{~km} \mathrm{SE}$ of CA Hwy 78, then $1.0 \mathrm{~km} \mathrm{SW} ; 32.92382^{\circ} \mathrm{N}$, 114.99087 W; 29 Apr.-2 May 2008; UCDC • 1 9; Imperial Co., Wash Road 11.3 km SE of CA Hwy 78 , then $1.0 \mathrm{~km} \mathrm{SW} ; 32.92347^{\circ} \mathrm{N}, 114.99039^{\circ} \mathrm{W}$; 6-10 Jul. 2008; UCDC 2 우영 same location as for preceding; 18-22 Sep. 2008; UCDC • 2 우; Imperial Co., Wash Road $11.3 \mathrm{~km} \mathrm{SE}$ of CA Hwy 78, then $1.2 \mathrm{~km} \mathrm{SW} ; 32.92335^{\circ} \mathrm{N}, 114.9919^{\circ} \mathrm{W} ; 30$ May-3 Jun. 2008; UCDC 2 2 + 9 ; same location as for preceding; 30 May-3 Jun. 2008; UCDC • 1 \%; Imperial Co., Wash Road 6.7 km SE of CA Hwy 78;

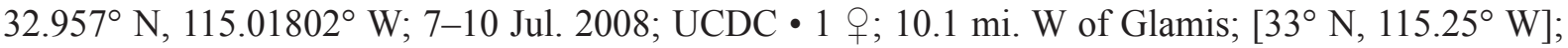
3 Apr. 1960; R.C. Dichman leg.; ex Larrea divaricata; UCRC 1 1 ; ; Winterhaven; [32.74 ${ }^{\circ} \mathrm{N}, 114.63^{\circ} \mathrm{W}$; 31 May 1930; Timberlake leg.; ex Sphaeralcea orcuttii; UCRC. - Nevada • 2 o 9 ; Clark Co., Kaolin Wash, S Overton; $36.5117^{\circ}$ N, $114.4442^{\circ}$ W; 3 Jun. 2001; D. Yanega leg.; ex Stephanomeria; UCRC • 3 우; Clark Co., Magnesite Wash, S Overton; $36.5117^{\circ} \mathrm{N}, 114.4442^{\circ} \mathrm{W} ; 25$ May 2009; D. Yanega leg.; UCRC • 1 क; Clark Co., Moapa Val., Kaolin Wash, E of Overton; $36.5119^{\circ}$ N, $114.4442^{\circ}$ W; 3 Jun. 2001; G.R. Ballmer leg.; ex Croton californicus; UCRC • 1 q; same location as for preceding; 3 Jun. 2001; G.R. Ballmer leg.; ex Chilopsis linearis; UCRC.

\section{Description}

\section{Female}

Colouration. Head and mesosoma blue to olive green; clypeus apical colour black, reddish brown, or orange; labrum reddish brown to orange; mandible orange with black basal spot or band and red tip; flagellum reddish brown dorsally, orange to pale yellow ventrally; pronotal lobe orange to pale yellow; metasoma reddish brown to pale yellow with discs of T1-5 metallic blue-green and dark spiracular spots on T2-4; legs black to reddish brown with femur-tibia joints, base and apex of tibiae, and tarsi orange to pale yellow; tegula orange to pale yellow; wing membrane hyaline, veins with subcosta brown to dark brown, otherwise orange to white.

PubesCENCE. Body hair colour white. Tomentum dense on paraocular area, lower frons, gena, pronotal collar and lobe, space between pronotal lobe and tegula, metanotum, preëpisternum, metepisternum, T1 laterally, T2 basally, and T3-4 throughout; sparse on supraclypeal area, scutum lateral margin, hypoepimeron, and mesepisternum. Scutum hair thin to densely plumose. Wing hairs light, long and sparse. Acarinarial fan complete, dense. T2 fringes sparse, T3 fringes sparse.

SuRfaCe SCULPTURE. Clypeus punctures sparse ( $\mathrm{i}=1-4 \mathrm{pd})$, becoming moderately dense in basal third ( $i=1-2$ pd), sculpture shiny, becoming weakly tessellate basally; supraclypeal area punctures dense ( $\mathrm{i} \leq 1 \mathrm{pd}$ ), sometimes sparse medially ( $\mathrm{i}=1-3 \mathrm{pd}$ ), sculpture tessellate, sometimes shiny medially; paraocular area punctures dense $(\mathrm{i}<1 \mathrm{pd})$, sculpture shiny, sometimes tessellate around antenna socket; frons punctures crowded $(i=0 \mathrm{pd})$, sculpture shiny; vertex punctures crowded $(\mathrm{i}=0 \mathrm{pd})$, becoming moderately dense medially $(i=1-2 \mathrm{pd})$, sculpture shiny; gena punctures fine, moderately dense ( $\mathrm{i}=1-2 \mathrm{pd}$ ), sculpture shiny; postgena sculpture imbricate to finely ruguloso-lineate, sometimes becoming smooth and shiny anteriorly; tegula punctures absent; scutum punctures dense $(i<1 \mathrm{pd})$, becoming slightly sparser submedially and anteromedially $(i=1-2 \mathrm{pd})$, sculpture shiny, becoming tessellate anteromedially; scutellum punctures dense marginally and on median line (i $<1 \mathrm{pd}$ ), 
moderately sparse submedially $(\mathrm{i}=1-2 \mathrm{pd}$ ), diversopunctate, sculpture shiny; metanotum sculpture rugulose, becoming shiny and finely, densely punctate anteromedially $(\mathrm{i}<1 \mathrm{pd})$; metapostnotum rugae strong, anastomosing or subparallel, reaching margin, sculpture imbricate or weakly so; preëpisternum sculpture rugulose; hypoepimeron punctures crowded ( $\mathrm{i}=0 \mathrm{pd}$ ), sculpture imbricate; mesepisternum punctures dense ( $i \leq 1 \mathrm{pd})$, becoming crowded dorsally ( $\mathrm{i}=0 \mathrm{pd})$, sculpture shiny; metepisternum sculpture lineate dorsally, imbricate ventrally; propodeum lateral face sculpture tessellate; propodeum posterior face sculpture tessellate; T1 anterior face sculpture coriarious; T1 dorsal surface punctures moderately dense ( $i=1-3 \mathrm{pd})$, absent or very sparse in large apicolateral oval patches, sculpture shiny; T2 disc punctures moderately dense $(i=1-3 \mathrm{pd})$, disc sculpture shiny, rim punctures fine, moderately sparse $(i=1-4 \mathrm{pd})$, rim sculpture shiny.

Structure. Face length/width ratio 0.85 ( $\pm 0.01 \mathrm{SD})$. Clypeus projecting $\sim 67 \%$ below suborbital tangent; clypeal area length/width ratio 0.44 ( $\pm 0.02 \mathrm{SD})$; apicolateral denticles rounded knobs; supraclypeal area length/width ratio 0.9 ( $\pm 0.06 \mathrm{SD})$. Forewing with 3 submarginal cells; pronotal angle obtuse; tegula shape normal. Intertegular distance $1.06( \pm 0.04 \mathrm{SD}) \mathrm{mm}$. Scutum length/width ratio $0.8( \pm 0.02 \mathrm{SD})$; scutum/scutellum length ratio 3.14 ( $\pm 0.12 \mathrm{SD})$; scutellum/metanotum length ratio $1.45( \pm 0.15 \mathrm{SD})$; metanotum/metapostnotum length ratio $0.74( \pm 0.05 \mathrm{SD})$. Propodeum lateral carinae not reaching dorsal margin; oblique carina weak (sometimes virtually absent), straight. T2 depressed apical rim length less than $50 \%$ of segment. $(n=10)$

\section{Male}

Colouration. Head and mesosoma blue to olive green; clypeus apical colour concolourous with base; labrum black to reddish brown; mandible orange to yellow with brown basal spot and red tip; flagellum orange to pale yellow, becoming reddish brown on dorsal surface toward base; pronotal lobe orange to pale yellow; metasoma black to reddish brown with rims of terga and sterna broadly pale yellow to translucent brown, discs of terga weakly metallic blue-green, and dark spiracular spots on T2-4; legs reddish brown with femur-tibia joints, base and apex of tibiae, and tarsi orange to pale yellow; tegula orange to pale amber; wing membrane hyaline, veins with subcosta dark brown, otherwise white to brown.

Pubescence. Body hair colour white. Tomentum dense on face below eye emargination and above clypeus, gena, pronotal angle and lobe, space between pronotal lobe and tegula, metepisternum, T1 laterally, T2 -3 basolaterally, and T4 -5 throughout; sparse on clypeus, scutum and scutellum marginally, preëpisternum, mesepisternum, and T6. Scutum hair thin to densely plumose. Sterna hair long (2-4 OD), moderately plumose, dense and erect. Wing hairs light, short and sparse.

SURFACE SCULPTURE. Clypeus punctures sparse ( $\mathrm{i}=1-5 \mathrm{pd})$, sculpture shiny; supraclypeal area punctures moderately sparse ( $i=1-3 \mathrm{pd})$, sculpture shiny; paraocular area punctures moderately dense $(i=1-2 \mathrm{pd})$, sculpture shiny; frons punctures dense $(\mathrm{i}<1 \mathrm{pd})$, sculpture shiny; vertex punctures dense laterally $(\mathrm{i} \leq 1 \mathrm{pd})$, sparse medially ( $i=1-3 \mathrm{pd}$ ), sculpture shiny; gena punctures moderately sparse ( $\mathrm{i}=1-2 \mathrm{pd}$ ), sculpture shiny; postgena sculpture imbricate and sometimes lineolate; tegula punctures absent; scutum punctures moderately dense ( $i=1-2 \mathrm{pd}$ ), becoming sparse submedially ( $\mathrm{i}=1-3 \mathrm{pd}$ ), sculpture shiny; scutellum punctures moderately dense $(i=1-2 \mathrm{pd}$ ), becoming sparse submedially ( $\mathrm{i}=1-3 \mathrm{pd})$, sculpture shiny; metanotum sculpture shiny and densely punctate $(i<1 \mathrm{pd})$, becoming rugulose laterally; metapostnotum rugae weak, subparallel, nearly reaching margin, sculpture shiny to weakly imbricate; preëpisternum sculpture areolate; hypoepimeron punctures crowded $(\mathrm{i}=0 \mathrm{pd})$, sculpture shiny; mesepisternum punctures dense $(\mathrm{i}<1 \mathrm{pd})$, becoming moderately sparse and diversopunctate ventrally $(\mathrm{i}=1-2 \mathrm{pd})$, sculpture shiny; metepisternum sculpture ruguloso-lineate dorsally, areolate-rugulose ventrally; propodeum lateral face punctures obscure, sculpture tessellate to rugulose; propodeum posterior face sculpture shiny; T1 anterior face sculpture coriarious; $\mathrm{T} 1$ dorsal surface punctures moderately sparse ( $\mathrm{i}=1-3 \mathrm{pd}$ ), becoming sparse in small apicolateral oval patches $(\mathrm{i}=1-4 \mathrm{pd})$ and absent on apical rim, sculpture shiny; $\mathrm{T} 2$ 


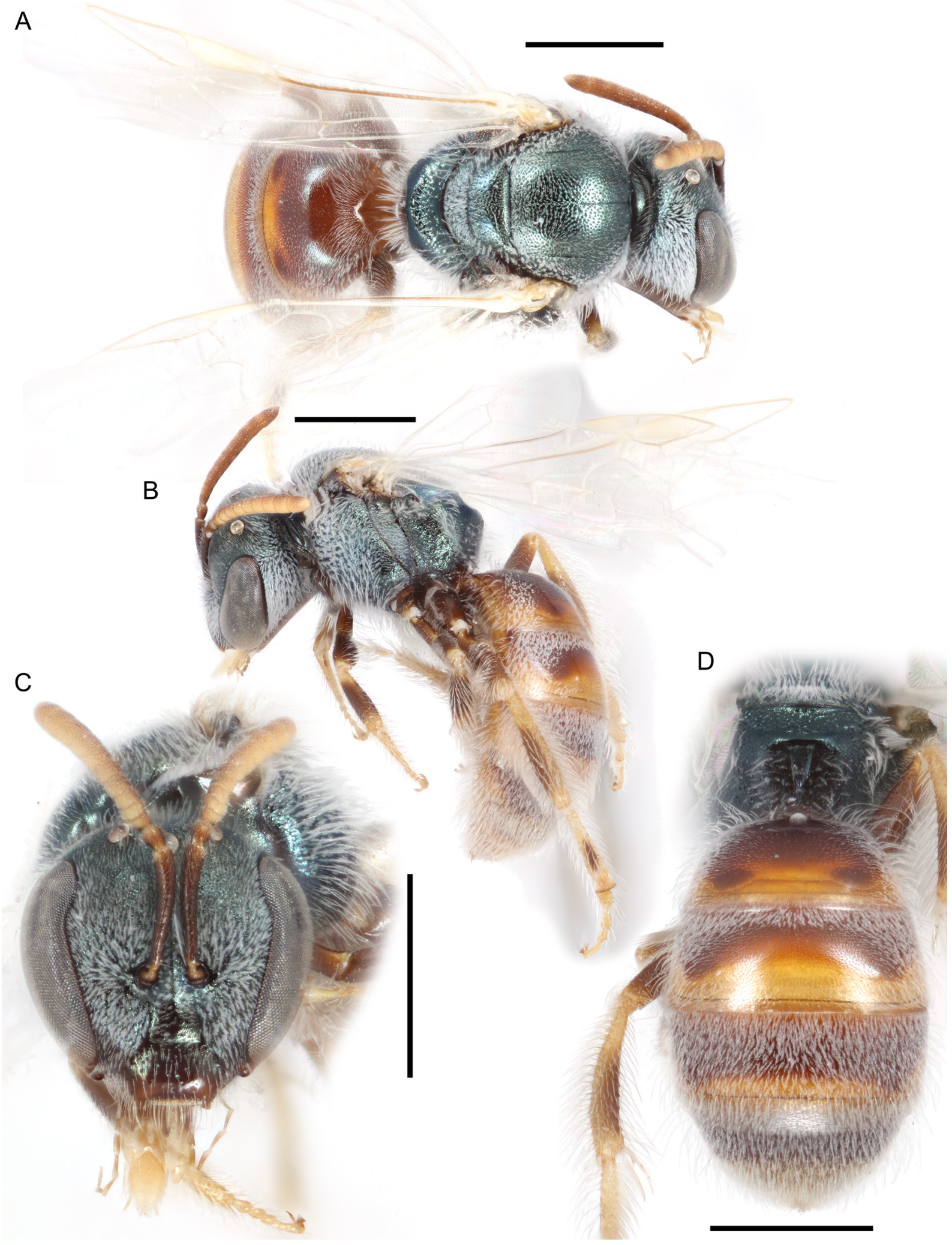

Fig. 75. Lasioglossum (D.) rufornatum sp. nov., + . A. Dorsal habitus. B. Lateral habitus. C. Face. D. Propodeum and metasoma. Scale bars: $1 \mathrm{~mm}$. 


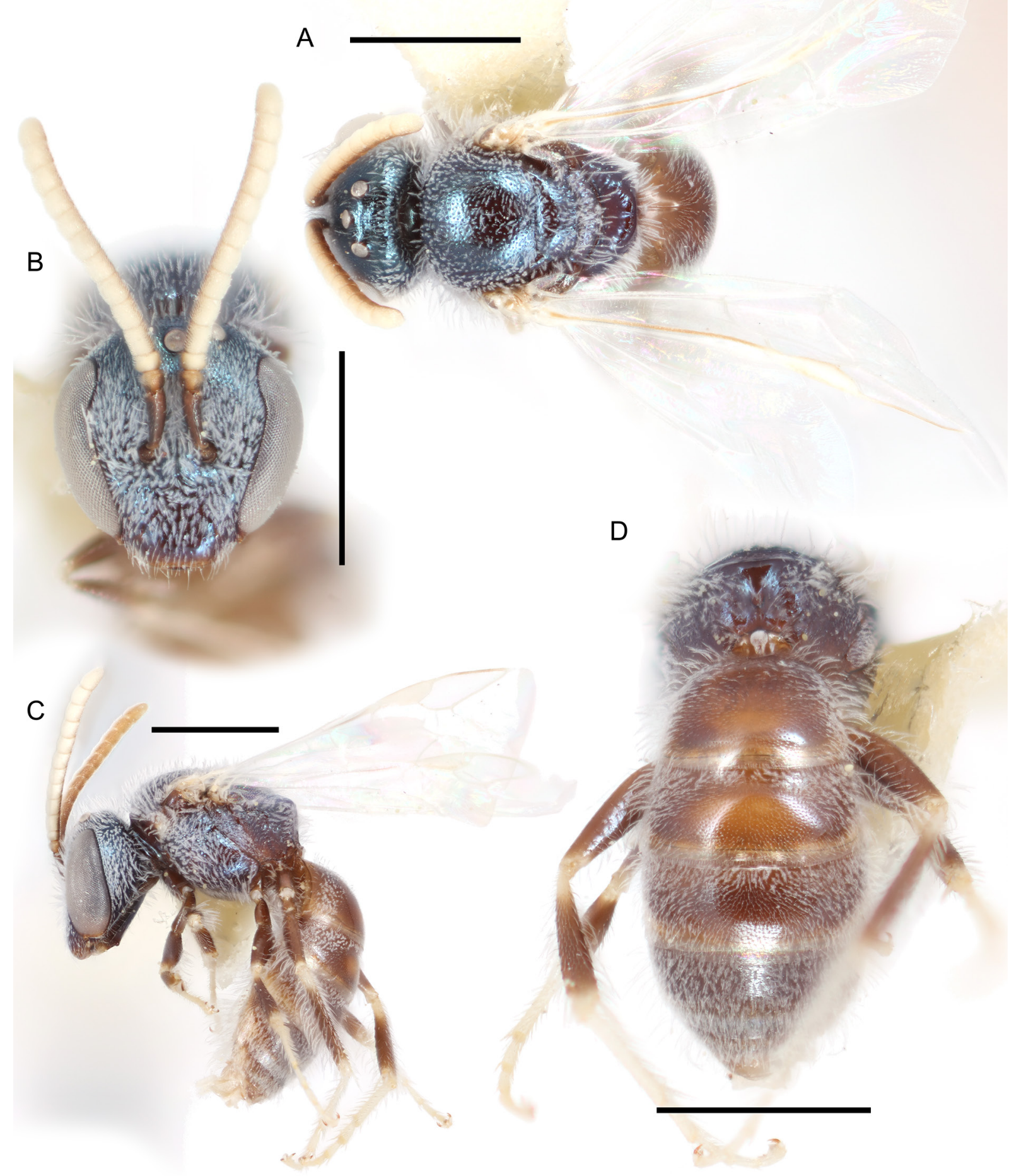

Fig. 76. Lasioglossum (D.) rufornatum sp. nov., đ. A. Dorsal habitus. B. Face. C. Lateral habitus. D. Propodeum and metasoma. Scale bars: $1 \mathrm{~mm}$. 
disc punctures moderately sparse $(\mathrm{i}=1-3 \mathrm{pd})$, disc sculpture shiny, rim punctures moderately sparse basomedially ( $\mathrm{i}=1-3 \mathrm{pd})$, otherwise absent, rim sculpture shiny.

STRUCTURE. Face length/width ratio $0.86( \pm 0.01 \mathrm{SD})$. F1:pedicel length ratio $0.89( \pm 0.06 \mathrm{SD}) ; \mathrm{F} 2: \mathrm{F} 1$ length ratio $1.55( \pm 0.12 \mathrm{SD}) ; \mathrm{F} 2$ length/width ratio $1.23( \pm 0.17 \mathrm{SD}) ; \mathrm{F} 9$ length/width ratio 0.98 ( $\pm 0.05 \mathrm{SD})$. Forewing with 3 submarginal cells; pronotal angle obtuse; tegula shape normal. Intertegular distance $1.02( \pm 0.16 \mathrm{SD}) \mathrm{mm}$. Scutum length/width ratio 0.77 ( $\pm 0.03 \mathrm{SD})$; scutum/scutellum length ratio $2.91( \pm 0.37 \mathrm{SD})$; scutellum/metanotum length ratio $1.61( \pm 0.16 \mathrm{SD}) ;$ metanotum/metapostnotum length ratio 0.63 ( $\pm 0.06 \mathrm{SD})$. Propodeum lateral carinae not reaching dorsal margin; oblique carina absent. $(n=5)$

Genitalia. As in Fig. 90M. Gonocoxite broad, rounded. Gonostylus very large, oblong, directed laterally beyond gonocoxite, with dense long hair. Retrorse lobe relatively narrow, ovoid, with dense long hair on margins.

\section{Range}

Colorado River valley from the Algodones Dunes north to Moapa (Fig. 77).

\section{Floral records}

APOCYNACEAE Juss. Asclepias L. A. subulata Decne. - ASTERACEAE Giseke: Baccharis: B. sarothroides A.Gray - Baileya: B. pleniradiata Harv. \& A.Gray ex A.Gray - Palafoxia: P. arida B.L. Turner \& M.I. Morris • P. linearis (Cav.) Lag. - Stephanomeria: S. pauciflora (Torr.) A. Nelson •

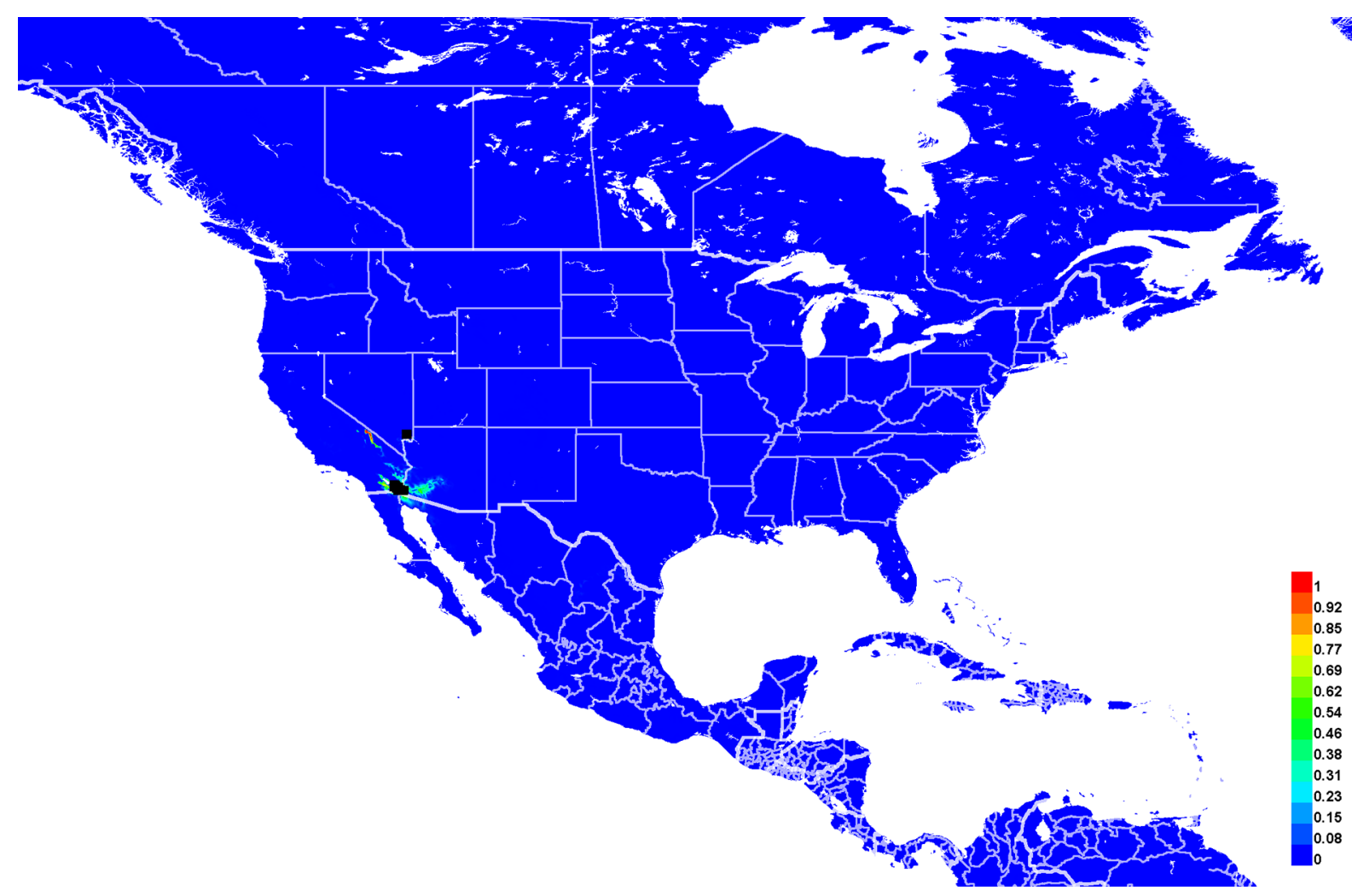

Fig. 77. Georeferenced collection records of Lasioglossum (D.) rufornatum sp. nov. (black squares) and predicted distribution by maximum entropy ecological niche modeling in Maxent (colour shading). Warmer colours indicate higher cloglog probability of occurrence. 
BIGONIACEAE Juss. Chilopsis D. Don: C. linearis (Cav.) Sweet - EUPHORBIACEAE Juss. Croton L. C. californicus Müll. Arg. $\bullet$ C. wigginsii L. C. Wheeler - FABACEAE Juss. Psorothamnus: P. emoryi (A.Gray) Rydb. - LOASACEAE Juss. Mentzelia L. M. longiloba J. Darl. • Petalonyx A.Gray • MALVACEAE Juss. Sphaeralcea A. St.-Hil. S. orcuttii Rose - POLYGONACEAE Juss. Eriogonum Michx. E. deserticola S. Watson - TAMARICACEAE Link: Tamarix L. T. ramosissima Ledeb. • ZYGOPHYLLACEAE R.Br. Larrea Cav. L. divaricata Cav. • L. tridentata (Sessé \& Moc. ex DC.) Coville.

\section{DNA barcodes}

Two sequences available (BOLD process IDs: DLIII198-20, DLIII199-20; BIN: BOLD:ACM2990). Lasioglossum rufornatum sp. nov. differs from all other western red-tailed L. (Dialictus) by 1 fixed substitution: 432(T) (Supplementary file 4).

\section{Remarks}

Lasioglossum rufornatum sp. nov. is extremely abundant in the Algodones Dunes of Imperial County, California, and becomes rarer further north along the Colorado River. No specimens have been seen from far beyond the Colorado River valley. It very likely also occurs in the Gran Desierto de Altar.

Lasioglossum (Dialictus) spivakae sp. nov. urn:1sid:zoobank.org:act:CECDA0EF-D202-45AB-8358-ADC1585CCA15 Figs $78-80,90 \mathrm{~N}$

\section{Diagnosis}

Females of Lasioglossum spivakae sp. nov. can be recognized by their small size (about $4 \mathrm{~mm}$ ), face, hypoepimeron, and mesepisternum shiny with uniformly large and dense punctures ( $\mathrm{i}<1 \mathrm{pd}$ ), scutum densely punctate except for small sparsely punctate areas just mesad of the parapsidal lines ( $i=1-2 \mathrm{pd})$, metasomal terga with tomentum sparse or absent except on $\mathrm{T} 4-5$, pronotal angle obtuse, $\mathrm{T} 2$ without dark spiracular spots, T1-3 usually red-orange with distinct, sharply delineated dark bands on T1 and T3, and T4-5 redddish brown to black. They are most similar to L. hudsoniellum and L. perditum sp. nov. Females of $L$. hudsoniellum have T1-5 with dense tomentum, scutum uniformly punctate, pronotal angle nearly 90 degrees, and metasoma orange to black but not distinctly banded. Females of L. perditum sp. nov. have the head and mesosoma sparsely punctate ( $\mathrm{i}=1-6 \mathrm{pd})$ and metasoma redorange without distinct bands.

Males of $L$. spivakae sp. nov. can be recognized by their small size (about $4 \mathrm{~mm}$ ), T1-3 uniformly shiny and moderately sparsely punctate $(i=1-2 \mathrm{pd})$ with no change in sculpture across the premarginal line, mesepisternum densely punctate $(\mathrm{i} \leq 1 \mathrm{pd}$ ), flagellomeres relatively short (F2 about 1.4 times as long as F1 and 1.25 times as long as broad), and metasoma all black. They are most similar to L. perditum sp. nov. and L. imbriumbrae sp. nov. Males of L. perditum sp. nov. have T1-3 rims more sparsely and less distinctly punctate than discs, T4 -5 with sparser tomentum, and flagellomeres relatively longer (F2 about 1.75 times as long as F1 and 1.4 times as long as broad). Males of L. imbriumbrae sp. nov. have T1-3 rims impunctate, metasomal terga with sparse tomentum, and metasoma usually partially reddish brown to orange.

\section{Etymology}

The specific epithet spivakae is a dedication to Marla Spivak, in recognition of her accomplishments as head of the University of Minnesota Bee Lab and her support during the lead author's years of work there. It is applied to this particular species because of the typical colour pattern of the female metasoma, which resembles that of a honey bee. An appropriate translation would be Spivak's sweat bee. 


\section{Material examined}

Holotype

UNITED STATES - Utah • P; Uintah Co., 15 mi. S of Roosevelt; $40.0834^{\circ} \mathrm{N}, 109.9781^{\circ} \mathrm{W} ; 15$ May 2012; S. C. Clark leg.; BBSL.

[Verbatim label: USA:UT Uintah Co. 15 mi. S of Roosevelt 12 587123E 4437512N 15 May 2012 S.C. Clark Pantrap, white / UBU-3 T-13 / BBSL821375 // BOLD / gard0142-UT / DLIII165-19 // HOLOTYPE / Lasioglossum (Dialictus) spivakae Gardner and Gibbs]

\section{Paratypes}

UNITED STATES - Arizona -2 q $\circ$; Mojave Co., $11 \mathrm{mi} . \mathrm{N}$ of Mt. Trumbull; $\left[36.56^{\circ} \mathrm{N}, 113.14^{\circ} \mathrm{W}\right]$; 9 Jun. 1994; G. Bryant leg.; ex Argemone; BBSL • 1 q; 24 mi. W of Cameron; [35.87 N, $\left.111.84^{\circ} \mathrm{W}\right]$; 3 Jul. 1952; R.H. and L.D. Beamer, C. Weiner, A. Wolf, C. Liang and W. LaBerge leg.; SEMC. Colorado - 1q; Fremont Co., 6 mi. NE of Florence; [38.45 N, 105.04 W]; 11 Aug. 1964; J. G., B. L. and K.C. Rozen leg.; AMNH - 1 q; Mesa Co., 5.5 air mi. ESE of De Beque; $39.3605^{\circ}$ N, 108.3134 ${ }^{\circ}$ W; $1725 \mathrm{~m}$ a.s.1.; 10 May 2012; J. Florez leg.; BBSL 1 क; same location as for preceding; 27 Apr. 2012; J. Florez leg.; BBSL • 1 q; same location as for preceding; 4 May 2012; J. Florez leg.; BBSL 1 क; Mesa Co., 5.58 air mi. ESE of De Beque; $39.3597^{\circ} \mathrm{N}, 108.3127^{\circ} \mathrm{W} ; 1727$ m a.s.1.; 4 May 2012; J. Florez leg.; BBSL • 1 q ; Mesa Co., 0.3 air mi. S of Pyramid Rock; $39.3043^{\circ}$ N, $108.2703^{\circ}$ W; 1534 m a.s.l.; 8 May 2012; J. Florez leg.; BBSL 35 우; Mesa Co.; $39.1019^{\circ}$ N, $108.7376^{\circ}$ W; $3-4$

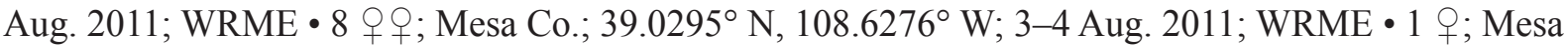

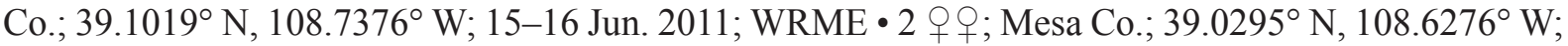

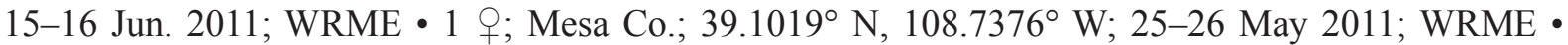

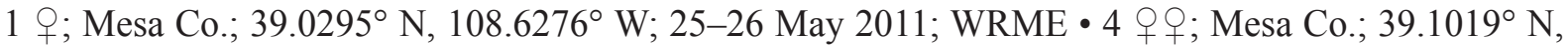
108.7376 ${ }^{\circ}$ W; 24-25 Aug. 2011; WRME • 1 क; Mesa Co.; 39.0295 N, $108.6276^{\circ}$ W; 24-25 Aug. 2011; WRME. - Nevada • 4 o $\circ$; Clark Co., 0.9 mi. E Indian Ridge; $36.4765^{\circ} \mathrm{N}, 115.6351^{\circ} \mathrm{W} ; 1396 \mathrm{~m}$ a.s.l.; 16 Jun. 2004; E. Ahlstrom leg.; ex Sphenosciadium capitellatum; BBSL • 1 o; Clark Co., 1.4 mi. E of Mule Spr.; $36.0313^{\circ}$ N, $115.5553^{\circ}$ W; 2 Jun. 2005; S. Higbee leg.; ex Fallugia paradoxa; PCYU • 2 o ; Clark Co., 2.7 mi. SE of Horse Sprs.; $36.2704^{\circ} \mathrm{N}, 115.8531^{\circ} \mathrm{W} ; 1535 \mathrm{~m}$ a.s.1.; 27 Aug. 2004; E. Rentz leg.; ex Stephanomeria pauciflora; BBSL • 5 o $\odot$; same location as for preceding; 21 Jul. 2004; S. Higbee leg.; ex Stanleya pinnata; BBSL・1 क; Clark Co., 16.91 mi. SE of Devils Hole Hills; $36.2433^{\circ}$ N, 115.8991 W; 20 Jun. 2005; R. Andrus leg.; ex Stanleya sp.; PCYU • 1 o; Clark Co., Peek-A-Boo Canyon; $36.5326^{\circ} \mathrm{N}, 115.1117^{\circ} \mathrm{W} ; 1760 \mathrm{~m}$ a.s.l.; 16 Aug. 2004; S. Higbee leg.; ex Eriogonum cernuum;

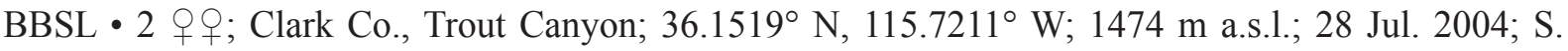
Higbee leg.; ex Eriogonum deflexum; BBSL 3 우; same location as for preceding; 28 Jul. 2004; D. Skandilis leg.; ex Eriogonum deflexum; BBSL • 1 \%; same location as for preceding; 28 Jul. 2004; E. Ahlstrom leg.; ex Eriogonum deflexum; BBSL • 1 \%; same location as for preceding; 28 Jul. 2004; E. Ahlstrom leg.; ex Eriogonum deflexum; PCYU • 1 \%; Clark Co., Willow Creek Campground, Charleston

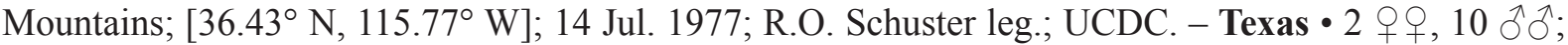
Jeff Davis Co., 0.5 mi. S of Kent; [31.06 ${ }^{\circ}$ N, $104.22^{\circ} \mathrm{W}$ ]; 29 May 1999; L. Packer leg.; PCYU. -

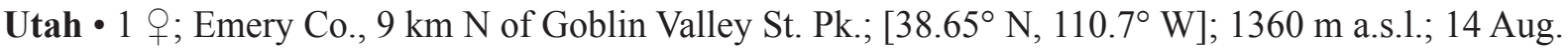
1992; D. Yanega leg.; SEMC • 1 \%; Emery Co., 1 air km SE of Gilson Butte; $38.587^{\circ} \mathrm{N}, 110.5922^{\circ} \mathrm{W}$; $1558 \mathrm{~m}$ a.s.1.; 16 Jun. 2000; F.D. Parker leg.; BBSL 1 क ; Garfield Co., 1.7 mi. WSW of Pioneer Mesa; $37.7876^{\circ} \mathrm{N}, 111.1682^{\circ} \mathrm{W}$; 6 May 2002; S. Higbee leg.; ex Mahonia fremontii; BBSL・ 1 \%; Kane Co., 1 mi. W of Wagon Box Mesa, nr 2A99; [37.82 ${ }^{\circ}$, $\left.111.09^{\circ} \mathrm{W}\right] ; 26$ May 2000; R. Andrus leg.; ex Purshia mexicana; PCYU • 1 ; ; Kane Co., Croton Canyon; [37.2 N, $111.3^{\circ} \mathrm{W}$ ]; 6 Jun. 2000; J. Grixti leg.; ex Tamarix sp.; BBSL • 1 क; same location as for preceding; 4 Jun. 2000; O. Messinger leg.; ex Tamarix sp.; PCYU • 1 q; Uintah Co., 15 mi. SW of Fort Duchesne; $40.0918^{\circ} \mathrm{N}, 109.914^{\circ} \mathrm{W} ; 11$ May 2012; S. C. Clark leg.; BBSL • 1 क; Uintah Co., Johnson Draw; 39.7619 N, $109.697^{\circ}$ W; 1819 m a.s.l.; 15 May 
2011; M. Lewis leg.; ex Hesperidanthus suffrutescens; BBSL • 1 \&; Wayne Co., 2 mi. W of Caineville; [38.33ํN, 111.06 W]; 28 Jun. 1999; L. Packer leg.; PCYU.

\section{Description}

\section{Female}

Colouration. Head and mesosoma blue to olive green; clypeus apical colour reddish brown to orange; labrum reddish brown to orange; mandible orange with black basal spot and red tip; flagellum reddish brown dorsally, light brown ventrally; pronotal lobe reddish brown; metasoma orange with base of T1, median band on T3, and T4-5 entirely brown, dark spiracular spots on T3-4; legs reddish brown with femur-tibia joints and tarsi mostly orange; tegula pale amber to brown; wing membrane hyaline, veins with subcosta brown, otherwise pale amber to light brown.

Pubescence. Body hair colour white. Tomentum dense on gena, pronotal collar and lobe, space between pronotal lobe and tegula, and T4-5; sparse on T3 apicolaterally. Scutum hair thin to slightly densely plumose. Wing hairs dark or light, short and dense. Acarinarial fan complete or incomplete, very sparse. $\mathrm{T} 2$ fringes sparse, $\mathrm{T} 3$ fringes sparse.

SuRfaCE SCULPTURE. Clypeus punctures dense on basal margin $(\mathrm{i} \leq 1 \mathrm{pd})$, large and irregularly sparse apically ( $\mathrm{i}<3 \mathrm{pd})$, sculpture shiny; supraclypeal area punctures sparse $(\mathrm{i}=1-3 \mathrm{pd})$, sculpture shiny; paraocular area punctures dense $(\mathrm{i} \leq 1 \mathrm{pd})$, sculpture shiny; frons punctures dense $(\mathrm{i}<1 \mathrm{pd})$, sculpture shiny; vertex punctures dense laterally $(i<1 \mathrm{pd})$, sparse medially $(\mathrm{i}=1-2 \mathrm{pd})$, sculpture shiny; gena punctures dense ( $i \leq 1 \mathrm{pd})$, sculpture shiny; postgena sculpture shiny, becoming weakly ruguloso-lineate to imbricate adjacent to hypostomal carina; tegula punctures absent; scutum punctures dense $(\mathrm{i}<1 \mathrm{pd})$, becoming sparse submedially and anteromedially ( $i=1-2 \mathrm{pd})$, sculpture tessellate, becoming shiny laterally and posteriorly; scutellum punctures dense marginally and on median line ( $i<1 \mathrm{pd})$, sparse submedially ( $\mathrm{i}=1-3 \mathrm{pd}$ ), sculpture imbricate marginally, shiny medially; metanotum sculpture imbricate; metapostnotum rugae strong, highly anastomosing, nearly reaching margin, sculpture imbricate; preëpisternum sculpture reticulate-punctate; hypoepimeron punctures crowded ( $\mathrm{i}=0 \mathrm{pd})$, sculpture shiny, sometimes ruguloso-punctate; mesepisternum punctures crowded ( $\mathrm{i}=0 \mathrm{pd}$ ), sculpture shiny, sometimes weakly reticulate; metepisternum sculpture lineate dorsally, rugulose medially, shiny with crowded punctures ventrally $(\mathrm{i}=0 \mathrm{pd})$; propodeum lateral face sculpture ruguloso-punctate anteriorly, becoming tessellate posteriorly; propodeum posterior face sculpture tessellate; T1 anterior face sculpture shiny; $\mathrm{T} 1$ dorsal surface punctures sparse $(\mathrm{i}=1-3 \mathrm{pd})$, impunctate in large apicolateral oval patches, sculpture shiny; $\mathrm{T} 2$ disc punctures dense ( $\mathrm{i} \leq 1 \mathrm{pd}$ ), sparse or impunctate in small apicolateral oval patches, disc sculpture shiny, rim punctures fine, sparse $(i=1-3 \mathrm{pd})$, rim sculpture shiny.

STRUCTURE. Face length/width ratio 0.87 ( $\pm 0.03 \mathrm{SD}$ ). Clypeus projecting $\sim 67 \%$ below suborbital tangent; clypeal area length/width ratio $0.39( \pm 0.02 \mathrm{SD})$; apicolateral denticles low rounded knobs; supraclypeal area length/width ratio 0.65 ( $\pm 0.04 \mathrm{SD})$. Forewing with 3 submarginal cells; pronotal angle obtuse; tegula shape normal. Intertegular distance $0.73( \pm 0.06 \mathrm{SD}) \mathrm{mm}$. Scutum length/width ratio $0.82( \pm 0.04 \mathrm{SD})$; scutum/scutellum length ratio 2.89 ( $\pm 0.27 \mathrm{SD})$; scutellum/metanotum length ratio 1.69 ( $\pm 0.16 \mathrm{SD})$; metanotum/metapostnotum length ratio $0.63( \pm 0.05 \mathrm{SD})$. Propodeum lateral carinae not reaching dorsal margin; oblique carina absent. T2 depressed apical rim length less than $50 \%$ of segment. $(n=10)$

\section{Male}

Colouration. Head and mesosoma blue; clypeus apical colour reddish brown; labrum reddish brown; mandible orange with black base and red tip; flagellum reddish brown dorsally, light brown ventrally; pronotal lobe reddish brown; metasoma black with rims of T3-5 and downcurved lateral areas of terga narrowly reddish brown, becoming transparent; legs dark reddish brown with tarsi light yellow-brown; tegula brown; wing membrane hyaline, veins with subcosta dark brown, otherwise light brown to pale amber. 


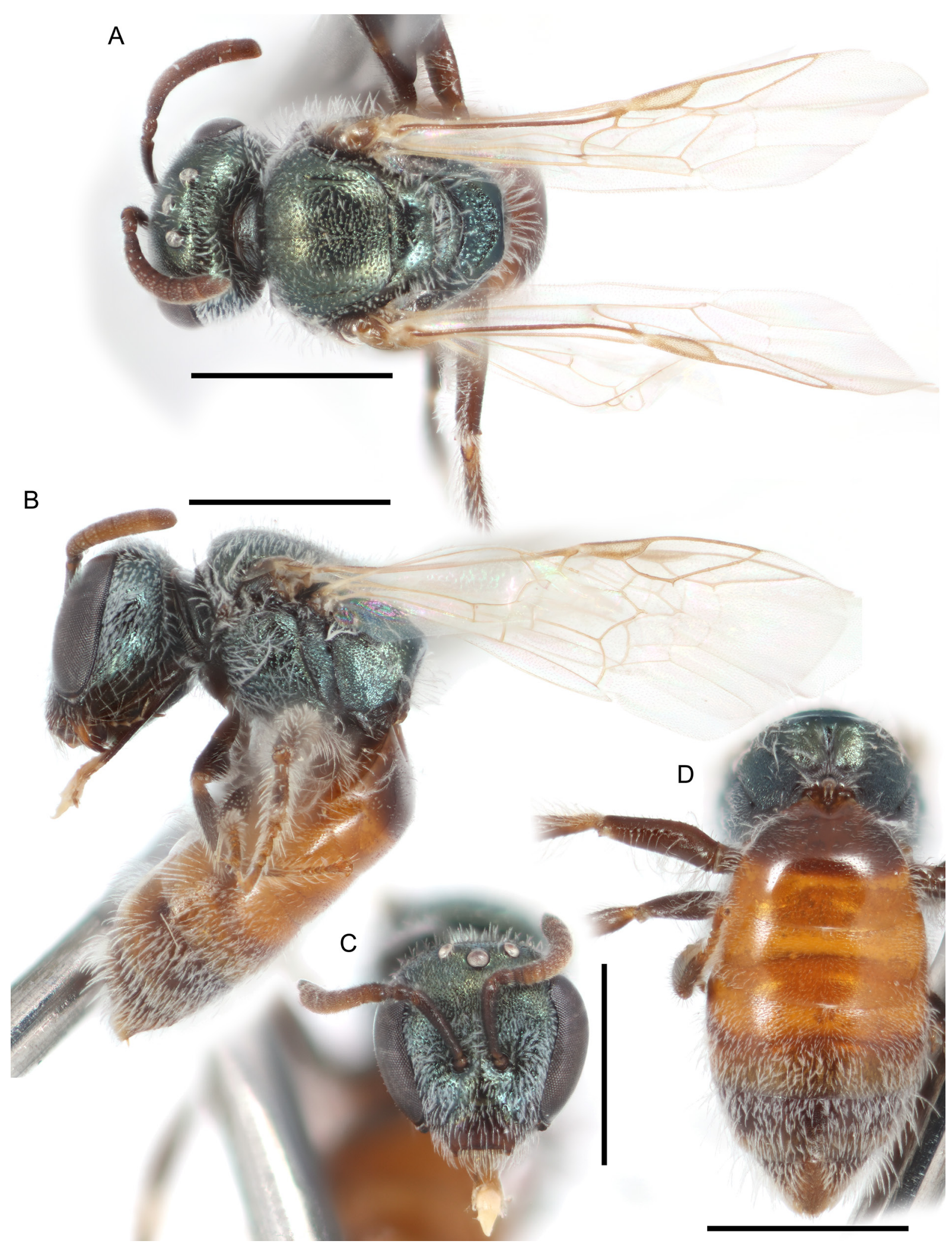

Fig. 78. Lasioglossum (D.) spivakae sp. nov., ․ A. Dorsal habitus. B. Lateral habitus. C. Face. D. Propodeum and metasoma. Scale bars: $1 \mathrm{~mm}$. 
A
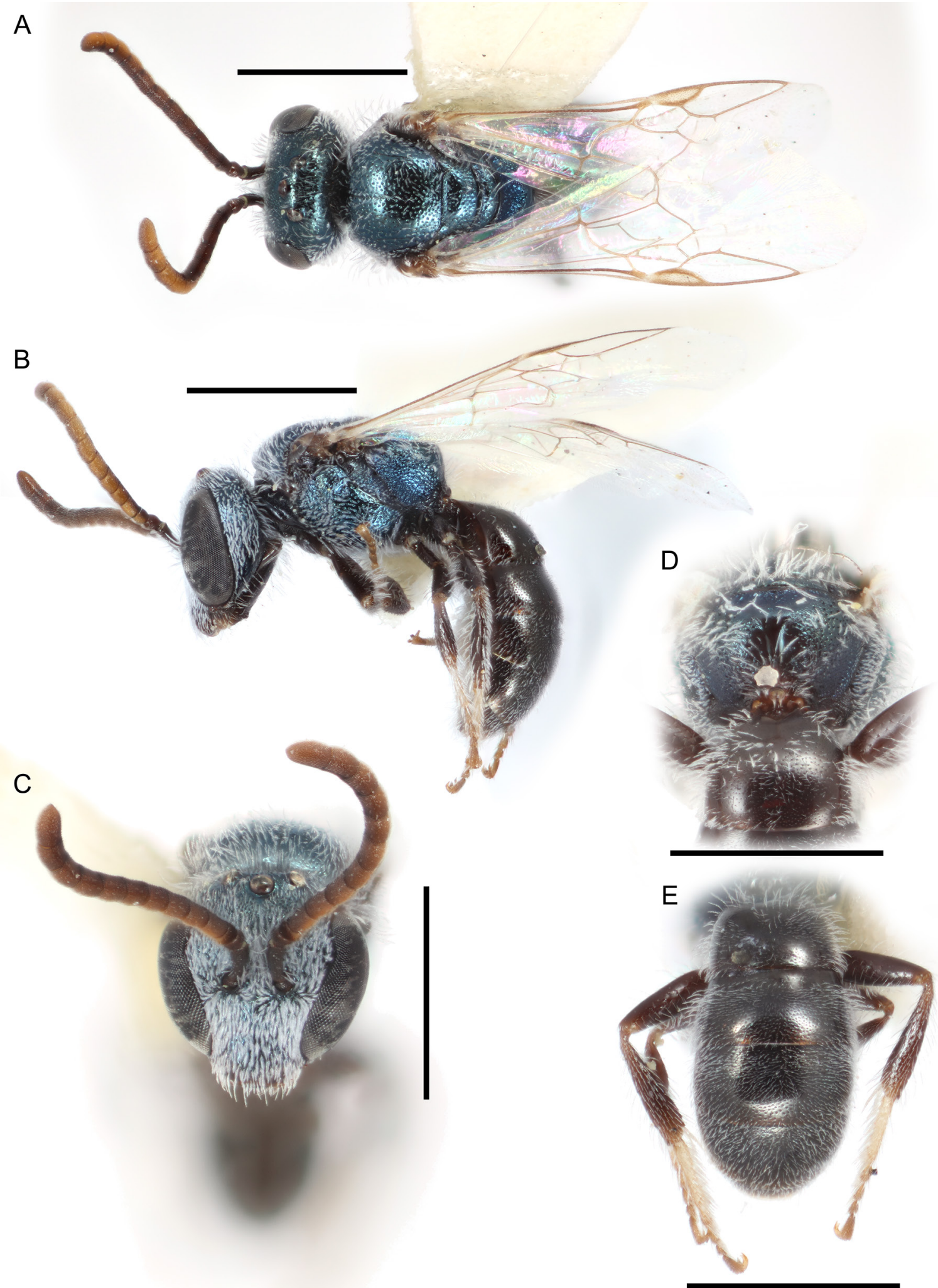

Fig. 79. Lasioglossum (D.) spivakae sp. nov., §. A. Dorsal habitus. B. Lateral habitus. C. Face. D. Propodeum. E. Metasoma. Scale bars: $1 \mathrm{~mm}$. 
Pubescence. Body hair colour white. Tomentum dense on face below ocelli, gena, pronotal collar and lobe, space between pronotal lobe and tegula, T2-3 basolaterally, and T4-5 throughout; sparse on thorax throughout, T1 laterally, T6, and rims of T1-3 laterally. Scutum hair moderately plumose. Sterna hair short (0.5-1.5 OD), densely plumose, dense and erect. Wing hairs light, short and sparse.

SuRfaCe SCUlPtURE. Clypeus punctures dense ( $\mathrm{i}<1 \mathrm{pd}$ ), sculpture shiny; supraclypeal area punctures dense $(\mathrm{i}<1 \mathrm{pd})$, sculpture shiny; paraocular area punctures dense $(\mathrm{i}<1 \mathrm{pd})$, sculpture shiny; frons punctures dense $(i<1 \mathrm{pd})$, sculpture shiny; vertex punctures dense laterally $(\mathrm{i}<1 \mathrm{pd})$, moderately sparse medially ( $\mathrm{i}=1-2 \mathrm{pd})$, sculpture shiny; gena punctures dense $(\mathrm{i}<1 \mathrm{pd})$, sculpture shiny; postgena sculpture shiny, becoming rugulose laterad of hypostomal carina and posteriorly; tegula punctures absent; scutum punctures dense ( $i \leq 1 \mathrm{pd})$, becoming sparse submedially ( $\mathrm{i}=1-3 \mathrm{pd})$, sculpture shiny; scutellum punctures dense marginally and on median line ( $\mathrm{i} \leq 1 \mathrm{pd}$ ), sparse submedially ( $\mathrm{i}=1-3 \mathrm{pd})$, sculpture shiny; metanotum sculpture shiny and finely, moderately sparsely punctate $(\mathrm{i}=1-2 \mathrm{pd})$; metapostnotum rugae strong, anastomosing, reaching or nearly reaching margin, sculpture shiny, sometimes weakly imbricate posteriorly; preëpisternum sculpture shiny with crowded punctures $(i=0 \mathrm{pd})$; hypoepimeron punctures crowded ( $\mathrm{i}=0 \mathrm{pd})$, sculpture shiny; mesepisternum punctures dense ( $\mathrm{i} \leq 1 \mathrm{pd}$ ), sculpture shiny; metepisternum sculpture lineate dorsally, reticulate-punctate ventrally; propodeum lateral face punctures crowded $(i=0 \mathrm{pd})$, becoming slightly sparser posteroventrally $(i<1 \mathrm{pd})$, sculpture imbricate, becoming ruguloso-punctate on dorsolateral slope; propodeum posterior face sculpture imbricate and densely punctate laterally $(\mathrm{i}<1 \mathrm{pd})$, becoming shiny and sparsely punctate medially $(\mathrm{i}=1-3 \mathrm{pd})$; $\mathrm{T} 1$ anterior face sculpture shiny; $\mathrm{T} 1$ dorsal surface punctures moderately sparse $(\mathrm{i}=1-2 \mathrm{pd})$, sparse in large apicolateral oval patches $(i=2-4 \mathrm{pd})$, sculpture shiny; $\mathrm{T} 2$ disc punctures moderately sparse $(\mathrm{i}=1-2 \mathrm{pd})$, disc sculpture shiny, rim punctures moderately sparse $(i=1-2 \mathrm{pd})$, rim sculpture shiny.

STRUCTURE. Face length/width ratio 0.88 ( $\pm 0.02 \mathrm{SD})$. F1:pedicel length ratio $1.3( \pm 1.09 \mathrm{SD})$; F2:F1 length ratio $1.41( \pm 0.41 \mathrm{SD}) ; \mathrm{F} 2$ length/width ratio $1.23( \pm 0.07 \mathrm{SD}) ; \mathrm{F} 9$ length/width ratio $1.09( \pm 0.08 \mathrm{SD})$. Forewing with 3 submarginal cells; pronotal angle obtuse; tegula shape normal. Intertegular distance $0.77( \pm 0.03 \mathrm{SD}) \mathrm{mm}$. Scutum length/width ratio $0.8( \pm 0.02 \mathrm{SD})$; scutum/scutellum length ratio 3.15 ( $\pm 0.4 \mathrm{SD})$; scutellum/metanotum length ratio 1.45 ( $\pm 0.3 \mathrm{SD})$; metanotum/metapostnotum length ratio 0.73 ( $\pm 0.07 \mathrm{SD})$. Propodeum lateral carinae not reaching dorsal margin; oblique carina absent. $(\mathrm{n}=10)$

Genitalia. As in Fig. 90N. Gonocoxite broad apically, narrowed at base. Gonostylus very long, attached subapically, with a few short hairs. Retrorse lobe relatively narrow, spatulate, covered with sparse short hairs apically.

\section{Range}

Western Colorado south to western Texas, west to Nevada (Fig. 80).

\section{Floral records}

APIACEAE Lindl. Sphenosciadium A.Gray: S. capitellatum A.Gray - ASTERACEAE Giseke: Stephanomeria: S. pauciflora (Torr.) A. Nelson • BERBERIDACEAE Juss. Mahonia Nutt. M. fremontii (Torr.) Fedde • BRASSICACEAE Burnett: Hesperidanthus (B.L. Rob.) Rydb. H. suffrutescens (Rollins) Al-Shehbaz - Stanleya Nutt. S. pinnata (Pursh) Britton - PAPAVERACEAE Juss. Argemone L. • POLYGONACEAE Juss. Eriogonum Michx. E. cernuum Nutt. $\bullet$ E. deflexum Torr. • ROSACEAE Juss. Fallugia Endl. F. paradoxa (D. Don) Endl. ex Torr. • Purshia DC. ex Poir. P. mexicana (D. Don) Henr. • TAMARICACEAE Link: Tamarix L.

\section{DNA barcodes}

Five sequences available (BOLD process IDs: DLIII100-18, DLIII165-19, DLIII166-19, DLIII167-19, LASNA021-08; BIN: BOLD:AAI1288). There is a small amount of divergence within these sequences 
(maximum p-distance 0.66\%). Lasioglossum spivakae sp. nov. differs from all other western red-tailed L. (Dialictus) by 3 fixed substitutions: 87(T), 142(A), and 144(T) (Supplementary file 4).

\section{Remarks}

There is some variation in the length of the face in L. spivakae sp. nov. A single specimen from Mojave County, Arizona, has an especially narrow and elongate head (face length/width ratio 0.95) with the postgena distinctly concave, but most specimens have a face length/width ratio closer to 0.85 and a normal postgena.

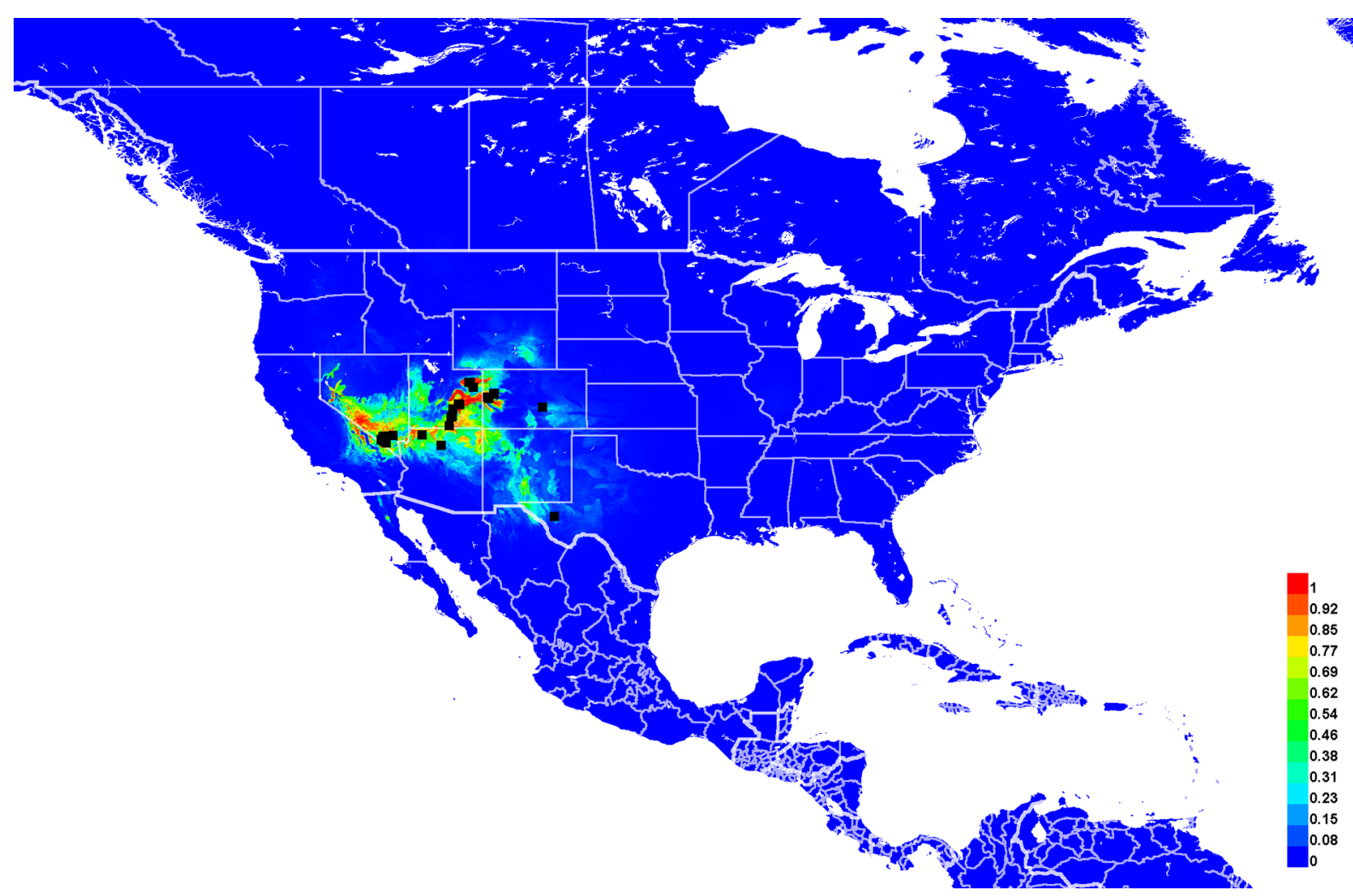

Fig. 80. Georeferenced collection records of Lasioglossum (D.) spivakae sp. nov. (black squares) and predicted distribution by maximum entropy ecological niche modeling in Maxent (colour shading). Warmer colours indicate higher cloglog probability of occurrence.

Lasioglossum (Dialictus) tessellatosum sp. nov. urn:1sid:zoobank.org:act:70FF0DE1-A7C4-4019-A346-41F5D5F6C6F4

Figs $81-83,93 \mathrm{~A}, 94 \mathrm{~A}$

\section{Diagnosis}

Females of Lasioglossum tessellatosum sp. nov. can be recognized by the head and mesosoma with very strong tessellate to reticulate microsculpture often obscuring the punctures, scutum with sparse punctures $(i=1-4 \mathrm{pd})$ abruptly becoming denser laterad of the parapsidal lines $(\mathrm{i}<2 \mathrm{pd})$, frons with relatively large and crowded punctures $(\mathrm{i}=0 \mathrm{pd}), \mathrm{T} 1$ anterior surface shiny, metasomal terga lacking tomentum, and T2 without dark spiracular spots. They are most similar to L. meteorum sp. nov. Females 
of $L$. meteorum sp. nov. have the frons with finer and distinctly separated punctures, scutum with slightly weaker (but still strong) microsculpture, $\mathrm{T} 1$ anterior surface coriarious, and metasomal terga with some sparse tomentum.

Males of L. tessellatosum sp. nov. can be recognized by the scutum tessellate with sparse punctures $(i=1-4 \mathrm{pd})$ abruptly becoming dense laterad of the parapsidal lines $(i \leq 1 \mathrm{pd})$, mesepisternum tessellate and densely punctate $(i \leq 1 \mathrm{pd})$, clypeus densely punctate $(\mathrm{i} \leq 1 \mathrm{pd})$ with very narrow black to brown apical band, frons very dull and densely punctate $(i=0 \mathrm{pd})$, and metasomal terga with broad impunctate rims. They are most similar to L. meteorum sp. nov. Males of L. meteorum sp. nov. have the clypeus and mesepisternum sparsely punctate $(i=1-4 \mathrm{pd})$, scutum and mesepisternum with weaker microsculpture, and clypeus without any non-metallic apical band.

\section{Etymology}

The specific epithet tessellatosum is formed from the Latin adjective 'tessellatum' ('inlaid with small square stones', a mosaic) and the suffix '-osum', indicating fullness or abundance. It refers to the very strong microsculpture of this species. An appropriate translation would be the tessellate sweat bee.

\section{Material examined}

Holotype

MEXICO - Chiapas • + ; Villa Corzo; 16.23 N, 93 W; 623 m a.s.1.; 3 Feb. 2016; Philippe Sagot leg.; ECOAB.

[Verbatim label: ECOAB.79796 / Mex., Chis / Villa Corzo / 632 m / 16,23 N ; 93 W / 2/3/2016 / Philippe Sagot // BOLD / DLIII117-18 / gard0051-MX // HOLOTYPE / Lasioglossum (Dialictus) tessellatosum Gardner and Gibbs]

\section{Paratypes}

GUATEMALA - Huehuetenango • 1 क; San Antonio Hulsta, Cajuil; $15.63746^{\circ} \mathrm{N}, 91.78722^{\circ} \mathrm{W}$; 1026 m a.s.1.; 5 Jul. 2010; Mario leg.; ex Coffea; ECOAB. - Quetzaltenango • 1 q; 5 mi. E of Coatepeque; [14. $\left.7^{\circ} \mathrm{N}, 91.8^{\circ} \mathrm{W}\right]$; 4 Jul. 1965; Slobodchikoff and Raske leg.; EMEC.

MEXICO - Chiapas • 1 q; Jaltenango Paz; $15.82^{\circ}$ N, 92.79 W; 692 m a.s.1.; 3 Feb. 2016; Philippe Sagot leg.; ECOAB • 1 q; La Trinitaria, San Francisco de Asis; $16.0232^{\circ}$ N, $92.0455^{\circ}$ W; 768 m a.s.1.; 16 Nov. 2011; Philippe Sagot leg.; ECOAB • 1 \%; Mapastepec, Camino A Santa Rosa; $15.3405^{\circ}$ N, 92.5757 W; 24 Feb. 2005; R. Ayala, R. Vandame and M. Guzmán leg.; ECOAB. - Chihuahua • 3 우; Santo Niño; [27 $7^{\circ}$, $107^{\circ}$ W]; 31 Aug. 1968; T.A. Sears, R.C. Gardner and C.S. Glaser leg.; UCDC. - Hidalgo 1 क; $14.5 \mathrm{~km}$ NW of Cardonal; $\left[20.73^{\circ} \mathrm{N}, 99.15^{\circ} \mathrm{W}\right.$ ]; $1850 \mathrm{~m}$ a.s.l.; 13 Jul. 1990; W. Bell, D. Conlon and R.L. Minckley leg.; ex Agave; SEMC. - Jalisco • 3 q ; ; Careyes; $19.4289^{\circ}$ N,

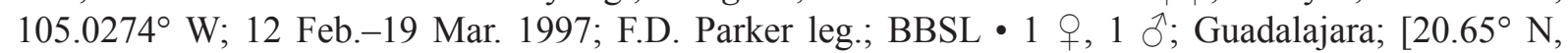
103.35 ${ }^{\circ} \mathrm{W}$; Crawford leg.; USNM • 1 क; Puerto Vallarta; $20.6214^{\circ} \mathrm{N}, 105.226^{\circ} \mathrm{W} ; 25$ Jan. 1984; G.E. Bohart leg.; BBSL. - Morelos • 1 क; 7.3 mi. S. Yautepec; $\left[18.78^{\circ}\right.$ N, $\left.99.06^{\circ} \mathrm{W}\right] ; 914 \mathrm{~m}$ a.s.1.; 15 Aug. 1962; Marston and Naumann leg.; SEMC • 1 क; same location as for preceding; 16 Aug. 1962; Ordway and Naumann leg.; SEMC • 1 \% ; Cañon de Lobos; [18.86 ${ }^{\circ}$ N, 99.12 $\left.{ }^{\circ} \mathrm{W}\right]$; $23-30$ Mar. 1961; C. Benschoter leg.; UMSP. - Veracruz • $1{ }^{\top}$; Orizaba; [18.85 N, $\left.97.1^{\circ} \mathrm{W}\right]$; Crawford leg.; USNM.

\section{Description}

\section{Female}

Colouration. Head and mesosoma blue-green to golden-green; clypeus apical colour black, reddish brown, or orange; labrum reddish brown to orange; mandible orange with black base and red tip; flagellum black to dark reddish brown dorsally, slightly lighter ventrally; pronotal lobe black, reddish brown, or 
orange; metasoma orange with $\mathrm{T} 1$ basal half and $\mathrm{T} 5$ sometimes black to brown, dark spiracular spots on T3-4; legs reddish brown with femur-tibia joints, tarsi, and sometimes tibiae mostly orange; tegula orange; wing membrane lightly infuscated, veins with subcosta dark brown, otherwise light brown to pale amber.

Pubescence. Body hair colour white to pale yellow. Tomentum dense on pronotal collar and lobe, space between pronotal lobe and tegula, and metepisternum ventral half; sparse on paraocular area, vertex, gena, and metanotum. Scutum hair thin. Wing hairs dark or light, short and dense. Acarinarial fan incomplete, sparse. T2 fringes sparse, T3 fringes sparse.

SuRfaCe SCUlPture. Clypeus punctures dense ( $\mathrm{i} \leq 1 \mathrm{pd}$ ), small on basal margin, otherwise large and somewhat irregular, sculpture tessellate in basal half, shiny apically; supraclypeal area punctures dense marginally ( $\mathrm{i} \leq 1 \mathrm{pd}$ ), sparse medially ( $\mathrm{i}=1-3 \mathrm{pd}$ ), sculpture tessellate, sometimes shiny medially; paraocular area punctures dense $(\mathrm{i}<1 \mathrm{pd})$, often obscure, sculpture shiny ventrally and in narrow line adjacent to compound eye, otherwise tessellate; frons punctures crowded ( $\mathrm{i}=0 \mathrm{pd}$ ), often indistinct, sculpture reticulate or ruguloso-punctate; vertex punctures crowded laterally ( $\mathrm{i}=0 \mathrm{pd}$ ), often obscure, absent medially, sculpture shiny or weakly tessellate; gena punctures fine, moderately dense ( $\mathrm{i}=1-2 \mathrm{pd})$, sculpture shiny; postgena sculpture shiny to tessellate, becoming ruguloso-lineate posteriorly; tegula punctures absent; scutum punctures sparse $(\mathrm{i}=1-4 \mathrm{pd})$, usually abruptly becoming dense to moderately dense laterad of parapsidal line ( $i<2$ pd), sculpture finely reticulate; scutellum punctures dense marginally and on median line ( $<<1 \mathrm{pd})$, sparse submedially ( $\mathrm{i}=1-2 \mathrm{pd})$; minute and hardly evident in anterior half, larger and more distinct posteriorly, sculpture finely reticulate; metanotum sculpture finely reticulate and moderately densely punctate $(i=1-2 \mathrm{pd})$; metapostnotum rugae weak or moderately strong, anastomosing, reaching about halfway to margin, sculpture tessellate; preëpisternum sculpture finely reticulate and densely but obscurely punctate $(i \leq 1 \mathrm{pd})$; hypoepimeron punctures dense $(\mathrm{i}<1 \mathrm{pd})$, obscure, sculpture finely reticulate; mesepisternum punctures dense dorsally $(i \leq 1 \mathrm{pd})$, becoming sparse ventrally ( $\mathrm{i}=1-2 \mathrm{pd})$, mostly obscured by microsculpture, sculpture finely reticulate; metepisternum sculpture ruguloso-lineate dorsally, becoming rugulose ventrally; propodeum lateral face sculpture tessellate; propodeum posterior face sculpture tessellate; T1 anterior face sculpture shiny; T1 dorsal surface punctures very fine and sparse $(i=2-6 \mathrm{pd})$, absent in median line and small apicolateral oval patches, sculpture shiny; T2 disc punctures fine, moderately sparse ( $\mathrm{i}=1-3 \mathrm{pd})$, disc sculpture shiny to weakly coriarious, rim punctures shallow and obscure, moderately sparse $(i=1-3 \mathrm{pd})$, rim sculpture weakly coriarious.

STRUCTURE. Face length/width ratio 0.81 ( $\pm 0.02 \mathrm{SD}$ ). Clypeus projecting $\sim 50 \%$ below suborbital tangent; clypeal area length/width ratio 0.43 ( $\pm 0.01 \mathrm{SD})$; apicolateral denticles rounded knobs; supraclypeal area length/width ratio $0.66( \pm 0.05 \mathrm{SD})$. Forewing with 3 submarginal cells; pronotal angle slightly obtuse, nearly right-angled; tegula shape normal. Intertegular distance $1( \pm 0.11 \mathrm{SD}) \mathrm{mm}$. Scutum length/width ratio $0.84( \pm 0.04 \mathrm{SD})$; scutum/scutellum length ratio 2.92 ( $\pm 0.13 \mathrm{SD})$; scutellum/metanotum length ratio $1.61( \pm 0.18 \mathrm{SD})$; metanotum/metapostnotum length ratio $0.62( \pm 0.08 \mathrm{SD})$. Propodeum lateral carinae not reaching dorsal margin; oblique carina absent. T2 depressed apical rim length less than $50 \%$ of segment. $(n=10)$

\section{Male}

Colouration. Head and mesosoma blue-green to olive green; clypeus apical colour narrowly black to reddish brown; labrum black to reddish brown; mandible brown with black basal band and red tip; flagellum reddish brown; pronotal lobe reddish brown; metasoma black to reddish brown; legs reddish brown; tegula brown; wing membrane lightly infuscated, veins dark brown. 

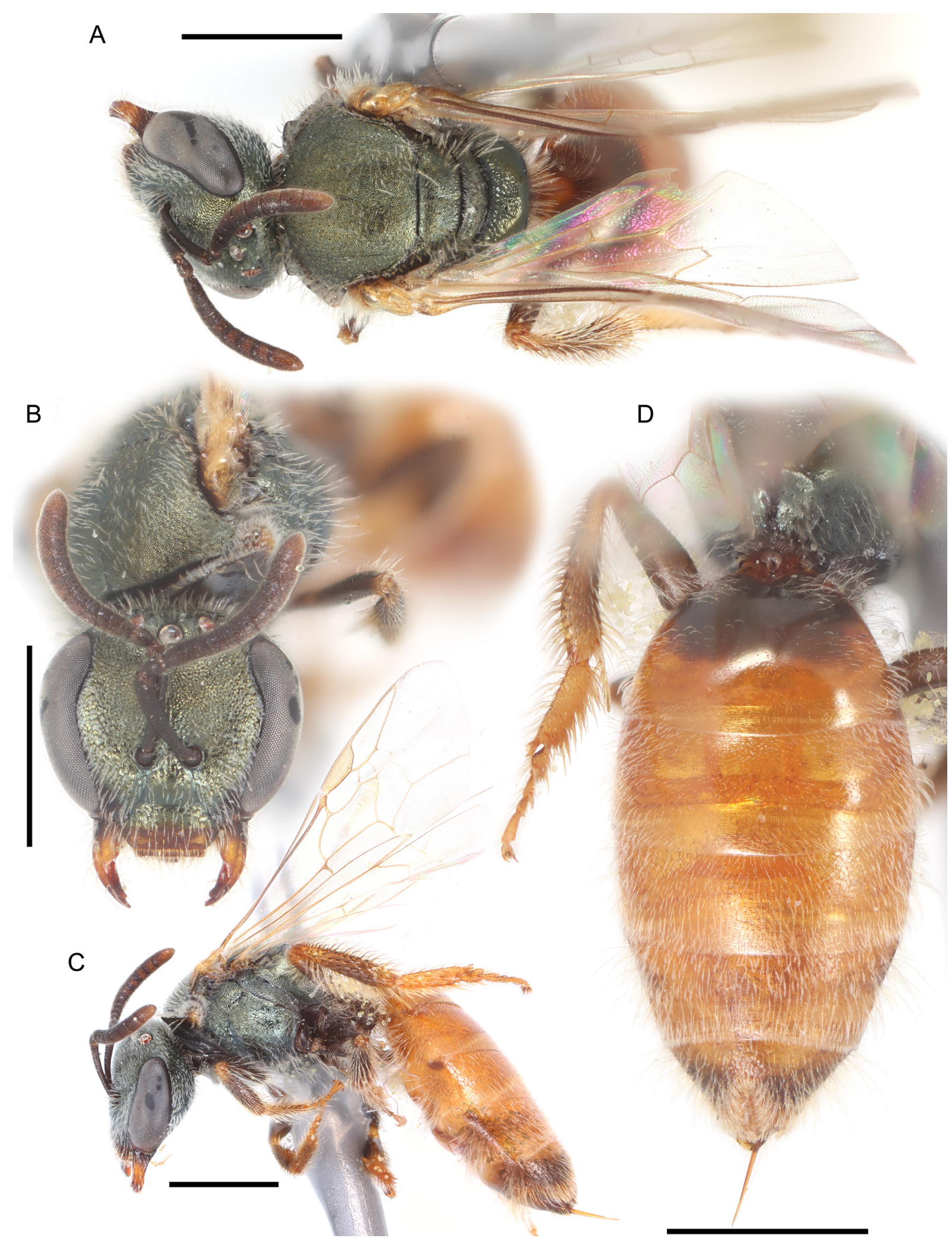

Fig. 81. Lasioglossum (D.) tessellatosum sp. nov., ${ }^{+}$. A. Dorsal habitus. B. Face. C. Lateral habitus. D. Propodeum and metasoma. Scale bars: $1 \mathrm{~mm}$. 


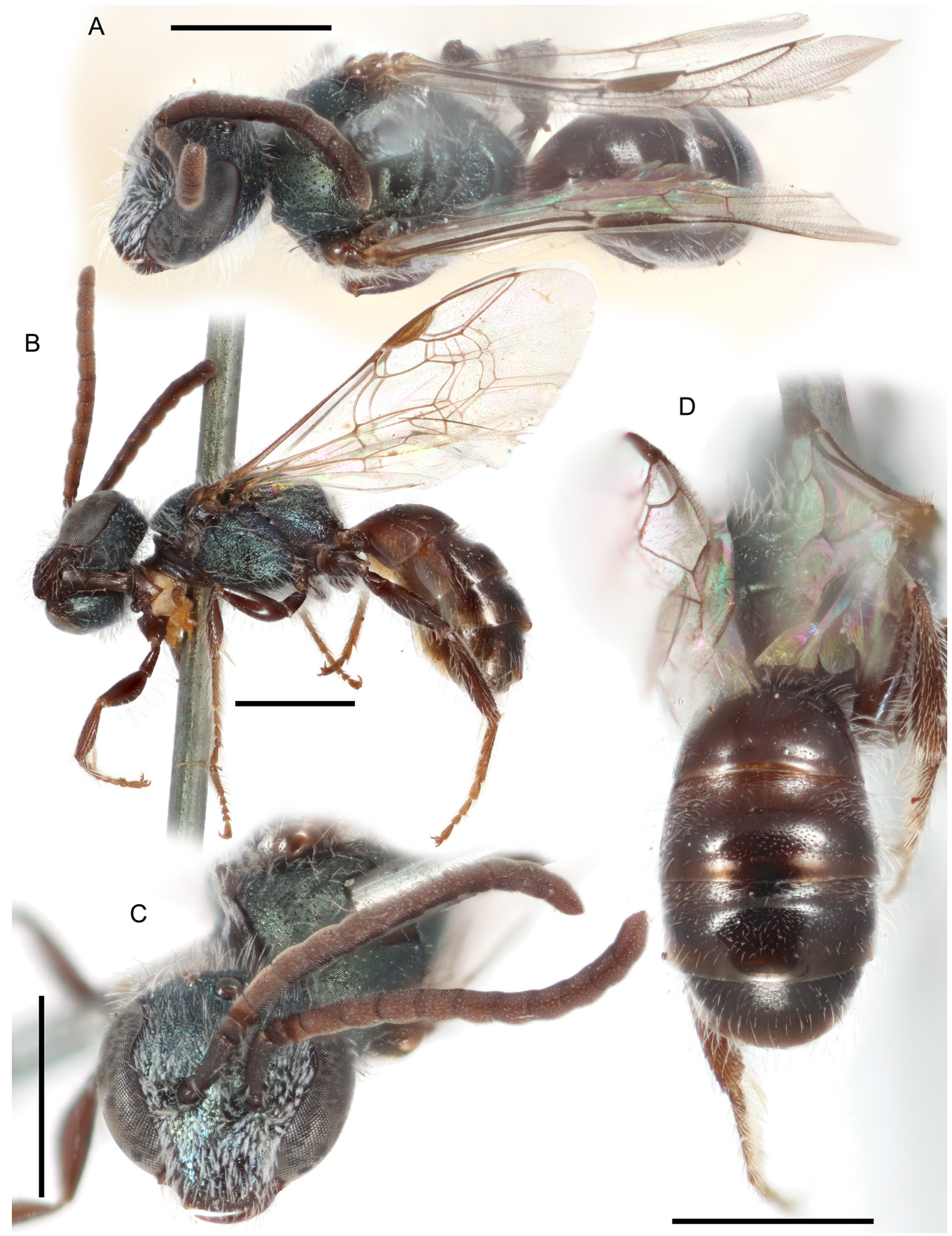

Fig. 82. Lasioglossum (D.) tessellatosum sp. nov., §ิ. A. Dorsal habitus. B. Lateral habitus. C. Face. D. Metasoma. Scale bars: $1 \mathrm{~mm}$. 
Pubescence. Body hair colour pale yellow to white. Tomentum dense in space between pronotal lobe and tegula; sparse on face below eye emargination, gena anteriorly, and pronotal angle and lobe. Scutum hair thin. Sterna hair moderately long (1-2 OD), moderately plumose, sparse and erect. Wing hairs dark, short and dense.

SuRfaCe SCULPTURE. Clypeus punctures dense ( $\mathrm{i} \leq 1 \mathrm{pd}$ ), diversopunctate, sculpture shiny; supraclypeal area punctures moderately sparse $(i=1-2 \mathrm{pd})$, obscure, sculpture tessellate; paraocular area punctures dense ( $\mathrm{i} \leq 1 \mathrm{pd})$, sculpture shiny; frons punctures crowded ( $\mathrm{i}=0 \mathrm{pd})$, sculpture reticulate-rugulose, becoming shiny to imbricate laterally; vertex punctures crowded laterally ( $\mathrm{i}=0 \mathrm{pd})$, dense $(\mathrm{i} \leq 1 \mathrm{pd})$ and obscure medially, sculpture imbricate to finely reticulate; gena punctures moderately sparse $(\mathrm{i}=1-2 \mathrm{pd})$, sculpture shiny, becoming imbricate posteriorly and ventrally; postgena sculpture shiny, becoming imbricate posteriorly and laterad of hypostomal carina; tegula punctures absent; scutum punctures sparse ( $\mathrm{i}=1-4 \mathrm{pd})$, abruptly becoming dense laterad of parapsidal lines $(\mathrm{i} \leq 1 \mathrm{pd})$, sculpture tessellate to finely reticulate; scutellum punctures dense marginally and on median line ( $\mathrm{i} \leq 1 \mathrm{pd})$, sparse submedially ( $i=1-4 \mathrm{pd}$ ), sculpture tessellate to finely reticulate, becoming shiny submedially; metanotum sculpture finely reticulate, sometimes becoming shiny medially, and moderately sparsely punctate $(i=1-2 \mathrm{pd})$; metapostnotum rugae strong, anastomosing, not reaching margin, sculpture imbricate; preëpisternum sculpture areolate-rugulose to rugose; hypoepimeron punctures dense $(\mathrm{i}<1 \mathrm{pd})$, sculpture tessellate to finely reticulate; mesepisternum punctures dense ( $i \leq 1 \mathrm{pd})$, sculpture tessellate to finely reticulate; metepisternum sculpture lineate dorsally, areolate ventrally; propodeum lateral face punctures obscure, sculpture tessellate to weakly rugulose; propodeum posterior face sculpture shiny, becoming imbricate and densely punctate laterally ( $\mathrm{i}<1 \mathrm{pd}$ ); T1 anterior face sculpture shiny; T1 dorsal surface punctures sparse $(i=1-4 \mathrm{pd})$, absent or very sparse in large apicolateral oval patches and on rim, sculpture shiny; $\mathrm{T} 2$ disc punctures sparse $(\mathrm{i}=1-3 \mathrm{pd})$, disc sculpture shiny, rim punctures absent, rim sculpture shiny.

STRUCTURE. Face length/width ratio 0.78 ( \pm 0 SD). F1:pedicel length ratio $0.9( \pm 0.14$ SD); F2:F1 length ratio $2.13( \pm 0.18 \mathrm{SD}) ; \mathrm{F} 2$ length/width ratio $1.49( \pm 0.1 \mathrm{SD}) ; \mathrm{F} 9$ length/width ratio $1.23( \pm 0.06 \mathrm{SD})$. Forewing with 3 submarginal cells; pronotal angle slightly obtuse, nearly right-angled; tegula shape normal. Intertegular distance $0.86( \pm 0.07 \mathrm{SD}) \mathrm{mm}$. Scutum length/width ratio 0.85 ( $\pm 0.01 \mathrm{SD})$; scutum/ scutellum length ratio 2.61 ( $\pm 0.12 \mathrm{SD})$; scutellum/metanotum length ratio $1.58( \pm 0.08 \mathrm{SD})$; metanotum/ metapostnotum length ratio $0.66( \pm 0.08 \mathrm{SD})$. Propodeum lateral carinae not reaching dorsal margin; oblique carina absent. $(n=2)$

Genitalia. Not examined.

\section{Range}

Tropics and transition zones from Chihuahua south to Guatemala (Fig. 83).

\section{Floral records}

ASPARAGACEAE Juss. Agave L. • RUBIACEAE Juss. Coffea L.

\section{DNA barcodes}

Two sequences available (BOLD process IDs: BBLEG173-17, DLIII117-18; BINs: BOLD:ADB1211, BOLD:ADH0657). Lasioglossum tessellatosum sp. nov. differs from all other western red-tailed L. (Dialictus) by 2 fixed substitutions: 45(A) and 381(T) (Supplementary file 4).

\section{Remarks}

Lasioglossum tessellatosum sp. nov. is extremely widespread throughout Mexico and occurs at least as far south as western Guatemala, but seems to be replaced by L. meteorum sp. nov. on the Yucatan 
Peninsula. It is most commonly found at high elevation in southern Mexico, with a disjunct series of specimens from Santo Niño, Chihuahua. The exact location of this 'Santo Niño' is not known (several locations in Chihuahua are known by that name), but somewhere in the less arid western part of the state is likely.

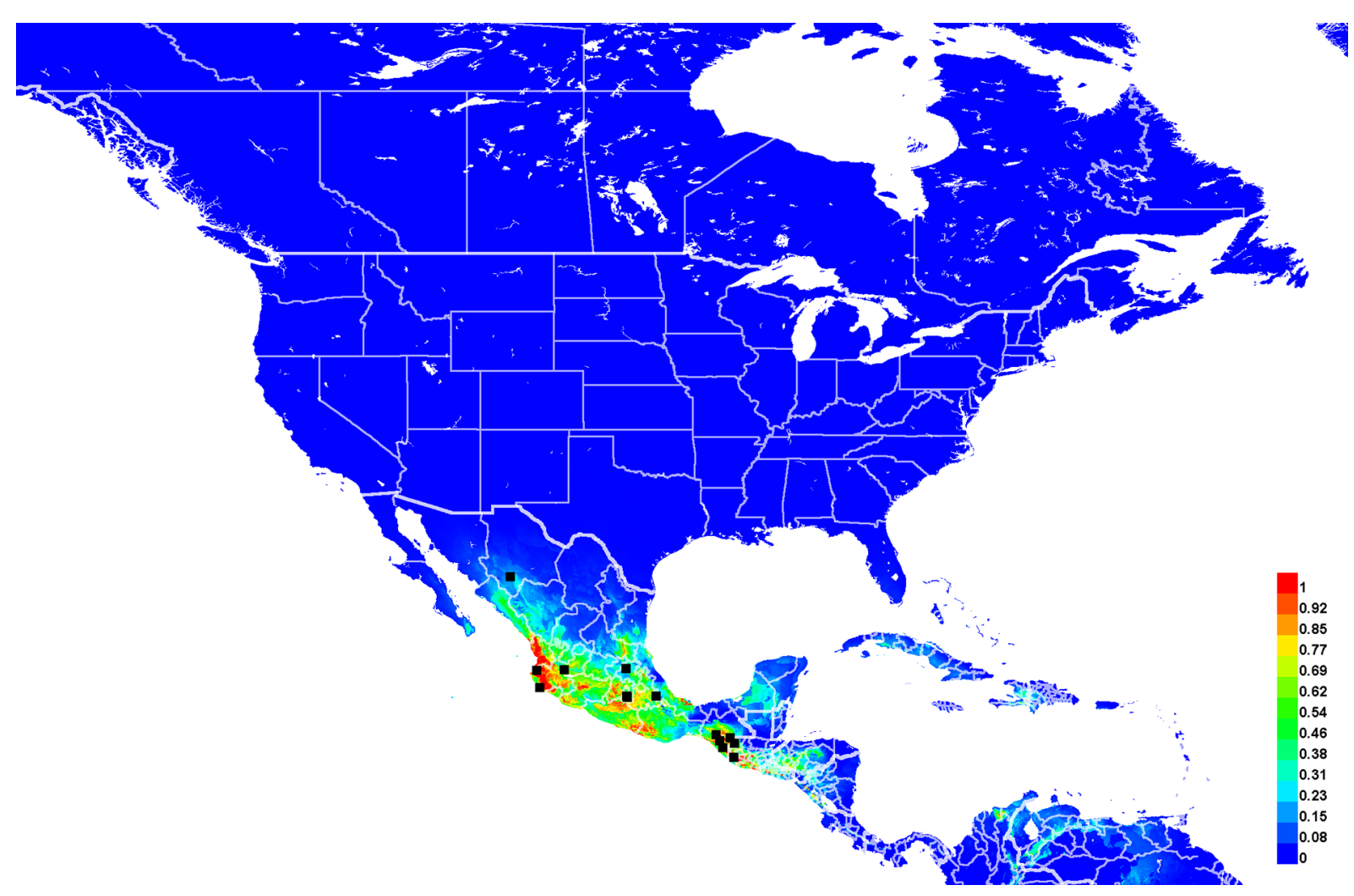

Fig. 83. Georeferenced collection records of Lasioglossum (D.) tessellatosum sp. nov. (black squares) and predicted distribution by maximum entropy ecological niche modeling in Maxent (colour shading). Warmer colours indicate higher cloglog probability of occurrence.

Lasioglossum (Dialictus) testaceum (Robertson, 1897)

Figs $84,900,96 \mathrm{~A}, 98 \mathrm{~A}$

Halictus testaceus Robertson, 1897: 323 (lectotype designated by W.E. LaBerge in Webb 1980, + , deposited in INHS, examined).

Halictus (Chloralictus) scrophulariae Cockerell, 1906: 428 (holotype,, , deposited in USNM, examined; synonymy by Gibbs 2011).

Halictus (Chloralictus) occultus Sandhouse, 1924: 27 (holotype, Ô, deposited in USNM, examined; name preoccupied due to primary homonymy with Halictus occultus Vachal, 1904).

Lasioglossum (Chloralictus) sandhouseae Michener, 1951: 1117 (catalog, replacement name for H. (C.) occultus Sandhouse, 1924; synonymy by Gibbs 2011).

Chloralictus testaceus - Robertson 1902b: 249 (key).

Halictus (Chloralictus) scrophulariae - Sandhouse 1924: 4 (key).

Lasioglossum (Chloralictus) scrophulariae - Michener 1951: 1117 (catalog). 
Lasioglossum (Chloralictus) testaceum - Michener 1951: 1118 (catalog).

Dialictus sandhouseae - Mitchell 1960: 418, fig. 102 (key, redescription). — Hurd 1979: 1971 (catalog). — Moure \& Hurd 1987: 129 (catalog).

Dialictus testaceus - Mitchell 1960: 424 (key, redescription). — Hurd 1979: 1972 (catalog). — Moure \& Hurd 1987: 135 (catalog).

Dialictus scrophulariae - Hurd 1979: 1971 (catalog). — Moure \& Hurd 1987: 129 (catalog).

Lasioglossum (Dialictus) sandhouseae - Krombein 1967: 466 (catalogue).

Lasioglossum testaceum - Donovall \& van Engeldorp 2010: 18 (checklist).

Lasioglossum (Evylaeus) testaceum - Gibbs 2010: 42, fig. 9b (key). — Scott et al. 2011: 32 (checklist). Lasioglossum (Dialictus) testaceum - Wolf \& Ascher 2009: 144 (checklist). — Gibbs 2011: 197, figs 213-217 (key, redescription).

\section{Diagnosis}

Females of Lasioglossum testaceum are readily identified by the propodeum with strong dorsoventral and oblique carinae, and T1 with sparse erect hairs and no acarinarial fan. This combination is unique among Nearctic L. (Dialictus) except for L. rufulipes (Cockerell, 1938), which has a dark metasoma, T4 with sparser tomentum, and a boreal distribution, and L. aquilae, which has the metasoma dark metallic green.

Males of L. testaceum can be recognized by the face short (length/width ratio $\sim 0.81$ ), clypeus apical half pale yellow, propodeum dorsolateral slope and posterior face rugose, propodeum lateral face smooth and tessellate, and retrorse lobe very narrow. They are most similar to L. zephyrus and L. pictum. Both of these species have the clypeus apical margin dark or much more narrowly yellow, retrorse lobes much broader, and face slightly longer. In addition, males of $L$. pictum have the scutum and mesepisternum with larger and denser punctures $(i=1-2 \mathrm{pd})$ and propodeum lateral face rugulose.

\section{Etymology}

Robertson (1897) named this species with the Latin adjective 'testaceus' (brick-like).

\section{Material examined}

\section{Lectotype}

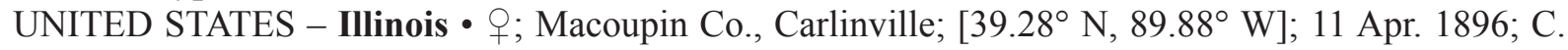
Robertson leg.; INHS.

\section{Other material}

CANADA - Alberta • 1 o ; Writing-On-Stone Prov. Pk.; [49.09 N, 111.62 ${ }^{\circ}$ W]; 26 May-6 Jun. 1990;

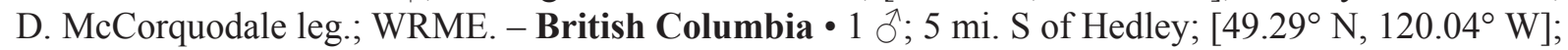
24 Jul. 1985; Finnamore and Thormin leg.; PMAE.

UNITED STATES - Colorado 22 + ; Alamosa Co., 4 mi. SW of Alamosa (intersection 370 and S106); 37.42738 ${ }^{\circ}$ N, $105.92846^{\circ}$ W; 2301 m a.s.1.; 25 May 2001; Va. Scott leg.; ex Salix (staminate); UCMC • 1 O; El Paso Co., Ramah; [39.12 ${ }^{\circ}$ N, $104.17^{\circ}$ W]; 15 Jun. 1971; Michener and Schorr leg.; ex Salix sp.; SEMC • 1 क; same location as for preceding; 15 Jun. 1971; C. Michener and R. Schorr leg.; ex Salix; SEMC • 2 우; Fremont Co., 5 mi. SW of Coaldale; [38.31 ${ }^{\circ} \mathrm{N}, 105.82^{\circ} \mathrm{W}$ ]; 10 Jun. 1971; C. Michener and R. Schorr leg.; ex Salix; SEMC • 1 क ; same location as for preceding; 10 Jun. 1971; C. Michener and R. Schorr leg.; ex Rhus trilobata; SEMC • 2 $q$; ; Fremont Co., Coaldale; [38.37 N, $\left.105.76^{\circ} \mathrm{W}\right] ; 11$ Jun. 1971; C. Michener and R. Schorr leg.; ex Salix; SEMC • 1 क; Jackson Co., Lake John; $40.7862^{\circ}$ N, 106.4692 ${ }^{\circ}$ W; $2461 \mathrm{~m}$ a.s.1.; 27 Jun. 2014; T. McIntyre and F.D. Parker leg.; BBSL • 1 क; Park Co., N. Fk. S. Platte R., 0.2 mi. E of Bailey; [39.404 N, $105.47^{\circ} \mathrm{W}$ ]; 11 Jun. 1988; E. Bergey leg.; CSUC • $1{ }^{\top}$; Routt Co., 4 mi. E of Clark; [40.71 ${ }^{\circ}$ N, 106.84 W]; 29 Aug. 1979; U.N. Lanham leg.; ex Solidago; UCMC • 1 क; Teller Co., S base of Blue Mtn., nr Florissant; [38.923 N, $\left.105.327^{\circ} \mathrm{W}\right] ; 2568 \mathrm{~m}$ a.s.l.; 1 
Aug. 1966; T. Emmel and M. Fosdick leg.; LACM • 1 क; Weld Co., 2-4 mi. W of Orchard; [ $40.33^{\circ} \mathrm{N}$, $104.18^{\circ}$ W]; 14 May 1992; M.G. Galindo and R.S. Beal leg.; ex Salix; CSUC • 3 do $^{\lambda} ; 8$ mi. NW of Steamboat Spgs.; [40.57 N, 106.94 W]; 28 Aug. 1979; U.N. Lanham leg.; ex Solidago; UCMC •

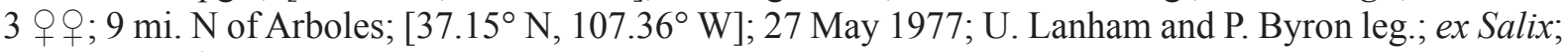

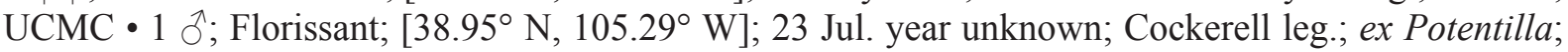
USNM - 1 \%; same location as for preceding; 25 Jul. year unknown; Cockerell leg.; ex Scrophularia; USNM. - Minnesota • 1 \%; $43.7509^{\circ} \mathrm{N}, 91.6541^{\circ} \mathrm{W}$; 20 Jun. 2014; S. Leddy leg.; ex Rudbeckia triloba; WRME. - Utah • 1 q; Uintah Co., Bonanza; [40.02 ${ }^{\circ}$ N, $109.18^{\circ} \mathrm{W}$ ]; Jul. 1977; G. Bohart leg.; BBSL. Wyoming • 1 ; ; Lincoln Co., Salt Creek; $42.4667^{\circ}$ N, $110.9678^{\circ}$ W; 2027 m a.s.1.; 14 Aug. 2014; J.B. Runyon leg.; MTEC.

\section{Description}

See Gibbs (2010) for a complete redescription and figures.

\section{Range}

Rocky Mountains from British Columbia southeast to New Mexico, east through the Great Plains to Illinois (Fig. 84).

\section{Floral records}

ANACARDIACEAE R.Br. Rhus L. $R$. trilobata Nutt. - APOCYNACEAE Juss. Asclepias L. A. speciosa Torr. (G11) - ASTERACEAE Giseke: Anaphalis (G11) - Nothocalais: N. cuspidata (Pursh) Greene (G11) - Rudbeckia: R. triloba L. - Solidago • Taraxacum (G11) - FABACEAE Juss.

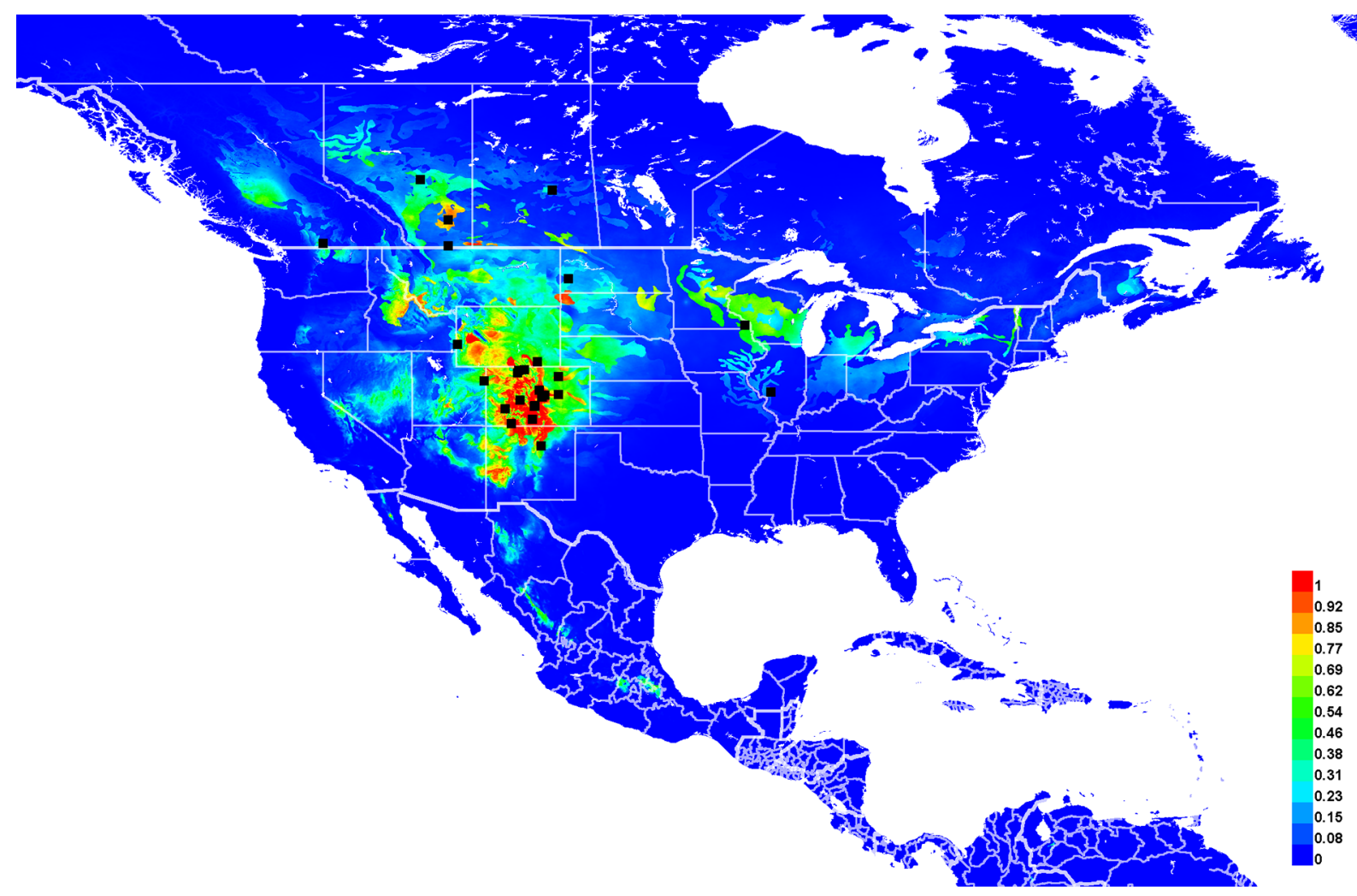

Fig. 84. Georeferenced collection records of Lasioglossum (D.) testaceum (Robertson, 1897) (black squares) and predicted distribution by maximum entropy ecological niche modeling in Maxent (colour shading). Warmer colours indicate higher cloglog probability of occurrence. 
Amorpha: A. canescens Pursh (G11) • GROSSULARIACEAE DC. Ribes L. R. valicola Greene (G11) • ONAGRACEAE Juss. Epilobium L. (G11) • POLYGONACEAE Juss. Eriogonum Michx. E. annuum Nutt. (G11) • ROSACEAE Juss. Dasiphora Raf. D. fruticosa (L.) Rydb. (G11) • Potentilla L. • Prunus L. (G11) • Spiraea L. (G11) • SALICACEAE Mirb. Salix L. S. brachycarpa Nutt. (G11) • S. eriocephala Michx. (G11) • SCROPHULARIACEAE Juss. Scrophularia L.

\section{DNA barcodes}

Not available. Lasioglosusm testaceum is morphologically similar to L. rufulipes, for which 3 sequences are available (BOLD process IDs: DLII1138-08, DLII1140-08, DLII1142-08). Barcodes of L. testaceum are expected to be similar to those of $L$. rufulipes, as these are very likely sister species. Phylogenetic analysis of COI barcodes and other genes of L. rufulipes suggest it is a distinct lineage relatively distantly related from the major clade of New World L. (Dialictus) (Gibbs et al. 2012b; Fig. 7).

Lasioglossum (Dialictus) torrens sp. nov. urn:1sid:zoobank.org:act:909EC02A-D47F-467F-96D2-387825DB1C89

Figs $85-86$

\section{Diagnosis}

Females of Lasioglossum torrens sp. nov. can be recognized by the head and mesosoma mostly covered in tessellate microsculpture, frons with crowded punctures $(\mathrm{i}=0 \mathrm{pd})$ contrasting with sparser paraocular area punctures $(\mathrm{i} \leq 1 \mathrm{pd})$, mesepisternum moderately sparsely punctate $(\mathrm{i}<2 \mathrm{pd})$, pronotal angle about 90 degrees, $\mathrm{T} 1$ anterior surface shiny with acarinarial fan sparse, ocelli normal, wings infuscated with dark veins and dark hairs, and most hair pale yellow to golden. Occasionally the scopa have black hairs intermixed. They are most similar to L. decorum sp. nov. and L. hudsoniellum. Females of L. decorum sp. nov. have $\mathrm{T} 1$ anterior surface coriarious with acarinarial fan dense, pronotal angle obtuse, and ocelli enlarged (separated by about $0.5 \mathrm{OD}$ ). Females of $L$. hudsoniellum have the head and mesosoma mostly shiny, frons with dense but distintly separated punctures ( $\mathrm{i}<1 \mathrm{pd}$ ), and mesepisternum densely punctate $(\mathrm{i}<1 \mathrm{pd})$.

\section{Etymology}

The specific epithet 'torrens' is a Latin present participle meaning 'scorching', 'roasting', or 'rushing', which is sometimes applied to lava. It is a reference to the yellow, red, and smoky colouration of this species, reminiscent of lava, and the type locality near the Colima volcano. An appropriate translation would be the scorching sweat bee.

\section{Material examined}

Holotype

MEXICO - Colima • o; Minatitlán; $19.43247^{\circ}$ N, $103.97724^{\circ}$ W; 11 Oct. 2010; S. Dumesh leg.; PCYU.

[Verbatim label: MEXICO Colima Minatitlán / N19.43247 W103.97724 / 1394m 11.x.10 S. Dumesh / PCYU-MEX10-0517 // BeeBOL / CCDB-09853 H08 / BOWMT377-10 // HOLOTYPE / Lasioglossum (Dialictus) torrens Gardner and Gibbs]

Paratypes

MEXICO - Colima • 1 q; Minatitlán; $19.42414^{\circ}$ N, $103.97286^{\circ}$ W; 11 Oct. 2010; S. Dumesh leg.; PCYU. - Morelos • 1 \%; Cuernavaca; [18.92 ${ }^{\circ}$ N, $99.22^{\circ}$ W]; Dec. 1944; N.L.H. Krauss leg.; USNM. -

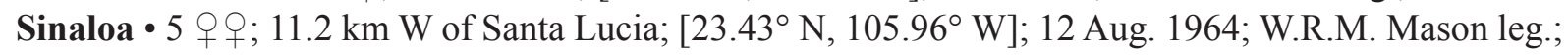
SEMC. 


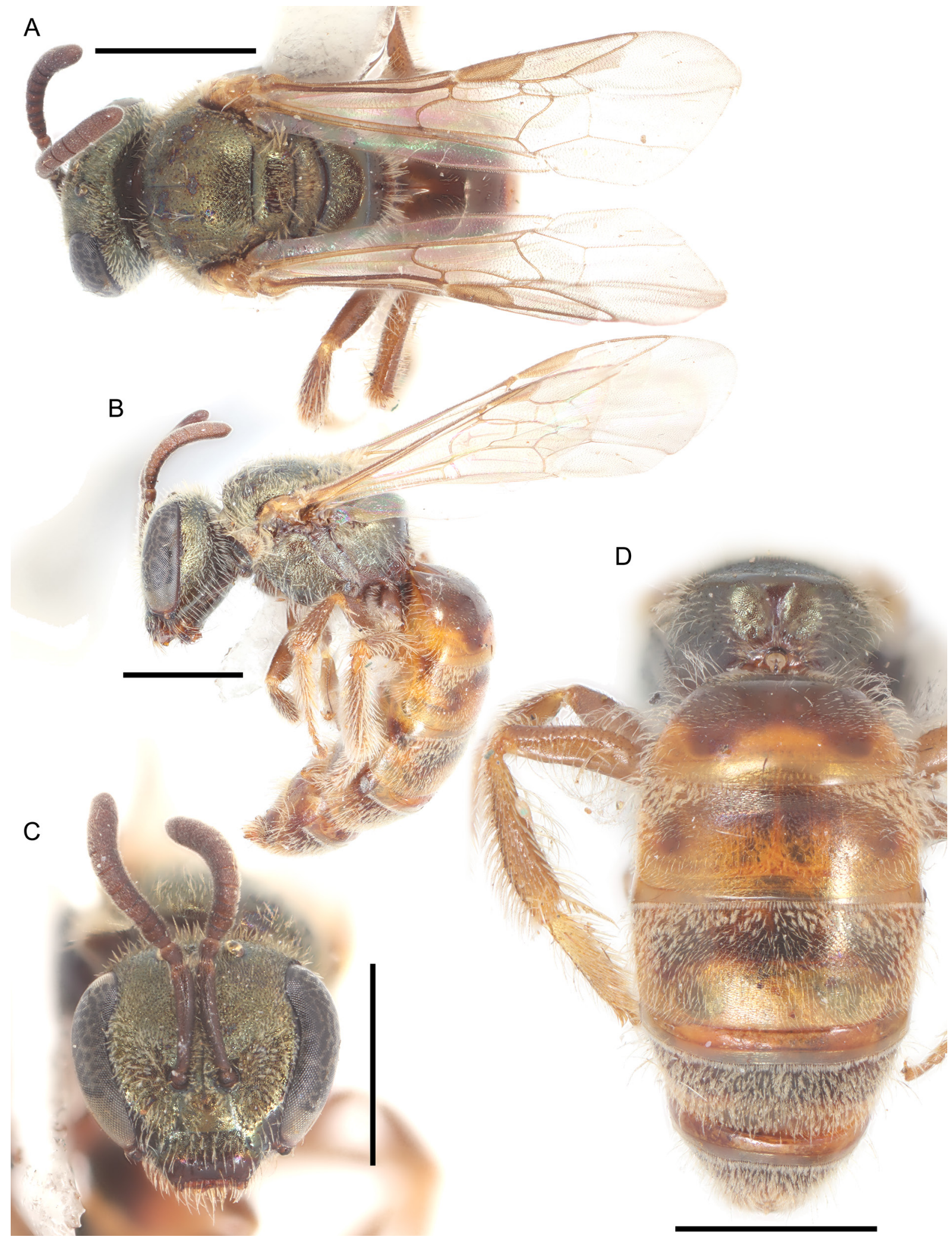

Fig. 85. Lasioglossum (D.) torrens sp. nov., †. A. Dorsal habitus. B. Lateral habitus. C. Face. D. Propodeum and metasoma. Scale bars: $1 \mathrm{~mm}$. 


\section{Description}

\section{Female}

Colouration. Head and mesosoma olive green to golden-green; clypeus apical colour black to reddish brown; labrum black to reddish brown; mandible orange with black basal band and red tip; flagellum black to reddish brown dorsally, reddish brown ventrally; pronotal lobe reddish brown to orange; metasoma orange to reddish brown with dark spiracular spots on T3-4 and sometimes T2; legs reddish brown with femur-tibia joints, base and apex of tibiae, and tarsi orange; tegula orange; wing membrane infuscated, veins brown to dark brown.

Pubescence. Body hair colour golden to pale yellow. Tomentum dense on gena, pronotal collar and lobe, space between pronotal lobe and tegula, metanotum medially, T2-3 basolaterally, and T4 throughout; sparse on face, metepisternum, and T5. Scutum hair thin to densely plumose. Wing hairs dark, long and dense. Acarinarial fan incomplete, very sparse. T2 fringes sparse, T3 fringes sparse.

SURFACE SCULPTURE. Clypeus punctures moderately sparse $(i=1-2 \mathrm{pd})$, sculpture imbricate or weakly so in basal half, shiny apically; supraclypeal area punctures sparse (i=1-4 pd), becoming dense laterally ( $\leq 1 \mathrm{pd}$ ), sculpture imbricate, sometimes shiny medially; paraocular area punctures dense ( $i \leq 1 \mathrm{pd}$ ), sculpture imbricate; frons punctures crowded ( $\mathrm{i}=0 \mathrm{pd}$ ), sculpture shiny to imbricate; vertex punctures crowded laterally ( $\mathrm{i}=0 \mathrm{pd}$ ), sparse medially ( $\mathrm{i}=1-3 \mathrm{pd}$ ), sculpture shiny to imbricate; gena punctures moderately sparse $(i=1-3 \mathrm{pd})$, sculpture shiny, becoming imbricate and sometimes weakly ruguloso-lineate posteriorly and ventrally; postgena sculpture imbricate, becoming shiny anteriorly; tegula punctures absent; scutum punctures dense $(i \leq 1 \mathrm{pd})$, becoming slightly sparser submedially and anteromedially ( $\mathrm{i}=1-3 \mathrm{pd}$ ), sculpture tessellate, sometimes becoming shiny posteromedially; scutellum punctures dense marginally and on median line $(\mathrm{i}<1 \mathrm{pd})$, sometimes finer and sparser anteriorly $(i=1-2 \mathrm{pd})$, sparse submedially $(i=1-3 \mathrm{pd})$, diversopunctate, sculpture shiny, sometimes becoming tessellate marginally and on median line; metanotum sculpture imbricate; metapostnotum rugae strong, anastomosing, sometimes reaching margin, sculpture imbricate to tessellate; preëpisternum sculpture rugulose; hypoepimeron punctures dense $(i<1 \mathrm{pd})$, sculpture imbricate; mesepisternum punctures dense to moderately sparse $(\mathrm{i}<2 \mathrm{pd})$, sculpture imbricate; metepisternum sculpture lineate dorsally, imbricate ventrally; propodeum lateral face sculpture tessellate; propodeum posterior face sculpture tessellate; $\mathrm{T} 1$ anterior face sculpture shiny; $\mathrm{T} 1$ dorsal surface punctures fine, sparse ( $\mathrm{i}=1-4 \mathrm{pd})$, absent in broad median line and large apicolateral oval patches, sculpture shiny; T2 disc punctures fine, moderately sparse ( $i=1-3 \mathrm{pd}$ ), becoming sparser medially ( $\mathrm{i}=1-4 \mathrm{pd})$, disc sculpture shiny, becoming coriarious basally, rim punctures fine, moderately sparse ( $\mathrm{i}=1-3 \mathrm{pd}$ ), becoming sparse $(\mathrm{i}=2-6 \mathrm{pd}$ ) or absent medially, rim sculpture shiny to weakly coriarious.

STRUCTURE. Face length/width ratio 0.83 ( $\pm 0.02 \mathrm{SD})$. Clypeus projecting $\sim 67 \%$ below suborbital tangent; clypeal area length/width ratio $0.41( \pm 0.02 \mathrm{SD})$; apicolateral denticles rounded knobs; supraclypeal area length/width ratio 0.72 ( $\pm 0.09 \mathrm{SD})$. Forewing with 3 submarginal cells; pronotal angle slightly obtuse, nearly right-angled; tegula shape normal. Intertegular distance 0.97 ( $\pm 0.02 \mathrm{SD}) \mathrm{mm}$. Scutum length/ width ratio $0.8( \pm 0.02 \mathrm{SD})$; scutum/scutellum length ratio 3.17 ( $\pm 0.13 \mathrm{SD})$; scutellum/metanotum length ratio 1.34 ( $\pm 0.07 \mathrm{SD})$; metanotum/metapostnotum length ratio $0.74( \pm 0.04 \mathrm{SD})$. Propodeum lateral carinae not reaching dorsal margin; oblique carina absent. T2 depressed apical rim length less than $50 \%$ of segment. $(n=8)$

\section{Male}

Unknown. 


\section{Range}

Sinaloa, Colima, and Morelos (Fig. 86).

\section{DNA barcodes}

Four sequences available (BOLD process IDs: BOWMT260-10, BOWMT377-10, BOWMT400-10, BOWMT401-10; BIN: BOLD:AAU5301). Lasioglossum torrens sp. nov. differs from all other western red-tailed L. (Dialictus) by 7 fixed substitutions: 120(A), 135(C), 177(C), 192(C), 231(C), 301(G), and 324(A) (Supplementary file 4).

\section{Remarks}

Rare. Only known from three localities in southern and central Mexico. Lasioglossum torrens sp. nov. is probably mostly Neotropical.

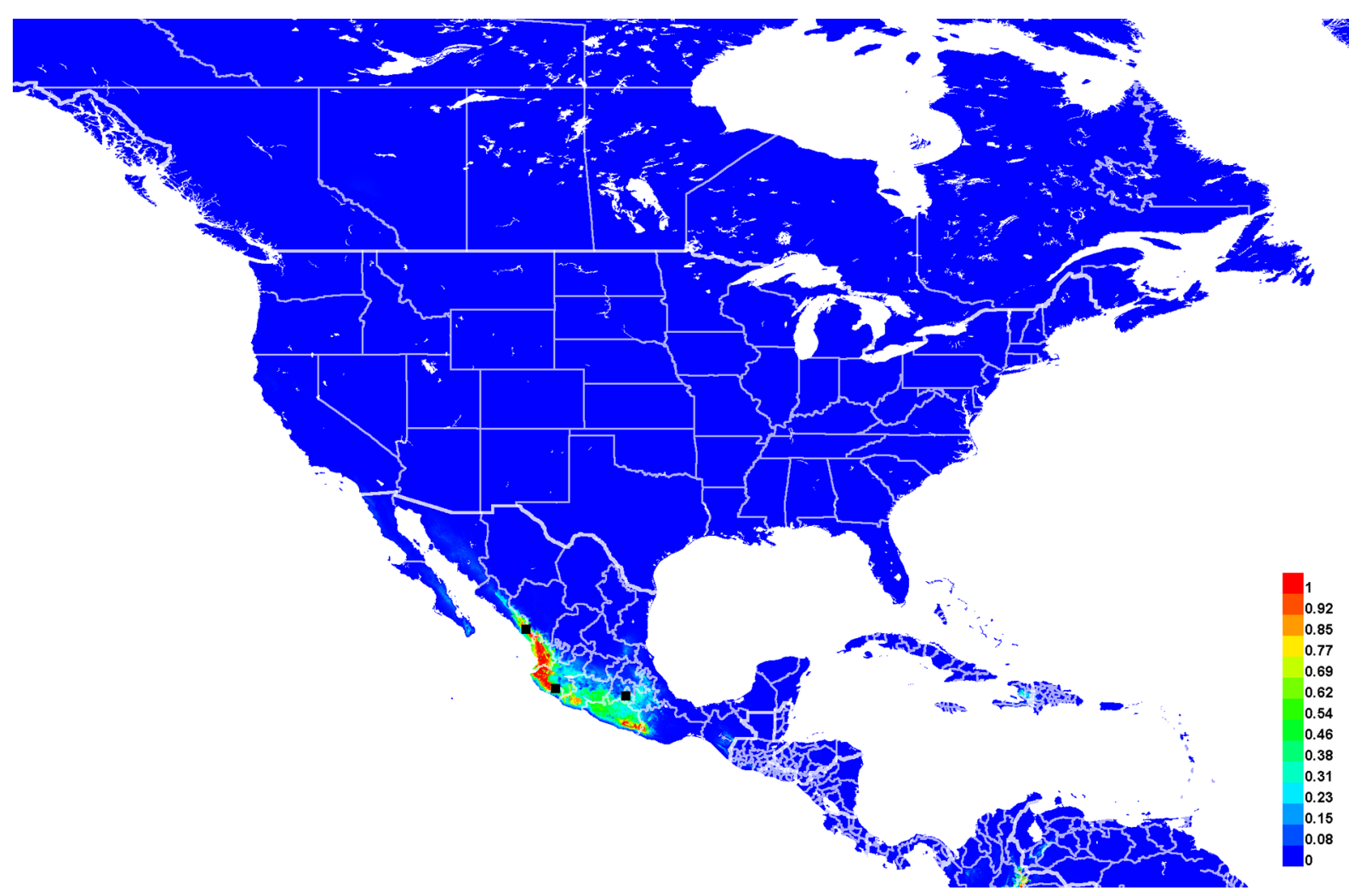

Fig. 86. Georeferenced collection records of Lasioglossum (D.) torrens sp. nov. (black squares) and predicted distribution by maximum entropy ecological niche modeling in Maxent (colour shading). Warmer colours indicate higher cloglog probability of occurrence. Due to the very small number of records, this distribution should be interpreted with caution. 


\section{Lasioglossum (Dialictus) tuolumnense Gibbs, 2009}

Fig. 87

Lasioglossum (Dialictus) tuolumnense Gibbs, 2009: 382, figs 10a-b, 11a-b, 12a-d (holotype, ㅇ, deposited in BBSL, examined).

Lasioglossum (Dialictus) tuolumnense - Heron \& Sheffield 2015 (distribution).

\section{Diagnosis}

Both sexes of Lasioglossum tuolumnense can be recognized by the characters for the Lasioglossum petrellum species complex (below), in addition to the characters given in the keys. Females are most similar to L. griswoldi, and probably males as well (the male of L. griswoldi is not described). Females of $L$. griswoldi have the malar space and supraclypeal area longer, and scutum slightly shinier and less densely punctate. In addition, the geographic ranges of these two species are not known to overlap: L. tuolumnense is only known from the mountains of California north to British Columbia, while L. griswoldi is only known from Utah and Colorado.

\section{Material examined}

\section{Holotype}

UNITED STATES - California • + ; Tuolumne Co., 2.2 mi. WNW of Mount Gibson; $38.01029^{\circ} \mathrm{N}$, $119.738^{\circ}$ W; $2036 \mathrm{~m}$ a.s.l.; 1 Aug. 2005; L. Fuerst and H. Briggs leg.; BBSL.

\section{Other material}

UNITED STATES - California • 1 क; Alpine Co., Woodfords; $\left[38.78^{\circ} \mathrm{N}, 119.82^{\circ} \mathrm{W}\right] ; 18$ Oct. 1962; W.R. Bauer leg.; LACM • 1 q; Amador Co., Bear River Reservoir; $\left[38.56^{\circ} \mathrm{N}, 120.22^{\circ} \mathrm{W}\right]$; 1 Aug. 1970; S.R. Sims leg.; UCDC • 1 क; El Dorado Co., Ice House Road (24 air mi. ENE of Placerville);

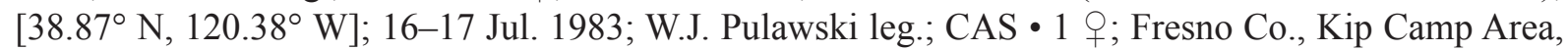

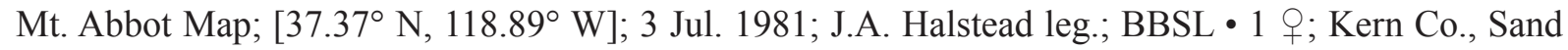

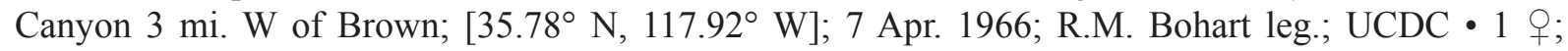
same location as for preceding; 7 Apr. 1966; R.O. Schuster leg.; UCDC • 1 \%; Mono Co., Convict Lake; [37.6 ${ }^{\circ} \mathrm{N}, 118.86^{\circ} \mathrm{W}$; $2530 \mathrm{~m}$ a.s.l.; 24 Jul. 1978; G.A. Levin leg.; ex Penstemon bridgesii; UCDC • 1 क; Mono Co., White Mountains, Crooked Creek Ranger Station; $37.4983^{\circ} \mathrm{N}, 118.17^{\circ} \mathrm{W}$; 3094 m a.s.l.; 4 Jul. 2004; F.D. Parker leg.; BBSL • 1 q; Tulare Co., Ash Mountain HQ; [36.49 N, $118.84^{\circ} \mathrm{W}$ ]; $518 \mathrm{~m}$ a.s.l.; 3 May 1979; J.A. Chemsak leg.; EMEC • 2 우; same location as for preceding; 3-4 May 1979; M.E. Buegler leg.; EMEC • 1 q; Tulare Co., Ash Mountain Kaweah Power Station \#3; [36.486 ${ }^{\circ}$ N, $118.837^{\circ}$ W]; 15 Aug. 1982; J.A. Halstead leg.; BBSL・ 1 क; Andreas Canyon; $\left[33.75^{\circ} \mathrm{N}, 116.6^{\circ} \mathrm{W}\right] ; 6$ Mar. 1933; H.L. McKenzie leg.; UCDC • 1 \%; Half Moon L., Tahoe; $\left[38.89^{\circ} \mathrm{N}\right.$, $120.13^{\circ} \mathrm{W}$; 23 Jul. 1915; E.P. Van Duzee leg.; USNM • 1 क; Julian; $33.1739^{\circ} \mathrm{N}, 116.6228^{\circ} \mathrm{W} ; 25$ Jun. 2016; K.J. Hung, Petterson, Matagual leg.; ex Calochortus weedii; WRME • 1 q; Mineralking; $\left[36.45^{\circ} \mathrm{N}, 118.595^{\circ} \mathrm{W}\right] ; 11 \mathrm{Jul}$. 1935; BBSL.

See also material in Gibbs (2009b).

\section{Description}

See Gibbs (2009b) for a complete description and figures.

\section{Range}

High elevations from southern California north to southern British Columbia (Fig. 87). 


\section{Floral records}

AMARYLliDACEAE J. St.-Hil. Allium L. A. yosemitense Eastw. (G09) - ASTERACEAE Giseke: Cirsium: C. occidentale (Nutt.) Jeps. C. o. var. californicum (A.Gray) D.J. Keil \& C.E. Turner (G09) • Eriophyllum: E. lanatum (Pursh) J. Forbes: E. L. var. integrifolium (Hook.) Smiley (G09) • Malacothrix (G09) - Senecio: S. scorzonella Greene (G09) • ERICACEAE Juss. Arctostaphylos Adans. A. viscida Parry (G09) • LILIACEAE Juss. Calochortus Pursh: C. weedii Alph. Wood - LIMNANTHACEAE R.Br. Limnanthes R.Br. L. montana Jeps. (G09) - PLANTAGINACEAE Juss. Keckiella Straw: K. breviflora (Lindl.) Straw (G09) • Penstemon Schmidel: P. bridgesii A.Gray • P. newberryi A.Gray (G09) • ROSACEAE Juss. Chamaebatia Benth. C. foliolosa Benth. (G09).

\section{DNA barcodes}

Seven sequences available (BOLD process IDs: DLII296-07, DLII302-07, DLII305-07, DLII922-07, DLII942-07, DLII944-07, DLII998-07; BIN: BOLD:AAD3535). Lasioglossum tuolumnense differs from L. droegei, L. griswoldi, and L. petrellum by 1 fixed substitution: 153(T) (Supplementary file 4). No unique fixed substitutions distinguish L. tuolumnense from all other western red-tailed L. (Dialictus).

\section{Remarks}

At least one specimen from the Cascade Mountains in Oregon has a completely black metasoma. This specimen was not available for physical study, but photographs taken by Joe Engler (FWSE) were examined and match L. tuolumnense except in colouration.

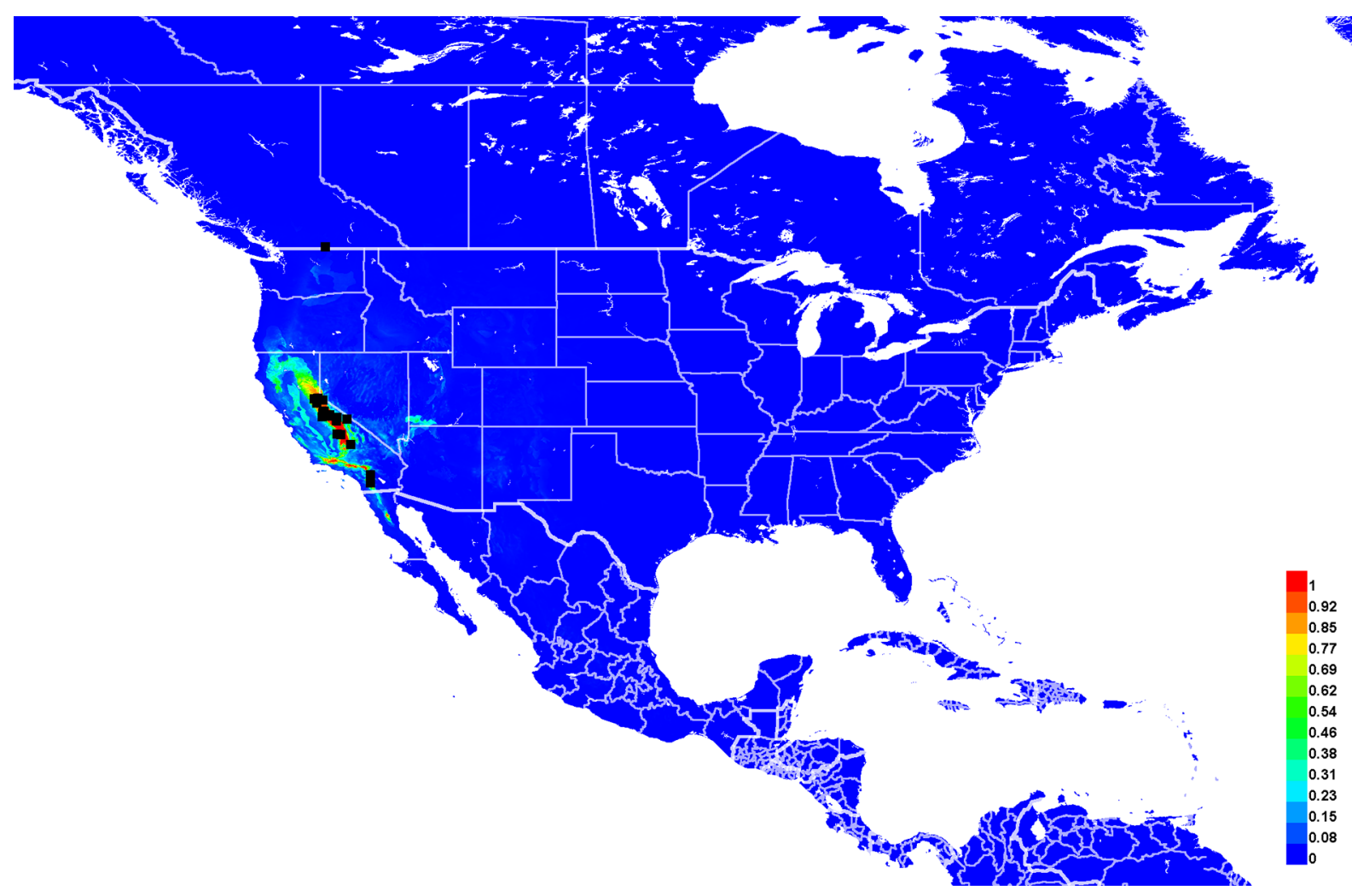

Fig. 87. Georeferenced collection records of Lasioglossum (D.) tuolumnense Gibbs, 2009 (black squares) and predicted distribution by maximum entropy ecological niche modeling in Maxent (colour shading). Warmer colours indicate higher cloglog probability of occurrence. Note that $L$. $(D$.) tuolumnense is also believed to occur in Oregon based on a photographed specimen. 


\section{Lasioglossum (Dialictus) vierecki (Crawford, 1904)}

Figs $88,90 \mathrm{P}, 92 \mathrm{~A}, 110 \mathrm{~A}, 111 \mathrm{~A}$

Halictus vierecki Crawford, 1904: 97 (holotype, 9 , deposited in ANSP, examined).

Halictus Vierecki-Graenicher 1910: 158 (description of male).

Halictus (Chloralictus) vierecki - Viereck et al. 1916: 707 (key).

Lasioglossum (Chloralictus) vierecki - Michener 1951: 1118 (catalog).

Dialictus vierecki-Mitchell 1960: 429, figs 101-102 (key, redescription). — Hurd 1979: 1973 (catalog).

— Moure \& Hurd 1987: 139 (catalog).

Lasioglossum (Dialictus) vierecki - Wolf \& Ascher 2009: 144 (checklist). — Gibbs 2010: 346, figs 233a-

d, 234a-e (key, redescription); 2011: 207 (key). — Gibbs et al. 2017: 87 (checklist).

\section{Diagnosis}

Females of Lasioglossum vierecki are readily identified by the head and mesosoma covered in very short, dense, thickly plumose, golden yellow hair and very dense punctures $(i<1 \mathrm{pd})$, face relatively long (length/width ratio $\sim 0.87$ ), metapostnotum dull and finely reticulate with weak rugae, metasomal terga with dense tomentum, and T2 without dark spiracular spots. Unworn specimens are not likely to be confused with any other species, but worn specimens can resemble L. arenisaltans sp. nov. Females of $L$. arenisaltans sp. nov. have the metapostnotum shiny with strong rugae, scutum with slightly sparser punctures (but still very dense), and T2 with dark spiracular spots.

Males of $L$. vierecki can be recognized by the scutum very densely punctate (all punctures touching with no distinct interspaces), metasomal terga uniformly densely punctate ( $\mathrm{i} \leq 1 \mathrm{pd})$, wings lightly infuscated, head and mesosoma golden-green with yellowish hair, and gonostylus short and broad with long hair. They are most similar to L. cactorum sp. nov. and L. spivakae sp. nov. Both of these species have the scutum more sparsely punctate $(\mathrm{i}=1-3 \mathrm{pd})$, wings hyaline, and head and mesosoma blue-green with white hair. In addition, males of L. cactorum sp. nov. have T1-4 rims strongly downcurved.

\section{Etymology}

Crawford (1904) dedicated this species to H.L. Viereck.

\section{Material examined}

\section{Holotype}

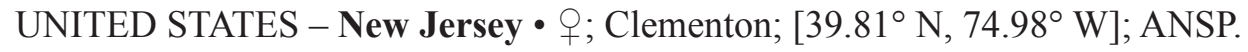

\section{Other material}

CANADA - Manitoba 1 1 ; Spirit Sands, SWPP (stabilized dune); $49.664^{\circ}$ N, $99.2951^{\circ} \mathrm{W} ; 30$ May 2019; T.M. Onuferko leg.; CMNC. - Quebec • 1 ते; CMN (Natural Heritage Campus), Gatineau; $45.4462^{\circ} \mathrm{N}, 75.8131^{\circ} \mathrm{W} ; 113$ m a.s.1.; 17 Jul. 2018; T.M. Onuferko leg.; CMNC.

UNITED STATES - Georgia • 1 q; Richmond Co., Augusta; [33.47 N, 82.01 W]; 6 Apr. 1959; R.R. Snelling leg.; LACM • $1 \mathrm{~J}^{\top}$; Richmond Co., Ft. Gordon; [33.42 $\left.\mathrm{N}, 82.14^{\circ} \mathrm{W}\right]$; 23 May 1958; R.R. Snelling leg.; LACM. - Kansas • 6 우; Reno Co., 5 mi. SE of Hutchinson; [38.01 $\left.{ }^{\circ} \mathrm{N}, 97.87^{\circ} \mathrm{W}\right] ; 2$ Sep. 1951; C.D. Michener and W.E. LaBerge leg.; ex Euphorbia; UNSM. - Minnesota • 1 o; Anoka Co., Bunker Hills Reg. Park; $45.2037^{\circ}$ N, 93.2792 ${ }^{\circ}$ W; 278 m a.s.1.; 8 Jun. 2015; J. Gardner leg.; UMSP • 1 क; same location as for preceding; 20 Jul. 2015; J. Gardner leg.; UMSP • 1 ; ; same location as for preceding; 10 Aug. 2015; J. Gardner leg.; UMSP • 1 क ; Anoka Co., Bunker Hills Reg. Park; 45.2059 $9^{\circ}$, $93.2777^{\circ}$ W; 277 m a.s.1.; 10 Aug. 2015; J. Gardner leg.; ex Liatris punctata; UMSP 4 우;

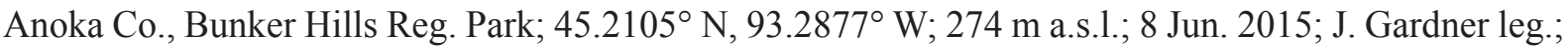


UMSP • 2 9 ; ; same location as for preceding; 1 Jul. 2015; J. Gardner leg.; UMSP • 1 영 same location as for preceding; 20 Jul. 2015; J. Gardner leg.; UMSP • 1 o; Anoka Co., Bunker Hills Reg. Park; 45.2145 ${ }^{\circ}$ N, 93.28 ${ }^{\circ}$ W; 278 m a.s.1.; 10 Aug. 2015; J. Gardner leg.; ex Dalea villosa; UMSP • 1 क; Anoka Co., Bunker Hills Reg. Park; 45.2159 N, 93.2848 ${ }^{\circ}$ W; 277 m a.s.1.; 20 Jul. 2015; J. Gardner leg.; ex Amorpha canescens; UMSP • 1 क; Anoka Co., Bunker Hills Reg. Park; 45.2177 N, 93.2903 W; 274 m a.s.l.; 20 Jul. 2015; J. Gardner leg.; ex Solidago speciosa; UMSP • 2 q ; ; Anoka Co., Bunker Hills Reg. Park; 45.2179 N, 93.2916 ${ }^{\circ}$ W; 8 Jun. 2015; J. Gardner leg.; ex Lithospermum canescens; UMSP • 1 क; same location as for preceding; 8 Jun. 2015; J. Gardner leg.; ex Berteroa incana; UMSP •

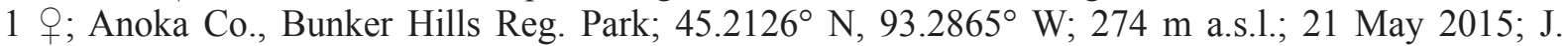
Gardner leg.; ex Barbarea vulgaris; UMSP • 1 q; Anoka Co., Bunker Hills Reg. Park; $45.21524^{\circ} \mathrm{N}$, 93.28744ํW; 276 m a.s.1.; 21 May 2015; J. Gardner leg.; ex Prunus virginiana; UMSP • 4 우; Anoka Co., Bunker Hills Reg. Park; 45.21632 N, 93.28838 W; 275 m a.s.1.; 21 May 2015; J. Gardner leg.; ex

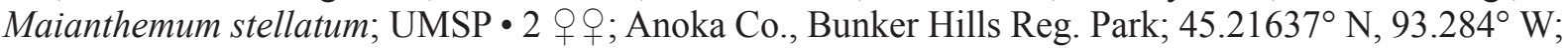
277 m a.s.l.; 21 May 2015; J. Gardner leg.; ex Arabis x divaricarpa; UMSP • 1 क; Anoka Co., Cedar Creek Ecosystem Science Reserve; 45.399 ${ }^{\circ}$ N, 93.1832 ${ }^{\circ}$ W; 280 m a.s.1.; 22 Jul. 2015; J. Gardner leg.; ex Solidago speciosa; UMSP • 2 + $\odot$; Anoka Co., Cedar Creek Ecosystem Science Reserve; $45.4029^{\circ} \mathrm{N}$, 93.1993 ${ }^{\circ}$ W; $278 \mathrm{~m}$ a.s.1.; 12 Jun. 2015; J. Gardner leg.; UMSP • 4 q $\odot$; same location as for preceding; 6 May 2016; J. Gardner leg.; UMSP • 1 क; Anoka Co., Cedar Creek Ecosystem Science Reserve; 45.40267 N, 93.19917 ${ }^{\circ}$ W; 255 m a.s.1.; 4 May 2015; E. Evans leg.; UMSP • 1 q; Anoka Co., Cedar Creek Ecosystem Science Reserve; 45.43096 ${ }^{\circ}$ N, 93.18936 W; 247 m a.s.1.; 4 May 2015; E. Evans leg.;

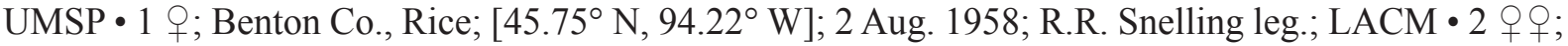
Houston Co., 1.5 mi. north Houston; [43.785 N, 91.56 ${ }^{\circ}$ W]; 18 May 1967; J.R. Powers leg.; EMEC •

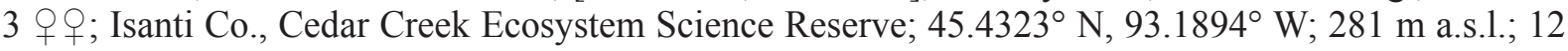
Jun. 2015; J. Gardner leg.; UMSP • 6 q ; ; same location as for preceding; 22 Jul. 2015; J. Gardner leg.; UMSP • 1 क; Isanti Co., Irving and John Anderson Co. Park; $45.4614^{\circ} \mathrm{N}, 93.0675^{\circ} \mathrm{W} ; 285 \mathrm{~m}$ a.s.1.; 21 May 2015; E. Evans leg.; UMSP • 1 क; Isanti Co., Irving and John Anderson Co. Park; $45.462^{\circ} \mathrm{N}$, 93.061 ${ }^{\circ} \mathrm{W} ; 261 \mathrm{~m}$ a.s.1.; $10 \mathrm{Jun} .2015$; E. Evans leg.; UMSP • 5 q $\odot$; same location as for preceding; 20 Jul. 2015; E. Evans leg.; UMSP • 1 q; Isanti Co., Irving and John Anderson Co. Park; $45.462^{\circ} \mathrm{N}$, 93.0537 ${ }^{\circ}$ W; 236 m a.s.l.; 10 Jun. 2015; E. Evans leg.; ex Rosa arkansana; UMSP • 1 \%; Isanti Co., Irving and John Anderson Co. Park; $45.4663^{\circ} \mathrm{N}, 93.0542^{\circ} \mathrm{W} ; 248 \mathrm{~m}$ a.s.l.; 10 Jun. 2015; E. Evans leg.; UMSP • 3 우우 Isanti Co., Irving and John Anderson Co. Park; $45.4614^{\circ} \mathrm{N}, 93.06754^{\circ} \mathrm{W} ; 285 \mathrm{~m}$ a.s.l.; 20 Jul. 2015; E. Evans leg.; UMSP • 4 우 ; Isanti Co., Irving and John Anderson Co. Park; $45.46627^{\circ} \mathrm{N}$, 93.05422 ${ }^{\circ}$ W; 248 m a.s.1.; 20 Jul. 2015; E. Evans leg.; UMSP・ 1 क; Ramsey Co., Bald Eagle Otter-Lks Reg. Park; $45.1005^{\circ}$ N, $93.0371^{\circ}$ W; 268 m a.s.1.; 5 Jun. 2015; E. Evans leg.; ex Trifolium repens; UMSP• 1 क; Sherburne Co., Uncas Dunes Scientific and Natural Area; $45.3951^{\circ} \mathrm{N}, 93.6658^{\circ} \mathrm{W} ; 258 \mathrm{~m}$ a.s.1.; 29 Apr. 2015; E. Evans leg.; UMSP • 1 क; Sherburne Co., Uncas Dunes Scientific and Natural Area;

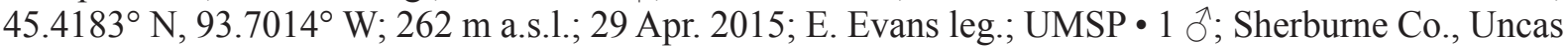
Dunes Scientific and Natural Area; $45.4208^{\circ}$ N, 93.7007 ${ }^{\circ}$ W; 264 m a.s.1.; 1 Sep. 2015; E. Evans leg.; ex Liatris punctata; UMSP • 1 \%; Sherburne Co., Uncas Dunes Scientific and Natural Area; $45.4219^{\circ}$ N, 93.6935 ${ }^{\circ}$ W; $271 \mathrm{~m}$ a.s.l.; 29 Jun. 2015; E. Evans leg.; UMSP • 4 우; Wabasha Co., Weaver Dunes; 44.27746 ${ }^{\circ}$ N, $91.93892^{\circ}$ W; 28 May 2015; M.J. Hatfield leg.; ex Ceanothus herbaceus; UMSP • 2 우; Wabasha Co., McCarthy Lake; $44.27413^{\circ}$ N, $91.94863^{\circ}$ W; 17 Jul. 2015; M.J. Hatfield leg.; UMSP • 1 \%; Washington Co., Belwin Conservancy; $44.924^{\circ}$ N, $92.79^{\circ}$ W; 247 m a.s.1.; 14 Aug. 2015; J. Gardner leg.; UMSP • 1 q; Washington Co., Belwin Conservancy; $44.9248^{\circ}$ N, $92.7911^{\circ} \mathrm{W} ; 260 \mathrm{~m}$ a.s.1.; 26 Jun. 2015; J. Gardner leg.; ex Rudbeckia hirta; UMSP • 2 o 9 ; Washington Co., Belwin Conservancy; $44.9248^{\circ}$ N, 92.7905 ${ }^{\circ} \mathrm{W} ; 221 \mathrm{~m}$ a.s.1.; 9 Jun. 2015; E. Evans leg.; ex Achillea millefolium; UMSP • 1 O; Washington Co., St. Croix Savanna Scientific and Natural Area; $44.9952^{\circ}$ N, $92.7784667^{\circ}$ W; 235 m a.s.1.; 21 Jul. 2015; E. Evans leg.; UMSP • 1 क; Washington Co., St. Croix Savanna Scientific and Natural Area; 44.9953 ${ }^{\circ}$ N, $92.7799^{\circ}$ W; 193 m a.s.1.; 4 Sep. 2015; E. Evans leg.; ex Solidago canadensis; UMSP • 1 ㅇ, 1 ơ; Washington Co., St. Croix Savanna Scientific and Natural Area; $44.9955^{\circ}$ N, $92.7788^{\circ}$ W; 192 m a.s.l.; 21 Jul. 2015; E. Evans leg.; ex Verbena stricta; UMSP • 1 क; Washington Co., St. Croix Savanna Scientific and Natural Area; $44.9957^{\circ}$ N, $92.7803^{\circ}$ W; 223 m a.s.1.; 30 Apr. 2015; J. Gardner leg.; UMSP • 6 우; Washington Co., St. Croix Savanna Scientific and Natural Area; $44.9971^{\circ}$ N, $92.7805^{\circ}$ W; 
262 m a.s.1.; 23 Jul. 2015; J. Gardner leg.; ex Dalea candida; UMSP. - Nebraska • 2 q ; McPherson Co., Sandhills Ag Lab; [41.62 ${ }^{\circ}$ N, 100.83 ${ }^{\circ}$ W]; 28 Jun.-10 Aug. 1972; UNSM • 1 $\odot$; same location as

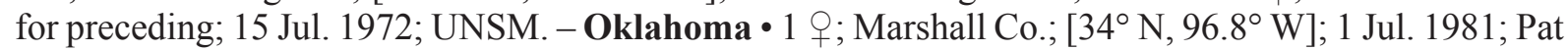
Terry leg.; ex Aphanostephus skirrhobasis; UNSM. - South Carolina • 1 क; Barnwell Co., Savannah River Site; 33.1607 ${ }^{\circ}$ N, $81.5072^{\circ}$ W; 23 May 2016; S. Breland leg.; WRME. - Texas • 1 ¿`; Bastrop Co., 6 mi. E of Bastrop; [30.11 ${ }^{\circ}$ N, 97.21 ${ }^{\circ} \mathrm{W}$ ]; 19 Jun. 1983; W.J. Pulawski leg.; CAS • 2 q ; ; same location as for preceding; 12-13 Jun. 1983; W.J. Pulawski leg.; CAS • 1 क; Bastrop Co., Camp Swift (Post Oak); $30.285^{\circ} \mathrm{N}, 97.287^{\circ} \mathrm{W} ; 20$ May 2004; J.L. Neff leg.; ex Ceanothus americanus; CTMI 3 q o+; Caldwell Co., 3-5 mi. S of Delhi; $29.772^{\circ} \mathrm{N}, 97.402^{\circ} \mathrm{W} ; 6$ Oct. 2008; A.W. Hook and J.L. Neff leg.; CTMI 1 1 ; Hemphill Co., 8 mi. NE of Canadian; [35.99 ${ }^{\circ}$ N $100.28^{\circ}$ W]; 23 Jun. 1970; L. and C.W. O'Brien leg.; LACM • 1 đ; Smith Co.; [32.3 N, 95.3 W]; 16 May 1948; A.R. Barr leg.; UMSP.

\section{Description}

See Gibbs (2010) for a complete redescription and figures.

\section{Range}

Sand dunes across southern Canada and the United States, east of about $100^{\circ}$ longitude (Fig. 88).

\section{Floral records}

ASPARAGACEAE Juss. Maianthemum F.H. Wigg. M. stellatum (L.) Link - ASTERACEAE Giseke: Achillea: A. millefolium L. Aphanostephus: A. skirrhobasis (DC.) Trel. ex Coville \& Branner $\bullet$ Aster (G10) • Euthamia: E. graminifolia (L.) Nutt. (G10) • Helianthus (G10) • Liatris: L. punctata Hook. •

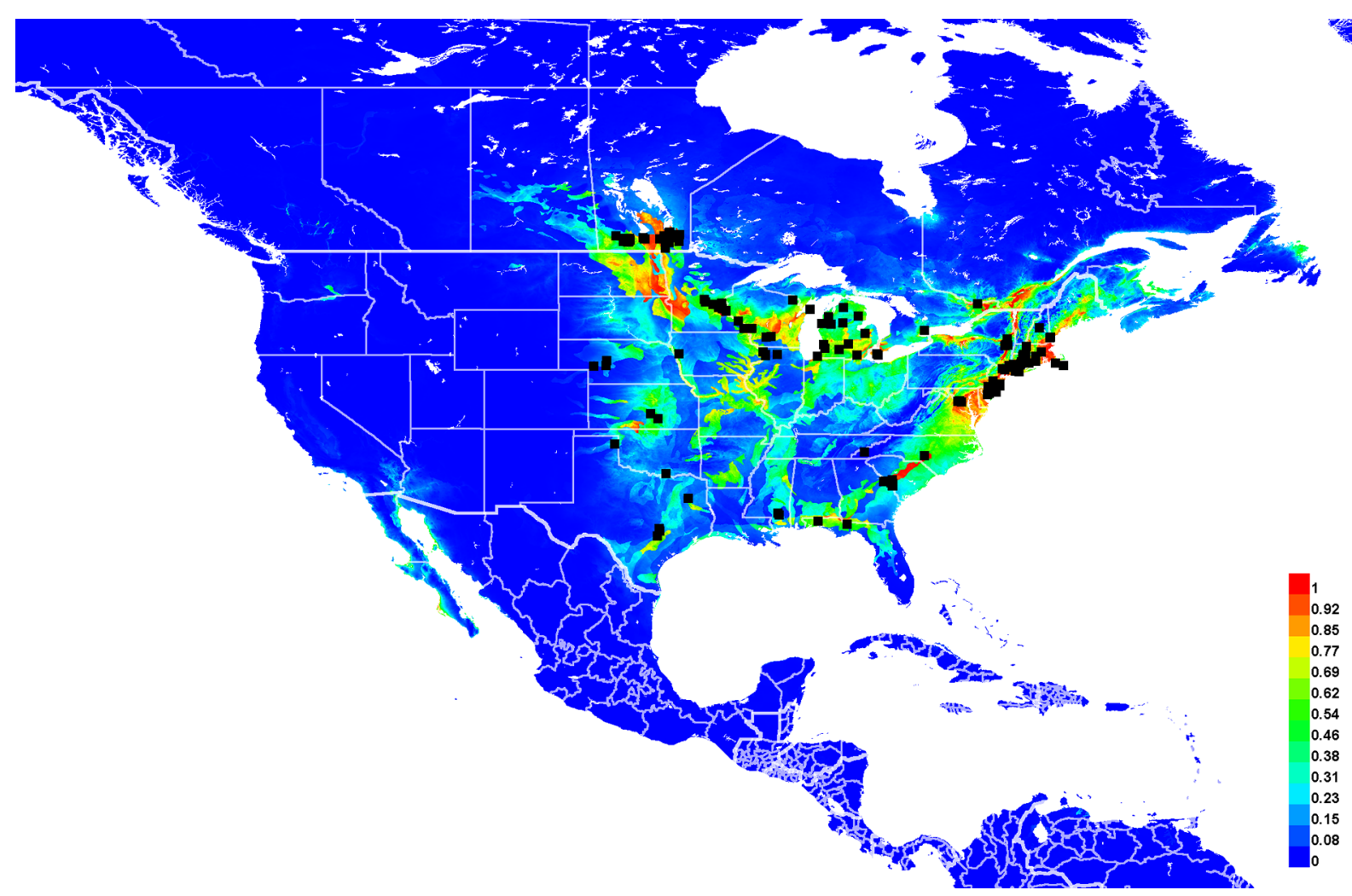

Fig. 88. Georeferenced collection records of Lasioglossum (D.) vierecki (Crawford, 1904) (black squares) and predicted distribution by maximum entropy ecological niche modeling in Maxent (colour shading). Warmer colours indicate higher cloglog probability of occurrence. 
Rudbeckia: R. hirta L. - Solidago: S. canadensis L. - S. speciosa A.Gray • BORAGINACEAE Juss. Lithospermum L. L. canescens (Michx.) Lehm. BRASSICACEAE Burnett: Arabis L. A. x divaricarpa A. Nelson (pro sp.) - Barbarea W.T. Aiton: B. vulgaris R.Br. Berteroa DC. B. incana (L.) DC. • Lepidium L. (G10) • CAMPANULACEAE Juss. Specularia Heist. ex A. DC. (G10) • CISTACEAE Juss. Crocanthemum Spach. C. canadense (L.) Britton (G10) • EUPHORBIACEAE Juss. Euphorbia L. - FABACEAE Juss. Amorpha: A. canescens Pursh - Dalea: D. candida Willd. - D. villosa (Nutt.) Spreng. - Melilotus (G10) - Trifolium: T. repens L. - HYDRANGEACEAE Dumort. Hydrangea L. (G10) - LAMIACEAE Martinov: Monarda L. M. punctata L. (G10) - RHAMNACEAE Juss. Ceanothus L. C. americanus L. $\bullet$. herbaceus Raf. - ROSACEAE Juss. Prunus L. P. virginiana L. • Rosa L. R. arkansana Porter $\bullet$ Rubus L. R. flagellaris Willd. (G10) • VERBENACEAE J. St.-Hil. Verbena L. V. stricta Vent.

\section{DNA barcodes}

Thirteen sequences available (BOLD process IDs: BWTWO1088-10, DLII314-07, DLII471-07, DIAL033-06, DIAL034-06, DIAL035-06, DIAL121-06, DIAL358-06, DIAL370-06, DIAL479-06, DIAL480-06, DIAL484-06, DIAL915-06; BIN: BOLD:AAB4651). No unique fixed substitutions distinguish $L$. vierecki from all other western red-tailed L. (Dialictus).

\section{Remarks}

Lasioglossum vierecki is a primarily eastern species, but it occurs sympatrically with several western species at the western edge of its range. It is believed to be a bivoltine solitary species (Knerer 1969; Packer 1993) and a sand habitat specialist.

\section{Lasioglossum (Dialictus) zephyrus (Smith, 1853)}

Figs 89, 90Q, 116A

Halictus Zephyrus Smith, 1853: 68 (holotype, ô, deposited in NHMUK).

Halictus (Chloralictus) academicus Sandhouse, 1924: 12 (holotype, , , deposited in USNM, examined; synonymy by Gibbs 2010).

Halictus (Chloralictus) vintonensis Sandhouse, 1924: 22 (holotype, + , deposited in USNM, examined; synonymy with academicus by Michener 1951, with zephyrus by Gibbs 2010).

Halictus zephyrus - Robertson 1895: 117 (female description). — Cockerell 1905: 352 (redescription). Chloralictus zephyrus - Robertson 1902: 248 (key).

Halictus (Chloralictus) zephyrus - Viereck et al. 1916: 706 (key). — Sandhouse 1924: 6 (key).

Lasioglossum (Chloralictus) academicum - Michener 1951: 1111 (catalog).

Lasioglossum (Chloralictus) zephyrus - Michener 1951: 1118 (catalog).

Dialictus zephyrus - Mitchell 1960: 431, figs 101-102 (key, redescription). - Hurd 1979: 1973 (catalog). — Moure \& Hurd 1987: 140 (catalog).

Lasioglossum (Dialictus) zephyrum (incorrect subsequent spelling) - Batra 1964 (biology); 1965 (associates); 1966 (biology). — Krombein 1967: 467 (catalog). — Michener et al. 1971a (biology); 1971b (biology). - Michener 1974 (biology); 1990 (biology). — Wolf \& Ascher 2009: 144 (checklist). — Gibbs 2010: 362, figs 242a-e, 243a-f (key, redescription); 2011: 211 (key). — Scott et al. 2011: 32 (checklist). — Dibble et al. 2017: 14 (checklist). — Gibbs et al. 2017: 88 (checklist).

Dialictus academicus - Hurd 1979: 1963 (catalog). — Moure \& Hurd 1987: 87 (catalog).

Evylaeus zephyrus - Pesenko et al. 2000: 42 (review).

Lasioglossum zephyrum - Donovall \& van Engeldorp 2010: 18 (checklist). 


\section{Diagnosis}

Females of Lasioglossum zephyrus can be recognized by the metapostnotum smooth with rugae reaching about halfway to the posterior margin, acarinarial fan dense and often complete, mesosoma with fine and often sparse punctures ( $\mathrm{i}=1-2 \mathrm{pd}$ ), metasoma mostly dark and often with metallic green terga, head often large with gena much broader than eye and ocelloccipital distance equal to or greater than interocellar distance, and pronotal ridge absent. They are most similar to L. kunzei and L. semibrunneum. Both of these species have the pronotal ridge carinate, acarinarial fan sparse, mesosoma very sparsely punctate $(i=1-6 \mathrm{pd})$, and gena sometimes with a ventral tubercle.

Males of L. zephyrus can be recognized by the propodeum dorsolateral slope rugose and lateral face smooth and tessellate, mesosoma with fine and often sparse punctures $(i=1-2 \mathrm{pd})$, paraocular area with dense tomentum, T2-5 with at least some tomentum, flagellomeres relatively long (F2 about 2 times as long as F1 and 1.75 times as long as broad), and pronotal ridge absent. They are most similar to L. testaceum, L. pictum, L. arenisaltans sp. nov., L. kunzei, and L. semibrunneum. Males of L.testaceum can be separated by the characters given in the key. Males of $L$. pictum and L. arenisaltans sp. nov. have the propodeum lateral face rugulose and mesosoma with larger and denser punctures ( $\mathrm{i}=1-2 \mathrm{pd}$ ). Males of L. kunzei and L. semibrunneum have the pronotal ridge carinate, flagellomeres relatively short (F2 about 1.3 times as long as F1 and 1.25 times as long as broad), and propodeum dorsolateral slope smooth and shiny.

\section{Etymology}

It is evident from his capitalization that Smith (1853) named this species after Zephyrus, the Greek god of the west wind. As a noun, it is not declinable to zephyrum, in contrast with common past usage (Batra 1964, 1965, 1966; Michener et al. 1971a, 1971b; Michener 1974, 1990; Wolf \& Ascher 2009; Scott et al. 2011; Gibbs 2010, 2011; Dibble et al. 2017; Gibbs et al. 2017). It has almost universally been spelled zephyrum in combination with Lasioglossum with the exception of Michener (1951).

\section{Material examined}

\section{Holotype}

UNITED STATES - Florida • ${ }^{\text {’ }}$; St. John's Bluff; $\left[30.38^{\circ} \mathrm{N}, 81.5^{\circ} \mathrm{W}\right]$; E. Doubleday leg.; NHMUK.

\section{Other material}

CANADA - Manitoba 1 क ; Carberry Sandhills $24 \mathrm{~km} \mathrm{~S}$ of Carberry; $49.4^{\circ}$ N, $99.16^{\circ} \mathrm{W} ; 340 \mathrm{~m}$ a.s.1.; 7 Aug. 2011; R.E. Wrigley leg.; WRME.

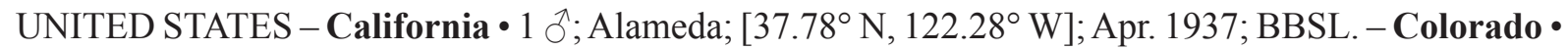
6 우; Douglas Co., 3 mi. SSW of Nighthawk; $39.332^{\circ}$ N, 105.1804 ${ }^{\circ} \mathrm{W} ; 1951$ m a.s.1.; 13 Jul. 2000; A.L. Hicks and Virginia Scott leg.; UCMC • 1 \%; Mesa Co., Colorado National Monument near Visitors Center; [39.101 ${ }^{\circ} \mathrm{N}, 108.735^{\circ} \mathrm{W}$ ]; $1737 \mathrm{~m}$ a.s.l.; 10 May 1988; U. Lanham and M. Weissman leg.; ex

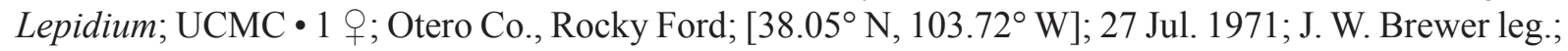
ex Citrullus lanatus; MTEC • 1 \%; Boulder; $\left[40.02^{\circ} \mathrm{N}, 105.27^{\circ} \mathrm{W}\right] ; 14$ May; Irene Bleasdale leg.;

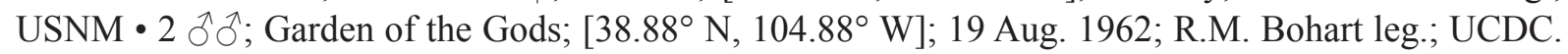
- Idaho • $1 \mathrm{O}^{\top}$; Ada Co., Boise; [43.62 ${ }^{\circ} \mathrm{N}, 116.2^{\circ} \mathrm{W}$ ]; 16 Aug. 1969; Carol Musgrave leg.; NMSU •

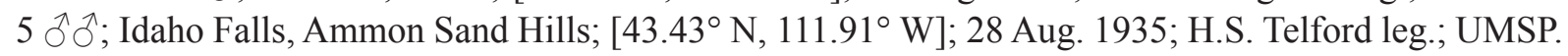

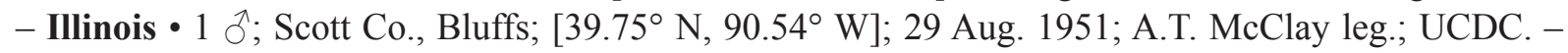
Iowa 1 1 ; Vinton; $\left[42.16^{\circ} \mathrm{N}, 92.02^{\circ} \mathrm{W}\right.$ ]; 23 Jun. 1922; Sandhouse leg.; USNM. - Kansas 1 \%; Decatur Co., 1 mi. E of Oberlin State Lake; [39.83 N, 100.46 W]; 27 Jun. 1963; N. Marston leg.; AMNH 11 \% ; Douglas Co., Lawrence; [38.97 N, 95.24 W]; 23 Sep. 1952; R.R. Snelling leg.; LACM •

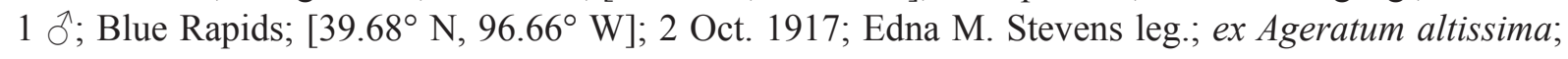


AMNH. - Michigan • 1 q; Allegan Co., Trevor Nichol Res. Ctr.; 42.5993 N, 86.1575 W; 12-23 May 2016; J. Gibbs leg.; WRME• 1 +; Allegan Co., Trevor Nichol Res. Ctr.; $42.6^{\circ}$ N, 86.155 W; 24-28 May 2015; J. Gibbs and Y. Nozoe leg.; WRME • 1 \%; same location as for preceding; 28 May-5 Jun. 2015; J. Gibbs and Y. Nozoe leg.; WRME 11 ; ; same location as for preceding; 26 Aug.-17 Sep. 2016; Gibbs

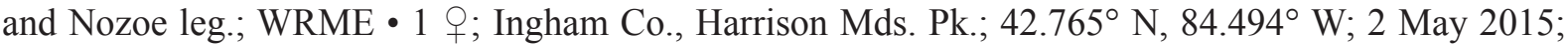

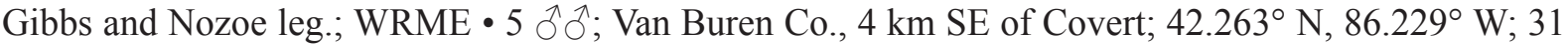
Jul. 2015; J. Gibbs leg.; WRME. - Minnesota • 1 क; Ramsey Co., Battle Creek Reg. Park; $44.9346^{\circ}$ N, 93.0141 ${ }^{\circ}$ W; 289 m a.s.1.; 10 Jun. 2015; J. Gardner leg.; ex Cornus sericea; UMSP • 1 q; Ramsey

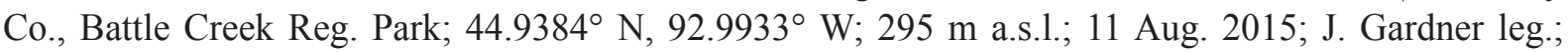

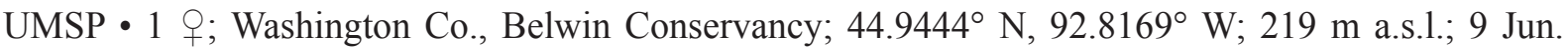
2015; E. Evans leg.; ex Zizea aurea; UMSP. - Missouri • 1 क; Boone Co., $12 \mathrm{mi}$. SW of Columbia; [38.83 N, 92.49 ${ }^{\circ}$ W]; 24 Apr. 1966; F.D. Parker leg.; UCDC. - Montana • 1 q; Cascade Co., Cascade; [47.27 $\left.\mathrm{N}, 111.7^{\circ} \mathrm{W}\right] ; 24$ Jun. 1954; R.C. Froeschner leg.; MTEC • 1 \%; Gallatin Co., Gallatin Grown; $45.7713^{\circ}$ N, $111.2953^{\circ}$ W; 30 Aug. 2013; C.M. Delphia leg.; ex Cucurbita; MTEC • 1 क ; same location as for preceding; 22 Aug. 2013; C.M. Delphia leg.; ex Helianthus; MTEC • 1 oे; same location as for preceding; 11 Sep. 2013; C.M. Delphia leg.; ex Cucurbita; MTEC • 1 O; same location as for preceding; 11 Sep. 2013; C.M. Delphia leg.; MTEC • 1 क; Gallatin Co.; 21 May 1953; Student leg.; MTEC. Nebraska $1 \delta^{\top} ; 1$ mi. E of Ravenna; $\left[41.03^{\circ} \mathrm{N}, 98.89^{\circ} \mathrm{W}\right]$; 24 Aug. 1958; Atyeo and Andresen leg.;

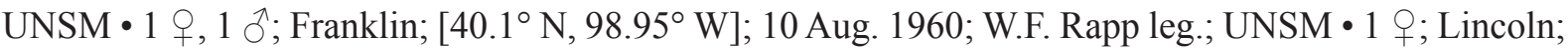
[40.81 $\left.{ }^{\circ} \mathrm{N}, 96.7^{\circ} \mathrm{W}\right] ; 31$ Jul. 1948; R.A. Larkin leg.; ex Medicago sativa; UNSM • 1 ; ; same location as for preceding; 3 Jul. 1948; R.A. Larkin leg.; ex Medicago sativa; UNSM • 1 क; McCool; [40.74 N,

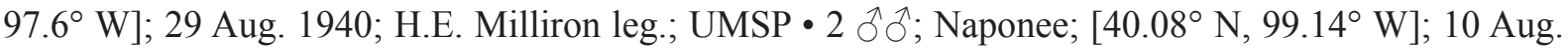

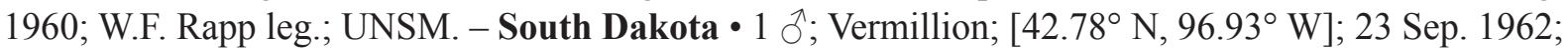

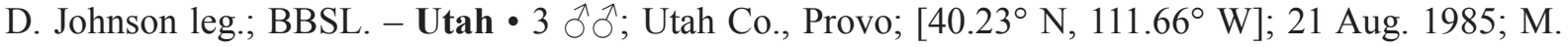
Khider leg.; BBSL 1 त; same location as for preceding; 4-11 Sep. 1985; BBSL 1 ô; Farmington;

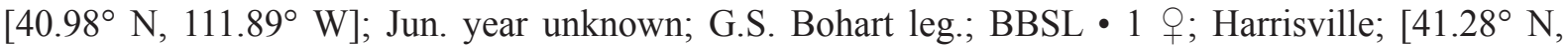
$111.99^{\circ} \mathrm{W}$; 6 May 1935; G.F. Knowlton leg.; BBSL • 1 क; Mantua; $41.515308^{\circ}$ N, $111.938108^{\circ} \mathrm{W}$;

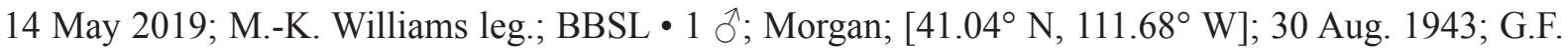
Knowlton and P.E. Telford leg.; BBSL • 1 क; Paradise; $41.550306^{\circ} \mathrm{N}, 111.829569^{\circ} \mathrm{W} ; 12$ May 2019; M-K. Williams leg.; BBSL • 1 q; Paradise; [41.550306 $\left.{ }^{\circ} \mathrm{N}, 111.829569^{\circ} \mathrm{W}\right] ; 11$ May 2019; MKFW leg.; BBSL • 1 q; Providence; $41.694842^{\circ}$ N, $111.826561^{\circ}$ W; 14 May 2019; A. Anderson leg.; BBSL • 1 \%; Salt Lake; [ $41^{\circ} \mathrm{N}, 113^{\circ} \mathrm{W}$ ]; 19 Jul. 1951; L. Dale Haws leg.; BBSL. - Washington • 1 q; Clark Co., Camas, 1641 NE Crown Lane; $45.591^{\circ} \mathrm{N}, 122.389^{\circ} \mathrm{W} ; 62 \mathrm{~m}$ a.s.1.; 17 May 2012; T.J. Zavortink leg.;

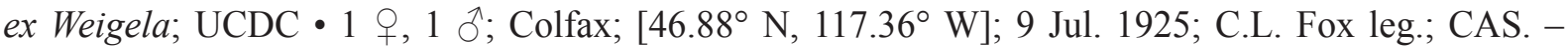

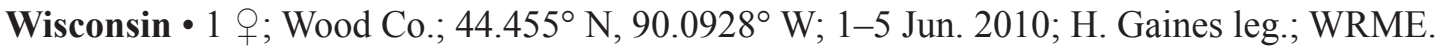

\section{Description}

See Gibbs (2010) for a complete redescription, figures, additional floral records, and reference to biological literature.

\section{Range}

Throughout most of the United States and southern Canada, southwest to northern California and Texas (Fig. 89).

\section{Floral records}

APIACEAE Lindl. Zizia W.D.J. Koch: Z. aurea Koch • ASTERACEAE Giseke: Ageratum: A altissima (L.) R.M. King \& H. Rob. • Helianthus $\bullet$ BRASSICACEAE Burnett: Lepidium L. $\bullet$ CAPRIFOLIACEAE Juss. Weigela Thunb. $\bullet$ CORNACEAE Bercht. ex J. Presl. Cornus L. C. sericea L. • CUCURBITACEAE 
Juss. Citrullus Schrad. C. lanatus (Thunb.) Matsum. \& Nakai - Cucurbita L. - FABACEAE Juss. Medicago: M. sativa L.

\section{DNA barcodes}

Sixteen sequences available (BOLD process IDs: ASBEE076-08, BOWGF1435-10, DLII675-07, DLII688-07，DLII702-07，DLII1512-08，DLII1519-08，DIAL070-06，DIAL071-06，DIAL606-06, DIAL725-06, DIAL952-06, DIAL1253-08, DIAL1254-08, DIAL1255-08，LASNA058-08; BIN: BOLD:AAD0272). No unique fixed substitutions distinguish L. zephyrus from all other western redtailed L. (Dialictus).

\section{Remarks}

Unlike other red-tailed L. (Dialictus), it is only the male of L. zephyrus that often has a red metasoma (Batra 1966).

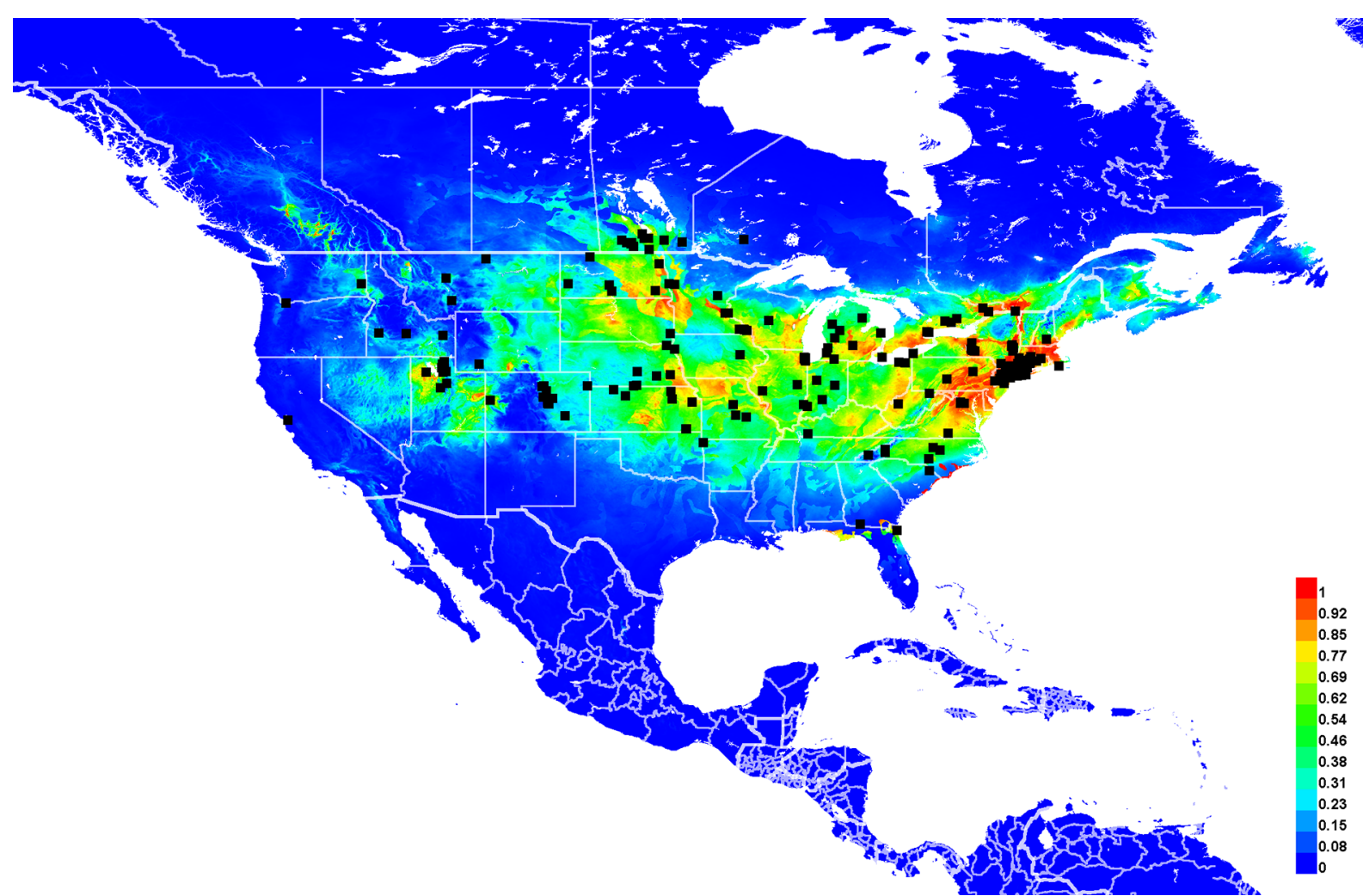

Fig. 89. Georeferenced collection records of Lasioglossum (D.) zephyrus (Smith, 1853) (black squares) and predicted distribution by maximum entropy ecological niche modeling in Maxent (colour shading). Warmer colours indicate higher cloglog probability of occurrence. 
A

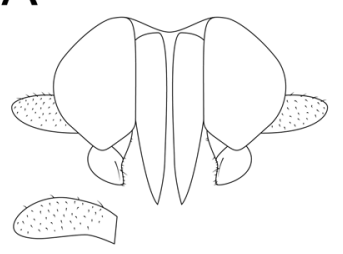

E

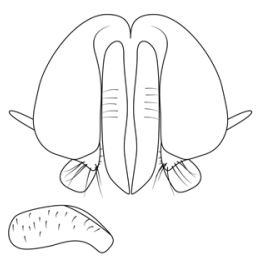

I

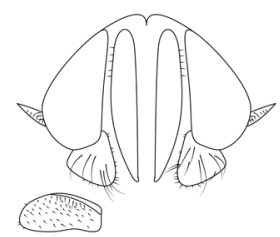

M
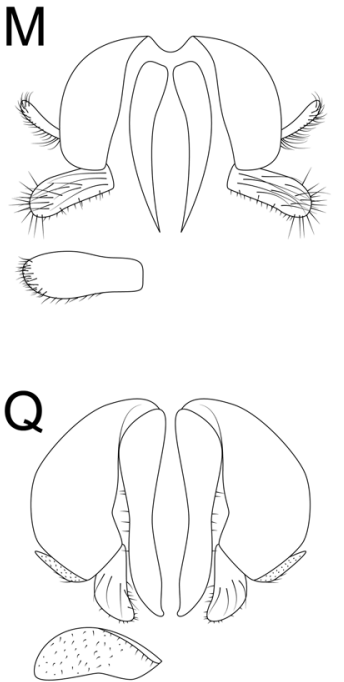

B

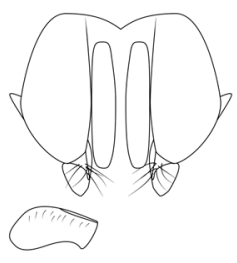

F

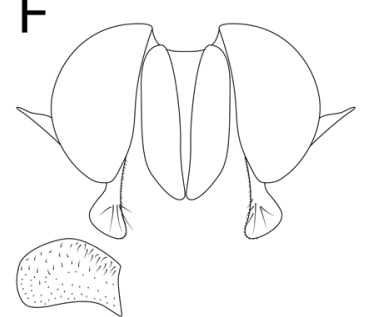

J

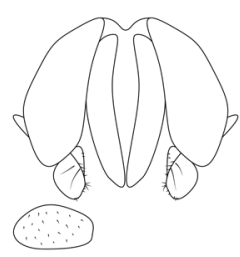

$\mathrm{N}$

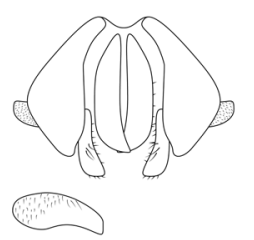

C

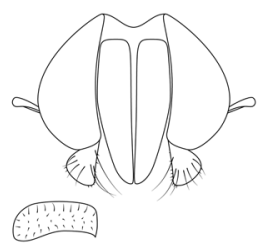

G

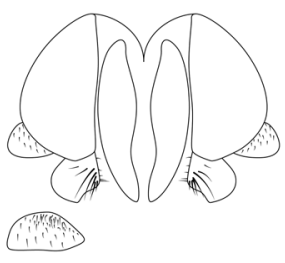

K

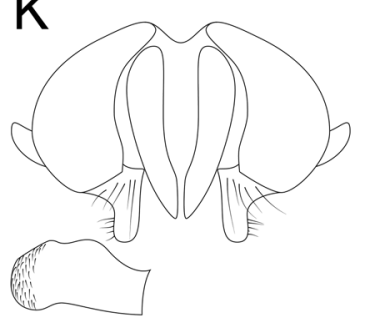

0

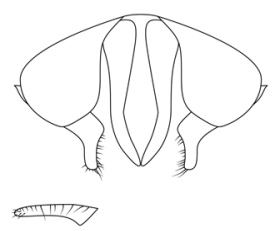

D

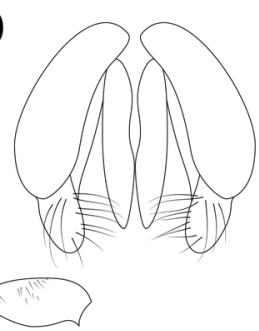

$\mathrm{H}$

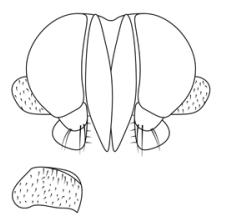

L

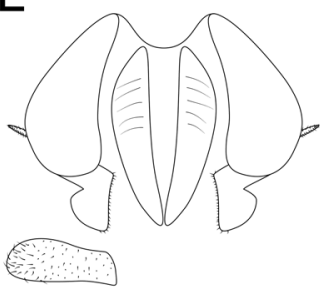

P

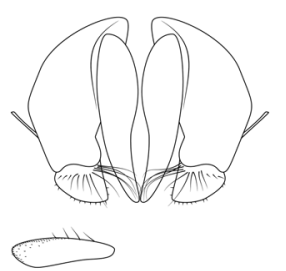

Fig. 90. Male genitalia. A. Lasioglossum (D.) arenisaltans sp. nov. B. L. (D.) clastipedion sp. nov. C. L. (D.) clematisellum (Cockerell, 1904). D. L. (D.) hudsoniellum (Cockerell, 1919). E. L. (D.) julipile sp. nov. F. L. (D.) lilianae sp. nov. G. L. (D.) mesillense (Cockerell, 1898). H. L. (D.) meteorum sp. nov. I. L. (D.) miltolepoides sp. nov. J. L. (D.) minckleyi sp. nov. K. L. (D.) pallidellum (Ellis, 1914). L. L. (D.) pictum (Crawford, 1902). M. L. (D.) rufornatum sp. nov. N. L. (D.) spivakae sp. nov. O. L. (D.) testaceum (Robertson, 1897). P. L. (D.) vierecki (Crawford, 1904). Q. L. (D.) zephyrus (Smith, 1853). Genitalia are illustrated in dorsal view and positioned such that the gonostylus is parallel to the plane of vision. Retrorse lobes are also illustrated separately to the bottom left of each figure in lateral view, positioned parallel to the plane of vision. 


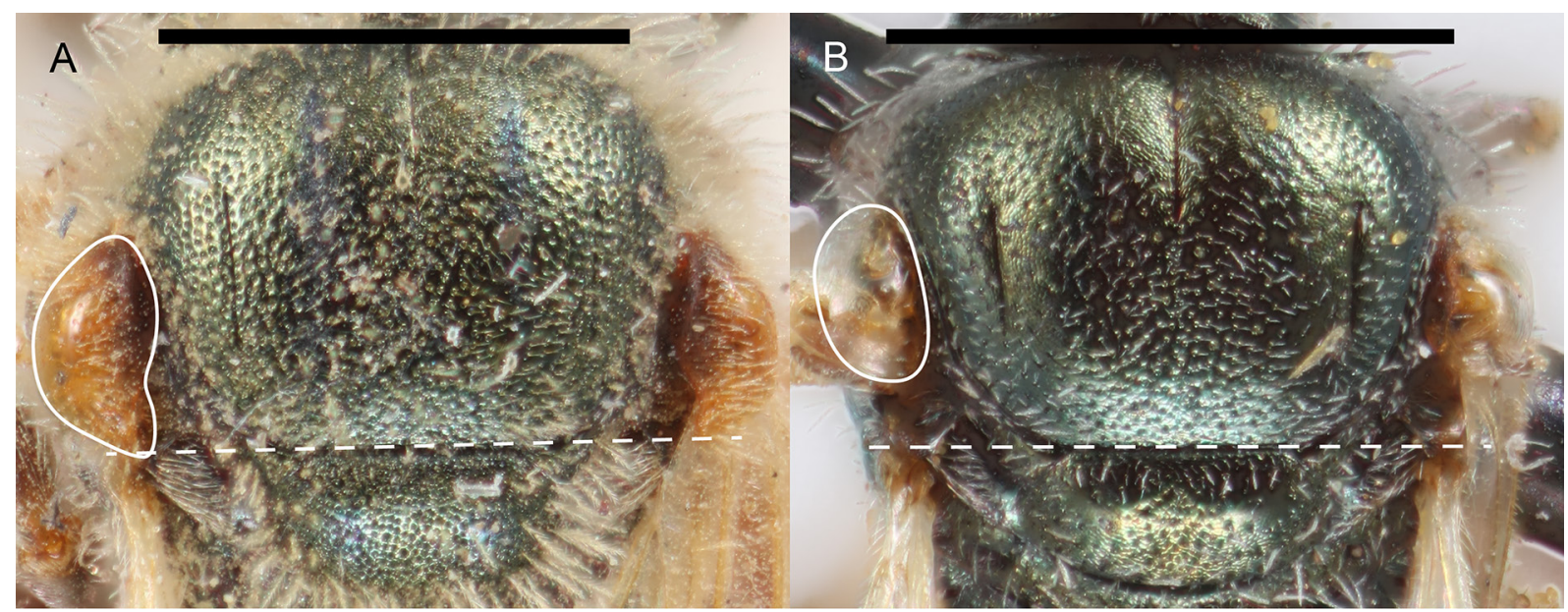

Fig. 91. Tegula. A. Lasioglossum (D.) hunteri (Crawford, 1932),, , enlarged, reaching scutellum, with concave inner posterior margin. B. L. (D.) clematisellum (Cockerell, 1904), $q$, ovoid and not reaching scutellum. Scale bars: $1 \mathrm{~mm}$.

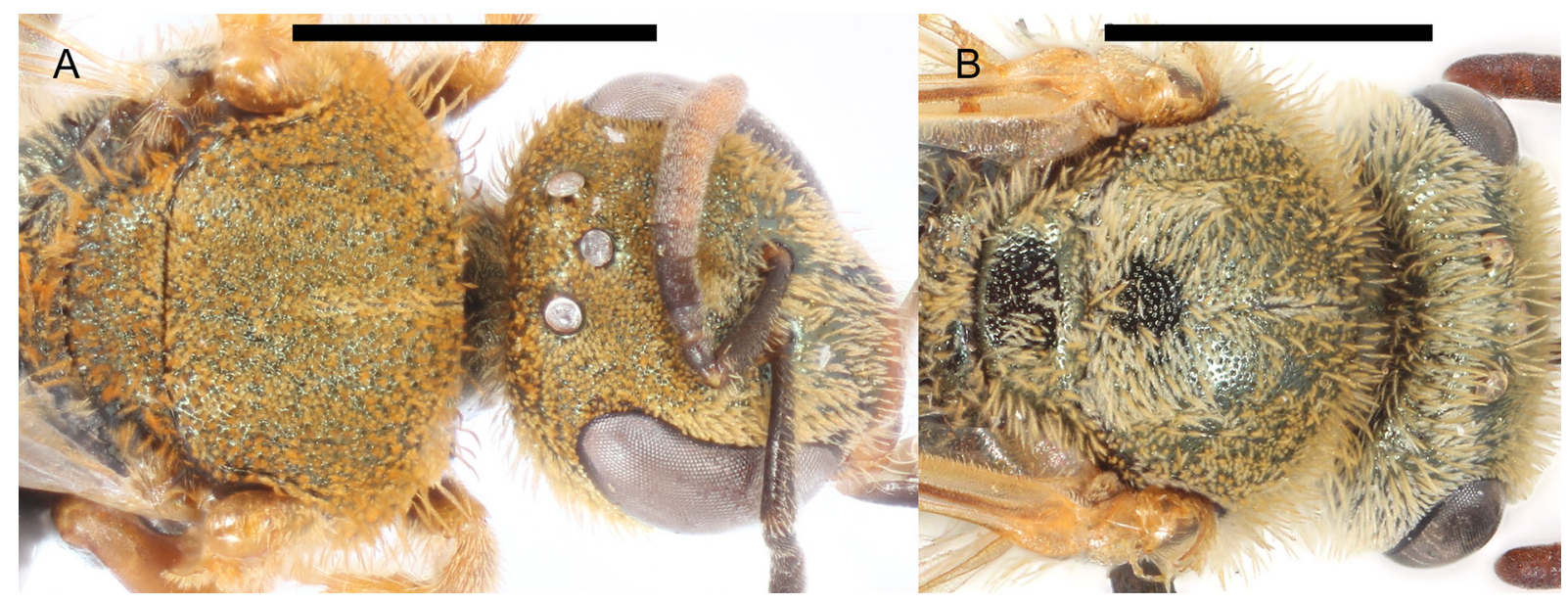

Fig. 92. Head and mesonotum. A. Lasioglossum (D.) vierecki (Crawford, 1904), $q$, with thickly plumose hair short and scale-like. B. L. (D.) cembrilacus sp. nov., o, with thickly plumose hair long. Scale bars: $1 \mathrm{~mm}$. 


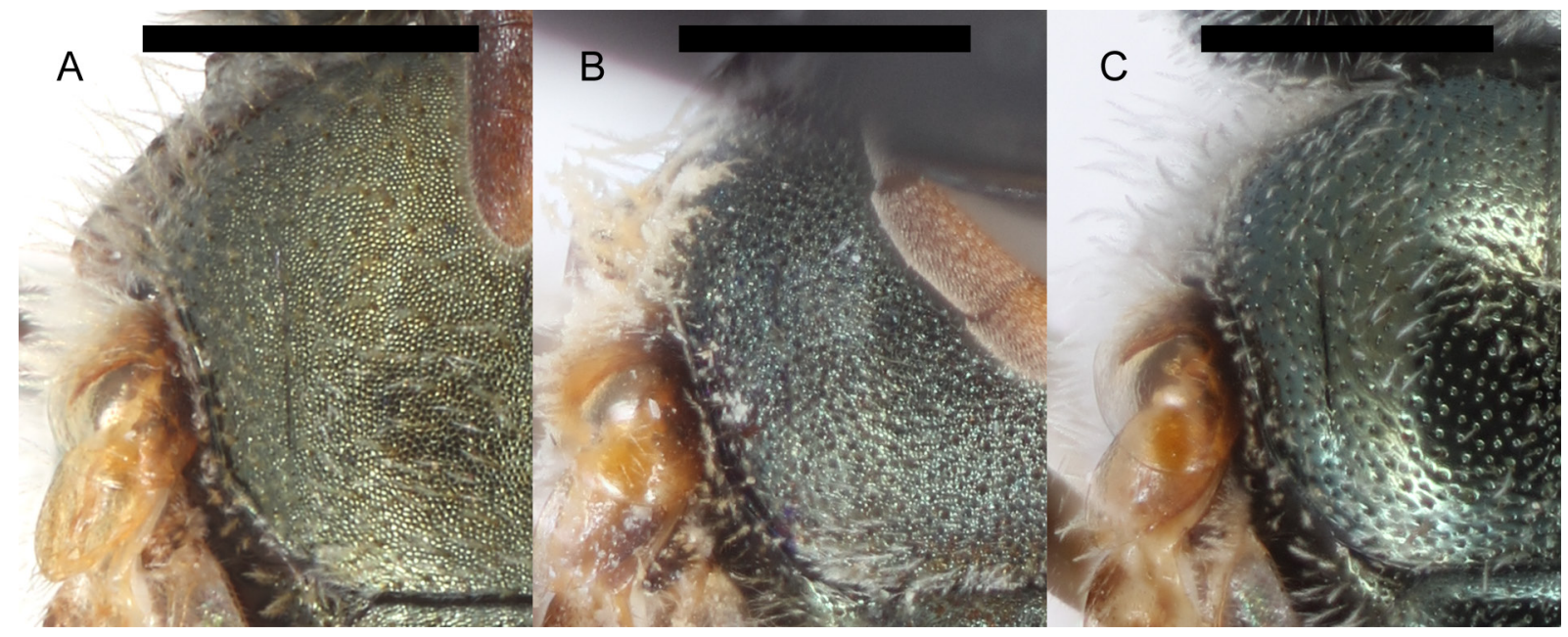

Fig. 93. Mesoscutum. A. Lasioglossum (D.) tessellatosum sp. nov., $q$, with strong microsculpture and sparse punctures. B. L. (D.) droegei Gibbs, 2009, §, with strong microsculpture and dense punctures. C. $L$. (D.) cactorum sp. nov.,, , shiny and sparsely punctate. Scale bars: $0.5 \mathrm{~mm}$.
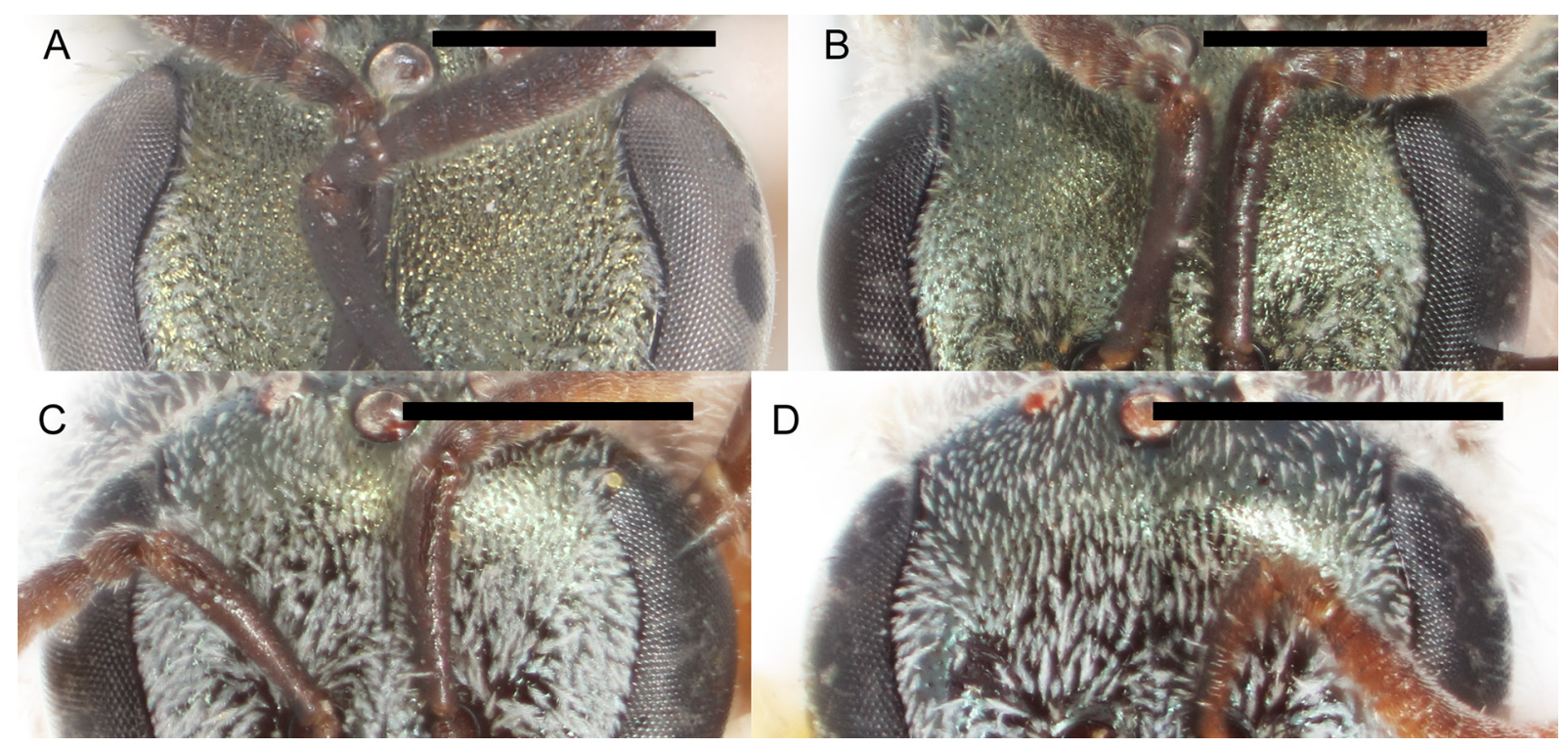

Fig. 94. Variation in frons punctation. A. Lasioglossum (D.) tessellatosum sp. nov., + , reticulatepunctate to ruguloso-punctate with crowded punctures. B. L. (D.) meteorum sp. nov., $q$, fine and dense but distinctly separated punctures. C. $L$. (D.) julipile sp. nov., + , moderately sparsely punctate. D. $L$. (D.) perditum sp. nov.,, , very sparsely punctate. Scale bars: $0.5 \mathrm{~mm}$. 


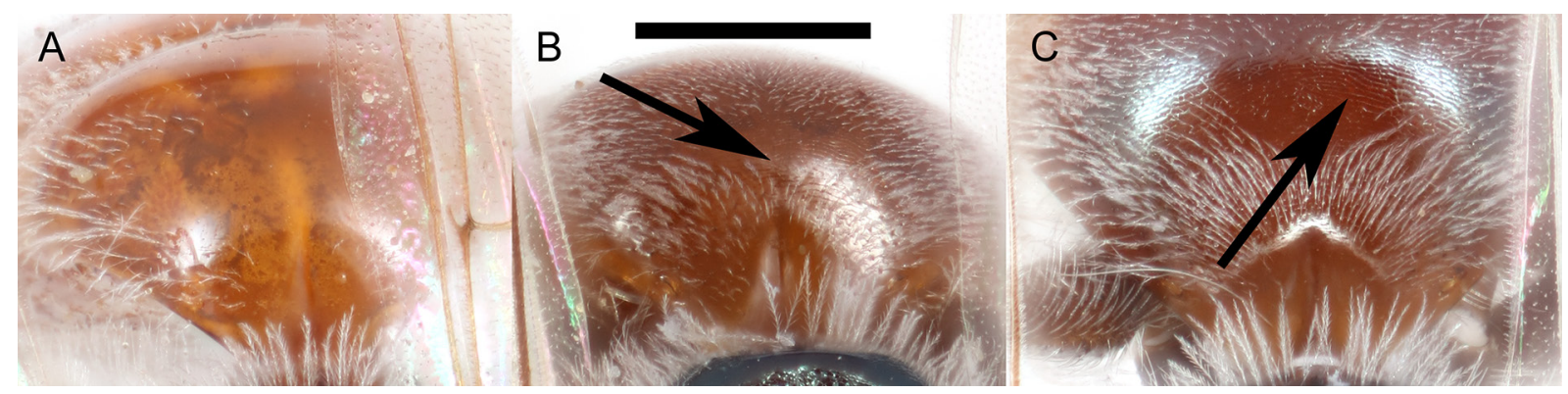

Fig. 95. T1 anterior face. A. Lasioglossum (D.) kunzei (Cockerell, 1898), $q$, shiny with weak and worn acarinarial fan. B. L. (D.) argammon sp. nov., ㅇ, weakly coriarious with sparse acarinarial fan. C. L. (D.) rufornatum sp. nov.,, , strongly coriarious with dense and well-developed acarinarial fan. Arrows point to areas where microsculpture is most easily visible. Scale bar is $0.5 \mathrm{~mm}$.
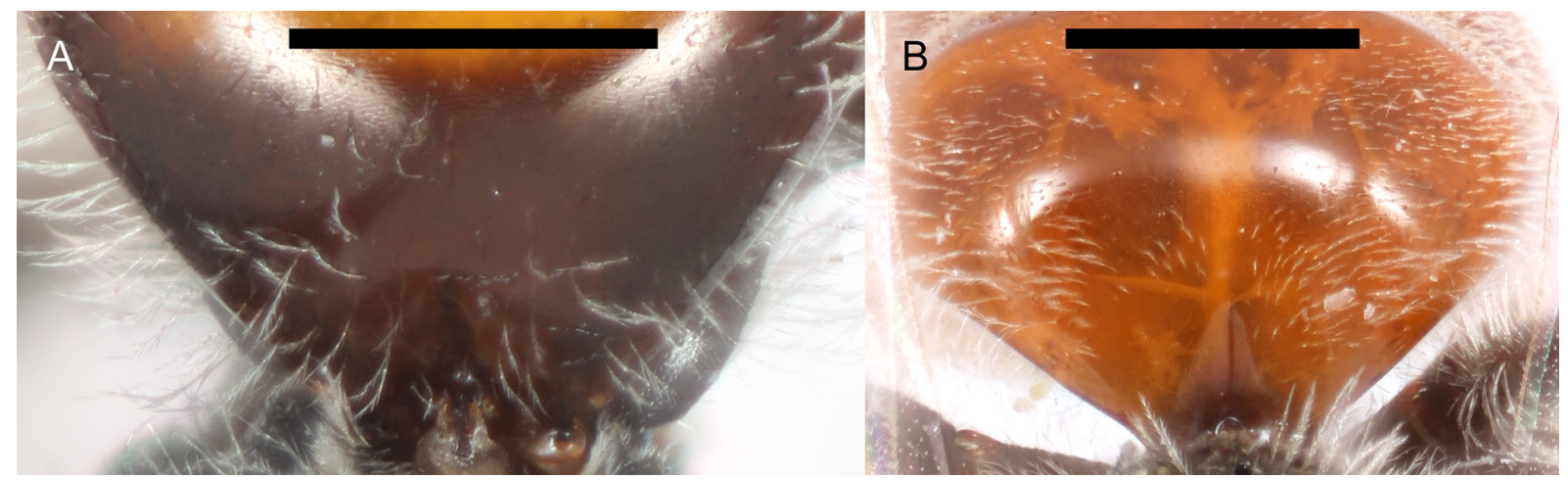

Fig. 96. T1 anterior face. A. Lasioglossum (D.) testaceum (Robertson, 1897), $q$, acarinarial fan absent with erect hairs throughout. B. L. (D.) clastipedion sp. nov.,, , acarinarial fan present but weak, with erect hairs restricted to lateral margins. Scale bars: $0.5 \mathrm{~mm}$. 

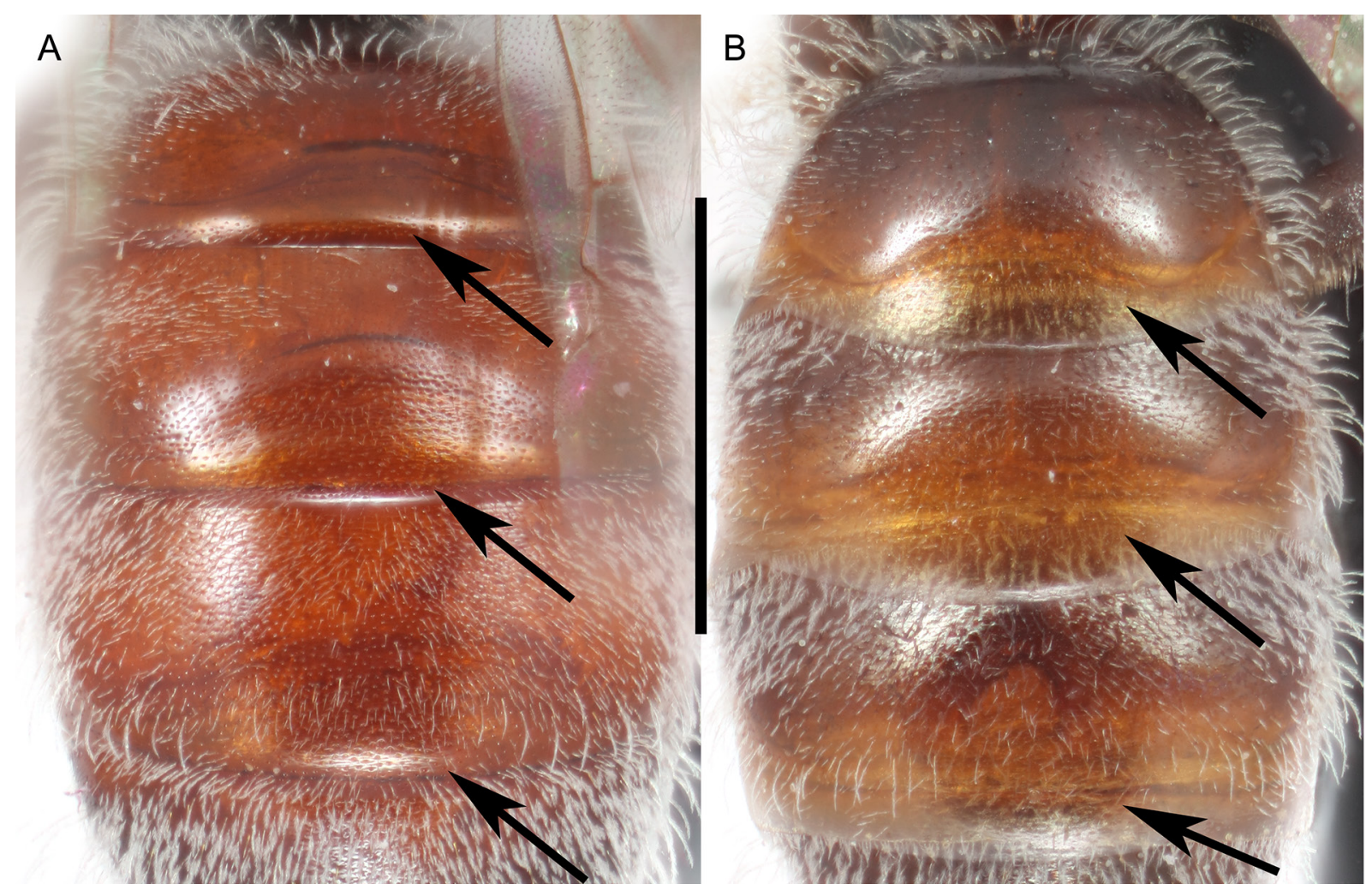

Fig. 97. T1-3. A. Lasioglossum (D.) cactorum sp. nov., $q$, with very narrow and abruptly downcurved apical rims and dense apical hair fringes. B. L. (D.) miltolepoides sp. nov.,, , with broad, flat, slightly depressed apical rims and sparse apical hair fringes. Arrows point to approximate lengthwise center of apical rims. Scale bar: $1 \mathrm{~mm}$.
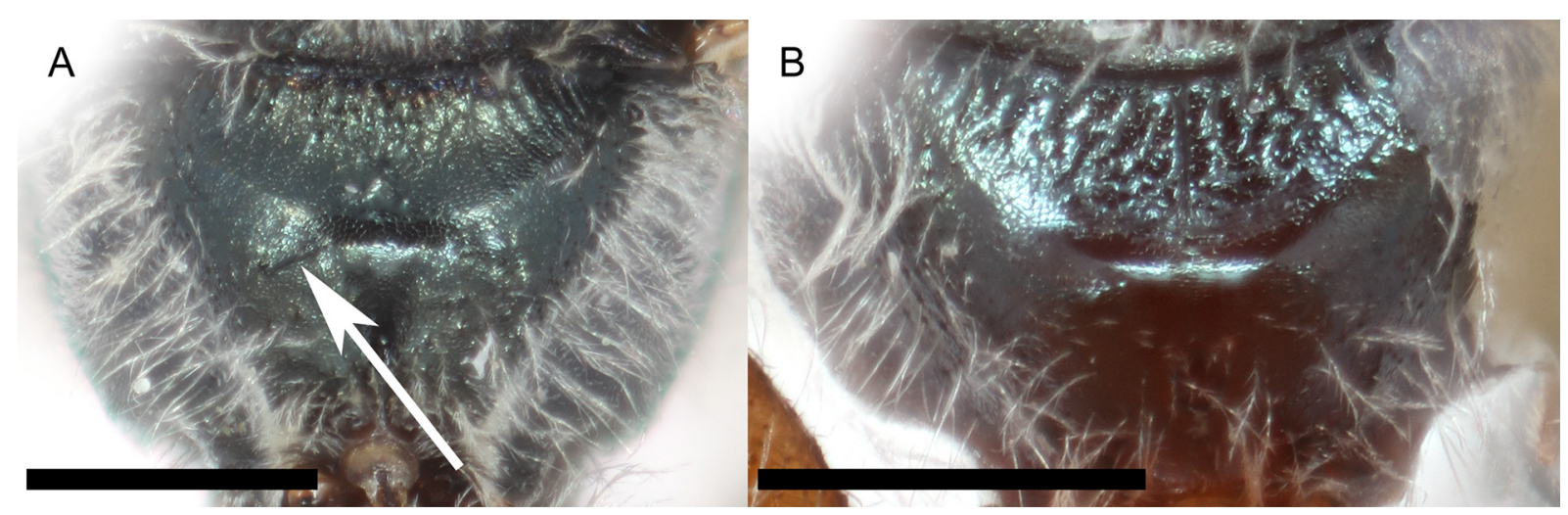

Fig. 98. Propodeum. A. Lasioglossum (D.) testaceum (Robertson, 1897), + , with strong oblique carina (arrow). B. L. (D.) minckleyi sp. nov.,, , without oblique carina. Scale bars: $0.5 \mathrm{~mm}$. 
A

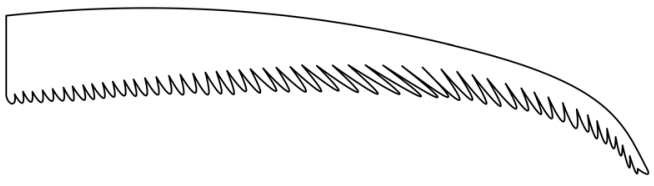

B

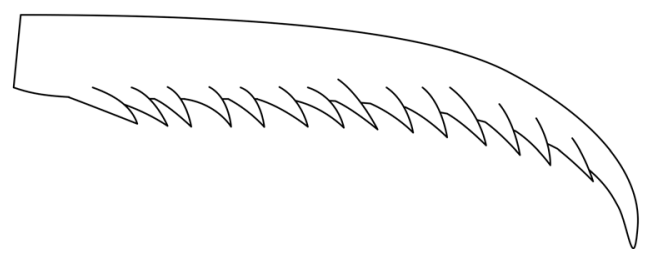

Fig. 99. Middle tibial spurs. A. Minutely serrate. B. Finely pectinate.

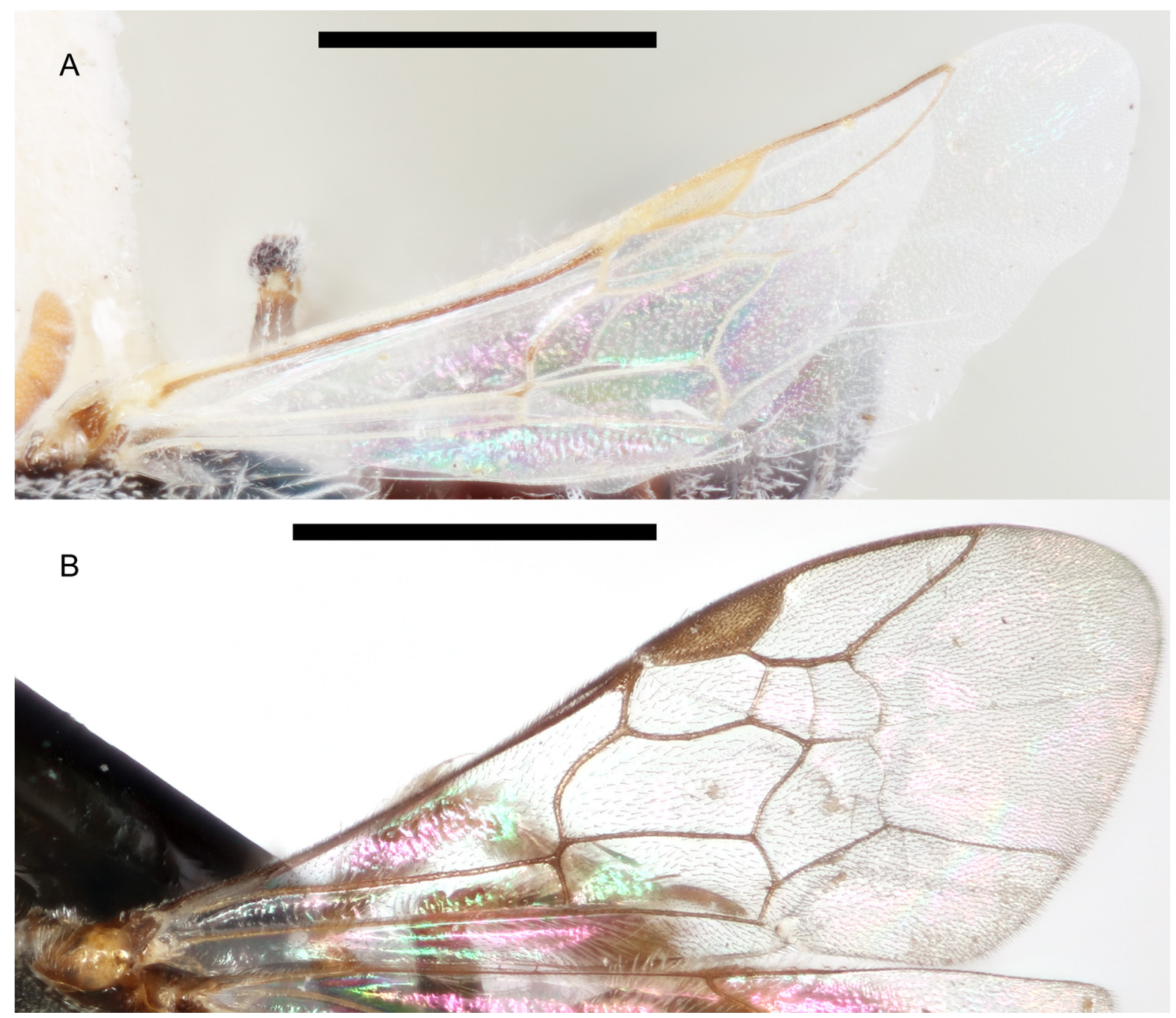

Fig. 100. Forewings. A. Lasioglossum (D.) pallidellum (Ellis, 1914), $\widehat{\jmath}$, with white hair, pale veins, and hyaline membrane. B. L. (D.) decorum sp. nov., ㅇ, with dark hair, dark veins, and lightly infuscated membrane. Scale bars: $1 \mathrm{~mm}$. 


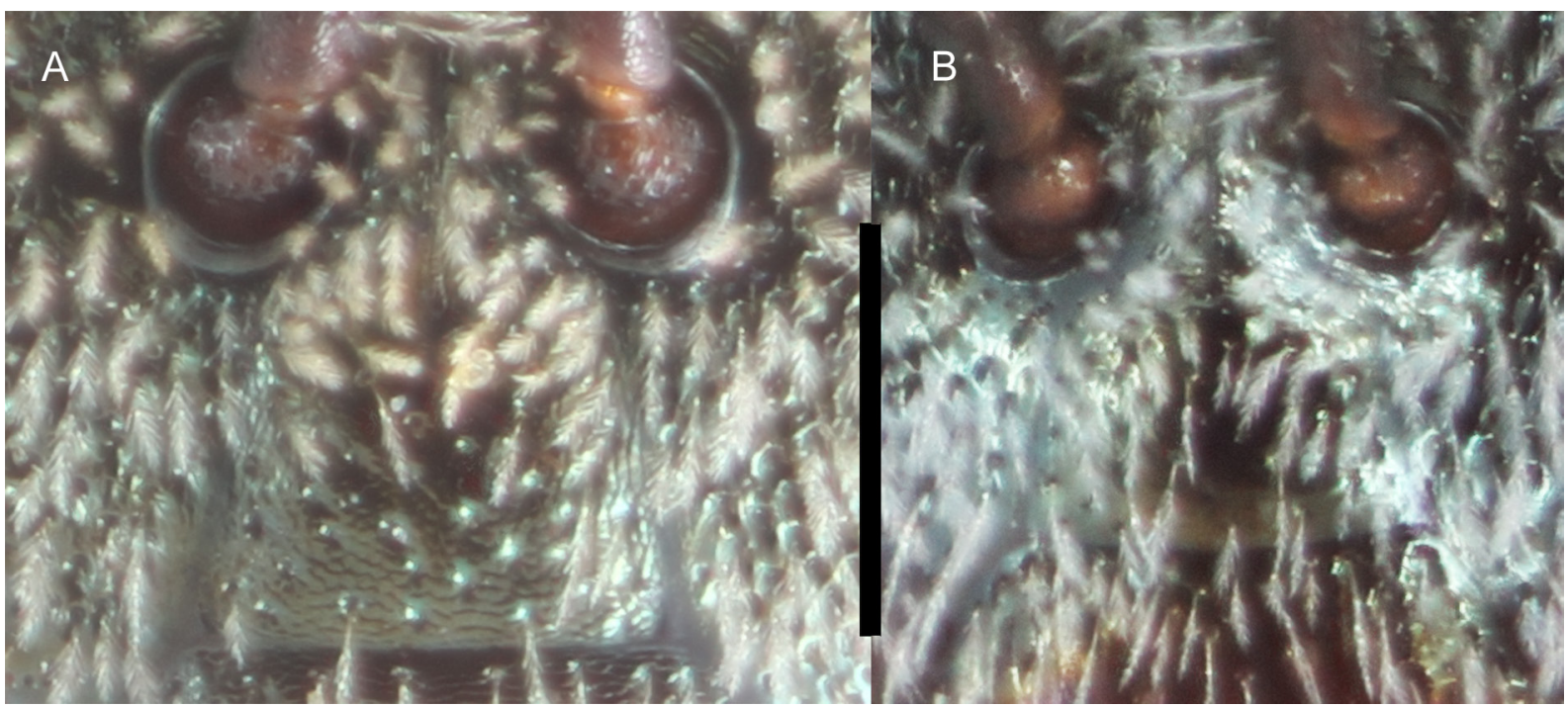

Fig. 101. Supraclypeal area. A. Lasioglossum (D.) arenisaltans sp. nov., + , mostly tessellate. B. L. (D.) mesillense (Cockerell, 1898),, , mostly shiny. Scale bar: $0.25 \mathrm{~mm}$.
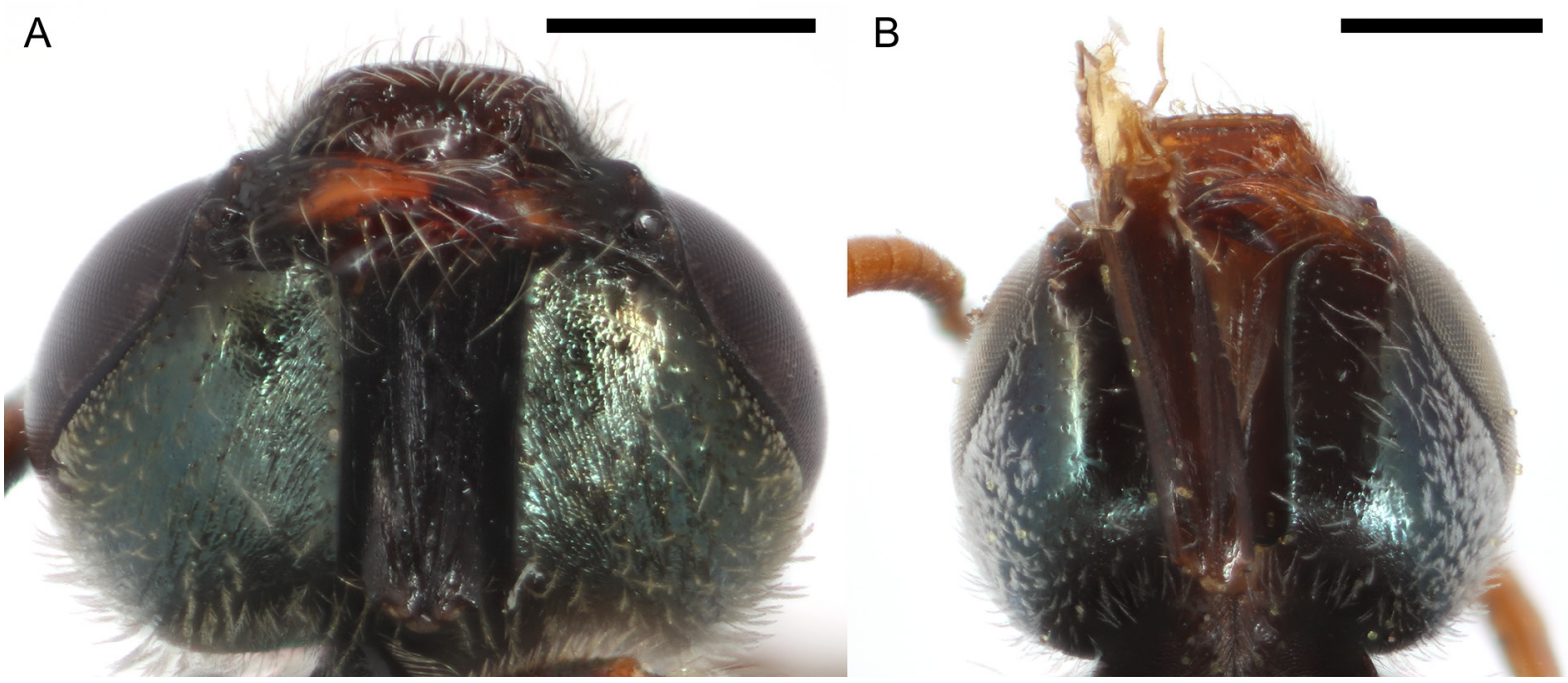

Fig. 102. Postgena. A. Lasioglossum (D.) pictum (Crawford, 1902), + , dull and lineate. B. L. (D.) lilianae sp. nov.,, , shiny. Scale bars: $0.5 \mathrm{~mm}$. 


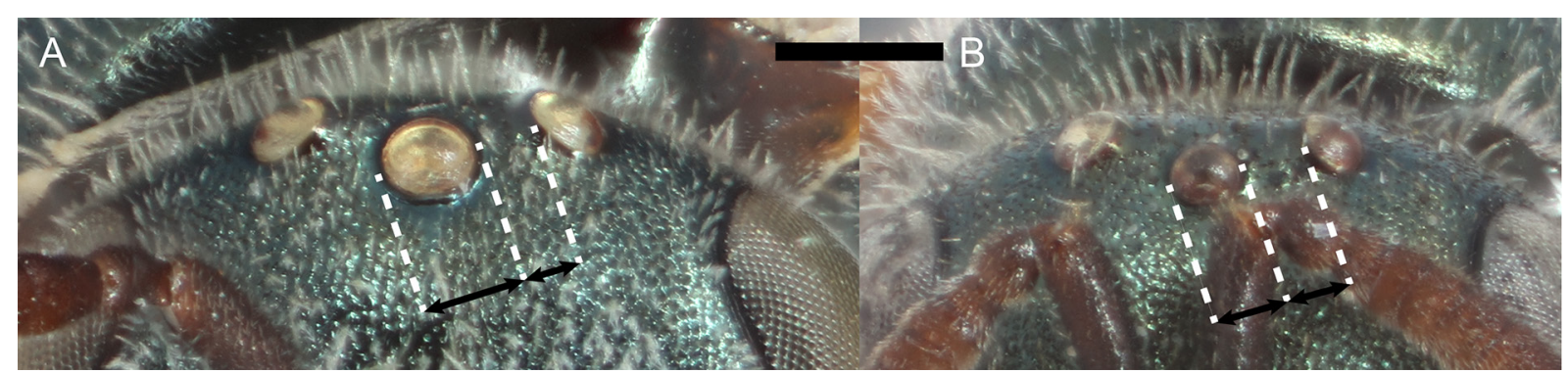

Fig. 103. Ocelli. A. Lasioglossum (D.) eophilus (Ellis, 1914),, , slightly enlarged and separated by less than two thirds of one ocellar diameter. B. L. (D.) miltolepoides sp. nov., , , normal size, separated by more than two thirds of one ocellar diameter. Scale bar: $0.25 \mathrm{~mm}$.

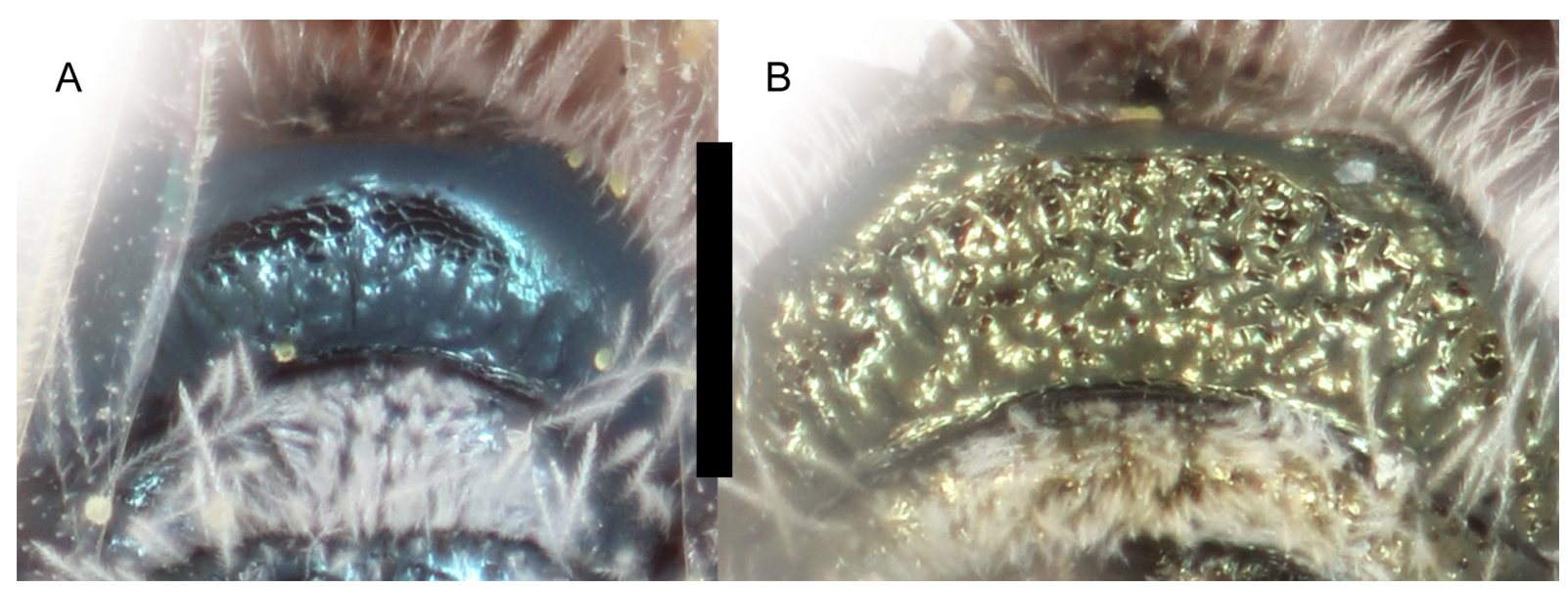

Fig. 104. Propodeum. A. Lasioglossum (D.) pallidellum (Ellis, 1914), $q$, with metapostnotal rugae not reaching the posterior margin. B. L. (D.) clastipedion sp. nov.,, , with metapostnotal rugae reaching the posterior margin. Scale bar: $0.25 \mathrm{~mm}$. 

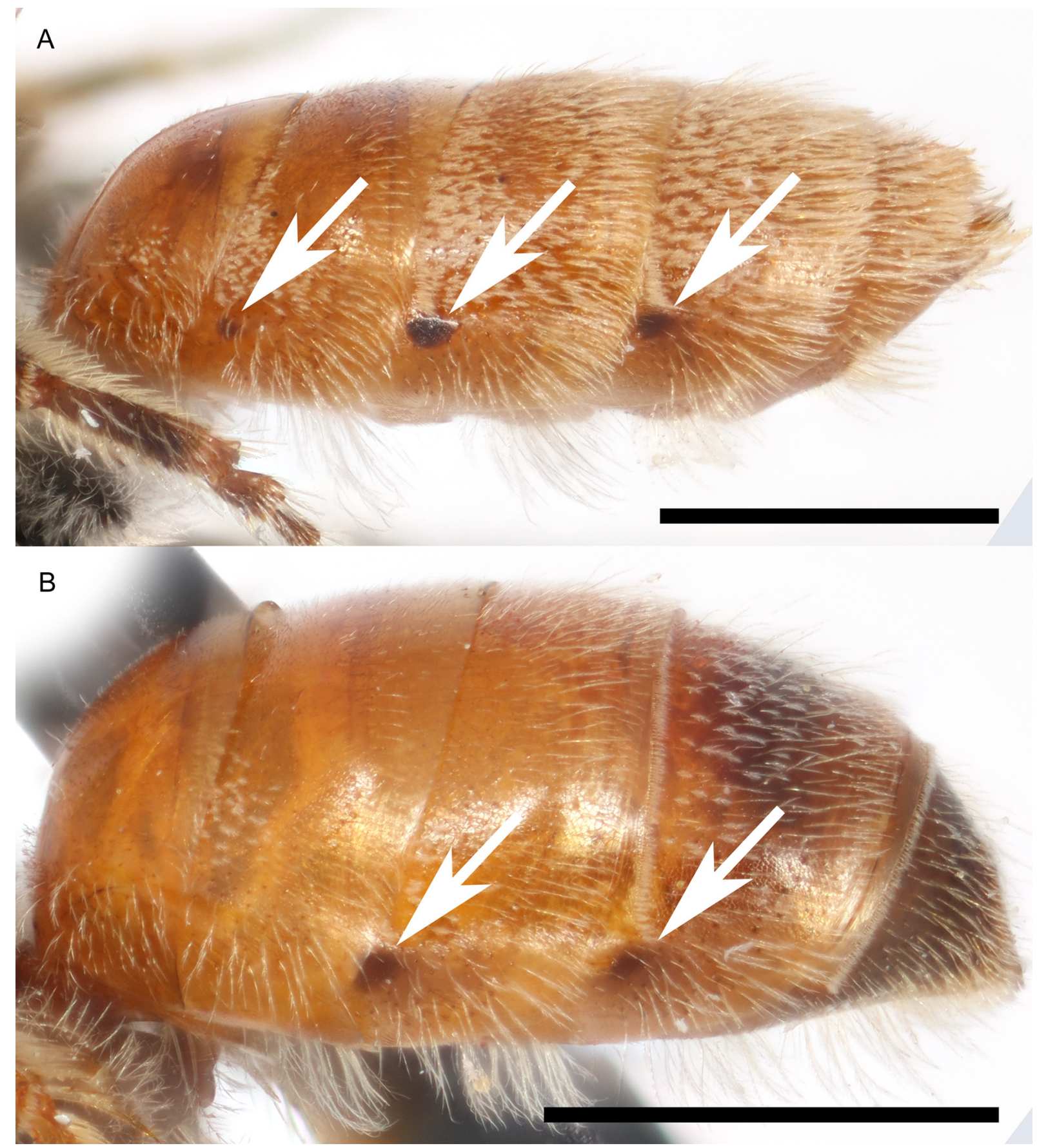

Fig. 105. Dark spiracular spots (arrows) on the metasoma. A. Lasioglossum (D.) pictum (Crawford, 1902),, , present on T2-4. B. L. (D.) meteorum sp. nov.,, , present on T3-4 only. Scale bars: $1 \mathrm{~mm}$. 


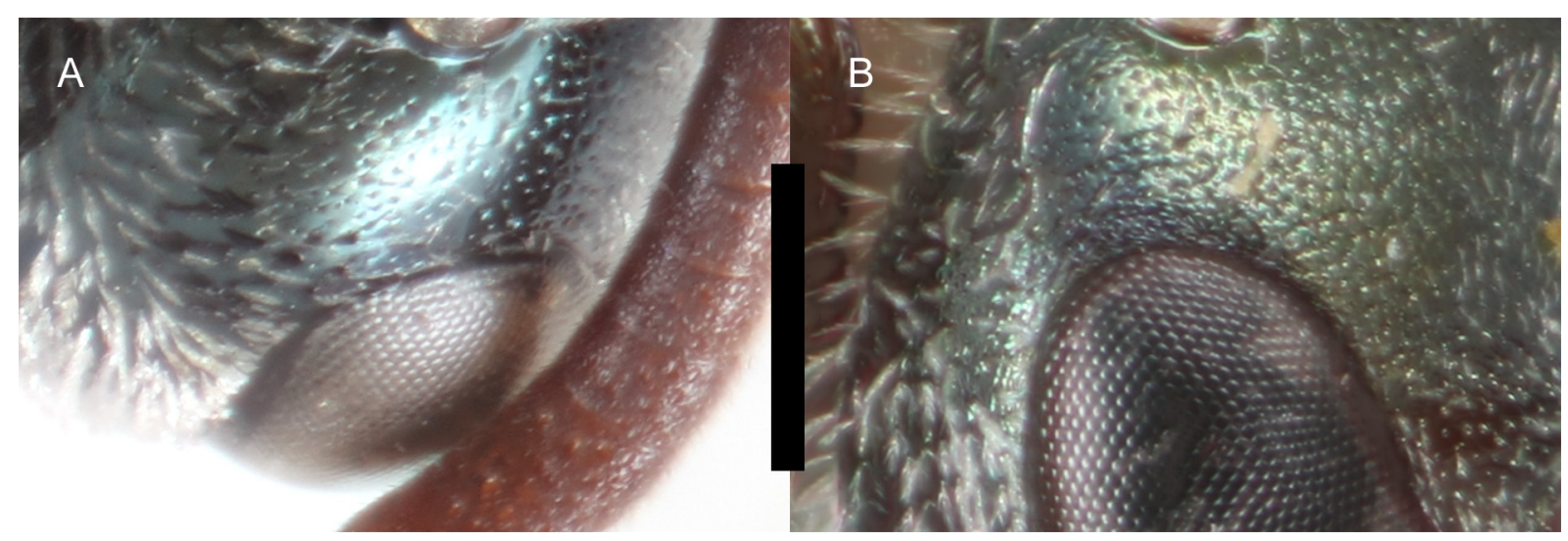

Fig. 106. Ocellocular area. A. Lasioglossum (D.) clavicorne sp. nov., $q$, sparsely and distinctly punctate. B. L. (D.) clematisellum (Cockerell, 1904),, , densely punctate with punctures somewhat obscured by microsculpture. Scale bar: $0.25 \mathrm{~mm}$.

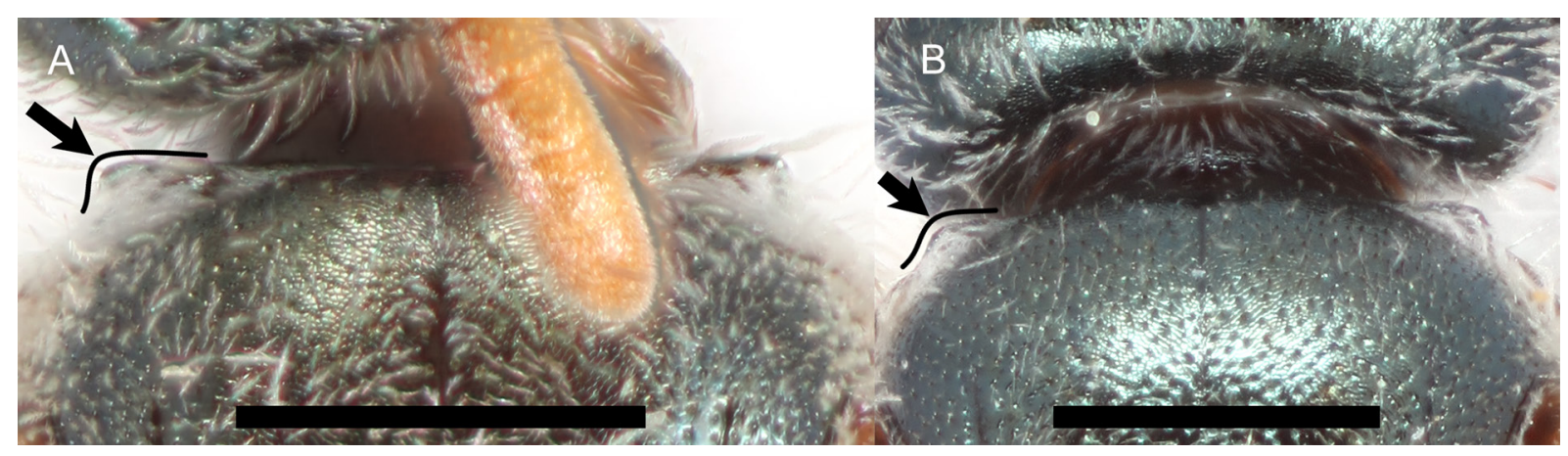

Fig. 107. Pronotal angle (arrow and outline). A. Lasioglossum (D.) hudsoniellum (Cockerell, 1919),, , nearly 90 degrees. B. L. (D.) minckleyi sp. nov.,, , obtuse. Scale bars: $0.5 \mathrm{~mm}$.

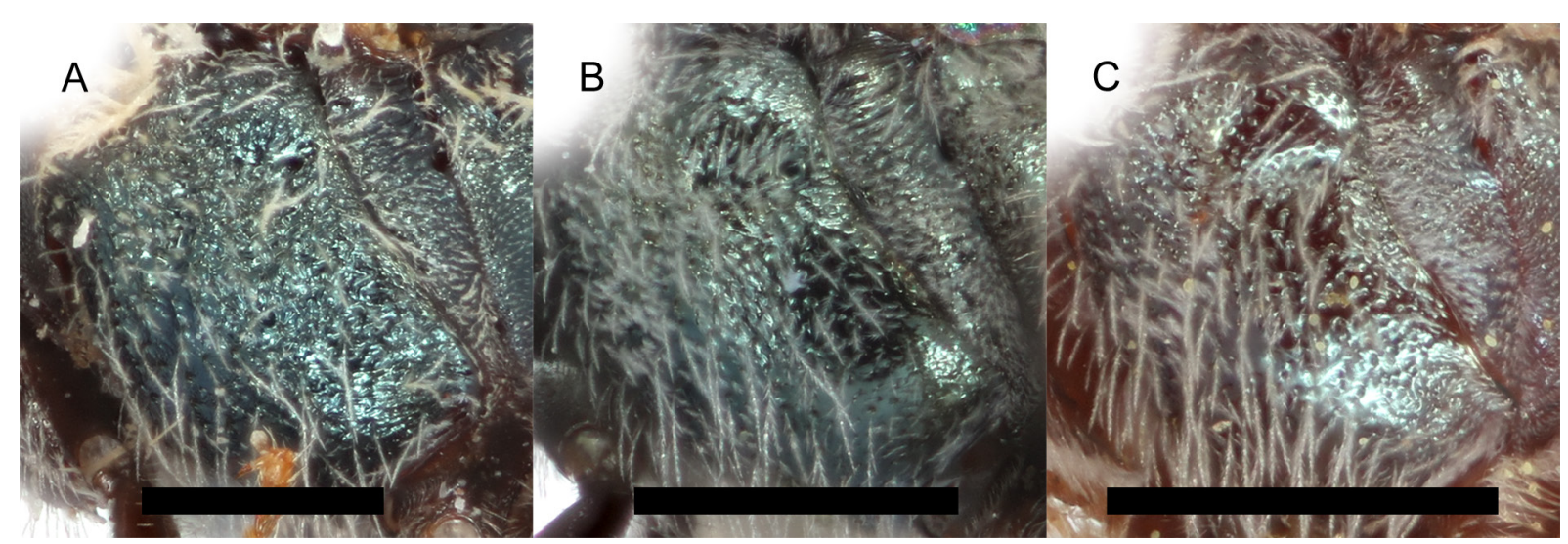

Fig. 108. Mesepisternum. A. Lasioglossum (D.) eophilus (Ellis, 1914), $q$, entirely rugose. B. L. (D.) mesillense (Cockerell, 1898), $ᄋ$, ruguloso-punctate in dorsal half, smooth and distinctly punctate in ventral half. C. L. (D.) imbriumbrae sp. nov.,, , entirely smooth and distinctly punctate. Scale bars: $0.5 \mathrm{~mm}$. 


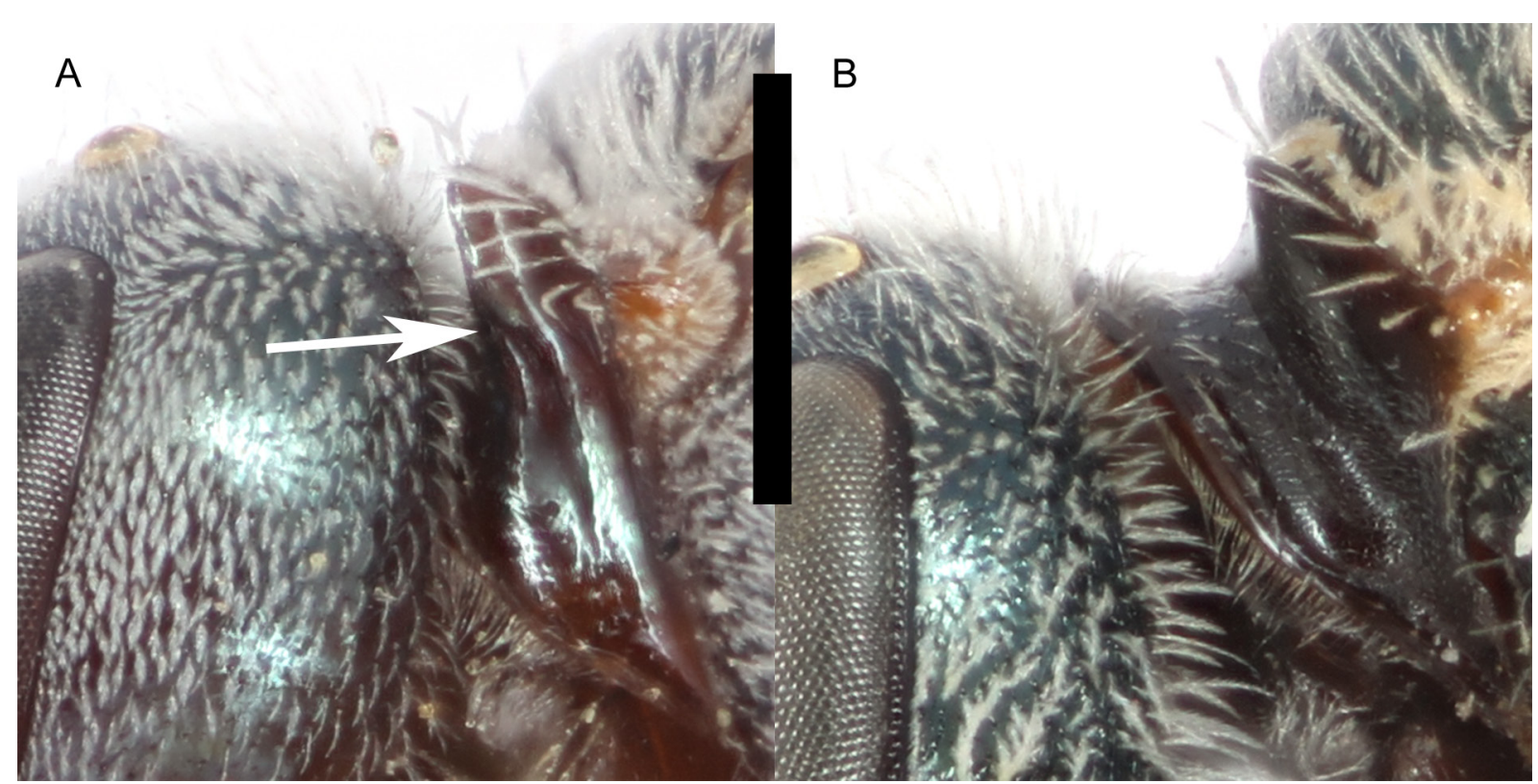

Fig. 109. Pronotal ridge (arrow). A. Lasioglossum (D.) kunzei (Cockerell, 1898), $\circ$, sharply carinate along full height of pronotum. B. L. (D.) eophilus (Ellis, 1914),, , absent. Scale bar: $0.5 \mathrm{~mm}$.
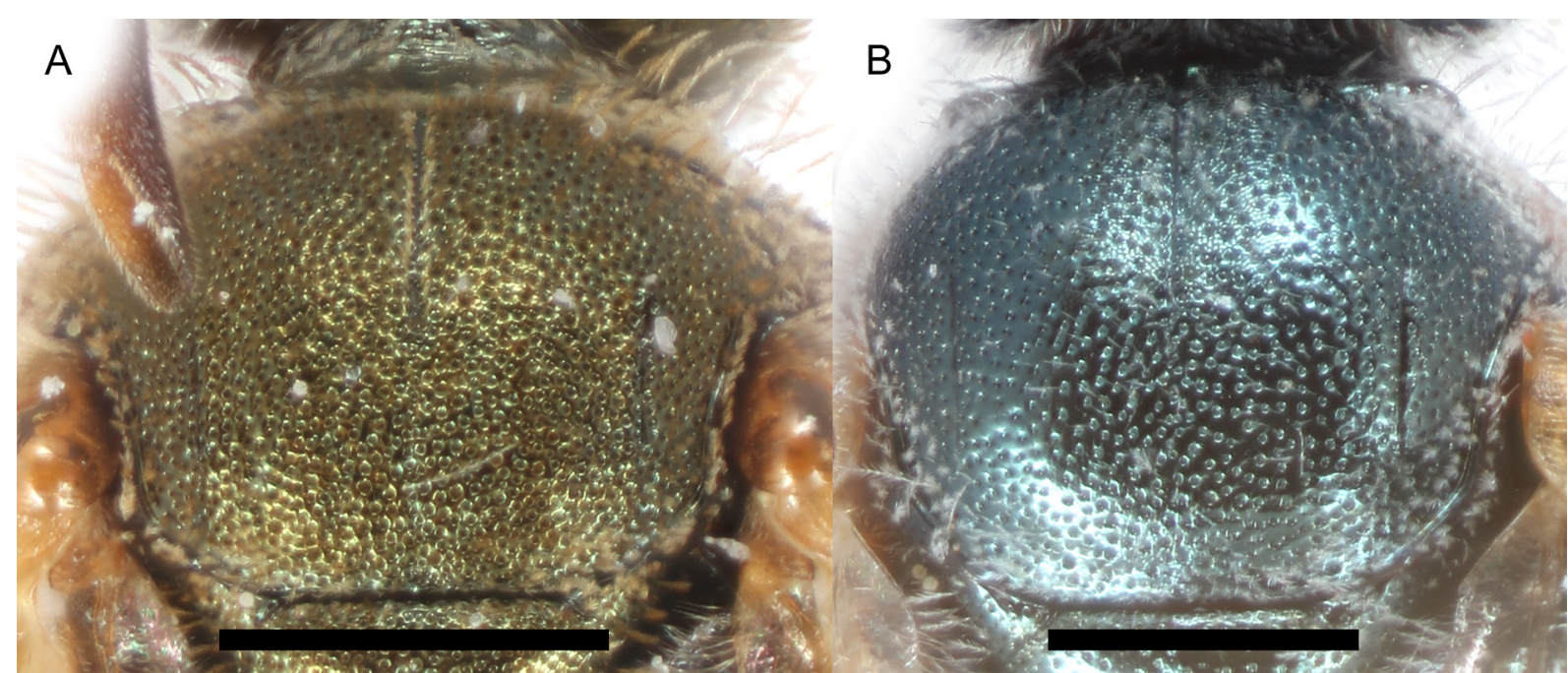

Fig. 110. Mesoscutum punctures. A. Lasioglossum (D.) vierecki (Crawford, 1904), ठ̂, all interspaces less than 1 puncture diameter. B. L. (D.) arenisaltans sp. nov., $\hat{\sigma}$, numerous interspaces of 1 puncture diameter or more. Scale bars: $0.5 \mathrm{~mm}$. 

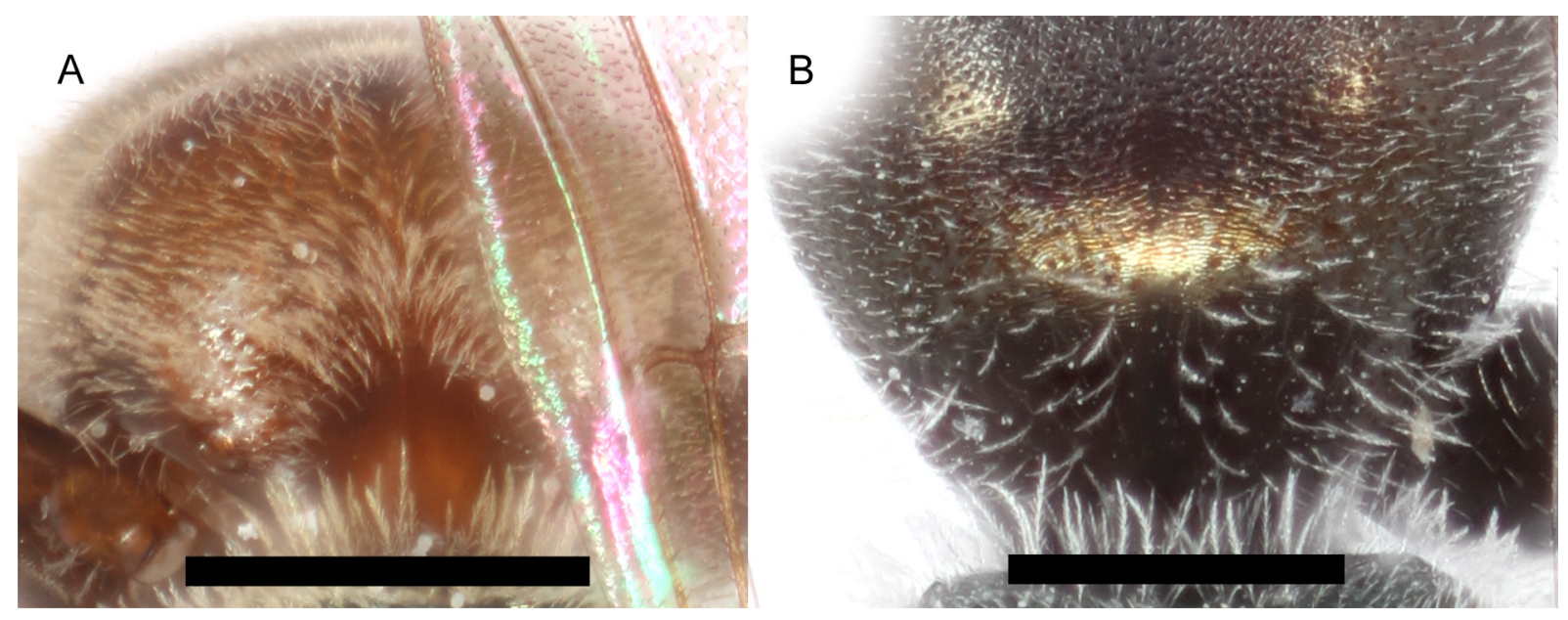

Fig. 111. T1 anterior surface. A. Lasioglossum (D.) vierecki (Crawford, 1904), §̂, with dense appressed hair. B. L. (D.) droegei Gibbs, 2009, ô, without appressed hair. Scale bars: $0.5 \mathrm{~mm}$.
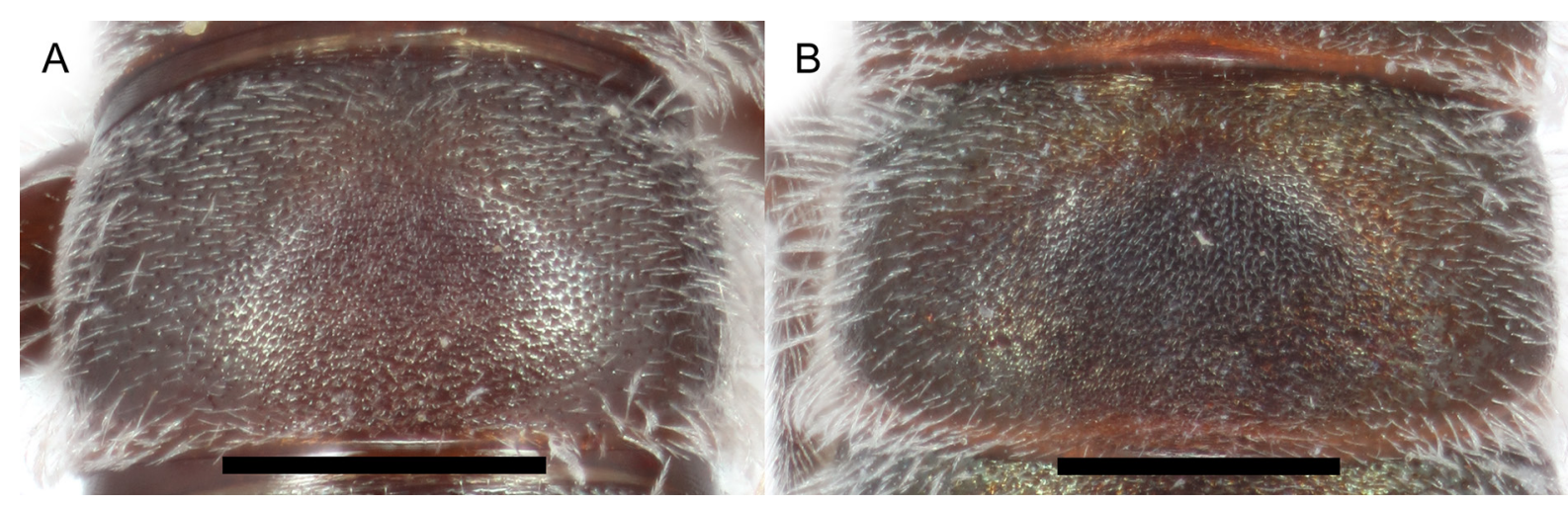

Fig. 112. T2. A. Lasioglossum (D.) petrellum (Cockerell, 1903), đ, with weaker microsculpture and distinct punctures throughout. B. L. (D.) droegei Gibbs, 2009, $\widehat{0}$, with strong microsculpture and obscure punctures towards apical rim. Scale bars: $0.5 \mathrm{~mm}$.

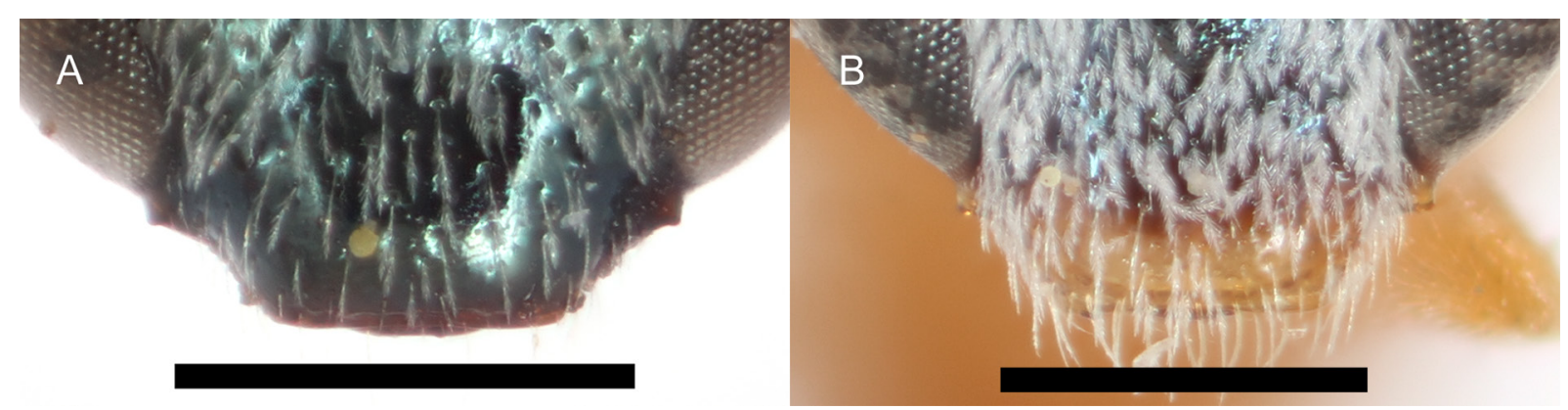

Fig. 113. Clypeus. A. Lasioglossum (D.) miltolepoides sp. nov., $\widehat{\jmath}$, sparsely punctate and entirely metallic. B. L. (D.) arenisaltans sp. nov., ô, with dense punctures and yellow-orange apical rim. Scale bars: $0.5 \mathrm{~mm}$. 


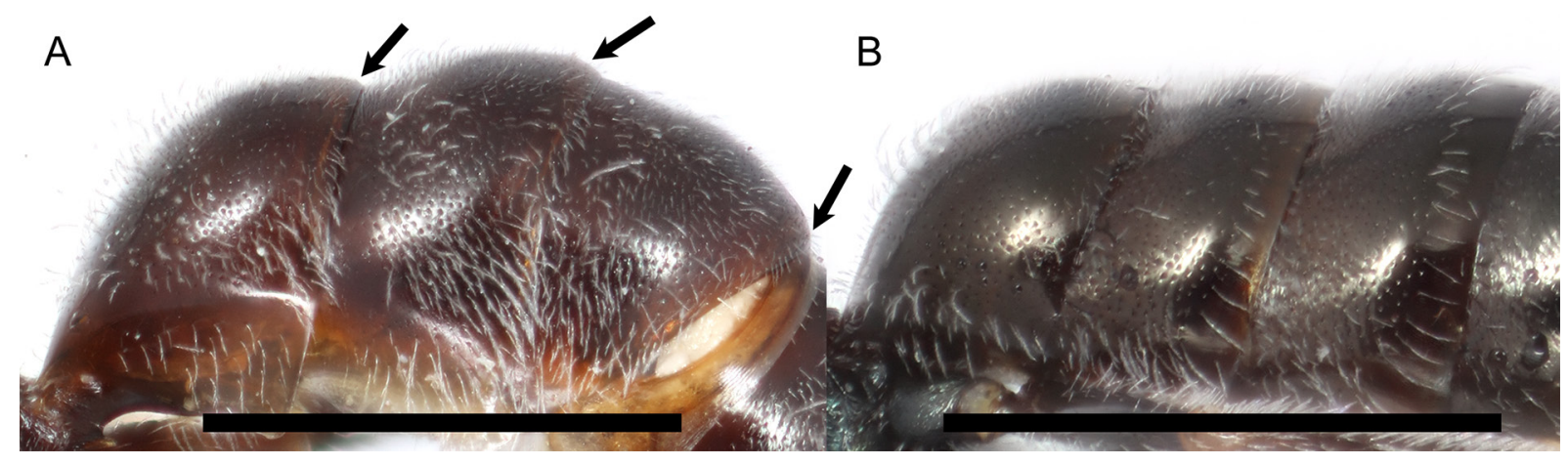

Fig. 114. T1-3. A. Lasioglossum (D.) cactorum sp. nov., ô, convex with rims strongly downcurved (arrows). B. L. (D.) clematisellum (Cockerell, 1904), đ̂, flat. Scale bars: $1 \mathrm{~mm}$.

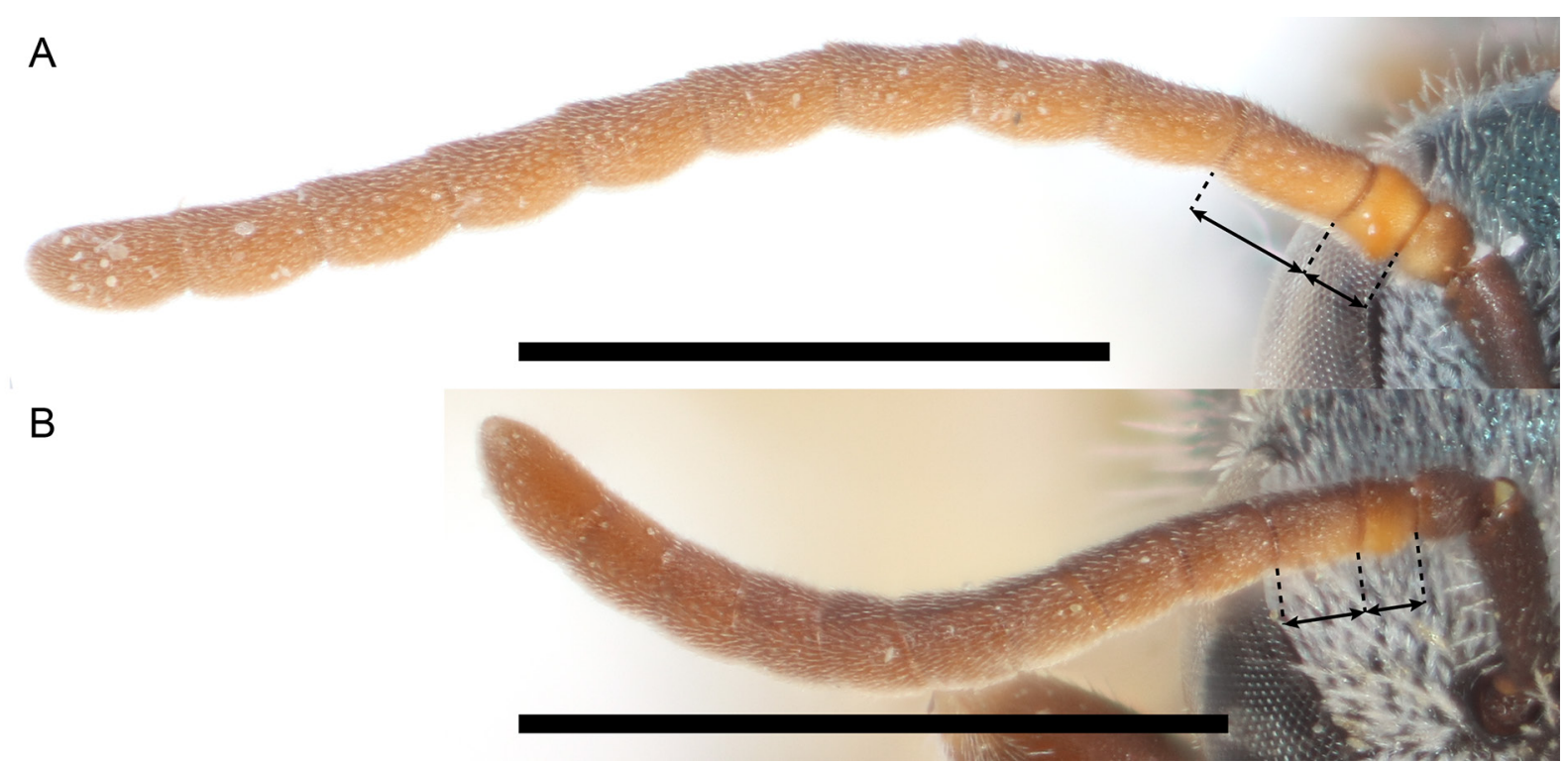

Fig. 115. Flagellomeres. A. Lasioglossum (D.) festinum sp. nov., đ, long. B. L. (D.) imbriumbrae sp. nov., $\widehat{\partial}$, short. Scale bars: $1 \mathrm{~mm}$. Arrows indicate relative lengths of F1 and F2. 
A

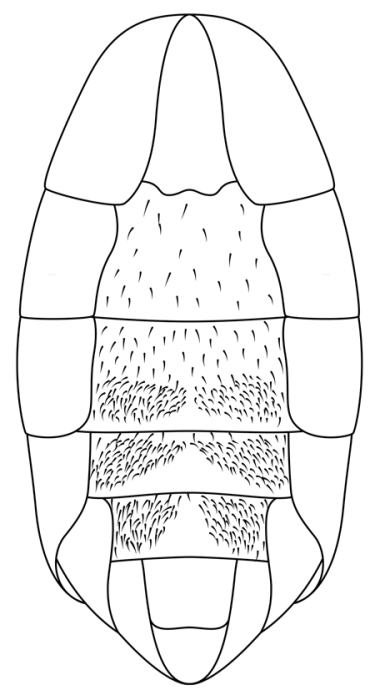

B

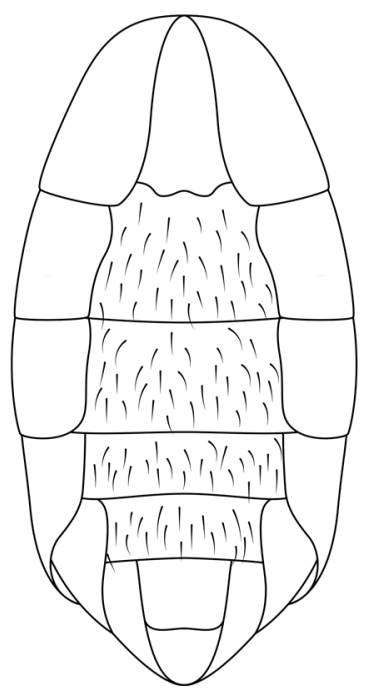

C

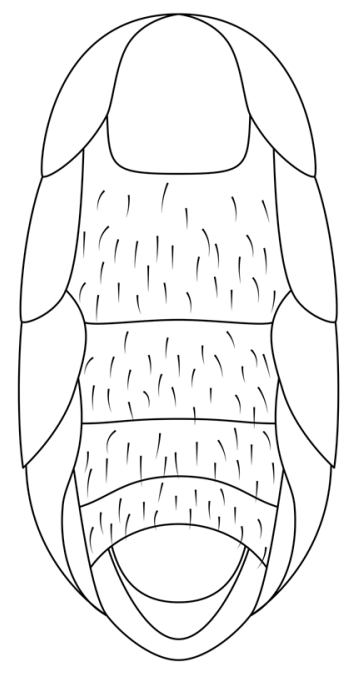

Fig. 116. Metasomal sterna, Ô. A. Lasioglossum (D.) zephyrus (Smith, 1853), with hair concentrated in apicolateral areas of S3-5. B. Typical red-tailed Lasioglossum (Dialictus), with hair evenly distributed and S5 straight. C. L. (D.) hudsoniellum (Cockerell, 1919), with S5 concave.

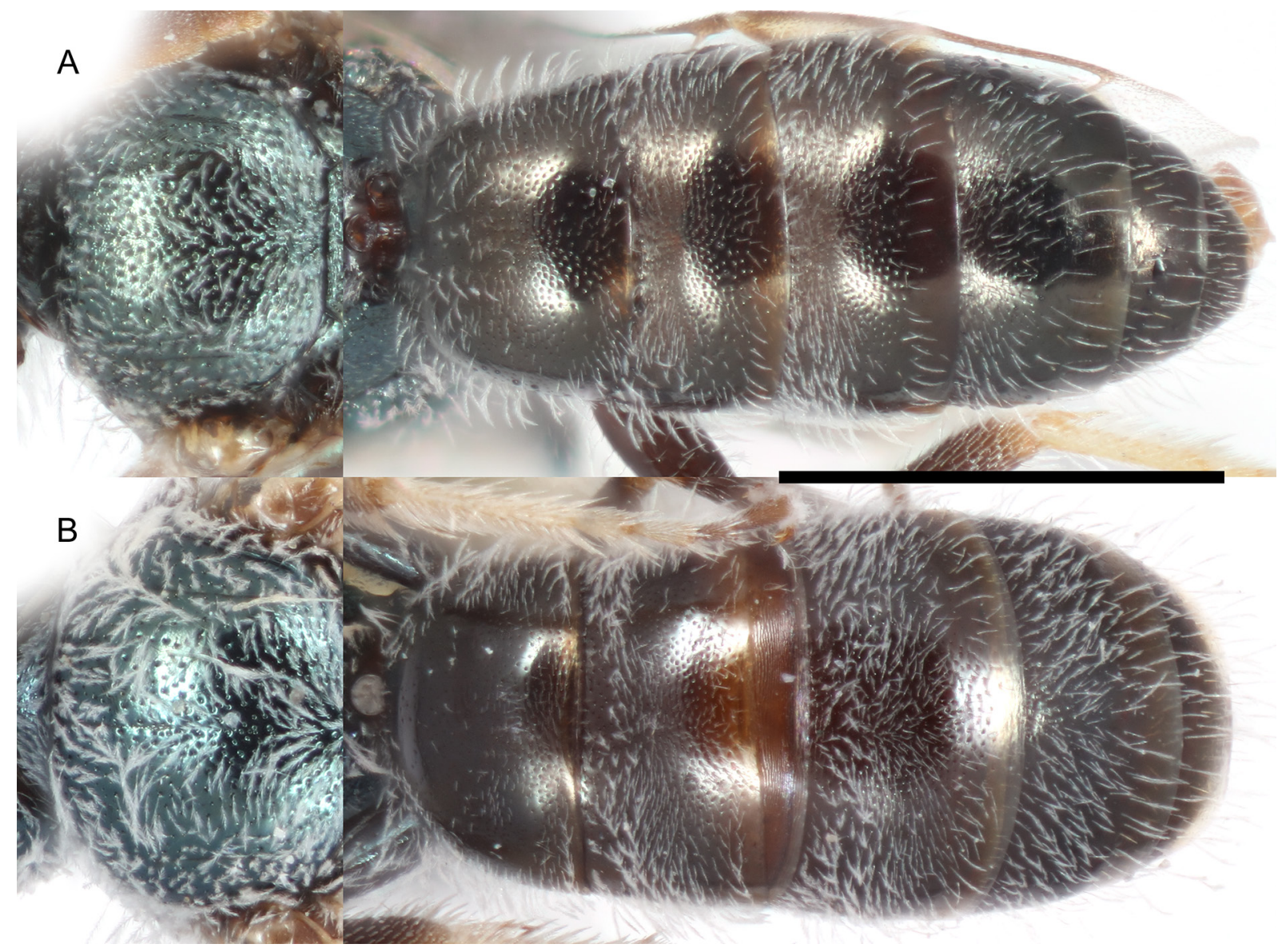

Fig. 117. Mesoscutum and metasoma. A. Lasioglossum (D.) clematisellum (Cockerell, 1904), with most hair thin and simple. B. L. (D.) julipile sp. nov., with most hair densely plumose. Scale bar: $1 \mathrm{~mm}$. 


\section{The Lasioglossum clematisellum species complex}

\section{Species included}

Lasioglossum clastipedion sp. nov., L. clavicorne sp. nov., L. clematisellum, L. julipile sp. nov., and L. perditum sp. nov.

\section{Diagnosis}

The Lasioglossum clematisellum species complex comprises several morphologically and genetically similar species found in deserts of the southwestern United States and northern Mexico. Females can be fairly easily diagnosed by the combination of wings pale with extremely short, inconspicuous white hairs, metapostnotal rugae strong and usually reaching the posterior margin, punctures of the face, hypoepimeron, and mesepisternum all distinctly separated, and T2 without dark spiracular spots. Males are small $(<5 \mathrm{~mm})$, with the metasoma relatively narrow and all black to brown, rims of T1-3 largely impunctate, flagellomeres relatively long (F2-11 1.5-2 times as long as broad), head and mesosoma usually shiny with distinctly separated punctures, and metapostnotum usually with strong rugae reaching the posterior margin and extending onto the dorsolateral slope. In addition, males of this group often lack the second submarginal crossvein in one or both forewings, therefore possessing only two submarginal cells, and in one species (L. perditum sp. nov.) this is the usual condition. The male of L. clavicorne sp. nov. is unusual and lacks many of these diagnostic characters; L. clavicorne sp. nov. is included in this group mainly based on female morphology.

These species are usually challenging to distinguish morphologically, but most are limited geographically and so collection locality may be used as an aid to identification. Lasioglossum clematisellum is the only species in this group with a very large distribution.

\section{The Lasioglossum petrellum species complex}

\section{Species included}

Lasioglossum droegei, L. griswoldi, L. petrellum, L. tuolumnense, and L. viridipetrellum Gibbs, 2009 [in Gibbs 2009b].

\section{Diagnosis}

The Lasioglossum petrellum species complex is an infrequently collected group of morphologically similar species, but each with distinct geographic distributions. It is often easier to identify specimens geographically than morphologically. This group was revised by Gibbs (2009b); users are referred to that work for additional details on the included species.

Females and males of the L. petrellum group can both be recognized by the head very elongate (length/ width ratio 0.88 or more), eyes strongly convergent ventrally, clypeus strongly protruding below the suborbital tangent, and most of the body covered in very fine, dense punctures and strong microsculpture. In addition, females lack an acarinarial fan, and males have the retrorse lobes very small.

\section{Identification keys to the western red-tailed species of Lasioglossum (Dialictus)}

\section{Key to females}

1. Tegula enlarged, reaching or nearly reaching scutellum, with inner posterior margin straight or concave, often punctate (Fig. 91A)...... L. hunteri (Crawford, 1932) and similar species ${ }^{1}$

- Tegula ovoid, impunctate, and clearly not reaching scutellum (Fig. 91B)

\footnotetext{
${ }^{1}$ L. parvum-tegulare species complex; not treated further herein. Specimens from desert and mountain regions are not likely true $L$. hunteri.
} 
2. Head (except clypeus and postgena) and mesonotum entirely covered in dense, yellowish, thickly plumose hairs which (unless worn) largely obscure the surface underneath (Fig. 92); face relatively long (length/width ratio $\sim 0.84$ )

- Head and mesonotum with thick hairs sparse or absent, or if more extensive, then hairs bright white and at least center of scutum largely exposed (Fig. 67A); face length variable

3. Thick hairs short and scale-like (Fig. 92A); body length 4.5-5 mm (sand dunes of eastern United States and Canada) ............................................................................. vierecki (Crawford, 1904)

- Thick hairs long (Fig. 92B); body length 5.5-6 mm (mountains of California and western Nevada)

L. cembrilacus sp. nov.

4. Mesonotum and mesepisternum entirely covered in strong tessellate to finely reticulate microsculpture, with fine, sparse punctures $(i=1-4 \mathrm{pd})$ which are often obscured by microsculpture (Fig. 93A) (Mexico)

- Mesonotum and/or mesepisternum usually shiny and distinctly punctate at least in part (Fig. 93C); if covered in strong microsculpture, then more densely punctate (Fig. 93B) (widespread) .............. 6

5. Frons punctures large (about as large as paraocular area punctures) and coarse, approaching a rugulose condition (Fig. 94A); T1 anterior surface shiny (Fig. 95A) (widespread throughout most of Mexico)

L. tessellatosum sp. nov.

- Frons punctures small (about half the size of paraocular punctures) and fine, at most very weakly rugulose above antennae (Fig. 94B); T1 anterior surface weakly coriarious (Fig. 95B) (Yucatan Peninsula)

L. meteorum sp. nov.

6. T1 with numerous erect hairs evenly distributed across anterior face; acarinarial fan absent (Fig. 96A) and/or metasomal terga with extremely narrow, abruptly downcurved rims usually with dense apicolateral fringes of long, plumose hair (Fig. 97A)

- T1 anterior face with erect hairs absent at least medially, usually limited to lateral margins; acarinarial fan present, although often weakly developed (Fig. 96B); metasomal terga with broad, flat rims with apicolateral fringes of short hair (Fig. 97B)

7. Scutum shiny and moderately sparsely punctate submedially ( $i=1-2$ pd) (Fig. 93C); face short (length/width ratio 0.88 or less).

- Scutum entirely finely and densely punctate ( $\mathrm{i} \leq 1 \mathrm{pd}$ ) and often dull (Fig. 93B); face long (length/ width ratio 0.88 or more).

8. Propodeum with strong V-shaped oblique carina (Fig. 98A); T1 anterior face with no appressed hairs.

- Oblique carina absent (Fig. 98B); T1 anterior face with some appressed hairs

9. Metasomal terga with extremely narrow, abruptly downcurved rims usually with dense apicolateral fringes of long, plumose hair (Fig. 97A); frons shiny with distinctly separated punctures (Fig. 94C); $\mathrm{T} 1$ anterior face with numerous long appressed hairs forming weak acarinarial fan.....

L. cactorum sp. nov.

- Metasomal terga with broad, flat rims with sparse apicolateral fringes of short, simple hair (Fig. 97B); frons densely reticulate-punctate (Fig. 94A); T1 anterior face with sparse, short appressed hairs laterally not forming distinct fan L. miltolepoides sp. nov.

10. Metasomal terga always, and scutum often mostly shiny; middle tibial spur and anterior hind tibial spur minutely serrate, appearing simple (Fig. 99A) (mountain species) .....

- Metasomal terga and scutum entirely dull; middle tibial spur and anterior hind tibial spur finely pectinate with teeth nearly half the width of the rachis (Fig. 99B) (desert species). 
11. Malar space as wide or wider than scape; supraclypeal area much longer than broad; scutum with slightly sparser punctures, with numerous interspaces up to 1 puncture diameter or slightly more, and more extensively shiny on average (Utah to Colorado).

L. griswoldi Gibbs, 2009

- Malar space less than width of scape; supraclypeal area only slightly longer than broad; scutum with denser punctures, with only a few interspaces up to 1 puncture diameter, and less shiny on average (sometimes completely dull) (California to British Columbia)....

L. tuolumnense Gibbs, 2009

12. T4 tomentum limited to narrow basal band; T2-3 with basolateral tomentum covering much less than half length of segment (Baja California to Arizona)

L. petrellum (Cockerell, 1903)

- T4 with sparse tomentum throughout; T2-3 with sparse basolateral tomentum covering about half length of segment laterally (New Mexico to Nuevo Leon)

L. droegei Gibbs, 2009

13. Forewing with all hairs white, often difficult to see; membrane hyaline; veins (excluding subcosta) usually pale amber (Fig. 100A) .

- Forewing with distinct, dark hairs in apical half or more; membrane sometimes infuscated; veins often dark brown (Fig. 100B)

14. Punctures of frons crowded (not all punctures are round), the surface thus appearing relatively dull (Fig. 94A), usually distinctly smaller and denser than punctures of paraocular area; and/or T1 anterior surface coriarious (Fig. 95B-C).

- Punctures of frons about the same size and density as punctures of paraocular area, with at least narrow shiny interspaces, the surface thus appearing shiny (Fig. 94C-D); T1 anterior surface shiny (Fig. 95A)

15. Supraclypeal area mostly or entirely tessellate (Fig. 101A); postgena mostly dull and/or lineate (Fig. 102A)

- Supraclypeal area (Fig. 101B) and postgena (Fig. 102B) mostly smooth and shiny ..

16. Scutum mostly shiny with most punctures separated by about one puncture diameter (Fig. 75A); discs of terga black to reddish brown with metallic blue-green reflections.... L. rufornatum sp. nov.

- Scutum mostly tessellate with all punctures separated by much less than one puncture diameter (Fig. 8A); metasoma usually entirely red to orange; if dark brown, then discs of terga not metallic

L. arenisaltans sp. nov. (in part)

17. Mesepisternum rugose in dorsal half or more, with thin, sparse hair (Fig. 36B); T1 anterior surface shiny (Fig. 95A); ocelli slightly enlarged, separated by much less than 1 ocellar diameter (Fig. 103A)

L. eophilus (Ellis, 1914) (in part)

- Mesepisternum with crowded punctures but not rugose, with abundant tomentum (Fig. 11B); T1 anterior surface at least weakly coriarious (Fig. 95B); ocelli normal, separated by nearly 1 ocellar diameter (Fig. 103B).

L. argammon sp. nov.

18. Forewing with two submarginal cells (rarely with three in one wing); size very small (body length $\sim 3.5 \mathrm{~mm}$; intertegular distance $\sim 0.67 \mathrm{~mm}$ ).

L. perditum sp. nov.

- Forewing with three submarginal cells; size usually larger (body length $\sim 4 \mathrm{~mm}$ or more; intertegular distance $\sim 0.73 \mathrm{~mm}$ or more).

19. Metapostnotal rugae usually not reaching posterior margin within 1 or 2 ridge widths (Fig. 104A); scutum often sparsely punctate on both sides of parapsidal lines ( $i=1-4 \mathrm{pd})$; dark spiracular spots present on T2-4 (Fig. 105A).

- Metapostnotal rugae usually uniformly strong and reaching posterior margin (within 1 or 2 ridge widths) (Fig. 104B); scutum usually more densely punctate on either side of parapsidal lines ( $\mathrm{i} \leq 2 \mathrm{pd}$ ); dark spiracular spots usually present on T3-4 only (Fig. 105B)..... 
20. Face long (length/width ratio $\sim 0.84$ ) with tomentum much sparser than on gena, surface mostly exposed (Fig. 52C); mesepisternum sometimes becoming areolate dorsally ........ L. lilianae sp. nov.

- Face much broader than long (length/width ratio $~ 0.78$ ) with tomentum as dense as on gena, largely obscuring surface (unless worn) (Fig. 67C); mesepisternum always distinctly punctate throughout.....

L. pallidellum (Ellis, 1914)

21. Ocellocular area with moderately sparse ( $\mathrm{i}=1-3 \mathrm{pd})$, distinct punctures (Fig. 106A); frons, ocellocular area, scutum, and mesepisternum entirely shiny (except small tessellate patch on scutum anteromedially). 22

- Ocellocular area with dense ( $i \leq 1 \mathrm{pd}$ ), sometimes obscure punctures (Fig. 106B); frons, ocellocular area, scutum, and/or mesepisternum usually more extensively dull

22. Scutum with all hairs thickly plumose (Fig. 46B); mesepisternum and hypoepimeron with punctures relatively small and fine (8-9 punctures along scrobal groove) (Fig. 46A) ..... L. julipile sp. nov.

- Scutum with many hairs thin and simple (Fig. 25A); face, mesepisternum, and hypoepimeron with punctures relatively large and coarse (6-7 punctures along scrobal groove) (Fig. 25B)

L. clavicorne sp. nov.

23. T1 and T2 with extensive lateral tomentum (Fig. 1); mesepisternum usually with crowded, indistinct punctures dorsally; pronotal angle usually about 90 degrees (Fig. 107A)

L. hudsoniellum (Cockerell, 1919) (in part)

- T1 and T2 with tomentum absent or limited to thin basal band; mesepisternum usually with distinct, round punctures throughout; pronotal angle obtuse (Fig. 107B).

24. Metasoma red-orange with the following areas usually brown: basal half of T1, median transverse band on T3, and T4-5 (Fig. 78D); scutum punctures relatively coarse (2-3 punctures present between posterior end of parapsidal line and lateral edge of scutum).

L. spivakae sp. nov. (in part)

- Metasoma entirely red-orange; scutum punctures relatively fine (3-5 punctures present between posterior end of parapsidal line and lateral edge of scutum).

25. T2-4 with tomentum absent or thin and not well differentiated from surrounding simple hairs (Fig. 28D); scutum hairs usually mostly thin and simple; anteromedial scutum punctures usually finer and less distinct than those elsewhere on scutum (Fig. 28A) ......L. clematisellum (Cockerell, 1904)

- Distinct tomentum present on T2 basolaterally, T3 basally, and T4 throughout (Fig. 22D); scutum hairs mostly densely plumose; anteromedial scutum punctures usually as distinct as those elsewhere on scutum (Fig. 22A) L. clastipedion sp. nov.

26. Mesepisternum rugulose or areolate with punctures absent or crowded and indistinct, at least in part (Fig. 108A-B).....

- Mesepisternum smooth with distinctly separated, round punctures throughout (Fig. 108C).......... 33

27. Supraclypeal area tessellate (Fig. 101A); postgena usually dull and lineate (Fig. 102A).............. 28

- Supraclypeal area (Fig. 101B) and postgena (Fig. 102B) mostly smooth and shiny ...................... 29

28. Scutum punctures dense but mostly distinctly separated; face broad (length/width ratio $\sim 0.82$ ); clypeus apical margin black or brown

L. pictum (Crawford, 1902)

- Scutum punctures very dense, almost all touching; face long (length/width ratio $\sim 0.85$ ); clypeus apical margin sometimes orange. L. arenisaltans sp. nov. (in part)

29. Ocelli slightly enlarged (separated by less than two thirds of one ocellar diameter) (Fig. 103A); mesepisternum mostly rugose to rugulose with few or no distinct punctures (Fig. 108A) ............. 30

- Ocelli normal (separated by more than two thirds of one ocellar diameter) (Fig. 103B); mesepisternum usually distinctly punctate at least in ventral half (Fig. 108B) 
30. T2-4 with extensive dense tomentum on basolateral areas of T2-3 and throughout T4 (Fig. 36D); clypeus apical margin black to reddish brown; legs and metasoma mostly reddish brown...

L. eophilus (Ellis, 1914) (in part)

- T2-4 with tomentum very sparse or absent (Fig. 38D); clypeus apical margin, legs, and metasoma often extensively orange

L. festinum sp. nov.

31. Pronotal angle usually about 90 degrees (Fig. 107A); dark spiracular spots present on T3-4 only (Fig. 105B); apical impressed areas of T2-3 usually shiny and distinctly punctate; clypeus with weak, obtuse apicolateral denticles (Fig. 3B).....

L. hudsoniellum (Cockerell, 1919) (in part)

- Pronotal angle obtuse (Fig. 107B); dark spiracular spots present on T2-4 (Fig. 105A); apical impressed area of T3 and sometimes T2 coriarious with punctures obscure; clypeus with strong, acute apicolateral denticles (Fig. 3A)

32. Frons punctures fine and dense but distinctly separated (Fig. 94B); T2-4 usually with extensive dense tomentum on basolateral areas of T2-3 and throughout T4 (Fig. 55C); scutum relatively long (length/width ratio usually $\geq 0.84$ )

L. mesillense (Cockerell, 1898) (in part)

- Frons punctures coarse and crowded (Fig. 94A); T2-4 with sparse tomentum limited to basal margins of T2-3 and basal half of T4 (Fig. 64D); scutum relatively broad (length/width ratio usually $\leq 0.8)$.

L. minckleyi sp. nov.

33. Hypoepimeron and upper mesepisternum densely punctate $(\mathrm{i}<1 \mathrm{pd})$; surface usually shiny........ 34

- Hypoepimeron and upper mesepisternum relatively sparsely punctate with many punctures separated by 1 puncture diameter or more, or if somewhat dense, then surface entirely imbricate.

34. Dark spiracular spots present on T3-4 only (Fig. 105B); apical impressed areas of T2-3 usually shiny and distinctly punctate

- Dark spiracular spots present on T2-4 (Fig. 105A); apical impressed area of T3 and sometimes T2 coriarious with punctures obscure 36

35. T2-3 with dense tomentum forming complete lateral and basal bands and apical band on T3 (Fig. 1); pronotal angle about 90 degrees (Fig. 107A); metasoma colour pattern highly variable

L. hudsoniellum (Cockerell, 1919) (in part)

- T2-3 with tomentum absent or limited to sparse lateral patches and apical band on T3 (Fig. 78D); pronotal angle obtuse (Fig. 107B); metasoma red-orange with basal half of T1, median transverse band on T3, and T4-5 usually brown (Fig. 78D)

L. spivakae sp. nov. (in part)

36. Hypoepimeron and upper mesepisternum punctures usually finer, more crowded, and less distinct than lower mesepisternum punctures (Fig. 108B); frons punctures uniformly fine and distinctly separated (Fig. 55D) .

L. mesillense (Cockerell, 1898) (in part)

- Hypoepimeron and mesepisternum punctures uniformly large and distinctly separated (Fig. 108C); frons punctures larger, becoming crowded medially (Fig. 43B)

L. imbriumbrae sp. nov.

37. Pronotal ridge sharply carinate along full height of pronotum (Fig. 109A); scutum and scutellum shiny and very sparsely punctate $(i=1-6$ pd); metapostnotum mostly shiny with rugae reaching halfway or less to posterior margin.

L. kunzei (Cockerell, 1898)

- Pronotal ridge absent (Fig. 109B); scutum and scutellum more extensively tessellate and densely punctate ( $\mathrm{i}=1-4 \mathrm{pd})$; metapostnotum dull with rugae usually reaching more than halfway to posterior margin

38. Acarinarial fan well-developed with dense appressed hairs, at least laterally (Fig. 95C); postgena usually dull and lineolate throughout (Fig. 102A).

- Acarinarial fan weak, with only a few sparse appressed hairs (Figs 95B, 96B); postgena usually smooth and shiny in apical half or more (Fig. 102B). 
39. Head, mesosoma, and $\mathrm{T} 1$ anterior surface mostly shiny; metasomal terga sometimes with metallic green reflections.

L. zephyrus (Smith, 1853)

- Head, mesosoma, and T1 anterior surface mostly dull; metasomal terga not metallic

L. decorum sp. nov.

40. T1 anterior surface coriarious (Fig. 95C); metasomal terga without tomentum (Fig. 13D); pronotal angle obtuse (Fig. 107B)

L. austerum sp. nov.

- T1 anterior surface shiny (Fig. 95A); T2-4 with extensive dense tomentum on basolateral areas of T2-3 and throughout T4 (Fig. 85D); pronotal angle about 90 degrees (Fig. 107A)

L. torrens sp. nov.

\section{Key to males}

1. Tegula enlarged, reaching or nearly reaching scutellum, with inner posterior margin straight or concave, often punctate (Fig. 91A).

L. hunteri (Crawford, 1932) and similar species ${ }^{2}$

- Tegula ovoid, impunctate, and clearly not reaching scutellum (Fig. 91B)..... 2

2. Scutum uniformly densely punctate with all punctures separated by less than 1 puncture diameter (Fig. 110A)......

- Scutum with punctures separated by 1 puncture diameter or more, at least submedially (Fig. 110B)

3. T1 anterior surface with dense appressed hair (Fig. 111A) (east of Rocky Mountains) .....

L. vierecki (Crawford, 1904)

- T1 anterior surface with no appressed hair (all hair erect) (Fig. 111B) (Rocky Mountains and westward).

4. Face short (length/width ratio $\sim 0.8$ ); clypeus with broad apical yellow band; hind basitarsus about 5 times as long as broad.

L. aquilae (Cockerell, 1898) ${ }^{3}$

- Face long (length/width ratio $\sim 0.9$ or greater); clypeus apical margin black to reddish brown; hind basitarsus less than 4 times as long as broad....

5. F2-10 about 1.5 times as long as broad; T1 entirely shiny (mountains of California to British Columbia) L. tuolumnense Gibbs, 2009

- F2-10 nearly twice as long as broad; T1 at least faintly coriarious to reticulate (deserts of southern California to Nuevo Leon)

6. T1-3 coriarious to imbricate with punctures distinct throughout (Fig. 112A) (Baja California to Arizona)

L. petrellum (Cockerell, 1903)

- T1-3 becoming reticulate with punctures somewhat obscure apically (Fig. 112B) (New Mexico to Nuevo Leon).....

7. Clypeus metallic blue-green all the way to the apical rim, sparsely punctate ( $i=1-5 \mathrm{pd}$ ), and with tomentum sparse or absent at least in part (Fig. 113A)

- Clypeus black, brown, or yellow in apical sixth or more, densely punctate $(\mathrm{i}<1 \mathrm{pd})$, and/or surface completely obscured by dense tomentum (Fig. 113B)

\footnotetext{
${ }^{2}$ L. parvum-tegulare species complex; not treated further herein. Specimens from desert and mountain regions are not likely true $L$. hunteri.

${ }^{3}$ Augochlora-like L. (Dialictus); not treated further herein.
} 
8. T1-6 with abundant tomentum and metallic blue-green at least on base of T1 (Fig. 76D); metasomal sterna with very long hair (2-4 OD); gonostylus very hairy and projecting laterally beyond gonocoxite (Fig. 90M)

L. rufornatum sp. nov.

- T1-6 with tomentum sparse or absent and without metallic reflections (Figs 59D, 61D); metasomal sterna with shorter hair (1-2 OD); gonostylus with sparse hair and not reaching lateral edge of gonocoxite (Fig. 90H-I)

9. Rims of T1-3 punctate (Fig. 62D); face with no tomentum; scutum usually mostly shiny and sparsely punctate (Fig. 62A) ( $\mathrm{i}=1-4 \mathrm{pd})$ (Utah to California)

L. miltolepoides sp. nov.

- Rims of T1-3 impunctate (Fig. 59D); face below eye emargination with sparse tomentum; scutum usually mostly tessellate and very sparsely punctate ( $i=1-6$ pd) (Fig. 59A) (Yucatan Peninsula).....

L. meteorum sp. nov.

10. Scutum between parapsidal lines entirely tessellate to finely reticulate and sparsely punctate (Fig. 93A) $(i=1-4$ pd) (Mexico).

L. tessellatosum sp. nov.

- Scutum between parapsidal lines shiny and/or densely punctate $(\mathrm{i} \leq 1 \mathrm{pd})$, at least in part (Figs 93C, 110B) (widespread).

11. T1-4 convex in lateral view, with rims strongly downcurved (Fig. 114A)....... L. cactorum sp. nov.

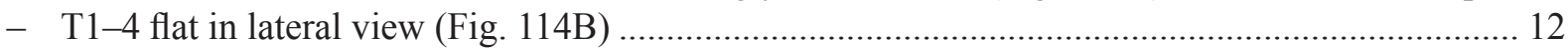

12. Antennae relatively long (as seen from below, length of F2 about twice length of F1, and F2-11 $1.5-2$ times as long as broad) (Fig. 115A).....

- Antennae relatively short (as seen from below, length of F2 less than twice length of F1, and F2-11 $1-1.5$ times as long as broad) (Fig. 115B).....

13. Clypeus apical half yellow; face short (length/width ratio $\sim 0.81$ ); retrorse lobe very narrow (Fig. 900)

L. testaceum (Robertson, 1897)

- Clypeus with apical yellow band absent or, if present, usually much less than half length of clypeus (if one third length of clypeus, then face long (length/width ratio $\sim 0.89$ )); retrorse lobe broad ..... 14

14. S2-5 with dense plumose hair concentrated on apicolateral portions of S3 and lateral margins of S4-5; hair of S2 thinner and sparser and S4-5 bare apicomedially (Fig. 116A).

L. zephyrus (Smith, 1853)

- S2-5 with hairs of uniform density and plumosity throughout (Fig. 116B).....

15. Clypeus orange to yellow apically (if covered in tomentum, best seen in ventral view); pronotal lobe orange to yellow

- Clypeus black to brown apically (if covered in tomentum, best seen in ventral view); pronotal lobe usually black to brown.

16. Head ovoid, face long (length/width ratio $\sim 0.89$ ) (Fig. 9C).

L. arenisaltans sp. nov.

- Head round, face short (length/width ratio $\sim 0.84-0.86$ ) (Figs 39C, 56B).

17. Face below eye emargination with dense tomentum obscuring the surface underneath (Fig. 56B); rims of T2-3 with distinct punctures in median third or less (Fig. 56D); pronotal collar and metepisternum dark.

L. mesillense (Cockerell, 1898)

- Face below eye emargination with sparse tomentum not obscuring the surface underneath (Fig. 39C); rims of T2-3 distinctly punctate nearly all the way to the lateral edge (Fig. 39D); pronotal collar and metepisternum sometimes with orange patches

L. festinum sp. nov. 
18. Tomentum sparse or absent on clypeus and supraclypeal area; metasoma partially red or metallic green; forewing with three submarginal cells.....

- Face below eye emargination entirely covered in dense tomentum; metasoma entirely black to brown; forewing sometimes with two submarginal cells

19. Propodeum dorsolateral slope rugose; frons, clypeus, and supraclypeal area with at least some tomentum; metasomal terga not metallic (sandy areas east of California).

L. pictum (Crawford, 1902) (in part)

- Propodeum dorsolateral slope shiny and distinctly punctate; face with tomentum restricted to lower paraocular area; metasomal terga partially metallic green (especially base of T1) (mountains of California and western Nevada).....

L. cembrilacus sp. nov.

20. Head sparsely punctate (frons and paraocular area with $i \geq 1$ pd and ocellocular area with $\mathrm{i} \geq 2 \mathrm{pd}$ ) (Fig. 94D); propodeum lateral face usually smooth and tessellate; forewing almost always with two submarginal cells; size small (body length $\sim 3.5 \mathrm{~mm}$, intertegular distance $\sim 0.65 \mathrm{~mm}$ ).

L. perditum sp. nov. (in part)

- Head more densely punctate (frons and paraocular area with $\mathrm{i} \leq 1 \mathrm{pd}$ and ocellocular area with $\mathrm{i} \leq 1.5 \mathrm{pd}$ ) (Fig. 94C); propodeum lateral face usually mostly rugulose; forewing often with three submarginal cells; usually larger (body length $\sim 4 \mathrm{~mm}$ or more, intertegular distance $\sim 0.75 \mathrm{~mm}$ or more).

21. Scutum and metasomal terga usually with many thin, weakly plumose hairs; T2 -3 with no basolateral tomentum (Fig. 117A)

L. clematisellum (Cockerell, 1904)

- Scutum and metasomal terga with most hairs thick and densely plumose; T2-3 usually with some basolateral tomentum (but often sparse) (Fig. 117B) .....

22. Postgena entirely dull and lineolate (Fig. 102A); posterior face of propodeum usually rugulose (Utah and northern Arizona) L. clastipedion sp. nov.

- Postgena weakly lineate and usually shiny in part (Fig. 102B); posterior face of propodeum shiny and distinctly punctate (eastern Arizona, New Mexico, and Chihuahua).

L. julipile sp. nov. (in part)

23. Metasomal terga with no tomentum; mesonotum and metasomal terga with most hairs thin and simple or weakly plumose (Fig. 117A)...

- Metasomal terga with at least some basolateral tomentum; mesonotum and metasomal terga with most hairs densely plumose and thick (Fig. 117B).....

24. Propodeum dorsolateral slope rugose

L. pictum (Crawford, 1902) (in part)

- Propodeum dorsolateral slope smooth....

25. Mesepisternum and hypoepimeron densely punctate $(\mathrm{i}<1 \mathrm{pd})$

- Mesepisternum and hypoepimeron sparsely punctate $(\mathrm{i} \geq 1 \mathrm{pd})$

26. Propodeum with weak microsculpture, appearing somewhat shiny; rims of $\mathrm{T} 1-3$ punctate basally (Fig. 65D); apical margin of clypeus and labrum usually orange

L. minckleyi sp. nov.

- Propodeum with strong dull microsculpture; rims of T1-3 almost completely impunctate (Fig. 44D); apical margin of clypeus and labrum reddish brown....

L. imbriumbrae sp. nov. (in part)

27. Rims of T1-3 impunctate (Fig. 32D) (southern Mexico)

L. decorum sp. nov.

- Rims of T1-3 punctate, at least basomedially (Fig. 14D) (Baja California peninsula)...... 
28. Pronotal ridge carinate (Fig. 109A); scutum and mesepisternum very finely, sparsely punctate $(\mathrm{i}=1-6 \mathrm{pd})$

- Pronotal ridge smoothly rounded (Fig. 109B); scutum and/or mesepisternum more densely punctate $(\mathrm{i} \leq 2 \mathrm{pd})$

29. Rim of S5 concave (Fig. 116C) L. hudsoniellum (Cockerell, 1919)

- Rim of S5 straight (Fig. 116B) 30

30. Antennae clavate (F7-11 about 1.3 times as wide as basal antenna segments) (Fig. 26C) L. clavicorne sp. nov.

- Antennae normal (all segments about equally wide) 31

31. Rims of T1-3 opaque with punctures as dense and distinct as those on discs and covering entire surface (Fig. 79D) L. spivakae sp. nov.

- Rims of T1-3 usually translucent, punctures finer and sparser than those on discs and/or restricted to basomedial area .....

32. Wings with membrane lightly infuscated, veins brown, and most hairs dark (Fig. 100B)

- Wings with membrane hyaline, veins light brown to amber contrasting with dark brown subcosta, and most hairs light (Fig. 100A)...

33. T1-5 with extensive tomentum; metasoma often partially or entirely orange

- Metasomal terga with tomentum limited to small basolateral patches on T2-4; metasoma all black.

34. Face long (length/width ratio $\sim 0.89$ ); clypeus usually sparsely punctate ( $i=1-4 \mathrm{pd}$ ), often with a broad orange apical band (Fig. 52C)

L. lilianae sp. nov.

- Face short (length/width ratio $~ 0.82$ ); clypeus moderately densely punctate (i=1-2 pd) without orange apical band (Fig. 68C) L. pallidellum (Ellis, 1914)

35. Head sparsely punctate (frons and paraocular area with $\mathrm{i} \geq 1$ pd and ocellocular area with $\mathrm{i} \geq 2$ pd) (Fig. 94D); propodeum lateral face usually smooth and tessellate; forewing almost always with two submarginal cells; size small (body length $\sim 3.5 \mathrm{~mm}$, intertegular distance

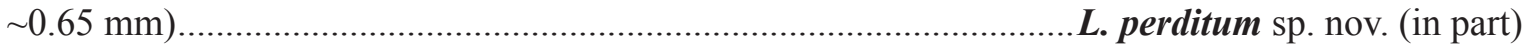

- Head more densely punctate (frons and paraocular area with $\mathrm{i} \leq 1 \mathrm{pd}$ and ocellocular area with $\mathrm{i} \leq 1.5 \mathrm{pd}$ ) (Fig. 94C); propodeum lateral face usually mostly rugulose; forewing often with three submarginal cells; slightly larger (body length $\sim 4 \mathrm{~mm}$, intertegular distance $\sim 0.77 \mathrm{~mm}$ ).

L. julipile sp. nov. (in part)

\section{Discussion}

This work is only the second attempt at a comprehensive taxonomic study of a well-defined group of $L$. (Dialictus) which includes the southwestern United States and northern Mexico. The first such study only dealt with a small complex of five species (Gibbs 2009b), while prior taxonomic work was opportunistic in nature and had virtually stopped by 1938. It is not surprising, then, that many new species and new distribution records should be discovered. Prior to the current work, 17 red-tailed species of L. (Dialictus) were known from the western Nearctic region. This work more than doubles the count, even after synonymizing L. mesillense, L. clarissimum, and L. perexiguum. Twenty new species are described, bringing the total number of western red-tailed L. (Dialictus) species to 35 (39 including four species from the eastern United States). This number may increase with additional study. Lasioglossum 
hunteri, a red-tailed member of the L. parvum-tegulare species complex, was not thoroughly revised in this study because it will be included in a forthcoming revision of that group. Preliminary data suggests that specimens historically identified as L. hunteri may comprise as many as five distinct species.

Fifteen species are newly described or recorded from the United States, fourteen from Mexico, and one from Guatemala. This is an especially significant contribution to the Mexican fauna: prior to this work, only $31 \mathrm{~L}$. (Dialictus) species (of any colour of metasoma) were recorded from the entire country (Ascher \& Pickering 2020). In addition, some old Mexican records were previously doubtful (L. mesillense has been frequently misidentified, and the only Mexican record of L. hudsoniellum was based on a low-resolution photograph posted to the iNaturalist website) and are here verified. This brings the total number of $L$. (Dialictus) of Mexico up to 45. However, at least seven of these Mexican records (L. aquilae, L. aspasia, L. aurora (Smith, 1879), L. biseptum (Vachal, 1904), L. cyanorugosum Engel et al., 2007, L. oaxacacola Engel et al., 2007, and L. terginum (Vachal, 1904)) are not believed to be 'true' L. (Dialictus).

Several interesting avenues for further research are suggested by the current work. Widespread and variable species such as L. hudsoniellum, L. mesillense, and L. pallidellum may be repositories of additional diversity and warrant deeper taxonomic study. For some, such as L. pallidellum, there were insufficient specimens available for study (particularly fresh ones suitable for DNA extraction), and its status as one or multiple species could be investigated simply by additional collecting and DNA barcoding (Gibbs 2009a, 2009b; Williams et al. 2012; Landaverde-González et al. 2017). For others, especially in cases where the DNA barcode gap is lacking (such as L. hudsoniellum), the use of additional molecular data such as ultraconserved elements (Faircloth et al. 2012; Pierce et al. 2017) may be needed.

The conservation status of $L$. cembrilacus sp. nov. should be assessed; about $95 \%$ of known specimens were collected before 2000, the last ones in 2005. As a high elevation habitat specialist, it may be threatened by shrinking habitat caused by climate change, but more recent evidence is needed. The scarcity of records in the last 20 years may be due to a lack of collecting effort in the Sierra Nevada range. Rare species with small distributions, such as L. argammon sp. nov., L. clavicorne sp. nov., L. imbriumbrae sp. nov., and L. perditum sp. nov., are also worth assessing.

The species pair L. kunzei and L. semibrunneum appear to have recently speciated due to allopatry. They would be an interesting case study in population genetics and speciation. The huge variation in head size and morphology within L. kunzei and L. semibrunneum suggests complex social behaviour with castebased allometry, as seen in L. (D.) umbripenne (Ellis, 1913) (Wille \& Orozco 1970). Halictus ligatus Say, 1837 has a similar head morphology to L. kunzei and L. semibrunneum, including genal protrusions in the largest individuals, and unusually aggressive intraspecific behaviour (Pabalan et al. 2000). Lasioglossum kunzei and L. semibrunneum might be similarly aggressive. Intriguingly, the enlarged head and mandibles, smooth surface sculpture, and especially the carinate pronotal ridge, are features more characteristic of socially parasitic L. (Dialictus) (Wcislo 1997; Gibbs 2010, 2011), yet L. kunzei and L. semibrunneum have well-developed nest-building and pollen-collecting structures.

The pollen-foraging preferences of L. lilianae sp. nov. should be investigated to determine if it is truly oligolectic on Mentzelia or not. Oligolecty is known in L. (Chilalictus) (Walker 1995), L. (Hemihalictus) (Daly 1961; Pesenko et al. 2000), L. (Lasioglossum) (Pesenko et al. 2000), and L. (Sphecodogastra) (McGinley 2003), but has never been demonstrated in L. (Dialictus). The "narrowly polylectic" species studied by Hurd (1970) was eventually synonymized with the widespread and broadly polylectic L. incompletum (Crawford, 1907).

The species described here occupy the full range of habitats in the western Nearctic, from hot deserts to cold mountaintops, which would allow for a wide range of nesting and social behaviours worthy of 
investigation. This revision should lay the necessary taxonomic foundation to begin this work, and gain a better understanding of one of the world's bee biodiversity hotspots.

\section{Acknowledgments}

We are indebted to Katherine Morgan (University of Manitoba), who spent many hours helping to collect morphometric measurements. We wish to thank all the museum curators and bee researchers (listed in Methods) who hosted us during physical visits to collections, and/or loaned us specimens.

The lead author was supported by the University of Manitoba Graduate Fellowship and the Douglas L. Campbell Graduate Fellowship. We acknowledge the financial support of the Natural Sciences and Engineering Research Council of Canada (NSERC), RGPIN-2018-05353 (awarded to the second author) and USDA National Institute of Food and Agriculture award 2017-68004-26323 (PI: R. Isaacs).

\section{References}

Allred D.M. 1969. Bees of the Nevada test site. Great Basin Naturalist 29 (1): 20-24.

Ascher J.S. 2020. Digital Bee Collections Network, 2014 (and updates). Ver. 13 May 2020. National Science Foundation grant DBI 0956388; contact: Ascher J.S. (dbsajs@nus.edu.sg).

Ascher J.S. \& Pickering J. 2020. Discover Life Bee Species Guide and World Checklist (Hymenoptera: Apoidea: Anthophila).

Available from http://www.discoverlife.org/mp/20q?guide=Apoidea_species [accessed 3 Apr. 2020].

Ayala R., Griswold T.L. \& Bullock S.H. 1993. The Native Bees of Mexico. In: Ramamoorthy T.P., Bye R., Lot A. \& Fa J. (eds) Biological Diversity of Mexico: Origins and Distribution: 179-227. Oxford University Press, New York.

Ballare K.M., Neff J.L., Ruppel R. \& Jha S. 2019. Multi-scalar drivers of biodiversity: local management mediates wild bee community response to regional urbanization. Ecological Applications 29 (3): e01869. https://doi.org/10.1002/eap.1869

Batáry P., Báldi A., Sárospataki M., Kohler F., Verhulst J., Knop E., Herzog F. \& Kleijn D. 2010. Effect of conservation management on bees and insect-pollinated grassland plant communities in three European countries. Agriculture, Ecosystems, and Environment 136: 35-39.

https://doi.org/10.1016/j.agee.2009.11.004

Batra S.W.T. 1964. Behavior of the social bee, Lasioglossum zephyrum, within the nest. Insectes Sociaux 11 (2): 159-186. https://doi.org/10.1007/BF02222935

Batra S.W.T. 1965. Organisms associated with Lasioglossum zephyrum (Hymenoptera: Halictidae). Journal of the Kansas Entomological Society 38 (4): 367-389.

Batra S.W.T. 1966. Life cycle and behavior of the primitively social bee, Lasioglossum zephyrum. University of Kansas Science Bulletin 46 (10): 359-423.

Batra S.W.T. 1987. Ethology of the vernal eusocial bee, Dialictus laevissimus (Hymenoptera: Halictidae). Journal of the Kansas Entomological Society 60 (1): 100-108.

Brown D.K. \& Kaul R.B. 1981. Floral structure and mechanism in Loasaceae. American Journal of Botany 68 (3): 361-372. https://doi.org/10.1002/j.1537-2197.1981.tb06373.x

Brown S.D.J., Collins R.A., Boyer S., Lefort M.C., Malumbres-Olarte J., Vink C.J. \& Cruickshank R.H. 2012. SPIDER: an R package for the analysis of species identity and evolution, with particular reference to DNA barcoding. Molecular Ecology Resources 12 (3): 562-565.

https://doi.org/10.1111/j.1755-0998.2011.03108.x 
Buri P., Humbert J.-Y. \& Arlettaz R. 2014. Promoting pollinating insects in intensive agricultural matrices: field-scale experimental manipulation of hay-meadow mowing regimes and its effects on bees. PLoS ONE 9 (1): e85635. https://doi.org/10.1371/journal.pone.0085635

Campbell J.W., Hanula J.L. \& Waldrop T.A. 2007. Effects of prescribed fire and fire surrogates on floral visiting insects of the blue ridge province in North Carolina. Biological Conservation 134: 393-404. https://doi.org/10.1016/j.biocon.2006.08.029

Cockerell T.D.A. 1898a. On some small bees from Arizona. The Canadian Entomologist 30 (9): 237239. https://doi.org/10.4039/Ent30237-9

Cockerell T.D.A. 1898b. Tables for the determination of New Mexico bees. Bulletin of the Scientific Laboratories of Denison University 11 (3): 41-73.

Cockerell T.D.A. 1903. New bees from southern California and other records. Bulletin of the Southern California Academy of Sciences 2: 84-94.

Cockerell T.D.A. 1904. Records of American bees. The Canadian Entomologist 36 (1): 13-14.

Cockerell T.D.A. 1905. Notes on some bees in the British Museum. Transactions of the American Entomological Society 31 (4): 309-364.

Cockerell T.D.A. 1906. The bees of Florissant, Colorado. Bulletin of the American Museum of Natural History 22: 419-455.

Cockerell T.D.A. 1916. The bees of the Coronado Islands. The Canadian Entomologist 48 (2): 54-58. https://doi.org/10.4039/Ent4854-2

Cockerell T.D.A. 1919. The bees of the Rocky Mountain National Park (Hymenop.). Entomological News, and Proceedings of the Entomological Section of the Academy of Natural Sciences of Philadelphia 30: 286-294.

Crane S. 2013. DNA Extraction from Archival Museum Insect Specimens.

Available from https://doi.org/10.6084/m9.figshare.741214.v4 [accessed 22 Oct. 2020].

Crawford J.C. 1902. Notes and descriptions of bees. The Canadian Entomologist 34 (9): 234-240. https://doi.org/10.4039/Ent34234-9

Crawford J.C. 1904. Two new Halictus from New Jersey. Entomological News, and Proceedings of the Entomological Section of the Academy of Natural Sciences of Philadelphia 15 (3): 97-99.

Creedy T.J., Norman H., Tang C.Q., Qing Chin K., Andujar C., Arribas P., O’Connor R.S., Carvell C., Notton D.G. \& Vogler A.P. 2020. A validated workflow for rapid taxonomic assignment and monitoring of a national fauna of bees (Apiformes) using high throughput DNA barcoding. Molecular Ecology Resources 20 (1): 40-53. https://doi.org/10.1111/1755-0998.13056

Daly H.V. 1961. Biological observations on Hemihalictus lustrans, with a description of the larva (Hymenoptera: Halictidae). Journal of the Kansas Entomological Society 34 (3): 134-141.

Danforth B.N., Conway L. \& Shuqing J. 2003. Phylogeny of eusocial Lasioglossum reveals multiple losses of eusociality within a primitively eusocial clade of bees (Hymenoptera: Halictidae). Systematic Biology 52 (1): 23-36. https://doi.org/10.1080/10635150390132687

DeSalle R., Egan M.G. \& Siddall M. 2005. The unholy trinity: taxonomy, species delimitation and DNA barcoding. Philosophical Transactions of the Royal Society of London Series B-Biological Sciences 360 (1462): 1905-1916. https://doi.org/10.1098/rstb.2005.1722

Dibble A.C., Drummond F.A., Stubbs C., Veit M. \& Ascher J.S. 2017. Bees of Maine, with a state species checklist. Northeastern Naturalist 24 (m15): 1-48. https://doi.org/10.1656/045.024.m1503 
Donovall L.R. \& van Engelsdorp D. 2010. A checklist of the bees (Hymenoptera: Apoidea) of Pennsylvania. Journal of the Kansas Entomological Society 83 (1): 7-24.

https://doi.org/10.2317/JKES808.29.1

Droege S., Tepedino V.J., LeBuhn G., Link W., Minckley R.L., Chen Q. \& Conrad C. 2010. Spatial patterns of bee captures in North American bowl trapping surveys. Insect Conservation and Diversity 3: 15-23. https://doi.org/10.1111/j.1752-4598.2009.00074.x

Ducke A. 1902. Ein neues Subgenus von Halictus Latr. Zeitschrift für Systematische Hymenopterologie und Dipterologie 2: 102-103.

Ebmer A.W. 2002. Asiatische Halictidae-10. Neue Halictidae aus China sowie diagnostische Neubeschreibungen der von Fan and Ebmer 1992 beschriebenen Lasioglossum-Arten (Insecta: Hymenoptera: Apoidea: Halictidae: Halictinae). Linzer Biologische Beiträge 34 (2): 819-934.

Eickwort G.C. 1986. First steps into eusociality: the sweat bee Dialictus lineatulus. Florida Entomologist 69 (4): 742-754. https://doi.org/10.2307/3495222

Ellis M.D. 1914a. New American bees of the genus Halictus (Hym.). Entomological News, and Proceedings of the Entomological Section of the Academy of Natural Sciences of Philadelphia 25 (4): 151-155.

Ellis M.D. 1914b. New bees of the genus Halictus (Hym.) from United States, Guatemala and Ecuador. Journal of the New York Entomological Society 22 (3): 218-223.

Engel M.S., Hinojosa-Díaz I.A. \& Yáñez-Ordóñez O. 2007. The Augochlora-like Dialictus from Guatemala and southern Mexico (Hymenoptera: Halictidae). Acta Zoológica Mexicana 23 (3): 125-134.

Faircloth B.C., McCormack J.E., Crawford N.G., Harvey M.G., Brumfield R.T. \& Glenn T.C. 2012. Ultraconserved elements anchor thousands of genetic markers spanning multiple evolutionary timescales. Systematic Biology 61 (5): 717-726. https://doi.org/10.1093/sysbio/sys004

FAO/UNESCO. 2007. Digital Soil Map of the World. Ver. 3.6. FAO, Rome.

Fick S.E. \& Hijmans R.J. 2017. WorldClim 2: new 1-km spatial resolution climate surfaces for global land areas. International Journal of Climatology 37 (12): 4302-4315. https://doi.org/10.1002/joc.5086

Folmer O., Black M., Hoeh W., Lutz R. \& Vrijenhoek R. 1994. DNA primers for amplification of mitochondrial cytochrome $c$ oxidase subunit I from diverse metazoan invertebrates. Molecular Marine Biology and Biotechnology 3 (5): 294-299.

Françoso E. \& Arias M.C. 2013. Cytochrome c oxidase I primers for corbiculate bees: DNA barcode and mini-barcode. Molecular Ecology Resources 13: 844-850. https://doi.org/10.1111/1755-0998.12135

Frankie G.W., Thorp R.W., Schindler M., Hernandez J., Ertter B. \& Rizzardi M. 2005. Ecological patterns of bees and their host ornamental flowers in two northern California cities. Journal of the Kansas Entomological Society 78 (3): 227-246. https://doi.org/10.2317/0407.08.1

Galbraith S.M., Cane J.H., Moldenke A.R. \& Rivers J.W. 2019. Wild bee diversity increases with local fire severity in a fire-prone landscape. Ecosphere 10 (4): e02668. https://doi.org/10.1002/ecs2.2668

Geroff R.K., Gibbs J. \& McCravy K.W. 2014. Assessing bee (Hymenoptera: Apoidea) diversity of an Illinois restored tallgrass prairie: methodology and conservation considerations. Journal of Insect Conservation 18: 951-964. https://doi.org/10.1007/s10841-014-9703-z

Gibbs J. 2009a. Integrative taxonomy identifies new (and old) species in the Lasioglossum (Dialictus) tegulare (Robertson) species group (Hymenoptera, Halictidae). Zootaxa 2032 (1): 1-38.

https://doi.org/10.11646/zootaxa.2032.1.1 
Gibbs J. 2009b. New species in the Lasioglossum petrellum species group identified through an integrative taxonomic approach. The Canadian Entomologist 141: 371-396.

https://doi.org/10.4039/n09-020

Gibbs J. 2010. Revision of the metallic species of Lasioglossum (Dialictus) in Canada (Hymenoptera, Halictidae, Halictini). Zootaxa 2591: 1-382. https://doi.org/10.11646/zootaxa.2591.1.1

Gibbs J. 2011. Revision of the metallic Lasioglossum (Dialictus) of eastern North America (Hymenoptera: Halictidae: Halictini). Zootaxa 3073 (1): 1-216. https://doi.org/10.11646/zootaxa.3073.1.1

Gibbs J. 2018a. Bees of the genus Lasioglossum (Hymenoptera: Halictidae) from Greater Puerto Rico, West Indies. European Journal of Taxonomy 400: 1-57. https://doi.org/10.5852/ejt.2018.400

Gibbs J. 2018b. DNA barcoding a nightmare taxon: assessing barcode index numbers and barcode gaps for sweat bees. Genome 61 (1): 21-31. https://doi.org/10.1139/gen-2017-0096

Gibbs J. 2020. Hylaeus (Hylaeana) dominicalis, a new species and the first colletid bee recorded from Dominica, Lesser Antilles. Journal of Melittology 93: 1-6.

Gibbs J., Albert J. \& Packer L. 2012a. Dual origins of social parasitism in North American Dialictus (Hymenoptera: Halictidae) confirmed using a phylogenetic approach. Cladistics 28: 195-207. https://doi.org/10.1111/j.1096-0031.2011.00373.x

Gibbs J., Brady S.G., Kanda K. \& Danforth B.N. 2012b. Phylogeny of halictine bees supports a shared origin of eusociality for Halictus and Lasioglossum (Apoidea: Anthophila: Halictidae). Molecular Phylogenetics and Evolution 65: 926-939. https://doi.org/10.1016/j.ympev.2012.08.013

Gibbs J., Packer L., Dumesh S. \& Danforth B.N. 2013. Revision and reclassification of Lasioglossum (Evylaeus), L. (Hemihalictus), and L. (Sphecodogastra) in eastern North America (Hymenoptera: Apoidea: Halictidae). Zootaxa 3672 (1): 1-117. https://doi.org/10.11646/zootaxa.3672.1.1

Gibbs J., Ascher J.S., Rightmyer M.G. \& Isaacs R. 2017. The bees of Michigan (Hymenoptera: Apoidea: Anthophila), with notes on distribution, taxonomy, pollination, and natural history. Zootaxa 4352 (1): 1-160. https://doi.org/10.11646/zootaxa.4352.1.1

Glad J.B. 1975. Taxonomy and Ecology of Mentzelia mollis Peck and Related Species. MSc thesis, Oregon State University.

Gonçalves R.B. \& Oliviera P.S. 2013. Preliminary results of bowl trapping bees (Hymenoptera, Apoidea) in a southern Brazil forest fragment. Journal of Insect Biodiversity 1 (2): 1-9.

https://doi.org/10.12976/jib/2013.1.2

Gonzalez V.H., Osborn A.L., Brown E.R., Pavlick C.R., Enríquez E., Tscheulin T., Petanidou T., Hranitz J.M. \& Barthell J.F. 2020. Effect of pan trap size on the diversity of sampled bees and abundance of bycatch. Journal of Insect Conservation 24: 1-12. https://doi.org/10.1007/s10841-020-00224-4

Grab H., Branstetter M.G., Amon N., Urban-Mead K.R., Park M.G., Gibbs J., Blitzer E.J., Poveda K., Loeb G. \& Danforth B.N. 2019. Agriculturally dominated landscapes reduce bee phylogenetic diversity and pollination services. Science 363 (6424): 282-284. https://doi.org/10.1126/science.aat6016

Graenicher S. 1910. Wisconsin bees-new and little-known species. The Canadian Entomologist 42 (5): 157-160. https://doi.org/10.4039/Ent42157-5

Griffin S.R., Brunninga-Socolar B., Kerr M.A., Gibbs J. \& Winfree R. 2017. Wild bee community change over a 26-year chronosequence of restored tallgrass prairie. Restoration Ecology 25 (4): 650660. https://doi.org/10.1111/rec.12481

Harris R.A. 1979. Glossary of surface sculpturing. Occasional Papers in Entomology 28: 1-31. 
Heron J. \& Sheffield C.S. 2015. First record of the Lasioglossum (Dialictus) petrellum species group in Canada (Hymenoptera: Halictidae). Journal of the Entomological Society of British Columbia 112: $88-91$.

Holmgren N.H. \& Holmgren P.K. 2002. New mentzelias (Loasaceae) from the intermountain region of western United States. Systematic Botany 27 (4): 747-762.

Hung K.-L.J., Ascher J.S. \& Holway D.A. 2017. Urbanization-induced habitat fragmentation erodes multiple components of temporal diversity in a Southern California native bee assemblage. PLOS ONE 12 (8): e0184136. https://doi.org/10.1371/journal.pone.0184136

Hurd P.D. 1970. A new, narrowly polylectic, autumnal species of Dialictus from the flowers of Jepsonia heterandra, an endemic California saxifrage (Hymenoptera: Apoidea). The Pan-Pacific Entomologist 46: 209-212.

Hurd P.D. 1979. Superfamily Apoidea. In: Krombein K.V., Hurd P.D., Smith D.R. \& Burks B.D. (eds) Catalog of Hymenoptera in America North of Mexico: 1741-2209. Smithsonian Institution Press, Washington, D.C.

International Commission of Zoological Nomenclature. 1997. Opinion 1882: Dialictus Robertson, 1902 and Chloralictus Robertson, 1902 (Insecta, Hymenoptera): given precedence over Paralictus Robertson, 1901. Bulletin of Zoological Nomenclature 54 (3): 201-202.

International Commission of Zoological Nomenclature. 1999. International Code of Zoological Nomenclature. The International Trust for Zoological Nomenclature, London.

Joshi N.K., Leslie T., Rajotte E.G., Kammerer M.A., Otieno M. \& Biddinger D.J. 2015. Comparative trapping efficiency to characterize bee abundance, diversity, and community composition in apple orchards. Annals of the Entomological Society of America 108 (5): 785-799.

https://doi.org/10.1093/aesa/sav057

Kalyaanamoorthy S., Minh B.Q., Wong T.K.F., von Haeseler A. \& Jermiin L.S. 2017. ModelFinder: Fast model selection for accurate phylogenetic estimates. Nature Methods 14: 587-589.

https://doi.org/10.1038/nmeth.4285

Kearse M., Moir R., Wilson A., Stones-Havas S., Cheung M., Sturrock S., Buxton S., Cooper A., Markowitz S., Duran C., Thierer T., Ashton B., Meintjes P. \& Drummond A. 2012. Geneious Basic: An integrated and extendable desktop software platform for the organization and analysis of sequence data. Bioinformatics 28 (12): 1647-1649. https://doi.org/10.1093/bioinformatics/bts199

Keeler K.H. 1981. Function of Mentzelia nuda (Loasaceae) postfloral nectaries in seed defense. American Journal of Botany 68 (2): 295-299. https://doi.org/10.1002/j.1537-2197.1981.tb12389.x

Klein A.-M., Vaissière B.E., Cane J.H., Steffan-Dewenter I., Cunningham S.A., Kremen C. \& Tscharntke T. 2007. Importance of pollinators in changing landscapes for world crops. Proceedings of the Royal Society B-Biological Sciences 274: 303-313. https://doi.org/10.1098/rspb.2006.3721

Knerer G. 1969. Synergistic evolution of halictine nest architecture and social behavior. Canadian Journal of Zoology 47: 925-930. https://doi.org/10.1139/z69-151

Knerer G. \& Atwood C.E. 1962. An annotated check list of the non-parasitic Halictidae (Hymenoptera) of Ontario. Proceedings of the Entomological Society of Ontario 92: 160-176.

Krijthe J.H. 2015. Rtsne: T-distributed Stochastic Neighbor Embedding using a Barnes-Hut Implementation. Available from https://rdrr.io/cran/Rtsne/man/Rtsne.html [accessed 16 Oct. 2020].

Krombein K.V. 1967. Superfamily Apoidea. In: Krombein K.V. \& Burks B.D. (eds) Hymenoptera of America North of Mexico-Synoptic Catalog, Second Supplement. United States Department of Agriculture, Washington, D. C. 
Kuhlman M. \& Burrows S. 2017. Checklist of bees (Apoidea) from a private conservation property in west-central Montana. Biodiversity Data Journal 5: e11506. https://doi.org/10.3897/BDJ.5.e11506

Landaverde-González P., Moo-Valle H., Murray T.E., Paxton R.J., Quezada-Euán J.J.G. \& Husemann M. 2017. Sympatric lineage divergence in cryptic Neotropical sweat bees (Hymenoptera: Halictidae: Lasioglossum). Organisms Diversity and Evolution 17 (1): 251-265.

https://doi.org/10.1007/s13127-016-0307-1

Lettow M.C., Brudvig L.A., Bahlai C.A., Gibbs J., Jean R.P. \& Landis D.A. 2018. Bee community responses to a gradient of oak savanna restoration practices. Restoration Ecology 26 (5): 882-890. https://doi.org/10.1111/rec.12655

Maddison W.P. \& Maddison D.R. 2019. Mesquite: a Modular System for Evolutionary Analysis. Ver. 3.61 .

Maechler M., Rousseeuw P., Struyf A., Hubert M. \& Hornik K. 2019. cluster: Cluster Analysis Basics and Extensions. Ver. R package ver. 2.1.0.

McGinley R.J. 2003. Studies of Halictinae (Apoidea: Halictidae), II: Revision of Sphecodogastra Ashmead, floral specialists of Onagraceae. Smithsonian Contributions to Zoology 610: 1-55.

Messinger-Carril O., Griswold T.L., Haefner J. \& Wilson J.S. 2018. Wild bees of Grand StaircaseEscalante National Monument: richness, abundance, and spatio-temporal beta-diversity. PeerJ 6: e5867. https://doi.org/10.7717/peerj.5867

Michener C.D. 1944. Comparative external morphology, phylogeny, and a classification of the bees (Hymenoptera). Bulletin of the American Museum of Natural History 82 (6): 151-326.

Michener C.D. 1951. Superfamily Apoidea. In: Muesebeck C.F.W., Krombein K.V. \& Townes H.K. (eds) Hymenoptera of America North of Mexico Synoptic Catalog: 1043-1255. United States Government Printing Office, Washington, D.C.

Michener C. D. 1960. Notes on the biology and supposed parthenogenesis of halictine bees from the Australian region. Journal of the Kansas Entomological Society 33 (2): 85-96.

Michener C.D. 1965. A classification of the bees of the Australian and South Pacific regions. Bulletin of the American Museum of Natural History 130: 1-362.

Michener C.D. 1966. The bionomics of a primitively social bee, Lasioglossum versatum (Hymenoptera: Halictidae). Journal of the Kansas Entomological Society 39 (2): 193-217.

Michener C.D. 1974. The Social Behavior of the Bees. The Belknap Press of Harvard University Press, Cambridge, MA.

Michener C.D. 1979. Biogeography of the bees. Annals of the Missouri Botanical Garden 66 (3): 277347. https://doi.org/10.2307/2398833

Michener C.D. 1990. Reproduction and castes in social halictine bees. In: Engels W. (ed.) Social Insects: An Evolutionary Approach to Castes and Reproduction: 77-121. Springer-Verlag, Berlin.

Michener C.D. 2007. The Bees of the World. The Johns Hopkins University Press, Baltimore, MD.

Michener C.D. \& Lange R.B. 1958. Observations on the behavior of Brasilian halictid bees. V. Chloralictus. Insectes Sociaux 5 (4): 379-407. https://doi.org/10.1007/BF02226855

Michener C.D. \& Wille A. 1961. The bionomics of a primitively social bee, Lasioglossum inconspicuum. University of Kansas Science Bulletin 42 (11): 1123-1202.

Michener C.D., Brothers D.J. \& Kamm D.R. 1971a. Interactions in colonies of primitively social bees: II, some queen-worker relations in Lasioglossum zephyrum. Journal of the Kansas Entomological Society 44 (2): 276-279. 
Michener C.D., Brothers D.J. \& Kamm D.R. 1971b. Interactions in colonies of primitively social bees: Artificial colonies of Lasioglossum zephyrum. Proceedings of the National Academy of Sciences 68 (6): 1241-1245. https://doi.org/10.1073/pnas.68.6.1241

Minckley R.L. 2008. Faunal composition and species richness differences of bees (Hymenoptera: Apiformes) from two north American regions. Apidologie 39 (1): 176-188.

https://doi.org/10.1051/apido:2007062

Mitchell T.B. 1960. Bees of the eastern United States, Volume I. North Carolina Agricultural Experiment Station Technical Bulletin 141: 1-538.

Mitchell T.B. 1962. Bees of the eastern United States, Volume II. North Carolina Agricultural Experiment Station Technical Bulletin 152: 1-557.

Morse R.A. 1960. The abundance of wild bees (Apoidea) in the northeastern United States. Journal of Economic Entomology 53 (4): 679-680. https://doi.org/10.1093/jee/53.4.679

Moure J.S. 1947. Novos agrupamentos genéricos e algumas espécies novas de abelhas sulamericanas. Museu Paranaense Publicações Avulsas 3: 1-37.

Moure J.S. 2001. Uma pequena abelha com cabeça e mandíbulas excepcionais (Hymenoptera, Halictidae). Revista Brasileira de Zoologia 18: 493-497.

Moure J.S. \& Hurd P.D. 1987. An Annotated Catalog of the Halictid Bees of the Western Hemisphere (Hymenoptera: Halictidae). Smithsonian Institution Press, Washington, D.C.

Murao R. \& Tadauchi O. 2011. Notes on colour variation of Lasioglossum (Evylaeus) politum pekingense (Hymenoptera, Halictidae). Japanese Journal of Systematic Entomology 17 (1): 55-58.

Oksanen J., Blanchet F.G., Friendly M., Kindt R., Legendre P., McGlinn D., Minchin P.R., O’Hara R.B., Simpson G.L., Solymos P., Stevens M.H.H., Szoecs E. \& Wagner H. 2018. Vegan: Community Ecology Package. Ver. 2.5.2.

Ollerton J., Winfree R. \& Tarrant S. 2011. How many flowering plants are pollinated by animals? Oikos 120: 321-326. https://doi.org/10.1111/j.1600-0706.2010.18644.x

Onuferko T.M., Skandilis D.A., León Cordero R. \& Richards M.H. 2017. Rapid initial recovery and long-term persistence of a bee community in a former landfill. Insect Conservation and Diversity 11 (1): 88-99. https://doi.org/10.1111/icad.12261

Pabalan N., Davey K.G. \& Packer L. 2000. Escalation of aggressive interactions during staged encounters in Halictus ligatus Say (Hymenoptera: Halictidae), with a comparison of circle tube behaviors with other halictine species. Journal of Insect Behavior 13 (5): 627-650. https://doi.org/10.1023/A:1007868725551

Packer L. 1992. The social organisation of Lasioglossum (Dialictus) laevissimum (Smith) in southern Alberta. Canadian Journal of Zoology 70: 1767-1774. https://doi.org/10.1139/z92-244

Packer L. 1993. Multiple-foundress associations in sweat bees. In: Keller L. (ed.) Queen Number and Sociality in Insects: 214-233. Oxford University Press, New York.

Packer L. 1994. Lasioglossum (Dialictus) tenax (Sandhouse) (Hymenoptera: Halictidae) as a solitary sweat bee. Insectes Sociaux 41: 309-313. https://doi.org/10.1007/BF01242302

Patenaude A. 2007. Diversity, Composition, and Seasonality of Wild Bees (Hymenoptera: Apoidea) in a Northern Mixed-grass Prairie Preserve. MSc thesis, University of Manitoba, Winnipeg, MB.

Pauly A. 1984. Classification des Halictidae de Madagascar et des îles voisines. I. Halictinae (Hymenoptera Apoidea). Verhandlungen der Naturforschenden Gesellschaft in Basel 94: 121-156. 
Pauly A., Gibbs J. \& Kuhlmann M. 2012. Capalictus, a new subgenus of Lasioglossum Curtis, 1833 from South Africa, with description of three new species (Hymenoptera, Apoidea, Halictidae). European Journal of Taxonomy 28: 1-28. https://doi.org/10.5852/ejt.2012.28

Pesenko Y.A. 2007. Subgeneric classification of the Palaearctic bees of the genus Evylaeus Robertson (Hymenoptera: Halictidae). Zootaxa 1500: 1-54. https://doi.org/10.11646/zootaxa.1500.1.1

Pesenko Y.A., Banaszak J., Radchenko V.G. \& Cierzniak T. 2000. Bees of the Family Halictidae (excluding Sphecodes) of Poland: Taxonomy, Ecology, Bionomics. Wydawnictwo Uczelniane Wyzszej Szkofy Pedagogicznej w Bydgoszczy, Bydgoszcz.

Phillips S.J., Dudík M. \& Schapire R.E. s.d. Maxent Software for Modeling Species Niches and Distributions. Ver. 3.4.1 [accessed 9 May 2020].

Phillips S.J., Anderson R.P. \& Schapire R.E. 2006. Maximum entropy modeling of species geographic distributions. Ecological Modelling 190: 231-259. https://doi.org/10.1016/j.ecolmodel.2005.03.026

Phillips S.J., Anderson R.P., Dudík M., Schapire R.E. \& Blair M.E. 2017. Opening the black box: an open-source release of Maxent. Ecography 40: 887-893. https://doi.org/10.1111/ecog.03049

Pierce M.P., Branstetter M.G. \& Longino J.T. 2017. Integrative taxonomy reveals multiple cryptic species within Central American Hylomyrma Forel, 1912 (Hymenoptera: Formicidae). Myrmecological News 25: 131-143. https://doi.org/10.25849/myrmecol.news_025:131

Plateaux-Quénu C. 1959. Un nouveau type de société d'insectes: Halictus marginatus Brullé (Hym., Apoidea). Année biologique 35: 325-444.

Portman Z.M., Brunninga-Socolar B. \& Cariveau D.P. 2020. The state of bee monitoring in the United States: a call to refocus away from bowl traps and towards more effective methods. Annals of the Entomological Society of America 113 (5): 1-6. https://doi.org/10.1093/aesa/saaa010

QGIS.org. 2020. QGIS Geographic Information System. Ver. 3.12. Open Source Geospatial Foundation Project, Beaverton, OR.

R Core Team. 2020. R: A Language and Environment for Statistical Computing. Ver. 4.0.0. R Foundation for Statistical Computing, Vienna, Austria.

Ratnasingham S. \& Hebert P.D.N. 2007. BOLD: the Barcode of Life Data System (www.barcodinglife.org). Molecular Ecology Notes 7 (3): 355-364. https://doi.org/10.1111/j.1471-8286.2007.01678.x

Ratnasingham S. \& Hebert P.D.N. 2013. A DNA-based registry for all animal species: the barcode index number (BIN) system. PLoS ONE 8 (7): e66213. https://doi.org/10.1371/journal.pone.0066213

Reilly J.R., Artz D.R., Biddinger D.J., Bobiwash K., Boyle N.K., Brittain C., Brokaw J., Campbell J.W., Daniels J., Elle E., Ellis J.D., Fleischer S.J., Gibbs J., Gillespie R.L., Gundersen K.B., Gut L., Hoffman G., Joshi N.K., Lundin O., Mason K., McGrady C.M., Peterson S.S., Pitts-Singer T.L., Rao S., Rothwell N., Rowe L., Ward K.L., Williams N.M., Wilson J.K., Isaacs R. \& Winfree R. 2020. Crop production in the USA is frequently limited by a lack of pollinators. Proceedings of the Royal Society B-Biological Sciences 287 (1931): e20200922. https://doi.org/10.1098/rspb.2020.0922

Rhoades P., Griswold T.L., Ikerd H., Waits L., Bosque-Pérez N.A. \& Eigenbrode S.D. 2017a. The native bee fauna of the Palouse Prairie (Hymenoptera: Apoidea). Journal of Melittology 66: 1-20.

https://doi.org/10.17161/jom.v0i66.5703

Rhoades P., Griswold T.L., Waits L., Bosque-Pérez N.A., Kennedy C.M. \& Eigenbrode S.D. 2017 b. Sampling technique affects detection of habitat factors influencing wild bee communities. Journal of Insect Conservation 21: 703-714. https://doi.org/10.1007/s10841-017-0013-0 
Richards M.H. 2000. Evidence for geographic variation in colony social organization in an obligately social sweat bee, Lasioglossum malachurum (Kirby) (Hymenoptera; Halictidae). Canadian Journal of Zoology 78: 1259-1266. https://doi.org/10.1139/z00-064

Robertson C. 1895. Notes on bees, with descriptions of new species. Transactions of the American Entomological Society 22 (2): 115-128.

Robertson C. 1897. North American bees-descriptions and synonyms. Transactions of the Academy of Science of St. Louis 7 (14): 315-356.

Robertson C. 1901. Some new or little-known bees. The Canadian Entomologist 33 (8): 229-231. https://doi.org/10.4039/Ent33229-8

Robertson C. 1902. Synopsis of Halictinae. The Canadian Entomologist 34 (9): 243-250.

https://doi.org/10.4039/Ent34243-9

Ronquist F., Teslenko M., Van Der Mark P., Ayres D.L., Darling A., Höhna S., Larget B., Liu L., Suchard M.A. \& Huelsenbeck J.P. 2012. MrBayes 3.2: efficient Bayesian phylogenetic inference and model choice across a large model space. Systematic Biology 61 (3): 539-542.

https://doi.org/10.1093/sysbio/sys029

Sakagami S.F. \& Munakata M. 1972. Distribution and bionomics of a transpalaearctic eusocial halictine bee, Lasioglossum (Evylaeus) calceatum, in northern Japan, with reference to its solitary life cycle at high altitude. Journal of the Faculty of Science, Hokkaido University, Series 6, Zoology 18 (3): 411-439.

Sakagami S.F., Laroca S. \& Moure J.S. 1967. Wild bee biocoenotics in São Jose dos Pinhais (PR), south Brazil. Preliminary report. Journal of the Faculty of Science, Hokkaido University, Series 6, Zoology 16: 253-291.

Sandhouse G.A. 1923. The bee-genus Dialictus. The Canadian Entomologist 55 (8): 193-195. https://doi.org/10.4039/Ent55193-8

Sandhouse G.A. 1924. New North American species of bees belonging to the genus Halictus (Chloralictus). Proceedings of the United States National Museum 65 (19): 1-43.

Schrottky C. 1911. Descripção de abelhas novas do Brazil e de regiões visinhas. Revista do Museu Paulista 8: 71-88.

Schwarz M.P., Richards M.H. \& Danforth B.N. 2007. Changing paradigms in insect social evolution: insights from halictine and allodapine bees. Annual Review of Entomology 52: 127-150.

https://doi.org/10.1146/annurev.ento.51.110104.150950

Scott V.L., Ascher J.S., Griswold T.L. \& Nufio C.R. 2011. The bees of Colorado. Natural History Inventory of Colorado 23: 1-100.

Smith B.A., Brown R.L., LaBerge W.E. \& Griswold T.L. 2012. A faunistic survey of bees (Hymenoptera: Apoidea) in the Black Belt Prairie of Mississippi. Journal of the Kansas Entomological Society 85 (1): 32-47. https://doi.org/10.2317/JKES111025.1

Smith F. 1853. Catalogue of Hymenopterous Insects in the Collection of the British Museum. Part I. Andrenidae and Apidae. Taylor and Francis, London.

Smyth B.B. 1897. Floral horologe for Kansas. In: Smyth B.B. (ed.) Transactions of the Annual Meetings of the Kansas Academy of Science: 106-108. JSTOR, Topeka, KS. https://doi.org/10.2307/3623709

Stockhammer K.A. 1967. Some notes on the biology of the blue sweat bee, Lasioglossum coeruleum (Apoidea: Halictidae). Journal of the Kansas Entomological Society 40: 177-189. 
Tepedino V.J., Bradley B.A. \& Griswold T.L. 2008. Might flowers of invasive plants increase native bee carrying capacity? Intimations from Capitol Reef National Park, Utah. Natural Areas Journal 28 (1): 44-50. https://doi.org/10.3375/0885-8608(2008)28\%5B44:MFOIPI\%5D2.0.CO;2

Toler T.R., Evans E.W. \& Tepedino V.J. 2005. Pan-trapping for bees (Hymenoptera: Apiformes) in Utah's West Desert: the importance of colour diversity. The Pan-Pacific Entomologist 81 (3/4): 103-113.

Tommasi D., Miro A., Higo H.A. \& Winston M.L. 2004. Bee diversity and abundance in an urban setting. The Canadian Entomologist 136 (6): 851-869. https://doi.org/10.4039/n04-010

van der Maaten L.J.P. 2014. Accelerating t-SNE using tree-based algorithms. Journal of Machine Learning Research 15: 3221-3245.

van der Maaten L.J.P. \& Hinton G.E. 2008. Visualizing high-dDimensional data using t-SNE. Journal of Machine Learning Research 9: 2579-2605.

Venables W.N. \& Ripley B.D. 2002. Modern Applied Statistics with S. Springer, New York.

Viereck H.L., MacGillivray A.D., Brues C.T., Wheeler W.M. \& Rohwer S.A. 1916. The Hymenoptera, or wasp-like insects, of Connecticut. In: Britton W.E. (ed.) Guide to the Insects of Connecticut: 824. Connecticut Geological and Natural History Survey, Hartford, CT.

Walker K. 1995. Revision of the Australian native bee subgenus Lasioglossum (Chilalictus) (Hymenoptera: Halictidae). Memoirs of the Museum of Victoria 55 (1-2): 1-423.

https://doi.org/10.24199/j.mmv.1995.55.01

Warncke K. 1975. Beiträge zur Systematik und Verbreitung der Furchenbienen in der Türkei (Hymenoptera, Apoidea, Halictus). Polskie Pismo Entomologiczne 45: 81-123.

Wcislo W.T. 1997. Invasion of nests of Lasioglossum imitatum by a social parasite, Paralictus asteris (Hymenoptera: Halictidae). Ethology 103: 1-11.

Webb D.W. 1980. Primary insect types in the Illinois Natural History Survey Collection, exclusive of the Collembola and Thysanoptera. Illinois Natural History Survey Bulletin 32 (2): 51-191.

WFO. 2020. World Flora Online. Available from http://www.worldfloraonline.org [accessed 9 May 2020].

Wiley E.O. \& Mayden R.L. 2000. The evolutionary species concept. In: Wheeler Q.D. \& Meier R. (eds) Species Concepts and Phylogenetic Theory: A Debate: 70-89. Columbia University Press, New York.

Wille A. \& Orozco E. 1970. The life cycle and behavior of the social bee Lasioglossum (Dialictus) umbripenne (Hymenoptera: Halictidae). Revista de Biologia Tropical 17 (2): 199-245.

Williams P. H., Brown M.J.F., Carolan J.C., An J., Goulson D., Aytekin A.M., Best L.R., Byvaltsev A.M., Cederberg B., Dawson R., Huang J., Ito M., Monfared A., Raina R.H., Schmid-Hempel P., Sheffield C.S., Šima P. \& Xie Z. 2012. Unveiling cryptic species of the bumblebee subgenus Bombus s. str. worldwide with COI barcodes (Hymenoptera: Apidae). Systematics and Biodiversity 10 (1): 21-56. https://doi.org/10.1080/14772000.2012.664574

Wilson J.S., Kelly M. \& Messinger-Carril O. 2018. Reducing protected lands in a hotspot of bee biodiversity: bees of Grand Staircase-Escalante National Monument. PeerJ 6: e6057. https://doi.org/10.7717/peerj.6057

Wolf A.T. \& Ascher J.S. 2009. Bees of Wisconsin (Hymenoptera: Apoidea: Anthophila). Great Lakes Entomologist 41: 129-168. 
Manuscript received: 20 May 2020

Manuscript accepted: 16 September 2020

Published on: 27 November 2020

Topic editor: Nesrine Akkari

Section editor: Gavin Broad

Desk editor: Kristiaan Hoedemakers

Printed versions of all papers are also deposited in the libraries of the institutes that are members of the EJT consortium: Muséum national d'histoire naturelle, Paris, France; Meise Botanic Garden, Belgium; Royal Museum for Central Africa, Tervuren, Belgium; Royal Belgian Institute of Natural Sciences, Brussels, Belgium; Natural History Museum of Denmark, Copenhagen, Denmark; Naturalis Biodiversity Center, Leiden, the Netherlands; Museo Nacional de Ciencias Naturales-CSIC, Madrid, Spain; Real Jardín Botánico de Madrid CSIC, Spain; Zoological Research Museum Alexander Koenig, Bonn, Germany; National Museum, Prague, Czech Republic.

\section{Supplementary material}

Supplementary file 1: Table showing the results of jackknife leave-one-out cross-validation on group predictions by linear discriminant analysis of morphometric data for females. Actual species are presented in rows, and predicted species membership is in columns. The last column is the percentage of correct predictions for each species. https://doi.org/10.5852/ejt.2020.725.1167.3201

Supplementary file 2: Table showing the results of jackknife leave-one-out cross-validation on group predictions by linear discriminant analysis of morphometric data for males. Actual species are presented in rows, and predicted species membership is in columns. The last column is the percentage of correct predictions for each species. Species for which only one male could be measured could not be assessed. https://doi.org/10.5852/ejt.2020.725.1167.3203

Supplementary file 3: Table showing the clusters assigned by PAM using qualitative and quantitative morphometric data. Actual species are presented in rows, and cluster assignments are in columns. The last column is the percentage of correct assignments for each species. Male Lasioglossum perditum were split into two clusters, resulting in no cluster corresponding to L. clavicorne. The two male L. perditum clusters were merged in this table. https://doi.org/10.5852/ejt.2020.725.1167.3205

Supplementary file 4: Available 654-bp DNA barcodes of western red-tailed Lasioglossum (Dialictus) species, plus L. semibrunneum for comparison with L. kunzei. Species with multiple haplotypes were merged into a single sequence with variable sites treated as ambiguities. Nucleotides diagnostic across all species are shaded black; nucleotides diagnostic within particular groups are outlined.

https://doi.org/10.5852/ejt.2020.725.1167.3207 